\title{
Topics on Lie pseudogroups
}

Pfaffian groups, Haefliger's cohomology and natural bundles 


\section{Thesis committee:}

Prof. dr. Christian Blohmann (Max Planck Institute for Mathematics, Germany)

Prof. dr. Ieke Moerdijk (Utrecht University, The Netherlands)

Prof. dr. Janez Mrčun (Univerza v Ljubljani, Slovenia)

Prof. dr. Xiang Tang (Washington University in St. Louis, USA)

Prof. dr. Chenchang Zhu (Georg-August-Universität Göttingen, Germany)

ISBN: 978-90-393-7399-6

Printed by: Ridderprint BV, www.ridderprint.nI 


\section{Topics on Lie pseudogroups}

Pfaffian groups, Haefliger's cohomology and natural bundles

\section{Onderwerpen over Lie pseudogroepen}

Pfaffiaanse groepen, Haefligercohomologie en natuurlijke bundels

(met een samenvatting in het Nederlands)

\section{Proefschrift}

ter verkrijging van de graad van doctor aan de Universiteit Utrecht op gezag van de rector magnificus, prof. dr. H. R. B. M. Kummeling, ingevolge het besluit van het college voor promoties in het openbaar te verdedigen op woensdag 29 september 2021 des middags te 12.15 uur

$$
\text { door }
$$

\section{Luca Accornero}

geboren op 13 mei 1993 te Vercelli, Italië 
Promotor: Prof. dr. M. N. Crainic

This thesis was made possible with financial support from the Nederlandse Organisatie voor Wetenschappelijk Onderzoek through the grant for the graduate program "Utrecht Geometry Centre" with number 022.006.004. Moreover, partial support through the VICI grant 639.033 .312 was also received. 
Ai miei nonni. 

Heráclito no sabe griego.

- Jorge Luis Borges, Heráclito 



\section{Contents}

1 A Pfaffian look at transitive pseudogroups 21

1.1 Preliminaries and motivation: transitive geometries . . . . . . . . . 21

1.2 The Pfaffian language . . . . . . . . . . . . . . . . . . . . . . . . 42

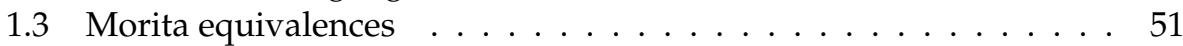

1.4 Cartan bundles . . . . . . . . . . . . . . . . . 63

2 Haefliger's differentiable cohomology 71

2.1 Introduction . . . . . . . . . . . . . . . 71

2.2 Overview of various characteristic classes . . . . . . . . . . . . . . 74

2.3 Haefliger's differentiable cohomology . . . . . . . . . . . . . . . . . . . . . . 94

2.4 Generalization: Haefliger cohomology . . . . . . . . . . . . . . . 106

2.5 The infinitesimal version of Haefliger cohomology . . . . . . . . . . . 112

2.6 Van Est maps . . . . . . . . . . . . . . . . . . . . . . . . . 124

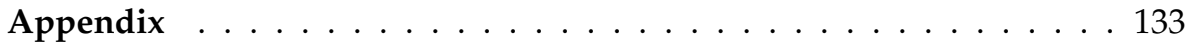

2.A Pro-finite manifolds . . . . . . . . . . . . . . . . 133

3 On regular actions of pseudogroups 153

3.1 Introduction . . . . . . . . . . . . . . . . 153

3.2 Pseudogroups, actions and natural bundles . . . . . . . . . . . 156

3.3 Smooth supports for étale groupoids. Lie algebra sheaves. . . . . . 179

3.4 Cartan resolutions of étale groupoids . . . . . . . . . . . . . . . . . . . . . . . . 189

3.5 Finite order problem à la Palais-Terng . . . . . . . . . . . . . . . . 206

Appendix . . . . . . . . . . . . . . . . . . . 218

3.A Some words on profinite dimensional differential geometry . . . . 218

3.B Pfaffian groupoids and their prolongations . . . . . . . . . . 226

3.C Basics on diffeologies . . . . . . . . . . . . . . . . . 232

$\begin{array}{ll}\text { Bibliography } & 239\end{array}$

$\begin{array}{ll}\text { Summary } & 245\end{array}$

$\begin{array}{ll}\text { Samenvatting } & 248\end{array}$

$\begin{array}{lr}\text { Acknowledgments } & 252\end{array}$ 


\section{Curriculum Vitae}




\title{
Introduction
}

\author{
1) Lasciate ogne speranza, voi ch'intrate.
}

— Dante Alighieri, Inferno, Canto III

This thesis deals with Lie pseudogroups and their differential geometry. Lie pseudogroups were introduced as "continuous transformation groups" by Sophus Lie and Friedrich Engels [44] at the end of the nineteenth century. Loosely speaking, a Lie pseudogroup on a manifold is a set of local transformations of the manifold itself, possessing group-like and sheaf-like properties and arising as the set of local solutions of some well behaved system of PDEs. Remarkable examples of Lie pseudogroups are given by sets of local symmetries of geometric structures and differential equations.

The thesis is divided into three chapters; each chapter is concerned with a specific topic and is written to be self contained and completely independent from the other two. Before going through the content of the chapters, we overview some of the common themes and concepts.

\section{What this thesis is about}

First of all, let us give the precise definition of pseudogroup, which is at the core of our work.

Definition. Let $\mathbf{X}$ be a smooth manifold and $\operatorname{Diff}_{\text {loc }}(\mathbf{X})$ be the set of locally defined diffeomorphisms of $\mathbf{X}$. A pseudogroup $\Gamma$ over $\mathbf{X}$ is a subset $\Gamma \subset \operatorname{Diff}_{\text {loc }}(\mathbf{X})$ such that

- $\Gamma$ contains the identity and is closed under inversion and composition of transformations;

- $\Gamma$ is closed under restriction and gluing of transformations.

By "closed under gluing of transformations" we mean that if $\varphi: U \subset \mathbf{X} \rightarrow$ $V \subset \mathbf{X}$ is a local transformation of $\mathbf{X}$ and there is a cover $\left\{U_{i}\right\}_{i \in I}$ of $U$ such that $\left.\varphi\right|_{U_{i}} \in \Gamma$, then $\varphi \in \Gamma$.

The class of examples we are mostly interested in is that of sets of symmetries. 
Example. Let us consider the euclidean space $\mathbb{R}^{2 k}, k \in \mathbb{N}$, with coordinates $\left(x_{1}, \ldots, x_{k}, y_{1}, \ldots, y_{k}\right)$. Let

$$
\omega_{\text {std }}=\sum_{i=1}^{k} d x_{i} \wedge d y_{i}
$$

be the canonical symplectic form on $\mathbb{R}^{2 k}$. A locally defined symplectomorphism of $\left(\mathbb{R}^{2 k}, \omega_{\text {std }}\right)$ is a local transformation $\varphi: U \rightarrow \phi(U)$, where $U \subset \mathbb{R}^{2 k}$ is an open subset, such that

$$
\varphi^{*}\left(\omega_{\text {std }}\right)=\omega_{\text {std }} .
$$

The set $\Gamma_{\mathrm{sp}}$ of locally defined symplectomorphisms of $\left(\mathbb{R}^{2 k}, \omega_{\mathrm{std}}\right)$ is a pseudogroup on $\mathbb{R}^{2 k}$. Observe that the fact that the identity

$$
\varphi^{*}\left(\omega_{\text {std }}\right)=\omega_{\text {std }}
$$

holds means precisely that $\varphi$ is a solution of a certain system of PDEs; $\Gamma_{\mathrm{sp}}$ can be equivalently described as the set of solutions of that system.

In the main body of the thesis we will present several examples of pseudogroups arising as set of symmetries of geometric structures on $\mathbb{R}^{n}$.

More in general, we care about pseudogroups that can be presented as set of solutions of systems of PDEs. Geometrically, this can be encoded as follows. When $\Gamma$ is a pseudogroup over $\mathbf{X}$, we can consider, for all $k \in \mathbb{N}$, the set $J^{k} \Gamma$ of $k$-jets of elements in $\Gamma$. The set $J^{k} \Gamma$ is a subset of $J^{k}(\mathbf{X}, \mathbf{X})$, the space of $k$-jets of maps from $\mathbf{X}$ to $\mathbf{X}$. There is a natural smooth structure on $J^{k}(\mathbf{X}, \mathbf{X})$; moreover, the canonical projections $J^{k}(\mathbf{X}, \mathbf{X}) \rightarrow J^{k-1}(\mathbf{X}, \mathbf{X})$, for $k \geq 1$, are surjective submersions. The important observation is that a submanifold $R \subset J^{k}(\mathbf{X}, \mathbf{X})$ can be seen as a geometric way for encoding a system of PDEs; the solutions of such a system are maps $\sigma: U \subset \mathbf{X} \rightarrow \mathbf{X}$ whose $k$-jet takes value in $R$.

Definition. A Lie pseudogroup over $\mathbf{X}$ is a pseudogroup $\Gamma$ over $\mathbf{X}$ such that for all $k \in \mathbb{N}$ the jet space $J^{k} \Gamma$ is an embedded submanifold of $J^{k}(\mathbf{X}, \mathbf{X})$ and the projection $J^{k} \Gamma \rightarrow J^{k-1} \Gamma$ is a surjective submersion.

The intuition behind the definition above is that a Lie pseudogroup can be described as the set of solutions of the system of PDEs corresponding to the embeddings $J^{k} \Gamma \hookrightarrow J^{k}(\mathbf{X}, \mathbf{X})$.

\section{The tools: Lie groupoids and multiplicative forms}

In [44], Lie and Engel focused mainly on the subclass of finite type Lie pseudogroups, see e.g. [69]. Their work can be seen as the origin of the modern notion of Lie group. Later on, Élie Cartan published two papers [13, 14] presenting a structure theory for Lie pseudogroups in full generality; his work contains great intuitions and underlies most of the later developments. See [69] for a modern formulation of Cartan's work.

The framework that we have chosen to deal with pseudogroups has been developed fairly recently $[15,55,69]$. The fundamental objects are Lie groupoids. When $\Gamma$ is a pseudogroup over $\mathbf{X}$ and $k \in \mathbb{N}$, the jet space $J^{k} \Gamma$ is a groupoid over 
$\mathbf{X}$ - the groupoid structure comes from the group-like properties of $\Gamma$. If $\Gamma$ is a Lie pseudogroup, $J^{k} \Gamma$ is also a submanifold of $J^{k}(\mathbf{X}, \mathbf{X})$ and, in fact, a Lie groupoid over X.

The key observation is that, for all $k \in \mathbb{N}, J^{k} \Gamma$ comes equipped with a canonical differential form, the so called Cartan form, denoted by $\omega^{k}$, which takes values in the vertical bundle of the projection $J^{k-1} \Gamma \rightarrow \mathbf{X}$ sending $j_{x}^{k-1} \varphi \in J^{k-1} \Gamma$ to $x \in \mathbf{X}$. Roughly speaking, the Cartan forms on the jet spaces $J^{k} \Gamma, k \in \mathbb{N}$, allow us to capture and describe geometrically the "PDE nature" of $\Gamma$. In particular, an element of $\Gamma$ induces, for each $k \in \mathbb{N}$, a section of the canonical projection

$$
J^{k} \Gamma \rightarrow \mathbf{X}, \quad j_{x}^{k} \varphi \rightarrow x,
$$

and this section has image tangent to the distribution $\operatorname{Ker}\left(\omega^{k}\right)$. Conversely, under reasonable assumptions, elements of $\Gamma$ are characterized by the fact that, for each $k \in \mathbb{N}$, the induced section of the projection $J^{k} \Gamma \rightarrow \mathbf{X}$ has image tangent to $\operatorname{Ker}\left(\omega^{k}\right)$. Furthermore, the Cartan forms $\omega^{k \prime}$ s are compatible with the group-like structure on $\Gamma$, in the sense that each $\omega^{k}$ is a multiplicative form on the groupoid $J^{k} \Gamma \rightrightarrows \mathbf{X}$; see [55, 69]. Lie groupoids equipped with multiplicative forms possessing some additional properties - called Pfaffian forms [55] - provide an abstract setting that is well suited for studying Lie pseudogroups. This setting is, in fact, a modern and quite natural evolution of Cartan's approach from [13, 14], as explained in [69].

\section{Geometric structures on manifolds via Lie pseudogroups}

One of the driving motivations for studying Lie pseudogroups is that there is a nice way of describing geometric structures on $n$-dimensional differentiable manifolds in terms of Lie pseudogroups on $\mathbb{R}^{n}$. The idea is rather simple: given a manifold $M$, one can consider atlases on $M$ such that the changes of coordinates belong to some pseudogroup $\Gamma$ on $\mathbb{R}^{n}$. An atlas with such a property is called a $\Gamma$-atlas; a maximal $\Gamma$-atlas on $M$ is called a $\Gamma$-structure on $M$. As the example below makes clear, $\Gamma$-structures on manifolds encode geometric structures.

Example. Let be $k \in \mathbb{N}$ and let $M$ be a $2 k$-dimensional manifold. Let us assume that $M$ admits a $\Gamma_{\mathrm{sp}}$-atlas (see the example above) denoted by $\left\{\left(U_{i}, \phi_{i}\right)\right\}_{i \in I}$. That is, $\left\{U_{i}\right\}_{i \in I}$ is a cover of $M$, the maps $\phi_{i}: U_{i} \rightarrow \mathbb{R}^{2 k}$ are charts on $M$ and the changes of coordinates $\varphi_{i j}$, for $i, j \in I$, are locally defined symplectomorphisms of $\left(\mathbb{R}^{2 k}, \omega_{\text {std }}\right)$.

The $\Gamma_{\mathrm{sp}}$-atlas $\left\{\left(U_{i}, \phi_{i}\right)\right\}_{i \in I}$ endows $M$ with the structure of symplectic manifold. In fact, for all $i \in I$, the pullback $\phi_{i}^{*}\left(\omega_{\text {std }}\right)$ is a symplectic form on the open set $U_{i} \subset M$. Since the change of coordinates $\varphi_{i j}$ is a symplectomorphism, $\phi_{i}^{*}\left(\omega_{\text {std }}\right)$ and $\phi_{j}^{*}\left(\omega_{\text {std }}\right)$ agree on $U_{i} \cap U_{j}$ and can be "glued" together. In other words, the collection $\left\{\phi_{i}^{*}\left(\omega_{\text {std }}\right)\right\}$ is given by the restrictions $\left.\omega\right|_{U_{i}}$ of a symplectic form $\omega$ on $M$.

There are several other remarkable examples of geometric structures that can be described as $\Gamma$-structures - e.g. flat Riemannian metrics, complex structures, foliations, contact structures. The recipe is always the same: one considers the 
"Euclidean" version of the structure on $\mathbb{R}^{n}$ and the induced pseudogroup of symmetries $\Gamma$. Reasoning as in the example above, a $\Gamma$-atlas on a manifold $M$ can be used to "pull-back" the structure from $\mathbb{R}^{n}$ to $M$.

More in general, one can require a manifold $M$ to admit an "order $k$ formal $\Gamma$-atlas"; that is, an ordinary atlas of $M$ where each change of coordinates is paired with a section of the $k$-jet space $J^{k} \Gamma$. Several geometric structures can be described via this kind of atlases - for instance: Riemannian metrics, almost complex structures and distributions. Intuitively, structures arising in this way are "curved versions" of the corresponding $\Gamma$-structures. They are called almost $\Gamma$-structures [15].

The groupoid perspective allows to describe $\Gamma$-structures and almost $\Gamma$ structures in an elegant and efficient way, extensively discussed in [15]. The key tool is given by principal groupoid bundles. In particular, $\Gamma$-structures on a manifold $M$ can be described as principal $\operatorname{Germ}(\Gamma)$-bundles over $M$ such that the map along which $\operatorname{Germ}(\Gamma)$ acts is étale; here, $\operatorname{Germ}(\Gamma)$ denotes the groupoid of germs of elements of $\Gamma$. Moreover, almost $\Gamma$-structures on $M$ can be described as principal $J^{k} \Gamma$-bundles over $M$ with some additional properties.

This principal bundle description of geometric structures, and, more in general, the study of actions of the groupoids induced by a pseudogroup, play a fundamental role in our work.

\section{The content of this thesis}

As we already mentioned, the three chapters of the thesis are written to be self contained and completely independent from each other. That said, we point out that the first section from Chapter 1 provides motivation and context that are relevant for the whole thesis. A similar remark could be made about Section 1.2: Pfaffian groupoids and principal Pfaffian bundles appear, explicitly or implicitly, throughout the thesis.

As a consequence of the fact that the chapters are self contained, some minor repetitions appear. This happens mainly with the background material concerning pseudogroups and groupoids - see Section 1.1, Section 2.3 and Section 3.2 and with the appendices concerning pf-manifolds - see Appendix 2.A and Appendix 3.A.

\section{Chapter 1}

Chapter 1 is joint work in progress with Francesco Cattafi. Our starting point is the description of geometric structures on differentiable manifolds in terms of Lie pseudogroups. Throughout the chapter, we focus on the case when the Lie pseudogroup is transitive - see Definition 1.1.3. In the literature, structures associated to transitive pseudogroups have been encoded into principal group bundles equipped with some additional object - a distribution/one form interacting nicely with the group action, e.g. [58, 64]. On the other hand, in [15], Pfaffian groupoids and principal Pfaffian bundles (Definitions 1.2.1 and 1.2.22) were shown to be a suitable framework to describe geometric structures associated to any Lie pseudogroup - being it transitive or not. We explore how the 
classical group picture is recovered from the groupoid one when the pseudogroup is transitive. We also investigate whether the groupoid approach could lead to additional insights. Part of this program was started in [16]. The approach that we provide here is complementary. In particular, we make use of the notion of Pfaffian Morita equivalence from [15].

In the first section, we recall the definitions of Lie pseudogroups and $\Gamma$ structures and provide various examples. We encode $\Gamma$-structures in terms of principal groupoid bundles, describe the groupoid structure on $J^{k} \Gamma$, and define almost $\Gamma$-structures in terms of principal $J^{k} \Gamma$-bundles, see Definition 1.1.28. We also recall the definition of the Cartan distribution on jet spaces - see Subsection 1.1.4.

In the second section, we introduce Pfaffian groupoids, Definitions 1.2.1 and 1.2.10. These are triples $(\Sigma, \omega, E)$ consisting of a Lie groupoid $\Sigma$, a representation $E$ and an $E$-valued one-form $\omega$ on $\Sigma$ that interacts nicely with the groupoid structure. The main example that we have in mind are the jet groupoids of Lie pseudogroups together with their Cartan form. We distinguish the important class of full Pfaffian groupoids - that is, Pfaffian groupoids $(\Sigma, \omega, E)$ such that $\omega$ is pointwise surjective. We carefully discuss also the example of Pfaffian groups which one encounters, for instance, by looking at the isotropy groups of Pfaffian groupoids, see Propositions 1.2.17 and 1.2.20. We introduce principal Pfaffian bundles [15]; they provide an abstract framework to discuss almost $\Gamma$-structures and pave the way for the introduction of the fundamental object in the chapter, principal Pfaffian bibundles.

In the third section we move to Pfaffian Morita equivalences, see Definition 1.3.1, that we encode in terms of principal Pfaffian bibundles, see Definition 1.3.5. The definition of Pfaffian Morita equivalence appeared first in [15]. We show that principal Pfaffian bundles of Pfaffian Morita equivalent groupoids are in one to one correspondence, up to isomorphisms, see Proposition 1.3.12. We show that transitive Pfaffian groupoids are Pfaffian Morita equivalent to their Pfaffian isotropy groups, see Proposition 1.3.15. We then discuss principal Pfaffian bundles for Pfaffian groups, that we call Cartan bundles, see Definition 1.3.19. Cartan bundles were already introduced, following a different approach, in [16]. By combining Propositions 1.3.12 and 1.3.15, we see that studying Cartan bundles is equivalent to studying principal Pfaffian bundles for transitive Pfaffian groupoids. In particular if $\Gamma$ is a transitive Lie pseudogroup, almost $\Gamma$-structures can be encoded into Cartan bundles.

In the fourth section, we provide some remarkable examples of Cartan bundles. We then analyze the case of flat Cartan bundles - which correspond to equivariant vertical Lie foliations on principal group bundles. Since Cartan bundles over $M$ are an abstract description of almost $\Gamma$-structures on $M$ in terms of principal group bundles, an important question to ask is how to encode integrability - i.e. how to describe the subclass of Cartan bundles that correspond to $\Gamma$-structures. It is still not completely clear to us how to do this in a way that fully takes into account the Pfaffian Morita equivalence between the jet groupoid of a transitive Lie pseudogroup and its Pfaffian isotropy Lie group. However, we propose an ad hoc approach that reproduces the expected outcome when tested on our main examples of Cartan bundles.

Here's a summary of the main contributions in Chapter 1. 
1. A new formulation of the definition of Pfaffian Morita equivalence from [15], see Definition 1.3.7.

2. A proof of the fact that if two Pfaffian groupoids are Pfaffian Morita equivalent, then their principal Pfaffian bundles are in one-to-one correspondence (up to isomorphisms), see Proposition 1.3.12.

3. A proof of the fact that Pfaffian Morita equivalent Pfaffian groupoids have Pfaffian isomorphic isotropy groups and representations, see Proposition 1.3.10.

4. A new approach to Cartan bundles [16] that makes use of Pfaffian Morita equivalences. A byproduct of this approach is that it becomes clear why studying Cartan bundles is equivalent to studying principal Pfaffian bundles of transitive Pfaffian groupoids.

5. A discussion of the relationship between Cartan bundles and equivariant Lie-foliations on principal bundles, see Section 1.4.

\section{Chapter 2}

Chapter 2, whose content is available on the arXiv [1], is joint work with Marius Crainic and deals with the construction of a "geometric" /Chern-Weil like characteristic map for geometric structures on manifolds. Our starting motivation was Haefliger's work on the "geometric" characteristic map for foliations $[6,7,11$, 35]. Haefliger constructed a differentiable cohomology for the set $\operatorname{Diff}_{\text {loc }}\left(\mathbb{R}^{q}\right)$ of locally defined diffeomorphisms of $\mathbb{R}^{q}$; furthermore, he described the "geometric" characteristic map for foliations as a map defined on such a cohomology [35].

We spend the second section recalling the construction of the characteristic map for foliations $[6,7]$ and pointing out the similarity with the theory of characteristic classes of principal bundles.

In the third section, we reinterpret Haefliger's differentiable cohomology in terms of the groupoid of infinite jets of elements of Diff ${ }_{\text {loc }}\left(\mathbb{R}^{q}\right)$. This reinterpretation allows to extend the definition to Lie pseudogroups, see equation (2.14) and the discussion below. We start by formulating a series of properties that a Haefliger-like cohomology on a Lie groupoid $\Sigma \rightrightarrows \mathbf{X}$ should have; then we prove that the necessary and sufficient structure needed to define such a cohomology is a flat multiplicative connection on $\Sigma$, see Definition 2.4.1.

The fourth section focuses on Lie groupoids $\Sigma \rightrightarrows \mathbf{X}$ possessing a flat multiplicative connection $\mathcal{C}$. Haefliger's cohomology is presented in this generality, see Definition 2.4.3. We then discuss principal $\Sigma$-bundles $P \rightarrow M$ possessing an Ehresmann connection $\mathcal{C}_{P}$ compatible with $\mathcal{C}$, i.e. multiplicative; see Definition 2.4.11. These are called flat $(\Sigma, \mathcal{C})$-structures. When $\Sigma$ is the infinite jet groupoid of a Lie pseudogroup, one recovers formally integrable "almost structures" [15], see Definition 2.4.16.

In the fifth section we discuss the infinitesimal counterpart of flat multiplicative connections on Lie groupoids, which are, by Theorem 1 in [23], flat infinitesimally multiplicative connections on Lie algebroids, see equation (2.57). A Lie algebroid $A \rightarrow \mathbf{X}$ equipped with such a connection $\nabla$ possesses an infinitesimal 
version of Haefliger cohomology. We spend some time discussing a nice characterization of flat infinitesimal multiplicative connections $\nabla$ on $A \rightarrow \mathbf{X}$ as Lie algebroid structures on $A \oplus T \mathbf{X}$. When $\nabla$ is interpreted in this way, the infinitesimal Haefliger cohomology of $(A, \nabla)$ becomes the Lie algebroid cohomology of $A \oplus T \mathbf{X}$.

In the sixth section, we construct a van Est-like map from the Haefliger cohomology of a Lie groupoid $\Sigma \rightrightarrows \mathbf{X}$ equipped with a flat multiplicative connection $\mathcal{C}$ to the infinitesimal Haefliger cohomology of its Lie algebroid equipped with the infinitesimal counterpart of $\mathcal{C}$. When $\Sigma$ is transitive, we prove a van Est-like isomorphism between the Haefliger cohomology of $\Sigma$ and the Lie algebra cohomology of the isotropy Lie algebra of $A \oplus T \mathbf{X}$. Furthermore, we construct a characteristic map for a flat- $(\Sigma, \mathcal{C})$-structure. When dealing with Lie pseudogroups, this map defines cohomological invariants for almost structures. In the special case of Diff ${ }_{\text {loc }}\left(\mathbb{R}^{q}\right)$, we recover the "geometric" characteristic map for foliations.

Throughout the chapter, the language of pro-finite manifolds is needed to deal with groupoids of infinite jets, which are, in general, infinite dimensional. We provide a detailed appendix introducing several fundamental constructions and proving various lemmata that are used in the main body.

Here is a summary of the main results and contributions in Chapter 2.

1. A novel definition - and a generalization to Lie pseudogroups - of the differentiable cohomology of $\operatorname{Diff}_{\text {loc }}\left(\mathbb{R}^{q}\right)$ introduced by Haefliger in [35]; see Definition 2.3.1.

2. A proof of the fact that a Haefliger-like bicomplex (2.36) on a Lie groupoid can be defined if and only if the Lie groupoid is equipped with a flat multiplicative connection.

3. An infinitesimal characterization of principal groupoid bundles equipped with a flat multiplicative connection in terms of a Maurer-Cartan equation, Proposition 2.5.18.

4. The construction of a characteristic map for principal groupoid bundles equipped with a flat multiplicative connection, see equation (2.63). In particular, we get a characteristic map for formally integrable "almost structures", see Proposition 2.6.3.

5. The construction of a van Est map for Haefliger cohomology, Proposition 2.6.5, and of a van Est isomorphism between the Haefliger cohomology of a transitive groupoid with a flat Cartan connection and the Lie algebra cohomology of its extended isotropy Lie algebra, Theorem 2.6.7.

6. A novel construction of the characteristic map for geometric structures from [11], obtained by combining our characteristic map for almost structures with our van Est isomorphism - see equation (2.67) and the discussion following it.

\section{Chapter 3}

In Chapter 3 we investigate the finite order problem for actions of pseudogroups. Our motivation is the finite order theorem for natural bundles by Palais and 
Terng [54] and Epstein and Thurston [28]. A natural bundle [53] is a functor $E$ from the category where the objects are $n$-dimensional manifolds and the arrows are open embeddings to the category of surjective submersions over $n$-dimensional manifolds, such that $E$ is a section of the base functor. The finite order theorem states that, if $E$ is a natural bundle and $\phi: M_{1} \rightarrow M_{2}$ is an open embedding between $n$-dimensional manifolds, the map

$$
E(\phi)_{x}:\left(E M_{1}\right)_{x} \rightarrow\left(E M_{2}\right)_{\phi(x)}, \quad x \in M_{1},
$$

depends only on the $k$-jet of $\phi$ at $x$, for some integer $k \in \mathbb{N}$ called the order of $E$. If one chooses a pseudogroup $\Gamma$ over $\mathbf{X}$, one can look at the category where objects are $n$-dimensional manifolds possessing a $\Gamma$-structure and arrows are given by open embeddings compatible with such a structure. Our aim is to investigate under which conditions a finite order theorem holds in this setting.

In the second section we give the precise definition of pseudogroup $\Gamma$ over a manifold $\mathbf{X}$, see Definition 3.2.1, and introduce the groupoid of germs $\operatorname{Germ}(\Gamma) \rightrightarrows \mathbf{X}$ of a pseudogroup. We recall Haefliger's correspondence [36] between pseudogroups $\Gamma$ over a manifold $\mathbf{X}$ and effective étale groupoids $\mathcal{G} \rightrightarrows \mathbf{X}$, which sends a pseudogroup $\Gamma$ to its germ groupoid. We introduce Lie pseudogroups, see Definition 3.2.17, and their canonical Cartan tower. We make use of Morita equivalences of groupoids to discuss the correspondence between actions of the germ groupoid of a pseudogroup $\Gamma$ - i.e. $\Gamma$-spaces $-T$ discussion following and natural $\Gamma$-bundles, Proposition 3.2.45. A natural $\Gamma$-bundle [5] is a functor $E$

- from the category of $n$-dimensional manifolds equipped with a $\Gamma$-structure, see Definition 3.2.28;

- to the category of surjective submersions over manifolds equipped with a $\Gamma$-structure;

which is a section of the base functor. We formulate the finite order problem for natural $\Gamma$-bundles in terms of actions of germ groupoids, see Question 1 on page 163. Finally, we extend our approach to generalized pseudogroups. Loosely speaking, if $\Sigma \rightrightarrows \mathbf{X}$ is a Lie groupoid and $\operatorname{Bisloc}_{\text {loc }}(\Sigma)$ is its set of locally defined bisections, a generalized pseudogroup $\Gamma$ supported by $\Sigma \rightrightarrows \mathbf{X}$ is a subset of the set $\operatorname{Bis}_{\text {loc }}(\Sigma)$ that satisfies pseudogroup-like properties; see Definition 3.2.51.

In the third section we introduce the support of an étale groupoid $\mathcal{G} \rightrightarrows \mathbf{X}$, see Definition 3.3.1. We explain how a support induces a diffeology on $\mathcal{G}$ and we introduce the notion of $C^{\infty}$-convergence on an étale groupoid with support, see Definition 3.4.15.

In the fourth section we introduce Cartan resolutions of étale groupoids, see Definition 3.4.5, modelled on the canonical Cartan tower of a Lie pseudogroup. Given an étale groupoid $\mathcal{G} \rightrightarrows \mathbf{X}$ and a resolution, we introduce two topologies on $\mathcal{G}$ induced by the resolution, Definitions 3.4.17 and 3.4.20, which are useful for a conceptual discussion of the finite order problem.

In the fifth section we formulate a finite order theorem for $\Gamma$-spaces, Theorem 3.5.2. We clearly state the key assumptions needed for the theorem to hold, which can roughly be divided in 
- assumptions on $\Gamma$ - three of topological nature and one of algebraic nature;

- assumptions on the $\Gamma$-space $E$ - namely $E$ is required to be Hausdorff and the map along which $\operatorname{Germ}(\Gamma)$ acts is required to be a surjective submersion;

- a further assumption on the action of $\Gamma$ on $E$, called regularity, which encompasses a continuity request and a smoothness request - see Definition 3.5.8.

We then proceed to give a proof of Theorem 3.5.2 that follows closely the arguments for $\Gamma^{n}$ in [54]. While doing so we also reinterpret conceptually some of the key lemmata and propositions from [54], explaining how they fit into our theoretical framework.

In Appendix 3.A (which is basically a shortened version of Appendix 2.A), we recall some fundamental concepts concerning pf-manifolds. In Appendix 3.B, we briefly discuss Pfaffian groupoids and Lie prolongations of Pfaffian groupoids - which are extensively used in the main body of the chapter. Finally, in Appendix 3.C we quickly go through the basics on diffeologies.

Here is a summary of the main contributions in Chapter 3.

1. A formulation of the classical finite order problem [28, 54, 65] (see also the more recent discussion [40]) in terms of actions of the germ groupoid $\operatorname{Germ}\left(\Gamma^{n}\right)$ and a generalization of the problem to (generalized) pseudogroups and actions of étale groupoids, see Section 3.2.

2. Inspired by Haefliger's correspondence, Proposition 3.2.8, and by the definition of generalized pseudogroup, we define supports $\Sigma \rightrightarrows \mathbf{X}$ of étale groupoid $\mathcal{G} \rightrightarrows \mathbf{X}$, see Definition 3.3.1. We introduce two natural diffeologies, on $\mathcal{G}$ and $\mathrm{Bis}_{\text {loc }}(\mathcal{G})$ respectively, induced by a support.

3. Inspired by the canonical Cartan tower of a pseudogroup and by the definition of Lie pseudogroup, see Definition 3.2.17, we define Cartan resolutions $\left(\Sigma^{*}, \mathrm{C}^{*}\right)$ of étale groupoids $\mathcal{G} \rightrightarrows \mathbf{X}$, see Definition 3.4.5. We also discuss two topologies on $\mathcal{G}$ that are induced by the limit of a Cartan resolution of $\mathcal{G} \rightrightarrows \mathrm{X}$, Definitions 3.4.17 and 3.4.20.

4. We formulate a finite order theorem, Theorem 3.5.2, in terms of actions of étale groupoids $\mathcal{G} \rightrightarrows \mathbf{X}$ equipped with a support and a Cartan resolution. When specialized to germ groupoids of classical pseudogroups, the result should be compared with other ones appearing in the literature, e.g. [5, 40, 62].

5. The proof of Theorem 3.5.2 makes use of ideas appearing elsewhere - most notably $[61,70]$ - but provides also new insights. In particular, we make explicit and carefully analyze the role of the diffeology induced by the support as well as the role of the topologies induced by the Cartan resolution. This also leads to a more conceptual reinterpretation of some of the key lemmata from [54]. 



\section{A Pfaffian look at transi- tive pseudogroups}

Bene, miei duri, bando agli scoramenti. Fora i petti, dritte l'armi, alte le insegne, baldanza!

- Brancaleone da Norcia, L'armata Brancaleone

The content of this chapter is joint work in progress with Francesco Cattafi. We explore the framework of principal actions of Pfaffian groupoids [55] introduced in [15] as a tool to encode the abstract properties of geometric structures on manifolds. In particular, we discuss the notion of Pfaffian Morita equivalence [15]. We focus on the transitive case and we prove that any transitive Pfaffian groupoid is Pfaffian Morita equivalent to a Lie group equipped with a right invariant and left equivariant form valued in a representation. As a consequence we encode geometric structures induced by transitive pseudogroup into principal bundles equipped with a vertical transversally parallelizable equivariant foliation. Our approach is complementary to [16].

\subsection{Preliminaries and motivation: transitive geome- tries}

\subsubsection{Geometry on manifolds}

In broad terms, by "geometric structure" on a manifold $M$ one means some additional piece of differential geometric data on $M$. A more precise definition depends, obviously, on the examples one has in mind/theoretical properties one wants to capture. Here, we will look at manifolds that locally look like $\mathbb{R}^{n}$ equipped with additional structure. In other words, we are interested in structures that

- can be "pulled-back" to $M$ from analogous structures on $\mathbb{R}^{n}$ by using that $M$ is locally modelled on $\mathbb{R}^{n}$;

- are defined by systems of (very) well behaved differential equations. 


\section{Local models}

Let $M$ be an $n$ dimensional manifold. The smooth structure on $M$ is defined by means of equivalence classes of atlases $\left(\left\{U_{i}\right\}_{i \in I},\left\{\phi_{i}\right\}_{i \in I}\right)$, where $\phi_{i}: U_{i} \rightarrow \mathbb{R}^{n}$ is a chart, with transition functions $\left\{\varphi_{i j}\right\}_{i, j \in I}$. We look at geometric structures defined by requiring the $\left\{\varphi_{i j}\right\}_{i, j \in I}$ 's to belong to a set of symmetries of some geometric structure on $\mathbb{R}^{n}$.

Definition 1.1.1. A pseudogroup $\Gamma$ on a manifold $X$ is a subset of the set of embeddings of open sets of $\mathbf{X}$ into $\mathbf{X}$ which is

- closed under the group-like operations, i.e. composition (when defined) and inversion, and containing the identity $i d_{\mathbf{X}}: \mathbf{X} \rightarrow \mathbf{X}$;

- local, i.e. such that restrictions of elements in $\Gamma$ belong to $\Gamma$;

- closed under gluings, i.e. if $\left\{V_{k}\right\}_{k \in K}$ covers an open $U$ and $\varphi_{k} \in \Gamma$ are the restrictions of an embedding $\varphi: U \hookrightarrow \mathbf{X}$ to the opens $V_{k}$ 's, then $\varphi \in \Gamma$.

If $\Gamma$ is a pseudogroup on $\mathbb{R}^{n}$, one calls $\Gamma$-atlas on a manifold $M$ an atlas on $M$ whose transition functions are elements of $\Gamma$. A $\Gamma$-structure on $M$ is the datum of a maximal $\Gamma$-atlas on $M$. The same definitions make sense when $\Gamma$ is defined on any manifold $\mathbf{X}$.

Definition 1.1.2. Let $\Gamma$ be a pseudogroup over a manifold $\mathbf{X}$. A $\Gamma$-atlas on a manifold $M$ is an atlas $\left\{U_{i}, \phi_{i}\right\}_{i \in I}$ of $M$ modelled on opens of $\mathbf{X}$ and such that the transition functions are elements of $\Gamma$. A maximal $\Gamma$-atlas on $M$ is called a $\Gamma$-structure on $M$.

Definition 1.1.3. Let $\Gamma$ be a pseudogroup on a manifold $\mathbf{X}$. The orbit $\mathcal{O}_{x}$ of $x \in \mathbf{X}$ is the set of $y \in \mathbf{X}$ for which there exists $\varphi \in \Gamma$ such that $\varphi(x)=y$.

A pseudogroup on $\mathbf{X}$ is called transitive if for all $x, y \in \mathbf{X}$ there is $\varphi \in \Gamma$ such that $\varphi(x)=y$, i.e. the only orbit is $\mathbf{X}$ itself.

Remark 1.1.4. When studying $\Gamma$-structures for transitive pseudogroups, one can restrict to pseudogroups over $\mathbb{R}^{n}$ without loss of generality.

We first observe that, if $\Gamma$ is a pseudogroup over $\mathbf{X}$ and $U \subset \mathbf{X}$ is an open subset, the set

$$
\left.\Gamma\right|_{U}=\{\varphi \in \Gamma: \operatorname{Dom}(\varphi) \subset U, \operatorname{Im}(\varphi) \subset U\}
$$

is a pseudogroup over $U$. Now, let $\Gamma$ be a transitive pseudogroup and $M$ be a manifold possessing a $\Gamma$-structure - i.e., a maximal $\Gamma$-atlas. For each $x \in \mathbf{X}$, we choose a chart $\phi: U_{x} \rightarrow \mathbf{X}$ defined on an open neighbourhood $U_{x}$ of $x$ and belonging to the maximal $\Gamma$-atlas. Since $\Gamma$ is transitive, there is an element $\varphi_{x} \in \Gamma$ such that $\left(\varphi_{x} \circ \phi_{x}\right)(x) \in U$. We can assume $U_{x}$ to be so small that $\phi_{x}\left(U_{x}\right) \subset$ $\operatorname{Dom}\left(\varphi_{x}\right)$ and $\varphi_{x} \circ \phi_{x}$ takes values in $U$. In this way, we see that $M$ admits a $\left.\Gamma\right|_{U}$-atlas; the charts are precisely the maps $\varphi_{x} \circ \phi_{x}$, for all $x \in \mathbf{X}$. Notice that each map $\varphi_{x} \circ \phi_{x}$ is in fact part of the maximal $\Gamma$-atlas on $M$. Notice also that different choices of charts $\phi_{x}$ (and different choices of transition functions $\varphi_{x}$ ) lead to compatible $\left.\Gamma\right|_{U}$ atlases, in the sense that they are part of the same maximal $\left.\Gamma\right|_{U}$-atlas on $M$. Finally, we observe that any $\left.\Gamma\right|_{U}$-atlas on $M$ is in particular a $\Gamma$ atlas on $\mathrm{M}$. 
Putting all together, we see that, when $\Gamma$ is a transitive pseudogroup over $\mathbf{X}$, there is a one to one correspondence between $\Gamma$-structures on a manifold $M$ and $\left.\Gamma\right|_{U}$-structures on the same manifold $M$, where $U \subset \mathbf{X}$ is any open subset. In particular, one can choose $U$ so that $U \cong \mathbb{R}^{n}$ and see $\left.\Gamma\right|_{U}$ as a pseudogroup on $\mathbb{R}^{n}$.

Explicit examples of pseudogroups on $\mathbb{R}^{n}$ can be obtained by considering symmetries of classical structures on $\mathbb{R}^{n}$.

Example 1.1.5. The simplest example of a pseudogroup on $\mathbb{R}^{n}$ is $\Gamma^{n}:=$ $\operatorname{Diff}_{\text {loc }}\left(\mathbb{R}^{n}\right)$, the set of all locally defined diffeomorphisms of $\mathbb{R}^{n}$. It can be thought of as the set of symmetries of the standard smooth structure of $\mathbb{R}^{n}$. A $\Gamma^{n}$-structure on a manifold $M$ is precisely a smooth structure on $M$; in fact, a $\Gamma^{n}$-atlas on $M$ is simply an ordinary atlas on $M$.

Example 1.1.6. A linear $G$-structure on $\mathbb{R}^{n}$ corresponds to the choice of a Lie subgroup of linear transformations $G \subset G L_{n}(\mathbb{R})$. Geometrically, we consider the set $\mathcal{S}_{G}$ of frames in the vector space $\mathbb{R}^{n}$ that can be obtained from the standard basis via a transformation by $G$. The group of linear transformations $G$ generates a pseudogroup $\Gamma_{G}$ on $\mathbb{R}^{n}$, given by those embeddings $\varphi: U \hookrightarrow \mathbb{R}^{n}$ whose differential at any point $x \in U$ lies in $G$. Notice that $\Gamma_{G}$ contains all translations; consequently, it is transitive.

$\Gamma_{G}$ should be interpreted as the set of (locally defined) symmetries of the linear $G$-structure on $\mathbb{R}^{n}$. For each $x \in \mathbb{R}^{n}$, fix the isomorphism $T_{x} \mathbb{R}^{n} \cong \mathbb{R}^{n}$ induced by the translation

$$
y \in \mathbb{R}^{n} \rightarrow y+x \in \mathbb{R}^{n} .
$$

Maps $\varphi \in \Gamma_{G}$ send frames of $T_{x} \mathbb{R}^{n} \cong \mathbb{R}^{n}$ lying in $\mathcal{S}_{G}$ to frames of $T_{\varphi(x)} \mathbb{R}^{n} \cong \mathbb{R}^{n}$ lying in $\mathcal{S}_{G}$.

By specifying $G$, one has more explicit examples; we list some of them below.

- If $G=O(n)$, then we are considering orthogonal frames of $\mathbb{R}^{n}$. The pseudogroup $\Gamma_{O(n)}$ is the set $\Gamma_{\text {eucl }}$ of locally defined isometries of the standard Euclidean metric on $\mathbb{R}^{n}$.

- If $n=2 k, G=G L_{k}(\mathbb{C})$, then we are considering complex frames of $\mathbb{C}^{k} \cong$ $\mathbb{R}^{2 k}$. The pseudogroup $\Gamma_{G l(k, \mathbb{C})}$ is the set $\Gamma_{\mathrm{cpx}}$ of locally defined biholomorphisms of the standard complex structure on $\mathbb{C}^{k}$.

- If $n=2 k, G=S p(k)$, then we are considering symplectic frames of $\mathbb{R}^{2 k}$. The pseudogroup $\Gamma_{S p(k)}$ is the set $\Gamma_{\mathrm{sp}}$ of locally defined symplectomorphism of the standard symplectic structure on $\mathbb{R}^{2 k}$.

- If $G=G L_{(k, n-k)}(\mathbb{R})$ is the group of invertible real matrices whose lower left $k \times(n-k)$ block is the null matrix, then we are considering frames of $\mathbb{R}^{n}$ whose first $(n-k)$ vectors are tangent to the subspace $\mathbb{R}^{n-k} \hookrightarrow \mathbb{R}^{n}$ given by the first $(n-k)$-coordinates. The pseudogroup $\Gamma_{G L_{(k, n-k)}(\mathbb{R})}$ is the set of symmetries of the codimension $k$-foliation by copies of $\mathbb{R}^{k}$.

- If $n=2 k+1, G=S p(k, 1)$ is the group of invertible matrices whose lower left $1 \times 2 k$ block is the null matrix and whose top left $2 k \times 2 k$ block lies in $S p(k)$, then we are considering frames of $\mathbb{R}^{n}$ whose first $2 k$ vectors are 
tangent to the subspace $\mathbb{R}^{2 k} \hookrightarrow \mathbb{R}^{n}$ given by the first $2 k$-coordinates and form a symplectic frame on $\mathbb{R}^{2 k}$. The pseudogroup $\Gamma_{S p(k, 1)}$ is the set of symmetries of the "linear" codimension 1-symplectic foliation by copies of $\mathbb{R}^{2 k}$ with the canonical symplectic structure.

$\Gamma$-structures on $M$ associated to the above pseudogroups are, in order:

- flat Riemannian structures;

- complex structures;

- symplectic structures;

- codimension $k$-foliations;

- codimension 1-symplectic foliations.

In all these cases, the structures on $M$ are obtained by pulling back the corresponding "linear" structures on $\mathbb{R}^{n}$ via charts in a $\Gamma$-atlas. In this way, one obtains local structures on $M$; since the transition functions of the atlas belong to $\Gamma$, one sees that these local structures glue correctly. The result is a global structure on $M$ that is locally isomorphic to the corresponding "linear" structure on $\mathbb{R}^{n}$.

Example 1.1.7. Structures on $\mathbb{R}^{n}$ that are not linear as the ones from the Example 1.1.6 have pseudogroups of symmetries which are not of the form $\Gamma_{G}$, where $G \subset G L_{n}(\mathbb{R})$ is a subgroup of linear transformations. For instance, let $n=2 k+1, k \in \mathbb{N}$ and let us consider coordinates $(\mathbf{x}, \mathbf{y}, z)$ on $\mathbb{R}^{2 k+1}$ where $\mathbf{x}=\left(x_{1}, \ldots x_{k}\right) \in \mathbb{R}^{k}$ and $\mathbf{y}=\left(y_{1}, \ldots y_{k}\right) \in \mathbb{R}^{k}$. Let $\xi_{\text {std }}=\operatorname{Ker}(d z-\mathbf{y} d \mathbf{x})$ be the standard contact structure on $\mathbb{R}^{2 k+1}$; we set

$$
\alpha_{\text {std }}:=\operatorname{Ker}(d z-\mathbf{y} d \mathbf{x}) .
$$

Let $\Gamma_{\text {cont }}$ be the pseudogroup of locally defined diffeomorphisms of $\mathbb{R}^{2 k+1}$ preserving $\alpha_{\text {std }}$; that is, a diffeomorphism onto its image $\phi: U \rightarrow \mathbb{R}^{2 k+1}$ defined on an open subset $U \subset \mathbb{R}^{2 k+1}$ belongs $\Gamma_{\text {cont }}$ if and only if $\phi^{*}\left(\left.\alpha_{\text {std }}\right|_{\phi(U)}\right)=\left.\alpha_{\text {std }}\right|_{U}$. $\Gamma_{\text {cont }}$ is a transitive pseudogroup, but it is not of the form $\Gamma_{G}$ for any $G \subset G L_{n}(\mathbb{R})$. In fact, $\Gamma_{\text {cont }}$ does not contain translations.

We still can describe explicitly some of the globally defined elements of $\Gamma_{\text {cont }}$ in terms of a Lie group. Recall that the Heisenberg group Hei $\cong \mathbb{R}^{2 k+1}$ is the Lie group structure on $\mathbb{R}^{2 k+1}$ with multiplication

$$
(\mathbf{x}, \mathbf{y}, z) \cdot\left(\mathbf{x}^{\prime}, \mathbf{y}^{\prime}, z^{\prime}\right)=\left(\mathbf{x}+\mathbf{x}^{\prime}, \mathbf{y}+\mathbf{y}^{\prime}, z+z^{\prime}+\left\langle\mathbf{x}, \mathbf{y}^{\prime}\right\rangle\right)
$$

where $\left\langle\mathbf{x}, \mathbf{y}^{\prime}\right\rangle$ denotes the standard scalar product. Hei acts on itself by right multiplication; the diffeomorphisms of $\mathbb{R}^{2 k+1}$ induced by this action belong to $\Gamma_{\text {cont }}$.

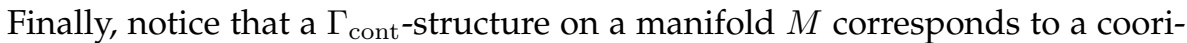
entable contact structure on $M$.

The pseudogroups from Examples 1.1.6 and 1.1.7 are transitive pseudogroups on $\mathbb{R}^{n}$. In both cases - pseudogroups of the form $\Gamma_{G}$, for some subgroup $G \subset$ $G L_{n}(\mathbb{R})$, and $\Gamma_{\text {cont }}$ - a way to realize that the pseudogroup under consideration is 
transitive is to observe that it contains elements that come from a free and transitive action of some Lie group on $\mathbb{R}^{n}$ (either the group of translations or the Heisenberg group).

Definition 1.1.8. Let $K$ be a Lie group acting freely (from the left) on a manifold $\mathbf{X}$ and let $\Gamma$ be a pseudogroup on $\mathbf{X}$. We say that $\Gamma$ contains $K$ if, for all $k \in K$, the diffeomorphism

$$
\varphi_{k}: \mathbf{X} \rightarrow \mathbf{X}, \quad x \rightarrow k \cdot x
$$

belongs to $\Gamma$.

The minimal pseudogroup generated by $K$, denoted by $\Gamma(K)$, is the pseudogroup generated by all the diffeomorphism of the form $\varphi_{k}, k \in K$.

"Generated" has the obvious meaning: $\Gamma(K)$ contains all the diffeomorphism of the form $\varphi_{k}, k \in K$, together with their restrictions to opens in $\mathbf{X}$ and all the possible gluings.

Example 1.1.9. The Lie group of translations of $\mathbb{R}^{n}$ can be identified with $\mathbb{R}^{n}$ equipped with the sum of vectors. It acts freely and transitively on $\mathbb{R}^{n}$. For any Lie subgroup $G \subset G L_{n}(\mathbb{R})$, we see that $\mathbb{R}^{n}$ is contained in $\Gamma_{G}$.

Example 1.1.10. As already mentioned in Example 1.1.7, the Heisenberg group Hei acts freely and transitively on $\mathbb{R}^{2 k+1}$ (it is, in fact, a Lie group structure on $\left.\mathbb{R}^{2 k+1}\right)$. The pseudogroup $\Gamma_{\text {cont }}$ contains Hei.

Example 1.1.11. Given any foliation $\mathcal{F}$ on $\mathbb{R}^{n}$ the pseudogroup $\Gamma_{\mathcal{F}}$ of leafwise diffeomorphisms (i.e. embeddings sending opens into leaves to opens into the same leaf) is very rarely transitive. In fact, the orbits of $\Gamma_{\mathcal{F}}$ are precisely the leaves of $\mathcal{F}$. By choosing $\mathcal{F}$ to be a singular foliation, one can exhibit pseudogroups whose orbits have a very complicated behaviour.

\section{Differential equations}

In our examples 1.1.6 and 1.1.7 above, our pseudogroups are defined by considering symmetries of global geometric objects on $\mathbb{R}^{n}$; a map belongs to the pseudogroup if it pulls back the given object to itself. On each specific example, this request translates into differential conditions / differential equations imposed on elements of the pseudogroup. Let us be precise about our framework for differential equations.

Definition 1.1.12. A geometric order $k$ differential equation $R$ is an embedded submanifold $R \subset J^{k}(M, N)$, where $M, N$ are manifolds and $J^{k}(M, N)$ denotes the space of $k$-jets of maps from $M$ to $N$. A local solution of $R$ is a map $f: U \subset$ $M \rightarrow N$ such that

$$
j^{k} f: U \rightarrow J^{k}(M, N), \quad x \rightarrow j_{x}^{k} f
$$

takes values in $R$.

Recall that the space $J^{k}(M, N)$ of $k$-jets consists of equivalence classes $j_{x}^{k} f$ of maps $f: M \rightarrow N$ up to contact of order $k$ at $x \in M$; i.e., $j_{x}^{k} f=j_{x}^{k} g, f, g: M \rightarrow N$, if and only if $f$ and $g$ have the same order $k$-Taylor expansion at $x . J^{k}(M, N)$ is always a smooth manifold - the coordinates are simply given by the coordinates 
on $M$ and $N$ together with the coefficients of order $k$-Taylor expansions - and surjects onto $J^{k-1}(M, N)$ and onto $M$. A section $U \subset M \rightarrow J^{k}(M, N)$ is a map $x \rightarrow j_{x}^{k} f_{x}$, where the representative $f_{x}$ depends on $x$; it is called holonomic when $f_{x}=f: U \rightarrow N$ does not depend on $x$. Holonomic sections are in bijection with locally defined maps from $M$ to $N$; a solution of an order $k$ differential equation $R \subset J^{k}(M, N)$ is a holonomic section taking values in $R$.

Now, let $\Gamma$ be a pseudogroup on $\mathbf{X}$; for any $k \in \mathbb{N}$ we can form the $k$-jet space

$$
J^{k} \Gamma=\left\{j_{x}^{k} \varphi: \varphi \in \Gamma\right\} \subset J^{k}(\mathbf{X}, \mathbf{X})
$$

obtaining a tower

$$
\cdots \rightarrow J^{k} \Gamma \rightarrow J^{k-1} \Gamma \rightarrow \cdots \rightarrow J^{0} \Gamma \rightarrow \mathbf{X}
$$

Here, $J^{0} \Gamma \subset \mathbf{X} \times \mathbf{X}$ encodes the orbits of $\Gamma$; that is, $(y, x) \in J^{0} \Gamma$ if and only if there is $\varphi \in \Gamma$ such that $\varphi(x)=y$. As example 1.1.11 shows, already at level $k=0$ there is no reason for $J^{k} \Gamma$ to be a submanifold of $J^{k}(\mathbf{X}, \mathbf{X})$.

Definition 1.1.13. A pseudogroup $\Gamma$ over $\mathrm{X}$ is called Lie pseudogroup when

- $J^{k} \Gamma$ is a submanifold of $J^{k}(\mathbf{X}, \mathbf{X})$, for all $k \in \mathbb{N}$;

- all the projections $J^{k} \Gamma \rightarrow J^{k-1} \Gamma$ are surjective submersions.

We call $\Gamma$ Lie pseudogroup of order $k$ if, additionally, $\phi \in \Gamma$ if and only if the holonomic section

$$
j^{k} \phi: \operatorname{Dom}(\phi) \rightarrow J^{k}(\mathbf{X}, \mathbf{X}), \quad x \rightarrow j_{x}^{k} \phi
$$

takes values in $J^{k} \Gamma$, i.e. if and only if $\phi$ is a solution of $J^{k} \Gamma \subset J^{k}(\mathbf{X}, \mathbf{X})$.

The intuition behind the above definition is simply that "Lie pseudogroups of order $k$ are pseudogroups arising as solutions of order $k$ PDEs".

There is some more structure on $J^{k} \Gamma$ that is worth mentioning, and plays a role in our approach to geometries on manifolds. A first piece of structure, that arises already at the level of jets, is a distribution detecting holonomic sections; we will discuss it at the end of this section.

The second piece of structure is directly inherited from the group-like properties of $\Gamma$. Namely

- one has a pair of projections $J^{k} \Gamma \rightrightarrows \mathbf{X}$ (the source and target of a $k$-jet, i.e. $s\left(j_{x}^{k} f\right)=x$ and $t\left(j_{x}^{k} f\right)=f(x)$, called source and target map);

- one has a "partial multiplication"

$$
J^{k} \Gamma{ }_{s}{ }_{t} J^{k} \Gamma \rightarrow J^{k} \Gamma, \quad\left(j_{\varphi(x)}^{k} \varphi^{\prime}, j_{x}^{k} \varphi\right) \rightarrow j_{\varphi(x)}^{k} \varphi^{\prime} \cdot j_{x}^{k} \varphi=j_{x}^{k}\left(\varphi^{\prime} \circ \varphi\right)
$$

such that

- there is a canonical global unit section $\mathbf{X} \rightarrow J^{k} \Gamma$ which is a bijection between points in $\mathbf{X}$ and units for the multiplication;

- each element has an inverse; 
- the multiplication is associative.

A groupoid $\Sigma$ over $\mathbf{X}$, denoted by $\Sigma \rightrightarrows \mathbf{X}$, is a set $\Sigma$ possessing a partial multiplication (and source and target maps) as above. $\Sigma$ is called arrow space while $\mathbf{X}$ is called unit space. The unit space $\mathbf{X}$ can be identified with the subset of $\Sigma$ given by the image the unit section $\mathbf{X} \rightarrow \Sigma$. Groupoids form a category: a morphism of groupoids, simply denoted by $\Phi: \Sigma_{1} \rightarrow \Sigma_{2}$ is a commutative diagram

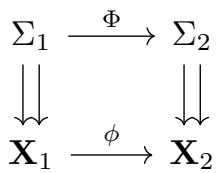

respecting the multiplication of arrows, i.e. such that $\Phi(g \cdot h)=\Phi(g) \cdot \Phi(h)$ for all $h, g \in \Sigma_{1}$ such that $t(h)=s(g)$.

Notice that, given a groupoid $\Sigma$ over $\mathbf{X}$, there is a natural notion of orbit: the orbit $\mathcal{O}_{x}$ of $x \in \mathbf{X}$ is the set of $y \in \mathbf{X}$ such that there is $g \in \Sigma$ with $s(g)=x, t(g)=$ $y$. Applied to $J^{k} \Gamma$, this recovers the notion of orbit of a pseudogroup. One also observes that the intersection $\Sigma_{x}=s^{-1}(x) \cap t^{-1}(x)$ possesses a group structure; it is called the isotropy group at $x$. Isotropy groups at points on the same orbits are isomorphic. Furthermore, the right action of $\Sigma_{x}$ on $s^{-1}(x)$ (respectively the left action of $\Sigma_{x}$ on $\left.t^{-1}(x)\right)$ has quotient in bijection with the orbit of $x$ and the quotient map can be identified with $t: s^{-1}(x) \rightarrow \mathbf{X}$. For details see, e.g., [49].

When dealing with the jet groupoid $J^{k} \Gamma \rightrightarrows \mathbf{X}$, we also see that both $J^{k} \Gamma$ and $\mathbf{X}$ carry smooth structures such that all the groupoid structure operations are smooth; and even more, $s$ and $t$ are surjective submersions. All in all $J^{k} \Gamma \rightrightarrows \mathbf{X}$ is a Lie groupoid [22, 49].

Definition 1.1.14. A Lie groupoid $\Sigma \rightrightarrows \mathbf{X}$ is a groupoid such that

- the arrow space is a smooth manifold (possibly non-Hausdorff and nonsecond countable);

- $\mathbf{X}$ is a smooth manifold (Hausdorff, second countable);

- all the structure maps (source and target, multiplication, unit section and inversion map) are smooth;

- $s$, hence $t$, is a surjective submersion (with Hausdorff fibers).

Remark 1.1.15. For any Lie groupoid $\Sigma \rightrightarrows \mathbf{X}$, the unit section $\mathbf{X} \rightarrow \Sigma$ is an embedding. We will often identify $\mathbf{X}$ with the submanifold of $\Sigma$ given by the image of the unit section.

Lie groupoids form a category as well: a morphism of Lie groupoids $\Sigma_{1} \rightrightarrows$ $\mathbf{X}_{1}$ and $\Sigma_{2} \rightrightarrows \mathbf{X}_{2}$ is a morphism of groupoids

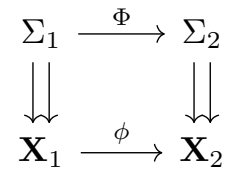

where both $\Phi$ and $\phi$ are smooth maps. When $\Sigma \rightrightarrows \mathbf{X}$ is a Lie groupoid 
- $s^{-1}(x)$ is an embedded submanifold of $\Sigma$, for all $x \in \mathbf{X}$;

- the isotropy group at $x, \Sigma_{x}=s^{-1}(x) \cap t^{-1}(x)$ is an embedded submanifold and a Lie group;

- the right action of $\Sigma_{x}$ on $s^{-1}(x)$ is smooth;

- the orbit of $x \in \mathbf{X}, \mathcal{O}_{x}$, is an immersed submanifold of $\mathbf{X}$;

- $t: s^{-1}(x) \rightarrow \mathbf{X}$ is a right principal $\Sigma_{x}$-bundle over $\mathcal{O}_{x}$ (a similar statement holds if we replace $s$ with $t$ - in that case we get a left principal bundle).

See [49] for details.

A Lie groupoid $\Sigma \rightrightarrows \mathbf{X}$ is called transitive when the anchor map

$$
(s, t): \Sigma \rightarrow \mathbf{X} \times \mathbf{X} \quad g \rightarrow(s(g), t(g))
$$

is a surjective submersion. Observed that surjectivity is equivalent to asking that for each $x \in \mathbf{X}, y \in \mathbf{X}$ there is $g \in \Sigma$ such that $s(g)=x$ and $t(g)=y$-i.e., $\Sigma$ possesses a single orbit: for each $x \in \mathbf{X}, \mathcal{O}_{x}=\mathbf{X}$. As a result, $t: s^{-1}(x) \rightarrow$ $\mathbf{X}$ is a principal $\Sigma_{x}$-bundle. Observe that the smooth structure on the quotient $s^{-1}(x) / \Sigma_{x}$ - the smooth structure on $\mathbf{X}$ as an orbit of $\Sigma-$ is diffeomorphic to the given smooth structure on $\mathbf{X}$ thanks to the fact that the anchor map is submersive. In fact, this implies that the restricted target map $t: s^{-1}(x) \rightarrow \mathbf{X}$ is submersive as well.

Remark 1.1.16. Observe that transitivity of a (not necessarily Lie) pseudogroup $\Gamma$ over $\mathbf{X}$ is equivalent to the map

$$
(s, t): J^{k} \Gamma \rightarrow \mathbf{X} \times \mathbf{X} \quad j_{x}^{k} \varphi \rightarrow(x, \varphi(x))
$$

being surjective.

Consequently, if the jet groupoid $J^{k} \Gamma \rightrightarrows \mathbf{X}$ is transitive, then $\Gamma$ is transitive.

Viceversa, if $\Gamma$ is transitive and a Lie pseudogroup, then $J^{k} \Gamma \rightrightarrows \mathbf{X}$ is transitive as well. In fact, transitivity of $\Gamma$ implies that

$$
(s, t): J^{k} \Gamma \rightarrow \mathbf{X} \times \mathbf{X} \quad j_{x}^{k} \varphi \rightarrow(x, \varphi(x))
$$

is surjective, but also that

$$
J^{0} \Gamma=\mathbf{X} \times \mathbf{X} .
$$

Since $\Gamma$ is a Lie pseudogroup, the map

$$
J^{k} \Gamma \rightarrow J^{0} \Gamma=\mathbf{X} \times \mathbf{X} \quad j_{x}^{k} \varphi \rightarrow(x, \varphi(x))
$$

is submersive by definition.

Example 1.1.17. All the pseudogroups from examples 1.1.6 and 1.1.7 are Lie pseudogroups of order one, since the differential conditions defining them are first order conditions.

Observe that the Lie group $G \subset G L_{n}(\mathbb{R})$ is isomorphic to the isotropy group (at any point) of $J^{1} \Gamma_{G}$. Actually

$$
J^{1} \Gamma_{G} \cong\left\{(x, y, l): l: \mathbb{R}^{n} \rightarrow \mathbb{R}^{n}, l \in G\right\} .
$$


On the other hand

$$
J^{1} \Gamma_{\mathrm{cont}} \cong\left\{(x, y, l): l: \mathbb{R}^{2 k+1} \rightarrow \mathbb{R}^{2 k+1}, l \in d_{y} \varphi_{y} \circ S p(k, 1) \circ d_{x} \varphi_{x}^{-1}\right\}
$$

where we identify $\mathbb{R}^{2 k+1}$ with the Heisenberg group Hei and denote by $\varphi_{x}$ and $\varphi_{y}$ the diffeomorphisms of $\mathbb{R}^{2 k+1}$ induced by right multiplication by $x \in \mathbb{R}^{2 k+1}$ and $y \in \mathbb{R}^{2 k+1}$ respectively. It follows that the isotropy group of $J^{1} \Gamma_{\text {cont }}$ is isomorphic to $S p(k, 1)$. Even more, it follows $J^{1} \Gamma_{\text {cont }} \cong J^{1} \Gamma_{S p(k, 1)}$.

\subsection{2 $\Gamma$-structures as principal bundles}

With the groupoid language at hand, one can conceptualize the notion of geometry on a manifolds in terms of familiar objects.

\section{The germ groupoid of a pseudogroup}

Let $\Gamma$ be a pseudogroup on $\mathbf{X}$. For the discussion in this subsection, we do not need $\Gamma$ to be a Lie pseudogroup. There is one further groupoid attached to $\Gamma$ : the germ groupoid $\operatorname{Germ}(\Gamma) \rightrightarrows \mathbf{X}$ with space of arrows

$$
\operatorname{Germ}(\Gamma)=\left\{\operatorname{germ}_{x}(\varphi): x \in \mathbf{X}, \varphi \in \Gamma\right\} .
$$

The source and target maps are given by

$$
s: \operatorname{germ}_{x}(\varphi) \in \operatorname{Germ}(\Gamma) \rightarrow x \in \mathbf{X}
$$

and

$$
t: \operatorname{germ}_{x}(\varphi) \in \operatorname{Germ}(\Gamma) \rightarrow \varphi(x) \in \mathbf{X}
$$

while the multiplication is defined by

$$
\operatorname{germ}_{\varphi(x)}\left(\varphi^{\prime}\right) \cdot \operatorname{germ}_{x}(\varphi):=\operatorname{germ}_{x}\left(\varphi^{\prime} \circ \varphi\right) \in \operatorname{Germ}(\Gamma),
$$

for $\operatorname{germ}_{\varphi(x)}\left(\varphi^{\prime}\right) \operatorname{germ}_{x}(\varphi) \in \operatorname{Germ}(\Gamma)$.

$\operatorname{Germ}(\Gamma)$ can be equipped with the étale topology, making $s$ and $t$ into local homeomorphisms. Since $\mathbf{X}$ is a manifold, $\operatorname{Germ}(\Gamma) \rightrightarrows \mathbf{X}$ is a Lie groupoid when given this topology.

Notice that all the source fibers $s^{-1}(x) \subset \operatorname{Germ}(\Gamma), x \in \mathbf{X}$, are discrete in the étale topology. Notice also that the manifold $\operatorname{Germ}(\Gamma)$ is in general non Hausdorff and non second countable.

Remark 1.1.18. If $\operatorname{Germ}(\Gamma) \rightrightarrows \mathbf{X}$ is a transitive Lie groupoid, then $\Gamma$ is transitive. The converse is not true (even for Lie pseudogroups!): if $\Gamma$ is transitive the map

$$
\operatorname{Germ}(\Gamma) \rightarrow \mathbf{X} \times \mathbf{X}, \quad \operatorname{germ}_{x}(\varphi) \rightarrow(x, \varphi(x))
$$

is surjective, but it is never submersive if $\operatorname{dim}(\mathbf{X}) \geq 1$, because the $s$-fibers of étale groupoids are necessarily discrete.

Compare with Remark 1.1.16.

We will use the notation $\mathcal{G} \rightrightarrows \mathbf{X}$ to denote étale groupoids - i.e. Lie groupoids such that the source and target maps are local homeomorphisms. 
Definition 1.1.19. Let $\Sigma \rightrightarrows \mathbf{X}$ be a Lie groupoid. A local section $\sigma: U \subset \mathbf{X} \rightarrow$ $\Sigma$ of the source map is called bisection if the composition $t \circ \sigma: U \rightarrow \mathbf{X}$ is a diffeomorphism onto its image.

Example 1.1.20. For any Lie groupoid $\Sigma \rightrightarrows \mathbf{X}$, the unit section $\mathbf{X} \rightarrow \Sigma$ is a bisection of $\Sigma \rightrightarrows \mathbf{X}$

Bisections of the jet groupoid $J^{k} \Gamma$ of a Lie pseudogroup $\Gamma$ on $\mathbf{X}$ that are also holonomic correspond precisely to elements of $\Gamma$. On the other hand any bisection of $\operatorname{Germ}(\Gamma)$ induce an element of $\Gamma$, and viceversa.

Definition 1.1.21. An étale groupoid $\mathcal{G} \rightrightarrows \mathbf{X}$ is called effective when, for all local bisections $\sigma, \sigma^{\prime}$, if $t \circ \sigma=t \circ \sigma^{\prime}$ then $\sigma=\sigma^{\prime}$.

All étale groupoids arising as germ of pseudogroups are effective. The converse is also true: the set

$$
\Gamma_{\mathcal{G}}=\{t \circ \sigma: \sigma \text { is a local bisection of } \mathcal{G}\}
$$

defines a pseudogroup and the morphism of groupoids

$$
\mathcal{G} \rightarrow \operatorname{Germ}\left(\Gamma_{\mathcal{G}}\right), \quad g \rightarrow \operatorname{germ}_{x}\left(t \circ \sigma_{g}\right),
$$

where $s(g)=x$ and $\sigma_{g}$ is a bisection defined around $x$ and such that $\sigma(x)=g$, is an isomorphism precisely when $\mathcal{G}$ is effective.

Theorem 1.1.22. There is a one to one correspondence between pseudogroups on $\mathbf{X}$ and effective étale groupoids over $\mathbf{X}$.

The above theorem is usually attributed to Haefliger. We are interested in using it to get a general and manageable description of geometries on manifolds.

Definition 1.1.23. Let $\Sigma \rightrightarrows \mathbf{X}$ be a Lie groupoid. A smooth left action of $\Sigma$ on a manifold $P$ along a map $\mu: P \rightarrow \mathbf{X}$, or, more briefly, a (left) $\Sigma$-space $\mu: P \rightarrow \mathbf{X}$, is a map

$$
a: \Sigma_{s} \times{ }_{\mu} P \rightarrow P
$$

such that

- $a\left(g_{1}, a\left(g_{2}, p\right)\right)=a\left(g_{1} g_{2}, p\right)$, for all $g_{1}, g_{2} \in \Sigma, s\left(g_{1}\right)=t\left(g_{2}\right), p \in P, \mu(p)=$ $s\left(g_{2}\right)$;

- $a\left(1_{x}, p\right)=p$ for all $p \in P$ such that $\mu(p)=x \in \mathbf{X}$, where $1_{x}$ denotes the identity in $\Sigma$ over $x$.

We will denote $a(g, p)$ by $g \cdot p$. The map $\mu$ is also called the moment map of the action.

The notion of right action/right $\Sigma$-space is defined analogously.

Given a $\Sigma$-space $\mu: P \rightarrow \mathbf{X}$, we will always assume the map $\mu$ to be a surjective submersion.

Given a $\Sigma$-space $\mu: P \rightarrow \mathbf{X}$, the orbit space of the action of $\Sigma$ is the quotient $P / \Sigma$ with respect to the equivalence relation

$$
p_{1} \sim p_{2} \quad \text { if and only if } \quad p_{2}=g \cdot p_{1}
$$

for some $g \in \Sigma, s(g)=\mu\left(p_{1}\right)$. 
Definition 1.1.24. Let $\Sigma \rightrightarrows \mathbf{X}$ be a Lie groupoid and $M$ be a manifold. A principal $\Sigma$-bundle over $M$ is a $\Sigma$-space $\mu: P \rightarrow \mathbf{X}$ together with a surjective submersion $\pi: P \rightarrow M$ such that

- $\pi$ is $\Sigma$-invariant, i.e. $\pi(g \cdot p)=\pi(p)$ for all $g \in \Sigma, p \in P$ such that $s(g)=\mu(p)$;

- the action is free and proper, i.e.

- the action is free: for all $p \in P, g \in \Sigma$ such that $s(g)=\mu(p)$ if $g \cdot p=p$ then $g=1_{\mu(p)}$;

- the action is proper: the map

$$
\Sigma_{s} \times{ }_{\mu} P \rightarrow P \times P, \quad(g, p) \rightarrow(g \cdot p, p)
$$

is a proper map.

The orbit space of a principal $\Sigma$-bundle over $M$ possesses a canonical manifold structure making the quotient projection into a surjective submersion; the map $P / \Sigma \rightarrow M$ induced by $\pi$ is a diffeomorphism when $P / \Sigma$ is given such a smooth structure.

Definition 1.1.25. A morphism of principal $\Sigma$-bundles $\mu_{1}: P_{1} \rightarrow \mathbf{X}, \mu_{2}: P_{2} \rightarrow \mathbf{X}$ over $M$ is a smooth map $F: P_{1} \rightarrow P_{2}$ commuting with the action of $\Sigma$, i.e.

- $\mu_{2} \circ F=\mu_{1}$;

- $F(g \cdot p)=g \cdot F(p)$ for all $g \in \Sigma, p \in P, \mu(p)=s(g)$.

The following follows from Proposition I.3.1 in [52]:

Proposition 1.1.26. There is a bijective correspondence between $\Gamma$-structures on $M$ and isomorphism classes of principal $\operatorname{Germ}(\Gamma)$-bundles whose moment map is étale (here $\operatorname{dim}(M)=\operatorname{dim}(\mathbf{X})$.

Let us review quickly how to pass from principal $\operatorname{Germ}(\Gamma)$-bundles to $\Gamma$-atlases and viceversa.

Notice that, as for principal group bundles, a trivialization of a principal $\Sigma$ bundle $\mu: P \rightarrow \mathbf{X}$ over $U \subset M$, where $\Sigma \rightrightarrows \mathbf{X}$ is a Lie groupoid, corresponds to a local section $\sigma: U \subset M \rightarrow P$ of the principal bundle projection $\pi: P \rightarrow M$. If $\sigma^{\prime}: V \subset M \rightarrow P$ is another section, the change of trivialization from $\sigma$ to $\sigma^{\prime}$ over $U \cap V$ is encoded by a map $\gamma: U \cap V \rightarrow \Sigma$, satisfying

$$
s(\gamma(x))=\mu(\sigma(x)), \quad x \in U \cap V,
$$

and

$$
\gamma(x) \cdot \sigma(x)=\sigma^{\prime}(x), \quad x \in U \cap V .
$$

Let now $\mu: P \rightarrow \mathbf{X}$ be a principal $\operatorname{Germ}(\Gamma)$-bundle over $M$ such that the map $\mu$ is étale, and let us choose a family of trivializations $\left\{\sigma_{i}\right\}_{i \in I}$ where $\sigma_{i}$ is defined over $U_{i} \subset M$ and $\left\{U_{i}\right\}_{i \in I}$ is an open cover of $M$. The composition $\mu \circ \sigma_{i}$ is (up to shrinking domain) a diffeomorphism onto its image. Combining this with the changes of trivializations $\gamma_{i j}: U_{i} \cap U_{j} \rightarrow \operatorname{Germ}(\Gamma)$, one gets a $\Gamma$-atlas. 
Viceversa, given a $\Gamma$-atlas $\left\{\left(U_{i}, \phi_{i}\right)\right\}_{i \in I}$ on $M$ with transition functions $\varphi_{i j} \in \Gamma$, a principal $\operatorname{Germ}(\Gamma)$-bundle is defined by considering the space

$$
\operatorname{Germ}(\Gamma) \times_{\mathbf{X}} \coprod_{i \in I} U_{i}=\left\{\left(\operatorname{germ}_{x}(\varphi), p, i\right) \mid \varphi \in \Gamma, i \in I, p \in U_{i}, x=\phi_{i}(p)\right\}
$$

and taking the quotient with respect to the equivalence relation

$$
\left(\operatorname{germ}_{x}(\varphi), p, i\right) \sim\left(\operatorname{germ}_{\varphi_{i j}^{-1}(x)}\left(\varphi \circ \varphi_{i j}\right), p, j\right)
$$

for all $p \in U_{i} \cap U_{j}, \varphi \in \Gamma$ with $x \in \operatorname{Dom}(\varphi), x=\phi_{i}(p)$. The moment map on such a quotient is defined by

$$
\mu\left[\left(\operatorname{germ}_{x}(\varphi), p, i\right)\right]=\varphi(x)
$$

while the action is defined by

$$
\operatorname{germ}_{y}\left(\varphi^{\prime}\right) \cdot\left[\left(\operatorname{germ}_{x}(\varphi), p, i\right)\right]:=\left[\left(\operatorname{germ}_{x}\left(\varphi^{\prime} \circ \varphi\right), p, i\right)\right], \quad \varphi(x)=y .
$$

This action defines in fact a principal bundle over $M$ with projection

$$
\pi\left(\left[\operatorname{germ}_{x}(\varphi), p, i\right]\right)=p .
$$

The fact that the isomorphism class of the principal bundle constructed in this way does not depend on the atlas chosen but only on the $\Gamma$-structure on $M$, and that $\Gamma$-atlases induced by isomorphic $\operatorname{Germ}(\Gamma)$-bundles define the same $\Gamma$ structure, is proven in [52], Proposition I.3.1.

Remark 1.1.27. Proposition I.3.1 in [52] is more general: it deals with the case when the moment map is not necessarily étale, $\operatorname{dim}(M)$ is not necessarily equal to $\operatorname{dim}(\mathbf{X})$ and $\operatorname{Germ}(\Gamma)$ is replaced with a - non necessarily effective - étale groupoid $\mathcal{G} \rightrightarrows \mathbf{X}$. It states that there is a bijective correspondence between isomorphism classes of principal $\mathcal{G}$-bundles over $M$ and Haefliger $\mathcal{G}$-structures on $M$. Defining Haefliger $\mathcal{G}$-structures goes beyond the scope of our discussion; $\Gamma$ structures are an example arising when $\mathcal{G}=\operatorname{Germ}(\Gamma)$.

The fact that Proposition I.3.1 in [52] specializes to Proposition 1.1.26 above can be seen from the discussion preceding the statement in [52]. In particular, one sees that the moment map of any representative in the isomorphism class of principal $\operatorname{Germ}(\Gamma)$-bundles induced by a $\Gamma$-structure is étale.

There is a relatively simple description of the principal $\operatorname{Germ}(\Gamma)$-bundle associated to a $\Gamma$-structure on $M$ that makes use of a maximal $\Gamma$-atlas $\mathcal{A}=\left\{\left(U_{i}, \phi_{i}\right)\right\}_{i \in I}$ with change of coordinates $\left\{\varphi_{i j}\right\}_{i, j \in I}, \varphi_{i j} \in \Gamma$. Define

$$
\operatorname{Germ}(\mathcal{A})=\left\{\operatorname{germ}_{p}\left(\phi_{i}\right) \mid p \in U_{i} \subset M\right\} .
$$

There is an action of $\operatorname{Germ}(\Gamma)$ on $\operatorname{Germ}(\mathcal{A})$ with moment map

$$
\mu: \operatorname{Germ}(\mathcal{A}) \rightarrow \mathbf{X}, \quad \operatorname{germ}_{p}\left(\phi_{i}\right) \rightarrow \phi_{i}(p) .
$$

The action is given by

$$
\operatorname{germ}_{\phi(p)}(\varphi) \cdot \operatorname{germ}_{p}\left(\phi_{i}\right)=\operatorname{germ}_{p}\left(\varphi \circ \phi_{i}\right)
$$


notice that $\operatorname{germ}_{p}\left(\varphi \circ \phi_{i}\right) \in \operatorname{Germ}(\mathcal{A})$ because $\mathcal{A}$ is a maximal atlas. This action is principal over $M$ with projection

$$
\pi: \operatorname{Germ}(\mathcal{A}) \rightarrow M, \quad \operatorname{germ}_{p}\left(\phi_{i}\right) \rightarrow p .
$$

We picture such a principal $\operatorname{Germ}(\Gamma)$-bundle as

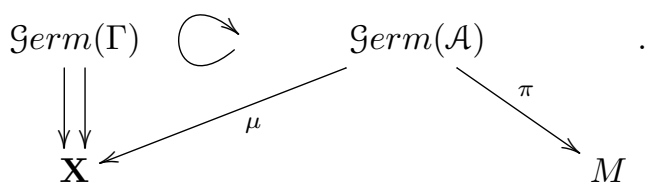

This explicit description will be useful below.

\section{Non integrable geometries}

Let $\Gamma$ be a Lie pseudogroup over $\mathbf{X}$, Definition 1.1.13, and let us consider a $\Gamma$ structure on $M$

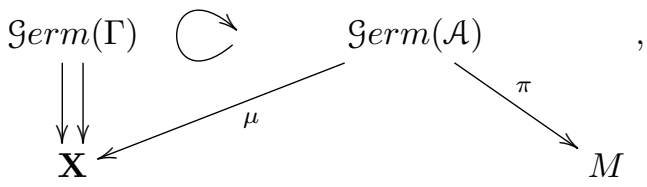

where $\mathcal{A}=\left\{\left(U_{i}, \phi_{i}\right)\right\}_{i \in I}$ is a maximal atlas with changes of coordinates $\left\{\varphi_{i j}\right\}_{i, j \in I}$, $\varphi_{i j} \in \Gamma$. One can make use of the tower of Lie groupoids

$$
\cdots \rightarrow J^{k} \Gamma \rightarrow J^{k-1} \Gamma \rightarrow \cdots \rightarrow J^{0} \Gamma \rightrightarrows \mathbf{X} .
$$

to look at the $k$-th order data of the atlas/the "projections" of the principal Germ $(\Gamma)$-bundle to $J^{k} \Gamma$. Precisely, one has principal bundles

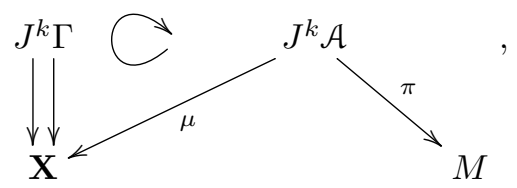

where now

$$
J^{k} \mathcal{A}=\left\{j_{p}^{k} \phi_{i} \mid p \in U_{i} \subset M\right\} .
$$

This point of view was extensively used in [15] to address the so called formal integrability problem for geometric structures on manifolds. We quickly go through the definitions that are relevant for our purposes; all the material in this subsection comes from [15], to which we refer for more details.

Notice that we have the following particular case of the above construction. If $M$ is a manifold and $\mathcal{A}_{M}$ is a maximal atlas, then, for each $k$, the space $J^{k} \mathcal{A}_{M}$ consisting of $k$-jets of charts is a principal $J^{k} \Gamma^{n}$-bundle (see Example 1.1.5 for the definition $\Gamma^{n}$ ) over $M$.

Let $\Gamma$ be a Lie pseudogroup over $\mathbf{X}$ and $M$ be a manifold. Assume $M$ to possess a Diff ${ }_{\text {loc }}(\mathbf{X})$-structure and denote the induced principal $J^{k} \operatorname{Diff}_{\text {loc }}(\mathbf{X})$-bundle 
by $\Pi^{k} \subset J^{k}(M, \mathbf{X})$. Recall that $\Pi^{k}$ is the space of germs of maps in a maximal Diff ${ }_{\text {loc }}(\mathbf{X})$-atlas. A $J^{k} \Gamma$-reduction of $\Pi^{k}$ is a principal $J^{k} \Gamma$-bundle $P$ over $M$ such that $P \subset \Pi^{k}$ and the moment map and action of $J^{k} \Gamma$ on $P$ are obtained restricting the moment map and action of $J^{k} \operatorname{Diff}_{\text {loc }}(\mathbf{X})$ on $\Pi^{k}$. Notice that any $\Gamma$-structure on $\mathbf{X}$ induces a $J^{k} \Gamma$-reduction of $\Pi^{k}$; in fact, a maximal $\Gamma$-atlas is necessarily contained in a maximal $\operatorname{Diff}_{\text {loc }}(\mathbf{X})$-atlas, since $\Gamma \subset \operatorname{Diff}_{\text {loc }}(\mathbf{X})$.

Definition 1.1.28. Let $\Gamma$ be a Lie pseudogroup over $\mathbf{X}$ and $M$ be a manifold. Assume $M$ to possess a Diff ${ }_{\text {loc }}(\mathbf{X})$-structure and denote the induced principal $J^{k} \operatorname{Diff}_{\text {loc }}(\mathbf{X})$-bundle by $\Pi^{k} \subset J^{k}(M, \mathbf{X})$. An almost $\Gamma$-structure of order $k$ on a manifold $M$ is a $J^{k} \Gamma$-reduction of $\Pi^{k}$.

An almost $\Gamma$-structure of order $k$ is called integrable if there is a $\Gamma$-structure on $M$ with maximal atlas $\mathcal{A}$ such that $P=J^{k} \mathcal{A}$.

Not all $\Gamma$-structures are integrable! The integrability corresponds precisely to existence of local models as explained above.

Example 1.1.29. Let $\Gamma_{\text {eucl }}$ be the pseudogroups of isometries of the euclidean met-

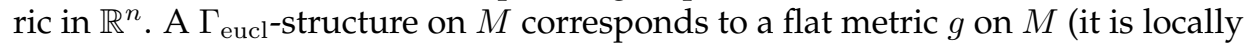
isometric to the Euclidean one by definition of $\Gamma$-atlas), see Example 1.1.6. If $\mathcal{A}$ is a maximal $\Gamma_{\text {eucl }}$-atlas, the induced principal $J^{1} \Gamma_{\text {eucl }}$-bundle $J^{1} \mathcal{A}$ can be encoded (passing to trivializations) by an atlas $\left(\left\{U_{i}\right\}_{i \in I},\left\{\phi_{i}\right\}_{i \in I}\right)$ where the charts can be chosen to be (induced by) holonomic sections of $J^{1} \mathcal{A}$ and are local isometries beween $(M, g)$ and $\left(\mathbb{R}^{n}, g_{\text {eucl }}\right)$.

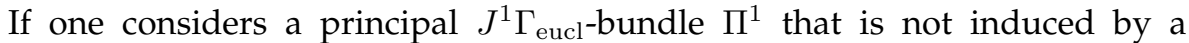
principal $\operatorname{Germ}\left(\Gamma_{\text {eucl }}\right)$-bundle and passes to trivializations, one still gets an atlas $\left(\left\{U_{i}\right\}_{i \in I},\left\{\phi_{i}\right\}_{i \in I}\right)$ of $M$; the charts $\phi_{i}{ }^{\prime}$ s are induced by sections $\sigma_{i}{ }^{\prime}$ s of $\Pi^{1}$ which are not necessarily holonomic. In fact, there is no guarantee that $\Pi^{1}$ has holonomic sections. We get a maximal atlas on $M$ whose transition functions $\varphi_{i j}$ may fail to be elements of $\Gamma_{\text {eucl }}$; however, they come paired with not necessarily holonomic sections $\psi_{i j}$ of $J^{1} \Gamma_{\text {eucl }}$. The pair $\left(\varphi_{i j}, \psi_{i j}\right)$ is a formal isometry: the map $\varphi_{i j}$ is not an isometry on its domain, but it comes paired with a smooth family of linear isometries of the tangent spaces defined on its domain. Namely, for $x \in \operatorname{Dom}\left(\varphi_{i j}\right), \psi_{i j}(x) \in J^{1} \Gamma_{\text {eucl }}$ defines a linear isometry of $\mathbb{R}^{n}$. One can use such an atlas to endow $M$ with a Riemannian metric $g$ which is, in general, not flat. The curvature of $g$ is precisely the obstruction to the existence of a $\operatorname{Germ}\left(\Gamma_{\text {eucl }}\right)$-principal bundle over the $J^{1} \Gamma_{\text {eucl }}$-one [15].

A similar discussion holds for all the $\Gamma_{G}$ 's from Example 1.1.6. The corresponding $J^{1} \Gamma_{G}$-principal bundles over $M$ are precisely $G$-structures on $M[18$, 64]; and they are flat if and only if the bundle is induced by a $\operatorname{Germ}\left(\Gamma_{G}\right)$-bundle. This is most appreciated when taking full account of transitivity, and we'll come back to it later on.

Example 1.1.30. A similar discussion holds true for $\Gamma_{\text {cont }}$ from Example 1.1.7. A principal $J^{1} \Gamma_{\text {cont }}$-bundle induces an atlas where the transition functions are formal contactomorphisms and the Darboux theorem holds precisely when the bundle is induced by a principal $\operatorname{Germ}\left(\Gamma_{\text {cont }}\right)$-bundle. 


\subsubsection{Morita equivalences}

\section{Transverse geometry}

The notion of Morita equivalence captures the idea of "transverse geometry" of a Lie groupoid $\Sigma \rightrightarrows \mathbf{X}[24,47,49]$.

Definition 1.1.31. A Morita equivalence ([68], Definition 2.1) between $\Sigma_{1} \rightrightarrows \mathbf{X}_{1}$ and $\Sigma_{2} \rightrightarrows \mathbf{X}_{2}$ is a principal bibundle, that is a space $P$ coming with two maps $\mu_{1}: P \rightarrow \mathbf{X}_{1}$ and $\mu_{2}: P \rightarrow \mathbf{X}_{2}$ such that

- $\mu_{1}: P \rightarrow \mathbf{X}_{1}$ is a left $\Sigma_{1}$-space and a principal $\Sigma_{1}$-bundle over $\mathbf{X}_{2}$, with projection $\mu_{2}: P \rightarrow \mathbf{X}_{2}$;

- $\mu_{2}: P \rightarrow \mathbf{X}_{2}$ is a right $\Sigma_{2}$-space and a principal $\Sigma_{2}$-bundle over $\mathbf{X}_{1}$, with projection $\mu_{1}: P \rightarrow \mathbf{X}_{1}$;

- the two actions commute.

We will write $\mathbf{X}_{1} \stackrel{\mu_{1}}{\leftarrow} P \stackrel{\mu_{2}}{\rightarrow} \mathbf{X}_{2}$.

Example 1.1.32. A transitive Lie groupoid is Morita equivalent to its isotropy group at any point. In fact, if $\Sigma \rightrightarrows \mathbf{X}$ is transitive, then the $s$-fiber $s^{-1}(x)$ at any point is a principal $\Sigma$ bundle over the point (along the restriction of the target map), but also a principal $\Sigma_{x}$-bundle over $M$ along the (constant) restriction of the source map.

The following proposition - which is well known, see e.g. Lemma 1.28 in [47] - makes precise the claim that Morita equivalent Lie groupoids have the same "transverse geometry". Recall that the orbit space of a Lie groupoid $\Sigma \rightrightarrows \mathbf{X}$ is the quotient of $\mathbf{X}$ with respect to the equivalence relation identifying two points $x, y \in \mathbf{X}$ when there is an arrow $g \in \Sigma$ such that $s(g)=x, t(g)=y$.

Proposition 1.1.33. Any Morita equivalence of Lie groupoids $\Sigma_{1} \rightrightarrows \mathbf{X}_{1}, \Sigma_{2} \rightrightarrows \mathbf{X}_{2}$, with bibundle $\mathbf{X}_{1} \leftarrow P \rightarrow \mathbf{X}_{2}$, induces:

- an homeomorphism of the orbit spaces

$$
\Phi_{P}: \mathbf{X}_{1} / \Sigma_{1} \cong \mathbf{X}_{2} / \Sigma_{2}
$$

- for each $p \in P$, an isomorphism of isotropy groups at points $x \in \mathbf{X}_{1}$ and $y \in \mathbf{X}_{2}$ such that $\mu_{1}(p)=x$ and $\mu_{2}(p)=y$ :

$$
\varphi_{p}:\left(\Sigma_{1}\right)_{x} \cong\left(\Sigma_{2}\right)_{y}, \quad g_{1} \rightarrow g_{2}=\varphi_{p}\left(g_{1}\right)
$$

where $g_{2}$ is the only element of $\Sigma_{y}$ such that $g_{1} \cdot p=p \cdot g_{2}$;

- an isomorphism of the normal representations at points whose orbits are related by $\Phi_{P}$ (see below). 
Let us comment on the last point. A representation of a groupoid $\Sigma \rightrightarrows \mathbf{X}$ is a vector bundle $E \rightarrow \mathbf{X}$ together with an action of $\Sigma$ along $E \rightarrow \mathbf{X}$ itself, such that each arrow acts by a linear isomorphism. If $\mathcal{O}_{x}$ is the orbit of $x$ in $\mathbf{X}$, the restriction $\left.\Sigma\right|_{\mathcal{O}_{x}}$ is a groupoid admitting a representation on the normal bundle $\nu_{\mathcal{O}_{x}}$ to the orbit. An arrow $\left.g \in \Sigma\right|_{\mathcal{O}_{x}}, s(g)=y \in \mathcal{O}_{x}$, acts by

$$
g \cdot\left[v_{y}\right]=\left[d t\left(V_{g}^{s}\right)\right]
$$

where $V_{g}^{s}$ is any $s$-lift of $v_{y}$ at $g$. This representation of $\left.\Sigma\right|_{\mathcal{O}_{x}}$ on $\nu_{\mathcal{O}_{x}}$ is called normal representation. By restricting it, one gets a representation of the isotropy group $\Sigma_{x}$ on the normal space $\nu_{x}$ to the orbit of $x$ at $x$; this latter representation is called normal representation as well.

Remark 1.1.34. Observe that, when $\mathcal{G} \rightrightarrows \mathbf{X}$ is an étale Lie groupoid, there is a representation of $\mathcal{G}$ on $T \mathbf{X}$, because $s$ and $t$ are local diffeomorphisms. In fact, one sets

$$
g \cdot v_{y}=d t\left(V_{g}^{s}\right), \quad g \in \mathcal{G}, s(g)=y, v_{y} \in T_{x} \mathbf{X}
$$

where now $V_{g}^{s}$ is the $s$-lift of $v_{y}$ at $g$ (which exists and is unique because $s$ is a local diffeomorphism). This defines a representation of $\mathcal{G}$ on $T \mathbf{X}$.

Such a representation can be used to reconstruct the normal representation of $\mathcal{G}_{\mathcal{O}_{x}}$ on $\nu_{\mathcal{O}_{x}}$ for all $x \in \mathbf{X}$. First of all, the representation of $\Sigma$ on $T \mathbf{X}$ induces a representation of $\left.\mathcal{G}\right|_{\mathcal{O}_{x}}$ on $i^{*}: T_{\mathbf{X}}$, where $i: \mathcal{O}_{x} \hookrightarrow \mathbf{X}$ is the inclusion. Let now $\left[v_{y}\right] \in \nu_{\mathcal{O}_{x}}, y \in \mathcal{O}_{x}, v_{y} \in T_{y} \mathbf{X}$, and $v_{y}+w_{y} \in T_{y} \mathbf{X}, w_{y} \in T_{y} \mathcal{O}_{x} \hookrightarrow T_{y} \mathbf{X}$ be any representative of $\left[v_{y}\right] \in \nu_{\mathcal{O}_{x}}$. It is straightforward to check that, for all $g \in \mathcal{G}$ with $s(g)=y$,

$$
g \cdot w_{y} \in T_{t(g)} \mathcal{O}_{x} .
$$

Consequently, the quotient projection $i^{*} T \mathbf{X} \rightarrow \nu_{\mathcal{O}_{x}}$ is a map of $\mathcal{G}_{\mathcal{O}_{x}}$ representations.

It turns out that any principal $\Sigma$-bundle $P \rightarrow M$ can be "completed" and made into a bibundle, in the sense of the following proposition. Recall that we always assume the moment map of a $\Sigma$-space to be surjective and submersive.

Proposition 1.1.35. Let $\pi: P \rightarrow M$ be a principal $\Sigma$-bundle along $\mu: P \rightarrow \mathbf{X}$. Then $\mu: P \rightarrow \mathbf{X}$ is a principal Gauge $(P)$ bundle along $\pi: P \rightarrow M$ where Gauge $(P) \rightrightarrows M$ is the groupoid with:

- the space of arrows is isomorphic to the quotient of $P \times P$ by the diagonal action of $\Sigma$;

- the source and target projection are induced by the first and second projections to $P$;

- the multiplication is induced by the multiplication

$$
(P \times P)_{p r_{1}} \times_{p r_{2}}(P \times P) \rightarrow P \times P, \quad\left(\left(p_{1}, p_{2}\right),\left(p_{2}, p_{3}\right)\right) \rightarrow\left(p_{1}, p_{3}\right)
$$

on the pair groupoid $P \times P \rightrightarrows P$. 
Example 1.1.36. In the particular case when $\Sigma=G$ is a Lie group acting principally on $P \rightarrow M$, one discovers the classical gauge groupoid $P \times P / G \rightrightarrows M$. It is always transitive over $M$, its isotropy group at any point is isomorphic to $G$ and its $s$-fiber is isomorphic to $P$. Compare with example 1.1.32; any transitive Lie groupoid is isomorphic to the gauge groupoid of its $s$-fiber acted from the right by the isotropy group - Proposition 5.14 in [49]. By making use of this fact, one can often rephrase constructions in terms of transitive Lie groupoids into more familiar constructions in terms of principal group bundles.

The following proposition, which is well known (e.g. the proof of Theorem 4.2 in [68]), was one of our starting points.

Proposition 1.1.37. The categories of principal bundles of Morita equivalent groupoids are equivalent.

The construction of the equivalence is not so mysterious. Let $P$ be a $\left(\Sigma_{1}, \Sigma_{2}\right)$ bibundle, with moment maps $\mu_{1}: P \rightarrow \mathbf{X}_{1}$ and $\mu_{2}: P \rightarrow \mathbf{X}_{2}$. Let $P_{1}$ be a principal $\Sigma_{1}$-bundle over a manifold $M$ and let $\tau_{1}: P \rightarrow \mathbf{X}_{1}$ be the moment map of the action of $\Sigma_{1}$ on $P_{1}$. In order to construct a principal $\Sigma_{2}$-bundle $P_{2}$ over $M$ out of $P_{1}$, one considers the pullback $\mu_{1}^{*} P_{1} \cong P_{1} \times_{\mathbf{x}_{1}} P$. One then notices that $\mu_{1}^{*} P_{1}$ carries the principal diagonal action of $\Sigma_{1} \ltimes P$ and the action

$$
\left(\left(p, g_{2}\right),\left(p_{1}, p\right)\right) \in\left(P \rtimes \Sigma_{2}\right) \times\left(P_{1} \times \mathbf{x}_{1} P\right) \rightarrow\left(p_{1}, p \cdot g_{2}\right)
$$

of $P \rtimes \Sigma_{2}$ on the second factor. The quotient by $\Sigma_{1} \ltimes P$ is then a $\Sigma_{2}$-space and, in fact, a principal $\Sigma_{2}$-bundle $P_{2}$ over $M$; the action is from the right but we can, of course, turn it into a left action by precomposing with the inversion.

\section{Transitive geometries}

Let us now go back to Lie pseudogroups $\Gamma$ on some manifold $\mathbf{X}$ and to almost $\Gamma$-structures on manifolds $M$. We now assume that $\Gamma$ is transitive. Consequently, the $k$-th jet groupoid $J^{k} \Gamma$ is transitive (Remark 1.1.16). An almost $\Gamma$-structure of order $k$ on a manifold $M$ is a principal bundle

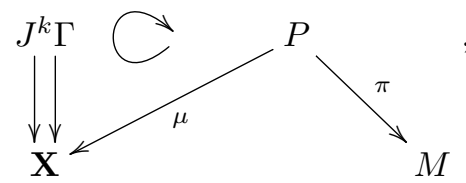

and, via proposition 1.1.37, this corresponds to a principal bundle

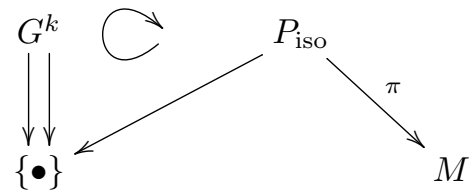

where $G^{k}:=\left(J^{k} \Gamma\right)_{x}$ is the isotropy group at some point $x \in M$. There is an explicit way of constructing $P_{\text {iso }}$ : we pick $x \in \mathbf{X}$ and restrict the action to the action of $\left(J^{k} \Gamma\right)_{x}$ on $P_{x}:=\mu^{-1}(x)$. Then we see that

$$
\left(J^{k} \Gamma\right)_{x}=\left\{j_{x}^{k} \varphi: \phi \in \Gamma, \phi(x)=x\right\} \subset G L_{n}^{k}(\mathbb{R}) .
$$


Notice that, if the structure is integrable, we have $P=J^{k} \mathcal{A}$ and we can write

$$
P_{x}:=\mu^{-1}(x)=\left\{j_{p}^{k} \phi: p \in M, \phi \in \mathcal{A}, \phi(p)=x\right\} \subset F r^{k}(M) .
$$

In other words, $P_{x}$ is a $G^{k}$-reduction of the bundle of $k$-frames of $M$; more precisely $P_{x}$ is given by those $k$-frames which are induced by the $\Gamma$-atlas.

When the structure is non integrable, we still have that $P$ is a reduction to $J^{k} \Gamma$ of the principal $J^{k} \Gamma^{n}$-bundle $J^{k} \mathcal{A}_{M}$, where $\mathcal{A}_{M}$ denotes a maximal atlas for $M$ and $\Gamma^{n}:=\operatorname{Diff}_{\text {loc }}\left(\mathbb{R}^{n}\right)$, Example 1.1.5. See Definition 1.1.28 and the discussion above it. This implies that, as in the integrable case, $P_{\text {iso }} \cong P_{x}:=\mu^{-1}(x)$ is a reduction of $F r^{k}(M)$ to $G^{k}=\left(J^{k} \Gamma\right)_{x}$. Reductions of frame bundles are well known to be a geometric mean of encoding structure on manifolds.

Definition 1.1.38. A $G$-structure of order $k$ on a manifold $M$ is a reduction of $F r^{k}(M)$ to a Lie subgroup $G \subset G L_{n}^{k}(\mathbb{R})$.

Example 1.1.39. Let $\Gamma=\Gamma_{G}$, where $G \subset G L_{n}(\mathbb{R})$ is a Lie subgroup (see Example 1.1.6). By considering almost $\Gamma$-structures of order one and passing to the isotropy group as explained above, we discover classical $G$-structures, see [18, 64]. For instance, the "almost" versions of the structures presented in Example 1.1.6 are:

- Riemannian metrics;

- almost complex structures;

- almost symplectic manifolds (i.e. manifolds with the choice of a nondegenerate 2-form - flatness corresponds to the form being close);

- codimension $k$-distributions;

- codimension 1-distributions with the choice of a 2-form which is non degenerate along the distribution.

Example 1.1.40. As for $\Gamma_{\text {cont }}$, see Example 1.1.7, the discussion from Example 1.1.17 shows that the corresponding $G$-structure is the same as the one from $\Gamma_{S p(k, 1)}$.

Let $\Gamma$ be a transitive pseudogroup on $\mathbf{X}$ and $M$ be a manifold. The discussion of this subsection shows that we can reinterpret almost $\Gamma$-structures of order $k$ on $M$ as principal $\left(J^{k} \Gamma\right)_{x}$-bundles over $M$. However, the last two examples suggest that, in general, this operation lead to some "loss of information". In fact, even though the $G$-structures induced by almost structures of order 1 for $\Gamma_{\text {cont }}$ and $\Gamma_{S p(k, 1)}$ are the same, the groupoids $J^{1} \Gamma_{\text {cont }}$ and $J^{1} \Gamma_{S p(k, 1)}$ (which are isomorphic, but not equal) behaves differently when one looks at integrability. Investigating this phenomenon is one of our motivations. Our starting approach is to use a stronger version of Morita equivalence, taking into account some additional structure on the jet groupoids, precisely the structure underlying the integrability problem. We briefly present such a structure below, and then proceed to give a more conceptual treatment in the following sections. 


\subsubsection{The Cartan distribution}

Recall that, when $R \subset J^{k}(M, N)$ is a PDE, a solution is a local section taking values in $R$ and being holonomic; that is, a map

$$
x \in U \subset M \rightarrow J^{k}(M, N), \quad x \rightarrow j_{x}^{k} f_{x}
$$

such that $f_{x}=f$ ( $U$ being some open in $M$ ). In simpler words, the section is a Taylor expansion of a map $f: U \rightarrow N$. Jet spaces come equipped with a canonical distribution detecting holonomic sections.

Definition 1.1.41. The Cartan distribution on the jet space $J^{k}(M, N)$ is the regular distribution $\mathrm{C}^{k}$ whose integral sections are precisely the holonomic sections.

Such a distribution indeed exists and is unique. We recall that it can be characterized as the kernel of the vector valued one form

$$
\omega^{k} \in \Omega^{1}\left(J^{k}(M, N), T^{s} J^{k-1}(M, N)\right), \quad \omega_{j_{x}^{k} f}^{k}=d p r^{k, k-1}-d\left(j^{k-1} f\right) \circ d s
$$

where $p r^{k, k-1}: J^{k}(M, N) \rightarrow J^{k-1}(M, N)$ is the canonical projection and $T^{s} J^{k-1}(M, N)$ is the vertical bundle of $s: J^{k-1}(M, N) \rightarrow M$. See [55, 69].

Lemma 1.1.42. Given a Lie pseudogroup $\Gamma$ over $\mathbf{X}$, the Cartan form $\omega^{k} \in$ $\Omega^{1}\left(J^{k}(\mathbf{X}, \mathbf{X}), T^{s} J^{k-1}(\mathbf{X}, \mathbf{X})\right)$ restricts to $J^{k} \Gamma$ as a regular one-form valued in $T^{s} J^{k-1} \Gamma$. Consequently, the Cartan distribution $\mathrm{e}^{k}$ restricts to $J^{k} \Gamma$ as a regular distribution.

See, e.g., [69]. We will keep the notation $\omega^{k}, \mathrm{e}^{k}$ to indicate the restrictions to $J^{k} \Gamma$ of the Cartan form/distribution on $J^{k}(\mathbf{X}, \mathbf{X})$.

The distribution $\mathrm{e}^{k}$ and the groupoid structure on $J^{k} \Gamma$ (see above) interact nicely; quite remarkably, this was noticed relatively late [55]. By "nicely" we mean that

- $\mathrm{e}^{k}$ is closed under multiplication (which is actually a consequence of the existence of local holonomic bisections around each point);

- $\mathrm{e}^{k} \cap \operatorname{Ker}(d s)$ is an involutive regular distribution.

In the terminology of [55], $\left(J^{k} \Gamma, \mathrm{C}^{k}\right)$ is a Pfaffian groupoid. The Pfaffian machinery is fundamental for our discussion. Since we will deal with it abstractly and in greater generality, we begin the next section with the precise definition of Pfaffian groupoid (Definition 1.2.1). We conclude this section by recalling the definition of Lie algebroid, that will be used in the rest of the chapter.

\subsubsection{Lie algebroids}

Recall that any Lie groupoid comes with an associated infinitesimal object, a Lie algebroid, i.e. a vector bundle $A \rightarrow \mathrm{X}$ equipped with

- a vector bundle map, called anchor, $\rho: A \rightarrow T M$;

- a Lie bracket on the space of sections $\Gamma(A)$; 
which satisfies the Leibniz identity

$$
[\alpha, f \beta]=f[\alpha, \beta]+L_{\rho(\alpha)}(f) \beta
$$

$\alpha, \beta \in \Gamma(A), f \in C^{\infty}(\mathbf{X})$. The Lie algebroid $A:=\operatorname{Lie}(\Sigma) \rightarrow \mathbf{X}$ of a Lie groupoid $\Sigma \rightarrow \mathbf{X}$ is constructed as follows:

- the total space $A$ is given by $\left.\operatorname{Ker}(d s)\right|_{\mathbf{X}}$ (recall that we can see $\mathbf{X}$ as an embedded submanifold of $\Sigma$ using the unit (bi)section of $\Sigma \rightrightarrows \mathbf{X}$ );

- the anchor map is the restriction $\left.d t\right|_{\operatorname{Ker}(d s) \mid \mathbf{x}} \rightarrow T \mathbf{X}$;

- the bracket on sections of $A$ is induced by the bracket of right invariant vector fields on $\Sigma \rightrightarrows \mathbf{X}$.

Let us clarify the last point. Given a Lie groupoid $\Sigma \rightrightarrows \mathbf{X}$, for any arrow $g \in \Sigma$ we have an induced smooth right multiplication

$$
R_{g}: s^{-1}(t(g)) \rightarrow s^{-1}(s(g)) .
$$

An $s$-vertical vector field $X$ on the arrow space $\Sigma$ is called right invariant if

$$
X_{R_{g}(h)}=d R_{g}\left(X_{h}\right)
$$

for all $h, g \in \Sigma$ such that $s(h)=t(g)$. There is a one to one correspondence between sections of the bundle $\left.\operatorname{Ker}(d s)\right|_{\mathbf{X}} \rightarrow \mathbf{X}$ and right invariant vector fields on $\Sigma$. One direction of the correspondence sends a section $\alpha$ of $\operatorname{Ker}(d s) \mid \mathbf{x} \rightarrow \mathbf{X}$ to the vector field $\alpha^{R}$ on $\Sigma$ defined by

$$
\alpha_{g}^{R}:=d R_{g} \alpha_{t(g)} ;
$$

the other direction of the correspondence sends a right invariant vector field $X$ on $\Sigma$ to the section of $\left.\operatorname{Ker}(d s)\right|_{\mathbf{X}} \rightarrow \mathbf{X}$ given by the restriction $\left.X\right|_{\mathbf{X}}$. Right invariant vector fields are closed with respect to the Lie bracket of vector fields on $\Sigma$. One then sets

$$
[\alpha, \beta]:=\left.\left[\alpha^{R}, \beta^{R}\right]\right|_{\mathbf{X}}, \quad \alpha, \beta \in \Gamma\left(\operatorname{Ker}(d s)_{\mathbf{X}}\right)
$$

and this defines a Lie bracket on the space of sections of $\left.\operatorname{Ker}(d s)\right|_{\mathbf{X}} \rightarrow \mathbf{X}$.

The construction of the Lie algebroid of a Lie groupoid is reminiscent of the construction of the Lie algebra of a Lie group, which is indeed a particular case any Lie group $G$ is a Lie groupoid over the point. Part of the classical Lie theory can be extended to Lie algebroids and Lie groupoids - e.g. [22] and references therein. In particular, we will make use of the fact that a Lie groupoid morphism induces a morphism of the corresponding Lie algebroids.

Let us first recall the definition of morphism of Lie algebroids. If $A_{1} \rightarrow \mathbf{X}_{1}$ and $A_{2} \rightarrow \mathbf{X}_{2}$ are Lie algebroids, then a morphism of Lie algebroids from $A_{1} \rightarrow$ $\mathbf{X}_{1}$ to $A_{2} \rightarrow \mathbf{X}_{2}$ is a vector bundle map

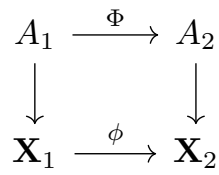

such that 
- $\rho_{A_{2}} \circ \Phi=d \phi \circ \rho_{A_{1}}$, where $\rho_{A_{1}}$ and $\rho_{A_{2}}$ are the anchor maps of $A_{1}$ and $A_{2}$ respectively;

- if $\alpha_{1}, \beta_{1}$ are sections of $A_{1} \rightarrow \mathbf{X}_{1}$ and

$$
\Phi \circ \alpha_{1}=\sum_{i} f_{i}\left(\alpha_{2}^{i} \circ \phi\right) \quad \Phi \circ \beta_{1}=\sum_{j} g_{j}\left(\beta_{2}^{j} \circ \phi\right),
$$

where $f_{i}, g_{j} \in C^{\infty}\left(\mathbf{X}_{1}\right), \alpha_{2}^{i}, \beta_{2}^{j}$ are sections of $A_{2} \rightarrow \mathbf{X}_{2}$, and both the sums are finite, then

$$
\begin{aligned}
\Phi\left(\left[\alpha_{1}, \beta_{1}\right]_{1}\right)=\sum_{i, j} f_{i} g_{j}[ & \left.\alpha_{2}^{i}, \beta_{2}^{j}\right]_{2} \circ \phi \\
& +\sum_{j} L_{\rho\left(\alpha_{1}\right)}\left(g_{j}\right)\left(\beta_{2}^{j} \circ \phi\right)-\sum_{i} L_{\rho\left(\beta_{1}\right)}\left(f_{i}\right)\left(\alpha_{2}^{i} \circ \phi\right),
\end{aligned}
$$

where $[,]_{1}$ and $[,]_{2}$ are the brackets of $A_{1}$ and $A_{2}$ respectively.

The last condition expresses the compatibility with the brackets of a morphism of Lie algebroids. Notice that, for any section $\alpha_{1} \in \Gamma\left(A_{1}\right)$, we can write $\Phi \circ \alpha_{1}$ as a finite combination

$$
\Phi \circ \alpha_{1}=\sum_{i} f_{i}\left(\alpha_{2}^{i} \circ \phi\right)
$$

where $f_{i} \in C^{\infty}\left(\mathbf{X}_{1}\right)$ and $\alpha_{2}^{i} \in \Gamma\left(A_{2}\right)$. This decomposition of $\Phi \circ \alpha_{1}$ is not unique; however, the condition expressing the compatibility with the brackets of a morphism of Lie algebroids does not depend on how sections of $A_{1} \rightarrow \mathbf{X}_{1}$ are decomposed.

Remark 1.1.43. Let $A_{1} \rightarrow \mathbf{X}$ and $A_{2} \rightarrow \mathbf{X}$ be Lie algebroids over the same base $\mathbf{X}$. A Lie algebroid morphism over the identity between $A_{1} \rightarrow \mathbf{X}$ and $A_{2} \rightarrow \mathbf{X}$ is a vector bundle map

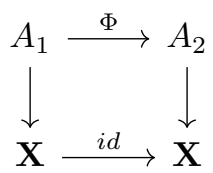

such that

- $\rho_{A_{2}} \circ \Phi=\rho_{A_{1}}$

- $[\Phi \circ \alpha, \Phi \circ \beta]_{2}=\Phi \circ[\alpha, \beta]_{1}$, for all $\alpha, \beta \in \Gamma\left(A_{1}\right)$.

Proposition 1.1.44. Let $\Sigma_{1} \rightrightarrows \mathbf{X}_{1}$ and $\Sigma_{2} \rightrightarrows \mathbf{X}_{2}$ be Lie groupoids and $A_{1} \rightarrow \mathbf{X}_{1}$, $A_{2} \rightarrow \mathbf{X}_{2}$ be their Lie algebroids. Let

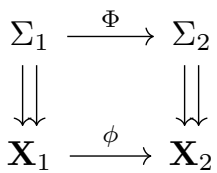


be a Lie groupoid morphism. The tangent map of $\Phi$ induces a Lie algebroid morphism

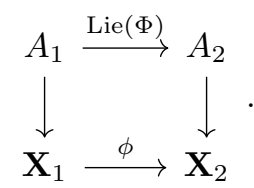

Furthermore, if $\Sigma_{3} \rightrightarrows \mathbf{X}_{3}$ is a Lie groupoid and there is a morphism $\Psi$ from $\Sigma_{2} \rightrightarrows \mathbf{X}_{2}$ to $\Sigma_{3} \rightrightarrows \mathbf{X}_{3}$ lifting $\psi: \mathbf{X}_{2} \rightarrow \mathbf{X}_{3}$, then

$$
\operatorname{Lie}(\Psi \circ \Phi)=\operatorname{Lie}(\Psi) \circ \operatorname{Lie}(\Phi) .
$$

We conclude this subsection by recalling that a Lie subalgebroid over $\mathbf{X}$ of a Lie algebroid $A \rightarrow \mathbf{X}$ is a sub-vector bundle $A^{\prime} \rightarrow \mathbf{X}$ such that the inclusion of $A^{\prime} \rightarrow \mathbf{X}$ into $A \rightarrow \mathbf{X}$ is a Lie algebroid morphism; in particular $\Gamma\left(A^{\prime}\right)$ is a subalgebra of $\Gamma(A)$. A subalgebroid $A^{\prime} \rightarrow \mathbf{X}$ of $A \rightarrow \mathbf{X}$ is called ideal if $\Gamma\left(A^{\prime}\right)$ is an ideal in $\Gamma(A)$.

Below, if the context is clear, we will often write $A \rightarrow \mathbf{X}$ in place of Lie $(\Sigma)$ to denote the Lie algebroid of a groupoid $\Sigma \rightrightarrows \mathbf{X}$.

\subsection{The Pfaffian language}

\subsubsection{Pfaffian groupoids}

Definition 1.2.1. A Pfaffian groupoid $(\Sigma, \omega, E)$ over $\mathbf{X}$ consists of a Lie groupoid $\Sigma \rightrightarrows \mathbf{X}$ together with a representation $E \rightarrow \mathbf{X}$ of $\Sigma$ and a differential form $\omega \in$ $\Omega^{1}\left(\Sigma, t^{*} E\right)$ such that

1. $\omega$ is multiplicative i.e.

$$
\left(m^{*} \omega\right)_{(g, h)}=\left(p r_{1}^{*} \omega\right)_{(g, h)}+g \cdot\left(p r_{2}^{*} \omega\right)_{(g, h)}, \quad(g, h) \in \Sigma \times_{\mathbf{X}} \Sigma
$$

2. $\omega$ has constant rank;

3. $\omega$ is $s$-transversal, i.e $T \Sigma=\operatorname{Ker}(\omega)+\operatorname{Ker}(d s) \subseteq T \Sigma$

4. the sub-bundle

$$
\mathfrak{g}_{\mathbf{X}}:=\left.(\operatorname{Ker}(\omega) \cap \operatorname{Ker}(d s))\right|_{\mathbf{x}} \subseteq A
$$

is a Lie subalgebroid of $A:=\operatorname{Lie}(\Sigma)$;

5. it holds

$$
\operatorname{Ker}(\omega) \cap \operatorname{Ker}(d t)=\operatorname{Ker}(\omega) \cap \operatorname{Ker}(d s) .
$$

We call $\mathfrak{g}_{\mathbf{X}}$ the symbol space of $(\Sigma, \omega, E)$. A Pfaffian groupoid $(\Sigma, \omega, E)$ is called full if the form $\omega$ is pointwise surjective. A holonomic bisection of $(\Sigma, \omega, E)$ is a local bisection $\sigma: U \subset \mathbf{X} \rightarrow \Sigma$ such that $\sigma^{*} \omega=0$.

The main examples to have in mind are the jet groupoids of a Lie pseudogroup together with their Cartan form - see Example 1.2.14 below. 
Remark 1.2.2. One can replace the condition on $\mathfrak{g}_{\mathbf{X}}$ with the involutivity of the distribution $\mathfrak{g}(\omega):=\operatorname{Ker}(\omega) \cap \operatorname{Ker}(d s)$.

Remark 1.2.3. When $(\Sigma, \omega, E)$ is a Pfaffian groupoid, the unit bisection $\mathbf{X} \hookrightarrow \Sigma$ is holonomic - as one readily sees from the multiplicativity equation.

Remark 1.2.4. Actually, the $s$-transversality condition in Definition 1.2.1 follows from the multiplicativity condition and the fact that $\omega$ has constant rank. See [15], Proposition 3.4.12.

We list below some properties of Pfaffian groupoids that are relevant for our discussion; see [55] for details.

Proposition 1.2.5. Let $(\Sigma, \omega, E), \omega \in \Omega\left(\Sigma, t^{*} E\right)$, be a Pfaffian groupoid. Then the following facts hold true.

- The quotient $A / \mathfrak{g}_{\mathbf{X}} \rightarrow \mathbf{X}$ is a representation of $\Sigma \rightrightarrows \mathbf{X}$. The action is given by

$$
g \cdot\left[V_{x}\right] \rightarrow\left[d R_{g^{-1}} \cdot d m\left(\operatorname{hor}_{g}^{\omega}\left(d t\left(V_{x}\right)\right), V_{x}\right)\right]
$$

where $g \in \Sigma, x=s(g),\left.\left[V_{x}\right] \in \operatorname{Ker}(d s)\right|_{\mathbf{X}} / \mathfrak{g}_{\mathbf{X}}$ and $\operatorname{hor}_{g}^{\omega}\left(d t\left(V_{x}\right)\right.$ is any s-lift of $d t\left(V_{x}\right)$ tangent to $\operatorname{Ker}(\omega)$ at $g$.

- The tangent space $T \mathbf{X} \rightarrow \mathbf{X}$ is a representation of $\Sigma \rightrightarrows \mathbf{X}$. The action is given by

$$
\Sigma \times \mathbf{X} T \mathbf{X} \rightarrow T \mathbf{X}, \quad\left(g, v_{x}\right) \rightarrow d t\left(\operatorname{hor}_{g}^{\omega}\left(v_{x}\right)\right)
$$

where $\operatorname{hor}_{g}^{\omega}\left(v_{x}\right)$ is any s-lift of $v_{x}$ tangent to $\operatorname{Ker}(\omega)$ at $g \in \Sigma$.

- The anchor map $\rho: A / \mathfrak{g}_{\mathbf{X}} \rightarrow T \mathbf{X}$ of $A / \mathfrak{g}_{\mathbf{X}}$ is a map of representations.

Remark 1.2.6. If $(\Sigma, \omega, E)$ is a Pfaffian groupoid, the representation on $T \mathbf{X}$ from the above proposition extends the normal representation.

Moreover, there is an induced representation of the isotropy group $\Sigma_{x}$ on $A_{x} / \mathfrak{g}_{\mathbf{X}}$. If $\mathfrak{g}_{x}$ denotes the Lie algebra of $\Sigma_{x}$ carrying the adjoint representation, we see that the map

$$
\mathfrak{g}_{x} \rightarrow A_{x} / \mathfrak{g}_{\mathbf{X}}
$$

induced by the quotient map is a map of representations.

In what follows, we denote by $T_{\mathbf{X}} \Sigma$ the pullback to $\mathbf{X}$ of $T \Sigma$ via the unit map.

Proposition 1.2.7. Let $(\Sigma, \omega, E), \omega \in \Omega\left(\Sigma, t^{*} E\right)$, be a Pfaffian groupoid. The image $\omega\left(T_{\mathbf{X}} \Sigma\right)$ is a vector bundle isomorphic to $A / \mathfrak{g}_{\mathbf{X}}$ and possesses a canonical Lie algebroid structure making

$$
\left.\omega\right|_{\operatorname{Ker}(d s) \mid \mathbf{x}}:\left.\operatorname{Ker}(d s)\right|_{\mathbf{x}} \rightarrow \omega\left(\left.\operatorname{Ker}(d s)\right|_{\mathbf{x}}\right)
$$

into a Lie algebroid map.

Furthermore, the inclusion $\omega\left(T_{\mathbf{X}} \Sigma\right) \hookrightarrow E$ is a map of representation.

Finally, the map

$$
\omega\left(T_{\mathbf{X}} \Sigma\right) \rightarrow A / \mathfrak{g}_{\mathbf{X}}, \quad \omega\left(v_{g}\right) \rightarrow\left[v_{g}^{\operatorname{Ker}(d s)}\right]
$$

where $v_{g} \in T_{g} \Sigma$ and $v_{g}=v_{g}^{\operatorname{Ker}(d s)}+v_{g}^{\operatorname{Ker}(\omega)}, v_{g}^{\operatorname{Ker}(d s)} \in \operatorname{Ker}_{g}(d s), v_{g}^{\operatorname{Ker}(\omega)} \in \operatorname{Ker}_{g}(\omega)$, is an isomorphism of representations. 
In particular, given a full Pfaffian groupoids $(\Sigma, \omega, E), \omega \in \Omega^{1}\left(\Sigma, t^{*} E\right)$, the representation $E \rightarrow \mathbf{X}$ possesses a Lie algebroid structure that depends only on $\Sigma$ and $\operatorname{Ker}(\omega)$.

Definition 1.2.8. An extended Pfaffian groupoid over $\mathbf{X}$ is a Pfaffian groupoid $(\Sigma, \omega, E)$ over $\mathbf{X}, \omega \in \Omega^{1}\left(\Sigma, t^{*} E\right)$, together with a Lie algebroid structure on the coefficient space $E \rightarrow \mathbf{X}$ such that

$$
A / \mathfrak{g}_{\mathbf{X}} \cong \omega\left(T_{\mathbf{X}} \Sigma\right) \hookrightarrow E
$$

is a map of Lie algebroids over $\mathbf{X}$.

For full Pfaffian groupoids $(\Sigma, \omega, E)$ we have $A / \mathfrak{g}_{\mathbf{X}} \cong E$; consequently, a full Pfaffian groupoid possesses one and only one structure of extended Pfaffian groupoid.

As Proposition 1.2.7 shows, given a full Pfaffian groupoid $(\Sigma, \omega, E)$, $\omega \in \Omega^{1}\left(\Sigma, t^{*} E\right)$, the representation $E \rightarrow \mathbf{X}$ can be reconstructed out of the groupoid $\Sigma$ and the distribution $\operatorname{Ker}(\omega)$. This suggests the following proposition.

Proposition 1.2.9. Let $(\Sigma, \omega, E), \omega \in \Omega^{1}\left(\Sigma, t^{*} E\right)$, be a full Pfaffian groupoid. The distribution $\mathrm{e}:=\operatorname{Ker}(\omega)$ is:

- multiplicative, in the sense that $\mathcal{C} \subset T \Sigma$ is a Lie subgroupoid of the tangent groupoid $T \Sigma \rightrightarrows T \mathbf{X}$ (whose structure maps are obtained differentiating those of $\Sigma \rightrightarrows \mathbf{X})$;

- $s$-transversal, in the sense that $\mathcal{C}+\operatorname{Ker}(d s)=T \Sigma$;

- such that $\mathfrak{g}(\mathcal{C})=\mathcal{C} \cap \operatorname{Ker}(d s)$ is involutive;

- such that $\mathcal{C} \cap \operatorname{Ker}(d s)=\mathcal{C} \cap \operatorname{Ker}(d t)$.

On the other hand, if

- $\Sigma \rightrightarrows \mathbf{X}$ is a Lie groupoid,

- $\mathcal{C} \subset T \Sigma$ is a distribution on $\Sigma$ having the four properties listed above,

- $p r: T \Sigma \rightarrow T \Sigma / \mathcal{C}$ is the quotient projection,

then the triple $(\Sigma, p r, T \Sigma / \mathcal{C})$ is a full Pfaffian groupoid.

See Propositions 6.1.13 and 6.1.14 in [55] for a proof.

Motivated by the Proposition 1.2.9, we give the following alternative definition of (not necessarily full) Pfaffian groupoid.

Definition 1.2.10. A Pfaffian groupoid $(\Sigma, \mathcal{C}, E)$ over $\mathbf{X}$ is the datum of

- a Lie groupoid $\Sigma \rightrightarrows \mathbf{X}$;

- a regular distribution $\mathcal{C}$ on $\Sigma$ that is:

- multiplicative;

- transversal to $s$; 
- such that $\mathfrak{g}(\mathcal{C}):=\mathcal{C} \cap \operatorname{Ker}(d s)$ is involutive;

- such that $\mathfrak{g}(\mathcal{C})=\mathcal{C} \cap \operatorname{Ker}(d t)$;

- a representation $E \rightarrow \mathbf{X}$ of $\Sigma \rightrightarrows \mathbf{X}$ admitting the following representation on the normal bundle $\nu_{\mathcal{C}} \mid \mathbf{X}$ as a sub-representation:

$$
g \cdot\left[V_{x}\right]:=\left[d R_{g^{-1}} \cdot d m\left(\operatorname{hor}_{g}^{\mathrm{e}}\left(d t\left(V_{x}\right)\right), V_{x}\right)\right],
$$

where $g \in \Sigma, x=s(g)$, and $\left[V_{x}\right] \in \nu_{\mathcal{C}} \mid \mathbf{x}$ is represented by $V_{x} \in \operatorname{Ker}(d s)$ - see the remark below.

Remark 1.2.11. Let us clarify the last point in Definition 1.2.10. First of all, $\operatorname{hor}_{g}^{\mathcal{C}}\left(d t\left(V_{x}\right)\right)$ denotes any $s$-lift of $d t\left(V_{x}\right)$ tangent to $\mathcal{C}$ at $g \in \Sigma$. We observe that, given a Lie groupoid $\Sigma \rightrightarrows \mathbf{X}$, the normal bundle $\nu_{\mathbf{X}}$ can be identified with $\left.\operatorname{Ker}(d s)\right|_{\mathbf{x}}$ (the total space of $A=\operatorname{Lie}(\Sigma)$ ). We also observe that, given a distribution $\mathcal{C}$ on $\Sigma$ that is multiplicative and transversal to $s,\left.\mathcal{C}\right|_{\mathbf{X}}$ contains $T \mathbf{X}$ as the submanifold of units with respect to the multiplication $d m$; in fact, multiplicativity implies that $\left.\mathcal{C}\right|_{\mathbf{X}}$ contains the vectors in $d s\left(\left.\mathcal{C}\right|_{\mathbf{X}}\right)$ as units with respect to the multiplication, while transversality to $s$ implies that $d s\left(\left.\mathcal{C}\right|_{\mathbf{X}}\right)=T \mathbf{X}$. Consequently, if $\mathcal{C}$ has the properties listed Definition 1.2.10, we may identify $\nu_{\mathrm{C}}$ with $\nu_{\mathbf{X}} / \mathfrak{g}(\mathcal{C})$, and it follows that equivalence classes in $\nu_{\mathcal{C}}$ can be represented by $s$-vertical vectors. The fact that the expression

$$
g \cdot\left[V_{x}\right]:=\left[d R_{g^{-1}} \cdot d m\left(\operatorname{hor}_{g}^{\mathcal{e}}\left(d t\left(V_{x}\right)\right), V_{x}\right)\right],
$$

where $g \in \Sigma, x=s(g)$, and $\left[V_{x}\right] \in \nu_{\mathcal{C}} \mid \mathbf{x}$ is represented by $V_{x} \in \operatorname{Ker}(d s)$, defines a representation of $\Sigma$ on $\left.\nu_{\mathrm{C}}\right|_{\mathrm{X}}$ can be checked directly using the properties of $\mathcal{C}$.

Definition 1.2.12. An extended Pfaffian groupoid over $\mathrm{X}$ is a Pfaffian groupoid $(\Sigma, \mathrm{C}, E)$ over $\mathbf{X}$ such that $E \rightarrow \mathbf{X}$ is a Lie algebroid and the injective representation map

$$
\left.\nu_{\mathcal{C}}\right|_{\mathbf{x}} \hookrightarrow E, \quad\left[V_{x}\right] \rightarrow \alpha_{x}
$$

is a Lie algebroid morphism (for the Lie algebroid structure on the left hand side see Proposition 1.2.7 and Proposition 1.2.9).

In what follows, we will alternatively use the form description (Definition 1.2.1) and the distribution one (Definition 1.2.10) according to convenience. We will often use the notations $(\Sigma, \omega)$ and $(\Sigma, \mathcal{C})$ when working with full Pfaffian groupoids. In that case, the reader should keep in mind that $\omega \in \Omega^{1}\left(\Sigma, t^{*} E\right)$ is a vector valued form and its coefficent space is the pullback of a representation $E \rightarrow \mathbf{X}$ of $\Sigma$ possessing a Lie algebroid structure (Proposition 1.2.7); moreover, $E \cong A / \mathfrak{g}_{\mathbf{X}}$ both as an algebroid and as a representation.

Definition 1.2.13. Let $\left(\Sigma_{1}, \omega_{1}, E_{1}\right)$ and $\left(\Sigma_{2}, \omega_{2}, E_{2}\right)$ be Pfaffian groupoids over $\mathbf{X}_{1}$ and $\mathbf{X}_{2}$ respectively. A morphism of Pfaffian groupoids from $\left(\Sigma_{1}, \omega_{1}, E_{1}\right)$ to $\left(\Sigma_{2}, \omega_{2}, E_{2}\right)$ is a pair $(\Phi, \Psi)$, where $\Phi$ is a Lie groupoid morphism from $\Sigma_{1}$ to $\Sigma_{2}$ and $\Psi$ is a morphism of representations $\Psi: E_{1} \rightarrow E_{2}$, such that $\Phi^{*} \omega_{2}=\Psi \circ \omega_{1}$ holds. If both groupoids are extended Pfaffian and $\Psi$ is also an morphism of Lie algebroids, then $(\Phi, \Psi)$ is a morphism of extended Pfaffian groupoids. 
Recall that a morphism of Lie groupoids $\Phi: \Sigma_{1} \rightarrow \Sigma_{2}$ covers a smooth map $f: \mathbf{X}_{1} \rightarrow \mathbf{X}_{2}$. By "morphism of representations" we mean the following.

- $\Psi$ is a morphism of vector bundles from $\pi_{1}: E_{1} \rightarrow \mathbf{X}_{1}$ to $\pi_{2}: E_{2} \rightarrow \mathbf{X}_{2}$ covering $f: \mathbf{X}_{1} \rightarrow \mathbf{X}_{2}$;

- one has

$$
\Psi\left(g_{1} \cdot e_{1}\right)=\Phi\left(g_{1}\right) \cdot \Psi\left(e_{1}\right)
$$

for all $e_{1} \in E_{1}, g_{1} \in \Sigma_{1}$ such that $\pi_{1}\left(g_{1}\right)=s\left(g_{1}\right)$.

If both $\left(\Sigma_{1}, \omega_{1}\right)$ and $\left(\Sigma_{2}, \omega_{2}\right)$ in Definition 1.2.13 are full Pfaffian groupoids, then $\Psi$ is uniquely determined by $\Phi$. In fact, after we make the identifications $E_{1} \cong A_{1} /\left(\mathfrak{g}_{1}\right) \mathbf{X}_{1}$ and $E_{2} \cong A_{2} /\left(\mathfrak{g}_{2}\right)_{\mathbf{X}_{2}}$ from the third part of Proposition 1.2.7, $\Psi$ is forced to be the map induced by the morphism of Lie algebroids $\operatorname{Lie}(\Phi): A_{1} \rightarrow$ $A_{2}$ (see Proposition 1.1.44).

Example 1.2.14. (Main example). As anticipated, when $\Gamma$ is a Lie pseudogroup over $\mathbf{X}$, the jet groupoid $J^{k} \Gamma \rightrightarrows \mathbf{X}$ is Pfaffian when equipped with the Cartan form $\omega^{k}$ from Subsection 1.1.4. The symbol space is given by the vertical bundle of the projection $J^{k} \Gamma \rightarrow J^{k-1} \Gamma$ restricted to $\mathbf{X}$ - as one readily checks using the explicit formula for $\omega^{k} .\left(J^{k} \Gamma, \omega^{k}\right)$ is full: the coefficient space of $\omega^{k}$ is the vertical bundle $T^{s} J^{k-1} \Gamma$ of the source projection of $J^{k-1} \Gamma$, which is isomorphic to the quotient of $T^{s} J^{k} \Gamma$ with respect to the vertical bundle of $J^{k} \Gamma \rightarrow J^{k-1} \Gamma$.

Notice that, for all $k \in \mathbb{N}, k \geq 1,\left(J^{k} \Gamma, \omega^{k}\right) \rightarrow\left(J^{k-1} \Gamma, \omega^{k-1}\right)$ is a morphism of Pfaffian groupoids.

\subsubsection{Pfaffian groups}

Recall that, when $\Sigma \rightrightarrows \mathbf{X}$ is a Lie groupoid, the Maurer-Cartan form $\omega_{M C}$ of $\Sigma$ is the $s$-foliated $t^{*} A$-valued form

$$
\omega_{M C}: \operatorname{Ker}(d s) \rightarrow t^{*} A \cong t^{*}\left(\left.\operatorname{Ker}(d s)\right|_{\mathbf{x}}\right), \quad V_{g} \rightarrow\left(g, d R_{g^{-1}}\left(V_{g}\right)\right)
$$

equivalently encoded into the bundle map

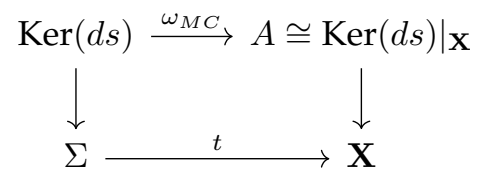

sending $V_{g}$ to $d R_{g^{-1}}\left(V_{g}\right) \in A_{t(g)}$.

Example 1.2.15. When $\Sigma \rightrightarrows \mathbf{X}$ is a Lie group $G$, the Maurer-Cartan form becomes the usual Maurer-Cartan form of a Lie group (defined using right multiplication).

Lemma 1.2.16. Let $(\Sigma, \omega, E)$ be a Pfaffian groupoid. The map $\left.\omega\right|_{\operatorname{Ker}(d s)}: \operatorname{Ker}(d s) \rightarrow E$ coincide with the composition $\left.\omega\right|_{\mathbf{X}} \circ \omega_{M C}$.

In the statement above, $\left.\omega\right|_{\mathbf{X}}$ denotes the restriction of $\omega$ to $T_{\mathbf{X}} \Sigma$, the pullback of $T \Sigma$ to $\mathbf{X}$ by the unit map. 
Proof. Given

$$
\omega: T \Sigma \rightarrow E, \quad v_{g} \rightarrow \omega\left(v_{g}\right) .
$$

we use the multiplicativity equation

$$
\left(m^{*} \omega\right)_{(g, h)}=\left(p r_{1}^{*} \omega\right)_{(g, h)}+g \cdot\left(p r_{2}^{*} \omega\right)_{g, h}
$$

on a pair of vectors of the form $\left(v_{g}, 0_{g^{-1}}\right), v_{g} \in \operatorname{Ker}_{g}(d s)$. We get

$$
\omega\left(d R_{g^{-1}}\left(v_{g}\right)\right)=\omega\left(v_{g}\right), \quad v_{g} \in \operatorname{Ker}_{g}(d s)
$$

that implies the claim.

Below, we adopt the following standard convention: when $\mathbf{X}$ is a manifold and $V$ is a vector space we write $\Omega^{1}(\mathbf{X}, V)$ for the space $\Omega^{1}(\mathbf{X}, \mathbf{X} \times V)$ of forms on $\mathbf{X}$ valued into the trivial bundle $\mathbf{X} \times V$. Moreover, $e$ will be used to denote the identity element in a Lie group. The following Proposition and its proof should be compared with Example 3.4.6 in [15].

Proposition 1.2.17. (Characterization of Pfaffian groups). Let $G$ be a Lie group and $V$ be a representation of $G$. Let be $\omega \in \Omega^{1}(G, V)$. Then $\omega$ makes the triple $(G, \omega, V)$ into a Pfaffian group over the point if and only if

- $\omega$ is right invariant;

- $\omega_{e}: \mathfrak{g} \rightarrow V$ is a map of representations (the left hand side carries the adjoint representation).

In fact, there is a one to one correspondence between Pfaffian group structures $(G, \omega, V)$ and representation maps

$$
l: \mathfrak{g} \rightarrow V .
$$

Proof. If $(G, \omega, V)$ is a Pfaffian group, then $\omega$ is right invariant and $\omega_{e}$ is a map of representations thanks to multiplicativity. In fact

$$
\left(m^{*} \omega\right)_{(g, h)}=\left(p r_{1}^{*} \omega\right)_{(g, h)}+g \cdot\left(p r_{2}^{*} \omega\right)_{(g, h)}, \quad g, h \in G
$$

implies:

- left equivariance

$$
\omega_{g h} \circ d L_{g}=g \cdot \omega_{h}, \quad g, h \in G
$$

- right invariance

$$
\omega_{g h} \circ d R_{h}=\omega_{g} \quad g, h \in G ;
$$

from which it follows

$$
\omega_{e} \circ d L_{g} \circ d R_{g^{-1}}=g \cdot \omega_{e}
$$

i.e. $\omega_{e}$ is a map of representations. If viceversa $\omega_{e}$ is a map of representations and $\omega$ is right invariant, then $\omega$ is also left equivariant. Multiplicativity of $\omega$ follows since

$$
\left(m^{*} \omega\right)_{(g, h)}\left(v_{g}, v_{h}\right)=\left(m^{*} \omega\right)_{(g, h)}\left(v_{g}, 0_{h}\right)+\left(m^{*} \omega\right)_{(g, h)}\left(0_{g}, v_{h}\right)
$$


notice that we are using the fact that $G$ is a Lie group - in particular, the unit space of $G$ is the point.

If now

$$
l: \mathfrak{g} \rightarrow V
$$

is a map of representations, then there is one and only one induced right invariant form $\omega_{l} \in \Omega^{1}(G, V)$ such that $\left(\omega_{l}\right)_{e}=l$. Consequently, the one to one correspondence from the claim holds true.

Corollary 1.2.18. Let $(G, \omega, V)$ be a Pfaffian group. Then

$$
\omega=l \circ \omega_{M C}
$$

where $l: \mathfrak{g} \rightarrow V$ is the map of representations from Proposition 1.2.17-i.e. $\omega_{e}$.

Proof. By Lemma 1.2.16 together with Proposition 1.2.17.

Remark 1.2.19. If $(\Sigma, \omega, E)$ is a Pfaffian groupoid, then multiplicativity implies that

- $\left.\omega\right|_{\operatorname{Ker}(d t)}$ is left equivariant;

- $\left.\omega\right|_{\operatorname{Ker}(d s)}$ is right invariant;

- for all $x \in \mathbf{X}$ the form $\left.\omega_{x}\right|_{\Sigma_{x}}: T_{x} \Sigma_{x} \rightarrow E_{x}$ is a map of representations;

via the same argument as in the proof of Proposition 1.2.17. However, these properties do not characterize Pfaffian forms on Lie groupoids. Notice that the fact that $\left.\omega_{x}\right|_{\Sigma_{x}}$ is a map of representations was already a consequence of Proposition 1.2.7. See also [15], Lemma 3.4.11.

Proposition 1.2.20. (Isotropies of Pfaffian groupoids). Let $(\Sigma, \omega, E)$ be a Pfaffian groupoid over $\mathbf{X}$. Then the triple $\left(\Sigma_{x},\left.\omega\right|_{\Sigma_{x}}, E_{x}\right)$ is a Pfaffian group. Furthermore for all $x, y \in \mathbf{X}$ and $g \in \Sigma$ such that $s(g)=x, t(g)=y$ the isomorphism of Lie groups

$$
\phi_{g}: \Sigma_{x} \rightarrow \Sigma_{y}, \quad h \rightarrow g h g^{-1}
$$

and the isomorphism of representations

$$
\psi_{g}: E_{x} \rightarrow E_{y}, \quad \alpha_{x} \rightarrow g \cdot \alpha_{y}
$$

make $\left(\psi_{g}, \phi_{g}\right)$ into an isomorphism of Pfaffian groups from $\left(\Sigma_{x},\left.\omega\right|_{\Sigma_{x}}, E_{x}\right)$ to $\left(\Sigma_{y},\left.\omega\right|_{\Sigma_{y}}, E_{y}\right)$.

Proof. The fact that the triple $\left(\Sigma_{x},\left.\omega\right|_{\Sigma_{x}}, E_{x}\right)$ is a Pfaffian group is immediate. The fact that the coniugation by $g$ is a Pfaffian isomorphism follows from the equivariance and invariance properties of $\omega$ coming from multiplicativity, see Remark 1.2.19.

Remark 1.2.21. Let $(\Sigma, \omega, E)$ be a full Pfaffian groupoid over $\mathbf{X}$ - i.e. $\omega$ is pointwise surjective. Its isotropy $\left(\Sigma_{x},\left.\omega\right|_{\Sigma_{x}}, E_{x}\right)$ is almost never a full Pfaffian group; that happens if and only if the Lie groupoid $\Sigma$ is a bundle of Lie groups over $\mathbf{X}$.

Let $(\Sigma, \omega, E)$ be an extended-Pfaffian groupoid over $\mathbf{X}$ - see Definition 1.2.8. The triple $\left(\Sigma_{x},\left.\omega\right|_{\Sigma_{x}}, E_{x}\right)$ is almost never an extended Pfaffian group in such a way that the inclusion map induces an extended Pfaffian morphism; that happens if and only if the Lie algebroid $E$ is a bundle of Lie algebras over $\mathbf{X}$. 


\subsubsection{Principal Pfaffian bundles}

Let $(\Sigma, \omega, E), \omega \in \Omega^{1}\left(\Sigma, t^{*} E\right)$ be a Pfaffian groupoid over $\mathbf{X}$.

Definition 1.2.22. A principal $(\Sigma, \omega, E)$-bundle over $M$ is a principal $\Sigma$-bundle $\pi: P \rightarrow M$ with moment map $\mu: P \rightarrow \mathbf{X}$ together with a one form $\theta \in$ $\Omega^{1}\left(P, \mu^{*} E\right)$ such that

- the action $a: \Sigma \times_{\mathbf{X}} P \rightarrow P$ of $\Sigma$ is multiplicative with respect to $\omega$ and $\theta$, i.e.

$$
\left(a^{*} \theta\right)_{(g, p)}=\left(p r_{1}^{*} \omega\right)_{(g, p)}+g \cdot\left(p r_{2}^{*} \theta\right)_{(g, p)}, \quad(g, p) \in \Sigma \times_{\mathbf{X}} P
$$

- $\operatorname{Ker}(d \mu) \cap \operatorname{Ker}(\theta)=\operatorname{Ker}(d \pi) \cap \operatorname{Ker}(\theta)$.

The principal bundle is called full if $\theta \in \Omega^{1}\left(P, \mu^{*} E\right)$ is pointwise surjective. A local section

$$
\sigma: U \subset M \rightarrow P
$$

is called holonomic if $\sigma^{*}(\theta)=0$.

When there is no need to emphasize the Pfaffian groupoid $(\Sigma, \omega, E)$, we will use the terminology principal Pfaffian bundle.

Example 1.2.23. If $\Gamma$ is a Lie pseudogroup over $\mathbf{X}$, an almost $\Gamma$-structure of order $k$, Definition 1.1.28,

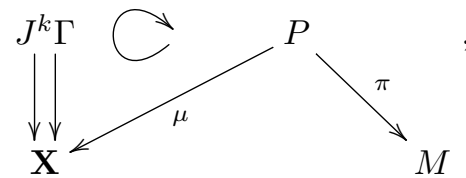

can be given the structure of principal Pfaffian bundle by taking the restriction to $P$ of the Cartan distribution on $J^{k}(M, \mathbf{X})$, see Subsection 1.1.4. If $P$ is integrable, then $P=J^{k} \mathcal{A}$ for some $\Gamma$-atlas $\mathcal{A}$ on $M$, Definition 1.1.28. In this case, around each point $j_{x}^{k} \phi \in J^{k} \mathcal{A}$ we have the section

$$
y \in \operatorname{Dom}(\phi) \subset M \rightarrow j_{y}^{k} \phi,
$$

which is holonomic. This property is in fact equivalent to integrability of the $\Gamma$ structure $P$. That is, if, for each $p$, one has a holonomic section of $(P, \theta)$ through $p$, then $P \subset J^{k}(M, \mathbf{X})$ is integrable in the sense of Definition 1.1.28. In fact, given an open cover $\left\{U_{i}\right\}_{i \in I}$ of $M$ such that for each $U_{i}$ one has a holonomic section $\sigma: U \subset M \rightarrow P \subset J^{k}(M, \mathbf{X})$, one can construct the $\Gamma$-atlas $\left\{\left(U_{i}, \mu \circ \sigma_{i}\right)\right\}_{i \in I}$.

Of course, an analogous definition can be given where the groupoid acts from the right - see also Remark 1.3.3 later on.

We will picture a principal $(\Sigma, \omega, E)$-bundle as

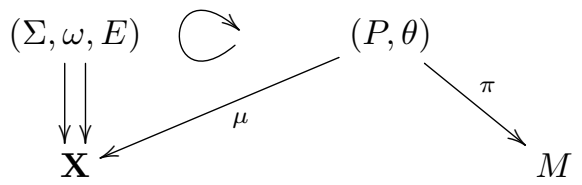

Observe that $\operatorname{Ker}(d \pi) \cap \operatorname{Ker}(\theta)$ is involutive since $\mathfrak{g}(\omega)$ is so and the infinitesimal action induces an isomorphism $\mu^{*} \operatorname{Ker}(d s) \cong \operatorname{Ker}(d \pi)$ which sends $\mathfrak{g}(\omega)$ to $\operatorname{Ker}(d \pi) \cap \operatorname{Ker}(\theta)$ because of multiplicativity. 
Remark 1.2.24. We observe that fullness of $(P, \theta, \Phi)$ follows from fullness of $(\Sigma, \omega, E)$. On the other hand, if $\operatorname{Ker}(\theta)$ is required to be transverse to $\operatorname{Ker}(d \mu)-$ i.e. if $(P, \theta)$ is a Pfaffian bundle according to [55], Definition 3.1.1 - then fullness of $(\Sigma, \omega, E)$ follows from fullness of $(P, \theta, \Phi)$. At a first sight, this seems to suggest that the definition of principal "Pfaffian" bundle should be modified to include such a property (which is satisfied by $(\Sigma, \omega, E)$ acting on itself from the left). However, modifying the definition in this way would rule out some examples that we think are of great importance.

Most notably, given a principal $(\Sigma, \omega, E)$-bundle over $\mathbf{X}$ and $x \in \mathbf{X}$, we can look at the action of the isotropy group $\Sigma_{x}$ on the source fiber $s^{-1}(x)$. If we equip $s^{-1}(x)$ with the form $\left.\omega\right|_{s^{-1}(x)}$, we get a principal $\left(\Sigma_{x},\left.\omega\right|_{\Sigma_{x}}, E_{x}\right)$-bundle according to Definition 1.2.22; however, $\operatorname{Ker}\left(\left.\omega\right|_{s^{-1}(x)}\right)$ is not transverse to $\operatorname{Ker}\left(d\left(\left.t\right|_{s^{-1}(x)}\right)\right)$ in fact, the two distributions coincide. If $(\Sigma, \omega, E)$ is full, so is $\left(s^{-1}(x),\left.\omega\right|_{s^{-1}(x)}\right)$; however, $\left(\Sigma_{x},\left.\omega\right|_{\Sigma_{x}}, E_{x}\right)$ is not full in general.

Compare also with Proposition 1.3.15.

In terms of distributions (see Definition 1.2.10) we have the following equivalent definition.

Definition 1.2.25. A principal $(\Sigma, \mathcal{C}, E)$-bundle over $M$ is a principal $\Sigma$-bundle $\pi: P \rightarrow M$ with moment map $\mu: P \rightarrow \mathbf{X}$ together with a distribution $\mathcal{C}_{P} \subset T P$ such that

- the action $a: \Sigma \times \mathbf{x} P \rightarrow P$ of $\Sigma$ is multiplicative with respect to $\mathcal{C}$ and $\mathcal{C}_{P}$, i.e. $\mathcal{C} \cdot \mathcal{C}_{P} \subset \mathcal{C}_{P}$ (here $\cdot$ denotes the differential of the action map);

- the injection $\mu^{*}(\mathcal{C} \cap \operatorname{Ker}(d s)) \hookrightarrow \mathcal{C}_{P} \cap \operatorname{Ker}(d \pi)$ induced by the infinitesimal action is an isomorphism;

- $\operatorname{Ker}(d \mu) \cap \mathcal{C}_{P}=\operatorname{Ker}(d \pi) \cap \mathcal{C}_{P}$

It is called full when the inclusion

$$
\nu_{\mathcal{C}_{P} \cap \operatorname{Ker}(d \pi)} \hookrightarrow \mu^{*} E
$$

of the normal bundle of $\mathcal{C}_{P} \cap \operatorname{Ker}(d \pi)$ in $\operatorname{Ker}(d \pi)$ induced by the infinitesimal action extends to an isomorphism

$$
\nu_{\mathcal{C}_{P}} \cong \mu^{*} E .
$$

We will use the notation

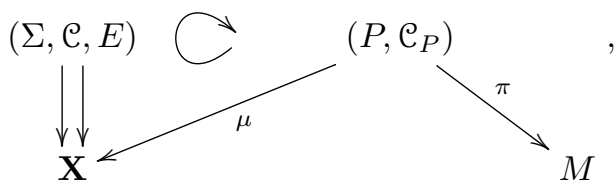

Remark 1.2.26. By infinitesimal action of $\Sigma$ on $P$ we mean the map

$$
t^{*} A \rightarrow \operatorname{Ker}(d \pi) \subset T P
$$


sending $\alpha_{x} \in A_{t(g)}=\operatorname{Ker}_{t(g)}(d s)$ to $a\left(\alpha_{x}, 0_{p}\right) \in \operatorname{Ker}_{p}(d \pi) \subset T_{p} P$. Observe that this map induces an isomorphism

$$
\mu^{*} A \rightarrow \operatorname{Ker}(d \pi), \quad \alpha_{\mu(p)} \rightarrow a\left(\alpha_{\mu(p)}, 0_{p}\right) .
$$

Recall also that $\left.\left(\nu_{\mathcal{C}}\right)\right|_{\mathbf{x}}$ is a sub-vector bundle of $E$ (by definition of Pfaffian groupoid, Definition 1.2.10). If we require the infinitesimal action to induce an isomorphism

$$
\mu^{*}(\mathcal{C} \cap \operatorname{Ker}(d s)) \cong \mathcal{C}_{P} \cap \operatorname{Ker}(d \pi)
$$

then the normal bundle of $\mathcal{C}_{P} \cap \operatorname{Ker}(d \pi)$ in $\operatorname{Ker}(d \pi)$ can be seen as a sub-vector bundle of $\mu^{*} E$ and the definition of full principal Pfaffian bundle makes sense.

The following statement, proven in [15], deals with the gauge construction in the Pfaffian setting.

Proposition 1.2.27. Let $(\Sigma, \omega, E)$ be a Pfaffian groupoid over $\mathbf{X}$ and $(P, \theta)$ a (left) principal $(\Sigma, \omega, E)$-bundle over $M$. The gauge groupoid Gauge $(\Sigma) \cong P \times_{M} P / \Sigma \rightrightarrows M$ can be endowed with a one-form $\omega_{\text {gauge }}$ and a representation $E_{\text {gauge }}$ in such a way that the right action on $P$ makes $(P, \theta)$ into a (right) principal (Gauge $\left.(\Sigma), \omega_{\text {gauge }}, E_{\text {gauge }}\right)$ bundle.

We conclude this section by defining morphisms of principal Pfaffian bundles.

Definition 1.2.28. Let $\left(P_{1}, \theta_{1}\right)$ and $\left(P_{2}, \theta_{2}\right)$ be principal $(\Sigma, \omega, E)$-bundles. A morphism of principal $(\Sigma, \omega, E)$-bundles

$$
\left(P_{1}, \theta_{1}\right) \rightarrow\left(P_{2}, \theta_{2}\right)
$$

consists of a morphism of principal $\Sigma$-bundles

$$
\Psi: P_{1} \rightarrow P_{2}
$$

such that $\Psi^{*}\left(\theta_{2}\right)=\theta_{1}$.

Remark 1.2.29. Just like for principal $\Sigma$-bundles, any morphism of principal $(\Sigma, \omega, E)$-bundles is an isomorphism.

\subsection{Morita equivalences}

\subsubsection{Morita equivalences of Pfaffian groupoids}

Let $\Sigma \rightrightarrows \mathbf{X}$ be a Lie groupoid. Recall that, when $\mu: P \rightarrow \mathbf{X}$ is a left $\Sigma$-space, we can form the action groupoid $\Sigma \ltimes P \rightrightarrows P$. The arrow space is given by $\Sigma \ltimes P=\Sigma_{s} \times{ }_{\mu} P$, the source map is the second projection and the target map sends $(g, p)$ with $\mu(p)=s(g)$ to $g \cdot p$. The multiplicaton is defined by

$$
(h, g \cdot p) \cdot(g, p)=(h g, p)
$$

for all $h, g \in \Sigma, p \in P$ with $\mu(p)=s(g), t(g)=s(h)$. Similarly, when $\mu: P \rightarrow \mathbf{X}$ is a right $\Sigma$-space, one can define the action groupoid $P \rtimes \Sigma$. 
Definition 1.3.1. Let $\left(\Sigma_{1}, \omega_{1}, E_{1}\right)$ and $\left(\Sigma_{2}, \omega_{2}, E_{2}\right)$ be Pfaffian groupoids over $\mathbf{X}_{1}$ and $\mathbf{X}_{2}$ respectively. A Pfaffian Morita equivalence between them is

- a Morita equivalence between $\Sigma_{1}$ and $\Sigma_{2}$, i.e. the datum of a principal bibundle $\mathbf{X}_{1} \stackrel{\mu_{1}}{\leftarrow} P \stackrel{\mu_{2}}{\rightarrow} \mathbf{X}_{2}$ as in Definition 1.1.31;

- a vector bundle isomorphism $\Phi: \mu_{1}^{*} E_{1} \rightarrow \mu_{2}^{*} E_{2}$;

- a one form $\theta \in \Omega^{1}\left(P, \mu_{1}^{*} E\right)$;

such that

1. $\Phi: \mu_{1}^{*} E_{1} \rightarrow \mu_{2}^{*} E_{2}$ is

- an isomorphism of (left) $\Sigma_{1} \ltimes P$-representations where

$$
\Sigma_{1} \ltimes P \times_{P} \mu_{1}^{*} E_{1} \rightarrow \mu_{1}^{*} E_{1}, \quad\left(\left(g_{1}, p\right),\left(p, \alpha_{\mu_{1}(p)}\right)\right) \rightarrow\left(g_{1} \cdot p, g_{1} \cdot \alpha_{\mu_{1}(p)}\right)
$$

is the representation naturally induced on $\mu_{1}^{*} E_{1}$, while $\mu_{2}^{*} E_{2}$ carries the trivial representation

$$
\Sigma_{1} \ltimes P \times_{P} \mu_{2}^{*} E_{2} \rightarrow \mu_{2}^{*} E_{2}, \quad\left(\left(g_{1}, p\right),\left(p, \alpha_{\mu_{2}(p)}\right)\right) \rightarrow\left(g_{1} \cdot p, \alpha_{\mu_{2}(p)}\right) ;
$$

- an isomorphism of (right) $P \rtimes \Sigma_{2}$-representations where

$$
P \rtimes \Sigma_{2} \times_{P} \mu_{2}^{*} E_{2} \rightarrow \mu_{2}^{*} E_{2}, \quad\left(\left(p, g_{2}\right),\left(p, \alpha_{\mu_{2}(p)}\right)\right) \rightarrow\left(p \cdot g_{2}, g_{2}^{-1} \cdot \alpha_{\mu_{2}(p)}\right)
$$

is the representation naturally induced on $\mu_{2}^{*} E_{2}$, while $\mu_{1}^{*} E_{1}$ carries the trivial representation

$$
P \rtimes \Sigma_{2} \times_{P} \mu_{1}^{*} E_{1} \rightarrow \mu_{1}^{*} E_{1}, \quad\left(\left(p, g_{2}\right),\left(p, \alpha_{\mu_{2}(p)}\right)\right) \rightarrow\left(p \cdot g_{2}, \alpha_{\mu_{1}(p)}\right) ;
$$

2. the left action of $\left(\Sigma_{1}, \omega_{1}\right)$ on $(P, \theta)$ is multiplicative;

3. the right action of $\left(\Sigma_{2}, \omega_{2}\right)$ on $(P, \Phi \circ \theta)$ is multiplicative;

4. $\operatorname{Ker}(\theta) \cap \operatorname{Ker}\left(d \mu_{1}\right)=\operatorname{Ker}(\theta) \cap \operatorname{Ker}\left(d \mu_{2}\right)$.

We will use the notation $(P, \theta, \Phi)$. If $\theta$ is pointwise surjective, both the Morita equivalence and the triple $(P, \theta, \Phi)$ are called full.

Remark 1.3.2. The properties of $\Phi$ are equivalently encoded into the formulas

$\Phi_{p \cdot g_{2}}\left(p \cdot g_{2}, \alpha_{\mu_{1}\left(p \cdot g_{2}\right)}\right)=g_{2}^{-1} \cdot \Phi_{p}\left(p, \alpha_{\mu_{1}(p)}\right), \quad\left(p, g_{2}\right) \in P \rtimes \Sigma_{2},\left(p, \alpha_{\mu_{1}(p)}\right) \in \mu_{1}^{*} E_{1}$

and

$$
\Phi_{p}\left(p, \alpha_{\mu_{1}(p)}\right)=\Phi_{g_{1} \cdot p}\left(g_{1} \cdot p, g_{1} \cdot \alpha_{\mu_{1}(p)}\right), \quad\left(g_{1}, p\right) \in \Sigma_{1} \ltimes P,\left(p, \alpha_{\mu_{1}(p)}\right) \in \mu_{1}^{*} E_{1} ;
$$

Remark 1.3.3. Recall that the multiplicativity equation for the left action

$$
\left(a_{\Sigma_{1}}^{*} \theta\right)_{\left(g_{1}, p\right)}=\left(p r_{1}^{*} \omega_{1}\right)_{\left(g_{1}, p\right)}+g_{1} \cdot\left(p r_{2}^{*} \theta\right)_{\left(g_{1}, p\right)}
$$

is an equality of forms on $\Sigma_{1} \ltimes P$. 
As for the right action, the multiplicativity equation becomes the following equality of forms on $P \rtimes \Sigma_{2}$ :

$$
a_{\Sigma_{2}}^{*}(\Phi \circ \theta)_{\left(p, g_{2}\right)}=g_{2}^{-1} \cdot\left(p r_{1}^{*}(\Phi \circ \theta)\right)_{\left(p, g_{2}\right)}+g_{2}^{-1}\left(p r_{2}^{*} \omega_{2}\right)_{\left(p, g_{2}\right)}
$$

This could look a bit puzzling. The reader is encouraged to look at the case of $(\Sigma, \omega)$ acting on itself from the left and from the right and, in particular, at the multiplicativity equation satisfied by $\left(i^{*} \omega\right)_{g}=-g^{-1} \cdot \omega$; the role of $\Phi$ is played by $\Phi: v_{t(g)} \rightarrow g^{-1} \cdot v_{t(g)}$.

Remark 1.3.4. The distribution point of view seems slightly clearer, because one can encode encode multiplicativity as $\mathcal{C}_{1} \cdot \mathcal{C}_{P} \subset \mathcal{C}_{P}$ and $\mathcal{C}_{P} \cdot \mathcal{C}_{2} \subset \mathcal{C}_{P}$.

Let $\left(\Sigma_{1}, \omega_{1}, E_{1}\right)$ and $\left(\Sigma_{2}, \omega_{2}, E_{2}\right)$ be Pfaffian Morita equivalent Pfaffian groupoids over $\mathbf{X}_{1}$ and $\mathbf{X}_{2}$ respectively. Let $(P, \theta, \Phi)$ be a triple representing a Pfaffian Morita equivalence. $(P, \theta)$ is a principal $\left(\Sigma_{1}, \omega_{1}, E_{1}\right)$-bundle, while $(P, \Phi \circ \theta)$ is a principal $\left(\Sigma_{2}, \omega_{2}, E_{2}\right)$-bundle.

Definition 1.3.5. Let $\left(\Sigma_{1}, \omega_{1}, E_{1}\right)$ and $\left(\Sigma_{2}, \omega_{2}, E_{2}\right)$ be Pfaffian groupoids over $\mathbf{X}_{1}$ and $\mathbf{X}_{2}$ respectively. A principal Pfaffian bibundle between them is a triple $(P, \theta, \Phi)$ where $\left.\mathbf{X}_{1} \leftarrow P \rightarrow \mathbf{X}\right) 2$ is a principal bibundle and

- $(P, \theta)$ is a left principal $\left(\Sigma_{1}, \omega_{1}, E_{1}\right)$-bundle;

- $\Phi: \mu_{1}^{*} E_{1} \rightarrow \mu_{2}^{*} E_{2}$ is an isomorphism of vector bundles;

such that

- $(P, \Phi \circ \theta)$ is a right principal $\left(\Sigma_{2}, \omega_{2}, E_{2}\right)$-bundle;

- $\Phi: \mu_{1}^{*} E_{1} \rightarrow \mu_{2}^{*} E_{2}$ is a morphism of $\Sigma_{1} \ltimes P$ and $P \rtimes \Sigma_{2}$ representation as in Definition 1.3.1.

The bibundle is called full if $\theta$ is pointwise surjective.

With this definition in mind, a Pfaffian Morita equivalence between two Pfaffian groupoids $\left(\Sigma_{1}, \omega_{1}, E_{1}\right)$ and $\left(\Sigma_{2}, \omega_{2}, E_{2}\right)$ corresponds to the datum of a principal Pfaffian bibundle between them. Notice that such a principal Pfaffian bibundle is, in particular, a pair $(P, \theta)$ which is

- a principal Pfaffian $\left(\Sigma_{1}, \omega_{1}, E_{1}\right)$-bundle;

- decorated by an isomorphism $\Phi: \mu_{1}^{*} E_{1} \rightarrow \mu_{2}^{*} E_{2}$ that makes $(P, \theta)$ into a principal Pfaffian $\left(\Sigma_{2}, \omega_{2}, E_{2}\right)$-bundle.

However, $\Phi$ satisfies an additional property - i.e. it is a representation map. This additional property should be thought of as a Pfaffian analogue of the compatibility of the actions in a principal bibundle/Morita equivalence of Lie groupoids, Definition 1.1.31.

Proposition 1.3.6. Let $\left(\Sigma_{1}, \omega_{1}, E_{1}\right)$ be a Pfaffian groupoid over $\mathbf{X}_{1}$ and $\left(\Sigma_{2}, \omega_{2}, E_{2}\right)$ be a Pfaffian groupoid over $\mathbf{X}_{2}$. Assume $\left(\Sigma_{1}, \omega_{1}, E_{1}\right)$ and $\left(\Sigma_{2}, \omega_{2}, E_{2}\right)$ to be Pfaffian Morita equivalent and let

$$
\mathbf{X}_{1} \stackrel{\mu_{1}}{\leftarrow}(P, \theta, \Phi) \stackrel{\mu_{2}}{\rightarrow} \mathbf{X}_{2}
$$


be the triple realizing the equivalence. Then $\Phi$ induces an isomorphism of $\Sigma_{2}$ representations

$$
\bar{\Phi}: \mu_{1}^{*} E_{1} /\left(\Sigma_{1} \ltimes P\right) \stackrel{\cong}{\rightrightarrows} E_{2} .
$$

Moreover, let $\left(\Sigma_{1}, \omega_{1}, E_{1}\right)$ be a Pfaffian groupoid over $\mathbf{X}_{1}$ and $\left(\Sigma_{2}, \omega_{2}, E_{2}\right)$ be a Pfaffian groupoid over $\mathbf{X}_{2}$. Let $(P, \theta, \Phi)$ be a triple where

- $(P, \theta)$ is a principal $\left(\Sigma_{1}, \omega_{1}, E_{1}\right)$-bundle;

- $\Phi: \mu_{1}^{*} E_{1} \rightarrow \mu_{2}^{*} E_{2}$ is the map induced on the pullback bundles by an isomorphism of $\Sigma_{2}$-representations

$$
\bar{\Phi}: \mu_{1}^{*} E_{1} /\left(\Sigma_{1} \ltimes P\right) \stackrel{\cong}{\rightrightarrows} E_{2} ;
$$

- $(P, \Phi \circ \theta)$ is a principal $\left(\Sigma_{2}, \omega_{2}, E_{2}\right)$-bundle.

Then $(P, \theta, \Phi)$ is a principal Pfaffian bibundle.

Proof. First of all notice that, given a bibundle $P$ between two Lie groupoids $\Sigma_{1}$ and $\Sigma_{2}$, the quotient $\mu_{1}^{*} E_{1} /\left(\Sigma_{1} \ltimes P\right)$ is indeed a vector bundle over $\mathbf{X}_{2}$, with projection

$$
\left[\left(p, \alpha_{\mu_{1}(p)}\right)\right] \in \mu_{1}^{*} E_{1} /\left(\Sigma_{1} \ltimes P\right) \rightarrow \mu_{2}(p)
$$

which is well defined because

$$
\mu_{2} \circ p r_{1}: \mu_{1}^{*} E_{1} \rightarrow \mathbf{X}_{2}
$$

is invariant under the action of $\Sigma_{1} \ltimes P$. The right representation of $P \rtimes \Sigma_{2}$ on $\mu_{1}^{*} E_{1}$ given by

$$
P \rtimes \Sigma_{2} \times_{P} \mu_{1}^{*} E_{1} \rightarrow \mu_{1}^{*} E_{1}, \quad\left(\left(p, g_{2}\right),\left(p, \alpha_{\mu_{1}(p)}\right)\right) \rightarrow\left(p \cdot g_{2}, \alpha_{\mu_{1}(p)}\right)
$$

is equivariant with respect to the action of $\Sigma_{1} \ltimes P$; the same holds for the left representation obtained acting with inverses, that is

$$
P \rtimes \Sigma_{2} \times_{P} \mu_{1}^{*} E_{1} \rightarrow \mu_{1}^{*} E_{1}, \quad\left(\left(p, g_{2}\right),\left(p \cdot g_{2}, \alpha_{\mu_{1}\left(p \cdot g_{2}\right)}\right)\right) \rightarrow\left(p, \alpha_{\mu_{1}\left(p \cdot g_{2}\right)}\right) .
$$

It follows that $\mu_{1}^{*} E_{1} /\left(\Sigma_{1} \ltimes P\right)$ carries a natural left $\Sigma_{2}$-representation (of course, it also carries a natural right $\Sigma_{2}$-representation and the left one is obtained from the right one acting with inverses). This uses the fact that $P$ is a bibundle: the compatibility of the $\Sigma_{1}$-action with the $\Sigma_{2}$-action is needed.

That said, the first part of the claim follows from the properties of $\Phi$ in Definition 1.3.1 - the explicit formulas from Remark 1.3.2 are useful.

First, $\Phi: \mu_{1}^{*} E_{1} \rightarrow \mu_{2}^{*} E_{2}$ is a morphism of $\Sigma_{1} \ltimes P$ representations - see Definition 1.3.1. This, in particular, implies that $\Phi$ descends to a map

$$
\bar{\Phi}: \mu_{1}^{*} E_{1} /\left(\Sigma_{1} \ltimes P\right) \rightarrow E_{2},
$$

because the orbit of the action of $\Sigma_{1}$ on $P$ are the $\mu$-fibers. $\bar{\Phi}$ is an isomorphism of vector bundles; by construction, it is an injective morphism between vector bundles of the same rank.

Furthermore, since $\Phi: \mu_{1}^{*} E_{1} \rightarrow \mu_{2}^{*} E_{2}$ is a morphism of $P \rtimes \Sigma_{2}$ representations - see Definition 1.3.1 - the induced map $\bar{\Phi}$ is a morphism of representations. 
As for the second part of the claim, if

$$
\bar{\Phi}: \mu_{1}^{*} E_{1} /\left(\Sigma_{1} \ltimes P\right) \rightarrow E_{2}
$$

is an isomorphism of $\Sigma_{2}$-representations then there is an induced map

$$
\Phi: \mu_{1}^{*} E_{1} \rightarrow \mu_{2}^{*} E_{2}, \quad\left(p, \alpha_{1}\right) \rightarrow\left(p, \bar{\Phi}\left(\left[p, \alpha_{1}\right]\right)\right) .
$$

This map is a morphism of $\Sigma_{1} \ltimes P$-representations by definition, and a morphism of (say, right) $P \rtimes \Sigma_{2}$-representations because $\bar{\Phi}$ is a morphism of (left) $\Sigma_{2}$-representations. In fact, if $\left(p, \alpha_{\mu_{1}}(p)\right) \in \mu_{1}^{*} E_{1}, g_{2} \in \Sigma_{2}, t\left(g_{2}\right)=\mu_{2}(p)$ then

$$
\begin{gathered}
\left(p, g_{2}\right) \cdot\left(p, \alpha_{\mu_{1}}(p)\right)=\left(p \cdot g_{2}, \alpha_{\mu_{1}}(p)\right) \\
\Phi\left(p \cdot g_{2}, \alpha_{\mu_{1}}(p)\right)=\left(p \cdot g_{2}, \bar{\Phi}\left(\left[p \cdot g_{2}, \alpha_{1}\right]\right)\right) ;
\end{gathered}
$$

we have

$$
\left[p \cdot g_{2}, \alpha_{1}\right]=g_{2}^{-1} \cdot\left[p, \alpha_{1}\right]
$$

hence

$$
\bar{\Phi}\left(\left[p \cdot g_{2}, \alpha_{1}\right]\right)=g_{2}^{-1} \cdot \bar{\Phi}\left(\left[p, \alpha_{1}\right]\right) .
$$

In the end

$$
\Phi\left(\left(p, g_{2}\right) \cdot\left(p, \alpha_{\mu_{1}}(p)\right)\right)=\left(p \cdot g_{2}, g_{2}^{-1} \cdot \bar{\Phi}\left(\left[p, \alpha_{1}\right]\right)\right)
$$

and the left hand side is exactly the formula for the right representation of $P \rtimes \Sigma_{2}$ on $\mu_{2}^{*} E_{2}$.

In view of the above proposition, we have the following alternative definition of Pfaffian Morita equivalence/Pfaffian bibundle.

Definition 1.3.7. Let $\left(\Sigma_{1}, \omega_{1}, E_{1}\right)$ be a Pfaffian groupoid over $\mathbf{X}_{1}$ and $\left(\Sigma_{2}, \omega_{2}, E_{2}\right)$ be a Pfaffian groupoid over $\mathbf{X}_{2}$. A Pfaffian Morita equivalence between $\left(\Sigma_{1}, \omega_{1}, E_{1}\right)$ and $\left(\Sigma_{2}, \omega_{2}, E_{2}\right)$ is the datum of a Pfaffian bibundle between $\left(\Sigma_{1}, \omega_{1}, E_{1}\right)$ to $\left(\Sigma_{2}, \omega_{2}, E_{2}\right)$, i.e.

- a space $P$ coming with two maps $\mu_{1}: P \rightarrow \mathbf{X}_{1}$ and $\mu_{2}: P \rightarrow \mathbf{X}_{2}$;

- a form $\theta \in \Omega^{1}\left(P, \mu_{1}^{*} E_{1}\right)$;

- a vector bundle isomorphism $\Phi: \mu_{1}^{*} E_{1} \rightarrow \mu_{2}^{*} E_{2}$;

such that

- the pair $(P, \theta)$ is a left principal $\left(\Sigma_{1}, \omega_{1}, E_{1}\right)$-bundle over $\mathbf{X}_{2}$ with moment map $\mu_{1}$ and projection $\mu_{2}$;

- the pair $(P, \Phi \circ \theta)$ is a right principal $\left(\Sigma_{2}, \omega_{2}, E_{2}\right)$-bundle over $\mathbf{X}_{2}$ with moment map $\mu_{2}$ and projection $\mu_{1}$;

- the two actions on $P$ commute and $\Phi$ induces an isomorphism

$$
\mu_{1}^{*} E_{1} /\left(\Sigma_{1} \ltimes P\right) \cong E_{2} .
$$


Pfaffian isomorphisms induce Pfaffian Morita equivalences:

Lemma 1.3.8. Let $\left(\Sigma_{1}, \omega_{1}, E_{1}\right),\left(\Sigma_{2}, \omega_{2}, E_{2}\right)$ be Pfaffian groupoids over $\mathbf{X}_{1}, \mathbf{X}_{2}$. An isomorphism of Pfaffian groupoids $(\Psi, \Phi)$ between them (Definition 1.2.13) induces naturally a Pfaffian Morita equivalence. If $\left(\Sigma_{1}, \omega_{1}, E_{1}\right)$ is full, so is the Pfaffian Morita equivalence.

Proof. The bibundle realizing the Morita equivalence is $\left(\Sigma_{1}, \omega_{1}, \hat{\Psi}\right)$ with right action of $\Sigma_{2}$ induced by the isomorphism of Lie groupoids $\Phi$, while the isomorphism $\hat{\Psi}$ is induced by $\Psi$ :

$$
\hat{\Psi}: t^{*} E_{1} \rightarrow s^{*} \phi^{*} E_{2}, \quad\left(g, e_{1}\right) \rightarrow\left(g,(\Phi(g))^{-1} \cdot\left(\Psi\left(e_{1}\right)\right)\right) .
$$

Here, $\phi: \mathbf{X}_{1} \rightarrow \mathbf{X}_{2}$ is the diffeomorphism of the base manifolds induced by $\Phi$.

Careful (but straightforward - starting with $\left(\Sigma_{1}, \omega_{1}, E_{1}\right)=\left(\Sigma_{2}, \omega_{2}, E_{2}\right)$ and $\Phi=i d$ is illuminating) computations show that all the properties of a Pfaffian Morita equivalence are satisfied.

The definition of Pfaffian Morita equivalence appeared first in [15]. We conclude this section by recalling the following result from [15].

Proposition 1.3.9. Pfaffian Morita equivalence is an equivalence relation (in particular, it is transitive).

The proof makes use of the Pfaffian gauge construction, Proposition 1.2.27.

\subsubsection{Some properties of Pfaffian Morita equivalences}

Proposition 1.1.33 describes the structure that is preserved under Morita equivalence of Lie groupoids. A Pfaffian Morita equivalence of Pfaffian groupoids between $\left(\Sigma_{1}, \omega_{1}, E_{1}\right)$ and $\left(\Sigma_{2}, \omega_{2}, E_{2}\right)$ is in particular a Morita equivalence between $\Sigma_{1}$ and $\Sigma_{2}$ - hence orbit spaces, isotropy groups and normal representations are preserved according to Proposition 1.1.33. There is additional Pfaffian structure preserved under Pfaffian Morita equivalence.

Proposition 1.3.10. Let $\left(\Sigma_{1}, \omega_{1}, E_{1}\right)$ be a Pfaffian groupoid over $\mathbf{X}_{1}$ and $\left(\Sigma_{2}, \omega_{2}, E_{2}\right)$ be a Pfaffian groupoid over $\mathbf{X}_{2}$. Assume $\left(\Sigma_{1}, \omega_{1}, E_{1}\right)$ and $\left(\Sigma_{2}, \omega_{2}, E_{2}\right)$ to be Pfaffian Morita equivalent and let

$$
\mathbf{X}_{1} \stackrel{\mu_{1}}{\leftarrow}(P, \theta, \Phi) \stackrel{\mu_{2}}{\rightarrow} \mathbf{X}_{2}
$$

be the triple realizing the equivalence. Then, if $x \in \mathbf{X}_{1}, y \in \mathbf{X}_{2}$ are points whose orbits are related by $P$ (in the sense of Proposition 1.1.33), the following facts hold true.

- The differential of the isomorphism from Proposition 1.1.33

$$
\varphi_{p}:\left(\Sigma_{1}\right)_{x} \rightarrow\left(\Sigma_{2}\right)_{y}
$$

induced by the point $p \in P$ sends

$$
\mathfrak{g}_{x}\left(\omega_{1}\right)=\operatorname{Ker}_{x}\left(\omega_{1}\right) \cap \operatorname{Ker}_{x}(d s) \subset \operatorname{Ker}_{x}(d t) \cap \operatorname{Ker}_{x}(d s)=T_{x}\left(\Sigma_{1}\right)_{x}
$$

to

$$
\mathfrak{g}_{y}\left(\omega_{2}\right)=\operatorname{Ker}_{y}\left(\omega_{2}\right) \cap \operatorname{Ker}_{y}(d s) \subset \operatorname{Ker}_{y}(d t) \cap \operatorname{Ker}_{y}(d s)=T_{y}\left(\Sigma_{2}\right)_{y} .
$$


- The representations of $\left(\Sigma_{1}\right)_{x}$ on $\left(E_{1}\right)_{x}$ and of $\left(\Sigma_{2}\right)_{y}$ on $\left(E_{2}\right)_{y}$ are isomorphic.

- The isomorphism $\varphi_{p}$ between $\left(\Sigma_{1}\right)_{x}$ and $\left(\Sigma_{2}\right)_{y}$ is an isomorphism of Pfaffian groups.

Proof. Let us proceed point by point.

- For the first point, recall that

$$
\varphi_{p}:\left(\Sigma_{1}\right)_{x} \rightarrow\left(\Sigma_{2}\right)_{y}, \quad g_{1} \rightarrow g_{2}=\varphi_{p}\left(g_{1}\right)
$$

where $g_{2} \in\left(\Sigma_{2}\right)_{x}$ is the only element such that $p \cdot g_{2}=g_{1} \cdot p$.

Let $v_{x} \in \mathfrak{g}_{x}\left(\omega_{1}\right)$; we need to show that $\omega_{2}\left(d_{x} \varphi_{p}\left(v_{x}\right)\right)=0$. Let

$$
\left.\gamma_{1}:\right]-1,1\left[\rightarrow\left(\Sigma_{1}\right)_{x}, \quad t \rightarrow \gamma_{1}(t)\right.
$$

be a curve in $\left(\Sigma_{1}\right)_{x}$ tangent to $v_{x}$ at $t=0$ and set

$$
\left.\gamma_{2}:=\varphi_{p} \circ \gamma_{1}:\right]-1,1\left[\rightarrow \Sigma_{1} .\right.
$$

Then $d_{x} \varphi_{p}\left(v_{x}\right)=\left.\frac{d}{d t}\right|_{t=0} \gamma_{2}(t)$. We have

$$
\omega_{1}\left(v_{x}\right)=\omega_{1}\left(\left.\frac{d}{d t}\right|_{t=0} \gamma_{1}(t)\right)=\theta\left(\left.\frac{d}{d t}\right|_{t=0}\left(\gamma_{1}(t) \cdot p\right)\right)
$$

by multiplicativity of the action of $\Sigma_{1}$ on $P$. Since

$$
\gamma_{1}(t) \cdot p=p \cdot \gamma_{2}(t)
$$

by definition, by using the multiplicativity equation of the action of $\Sigma_{2}$ on $P$ we see that $\omega_{2}\left(\left.\frac{d}{d t}\right|_{t=0} \gamma_{2}(t)\right)=0$.

- The isomorphism $\Phi: \mu_{1}^{*} E_{1} \rightarrow \mu_{2}^{*} E_{2}$ induces an isomorphism of vector spaces $\Phi_{p}:\left(E_{1}\right)_{x} \rightarrow\left(E_{2}\right)_{y}$. The properties of $\Phi-$ see Definition 1.3.1 and Remark 1.3.2 - imply immediately that the pair $\left(\Phi_{p}, \varphi_{p}\right)$ is an isomorphism of representations.

- The third point follows by definition from the previous two.

At this point we have

Corollary 1.3.11. Let $\left(G_{1}, \omega_{1}, V_{1}\right),\left(G_{2}, \omega_{2}, V_{2}\right)$ be Pfaffian groups. Then they are Pfaffian Morita equivalent if and only if they are Pfaffian isomorphic.

Proof. On the one hand we have simply Lemma 1.3.8: a Pfaffian isomorphism naturally induce a Morita equivalence. On the other hand, the third point in Proposition 1.3.10 implies, in particular, that Pfaffian Morita equivalent Pfaffian groups are Pfaffian isomorphic. 


\subsubsection{Interaction with principal bundles}

We aim at a version of Proposition 1.1.37 in the Pfaffian setting.

Proposition 1.3.12. Let $\left(\Sigma_{1}, \omega_{1}, E_{1}\right)$ and $\left(\Sigma_{2}, \omega_{2}, E_{2}\right)$ be Pfaffian groupoids. There is a one to one correspondence

$$
\left\{\begin{array}{c}
\text { Principal }\left(\Sigma_{1}, \omega_{1}, E_{1}\right) \text {-bundles } \\
\text { up to isomorphisms }
\end{array}\right\} \stackrel{1-1}{\longleftrightarrow}\left\{\begin{array}{c}
\text { Principal }\left(\Sigma_{2}, \omega_{2}, E_{2}\right) \text {-bundles } \\
\text { up to isomorphisms }
\end{array}\right\}
$$

whenever $\left(\Sigma_{1}, \omega_{1}, E_{1}\right)$ and $\left(\Sigma_{2}, \omega_{2}, E_{2}\right)$ are Pfaffian Morita equivalent.

Proof. Let us consider a principal bibundle $\mathbf{X}_{1} \leftarrow P \rightarrow \mathbf{X}_{2}$ between $\Sigma_{1}$ and $\Sigma_{2}$ supporting a Pfaffian Morita equivalence, Definition 1.3.1. We denote by $\mu_{i}^{*} P \rightarrow$ $\mathbf{X}_{i}$ the moment map of the action by $\Sigma_{i}$. Since $P$ supports a Pfaffian Morita equivalence, we have a form $\theta \in \Omega^{1}\left(P, \mu_{1}^{*} E\right)$ and an isomorphism $\Phi: \mu_{1}^{*} E_{1} \rightarrow$ $\mu_{2}^{*} E_{2}$ as in Definition 1.3.1.

Let now $P_{1}$ be a principal $\Sigma_{1}$-bundle, with moment map $\tau_{1}: P_{1} \rightarrow \mathbf{X}_{1}$. Recall (see the discussion after Proposition 1.1.37) that we can construct a principal $\Sigma_{2}$ bundle $P_{2}$ by taking the pullback $\mu_{1}^{*} P_{1} \cong P_{1} \times_{\mathbf{X}_{1}} P$ and then the quotient by the principal action of $\Sigma_{1} \ltimes P$ induced by the diagonal action of $\Sigma_{1}$. The action of $P \rtimes \Sigma_{2}$ induced by $\Sigma_{2}$ acting on the second factor makes the quotient into a principal $\Sigma_{2}$-bundle $P_{2}$. We use $\tau_{2}: P_{2} \rightarrow \mathbf{X}_{2}$ to denote the moment map of $P_{2}$.

What one has to do is constructing a form $\theta_{2} \in \Omega^{1}\left(P_{2}, \mu_{2}^{*} E_{2}\right)$ which makes the action of $\Sigma_{2}$ a principal Pfaffian bundle. We can consider the form $\hat{\theta}=p r_{2}^{*} \theta-$ $p r_{1}^{*} \theta_{1}$ on $\mu_{1}^{*} P$. Notice that, post-composing with the isomorphism of coefficents induced by $\Phi, p r_{2}^{*} \theta$ can be thought of as $p r_{2}^{*} \mu_{2}^{*} E_{2}$-valued; the same is true for $p r_{1}^{*} \theta_{1}$ since $\Phi$ induces the isomorphism (still denoted by $\Phi$ )

$$
\Phi: p r_{1}^{*} \tau_{1}^{*} E_{1} \rightarrow p r_{1}^{*} \mu_{2}^{*} E_{2}, \quad\left(p, a_{1}, p_{1}\right) \rightarrow\left(p, \Phi\left(p, a_{1}\right), p_{1}\right) .
$$

In what follows, $a^{P_{1}}$ denotes the action of $\Sigma_{1}$ on $P_{1}, a_{1}^{P}$ denotes the action of $\Sigma_{1}$ on $P$ and $a_{2}^{P}$ denotes the action of $\Sigma_{2}$ on $P$.

1. First, we claim that $\hat{\theta}$ is $\Sigma_{1} \ltimes P$-basic.

- $\hat{\theta}$ is horizontal. To see horizontality, first observe that the space tangent to the orbits is given by vectors of the form

$$
\hat{v}=\left(d a^{P_{1}}(v, 0), d a_{1}^{P}(v, 0)\right)
$$

where $v \in \operatorname{Ker}(d s) \subset T \Sigma_{1}$; then $\hat{\theta}(\hat{v})=0$ follows from multiplicativity of $\theta_{1}$ and $\theta$.

- $\hat{\theta}$ is equivariant. Here we need to observe that $\Sigma_{1} \ltimes P$-equivariance is in principle well defined only in the direction normal to $\Sigma_{1} \ltimes P$-orbits and with respect to the normal representation of $\left(\Sigma_{1} \ltimes P\right) \ltimes \mu_{1}^{*} P$. However, by using the Pfaffian forms, the normal representation can be extended to a representation on the whole $\mu_{1}^{*} P$; equivariance holds in this last (stronger!) sense. The action of $\left(g_{1}, p\right)$ on $\left(v_{1}, v\right) \in \mu_{1}^{*} P_{1}$ is given by

$$
\left.\left(d a^{P_{1}}\left(d \tau_{1}\left(v_{1}\right)_{g}^{\omega_{1}}, v_{1}\right)\right),\left(d a_{1}^{P}\left(d \mu_{1}(v)_{g}^{\omega^{1}}, v\right)\right)\right)
$$


where $d \tau_{1}\left(v_{1}\right)_{g}^{\omega_{1}}$ and $d \mu_{1}(v)_{g}^{\omega_{1}}$ denote $s$-lifts at $g$ tangent to $\operatorname{Ker}\left(\omega_{1}\right)$. One then sees, again using multiplicativity of $\theta_{1}$ and $\theta$ with respect to $\omega_{1}$, that $\hat{\theta}$ is equivariant.

Since $\hat{\theta}$ is basic, so is $\Phi \circ \hat{\theta}$; a form $\theta_{2}$ is induced on the quotient $P_{2}$.

2. $\theta_{2}$ is multiplicative. Recall that we turn $P_{2}$ into a left $\Sigma_{2}$-space by acting with inverses. The left action of $\Sigma_{2}$ on $P_{2}$ is denoted by $a^{P_{2}}$. If $v_{2} \in T P_{2}$, we know that $\theta_{2}\left(v_{2}\right)=\Phi \circ \hat{\theta}\left(\hat{v}_{2}\right)$ for any lift $\hat{v}_{2}$ with respect to the quotient projection. Because of how the action is constructed, the lift of $d a^{P_{2}}\left(w_{2}, v_{2}\right)$ at $\left(p_{1}, p\right)$, where $w_{2} \in T_{g_{2}} \Sigma_{2}$ is a compatible vector, is computed as

$$
d a\left(\hat{v_{2}}, \widehat{d i\left(w_{2}\right)}\right)
$$

where $\widehat{d i\left(w_{2}\right)}$ is the lift of $d i\left(w_{2}\right)$ at $\left(p \cdot g_{2}, g_{2}^{-1}\right)$ compatible with $\hat{v}_{2}$ and $a$ is the right action of $P \rtimes \Sigma_{2}$ on $\mu_{1}^{*} P_{1}$. With this remark at hand, the multiplicativity equation for $\theta_{2}$ is obtained from the multiplicativity of $\theta$ with respect to $\omega_{2}$ and the properties of $\Phi$. In fact

$$
\begin{array}{r}
\left(\theta_{2}\right)_{\mu_{2}(p)}\left(d a^{P_{2}}\left(v_{2}, w_{2}\right)\right)=\Phi \circ\left(p r_{2}^{*} \theta-p r_{1}^{*} \theta_{1}\right)_{\left(p_{1}, p\right)}\left(d a\left(\hat{v_{2}}, \widehat{d i\left(w_{2}\right)}\right)\right) \\
=g_{2} \cdot(\theta)_{p}\left(v_{P}\right)+\left(\omega_{2}\right)_{g_{2}}\left(w_{2}\right)-g_{2} \cdot\left(\theta_{1}\right)_{p_{1}}\left(v_{1}\right)
\end{array}
$$

where we write $\hat{v_{2}}=\left(v_{1}, v_{p}\right) \in T\left(P_{1} \times \mathbf{x}_{1} P\right)$. On the other hand

$$
\left(\omega_{2}\right)_{g_{2}}\left(w_{2}\right)+g_{2} \cdot\left(\theta_{2}\right)_{\mu_{2}(p)}\left(v_{2}\right)=\left(\omega_{2}\right)_{g_{2}}\left(w_{2}\right)+g_{2} \cdot\left((\theta)_{p}\left(v_{P}\right)-\left(\theta_{1}\right)_{p_{1}}\left(v_{1}\right)\right) \text {. }
$$

3. $\theta_{2}$ is Pfaffian. Now one has to show that $\operatorname{Ker}\left(d \tau_{2}\right) \cap \operatorname{Ker}\left(\theta_{2}\right)=\operatorname{Ker}\left(d \pi_{2}\right) \cap$ $\operatorname{Ker}\left(\theta_{2}\right)$, where we use $\pi_{i}: P_{i} \rightarrow M_{i}$ for the quotient projections. The intersection $\operatorname{Ker}\left(d \tau_{2}\right) \cap \operatorname{Ker}\left(\theta_{2}\right)$ is computed as the quotient of the intersection of $\operatorname{Ker}(\hat{\theta})$ with the vertical bundle of the projection $\mu_{1}^{*} P_{1} \rightarrow P$, which is readily seen to be $\operatorname{Ker}\left(d \tau_{1}\right) \cap \operatorname{Ker}\left(\theta_{1}\right)$. As for the intersection $\operatorname{Ker}\left(d \mu_{2}\right) \cap \operatorname{Ker}\left(\theta_{2}\right)$, it is isomorphic to $\operatorname{Ker}\left(d \tau_{1}\right) \cap \operatorname{Ker}\left(\theta_{1}\right)$, since the quotient projection $\pi_{2}$ is induced by the projection $\mu_{1}^{*} P_{1} \rightarrow M_{1}$ and we have

$$
\begin{aligned}
& \operatorname{Ker}\left(d \mu_{1}\right) \cap \operatorname{Ker}(\theta)=\operatorname{Ker}\left(d \mu_{2}\right) \cap \operatorname{Ker}(\theta), \\
& \operatorname{Ker}\left(d \mu_{2}\right) \cap \operatorname{Ker}(\theta) \cong \operatorname{Ker}\left(d \pi_{1}\right) \cap \operatorname{Ker}\left(\theta_{1}\right),
\end{aligned}
$$

and

$$
\operatorname{Ker}\left(d \pi_{1}\right) \cap \operatorname{Ker}\left(\theta_{1}\right)=\operatorname{Ker}\left(d \tau_{1}\right) \cap \operatorname{Ker}\left(\theta_{1}\right) .
$$

Performing the construction backwards, one gets a principal Pfaffian bundle $\left(P_{1}, \theta_{1}^{\prime}\right)$ which is isomorphic to $P_{1}$ via a map preserving the form (it is worth noticing here that the base manifolds of $P_{1}$ and of $P_{2}$ are diffeomorphic).

Remark 1.3.13. Let us assume $\left(\Sigma_{1}, \omega_{1}, E_{1}\right)$ and $\left(\Sigma_{2}, \omega_{2}, E_{2}\right)$ to be Pfaffian isomorphic. The Pfaffian isomorphism

$$
(\Psi, \Phi):\left(\Sigma_{1}, \omega_{1}, E_{1}\right) \rightarrow\left(\Sigma_{2}, \omega_{2}, E_{2}\right)
$$


induces a one to one correspondence between $\left(\Sigma_{1}, \omega_{1}, E_{1}\right)$-bundles and principal $\left(\Sigma_{2}, \omega_{2}, E_{2}\right)$-bundles. If $\left(P_{1}, \theta_{1}\right)$ is a principal $\left(\Sigma_{1}, \omega_{1}, E_{1}\right)$-bundle, then $\left(P_{1}, \Psi \circ \theta_{1}\right)$ is a principal $\left(\Sigma_{2}, \omega_{2}, E_{2}\right)$-bundle - where $\Sigma_{2}$ acts on $P_{1}$ through $\Phi$ in the obvious sense - and this clearly defines a one to one correspondence. Using the Morita equivalence from Lemma 1.3.8, we see that the principal $\left(\Sigma_{2}, \omega_{2}, E_{2}\right)$-bundle constructed out of a principal $\left(\Sigma_{1}, \omega_{1}, E_{1}\right)$-bundle by following the lines of the proof of Proposition 1.3.12 is actually isomorphic to $\left(P_{1}, \Psi \circ \theta_{1}\right)$.

\subsubsection{Morita equivalences and transitive Pfaffian groupoids}

In this subsection, we focus on the case when the Pfaffian groupoid $(\Sigma, \omega, E)$ is in particular a transitive Lie groupoid. Recall that $\Sigma \rightrightarrows \mathbf{X}$ is transitive when

$$
(s, t): \Sigma \rightarrow \mathbf{X} \times \mathbf{X} \quad g \rightarrow(s(g), t(g))
$$

is a surjective submersion. When the arrow space $\Sigma$ is second countable, if $(s, t)$ is surjective then it is also submersive. For the rest of this chapter, we do not need to work with groupoids of germs anymore. Consequently, we make the following assumption.

Assumption. Let $\Sigma \rightrightarrows \mathbf{X}$ be a Lie groupoid. From now on, unless otherwise specified, the arrow space $\Sigma$ is assumed to be second countable.

In this setting, $\Sigma$ is transitive if and only if for all $x \in \mathbf{X}$ :

for all $y \in \mathbf{X}$ there is $g \in \Sigma$ such that $s(g)=x, t(g)=y$.

It is well known that

Proposition 1.3.14. A transitive Lie groupoid $\Sigma \rightrightarrows \mathbf{X}$ is Morita equivalent to its isotopy Lie group $\Sigma_{x}$, for any $x \in \mathbf{X}$.

The bibundle realizing the equivalence between $\Sigma$ and the isotropy $\Sigma_{x}$ at (say) $x \in \mathbf{X}$ is the $s$-fiber $s^{-1}(x)$ over $x$.

It makes sense to investigate how to make the above statement Pfaffian. The outcome is

Proposition 1.3.15. A full transitive Pfaffian groupoid $(\Sigma, \omega, E)$ is Pfaffian Morita equivalent to the non full Pfaffian group (oid) $\left(G, \bar{\omega}_{M C}, V\right)$ where

- $G=\Sigma_{x}$ the isotropy group of $\Sigma$ at $x \in \mathbf{X}$;

- $V=E_{x}$;

- $\bar{\omega}_{M C}$ is the composition of the Maurer-Cartan form $\omega_{M C}: G \rightarrow \mathfrak{g}$ with the map $\mathfrak{g} \rightarrow V$ induced by $\left.\omega\right|_{x}$.

See also Propositions 1.2.17 and 1.2.20 and Corollary 1.2.18.

Remark 1.3.16. Because of Corollary 1.2.18, we will denote Pfaffian groups also by $(G, l, V)$, where $l: \mathfrak{g} \rightarrow V$ is a representation map (corresponding to $\omega_{e}$ ). Alternatively we will use the notation $(G, \mathfrak{h}, V)$, where $\mathfrak{h}$ is the Lie subalgebra and, by Proposition 1.2.7, the ideal - of $\mathfrak{g}$ given by the symbol space. When we want to emphasize that $l: \mathfrak{g} \rightarrow V$ has kernel $\mathfrak{h}$, we will denote $l$ by $l_{\mathfrak{h}}$; analogously, we will write $\mathfrak{h}_{l}$ to stress that $\mathfrak{h}$ is the kernel of the map $l: \mathfrak{g} \rightarrow V$. 
The bibundle needed to prove Proposition 1.3.15 is not hard to guess.

Proof. One fixes $x \in \mathbf{X}$. The triple $\left(s^{-1}(x),\left.\omega\right|_{s^{-1}(x)}, \Phi\right)$ realizes a Pfaffian Morita equivalence between $(\Sigma, \omega)$ and the Pfaffian group $\left(G_{x},\left.\omega\right|_{G_{x}}, E_{x}\right)$. Here,

$$
\Phi: t^{*} E \rightarrow s^{-1}(x) \times E_{x}
$$

is given by

$$
\left(g, \alpha_{t(g)}\right) \rightarrow\left(g, g^{-1} \cdot \alpha_{t(g)}\right) .
$$

In fact, careful but straightforward computations show that $\left(s^{-1}(x),\left.\omega\right|_{s^{-1}(x)}\right)$ is a (right) principal $\left(G_{x},\left.\omega\right|_{G_{x}}, E_{x}\right)$-bundle and that all the properties defining a principal Pfaffian bibundle are satisfied. See also [15].

By Lemma 1.2.16, $\left.\omega\right|_{G_{x}}$ coincide with $\bar{\omega}_{M C}$, where the map $l: \mathfrak{g}_{x} \rightarrow E_{x}$ is a map of representations. Notice that the Pfaffian Morita equivalence class of $\left(G_{x},\left.\omega\right|_{G_{x}}, E_{x}\right)$ does not depend on the choice of $x \in \mathbf{X}$, see Proposition 1.2.20 and Corollary 1.3.11.

Recall here Remark 1.2.21: $\left(G, \bar{\omega}_{M C}, V\right)$ is, in general, neither full nor extended even when $(\Sigma, \omega, E)$ is so.

Remark 1.3.17. Let us combine Proposition 1.3.12 with Proposition 1.3.15. Given a full transitive Pfaffian groupoid $(\Sigma, \omega, E)$ over $\mathbf{X}$, a principal $(\Sigma, \omega, E)$-bundle $(P, \theta)$ over $M$ induces a principal $\left(\Sigma_{x},\left.\omega\right|_{x} \circ \omega_{M C}, E_{x}\right)$-bundle $\left(P_{x}, \theta_{P_{x}}\right)$ over $M$ following the construction in the proof of Proposition 1.3.12. However, if $\mu$ denotes the moment map of the action of $\Sigma$ on $P$, the pair $\left(\mu^{-1}(x),\left.\theta\right|_{\mu^{-1}(x)}\right)$ is also a principal $\left(\Sigma_{x},\left.\omega\right|_{x} \circ \omega_{M C}, E_{x}\right)$-bundle over $M$.

$\left(P_{x}, \theta_{P_{x}}\right)$ can also be obtained from $\left(\mu^{-1}(x),\left.\theta\right|_{\mu^{-1}(x)}\right)$ by restricting the bibundle $\left(s^{-1}(x),\left.\omega\right|_{s^{-1}(x)}, \Phi\right)$ from above to a bibundle between $\left(\Sigma_{x},\left.\omega\right|_{x} \circ \omega_{M C}, E_{x}\right)$ and $\left(\Sigma_{x},\left.\omega\right|_{x} \circ \omega_{M C}, E_{x}\right)$ itself and following the construction in the proof of Proposition 1.3.12.

In conclusion, there is an isomorphism of principal $\left(\Sigma_{x},\left.\omega\right|_{x} \circ \omega_{M C}, E_{x}\right)$ bundles

$$
\left(P_{x}, \theta_{P_{x}}\right) \rightarrow\left(\mu^{-1}(x),\left.\theta\right|_{\mu^{-1}(x)}\right) .
$$

over the identity $M \rightarrow M$, see Remark 1.3 .13 .

\subsubsection{Principal actions of transitive Pfaffian groupoids}

Below, our purpose will be to study actions of full transitive Pfaffian groupoids over $\mathbb{R}^{n}$ - more precisely, jet groupoids of transitive pseudogroups over $\mathbb{R}^{n}$ with their Cartan distribution. Propositions 1.3.12 and 1.3.15 imply that it is enough to study principal $(G, \omega, V)$-bundles, where $(G, \omega, V)$ is a Pfaffian group (see Subsection 1.2.2 for Pfaffian groups). If one wants to implement this idea, one needs to allow for $(G, \omega, V)$ to be neither full nor extended, see Remark 1.2.21. On the other hand, it is not restrictive to assume principal $(G, \omega, V)$-bundles $(P, \theta)$ to be full (i.e. $\theta \in \Omega^{1}(P, V)$ to be pointwise surjective).

Assumption. From now on, unless otherwise specified, principal Pfaffian bundles $(P, \theta)$ are assumed to be full - i.e. $\theta$ is assumed to be pointwise surjective, 
see Definition 1.2.22. In terms of distributions, given a principal $(\Sigma, \mathcal{C}, E)$-bundle $\left(P, \mathcal{C}_{P}\right)$, the inclusion

$$
\nu_{\mathrm{e}_{P} \cap \operatorname{Ker}(d \pi)} \hookrightarrow \mu^{*} E
$$

induced by the infinitesimal action is assumed to extend to an isomorphism

$$
\nu_{\mathcal{C}_{P}} \cong \mu^{*} E
$$

see Definition 1.2.25.

In what follows, we will often describe Pfaffian groups in terms of distributions - i.e. as triples $(G, \mathfrak{h}, V)$, where $\mathfrak{h}$ is an ideal of the Lie algebra $\mathfrak{g}$ of $G$ and $V$ is a representation of $G$ admitting $\mathfrak{g} / \mathfrak{h}$ as a sub-representation; see Definition 1.2.10, and Remark 1.3.16 for the notation.

When $p: Y \rightarrow M$ is a surjective submersion and $\mathcal{F}$ is a foliation on $Y$, we say that $\mathcal{F}$ is vertical if the leaves of $\mathcal{F}$ are tangent to the vertical bundle of $p$.

Proposition 1.3.18. A principal $(G, \mathfrak{h}, V)$-bundle over $M$ is equivalent to the datum of a principal $G$-bundle $P$ over $M$ (action from the left) together with a $G$-equivariant vertical foliation $\mathcal{F}_{\mathfrak{h}}$ and an isomorphism $\Phi: \nu_{\mathcal{F}_{\mathfrak{h}}} \cong P \times V$ such that

$$
\Phi\left(\left[\hat{v}_{p}\right]\right)=\left(p, l_{\mathfrak{h}}(v)\right), \quad p \in P, v \in \mathfrak{g},
$$

where $\nu_{\mathcal{F}_{\mathfrak{h}}}$ denotes the normal bundle of $\mathcal{F}_{\mathfrak{h}}$ and $\hat{v}_{p}$ denotes the image of $v \in \mathfrak{g}$ under the infinitesimal action at $p \in P$.

Alternatively, a principal $(G, \mathfrak{h}, V)$-bundle over $M$ is equivalent to the datum of a principal $G$-bundle $P$ over $M$ together with an equivariant one form $\theta_{\mathfrak{h}} \in \Omega^{1}(P, V)$ such that $\operatorname{Ker}\left(\theta_{\mathfrak{h}}\right)$ is involutive and

$$
\theta_{\mathfrak{h}}\left(\hat{v}_{p}\right)=l_{h}(v), \quad p \in P, v \in \mathfrak{g} .
$$

Proof. A principal $(G, \mathfrak{h}, V)$-bundle is given by a principal $G$-bundle $P$ with a vertical distribution $\mathcal{C}_{P}$ such that the action is Pfaffian - see Definition 1.2.25. By using multiplicativity, we see that the distribution $\mathcal{C}_{P}$ is equivariant. Since $\mathcal{C}_{P}$ is vertical, multiplicativity implies that $\mathcal{C}_{P}$ at $p \in P$ is equal to the image of $\mathfrak{h}$ under the infinitesimal action at $p$. It follows that the distribution $\mathcal{C}_{P}$ is necessarily involutive, because $\mathfrak{h}$ is a subalgebra of $\mathfrak{g}$; consequently, $\mathcal{C}_{P}$ is tangent to an equivariant vertical foliation $\mathcal{F}_{\mathfrak{h}}$. The isomorphism $\Phi: \nu_{\mathcal{F}_{\mathfrak{h}}} \cong P \times V$ from the claim comes from the fact that $\left(P, \mathcal{C}_{P}\right)$ is a full principal $(G, \mathfrak{h}, V)$-bundle - see Definition 1.2.25. Conversely, given a foliation $\mathcal{F}_{\mathfrak{h}}$ as in the claim, the distribution $\mathcal{C}_{P}$ tangent to $\mathcal{F}_{\mathfrak{h}}$ makes $\left(P, \mathcal{C}_{P}\right)$ into a full principal $(G, \mathfrak{h}, V)$-bundle.

The form description is dual to the foliation one. Notice that, given a full principal $(G, \omega, V)$-bundle $P, \theta$, the condition

$$
\theta_{\mathfrak{h}}\left(\hat{v}_{p}\right)=l_{h}(v), \quad p \in P, v \in \mathfrak{g}
$$

can be immediately seen as a consequence of multiplicativity.

Definition 1.3.19. Principal $(G, \mathfrak{h}, V)$-bundles over manifolds $M$ are called Cartan bundles. We use the notation $\left(P, \mathcal{F}_{\mathfrak{h}}, \Phi\right)$ or $(P, \theta), \theta \in \Omega^{1}(P, V)$.

When $\left(P, \mathcal{F}_{\mathfrak{h}}, \Phi\right)$ is a principal $(G, \mathfrak{h}, V)$-bundle, we will also write Cartan $(G, \mathfrak{h}, V)$-bundle to emphasize the Pfaffian group $(G, \mathfrak{h}, V)$. 


\subsection{Cartan bundles}

\subsubsection{Some remarkable examples}

Let $(G, \mathfrak{h}, V)$ be a Pfaffian group and $\left(P, \mathcal{F}_{\mathfrak{h}}, \Phi\right)$ be a Cartan $(G, \mathfrak{h}, V)$-bundle.

\section{G-structures}

We start looking at the case when $\mathcal{F}_{\mathfrak{h}}$ consist of the whole foliation by fibers of $P \rightarrow M$; this happens if and only if $\mathfrak{h}=\mathfrak{g}$. We refer to this as simple case, since $\mathcal{F}_{\mathfrak{h}}$ is a simple foliation on $P$ and the leaf space is canonically diffeomorphic to $M$. From the form point of view, this case corresponds to an equivariant form $\theta$ on $P$ that vanishes on vertical vectors; since we ask for $\theta$ to be pointwise surjective, $\operatorname{dim}(V)=\operatorname{dim}(M)$.

This is the setting of abstract $G$-structures, i.e. principal $G$-bundles equipped with a representation of $G$ on $\mathbb{R}^{n}(n=\operatorname{dim}(M))$ and an equivariant $\mathbb{R}^{n}$-valued form whose kernel is the vertical bundle.

Proposition 1.4.1. A simple Cartan bundle induces an abstract $G$-structure.

One recovers classical $G$-structures, see Example 1.1.39 and [18, 64], by looking at the frame bundle $p: \operatorname{Fr}(M) \rightarrow M$ equipped with the tautological form $\theta_{\text {taut }} \in \Omega^{1}\left(\operatorname{Fr}(M), \mathbb{R}^{n}\right)$; recall that $\theta_{l}\left(V_{l}\right)$ is given by the components of $d p\left(V_{l}\right)$ with respect to the frame $l \in \operatorname{Fr}(M)$. If $G \subset G L_{n}(\mathbb{R})$ is a Lie subgroup and $p: P \rightarrow M$ is a $G$-reduction, the restriction of $\theta_{\text {taut }}$ to $P$, which we keep denoting by $\theta_{\text {taut }}$, makes $P$ into a simple Cartan bundle.

\section{Cartan geometries}

On the other extremum of the spectrum we have the case when $\mathcal{F}_{\mathfrak{h}}$ is the foliation by points. We refer to this case as parallelizable, since $\theta \in \Omega^{1}(P, V)$ is now an absolute parallelism on $P$; that is, we have $\operatorname{dim}(V)=\operatorname{dim}(P)$ and

$$
\theta: T P \rightarrow P \times V
$$

is an isomorphism. Notice that $\theta$ is equivariant and it coincides with the identity when composed with the infinitesimal action on $P$, thanks to multiplicativity. In other words, $(P, \theta)$ is a Cartan geometry.

Definition 1.4.2. A Cartan geometry on $M$ is the datum of

- a principal $G$-bundle $P \rightarrow M$;

- a $G$-representation $V$ containing the adjoint representation of $G$ on $\mathfrak{g}$ as a sub-representation;

- an equivariant form $\theta \in \Omega^{1}(P, V)$, called Cartan connection, which is a pointwise isomorphism and such that

$$
\theta_{p}\left(\hat{v}_{p}\right)=v, \quad p \in P, v \in \mathfrak{g},
$$

where $\hat{v}_{p}$ is the image of $v \in \mathfrak{g}$ under the infinitesimal action at $p \in P$. 
Proposition 1.4.3. Parallelizable Cartan bundles are in one to one correspondence with Cartan geometries.

Cartan geometries are extensively studied - see e.g. [3, 12, 58] for detailed introductions and further references. They were already encoded into transitive Lie algebroids in [8]. They may be thought of as curved versions of Klein geometries (see also the next two subsections); this perspective is explored in [58].

Example 1.4.4. Simple examples of Cartan geometries are given by classical $G$ structures $P \rightarrow M$ equipped with a compatible connection - that is, a connection whose parallel transport sends frames in $P \subset \operatorname{Fr}(M)$ to frames in $P \subset$ $\operatorname{Fr}(M)$. Such a connection can be encoded into a principal connection one form $\omega \in \Omega^{1}(P, \mathfrak{g})$ on the principal bundle $P$. The form $\theta=\theta_{\text {taut }}+\omega$ is a Cartan connection.

\section{Higher order $G$-structures}

By considering higher order $G$-structures, see Definition 1.1.38 and the discussion preceeding it, one gets Cartan bundles which are neither simple nor parallelizable.

Let $P \subset F r^{k}(M) \rightarrow M$ be a $G$-structure of order $k$ (hence, $G \subset G L_{n}^{k}(\mathbb{R})$ ). Below, we will consider the tautological form $\theta_{\text {taut }}$ on the higher order frame bundle $p: F r^{k}(M) \rightarrow M$. The form $\theta_{\text {taut }}$ is defined as in the $k=1$ case: for $l \in \operatorname{Fr}^{k}(M)$ and $V_{l} \in T_{l} F r^{k}(M)$, we declare $\theta_{l}\left(V_{l}\right) \in \mathbb{R}^{n}$ to be given by the components of $d p\left(V_{l}\right)$ with respect to the frame obtained by projecting $l \in F r^{k}(M)$ to $F r^{1}(M)$. The restriction of $\theta_{\text {taut }}$ to $P \subset F r^{k}(M)$ is still denoted by $\theta_{\text {taut }}$.

We consider the sequence of Lie groups

$$
G L_{n}^{k}(\mathbb{R}) \rightarrow G L_{n}^{k-1}(\mathbb{R}) \rightarrow \ldots G L_{n}(\mathbb{R}) .
$$

If we rebaptize $G$ as $G^{k}$, we get the sequence of Lie groups

$$
G^{k} \rightarrow G^{k-1} \rightarrow \ldots G^{1}
$$

by restricting maps. We denote the map $G^{k} \rightarrow G^{k-1}$ by $\tau^{k, k-1}$. We set $\mathfrak{g}^{k, k-1}:=$ $\operatorname{Ker}\left(d \tau^{k, k-1}\right) \subset \mathfrak{g}^{k}$ and

$$
\omega^{k}:=\omega_{M C} \circ d \tau^{k, k-1}: T G^{k} \rightarrow \mathfrak{g}^{k-1} .
$$

Notice that $\omega^{k}$ is equivariant. When $k=1$ one gets the 0 -form. We can postcompose $\omega^{k}$ with the map $\mathfrak{g}^{k-1} \rightarrow \mathfrak{g}^{k-1} \times \mathbb{R}^{n}$ sending $a \in \mathfrak{g}^{k-1}$ to $(a, 0)$; the resulting form, which we keep denoting by $\omega^{k}$, is in fact a Pfaffian form on $G^{k}$, with image given by $G^{k} \times \mathfrak{g}^{k-1}$ and kernel given by the equivariant distribution on $G^{k}$ generated by $\mathfrak{g}^{k, k-1}$. Similarly, if we rebaptize $P$ as $P^{k}$, we have the sequence of principal bundles

$$
P^{k} \rightarrow P^{k-1} \rightarrow \ldots P^{1} \rightarrow M,
$$

where $P^{i} \subset F r^{i}(M), 1 \leq i<k$, is the $G^{i}$-reduction of $F^{i}(M)$ obtained by projecting $P^{k} \subset F r^{k}(M)$ to $F r^{i}(M)$. We will denote the map $P^{k} \rightarrow P^{k-1}$ by 
$\pi^{k, k-1}$ and the map $P^{k} \rightarrow P^{1}$ by $\pi^{k, 1} . P^{k}$ is a Cartan $\left(G^{k}, \mathfrak{g}^{k, k-1}, \mathfrak{g}^{k-1} \times \mathbb{R}^{n}\right)$ bundle when equipped with the form

$$
\left(\theta^{k}\right)_{l^{k}}:=\left(\phi_{l^{k}} \circ d \pi^{k, k-1}, \theta_{\text {taut }}\right): T P^{k} \rightarrow \mathfrak{g}^{k-1} \times \mathbb{R}^{n} .
$$

Here, $\phi_{l_{k}}$ denotes the left splitting of the sequence

$$
0 \rightarrow \operatorname{Ker}\left(d\left(P^{k-1} \rightarrow M\right)\right) \rightarrow T P^{k-1} \rightarrow T M \rightarrow 0
$$

induced by $l^{k}=j_{x}^{k} \psi$, where $x \in M$ and $\psi: U \subset M \rightarrow \mathbb{R}^{n}$ is an isomorphism sending $x$ to 0 .

\section{Fibers of transitive Pfaffian groupoids}

If $(\Sigma, \omega)$ is a transitive full Pfaffian groupoid, the $t$-fiber $t^{-1}(x)$ at any point is a Cartan bundle when equipped with $\theta:=\left.\omega\right|_{t^{-1}(x)}$. Alternatively, one can look at the $s$-fibers with the form $\theta_{g}:=\left.g^{-1} \cdot \omega\right|_{s^{-1}(x)}$. This was used in Proposition 1.3.15. Keeping in mind the gauge construction from Proposition 1.2.27, this example can be seen as a different way to discover Cartan bundles - a way that was explored in [16].

\subsubsection{The flat case: equivariant vertical Lie foliations}

Definition 1.4.5. Let $(G, \mathfrak{h}, V)$ be a Pfaffian group. We denote by $\mathfrak{k}$ the vector space $V$ together with the choice of a Lie bracket $[$,$] on V$. We say that $\mathfrak{k}$ is a transverse infinitesimal model on $V$ if the map $\mathfrak{g} \rightarrow \mathfrak{k}$ sending $\mathfrak{g}$ to $\mathfrak{g} / \mathfrak{h}$ is a Lie algebra morphism. If

- $K$ is the 1-connected Lie group integrating $\mathfrak{k}$;

- there is a morphism $\psi: G \rightarrow K$ integrating $\mathfrak{g} \rightarrow \mathfrak{g} / \mathfrak{h} \subset \mathfrak{k}$ and having closed image;

we call $K$ a transverse model.

From now on, we assume to have chosen an infinitesimal model, unless otherwise specified.

Remark 1.4.6. Choosing an infinitesimal transverse model corresponds precisely to endowing the Pfaffian group $(G, \mathfrak{h}, V)$ with the additional structure needed to make it extended Pfaffian, Definitions 1.2.8 and 1.2.12.

Remark 1.4.7. Since $\mathfrak{h}$ is an ideal in $\mathfrak{g}$, the Lie algebra structure on $\mathfrak{g} / \mathfrak{h}$ is fixed. An infinitesimal transverse model is the choice of an inclusion $\mathfrak{g} / \mathfrak{h} \hookrightarrow \mathfrak{k}$.

The adjoint representation of $G$ on $\mathfrak{g}$ passes to the quotient, inducing a representation on $\mathfrak{g} / \mathfrak{h} \subset \mathfrak{k}$; the representation on $\mathfrak{k}$, which is part of the datum of a Pfaffian group, is an extension of such a representation. The corresponding infinitesimal representation of $\mathfrak{g}$ on $\mathfrak{k}$ can be equivalently seen as an extension of the composition of the adjoint representation of the Lie algebra $\mathfrak{g} / \mathfrak{h}$ with the map $\mathfrak{g} \rightarrow \mathfrak{g} / \mathfrak{h}$.

If we deal with (non infinitesimal) transverse models, the (closed) image of the morphism $G \rightarrow K$ acts by coniugation on $K$; composing the induced representation on $\mathfrak{k}$ with the map $G \rightarrow K$ we get the representation of $G$ on $\mathfrak{k}$. 
Definition 1.4.8. Let $(P, \theta), \theta \in \Omega^{1}(P, V)$ be a Cartan bundle and $\mathfrak{k}$ a model. The $\mathfrak{k}$-curvature - or simply curvature if $\mathfrak{k}$ is clear from context - is the two-form

$$
\Omega^{\mathfrak{k}}:=d \theta+\frac{1}{2}[\theta, \theta] .
$$

We say that a Cartan bundle is flat with respect to the model $\mathfrak{k}$ - or simply flat if there is no need to emphasize the model - if $\theta$ satisfies the Maurer-Cartan equation $\Omega^{\mathfrak{k}}=0$.

In the definition above, $[\theta, \theta](V, W)=2[\theta(V), \theta(W)]$ (the bracket of $\mathfrak{k}$ is used), while $d \theta(V, W)=\nabla_{W}(\theta(V))-\nabla_{V}(\theta(W))-\theta[V, W], \nabla$ being the trivial connection on $P \times \mathfrak{k}$.

In investigating flatness from the point of view of distributions, one is lead to a more general setting. Recall that a Maurer-Cartan form on a manifold $M$ with coefficents in a Lie algebra $\mathfrak{k}$ is a one form $\theta \in \Omega^{1}(M, \mathfrak{k})$ satisfying the MaurerCartan equation

$$
d \theta+\frac{1}{2}[\theta, \theta]=0 .
$$

The form $\theta$ is called non singular if, for all $x \in M, \theta_{x}: T_{x} M \rightarrow \mathfrak{k}$ has maximal rank; we will always deal with non singular forms. A flat Cartan bundle is in particular a manifold $P$ with a Maurer-Cartan form $\theta$. Maurer-Cartan forms on manifolds are known to correspond to a particular class of transversally parallelizable foliations, called Lie foliations $[49,51]$.

Proposition 1.4.9. If $\theta \in \Omega^{1}(M, \mathfrak{k})$ is a non singular Maurer-Cartan form on $M$, then $\operatorname{Ker}(\theta)$ is involutive. Furthermore, it is transversally parallelizable - i.e. it admits transverse vector fields trivializing its normal bundle - with trivialization of the normal bundle

$$
\nu_{\operatorname{Ker}(\theta)} \rightarrow M \times \mathfrak{k}, \quad\left[V_{x}\right] \rightarrow\left(x, \theta\left(V_{x}\right)\right) .
$$

Hence

Corollary 1.4.10. The foliation $\mathcal{F}_{\mathfrak{h}}$ of a flat Cartan bundle $\left(P, \mathcal{F}_{\mathfrak{h}}, \mathfrak{k}\right)$ is an equivariant vertical Lie foliation.

\subsubsection{Monodromy maps}

When dealing with Lie foliations, one can attach a monodromy homomorphism $\Psi_{\theta}: \pi_{1}\left(M, x_{0}\right) \rightarrow K$ to the Maurer-Cartan form $\theta \in \Omega^{1}(M, \mathfrak{k})$ on $M$.

Lemma 1.4.11. Let $I$ be the unit interval and $\gamma: I \rightarrow M$ a path. For any $k \in K$ there exists a unique $\widetilde{\gamma}: I \rightarrow K$ such that $\gamma(0)=k$ and $\gamma^{*} \theta=\widetilde{\gamma}^{*} \omega_{M C}$.

See [51] or even [58] for the case when $\theta$ is a pointwise isomorphism. Since the Maurer-Cartan form $\omega_{M C}$ of a Lie group is a pointwise isomorphism, the path $\widetilde{\gamma}$ in the statement above is determined infinitesimally, and can be globally constructed by integration. Out of the developing map, one constructs the monodromy homomorphism $\Psi_{\theta}: \pi_{1}\left(M, x_{0}\right) \rightarrow K$ by taking the endpoint of the development of paths with starting point $e \in K$. The Maurer-Cartan equation 
implies that the map descends to the homotopy group, and one can check that it is indeed a group homomorphism.

In the Cartan bundle setting, where we replace $M$ with a principal $G$-bundle $\pi: P \rightarrow M$, the multiplicativity of $\theta$ /equivariance of $\mathcal{F}_{\mathfrak{h}}$ allow to obtain a developing map defined on the quotient $M$. In the case of Cartan geometries - i.e. when $\theta$ is a pointwise isomorphism - this is carefully explained in [58]. In general, assuming $(G, \mathfrak{h}, K)$ to be a model, we have

Lemma 1.4.12. For any path $\gamma: I \rightarrow P$ in $P$ and any function $g: I \rightarrow G$ one has $\widetilde{g \cdot \gamma}=\psi(g) \cdot \widetilde{\gamma}$ (see the Definition 1.4.5), provided that the left hand side starts at $\psi(g) \cdot \widetilde{\gamma}(0)$.

Proof. We provisionally use the notiation $\omega_{M C}^{K}$ and $\omega_{M C}^{G}$ to distinguish the Maurer-Cartan forms on $K$ and $G$. We also denote $\psi(g): I \rightarrow K$ by $k: I \rightarrow K$.

First of all, we observe that the classical gauge formula

$$
(k \cdot \widetilde{\gamma})^{*}\left(\omega_{M C}^{K}\right)=k^{*} \omega_{M C}^{K}+A d(k) \circ \widetilde{\gamma}^{*} \omega_{M C}^{K}
$$

holds. By definition

$$
(\widetilde{g \cdot \gamma})^{*} \omega_{M C}^{K}=(g \cdot \gamma)^{*} \theta
$$

Now

$$
(g \cdot \gamma)^{*} \theta=(a \circ(g, \gamma) \circ \Delta)^{*} \theta
$$

where $\Delta$ is the diagonal $I \rightarrow I \times I$ and $a$ is the action $G \times P \rightarrow P$. We apply multiplicativity

$$
\left(a^{*} \theta\right)_{(g, p)}=\left(p r_{1}^{*} \bar{\omega}_{M C}^{G}\right)_{(g, p)}+g \cdot p r_{2}^{*}(\theta)_{(g, p)}, \quad(g, p) \in G \times P,
$$

where $\bar{\omega}_{M C}^{G}$ denotes the Pfaffian form on $G$, which is the composition of the map $\mathfrak{g} \rightarrow \mathfrak{k}$ with $\omega_{M C}^{G}$. We see that

$$
(g \cdot \gamma)^{*} \theta=g^{*} \bar{\omega}_{M C}^{G}+A d(k) \cdot \widetilde{\gamma}^{*} \omega_{M C}^{K} .
$$

since, by definition of model, the action of $g$ on $\mathfrak{k}$ is given by the action of $k$ on $\mathfrak{k}$, which is by the adjoint map. Furthermore

$$
g^{*} \bar{\omega}_{M C}^{G}=k^{*} \omega_{M C}^{K}
$$

by definition of $g$ and $k$ and the fact that $G \rightarrow K$ is a groupoid morphism. It follows that

$$
(k \cdot \widetilde{\gamma})^{*}\left(\omega_{M C}^{K}\right)=(\widetilde{g \cdot \gamma})^{*} \omega_{M C}^{K}
$$

which implies the claim by Lemma 1.4.11.

Below, we use $\psi(G) \backslash K$ to denote the space of left cosets of $\psi(G)$ in $K$. By the lemma above, the following definition makes sense.

Definition 1.4.13. Let $\gamma: I \rightarrow M$ be a path. The development of $\gamma$ in $\psi(G) \backslash K$ is the projection to $\psi(G) \backslash K$ of the development of a lift $\hat{\gamma}: I \rightarrow P$ of $\gamma$.

Via the homotopy lifting property of fiber bundles, one has 
Proposition 1.4.14. Let $(P, \theta, K)$ be a flat Cartan bundle. The development in $\psi(G) \backslash K$ of a path in $\gamma: I \rightarrow M$, depends only on the homotopy class of $\gamma$.

Consequently, the reduced monodromy map

$$
\Psi_{\text {red }}: \pi_{1}\left(M, x_{0}\right) \rightarrow \psi(G) \backslash K
$$

is well defined.

\subsubsection{Integrability and Cartan bundles}

Below, $(G, \omega, \mathfrak{k}), \mathfrak{h}=\operatorname{Ker}_{e}(\omega)$, is an extended Pfaffian group; that is, $(G, \omega, V)$ is a Pfaffian group and we choose a transverse infinitesimal model $\mathfrak{k}$ on $V$, see Definition 1.4.5. This simply means that $V$ possesses a Lie algebra structure, denoted by $\mathfrak{k}$, such that $\mathfrak{g} / \mathfrak{h} \subset \mathfrak{k}$ is a subalgebra.

Definition 1.4.15. Let $\mathfrak{a}$ be an extension of $\mathfrak{h}$ by $\mathfrak{k}$ - i.e. there is a short exact sequence of Lie algebras

$$
0 \rightarrow \mathfrak{k} \rightarrow \mathfrak{g} \rightarrow \mathfrak{h} \rightarrow 0 .
$$

Let $(P, \theta)$ be a full Cartan $(G, \omega, \mathfrak{k})$-bundle - hence $\theta$ is pointwise surjective. An $\mathfrak{a}$-lift of $\theta$ is a Cartan connection $\eta \in \Omega^{1}(P, \mathfrak{a})$ - see Subsection 1.4.1 - such that the corresponding parallelism $\eta: T P \rightarrow P \times \mathfrak{a}$ factors through the trivialization $\nu_{\mathrm{Ker}(\theta)} \rightarrow P \times \mathfrak{k}$ induced by $\theta$.

Definition 1.4.16. A Cartan bundle $(P, \theta), \theta \in \Omega^{1}(P, \mathfrak{k})$, is called $\mathfrak{a}$-flat, or flat with respect to $\mathfrak{a}$, if $\mathfrak{a}$ is an extension of $\mathfrak{h}$ by $\mathfrak{k}$ such that $\theta$ admits a flat $\mathfrak{a}$-lift $\eta \in \Omega^{1}(P, \mathfrak{a})$.

Example 1.4.17. Of course, when $\mathfrak{h}=0$ extending $\mathfrak{h}$ by $\mathfrak{g}$ is a trivial matter; consequently, for Cartan geometries $\mathfrak{a}=\mathfrak{g}$-flatness reduces to ordinary flatness, see the previous subsection.

Example 1.4.18. Consider a classical $G$-structure $\left(P, \theta_{\text {taut }}\right), P \subset \operatorname{Fr}(M) \rightarrow M$. We see $\theta_{\text {taut }} \in \Omega^{1}\left(P, \mathbb{R}^{n}\right)$ as valued in the abelian Lie algebra $\mathbb{R}^{n}$ - i.e. our infinitesimal transverse model is given by the trivial Lie bracket on $\mathbb{R}^{n}$. One can take the extension bracket on $\mathfrak{a}=\mathfrak{g} \oplus \mathfrak{k}$ to be given by the semidirect sum bracket

$$
[(\alpha, v),(\beta, w)]=\left([\alpha, \beta], \nabla_{\alpha}(w)-\nabla_{\beta}(v)\right) .
$$

Here, $\nabla$ is given by the representation of $\mathfrak{g}$ on $\mathfrak{k}$. Flatness with respect to this extension corresponds to integrability of the underlying $G$-structure. In fact, an $\mathfrak{a}$-lift of $\theta_{\text {taut }}$ can be constructed by choosing a principal connection $\tau \in \Omega^{1}(P, \mathfrak{g})$ and taking $\eta=\theta_{\text {taut }}+\tau$. Notice now that the Maurer-Cartan equation

$$
d \eta+\frac{1}{2}[\eta, \eta]_{\mathfrak{a}}=0
$$

can be splitted according to the decomposition $T P=\operatorname{Ker}(\omega) \oplus P \times \mathfrak{k}$ giving

$$
d \tau+\frac{1}{2}[\tau, \tau]_{\mathfrak{g}}=0, \quad \text { (flatness of } \tau \text { ) }
$$

and

$$
d \theta_{\text {taut }}+\tau \wedge \theta_{\text {taut }}=0 .
$$


The latter equation, when pulled back to an open $U \subset M$ via a section $\sigma$ such that $\sigma^{*}(\tau)=0$ gives

$$
d \sigma^{*} \theta_{\text {taut }}=0
$$

since $\sigma^{*} \theta_{\text {taut }}\left(v_{x}\right)$ is given by the components of the vector $v_{x}$ with respect to the frame $\sigma(x)$; consequently, the equation shows that the frame $x \rightarrow \sigma(x)$ is by commuting vector fields. The existence of such a frame in $P$ around each point of $M$ is equivalent to the flatness of the given $G$-structure/integrability of the associated $\Gamma_{G}$-structure, see Section 1.1.

Example 1.4.19. The above example can be slightly generalized to deal with $G$ structures $\left(P, \theta_{\text {taut }}\right)$ which are integrable with respect to a given Lie algebra model $\mathfrak{k}$ - where $\mathfrak{k}$ denotes $\mathbb{R}^{n}$ together with the choice of a Lie bracket. A $G$-structure $P \subset \operatorname{Fr}(M) \rightarrow M$ is k-integrable when, for all $x \in M$ there is an open $U_{x} \subset M$ and a local frame $\left(V_{1}, \ldots V_{n}\right)$ defined over $U_{x}$ such that

- $\left(V_{1}, \ldots V_{n}\right): U_{x} \rightarrow \operatorname{Fr}(M)$ takes values in $P$;

- the span over $\mathbb{R}$ of $\left\{V_{1}, \ldots V_{n}\right\}$, denoted by $\operatorname{span}_{\mathbb{R}}\left\{V_{1}, \ldots V_{n}\right\}$, is closed under the Lie bracket of vector fields over $U_{x}$;

- $\left(\operatorname{span}_{\mathbb{R}}\left\{V_{1}, \ldots V_{n}\right\},[],\right)$ is isomorphic to $\mathfrak{k}$.

The notion of $\mathfrak{k}$-integrability appears in [2].

Let us assume that $\mathfrak{g}$ acts on $\mathfrak{k}$ by derivations. In this case, we can still proceed as above; we form the semidirect sum $\mathfrak{a}=\mathfrak{g} \oplus \mathfrak{k}$ with bracket

$$
[(\alpha, v),(\beta, w)]=\left([\alpha, \beta],[v, w]+\nabla_{\alpha}(w)-\nabla_{\beta}(v)\right) .
$$

Then, if $\tau \in \Omega^{1}(P, \mathfrak{g})$ is a principal connection, the sum $\eta:=\theta_{\text {taut }}+\tau$ is a Cartan connection and an a-lift of $\theta_{\text {taut }}$. If $\eta$ is flat, then the Maurer-Cartan equation

$$
d \eta+\frac{1}{2}[\eta, \eta]_{\mathfrak{a}}=0
$$

splits into

$$
d \tau+\frac{1}{2}[\tau, \tau]_{\mathfrak{g}}=0, \quad \text { (flatness of } \tau \text { ) }
$$

and

$$
d \theta_{\text {taut }}+\frac{1}{2}\left[\theta_{\text {taut }}, \theta_{\text {taut }}\right]_{\mathfrak{k}}+\tau \wedge \theta_{\text {taut }}=0 .
$$

Reasoning as above, we discover that if $\left(P, \theta_{\text {taut }}\right)$ is $\mathfrak{a}$-flat, then it is integrable with respect to $\mathfrak{k}$.

Example 1.4.20. A standard example of $G$-structures integrable with respect to a Lie algebra are coorientable contact structures on manifolds $M, \operatorname{dim}(M)=$ $2 k+1$; see [2]. Underlying a coorientable contact structure on $M$ there is always a $S p(k, 1)$-structure, where $S p(k, 1)$ is the group of linear symmetry of the canonical symplectic foliation on $\mathbb{R}^{2 k+1}$, see Example 1.1.6. This underlying $S p(k, 1)$ structure is never flat in the usual sense (a flat $S p(k, 1)$-structure needs to be a symplectic foliation). However, thanks to the Darboux theorem, the $S p(k, 1)$ structures underlying coorientable contact structures are integrable with respect to the $(2 k+1)$-dimensional Heisenberg Lie algebra hei: around each point there is a local frame that behaves like $\mathfrak{h e i}$ under the Lie bracket. 



\title{
2 Haefliger's differentiable cohomology
}

\author{
$1 /$ - Come sto andando scusi? \\ - Male, per Dio! \\ - Ragionier Ugo Fantozzi e Direttore Conte \\ Corrado Maria Lobbiam, Il secondo tragico \\ Fantozzi
}

The content of this chapter is available as a preprint on the arXiv [1] and is joint work with Marius Crainic. We review Haefliger's differentiable cohomology for the pseudogroup of diffeomorphisms of $\mathbb{R}^{q}$ [35]. We investigate the structure needed to define such a cohomology, which, remarkably, is related to the so called Cartan distribution underlying the geometric study of PDE. We define an analogue of Haefliger differentiable cohomology for flat Cartan groupoids, investigate its infinitesimal counterpart and relate the two by a van Est-like map. Finally, we define a characteristic map for geometric structures on manifolds $M$ associated to flat Cartan groupoids. The outcome generalizes the existing approaches to characteristic classes for foliations $[6,7,11,35]$.

\subsection{Introduction}

Various geometric structures on manifolds $M$ come with invariants that live in the cohomology of the ambient space $M$, and which are organised into a map from a certain "universal space" (associated to the type of structure one is looking at) to the cohomology of $M$; such maps are called generically "characteristic maps". For instance, for vector bundles one has Pontryagin/Chern classes and the Chern-Weil homomorphisms. To help the reader, but also to put things into perspective, in the first section of this chapter we overview some of the standard characteristic maps (for principal bundles, for flat ones, for foliations and then for more general $\Gamma$-structures).

Haefliger's differentiable cohomology arose in the development of characteristic classes for foliations $\mathcal{F}$ in analogy with the one for flat principal bundles. The theory was producing: 
- explicit/geometric characteristic maps $\kappa^{\mathcal{F}}$, in the spirit of Chern-Weil theory, defined on certain Lie algebra cohomologies that can be computed explicitly (see Subsection 2.2.3),

- abstract characteristic maps $\kappa_{\text {abs }}^{\mathcal{F}}$ built via classifying spaces and maps, defined on a rather huge and complicated cohomology $H^{*}\left(B \Gamma^{q}\right)$ (see Subsection 2.2.4),

- a universal characteristic map $\kappa^{\text {univ }}$ (see Subsection 2.3.1),

fitting together in a commutative diagram

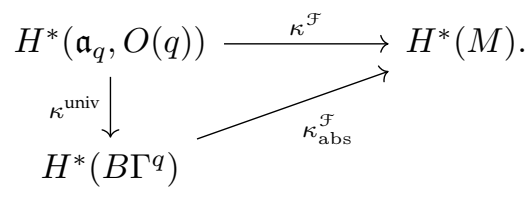

As the name indicates, $\kappa_{\text {abs }}^{\mathcal{F}}$ was constructed rather abstractly and even its domain $H^{*}\left(B \Gamma^{q}\right)$ is rather huge and unmanageable. There have been several more explicit descriptions of this cohomology, and of the abstract characteristic map, most notably:

- as a "de Rham-like" cohomology based on the Bott-Shulman complex (see Subsection 2.2.6),

- via sheaf cohomology and then, based on explicit bar-type resolutions, via group-like cochains on $\Gamma^{q}$, the groupoid of germs of diffeomorphisms of $\mathbb{R}^{q}$. This is in complete analogy with cohomology of discrete groups which, themselves, play a similar role in characteristic maps for flat principal $G$ bundles.

While everything was very similar to the case of flat principal bundles, there was still a question left: whether one could make sense, inside the complex computing the cohomology $H^{*}\left(B \Gamma^{q}\right)$, of a "differentiable complex of $\Gamma^{q "}$ giving rise to a "differentiable cohomology"

$$
H_{\text {diff }}^{*}\left(\Gamma^{q}\right)
$$

and whether one could prove a "van Est isomorphism" between this cohomology and $H^{*}\left(\mathfrak{a}_{q}, O(q)\right)$, so that the previous diagram becomes

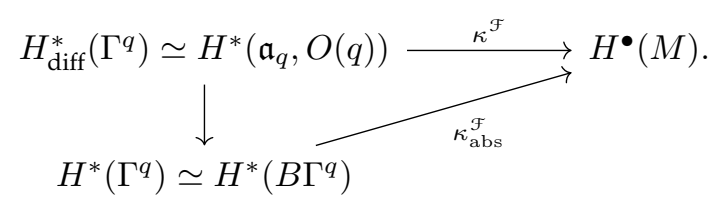

Of course, the vertical map would be simply the one induced by the inclusion of differentiable cocycles into the general ones. This is precisely the task undertaken by Haefliger in [35]. Given the fact that he was concentrating on the special case of $\Gamma^{q}$, and perhaps also because of the apparently ad-hoc nature of the construction, his construction of $H_{\text {diff }}^{*}\left(\Gamma^{q}\right)$ (and of his van Est-like isomorphism) did not receive 
much attention and seems to be forgotten. One of the aims of this chapter is to explain and provide more insight into Haefliger's differentiable cohomology.

As it was/is interesting to generalise such constructions from foliations to general $\Gamma$-structures (also called $\Gamma$-foliations), we will proceed in this generality. But it is fair to say that everything was in place in this generality already during Haefliger's work mentioned above. The only little problems were that

- one usually assumed that $\Gamma$ was transitive,

- while in principle Haefliger's differentiable cohomology $H_{\text {diff }}^{*}\left(\Gamma^{q}\right)$ could just be copied for any pseudogroup $\Gamma$, the situation is actually a bit more subtle (and, as we shall point out, it is for Lie pseudogroups $\Gamma$ that everything works).

Furthermore, looking at Haefliger's definition, it is clear right away that the outcome does not depend on $\Gamma$, but rather on the associated infinite jet groupoid $J^{\infty} \Gamma$ (and certain structure on it). One of the main driving questions behind this chapter was to find the structure on $J^{\infty} \Gamma$ that makes Haefliger's differentiable cohomology work. The outcome is that of "flat Cartan groupoid $(\Sigma, \mathcal{C})$ ", whose resulting cohomology we called Haefliger cohomology

$$
H_{\text {Haef }}^{*}(\Sigma, \mathcal{C}) \text {. }
$$

For $\Sigma=J^{\infty} \Gamma$ we find out that the extra-structure that we were after is precisely the well-known Cartan distribution $\mathcal{C}$ on infinite jet spaces plus the (less known) understanding of its compatibility with the groupoid structure; and, of course,

$$
H_{\text {Haef }}^{*}\left(J^{\infty} \Gamma, \mathcal{C}\right)=H_{\text {diff }}^{*}(\Gamma) .
$$

We must add here that the notion of Cartan groupoid (and variations) came already to our attention from our study of Lie pseudogroups from the point of view of PDEs, and of the corresponding geometric structures [15, 55, 69]. The fact that such different aspects point out to the same structure may seem quite surprising/remarkable at first; at a second thought however, they just show that it is always the same structure that make the jet spaces interesting and useful over and over again. This rather philosophical insight is actually used several times inside the chapter. For instance, the theory of almost geometric structures reveals the notion of flat "principal Cartan groupoid-bundle" or, in the terminology of Section 2.4, of flat $(\Sigma, \mathcal{C})$-structure. We use that notion to point out that some of the characteristic maps associated to geometric structures depend only on the underlying almost structures. In turn, this may have interesting applications to the study of almost structures themselves: as pointed out in Remark 2.6.4, the resulting Haefliger characteristic maps may be used to detect almost $\Gamma$-structures that are not integrable.

In Section 2.2, we briefly review the connection-curvature construction and the classifying space approach to characteristic classes for principal bundles and flat principal bundles, and the similar machinery for characteristic classes for foliations. We introduce pseudogroups and recall the equivalence between the category of pseudogroups and the category of effective étale groupoids. We review the Bott-Shulman model and the sheaf theoretical approach to the cohomology of the classifying space of an étale groupoid. In Section 2.3, we adapt 
Haefliger's definition of $H_{\text {diff }}^{*}\left(\Gamma^{q}\right)$ to a general Lie pseudogroup $\Gamma$. We proceed to investigate the minimal structure that allows to construct an analogous cohomology on a general Lie groupoid $\Sigma \rightrightarrows \mathbf{X}$, discovering flat Cartan connections; we then briefly review the canonical flat Cartan connection on $J^{\infty} \Gamma$. In Section 2.4 we depart from Lie pseudogroup to work with Lie groupoids equipped with a flat Cartan connection, introducing what we called "Haefliger cohomology" and discussing some examples. We also study flat principal bundles in this context, which include formally integrable "almost geometric structures" as a particular example. In Section 2.5 we present the infinitesimal picture of the theory discussed in Sections 2.3 and 2.4. Finally, in Section 2.6, we discuss a van Est theorem for groupoids equipped with a flat Cartan connection - generalizing a similar result proved by Haefliger for $\operatorname{Diff}_{\text {loc }}\left(\mathbb{R}^{q}\right)$. We conclude presenting a characteristic map for "flat principal actions" of groupoids with a flat Cartan connection. Specializing to $J^{\infty} \Gamma^{q}$ with its canonical Cartan connection, we recover Haefliger's approach to characteristic classes of foliations. Throughout the chapter, we need to work with pro-finite (dimensional) manifolds and pro-finite Lie groupoids. Since some of the properties that we make use of are not explicitly spelled out in the literature, we provide a detailed appendix.

\subsection{Overview of various characteristic classes}

\subsubsection{Geometric/abstract characteristic classes for principal bun- dles}

Characteristic classes for principal $G$-bundles $P \rightarrow M$ ( $G$ being a Lie group and $P, M$ smooth manifolds) are usually introduced either:

(geom): more explicitly/geometrically: one uses connections to produce explicit cohomology classes on the base $M$ of the principal $G$-bundle. Known as the Chern-Weil theory, it produces the so-called Chern-Weil map

$$
\kappa^{P}: \operatorname{Inv}(\mathfrak{g}) \rightarrow H^{*}(M)
$$

defined on the space of $G$-invariant polynomials $\operatorname{Inv}(\mathfrak{g})=S\left(\mathfrak{g}^{*}\right)^{G}$ on the Lie algebra $\mathfrak{g}$ of $G$.

(abs): more abstractly/topologically: this is based on the existence of a certain principal $G$-bundle $E G \rightarrow B G$ which is universal in the sense that any principal $G$-bundle $P \rightarrow M$ arises as the pull-back of $E G$ via a classifying map $M \rightarrow B G$. This map is unique up to homotopy, hence one has a uniquely defined characteristic map in cohomology:

$$
\kappa_{\mathrm{abs}}^{P}: H^{*}(B G) \rightarrow H^{*}(M) .
$$

In the more abstract approach one concentrates on the classifying space $B G$ and its cohomology and one would like to have more explicit models. While $B G$ is unique up to homotopy, there are various known models for it; however, in general, $B G$ still remains rather abstract. Of course, its cohomology may be simpler and one hopes for more explicit models for realising $H^{*}(B G)$ and $\kappa_{\text {abs }}^{P}$. 
Of course, the Chern-Weil map $\kappa^{P}$ fits this scheme. First of all one has a natural map

$$
\kappa^{\text {univ }}: \operatorname{Inv}(\mathfrak{g}) \rightarrow H^{*}(B G)
$$

that can be thought of as the Chern-Weil map associated to the universal bundle $E G$, and all the maps fit in a commutative diagram:

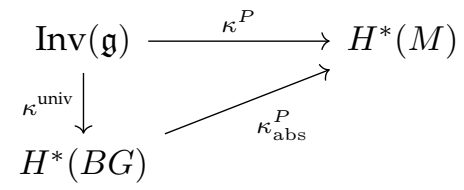

Furthermore, it is known (see e.g. [26]) that, for compact Lie groups $G, \kappa^{\text {univ }}$ is an isomorphism and, therefore, the two characteristic maps $\kappa^{P}$ and $\kappa_{\text {abs }}^{P}$ become identified:

$$
H^{*}(B G) \cong \operatorname{Inv}(\mathfrak{g})
$$

When $G$ is compact and connected, by restricting to a maximal torus $T \subset G$ (with Lie algebra $\mathfrak{t} \subset \mathfrak{g}$ ), $\operatorname{Inv}(\mathfrak{g})$ becomes isomorphic with the algebra of polynomials on $\mathfrak{t}$ that are invariant under the action of the corresponding Weyl group $W$, i.e. $H^{*}(B G) \cong S\left(\mathfrak{t}^{*}\right)^{W}$. More in general, when $G$ is compact with connected component of the identity denoted by $G_{0}$, one has an action of $G / G_{0}$ on $S\left(\mathfrak{t}^{*}\right)^{W}$ and

$$
H^{*}(B G) \cong \operatorname{Inv}_{G / G_{0}}\left(S\left(\mathfrak{t}^{*}\right)^{W}\right) .
$$

Recall also that, for general Lie groups $G$, the cohomology of $B G$ is canonically isomorphic to the cohomology of $B K$, the classifying space of the maximal compact subgroup $K$ of $G$. Therefore, for Lie groups, the rather abstract $H^{*}(B G)$ can be made actually quite explicit.

Example 2.2.1. When looking at complex vector bundles $E \rightarrow M$ interpreted as principal $G L_{n}(\mathbb{C})$-bundles $\operatorname{Fr}(E) \rightarrow M, K=U(n)$ with maximal torus $T^{n}$ (diagonal matrices), and the Weyl group $W=S_{n}$ permuting the entries, we have

$$
H^{*}\left(B G L_{n}(\mathbb{C})\right) \cong H^{*}(B U(n))=\mathbb{R}\left[x_{1}, \ldots, x_{n}\right]^{S_{n}}=\mathbb{R}\left[c_{1}, \ldots, c_{n}\right],
$$

where the $x_{i}{ }^{\prime}$ 's are degree one variables and the $c_{i}{ }^{\prime}$ 's are the fundamental symmetric polynomials on the $x_{i}$ 's. Of course, for a rank $n$ complex vector bundle $E \rightarrow M$,

$$
c_{i}(E)=\kappa_{\mathrm{abs}}^{F r(E)}\left(c_{i}\right) \in H^{2 i}(M)
$$

are the usual Chern classes of $E$.

Example 2.2.2. A similar discussion applies to the Pontryagin classes of real vector bundles $F \rightarrow M$,

$$
p_{j}(F) \in H^{4 j}(M), \quad\left(0 \leq j \leq k:=\left[\frac{n}{2}\right], n=\operatorname{rank}(F)\right),
$$

which are usually defined as the even Chern classes $c_{2 j}\left(F_{\mathbb{C}}\right)$ of the complexification $F_{\mathbb{C}}$ of $F$ (with the footnote that the odd classes vanish). The relevant group is $G=G L_{n}(\mathbb{R})$ and

$$
H^{*}\left(B G L_{n}(\mathbb{R})\right)=H^{*}(B O(n))=\mathbb{R}\left[y_{1}, \ldots, y_{k}\right]^{S_{n}}=\mathbb{R}\left[p_{1}, \ldots, p_{k}\right],
$$

where $k=\left[\frac{n}{2}\right]$ and $y_{j}$ has degree $2 j$. Of course, with the mind at the definition $p_{j}(F)=c_{2 j}\left(F_{\mathbb{C}}\right)$, one may think that $y_{j}=x_{j}^{2}$. 


\subsubsection{Geometric/abstract characteristic classes for flat bundles}

The previous discussion serves as guideline in various other contexts. For instance, a related (and actually simpler) discussion is that of characteristic classes for flat $G$-bundles $P \rightarrow M$ :

(geom): more explicitly: the flatness is encoded in a flat connection 1-form $\omega \in$ $\Omega^{1}(P, \mathfrak{g})$. This is immediately turned into a chain map from the ChevalleyEilenberg complex of $\mathfrak{g}$ to the de Rham complex of $P$ and then to a map in cohomology,

$$
\kappa^{\omega}: H^{*}(\mathfrak{g}, K) \rightarrow H^{*}(M),
$$

defined on the Lie algebra cohomology of $\mathfrak{g}$ relative to the maximal compact subgroup $K$ of $G$.

(abs): abstractly/topologically: the flatness of $P$ ensures that it arises from a principal $G^{\delta}$-bundle ( $G$ endowed with the discrete topology) hence, by the discussion above, the relevant characteristic map is

$$
\kappa_{\mathrm{abs}}^{\omega}: H^{*}\left(B G^{\delta}\right) \rightarrow H^{*}(M)
$$

Again, the two theories are related to each other via a "universal characteristic map"

$$
\kappa_{\delta}^{\text {univ }}: H^{*}(\mathfrak{g}, K) \rightarrow H^{*}\left(B G^{\delta}\right)
$$

Actually, everything can be made even more explicit since:

- there is a simple explicit model computing $H^{*}\left(B G^{\delta}\right)$ namely the group cohomology $H_{\delta}^{*}(G)$ of $G$ viewed as a discrete group; recall that the relevant complex is given by group cochains $C_{\delta}^{p}(G)=C^{p}\left(G^{\delta}\right)=\left\{c: G^{p} \rightarrow \mathbb{R}\right\}$ (which ignore the topology on $G$ ), while the differential is the group differential given by

$$
\begin{aligned}
\delta(c)\left(g_{1}, \ldots, g_{p+1}\right)= & c\left(g_{2}, \ldots, g_{p+1}\right)+\sum_{i=1}^{p}(-1)^{i} c\left(g_{1}, \ldots, g_{i} \cdot g_{i+1}, \ldots, \ldots g_{p+1}\right) \\
& +(-1)^{p+1} c\left(g_{1}, \ldots, g_{p}\right)
\end{aligned}
$$

- of course, inside $C_{\delta}^{*}(G)$ one has the subcomplex of differentiable cochains

$$
C_{\text {diff }}^{*}(G) \subset C_{\delta}^{*}(G)
$$

and then the so-called differentiable cohomology $H_{\text {diff }}^{*}(G)$ mapping naturally into $H_{\delta}^{*}(G)=H^{*}\left(B G^{\delta}\right)$.

- and one has the so-called van Est isomorphism (assuming $G$ to be connected),

$$
V E: H_{\text {diff }}^{*}(G) \stackrel{\simeq}{\longrightarrow} H^{*}(\mathfrak{g}, K)
$$


All together, everything fits in one commutative diagram analogous to (2.4):

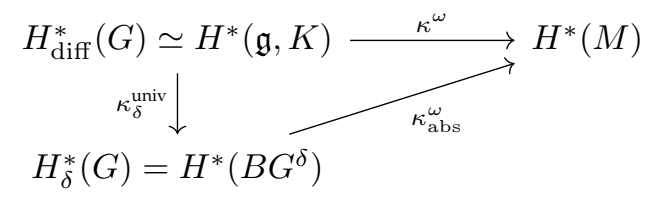

Example 2.2.3. For flat complex vector bundles one deals with $G=G L_{n}(\mathbb{C})$,

$$
H_{\text {diff }}^{*}\left(G L_{n}(\mathbb{C})\right) \cong H^{*}\left(\mathfrak{g l}_{n}(\mathbb{C}, U(n)) \cong \Lambda\left(h_{1}, \ldots, h_{2 n-1}\right),\right.
$$

the exterior algebra in generators $h_{2 i-1}$ of degree $2 i-1$. In some sense, the vanishing of the Chern classes (in degree $2 i$ ) give rise to "secondary" classes in odd degrees. A completely analogous story holds for real vector bundles. See, e.g., [39].

\subsubsection{Geometric characteristic classes for foliations}

The explicit/geometric approach to characteristic classes for foliations has an outcome similar to what has been described above: for a codimension $q$ foliation $\mathcal{F}$ on a manifold $M$ there is an explicit characteristic map

$$
\kappa^{\mathcal{F}}: G F_{q}^{*} \rightarrow H^{*}(M)
$$

defined on certain cohomology groups $G F_{q}^{*}$. Here we use the notation "GF" to illustrate the fact that these groups are usually referred to as "Gel'fand-Fuks cohomology groups"1. Themselves rather explicit/computable, they have several different descriptions, depending on the way one approaches characteristic classes for foliations.

The most explicit way of describing codimension $q$ foliations on a manifold $M$ is as codimension $q$ sub-bundles $\mathcal{F} \subset T M$ which are involutive (closed under taking Lie brackets). And the explicit approach to $\kappa^{\mathcal{F}}$ arises by looking at the associated normal bundle $\nu=T M / \mathcal{F}$. Actually, one of the origins of the characteristic classes for foliations is Bott's vanishing theorem which says that all the usual characteristic classes (polynomial expressions in the Pontryagin classes) of $\nu$ vanish in degrees $>2 q$. As for flat bundles, this gives rise to "secondary classes" which Bott constructs (see [9]) in the spirit of the Chern-Weil theory. The vanishing result indicates that, instead of the polynomial algebra $\mathbb{R}\left[c_{1}, \ldots, c_{q}\right]$ one should be using

$$
\mathbb{R}_{q}\left[c_{1}, \ldots, c_{q}\right]:=\mathbb{R}\left[c_{1}, \ldots, c_{q}\right] /\{\mathcal{P}: \operatorname{deg}(\mathcal{P})>2 q\} .
$$

Combined with the fact that the odd Chern classes of $\nu_{\mathbb{C}}$ vanish, one eventually discovers the differential graded algebra

$$
W O(q):=\Lambda\left(h_{1}, h_{3}, \ldots, h_{\langle q\rangle}\right) \otimes \mathbb{R}_{q}\left[c_{1}, \ldots, c_{q}\right]
$$

\footnotetext{
${ }^{1}$ We warn the reader that the transliterations "Gelfand" and "Fuchs" are widely used; our choice is consistent with the choice made in [31].
} 
where $c_{i}$ have degree $2 i, h_{i}$ have degree $2 i-1$ (and defined only when $i$ itself is odd), $\langle q\rangle$ is the largest odd number with $2\langle q\rangle-1 \leq q$, endowed with the differential

$$
d\left(c_{i}\right)=0, \quad d\left(h_{i}\right)=c_{i}
$$

With these,

$$
G F_{q}^{*}:=H^{*}(W O(q))
$$

Another rather explicit but more conceptual standard description of $G F_{q}^{*}$ is as the cohomology of the Lie algebra $\mathfrak{a}_{q}$ of formal vector fields on $\mathbb{R}^{q}$, i.e. expressions of type

$$
X=\sum_{i}^{q} f^{i}\left(x^{1}, \ldots x^{q}\right) \frac{\partial}{\partial x^{i}}
$$

where the $f^{i}$ 's are formal power series in the variables $x^{i \prime}$. We endow $\mathfrak{a}_{q}$ with the power series topology and we consider the resulting (continuous) Lie algebra cohomology $H^{*}\left(\mathfrak{a}_{q}\right)$ and the relative version $H^{*}\left(\mathfrak{a}_{q}, O(q)\right)$. As explained in [10] there is a canonical map from $W O(q)$ to the relative Chevalley-Eilenberg complex, which induces an isomorphism

$$
H^{*}(W O(q)) \cong H^{*}\left(\mathfrak{a}_{q}, O(q)\right) .
$$

With this in mind, $\kappa^{\mathcal{F}}$ can be constructed in the spirit of (2.5).

\subsubsection{Abstract characteristic classes for foliations. Pseudogroups and $\Gamma$-structures}

The abstract characteristic map for foliations is based on the reinterpretation of foliations as principal bundles where, instead of Lie groups as structure groups one has to allow for pseudogroups/étale groupoids.

To recall this we start from the very definition of (codimension $q$ ) foliations $\mathcal{F}$ on $M$ : as partitions of $M$ by connected immersed submanifolds (leaves) of codimension $q$ which, locally, look like

$$
\mathbb{R}^{n}=\mathbb{R}^{p} \times \mathbb{R}^{q}=\cup_{y \in \mathbb{R}^{q}} \mathbb{R}^{p} \times\{y\} \quad(p=n-q) .
$$

This is realised by "foliation charts" on opens $U_{i} \subset M$ (covering $M$ ),

$$
\chi_{i}: U_{i} \rightarrow \mathbb{R}^{n}
$$

the fact that the leaves are preserved translates into the fact that the changes of coordinates $\chi_{j} \circ \chi_{i}^{-1}$ are, locally, of type

$$
(x, y) \mapsto\left(h_{i j}(x, y), g_{i j}(y)\right)
$$

Of course, this definition is equivalent to the description as an involutive subbundle of $T M$ thanks to Frobenius theorem.

A slight variation is obtained by realizing that the leaves are induced by (i.e. are just the connected components of fibers of) submersions

$$
f_{i}: U_{i} \rightarrow \mathbb{R}^{q}
$$


and the different submersions $f_{i}$ are, locally, related by

$$
f_{j}=g_{i j} \circ f_{i} .
$$

These are the same $g_{i j}$ 's as before, and they are diffeomorphisms between opens inside $\mathbb{R}^{q}$ - the collection of which we denote by $\operatorname{Diff}_{\text {loc }}\left(\mathbb{R}^{q}\right)$. Notice that these $g_{i j}$ 's are there only locally: even when $i$ and $j$ are fixed, as $U_{i} \cap U_{j}$ may be disconnected. One can elegantly encode this situation by passing to germs:

$$
\Gamma^{q}:=\left\{\operatorname{germ}_{x}(\phi): \phi \in \operatorname{Diff}_{\text {loc }}\left(\mathbb{R}^{q}\right), x \in \operatorname{Domain}(\phi)\right\} .
$$

This has the so-called germ topology, in which the basic opens are the ones consisting of germs of any given $\phi \in \operatorname{Diff}_{\text {loc }}\left(\mathbb{R}^{q}\right)$ :

$$
U(\phi):=\left\{\operatorname{germ}_{x}(\phi): x \in \operatorname{Domain}(\phi)\right\} .
$$

With this one may say, with a slight abuse of notation, that

$$
g_{i j}: U_{i} \cap U_{j} \rightarrow \Gamma^{q}
$$

are smooth maps, bringing foliations closer to principal bundles. Note, in particular, that the cocycle condition

$$
g_{j k} g_{i j}=g_{i k}
$$

is satisfied, as it follows from (2.9) and the fact that the $f_{i}$ s are submersions. Understanding what allows one to write down such an equation reveals the relevant structure behind this discussion. In particular, one discovers the more general setting of pseudogroups, étale groupoids and $\Gamma$-structures. We stress that this general framework not only allows us to get an abstract characteristic map for foliation in complete analogy with (2.3), but provide us with a unifying approach that:

- produces more general constructions, i.e. the characteristic map is defined for $\Gamma$-structures, Definition 2.2.6 below;

- is well suited to describe a geometric map as well, see Subsection 2.2.5 and Section 2.3;

- paves the way for a further generalization of the various concepts involved, see Section 2.4, Section 2.5 and Section 2.6, that makes the structure behind both maps and their interplay conceptually clear.

We start by summing up the main definitions and constructions.

First of all, a pseudogroup on a manifold $\mathbf{X}$ is a subset $\Gamma \subset \operatorname{Diff}_{\text {loc }}(\mathbf{X})$ of the set of diffeomorphisms between opens in $\mathbf{X}$ satisfying the following axioms:

- $\Gamma$ is closed under composition, inversion and it contains the identity $i d_{\mathbf{X}}$.

- $\Gamma$ is closed under restriction to smaller opens.

- Let $\mathcal{U}$ be an open cover of an open set $\tilde{U} \subset \mathbf{X}$ and $\phi$ a diffeomorphism defined on $\tilde{U}$ and such that $\left.\phi\right|_{U} \in \Gamma$ for all $U \in \mathcal{U}$. Then $\phi \in \Gamma$. 
Of course, the basic example for us is $\operatorname{Diff}_{\text {loc }}\left(\mathbb{R}^{q}\right)$. As indicated by the discussion above, to talk about cocycles (and principal bundles) it is useful to pass to germs

$$
\operatorname{Germ}(\Gamma):=\left\{\operatorname{germ}_{x}(\phi): \phi \in \Gamma, x \in \operatorname{Domain}(\phi)\right\}
$$

endowing $\operatorname{Germ}(\Gamma)$ with the germ topology and the induced smooth structure as above. The structure present on $\operatorname{Germ}(\Gamma)$ that is relevant to our discussion is that of groupoid over $\mathbf{X}$ - schematically denoted as

$$
\operatorname{Germ}(\Gamma) \rightrightarrows \mathbf{X}
$$

In more detail: it comes with source and target maps

$$
s, t: \operatorname{Germ}(\Gamma) \rightarrow \mathbf{X}
$$

which send an element germ $x_{x}(\phi)$ to $x$, and $\phi(x)$, respectively; and, using composition of functions, any two elements $\gamma_{1}, \gamma_{2} \in \operatorname{Germ}(\Gamma)$ which match (i.e. $s\left(\gamma_{1}\right)=$ $\left.t\left(\gamma_{2}\right)\right)$ can be composed to give $\gamma_{1} \cdot \gamma_{2} \in \operatorname{Germ}(\Gamma)$. Indeed, writing $\gamma_{i}=\operatorname{germ}_{x_{i}}\left(\phi_{i}\right)$, the matching condition is $x_{1}=\phi\left(x_{2}\right)$ and then $\phi:=\phi_{1} \circ \phi_{2}$ is well defined around $x:=x_{2}$ hence we can define $\gamma_{1} \cdot \gamma_{2}:=\operatorname{germ}_{x}(\phi)$.

There is one important property of the groupoid $\operatorname{Germ}(\Gamma)$ to be noticed: it is an étale groupoid, in the sense that its source and target maps are local diffeomorphisms. The fact that étale groupoids $\mathcal{G} \rightrightarrows \mathbf{X}$ are closely related to pseudogroups on $\mathbf{X}$ is seen right away by looking at bisections of $\mathcal{G}$, i.e. submanifolds $\mathcal{B} \subset \mathcal{G}$ on which both $s$ and $t$ restrict to diffeomorphism onto opens inside $\mathbf{X}, U:=s(\Sigma)$ and $V:=t(\Sigma)$. One can re-interpret such bisections as sections of $s$

$$
b: U \rightarrow \mathcal{G}
$$

with the property that $t \circ b$ is a diffeomorphism into its image ( $b$ is the inverse of $\left.s\right|_{\mathcal{B}}$, and, reversely, $\mathcal{B}$ is the image of $b$ ). Any bisection gives rise to a diffeomorphism

$$
\phi_{\mathcal{B}}=\phi_{b}:=\operatorname{germ}_{g}(t) \circ \operatorname{germ}_{g}(s)^{-1}: U \rightarrow V
$$

and, all together, the diffeomorphisms arising in this way form the pseudogroup $\Gamma_{\mathcal{G}}$ associated to $\mathcal{G}$. Note that:

- the pseudogroup associated to an étale groupoid of type $\operatorname{Germ}(\Gamma)$ is $\Gamma$ itself;

- for a general étale groupoid $\mathcal{G} \rightrightarrows \mathbf{X}$, there is a canonical surjection from $\mathcal{G}$ into the germ groupoid associated to $\Gamma_{\mathcal{G}}$,

$$
\mathcal{G} \rightarrow \operatorname{Germ}\left(\Gamma_{\mathcal{G}}\right), \quad g \mapsto \xi_{g},
$$

where $\xi_{g}$ is represented by a/any open neighborhood $\Sigma_{g}$ of $g$ on which $s$ and $t$ restrict to diffeomorphisms - more directly, by the germ of $\phi_{\mathcal{B}}$.

It is customary to call an étale groupoid $\mathcal{G} \rightrightarrows \mathbf{X}$ effective if, for $g \in \mathcal{G}$, the germ $b_{g}$ is the identity only if $g$ is a unit arrow. All together, one obtains

$$
\left\{\begin{array}{c}
\text { pseudogroups } \\
\Gamma \text { on } \mathbf{X}
\end{array}\right\} \stackrel{1-1}{\longleftrightarrow}\left\{\begin{array}{c}
\text { effective étale groupoids } \\
\mathcal{G} \rightrightarrows \mathbf{X}
\end{array}\right\}
$$


up to isomorphism or, more precisely, an equivalence of categories. However, many notions involving pseudogroups $\Gamma$ (e.g. equivalences, cocycles, actions) are simpler and more elegantly described passing to the associated germ groupoids $\operatorname{Germ}(\Gamma)$.

Definition 2.2.4. Let $\Gamma$ be a pseudogroup on $\mathbf{X}$ and let $M$ be another manifold. A $\Gamma$-cocycle on $M$ consists of a cover $\mathcal{U}=\left\{U_{i}\right\}_{i \in I}$ of $M$ and smooth maps

$$
\gamma_{i j}: U_{i} \cap U_{j} \rightarrow \operatorname{Germ}(\Gamma)
$$

satisfying

- for $i=j$, each $\gamma_{i i}(x)$ is a unit (hence can be seen as a point in $\mathbf{X}$ ),

- for arbitrary $i$ and $j$ and $x \in U_{i} \cap U_{j}, \gamma_{i j}(x)$ is an arrow from $\gamma_{i i}(x)$ to $\gamma_{j j}(x)$, and such that the cocycle condition

$$
\gamma_{j k}(x) \cdot \gamma_{i j}(x)=\gamma_{i k}(x)
$$

is satisfied for all $x \in U_{i} \cap U_{j} \cap U_{k}$. Similarly we talk about $\mathcal{G}$-cocycles for any groupoid $\mathcal{G} \rightrightarrows \mathbf{X}$ by replacing above $\operatorname{Germ}(\Gamma)$ with $\mathcal{G}$.

Note that the dotted conditions actually follow from the cocycle one and the fact that it should make sense. Spelling them out, the first condition encodes maps

$$
f_{i}:=\gamma_{i i}: U_{i} \rightarrow \mathbf{X}
$$

while the second condition means that, around each $x \in U_{i} \cap U_{j}$, one can find $g_{i j} \in \Gamma$ representing $\gamma_{i j}(x)$ and such that, on its domain,

$$
f_{j}=g_{i j} \circ f_{i} .
$$

When we want to emphasize the $f_{i}$ 's, we also say that $\left(\mathcal{U}, f_{i}, \gamma_{i j}\right)_{i, j \in I}$ is a $\Gamma$ cocycle. If the maps $f_{i}$ are submersions, then the cocycle condition follows automatically, but not in general.

One recognizes the discussion from foliations when $\mathbf{X}=\mathbb{R}^{q}, \Gamma=\Gamma^{q}$ and the $f_{i}$ 's are submersions. General $\Gamma^{q}$-cocycles can be thought of as "singular foliations".

Remark 2.2.5. Any open cover $\mathcal{U}=\left\{U_{i}\right\}_{i \in I}$ of a manifold $M$ gives rise to a Mayer-Vietoris groupoid $M_{\mathfrak{U}}$ which allows to re-interpret the notion of cocycle a bit more conceptually. The groupoid $M_{U}$ is defined over the disjoint union of the $U_{i}$ as the pull-back of the unit groupoid $M \rightrightarrows M$. More explicitly, $M_{u}$ is the groupoid

$$
\bigsqcup_{i} U_{i} \cap U_{j} \rightrightarrows \bigsqcup_{i} U_{i}
$$

where to describe the groupoid structure we write the elements in the base as pairs $(i, x)$ with $i \in I$ and $x \in U_{i}$, and the elements in the total space as triples $(i, x, j)$ with $i, j \in I$ and $x \in U_{i} \cap U_{j}$; in these notations, the target, source and composition are:

$$
t(i, x, j)=(i, x), \quad s(i, x, j)=(j, x), \quad(i, x, j) \cdot(j, x, k)=(i, x, k) .
$$


With this, for any étale groupoid $\mathcal{G} \rightrightarrows \mathbf{X}$, a $\mathcal{G}$-cocycle $\gamma=\left\{\gamma_{i j}\right\}$ on $M$ over an open cover $\mathcal{U}=\left\{U_{i}\right\}$ of $M$ is the same thing as a morphism of groupoids

$$
\gamma: M_{u} \rightarrow \mathcal{G}
$$

There is also a straightforward analogue of the notion of equivalence of cocycles for Lie groups, called equivalence of $\Gamma$-cocycles. Compactly: two $\Gamma$-cocycles indexed by $I$ and $J$ are equivalent if they are part of a larger $\Gamma$-cocycle indexed by $I \sqcup J$.

Definition 2.2.6. A Haefliger $\Gamma$-structure on a manifold $M$ is an equivalence class of $\Gamma$-cocycles. A $\Gamma$-structure on $M$ is a Haefliger $\Gamma$-structure induced by a $\Gamma$ cocycle for which all the $f_{i}$ 's are submersions.

Example 2.2.7. In some of the existing literature, the term " $\Gamma$-structure" is reserved to the case when the dimension of $M$ equals to the dimension of $\mathbf{X}$ (on which $\Gamma$ lives) and is defined directly via the notion of $\Gamma$-atlas on $M$, i.e. a smooth atlas modelled on opens in $\mathbf{X}$ with the property that the coordinate changes are elements of $\Gamma$. Of course, this is just the notion of $\Gamma$-cocycle which becomes simpler due to the condition $\operatorname{dim}(M)=\operatorname{dim}(\mathbf{X})$.

On the other hand, $\Gamma$-structures are sometimes also called $\Gamma$-foliations. This terminology emphasises the fact that a $\Gamma$-structure on $M$ induces a foliation (built out of the fibers of the submersions $f_{i}$ from the cocycle). In some sense, a $\Gamma$ structure on $M$ is a foliation together with extra-structure in the transversal direction.

For instance, when $\mathbf{X}=\mathbb{C}^{k}=\mathbb{R}^{2 k}$ and $\Gamma$ is the pseudogroup of holomorphic diffeomorphisms, a $\Gamma$-structure on an $2 k$-dimensional manifold $M$ is the same thing as a complex structure on $M$; if $\operatorname{dim}(M)>2 k$, one is looking at transversally holomorphic foliations on $M$ of codimension $n-2 k$.

As for cocycles with values in a Lie group $G$, one can re-encode everything into more global objects: principal bundles. In particular, this allows one not to worry anymore about the choice of coverings (and the corresponding notion of equivalence).

This can be done (and will be needed) in the generality of Lie groupoids. A Lie groupoid is a groupoid $\Sigma \rightrightarrows \mathbf{X}$ where both the arrow space and the unit space are smooth manifolds, all the defining maps are smooth and both the source map and the target map are surjective submersions. We will use $\mathcal{G}$ to denote arrow spaces of étale groupoids and $\Sigma$ to denote arrow spaces of more general, non étale, Lie groupoids.

For a Lie groupoid $\Sigma \rightrightarrows \mathbf{X}$, a principal $\Sigma$-bundle over a manifold $M$, schematically described as

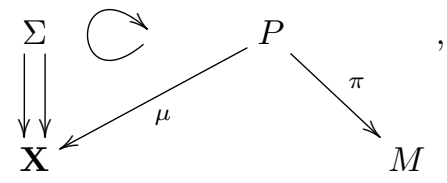

is a manifold $P$, together with a submersion $\pi: P \rightarrow M$ and a free and proper action of $\Sigma$ on $P$ from the left, along some smooth map $\mu: \mathcal{P} \rightarrow \mathbf{X}$, such that $\pi$ is 
identified with the resulting quotient map $P \rightarrow P / \Sigma$. Recall here that an action of $\Sigma$ on $P$ means that any arrow $g \in \Sigma$ from $x$ to $y$ gives rise to "multiplication by $g^{\prime \prime}$ between the resulting $\mu$-fibers

$$
\mu^{-1}(x) \rightarrow \mu^{-1}(y), \quad p \mapsto g \cdot p
$$

satisfying the usual identities for actions. Of course, everything is required to be smooth - i.e. $(g, p) \mapsto g \cdot p$ is smooth as a map defined on the submanifold $\Sigma \ltimes P \subset \Sigma \times P$ consisting of pairs $(g, p)$ with $\mu(p)=s(g)$. Similarly one can talk about actions from the right and right principal $\Sigma$-bundles:

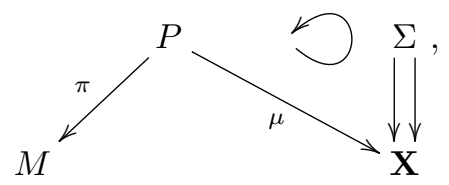

when the multiplication $p \mapsto p \cdot g$ by an arrow $g \in \Sigma$ from $x$ to $y$ takes $\mu^{-1}(y)$ to $\mu^{-1}(x)$. The action is free if the equalities of type $g \cdot p=p$ hold only when $g$ is a unit arrow; and the action is proper if the map $\Sigma \ltimes P \rightarrow P \times P,(g, p) \mapsto(g \cdot p, p)$ is a proper map. These conditions can be slightly reformulated by saying that one has a smooth action of $\Sigma$ on $P$ such that the map

$$
\Sigma \ltimes P \rightarrow P \times_{M} P
$$

is a diffeomorphism.

Of course, the entire discussion is completely similar to that from principal bundles with Lie structural group. And so is the relationship with cocycles: given such a principal $\Sigma$-bundle $\pi: P \rightarrow M$, one chooses a cover $\left\{U_{i}\right\}_{i \in I}$ of $M$ by opens $U_{i}$ on which $P$ admits sections $\sigma_{i}: U_{i} \rightarrow P$ and then, on points $x \in U_{i} \cap U_{j}$ in the overlaps, one can write

$$
\sigma_{i}(x)=\gamma_{i j}(x) \cdot \sigma_{j}(x)
$$

for some $\gamma_{i j}(x) \in \Sigma$. The $\sigma_{i}{ }^{\prime}$ s induce maps into $\mathbf{X}$, defined by

$$
f_{i}=\mu \circ \sigma_{i}: U_{i} \rightarrow \mathbf{X}
$$

and one ends up with a $\Sigma$-cocycle. And, still proceeding as for Lie groups to define the notion of equivalence of coycles (see [52]), one obtains

$$
\left\{\begin{array}{c}
\Sigma \text {-cocycles on } M \\
\text { (up to equivalence) }
\end{array}\right\} \stackrel{1-1}{\longleftrightarrow}\left\{\begin{array}{c}
\text { principal } \Sigma \text {-bundles on } M \\
\text { (up to isomorphism) }
\end{array}\right\}
$$

And, again as for Lie groups, for any Lie groupoid $\Sigma$ one can talk about its classifying space $B \Sigma$, for which there are various homotopically equivalent models. Principal $\Sigma$-bundles $\pi: P \rightarrow M$ come together with classifying maps $M \rightarrow B \Sigma$ uniquely defined up to homotopy. A standard approach can be found in [56]; $B \Sigma$ can be defined as the thick geometric realization of the nerve of $\Sigma$ (see also Subsection 2.2.6).

Observe that, when the Lie groupoid $\Sigma \rightrightarrows \mathbf{X}$ is an étale groupoid $\mathcal{G} \rightrightarrows \mathbf{X}$, the projection $\pi$ is an étale map. To stress this, we will use the notation $\mathcal{P}$ for the total space of a principal $\mathcal{G}$-bundle. We focus now on this case. 
As a consequence of the existence of a classifying space, one has abstract characteristic maps

$$
\kappa_{\mathrm{abs}}^{\mathcal{P}}: H^{*}(B \mathcal{G}) \rightarrow H^{*}(M) .
$$

An explicit construction is described/recalled a bit later, in Example 2.2.20. For $\mathcal{G}=\operatorname{Germ}(\Gamma)$ associated to a pseudogroup $\Gamma$, one uses the notation $B \Gamma$. Finally, applied to codimension $q$ foliations $\mathcal{F}$ on $M$ interpreted as $\Gamma^{q}$-cocycles, one obtains the resulting abstract characteristic maps that are of interest for us:

$$
\kappa_{\mathrm{abs}}^{\mathcal{F}}: H^{*}\left(B \Gamma^{q}\right) \rightarrow H^{*}(M) .
$$

\subsubsection{Intermezzo: back to geometric characteristic classes (for $\Gamma$ - structures)}

While the discussion of the abstract characteristic classes map led us to the more general context of pseudogroups $\Gamma$ and $\Gamma$-structures, it is natural to wonder whether also the geometric characteristic map (2.8) can be adapted to this more general context. The answer is "yes", at least in the case of pseudogroups $\Gamma$ that are Lie (see below) and transitive in the sense that for any two points in the base, $x, y \in \mathbf{X}$, there exists $\phi \in \Gamma$ such that $\phi(x)=y$. The key is to look at the infinitesimal counterpart of diffeomorphisms, i.e. at vector fields.

Definition 2.2.8. Let $\Gamma$ be a pseudogroup on $\mathbf{X}$.

- A (local) $\Gamma$-vector field is any (local) vector field $X$ on $\mathbf{X}$ with the property that its associated flow of diffeomorphisms $\phi_{X}^{t}$ all belong to $\Gamma$. Denote by $\mathfrak{X}_{\Gamma}(U)$ the space of such vector fields defined over $U \subset \mathbf{X}$ open.

- A formal $\Gamma$-vector field at $x$, for $x \in \mathbf{X}$, is an infinite jet at $x$ of a local $\Gamma$-vector field defined around $x$. Denote by $\mathfrak{a}_{x}(\Gamma)$ the space of such.

- When $\Gamma$ is a transitive pseudogroup on $\mathbf{X}=\mathbb{R}^{q}, x=0$, the space $\mathfrak{a}_{0}(\Gamma)$ can be equipped with the Lie bracket defined by

$$
\left[j_{0}^{\infty} X, j_{0}^{\infty} Y\right]=j_{0}^{\infty}[X, Y]
$$

where $X, Y$ are local $\Gamma$-vector fields around 0 ; it will be called the algebra of $\Gamma$-vector fields on $\mathbb{R}^{q}$, or the formal algebra of $\Gamma$, and will be denoted $\mathfrak{a}(\Gamma)$.

Concerning the third point, it is not difficult to see that, when $\Gamma$ is transitive, all the formal algebras $\mathfrak{a}_{x}(\Gamma)$ are isomorphic to each other hence, up to isomorphism, there is an unambiguously defined formal Lie algebra $\mathfrak{a}(\Gamma)$; however, to work with a concrete model, one has to fix a base point $x \in \mathbf{X}$. When $\mathbf{X}=\mathbb{R}^{q}$ and $\Gamma$ is the pseudogroup $\operatorname{Diff}_{\text {loc }}\left(\mathbb{R}^{q}\right)$, the resulting Lie algebra is denoted by $\mathfrak{a}_{q}$. Notice that in this case, formal vector fields $X \in \mathfrak{a}_{q}$ are simply expressions

$$
X=\sum_{i}^{n} f^{i}\left(x^{1}, \ldots x^{q}\right) \frac{\partial}{\partial x^{i}}
$$

where each $f^{i}$ is a formal power series in $x^{1}, \ldots, x^{q}$. 
For a general pseudogroup $\Gamma$, germs of elements from $\Gamma$ gave rise to the groupoid $\operatorname{Germ}(\Gamma)$. Similarly, for $l \in \mathbb{N}$, $l$-jets of elements from $\Gamma$ gives rise to the groupoid of $l$-jets $J^{l} \Gamma$ and one has the tower of groupoids

$$
J^{\infty} \Gamma \rightarrow \ldots \rightarrow J^{l} \Gamma \rightarrow J^{l-1} \Gamma \rightarrow \ldots \rightarrow J^{1} \Gamma \rightarrow J^{0} \Gamma .
$$

For instance, $J^{0} \Gamma \subset \mathbf{X} \times \mathbf{X}$ and, if $\Gamma$ is transitive, this inclusion becomes equality. For general $l, J^{l} \Gamma$ is a subspace of the manifold $J^{l}(\mathbf{X}, \mathbf{X})$ of $l$-jets of diffeomorphisms of $\mathbf{X}$. When all these are smooth submanifolds and all the projections in the tower are surjective submersions, one says that $\Gamma$ is a Lie pseudogroup. Lie pseudogroups are best discussed in the framework of profinite dimensional differential geometry, which allows to make sense of $J^{\infty} \Gamma$ as a pf Lie groupoid. We refer to the Appendix; in particular, Example 2.A.38 provides a definition of Lie pseudogroup that fit into this framework and explores some of the features of the tower above. Using terminology from the appendix, we point out that we will always assume the tower above to be a normal pf-atlas of $J^{\infty} \Gamma$, i.e. that the natural $\operatorname{map} J^{\infty} \Gamma \rightarrow \lim J^{l} \Gamma$ is a bijection. Here, we are especially interested in the inverse system of the isotropy Lie groups $J^{l} \Gamma_{x}$ at any $x \in \mathbf{X}$, for $l \in \mathbb{N}$. The isotropy group $J^{\infty} \Gamma_{x}$ is isomorphic to the limit of this system via an isomorphism of pf-Lie groups. Thanks to the Lie assumption one can find, at each $x \in \mathbf{X}$, a subgroup $K \subset J^{\infty} \Gamma_{x}$ such that its projection at each finite order $l$ is a maximal compact subgroup of $J^{l} \Gamma$. Such a $K$ will be called a maximal compact subgroup at $b$; they are unique up to conjugation. As before, when $\Gamma$ is transitive, one can move from $x$ to any other point in $\mathbf{X}$ and carry $K$ to similar groups at other points. In particular, when $\Gamma$ is a transitive Lie pseudogroup over $\mathbf{X}=\mathbb{R}^{q}$, we use $x=0$ and we talk about maximal compact subgroups for $\Gamma$.

For instance, when $\Gamma$ is the entire pseudogroup $\operatorname{Diff}_{\text {loc }}\left(\mathbb{R}^{q}\right)$, one has $J^{1} \operatorname{Diff}_{\text {loc }}\left(\mathbb{R}^{q}\right)_{x} \cong G L_{q}(\mathbb{R})$, with maximal compact subgroup $O(q)$. The orthogonal group $O(q)$ can be embedded in $J^{\infty} \operatorname{Diff}_{\text {loc }}\left(\mathbb{R}^{q}\right)_{x}$ by taking infinite jets of linear orthogonal maps, and in fact the maximal compact subgroup $K$ of $J^{\infty} \operatorname{Diff}_{\text {loc }}\left(\mathbb{R}^{q}\right)_{x}$ is isomorphic to $O(q)$.

Theorem 2.2.9. For any transitive Lie pseudogroup $\Gamma$ on $\mathbb{R}^{q}$ and for any choice of a maximal compact $K$, any principal $\mathcal{G}=\operatorname{Germ}(\Gamma)$-bundle $\mathcal{P} \rightarrow M$ can be associated to a map in cohomology

$$
\kappa^{\mathcal{P}}: H^{*}(\mathfrak{a}(\Gamma), K) \rightarrow H^{*}(M)
$$

such that:

- when $\Gamma=\operatorname{Diff}_{l o c}\left(\mathbb{R}^{q}\right)$ and $\mathcal{P}$ describes a codimension $q$ foliations, one has a canonical isomorphism

$$
H^{*}\left(\mathfrak{a}_{q}, O(q)\right) \cong H^{*}(W O(q))
$$

and we recover the geometric characteristic map $\kappa^{\mathcal{F}}(2.8)$ of the foliation.

- if $\mathcal{P}$ encodes a $\Gamma$-structure, with induced foliation $\mathcal{F}$ on $M$, then the composition of $\kappa^{\mathcal{P}}$ with the canonical map induced by the inclusion $i: \mathfrak{a}(\Gamma) \hookrightarrow \mathfrak{a}$ is precisely the 
geometric characteristic map $\kappa^{\mathcal{F}}(2.8)$.

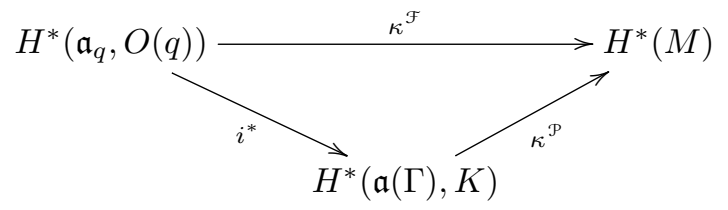

Incidentally, let us point out here that the obvious map from $\mathfrak{X}\left(\mathbb{R}^{q}\right)$ to $\mathfrak{a}_{q}$ (taking the infinite jet at the origin) is known to induce isomorphisms in (continuous) Lie algebra cohomology (see [31]), and this provides yet another description of the Gel'fand-Fuks cohomology. In fact, this is the original approach of Gel'fand and Fuks.

\subsubsection{Cohomology of classifying spaces: the Bott-Shulman model}

With the construction of the abstract characteristic map in mind (see the conclusion of Subsection 2.2.4) we see that, as for Lie groups, what really matters for us is just the cohomology of the classifying spaces rather than the classifying spaces themselves. There are various more explicit models available- each one of them providing a more or less explicit description of the characteristic map. One such model is the Bott-Shulman double complex $\Omega^{*}\left(\Sigma^{*}\right)$, defined for any Lie groupoid $\Sigma \rightrightarrows \mathbf{X}$. It is based on the so-called nerve of $\Sigma$, whose construction we briefly recall. One considers the spaces $\Sigma^{(p)} \subset \Sigma^{p}$ of $p$-strings $\left(g_{1}, \ldots, g_{p}\right)$ of composable arrows of $\Sigma$, related by the maps:

$d_{i}: \Sigma^{(p)} \rightarrow \Sigma^{(p-1)}, \quad d_{i}\left(g_{1}, \ldots, g_{p}\right)=\left\{\begin{array}{cc}\left(g_{2}, \ldots, g_{p}\right) & \text { if } i=0 \\ \left(g_{1}, \ldots, g_{i} g_{i-1}, \ldots, g_{p}\right) & \text { if } 1 \leq i \leq p-1 \\ \left(g_{1}, \ldots, g_{p-1}\right) & \text { if } i=p\end{array}\right.$

Note that these maps show up already when looking at group cohomology, the differential (2.6) being simply

$$
\delta=\sum_{i}(-1)^{i} d_{i}^{*}
$$

The same formula, but using pull-backs of forms, defines a differential which, together with de Rham differential, forms a double complex

$$
\left(\Omega^{*}\left(\Sigma^{(*)}\right), \delta, d_{\mathrm{dR}}\right)
$$

with associated total complex

$$
\operatorname{Tot}^{k} \Omega^{*}\left(\Sigma^{(*)}\right):=\bigoplus_{p+q=k} \Omega^{q}\left(\Sigma^{(p)}\right), \quad D_{\text {tot }}=\delta+(-1)^{p} d_{\mathrm{dR}} \text { on } \Omega^{q}\left(\Sigma^{(p)}\right) .
$$

We refer to both of them as the Bott-Shulman complex of the Lie groupoid $\Sigma \rightrightarrows$ $\mathrm{X}$. 
Definition 2.2.10. The cohomology of the Bott-Shulman complex is called the de Rham cohomology of the Lie groupoid $\Sigma \rightrightarrows \mathbf{X}$, denoted $H_{\mathrm{dR}}^{*}(\Sigma)$.

By a folklore theorem, for Hausdorff groupoids, this is isomorphic to the singular cohomology of $B \Sigma$ (see below). Furthermore, also the product structure on cohomology can be exhibited directly on the Bott-Shulman complex:

$$
-\cup_{\text {tot }}-: \Omega^{q}\left(\Sigma^{(p)}\right) \times \Omega^{q^{\prime}}\left(\Sigma^{\left(p^{\prime}\right)}\right) \rightarrow \Omega^{q+q^{\prime}}\left(\Sigma^{\left(p+p^{\prime}\right)}\right)
$$

where

$$
\omega \cup_{\text {tot }} \omega^{\prime}:=(-1)^{q p^{\prime}} \text { first }_{p}^{*}(\omega) \wedge \operatorname{last}_{p^{\prime}}^{*}\left(\omega^{\prime}\right) .
$$

Here, first f $_{p}: \Sigma^{\left(p+p^{\prime}\right)} \rightarrow \Sigma^{(p)}$ keeps the first $p$ arrows, last $p^{\prime}$ keeps the last $p^{\prime}$. Of course, the signs are chosen so that $D_{\text {tot }}$ satisfies the Leibniz identity w.r.t. the total degree:

$$
D_{\text {tot }}\left(\omega \cup_{\text {tot }} \omega^{\prime}\right)=D_{\text {tot }}(\omega) \cup_{\text {tot }} \omega^{\prime}+(-1)^{k} \omega \cup_{\text {tot }} D_{\text {tot }}\left(\omega^{\prime}\right)
$$

where $k=p+q$ is the total degree of $\omega \in \Omega^{q}\left(\Sigma^{(p)}\right)$. Therefore:

$$
\left(\operatorname{Tot} \Omega^{*, *}(\Sigma), D_{\text {tot }}, \cup_{\text {tot }}\right)
$$

becomes a DGA and the isomorphism with $H^{*}(B \Sigma)$ mentioned above is an isomorphism of algebras.

Recall also that, next to the maps $d_{i}$ there are also the degeneracy maps

$$
s_{i}: \Sigma^{(p)} \rightarrow \Sigma^{(p+1)}, \quad s_{i}\left(g_{1}, \ldots, g_{p}\right)=\left(\ldots, g_{i}, 1, g_{i+1}, \ldots\right) \quad(0 \leq i \leq p)
$$

All together, they form the nerve of $\Sigma$, which is a simplicial manifold, in the sense that the simplicial identities

$$
\left\{\begin{array}{cl}
d_{i} d_{j}=d_{j-1} d_{i}, & \text { if } i<j \\
s_{i} s_{j}=s_{j} s_{i-1} & \text { if } i>j
\end{array} \quad d_{i} s_{j}=\left\{\begin{array}{cc}
s_{j-1} d_{i} & \text { if } i<j \\
\text { id } & \text { if } i \in\{j, j+1\} \\
s_{j} d_{i-1} & \text { if } i>j+1
\end{array}\right.\right.
$$

are satisfied. The main point is that to any simplicial manifold one can associate a space, called geometric realization - a construction which applied to the nerve of $\Sigma$ produces precisely $B \Sigma$. See [56].

We are interested in the case of étale groupoids $\mathcal{G} \rightrightarrows \mathbf{X}$ such as $\operatorname{Germ}(\Gamma)-$ where there are a couple of remarks to be made.

Remark 2.2.11. So far we have ignored one issue: some of our groupoids, especially the ones that use the germ topology such as $\operatorname{Germ}(\Gamma)$, have a space of arrows which is a non-Hausdorff manifold. Often one just ignores at first this aspect, carries on and returns later adapting the arguments/constructions to include the non-Hausdorff case. This affects our discussions here because the folklore theorem that the Bott-Shulman complex of $\mathcal{G}$ computes the cohomology of $B \mathcal{G}$ requires $\mathcal{G}$ to be Hausdorff. The reason is very simple: for non-Hausdorff manifolds $M$, differential forms still provide a resolution of the constant sheaf, but it is no longer a "good" resolution (not even acyclic); in other words, while 
there still is a canonical map from de Rham cohomology to sheaf cohomology (with coefficients in $\mathbb{R}$, say), this may fail to be an isomorphism if $M$ is not Hausdorff. However, pin-pointing the problem also indicates the way out: just build an analogue of the Bott-Shulman complex which uses "good resolutions". However, as indicated above, for the reader that is not comfortable with nonHausdorff manifolds one may just assume first that we work only with pseudogroups for which $\operatorname{Germ}(\Gamma)$ is Hausdorff.

The other remark to be made is that, for étale groupoids, there is more structure available that allows one to re-interpret the Bott-Shulman complex (and variations if it). Very briefly: the sheaves of differential forms on the base carry an action of the groupoid (using the germs (2.10) induced by arrows). This brings us to another approach to the cohomology of $B \mathcal{G}$ which, in this context, was first considered by Haefliger: via $\mathcal{G}$-sheaves and their cohomology.

\subsubsection{Cohomology of classifying spaces: sheaf theoretical approach}

The notion of $\Gamma$-sheaf for a pseudogroup $\Gamma$ over $\mathbf{X}$ should be clear:

Definition 2.2.12. Given a pseudogroup $\Gamma$ over $\mathbf{X}$, a $\Gamma$-sheaf is any sheaf $\mathcal{S}$ over $\mathbf{X}$ together with an action of $\Gamma$ on $\mathcal{S}$, i.e. a collection of maps

$$
\phi^{*}: \mathcal{S}(V) \rightarrow \mathcal{S}(U), \quad U, V \text { opens in } \mathbf{X}
$$

- one for any element $\phi: U \rightarrow V$ of $\Gamma$, where $V=\phi(U)$ - satisfying the following conditions:

- compatibility with restrictions, i.e. for all $\phi: U \rightarrow V$ in $\Gamma$ and for all open subsets $W \subset U$,

$$
\left(\left.\phi\right|_{W}\right)^{*} \circ \rho_{\phi(W), V}=\rho_{W, U} \circ \phi^{*}
$$

where $\rho_{\phi(W), V}$ and $\rho_{W, U}$ are the restriction morphisms of the sheaf $\mathcal{S}$;

- (contravariant) functoriality, i.e.

$$
\phi^{*} \circ \psi^{*}=(\psi \circ \phi)^{*}, \quad i d^{*}=i d
$$

for any $\phi, \psi \in \Gamma$ composable.

We denote by $\operatorname{Sh}(\Gamma)$ the resulting category of $\Gamma$-sheaves (where morphisms are morphisms of sheaves that commute with all $\phi^{* \prime}$ ). Similarly, we introduce the category $\operatorname{Ab}(\Gamma)$ of abelian $\Gamma$-sheaves, or the category $\operatorname{Vect}_{\mathbb{R}}(\Gamma)$ of $\Gamma$-sheaves of vector spaces.

Note that, in some sense, we are enlarging the lattice of opens $\mathcal{O} p(\mathbf{X})$ in $\mathbf{X}$ by $\Gamma$. We interpret $\mathcal{O} p(\mathbf{X})$ as the category with the opens $U \subset \mathbf{X}$ as objects, and inclusions $U \hookrightarrow V$ as morphisms - so that a (pre-)sheaf on $\mathbf{X}$ can be interpreted as a functor $\mathcal{O} p(\mathbf{X}) \rightarrow$ Set. Any pseudogroup $\Gamma$ gives rise to a similar, but larger category $\mathcal{O} p_{\Gamma}(\mathbf{X})$, with the same objects, but where the morphisms are all the smooth maps $\phi: U \rightarrow V$ between opens in $\mathbf{X}$ such that, as a map from $U$ to 
$\phi(U), \phi$ belongs to $\Gamma$. Intuitively, one should think of $\mathcal{O} p_{\Gamma}(\mathbf{X})$ as representing the lattice of opens in the quotient space $\mathbf{X} / \Gamma$ of $\Gamma$-orbits - where the orbit of $x \in \mathbf{X}$ is defined as the set of $y \in \mathbf{X}$ such that $\phi(x)=y$ for some $\phi \in \Gamma$. With this, $\Gamma$-sheaves can be interpreted as contravariant functors

$$
\mathcal{S}: \mathcal{O} p_{\Gamma}(\mathbf{X}) \rightarrow \text { Set }
$$

satisfying the gluing condition. Of course, the sheaves of sections of natural bundles [53] are automatically $\Gamma$-sheaves for any $\Gamma$. One example to have in mind are differential forms.

Sheaf cohomology of topological spaces $\mathbf{X}$ arises taking the right derived functors of the global sections functor $\mathcal{O} p(\mathbf{X}) \rightarrow \mathrm{Ab}$. For $\Gamma$-sheaves, one considers invariant global sections:

Definition 2.2.13. For a $\Gamma$-sheaf $\mathcal{S}$, a global section $s \in \mathcal{S}(\mathbf{X})$ is called invariant if, for any $\phi: U \rightarrow V$ in $\Gamma$,

$$
\phi^{*}\left(\left.s\right|_{V}\right)=\left.s\right|_{U}
$$

We denote by $\mathcal{S}^{\text {inv }}(\mathbf{X})$ the space of such sections.

It is not difficult to see that the category $\mathrm{Ab}(\Gamma)$ of abelian $\Gamma$-sheaves is abelian and $\mathcal{S} \mapsto \mathcal{S}^{\operatorname{inv}}(\mathbf{X})$ is a left-exact functor; one can also show that $A b(\Gamma)$ has enough injectives (see [34]).

Definition 2.2.14. Given a pseudogroup $\Gamma$ over $\mathbf{X}$, the resulting right derived functors of $\mathcal{S} \mapsto \mathcal{S}^{\text {inv }}(\mathbf{X})$ are denoted

$$
H^{p}(\Gamma, \cdot): \operatorname{Ab}(\Gamma) \rightarrow \mathrm{Ab}, \quad \mathcal{S} \mapsto H^{p}(\Gamma, \mathcal{S})
$$

$H^{p}(\Gamma, \mathcal{S})$ is called the $p$-cohomology group of $\Gamma$ with coefficients in $\mathcal{S}$.

Hence, in order to compute the cohomology with coefficients in $\mathcal{S}$ explicitly one needs an injective resolution

$$
0 \rightarrow \mathcal{S} \rightarrow \mathcal{J}^{*}
$$

by $\Gamma$-sheaves of $\mathcal{S}$ and $H^{*}(\Gamma, \mathcal{S})$ is the cohomology of the complex:

$$
0 \rightarrow \mathcal{J}^{*, \operatorname{inv}}(\mathbf{X})
$$

For the constant sheaf $\mathcal{S}=\mathbb{R}$ we simplify the notation to $H^{*}(\Gamma)$. As for usual cohomology, concrete models are obtained using various injective resolutions of $\mathbb{R} \in \operatorname{Ab}(\Gamma)$. And this provides explicit models for $H^{*}(B \Gamma)$ since:

Theorem 2.2.15. For any pseudogroup $\Gamma, H^{*}(\Gamma)$ is canonically isomorphic to $H^{*}(B \Gamma)$.

It is interesting to rewrite the entire discussion about cohomology in terms of $\operatorname{Germ}(\Gamma)$ rather than $\Gamma$ itself. Of course, an outcome is a slight generalisation to the more general context of étale groupoids $\mathcal{G} \rightrightarrows \mathbf{X}$. One way to proceed is to consider the analogue of $\mathcal{O} p_{\Gamma}(\mathbf{X})$ known as the embedding category of $\mathcal{G}$ and denoted $\operatorname{Emb}(\mathcal{G})$. Its objects are opens $U \subset M$, while arrows from $U$ to $V$ 
are bisections $\sigma: U \rightarrow \mathcal{G}$ with the property that $t(\sigma(U)) \subset V$. The product of $\sigma_{1}: U_{1} \rightarrow \mathcal{G}$ from $U_{1}$ to $U_{2}$ with $\sigma_{2}: U_{2} \rightarrow \mathcal{G}$ from $U_{2}$ to $U_{3}$ is given by

$$
\sigma_{2} \cdot \sigma_{1}(x)=\sigma_{2}\left(\sigma_{1}(x)\right) \cdot \sigma_{1}(x)
$$

This gives rise to the resulting categories of $\mathcal{G}$-sheaves $\operatorname{Sh}(\mathcal{G}), \mathrm{Ab}(\mathcal{G})$, etc. Also the discussion about cohomology carries on without any change, giving rise to

$$
H^{*}(\mathcal{G}, \cdot): \mathrm{Ab}(\mathcal{G}) \rightarrow \mathrm{Ab}
$$

and the analogue of Theorem 2.2.15 continues to hold; see [48].

We would like to emphasize that, even if one is interested only in the case $\mathcal{G}=$ $\operatorname{Germ}(\Gamma)$ for pseudogroups $\Gamma$, the point of view of étale groupoids provides extrainsight. For instance, the usual interpretation of (standard) sheaves $\mathcal{S}$ on a space $\mathbf{X}$ as étale spaces $\hat{\mathcal{S}} \rightarrow \mathbf{X}$ (made of germs of sections) has a now straightforward generalization: $\mathcal{G}$-shaves become étale spaces $\hat{\mathcal{S}} \rightarrow \mathbf{X}$ together with a (continuous) action of $\mathcal{G}$ on $\hat{\mathcal{S}}$ - say from the right. In particular, any arrow of $\mathcal{G}, g: x \rightarrow y$, induces an action by $g, g^{*}: \mathcal{S}_{y} \rightarrow \mathcal{S}_{x}$. While sections of the sheaf correspond to sections of $\hat{\mathcal{S}} \rightarrow \mathbf{X}$, for such a (global) section $s$ the meaning of invariance should be clear:

$$
g^{*}(s(y))=s(x)
$$

for any arrow $g: x \rightarrow y$ of $\mathcal{G}$.

Another illustration of the use of étale groupoids is the fact that they provide bar-type complexes computing the cohomology. Ultimately, this is what allows one to relate this cohomology to the Bott-Shulman complex (and to overcome the non-Hausdorfness problem). Furthermore, this also provides a framework that puts together the characteristic classes for foliations with the ones for flat bundles; note here that, when $\mathcal{G}$ is a discrete group (interpreted as an étale groupoid over a point), one just recovers the usual group cohomology (Example 2.2.19).

To describe the bar-type complexes we follow [35]. As we already recalled above, for any groupoid $\mathcal{G} \rightrightarrows \mathbf{X}$ one denotes by $\mathcal{G}^{(p)}$ the space of strings of $p$ composable arrows

$$
\mathcal{G}^{(p)}=\mathcal{G}_{s} \times{ }_{t} \cdots{ }_{s} \times{ }_{t} \mathcal{G} .
$$

A $p$-cochains on $\mathcal{G}$ with values in a $\mathcal{G}$-sheaf $\mathcal{S}$ is any global section of the pull-back $t^{*} \mathcal{S}$ of the sheaf $\mathcal{S}$ to $\mathcal{G}^{(p)}$ where $t: \mathcal{G}^{(p)} \rightarrow \mathbf{X}$ takes the target of the first element of a $p$-string. The corresponding space is denoted by $C^{p}(\mathcal{G}, \mathcal{S})$. Hence

$$
C^{p}(\mathcal{G}, \mathcal{S})=\Gamma\left(\mathcal{G}^{(p)}, t^{*} \mathcal{S}\right)
$$

Equivalently, in terms of the stalks $\mathcal{S}_{x}$ (i.e. the associated étale spaces of germs $\hat{\mathcal{S}} \rightarrow \mathbf{X})$, a $p$-cochain $c \in C^{p}(\mathcal{G}, \mathcal{S})$ is a map

$$
\mathcal{G}^{(p)} \ni\left(g_{1}, \ldots, g_{p}\right) \mapsto c\left(g_{1}, \ldots, g_{p}\right) \in \mathcal{S}_{t\left(g_{1}\right)}
$$

which is continuous. The groupoid differential

$$
\delta: C^{p}(\mathcal{G}, \mathcal{S}) \rightarrow C^{p+1}(\mathcal{G}, \mathcal{S})
$$


is defined by the adaptation of formula (2.6), where we use the action by $g_{1}$ so that the resulting formula lends in the desired space (germs at $t\left(g_{1}\right)$ ):

$$
\begin{aligned}
\delta(c)\left(g_{1}, \ldots, g_{p+1}\right)= & g_{1} \cdot c\left(g_{2}, \ldots, g_{p+1}\right)+\sum_{i=1}^{p}(-1)^{i} c\left(g_{1}, \ldots, g_{i} \cdot g_{i+1}, \ldots, \ldots g_{p+1}\right) \\
& +(-1)^{p+1} c\left(g_{1}, \ldots, g_{p}\right)
\end{aligned}
$$

Notice that, if $\Phi: \mathcal{S}^{1} \rightarrow \mathcal{S}^{2}$ is a morphism of $\mathcal{G}$-sheaves, there is an induced morphism of complexes $C^{*}\left(\mathcal{G}, S^{1}\right) \rightarrow C^{*}\left(\mathcal{G}, S^{2}\right)$.

In particular, for pseudogroups one obtains the following notion of cocycle, which would not have been so natural without the viewpoint of the germ groupoid.

Definition 2.2.16. Given a pseudogroup $\Gamma$ over $\mathbf{X}$, by a continuous $p$-cochain on $\Gamma$ we mean any $p$-cochain on the germ groupoid $\operatorname{Germ}(\Gamma) \rightrightarrows \mathbf{X}$, with resulting complex denoted

$$
C_{\text {cont }}^{*}(\Gamma, \mathcal{S}):=C^{*}(\operatorname{Germ}(\Gamma), \mathcal{S})
$$

for any $\Gamma$-sheaf $\mathcal{S}$.

Returning to general étale groupoids $\mathcal{G} \rightrightarrows \mathbf{X}$, it is not so easy to "resolve" an arbitrary sheaf $\mathcal{S}$ by a resolution consisting of sheaves which are injective as $\mathcal{G}$-sheaves (or just acyclic w.r.t. the cohomology of $\mathcal{G}$-sheaves). One reason is that the injectivity (or acyclicity) as a sheaf over $\mathbf{X}$ does not imply the injectivity (or acyclicity) as a $\mathcal{G}$-sheaf. The bar-complexes arise by "resolving" the sheaves in steps. The outcome is the following:

Theorem 2.2.17. Let $\mathcal{S}$ be a $\mathcal{G}$-sheaf and

$$
0 \rightarrow \mathcal{S} \rightarrow \mathcal{F}^{*}
$$

a resolution by $\mathcal{G}$-sheaves which are acyclic as sheaves on $\mathbf{X}$ and also when pulled back to the spaces $\mathcal{G}^{(p)}$ via $t$. Then, for all $k$,

$$
H^{k}(\mathcal{G}, \mathcal{S}) \cong H^{k}\left(C^{*}\left(\mathcal{G}, \mathcal{F}^{*}\right)\right)
$$

where the right hand side is the $k$-th cohomology group of the double complex $\left(\left(C^{p}\left(\mathcal{G}, \mathcal{F}^{q}\right), \delta, d\right)\right)_{p, q}$ and $d$ is induced by the differential of the resolution $\mathcal{F}^{*}$.

See [34] for a proof; in [35], the above theorem is used to define continuous cohomology via the Godement resolution of a sheaf. The advantage of the Godement resolution

$$
0 \rightarrow \mathcal{S} \rightarrow \mathcal{C}^{0} \mathcal{S} \rightarrow \mathcal{e}^{1} \mathcal{S} \rightarrow \mathcal{C}^{2} \mathcal{S} \ldots
$$

(defined over any space) is that it is flabby (hence also acyclic) and is preserved by taking pull-backs via étale maps - hence the previous theorem applies.

The above theorem only needs the base $\mathbf{X}$ to be a topological space. When $\mathbf{X}$ is a manifold and we deal with real coefficients, we have also the de Rham resolution

$$
0 \rightarrow \mathbb{R}_{\mathbf{X}} \rightarrow \Omega_{\mathbf{X}}^{0} \rightarrow \Omega_{\mathbf{X}}^{1} \rightarrow \ldots
$$


by the $\mathcal{G}$-sheaves of differential forms. Since the maps $t: \mathcal{G}^{(p)} \rightarrow \mathbf{X}$ are étale, they pull-back the sheaf of differential forms on $\mathbf{X}$ to sheaves of differential forms on the manifolds $\mathcal{G}^{(p)}$. Hence one obtains the following, which gives a re-interpretation of the Bott-Shulman complex:

$$
\Omega^{q}\left(\mathcal{G}^{(p)}\right)=\mathcal{e}^{p}\left(\mathcal{G}, \Omega_{\mathbf{X}}^{q}\right) .
$$

Recall that the sheaf of differential forms of any degree is acyclic whenever we work over Hausdorff manifolds. Consequently, one obtains the relationship with de Rham cohomology mentioned above (see Definition 2.2.10) [34,35]:

Lemma 2.2.18. For any étale groupoid $\mathcal{G} \rightrightarrows \mathbf{X}$ there is a canonical map

$$
\iota: H_{\mathrm{dR}}^{*}(\mathcal{G}) \rightarrow H^{*}(\mathcal{G}, \mathbb{R})
$$

and, if $\mathcal{G}$ is Hausdorff, this map is an isomorphism.

In the non-Hausdorff case the situation is a bit more delicate and Theorem 2.2.17 may not apply to the de Rham resolution; hence in that case one obtains a map between the two cohomologies, but it may fail to be an isomorphism.

Example 2.2.19. It is clear that when $\mathcal{G}=G$ is a discrete group viewed as an étale groupoid over a point, one recovers the usual cohomology of $G$ with coefficients in $G$-modules.

At the other extreme one has that, for any manifold $M$, the cohomology of the unit groupoid $M \rightrightarrows M$ (consisting only of units) is the standard sheaf cohomology of $M$.

A related example is the groupoid $M_{\mathcal{U}}$ associated to an open cover $\mathcal{U}$ of $M$ (see Remark 2.2.5). Due to the gluing condition on sheaves, there is an immediate equivalence of categories

$$
\mathrm{Ab}\left(M_{u}\right) \cong \mathrm{Ab}(M)
$$

and it is not difficult to see that also the cohomology is preserved:

$$
H^{*}(M, \mathcal{S}) \cong H^{*}\left(M_{\mathcal{U}}, \mathcal{S}_{\mathcal{U}}\right)
$$

Example 2.2.20 (back to the abstract characteristic map (2.12)). The very last example gives, as promised, a more concrete model for the abstract characteristic map (2.12). To achieve that, recall that any $\mathcal{G}$-cocycle $\gamma=\left\{\gamma_{i j}\right\}$ over the cover $\mathcal{U}$ can be interpreted as a morphism of groupoids $\gamma: M_{\mathcal{U}} \rightarrow \mathcal{G}$ (cf. Remark 2.2.5). Furthermore, it is clear that the Bott-Shulman complex (and the de Rham cohomology) of groupoids is functorial with respect to groupoid morphisms. Hence, a cocycle $\gamma$ induces

$$
\begin{aligned}
\gamma^{*}: C^{p}\left(\mathcal{G}, \Omega_{\mathbf{X}}^{q}\right) & \rightarrow C^{p}\left(M_{\mathfrak{U}}, \Omega_{M}^{q}\right) \\
c & \rightarrow \gamma^{*}(c)
\end{aligned}
$$

where, explicitly,

$$
\gamma^{*}(c)_{i_{0}, \ldots i_{p}}(x)=f_{i}^{*}\left(c\left(\gamma_{i_{p-1} i_{p}}(x), \ldots, \gamma_{i_{0} i_{1}}(x)\right)\right) .
$$


Here, we are making use of the fact that a groupoid cochain in $M_{\mathfrak{U}}$ is the same thing as a Čech cochain. Passing to cohomology,

$$
\gamma^{*}: H_{\mathrm{dR}}^{*}(\mathcal{G}) \rightarrow H^{*}(M)
$$

When $\mathcal{G}$ is Hausdorff so that we can apply Lemma 2.2.18 (see also Theorem 2.2.15), this becomes precisely the promised explicit description of the characteristic map (2.12). In the general case, this is the composition of $\iota: H_{\mathrm{dR}}^{*}(\mathcal{G}) \rightarrow$ $H^{*}(\mathcal{G}, \mathbb{R}) \cong H^{*}(B \mathcal{G})$ with the characteristic map (2.12)

$$
\kappa_{\mathrm{abs}}^{\mathcal{P}}: H^{*}(B \mathcal{G}) \rightarrow H^{*}(M) .
$$

Remark 2.2.21 (product structure). One of the advantages of the bar-complexes is that they allow one to exhibit explicitly the product structures present in cohomology. For instance, for any three abelian $\mathcal{G}$-sheaves $\mathcal{S}_{1}, \mathcal{S}_{2}$ and $\mathcal{S}$ and any map of abelian $\mathcal{G}$-sheaves $w: \mathcal{S}_{1} \otimes \mathcal{S}_{2} \rightarrow \mathcal{S}$, one has an induced operation

$$
\begin{gathered}
-\cup_{w}-: C^{p}\left(\mathcal{G}, \mathcal{S}_{1}\right) \times C^{p^{\prime}}\left(\mathcal{G}, \mathcal{S}_{2}\right) \rightarrow C^{p+p^{\prime}}(\mathcal{G}, \mathcal{S}) \\
\left(c_{1} \cup_{w} c_{2}\right)\left(g_{1}, \ldots, g_{p+p^{\prime}}\right)=w\left(c\left(g_{1}, \ldots, g_{p}\right), g_{1} \cdot \ldots \cdot g_{p} \cdot c^{\prime}\left(g_{p+1}, \ldots, g_{p+p^{\prime}}\right)\right) \in \mathcal{S}_{t\left(g_{1}\right)} .
\end{gathered}
$$

In particular:

- when $\mathcal{S}_{1}=\mathcal{S}_{2}=\mathcal{S}=\mathbb{R}$ and $\omega$ is the identity, one obtains the so-called cup-product

$$
-\cup-: C^{p}(\mathcal{G}) \times C^{p^{\prime}}(\mathcal{G}) \rightarrow C^{p+p^{\prime}}(\mathcal{G}),
$$

making $C^{*}(\mathcal{G})$ into a graded algebra. Together with $\delta$, it is actually a differential graded algebra, i.e. $\delta$ satisfies the derivation rule

$$
\delta\left(c \cup c^{\prime}\right)=\delta(c) \cup c^{\prime}+(-1)^{p} c \cup \delta\left(c^{\prime}\right) ;
$$

- similarly, when $\mathcal{S}_{1}$ is $\mathbb{R}$ and $\mathcal{S}_{2}$ is an arbitrary $\mathcal{S}$ one obtains that $C^{p}(\mathcal{G}, \mathcal{S})$ comes with a left and a right action of $C^{*}(\mathcal{G})$, making it into a (differential) graded module;

- when $\mathcal{S}_{1}=\Omega_{\mathbf{X}}^{q}, \mathcal{S}_{2}=\Omega_{\mathbf{X}}^{q^{\prime}}$ are sheaves of differential forms and $w$ is the usual wedge operation, one obtains an induced operation

$$
-\cup-: \Omega^{q}\left(\mathcal{G}^{(p)}\right) \times \Omega^{q^{\prime}}\left(\mathcal{G}^{\left(p^{\prime}\right)}\right) \rightarrow \Omega^{q+q^{\prime}}\left(\mathcal{G}^{\left(p+p^{\prime}\right)}\right) .
$$

It is immediate to see that this is precisely the unsigned version of the total cupproduct (2.16), a version that interacts nicer with $\delta$ and $d$ (rather than the total differential). More precisely one has:

$$
\begin{aligned}
& \delta\left(\omega \cup \omega^{\prime}\right)=\delta(\omega) \cup \omega^{\prime}+(-1)^{p} \omega \cup \delta\left(\omega^{\prime}\right) \\
& d\left(\omega \cup \omega^{\prime}\right)=d(\omega) \cup \omega^{\prime}+(-1)^{q} \omega \cup d\left(\omega^{\prime}\right)
\end{aligned}
$$

for all $\omega \in \Omega^{p, q}, \omega^{\prime} \in \Omega^{p^{\prime}, q^{\prime}}$. Of course, the last equation is a consequence of the Leibniz rule for the usual wedge product:

$$
d\left(\omega \wedge \omega^{\prime}\right)=d(\omega) \wedge \omega^{\prime}+(-1)^{q} \omega \wedge d\left(\omega^{\prime}\right)
$$




\subsection{Haefliger's differentiable cohomology}

\subsubsection{Haefliger's differentiable cohomology of pseudogroups}

Let us return to the two characteristic maps for foliations: the geometric one $\kappa^{\mathcal{F}}$ from (2.8) and the abstract one $\kappa_{\text {abs }}^{\mathcal{F}}$ from (2.13). As for principal bundles with corresponding commutative diagram (2.4), and as for discrete bundles with corresponding commutative diagram (2.7), one has a similar commutative diagram

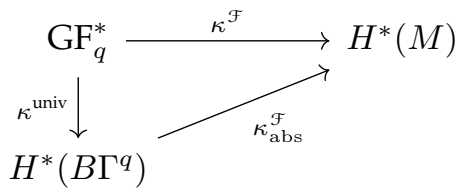

Here, the existence of vertical map $\kappa^{\text {univ }}$ making the previous diagram commutative can be obtained using the universality of $B \Gamma^{q}$, or explicitly using the barcomplex (see also below for a different, simpler, description). We have seen that, in this diagram:

- the domain of the abstract characteristic map could be interpreted as the cohomology of the groupoid $\Gamma^{q}$.

- the domain $\mathrm{GF}_{q}^{*}$ of the geometric characteristic map has several descriptions - e.g. very explicitly using the complex $W O(q)$ or, still explicit but more conceptual, using the Lie algebra $\mathfrak{a}_{q}$ of formal vector fields.

Given the striking analogy with the discussion of characteristic classes for flat bundles, there is an obvious question: can one interpret the domain of the geometric characteristic map as a certain "differentiable cohomology"

$$
H_{\text {diff }}^{*}\left(\Gamma^{q}\right)
$$

so that the previous diagram appears as a variation of (2.7):

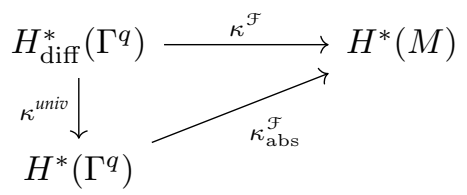

combined with a "van Est isomorphism"

$$
V E: H_{\text {diff }}^{*}\left(\Gamma^{q}\right) \stackrel{\simeq}{\rightrightarrows} G F_{q}^{*}
$$

The construction of the "differentiable cohomology (2.30)" was carried out by Haefliger [35]. However, it seems to be forgotten. It does look a bit ad-hoc in the sense that it is described only for $\Gamma^{q}$ and furthermore, it is not clear what is the structure that makes the definition work. Our aim in this chapter is to clarify this construction and, in particular, provide the general conceptual framework to which it belongs. 
Let us go through Haefliger's definition, also making sure that it makes sense for more general pseudogroups $\Gamma$ - hence defining $H_{\text {diff }}^{*}(\Gamma)$. Haefliger's idea is very simple: while "continuous cohomology" of $\Gamma$ is, modulo the Hausdorfness issue, computed by the Bott-Shulman complex

$$
\Omega^{q}\left(\mathcal{G}^{(p)}\right)=C^{p}\left(\mathcal{G}, \Omega_{\mathbf{X}}^{q}\right) \quad(\mathcal{G}=\operatorname{Germ}(\Gamma)),
$$

the differentiable cohomology should be computed by a sub-complex consisting of "differentiable cohains". The notion of "differentiable cochain" makes sense for cochains

$$
c \in C^{p}(\mathcal{G}, \mathcal{E})
$$

whenever $\mathcal{E}$ is the sheaf of sections of a smooth vector bundle $E \rightarrow \mathbf{X}$ : it means that at any

$$
g=\left(g_{1}, \ldots, g_{p}\right)=\left(\operatorname{germ}_{x_{1}}\left(\phi_{1}\right), \ldots, \operatorname{germ}_{x_{p}}\left(\phi_{p}\right)\right) \in \mathcal{G}^{(p)},
$$

the value $c(g) \in E_{t\left(g_{1}\right)}$ depends only on

$$
j^{\infty}(g):=\left(j_{x_{1}}^{\infty}\left(\phi_{1}\right), \ldots, j_{x_{p}}^{\infty}\left(\phi_{p}\right)\right)
$$

and it is "smooth in $j^{\infty}(g)$ ". More precisely, we define

$$
C_{\text {diff }}^{p}(\Gamma, E):=C^{p}\left(J^{\infty} \Gamma, E\right),
$$

the space of smooth sections of the pull-back of the vector bundle $E$ via $t: J^{\infty} \Gamma \rightarrow$ $\mathbf{X}$ that takes the target of the first arrow. We recall once more that the "smooth structure" on $J^{\infty} \Gamma$ is the one of profinite dimensional manifold; see the appendix for details. Observe that, to have a profinite dimensional smooth structure on $J^{\infty} \Gamma$, we need to assume that $\Gamma$ is a Lie pseudogroup, see (2.14) and the following discussion; recall also that we take $J^{\infty} \Gamma \cong \lim _{\longleftarrow} J^{l} \Gamma$ as part of our definition of Lie pseudogroup.

Of course, via the infinite jet map $j^{\infty}: \mathcal{G} \rightarrow J^{\infty} \Gamma$, we have

$$
C_{\text {diff }}^{p}(\Gamma, E) \subset C^{p}(\mathcal{G}, \mathcal{E}) \quad(\mathcal{E} \text { being the sheaf of sections of } E) .
$$

Definition 2.3.1. The Haefliger's differentiable cohomology of a Lie pseudogroup $\Gamma$, denoted by $H_{\text {diff }}^{*}(\Gamma)$, is the cohomology of the total complex associated to the double complex

$$
C_{\text {diff }}^{p, q}(\Gamma):=C_{\text {diff }}^{p}\left(\Gamma, \Lambda^{q} T^{*} \mathbf{X}\right)=C^{p}\left(J^{\infty} \Gamma, \Lambda^{q} T^{*} \mathbf{X}\right)
$$

which, via the map induced by $j^{\infty}: \mathcal{G} \rightarrow J^{\infty} \Gamma$, is a subcomplex of the BottShulman complex associated to $\mathcal{G}=\operatorname{Germ}(\Gamma)$. We will denote by $j^{*}: C_{\text {diff }}^{p, q}(\Gamma) \hookrightarrow$ $\Omega^{q}\left(\mathcal{G}^{(p)}\right)$ the corresponding inclusion and, similarly the map induced in cohomology

$$
j^{*}: H_{\mathrm{diff}}^{*}(\Gamma) \rightarrow H_{\mathrm{dR}}^{*}(\mathcal{G}) \quad(\mathcal{G}=\operatorname{Germ}(\Gamma)) .
$$

Finally, for a $\Gamma$-structure $\mathcal{P}$ on a manifold $M$ (represented by some cocycle $\gamma$, c.f. Definition 2.2.4), the composition of $j^{*}$ with the characteristic map $\gamma^{*}(2.24)$ is denoted

$$
\kappa_{\text {diff }}^{\mathcal{P}}: H_{\text {diff }}^{*}(\Gamma) \rightarrow H^{*}(M)
$$

and is called the differentiable characteristic map associated to the $\Gamma$-structure $\mathcal{P}$ on $\mathrm{M}$. 
The definition of the differentiable cohomology above is precisely Haefliger's definition [35]. Being a subcomplex means that it is preserved by the differentials $\delta$ (along $p$ ) and $d$ (along $q$ ) of the Bott-Shulman complex, therefore giving rise to similar differentials

$$
\delta: C_{\text {diff }}^{p, q}(\Gamma) \rightarrow C_{\text {diff }}^{p+1, q}(\Gamma), \quad d: C_{\text {diff }}^{p, q}(\Gamma) \rightarrow C_{\text {diff }}^{p, q+1}(\Gamma)
$$

On the other hand, it is immediate to see that this subcomplex is closed also under the product structure (see Remark 2.2.21); actually, the induced cup-product on the differentiable complex fits precisely the scheme described in Remark 2.2.21, but for the groupoid $J^{\infty} \Gamma$ and with coefficients in vector bundle representations rather than in sheaves. Therefore, $C_{\text {diff }}^{*, *}(\Gamma)$ inherits from the Bott-Shulman complex the same type of structure/properties; this will be made more precise later on but, for now, let us mention the fact that the Leibniz-type identities (2.17), (2.26) and (2.27) will be inherited.

Strictly speaking, the fact that the differentiable complex is a subcomplex is not immediate and requires a proof. Even more puzzling is to understand "why" this happens and unravel the structure on $J^{\infty} \Gamma$ that governs this construction. More precisely, while the differentiable complex is defined using cochains on $J^{\infty} \Gamma$, it is natural to look at general Lie groupoids $\Sigma \rightrightarrows \mathbf{X}$ and:

Problem: investigate the structure on $\Sigma \rightrightarrows \mathbf{X}$ that is needed in order to be able to form a bicomplex

$$
\left(C^{p}\left(\Sigma, \Lambda^{q} T^{*} \mathbf{X}\right), \delta, d\right)
$$

with "basic properties" similar to those of the differentiable complex; and, of course, $C_{\mathrm{diff}}^{*, *}(\Gamma)$ should be obtained in the particular case when $\Sigma=J^{\infty} \Gamma$. Here, by basic properties we mean that (2.36) is a double complex, the Leibniz identities (2.17), (2.26) and (2.27) still hold and, in low degrees:

- for $q=0, \delta$ is the usual groupoid differential on $C^{*}(\Sigma)$

- for $p=0, d$ is the de Rham differential on $\Omega^{*}(\mathbf{X})$.

It is remarkable that the outcome is precisely the structure that also shows up in the study of partial differential equations from a geometric point of view, i.e. the study of the Cartan distribution on jet spaces and their submanifolds, see [32, 41].

Below, we explain how one can slowly discover this structure when trying to construct the complex (2.36).

\subsubsection{The horizontal differential $\delta$ and groupoid actions}

The fact that differentials $\delta$ can be seen as an algebraic way to encode actions is rather standard - e.g. one has the following general result.

Lemma 2.3.2. For any groupoid $\Sigma \rightrightarrows \mathbf{X}$ and any vector bundle $E \rightarrow \mathbf{X}$ there is a 1-1 correspondence between actions of $\Sigma$ on $E$ (making $E$ into a representation) and differentials

$$
\delta: C^{*}(\Sigma, E) \rightarrow C^{*+1}(\Sigma, E)
$$


which make $C^{*}(\Sigma, E)$ into a DG module over $\left(C^{*}(\Sigma), \delta\right)$ (i.e.(2.26) holds for $\omega \in C^{p}(\Sigma)$, $\omega^{\prime} \in C^{p^{\prime}}(\Sigma, E)$ ) and such that $\delta$ preserves the subcomplex of normalised cochains (i.e. cochains that vanish whenever one of the entries is a unit).

This appears e.g. as Lemma 2.6 in [4], but the proof is rather obvious. Explicitly, $\delta$ is given by the standard formula (2.20), while the action can be recovered by what $\delta$ does on elements $\xi \in C^{0}(\Sigma, E)$, i.e. sections of $E$ : for $g: x \rightarrow y$ arrow of $\Sigma$ and $v \in E_{x}$, one chooses $\xi$ with $\xi(x)=v$ and then $g \cdot v=\xi(y)-\delta(\xi)(g)$.

Returning to our main problem we see that, in order to have the differential $\delta$, we need actions of $\Sigma$ on $\Lambda^{q} T^{*} \mathbf{X}$ (at least if we add the condition on normalised cochains); furthermore, taking advantage of the derivation identity (2.47) for all $q$ and $q^{\prime}$ (not only for $q=0$ ), it is not difficult to see that the actions on $\Lambda^{q} T^{*} \mathbf{X}$ must be the induced (diagonal) action on $\Lambda^{1} T^{*} \mathbf{X}=T^{*} \mathbf{X}$. Dualising, we need a right action of $\Sigma$ on $T \mathbf{X}$ - so that any arrow $g \in \Sigma$ acts as a linear map

$$
g^{*}: T_{t(g)} \mathbf{X} \rightarrow T_{s(g)} \mathbf{X} .
$$

Of course, using the fact that we deal with groupoids, i.e. using the presence of inverses, one can always turn such a right action into a left one by $g \cdot v:=$ $\left(g^{-1}\right)^{*}(v)$. The conclusion is that what we need is: an action of $\Sigma$ on $T \mathbf{X}$.

Returning to the infinite jet groupoid, $T \mathbf{X}$ carries an obvious action of $J^{\infty} \Gamma$ (in fact, of $J^{k} \Gamma$, for any $k \geq 1$ ): $j_{x}^{\infty} f \in J^{\infty} \Gamma$ acts on tangent vectors via $(d f)_{x}$. In turn this induces actions on the exterior powers $\Lambda^{q} T^{*} \mathbf{X}\left(j_{x}^{\infty} f \in J^{\infty} \Gamma\right.$ acts on a $q$-linear form at $x$ via the pullback by $f^{-1}$ ) and then it is not difficult to see that, indeed, $\delta$ of the differentiable complex becomes just the corresponding differential.

\subsubsection{The vertical differential $d$ and connections}

The differential $d$ is a bit more subtle as it reveals certain types of connections. Again, this is based on a rather standard lemma that holds in the very general context of surjective submersions $t: P \rightarrow \mathbf{X}$ when one is looking for operators acting on horizontal forms

$$
D: \Omega_{\text {hor }}^{*}(P) \rightarrow \Omega_{\text {hor }}^{*+1}(P) .
$$

Recall here that a form $\omega \in \Omega^{q}(P)$ is said to be horizontal if, $i_{V}(\omega)=0$ whenever $V$ is a vector tangent to the $t$-fibers (i.e. $V \in \operatorname{Ker}(d t))$. To make the relationship with complexes of type (2.36) more transparent, note that there is an identification

$$
\Omega_{\mathrm{hor}}^{*}(P) \cong \Gamma\left(P, t^{*} \Lambda^{*} T^{*} \mathbf{X}\right) .
$$

On the other hand, recall that:

Definition 2.3.3. An Ehresmann connection on $t: P \rightarrow \mathbf{X}$ is a vector subbundle $\mathcal{C} \subset T P$ that is complementary to the sub-bundle of vertical vectors $T^{\mathrm{v}} P=\operatorname{Ker}(d t)$.

Such a $\mathcal{C}$ gives rise to (and can be reinterpreted as) an operation of horizontal lifting of vector fields

$$
\operatorname{hor}^{\mathfrak{e}}: \mathfrak{X}(\mathbf{X}) \rightarrow \mathfrak{X}(P)
$$


which is actually defined pointwise: at each $x \in P$, hor ${ }_{x}^{e}$ sends a vector $v \in T_{t(x)} \mathbf{X}$ to the unique vector in $\mathrm{C}_{x}$ that project via $t$ to $v$. Using the projection on $\mathrm{C}, \mathrm{pr}_{\mathrm{C}}$ : $T P \rightarrow \mathcal{C}$, the de Rham operator on $\Omega^{*}(P)$ induces an operator $d_{\mathcal{C}}$ on $\Omega_{\text {hor }}^{*}(P)$ by

$$
d_{\mathcal{C}}(\omega)\left(X^{1}, \ldots, X^{q}\right):=\left(d_{\mathrm{dR}} \omega\right)\left(\operatorname{pr}_{\mathcal{C}}\left(X^{1}\right), \ldots, \operatorname{pr}_{H}\left(X^{q}\right)\right) .
$$

or, interpreting horizontal forms as sections of $t^{*} \Lambda^{*} T^{*} \mathbf{X}$, for $v^{1}, \ldots, v^{q} \in T_{t(x)} \mathbf{X}$ :

$$
d_{\mathcal{C}}(\omega)\left(v^{1}, \ldots, v^{q}\right):=\left(d_{\mathrm{dR}} \omega\right)\left(\operatorname{hor}_{x}^{\mathcal{C}}\left(v^{1}\right), \ldots, \operatorname{hor}_{x}^{\mathcal{C}}\left(v^{p}\right)\right) .
$$

Lemma 2.3.4. For any bundle $t: P \rightarrow \mathbf{X}$, the construction $\mathcal{C} \mapsto d_{\mathcal{C}}$ gives a 1-1 correspondence between Ehresmann connection and linear operators (2.38) satisfying the following two properties:

- They satisfy the Leibniz derivation identity (2.48) for all $\omega \in \Omega^{q}(\mathbf{X})$, $\omega \in \Omega_{\text {hor }}^{q^{\prime}}(P)$.

- On basic forms, i.e. of type $t^{*}(\omega)$ with $\omega \in \Omega^{*}(\mathbf{X}), D\left(t^{*}(\omega)\right)=t^{*}\left(d_{\mathrm{dR}} \omega\right)$.

Moreover, $d_{\mathfrak{C}}^{2}=0$ if and only if $\mathcal{C}$ is a flat connection, i.e. the induced operation

$$
\operatorname{hor}^{\mathcal{E}}: \mathfrak{X}(\mathbf{X}) \rightarrow \mathfrak{X}(\mathrm{P})
$$

of lifting vector fields from the base preserves the Lie brackets.

This lemma is relevant for us when applied to the maps

$$
t: \Sigma^{(p)} \rightarrow \mathbf{X}
$$

Note that having operators $d$ with the properties that we required in the Problem, hence also satisfying (2.27), implies that the conditions of the lemma are satisfied. Indeed, for $p=0$ and $\omega$ arbitrary (hence $\omega \in \Omega^{q}(\mathbf{X})$ ), $q^{\prime}=0$ and $\omega^{\prime}=1$ (the constant 0 -form on $\Sigma^{(p)}$ with $p$-arbitrary), since

$$
\omega \cup 1=t^{*}(\omega)
$$

identity (2.27) becomes the second condition in the previous lemma. All together, one ends up with Ehresmann connections

$$
\mathcal{C}^{(p)} \subset T \Sigma^{(p)} .
$$

Of course, they will not be independent, as one discovers using the entire derivation identities (see Subsection 3.5). Before we discuss that, we stare a bit at the case $p=1$. While the way $\mathcal{C} \subset T \Sigma$ is obtained appeals only to the submersion $t: \Sigma \rightarrow \mathbf{X}$, one can slowly bring in more of the groupoid structure. First of all, using also the source map we obtain an "action-like" operation induced by $\mathrm{C}$ : any arrow $g$ from $x$ to $y$ induces an operation

$$
\lambda_{g}^{*}: T_{y} \mathbf{X} \rightarrow T_{x} \mathbf{X}, \quad \lambda_{g}^{*}(v)=(d s)_{g}\left(\operatorname{hor}_{g}^{\mathrm{e}}(v)\right) .
$$

There is no surprise that this is basically the same action as in the previous subsection. 


\subsubsection{Compatibility of $\delta$ and $d$}

The reason that the two actions coincide is precisely the compatibility of $\delta$ and $d_{e}$

$$
d_{\mathfrak{e}} \circ \delta=\delta \circ d_{\mathfrak{e}},
$$

applied in low degrees $p$ (i.e. for $p=0,1$ ):

Lemma 2.3.5. Assume we are given a Lie groupoid $\Sigma \rightrightarrows \mathbf{X}$ together with an Ehresmann connection $\mathcal{C}$ along $t: \Sigma \rightarrow \mathbf{X}$ and an action of $\Sigma$ on the vector bundle $T \mathbf{X}$. Then the corresponding operators $\delta$ and $d_{\mathrm{e}}$ are compatible, i.e. the following diagram commutes:

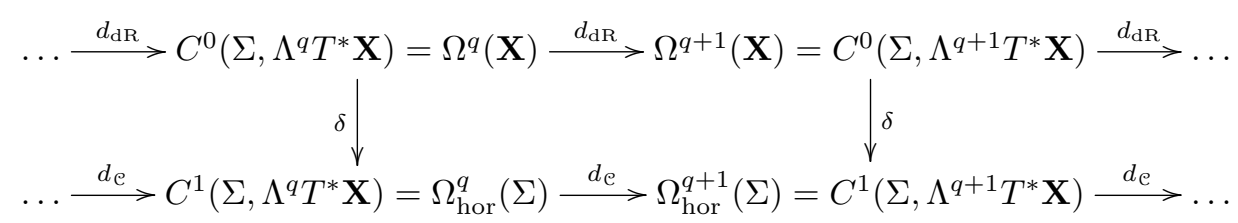

if and only if action-like operation (2.40) induced by $\mathrm{C}$ coincides with the dual (2.37) of the original action.

Proof. Writing the compatibility condition for $q=0$ and applying it to arbitrary functions $f \in C^{\infty}(\mathbf{X})$ one obtains

$$
d_{\mathcal{C}}\left(s^{*} f-t^{*} f\right)=g \cdot s^{*}(d f)-t^{*}(d f),
$$

where the $t$-terms coincide by the properties of $d_{\mathfrak{e}}$. We are left with the $s$-terms which, applied to arbitrary $v \in T_{t(g)} \mathbf{X}$ becomes

$$
(d f)(d s)_{g}\left(\operatorname{hor}_{g}^{\mathrm{e}}(v)\right)=(d f)(d s)\left(g^{-1} \cdot v\right) .
$$

Conversely, if the two actions coincide it is clear that the diagram is commutative when applied on functions (i.e. at $q=0$ ). Since $d_{e} \circ \delta-\delta \circ d$ commutes with $d_{\mathcal{e}}$, the same happens when applied on exact 1-forms $d f$. Using the derivation identities it follows that it happens on all $q$-forms on $\mathbf{X}$.

\subsubsection{Only one (multiplicative) connection $\mathcal{C}$}

The conclusion of Subsection 2.3.3 was that we need Ehresmann connections $\mathcal{C}^{(p)} \subset T \Sigma^{(p)}$, each one of them giving rise to $d=d_{\mathcal{C}(p)}$ acting on $C^{p}\left(\Sigma, \Lambda^{*} T^{*} \mathbf{X}\right)$. However, these will be related if we want the derivation identity (2.27) to hold.

Lemma 2.3.6. Assume that $\mathrm{C}^{(p)}$ are Ehresmann connections on $t: \Sigma^{(p)} \rightarrow \mathbf{X}$ so that the resulting operators $d=d_{\mathcal{C}^{(p)}}$ together satisfy the derivation identity (2.27). Then each $\mathrm{C}^{(p)}$ is determined by $\mathrm{C}:=\mathrm{C}^{(1)}$ as

$$
\mathcal{C}^{(p)}=\left\{\left(v^{1}, \ldots, v^{p}\right) \in T \Sigma^{(p)}: v^{1}, \ldots, v^{p} \in \mathcal{C}\right\} .
$$

Equivalently, the induced horizontal lifting map is given by

$$
\operatorname{hor}_{g_{1}, \ldots, g_{p}}^{\mathrm{e}^{(p)}}(v)=\left(\operatorname{hor}_{g_{1}}^{\mathfrak{e}}(v), \operatorname{hor}_{g_{2}}^{\mathrm{e}}\left(g_{1}^{*} v\right), \ldots, \operatorname{hor}_{g_{p}}^{\mathfrak{e}}\left(g_{p-1}^{*} \ldots g_{1}^{*} v\right)\right)
$$


Proof. We concentrate on the last formula (which implies the other part by dimension counting). Also, for notational simplicity, we assume $p=2$. It suffices to use (2.27) in a very extreme case: for 0 -forms, i.e. functions, $f_{1}, f_{2} \in C^{\infty}(\Sigma)=$ $C^{1}\left(\Sigma, \Lambda^{0} T^{*} \mathbf{X}\right)$ :

$$
d_{\mathcal{C}^{(2)}}\left(f_{1} \cup f_{2}\right)=d_{\mathcal{C}}\left(f_{1}\right) \cup f_{2}+f_{1} \cup d_{\mathfrak{C}}\left(f_{2}\right)
$$

which, in turn, can be rewritten as

$$
d_{\mathcal{C}^{(2)}}\left(\operatorname{pr}_{1}^{*} f_{1} \cup \operatorname{pr}_{2}^{*} f_{2}\right)=\operatorname{pr}_{1}^{*} d_{\mathfrak{C}}\left(f_{1}\right) \cup \operatorname{pr}_{2}^{*} f_{2}+\operatorname{pr}_{1}^{*} f_{1} \cup\left(1 \cup d_{\mathcal{C}}\left(f_{2}\right)\right) .
$$

Taking $f_{2}=1$ and $f_{1}=f \in C^{\infty}(\Sigma)$ arbitrary, and then $f_{1}=1$ and $f_{2}=f$, we obtain:

$$
d_{\mathcal{C}^{(2)}}\left(\mathrm{pr}_{1}^{*} f\right)=\mathrm{pr}_{1}^{*} d_{\mathcal{C}}(f), \quad d_{\mathcal{C}^{(2)}}\left(\mathrm{pr}_{2}^{*} f\right)=1 \cup d_{\mathcal{C}}(f) .
$$

Writing out $d_{\mathcal{e}}$ in terms of the horizontal lifts, the first equation translates into the fact that for $\left(g_{1}, g_{2}\right) \in \Sigma^{(2)}, v \in T_{t\left(g_{1}\right)} \mathbf{X}$, the first component of hor ${ }_{g_{1}, g_{2}}^{\mathrm{e}^{(2)}}(v)$ is hor $_{g_{1}}^{\mathrm{e}}(v)$. Similarly, the second equation implies that the second component of $\operatorname{hor}_{g_{1}, g_{2}}^{\mathrm{e}^{(2)}}(v)$ is hor $\mathrm{g}_{2}^{\mathrm{e}}\left(g_{1}^{*} v\right)$.

And yet another property that we addressed only in degrees 0 and 1 , namely the compatibility of $\delta$ and $d$ from Lemma 2.3.5, has more implications on $\mathcal{C}$ if one moves one line higher. To state it we make use of the fact that for any groupoid $\Sigma \rightrightarrows \mathbf{X}$, by taking the differentials of the structure maps of $\Sigma$ (source, target, multiplication), one obtains a new groupoid

$$
T \Sigma \rightrightarrows T \mathbf{X}
$$

called the tangent groupoid of $\Sigma$. With this,

Definition 2.3.7. A sub-bundle $\mathcal{C} \subset T \Sigma$ (as vector bundles over $\Sigma$ ) is said to be multiplicative if it is a full sub-groupoid of $T \Sigma$.

This boils down to the following explicit conditions:

$\mathrm{m} 1: d s, d t: \mathcal{C} \rightarrow T \mathbf{X}$ are surjective.

$\mathrm{m} 2$ : for $v, w \in \mathcal{C}$ composable (i.e. with $d s(v)=d t(w)), v \circ w:=d m(v, w) \in \mathcal{C}$.

m3: at any $x \in \mathbf{X}, \mathrm{e}_{1_{x}}$ contains $T_{x} \mathbf{X}$ (interpreted as a subspace of $T_{1_{x}} \Sigma$ via the unit map).

m4: the differential of the inversion map $\tau: \Sigma \rightarrow \Sigma$ takes $\mathcal{C}$ to itself.

While these conditions make sense for more general sub-bundles $\mathcal{C}$, for Ehresmann connections they can be expressed in terms of the operation of horizontal lifting as:

$$
d m\left(\operatorname{hor}_{g_{1}}^{\mathrm{e}}(v), \operatorname{hor}_{g_{2}}^{\mathrm{e}}\left(g_{1}^{*} v\right)\right)=\operatorname{hor}_{g_{1} g_{2}}^{\mathrm{e}}(v),
$$

for all $g_{1}, g_{2} \in \Sigma$ composable, $v \in T_{t\left(g_{1}\right)}$,

$$
\operatorname{hor}_{1_{x}}^{\mathrm{e}}(v)=v
$$


for all $v \in T_{x} \mathbf{X} \subset T_{1_{x}} \Sigma$,

$$
\operatorname{hor}_{g^{-1}}^{\mathrm{e}}\left(\lambda_{g}^{*} v\right)=(d \tau)\left(\operatorname{hor}_{g}^{\mathrm{e}}(v)\right)
$$

for all $g \in \Sigma, v \in T_{t(g)} \mathbf{X}$. Here, $\lambda_{g}^{*}$ denotes the induced quasi-action (2.40). See also the proof below. Moreover, condition $\mathrm{m} 1$ can be rephrased into the condition that all the $\lambda_{g}^{*}$ are isomorphisms; the rest of the conditions imply, of course, that the quasi-action is actually an action.

Lemma 2.3.8. With the assumptions from Lemma 2.3.6 and Lemma 2.3.5, the following diagram is commutative

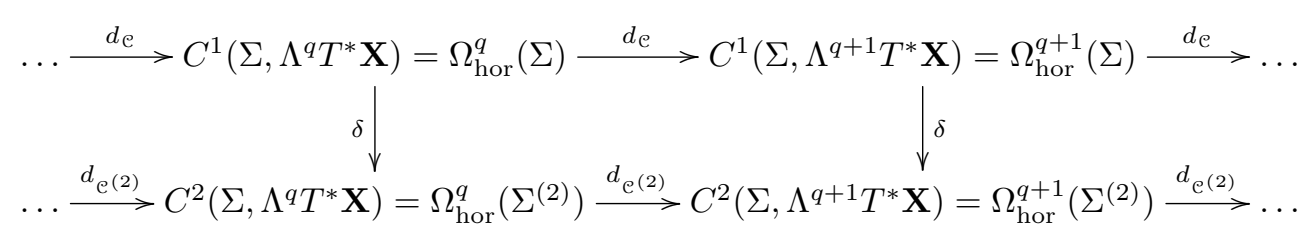

if and only if $\mathrm{C}$ is multiplicative (actually, if $\mathrm{m} 2$ holds).

Proof. For simplicity, let us work just with functions $f \in C^{\infty}\left(\Sigma^{(2)}\right)$ and write down the compatibility

$$
d_{\mathcal{C}^{(2)}}\left(\mathrm{pr}_{1}^{*} f+\mathrm{pr}_{2}^{*} f-m^{*} f\right)=\delta d_{\mathcal{C}}(f) .
$$

Using the two equations from (2.42), we are left with

$$
d_{\mathcal{C}^{(2)}}\left(m^{*} f\right)=m^{*} d_{\mathfrak{C}}(f)
$$

Writing this out in terms of horizontal lifts one obtains

$$
d m\left(\operatorname{hor}_{g_{1}, g_{2}}^{\mathrm{e}^{(2)}}(v)\right)=\operatorname{hor}_{g_{1} g_{2}}^{\mathrm{e}}(v), \quad \text { for }\left(g_{1}, g_{2}\right) \in \Sigma^{(2)}, v \in T_{t\left(g_{1}\right)} \mathbf{X} .
$$

Since the image of hor ${ }^{\mathcal{C}^{(2)}}$ is $\mathcal{C}^{(2)}=T \Sigma^{(2)} \cap \mathcal{C} \times \mathcal{C}$, we see that the condition $\mathrm{m} 2$ for multiplicativity implies the commutativity of the diagram and viceversa if the diagram is commutative $\mathrm{m} 2$ follows. Note that, with the formula for hor ${ }^{\mathrm{e}^{(2)}}$ from the previous lemma, we obtain precisely (2.43). It remains to show that $\mathrm{m} 1, \mathrm{~m} 3$ and $\mathrm{m} 4$ can be derived from $\mathrm{m} 2$ and the assumptions. Condition $\mathrm{m} 1$ follows from the fact that the quasi-action is an action, by assumption (Lemma 2.3.5). For m3 one makes use of (2.43) written at units to derive (2.44). Alternatively, m3 can also be derived from the rest because, in the tangent groupoid, we can write units as $V \circ V^{-1}$ with $V$ chosen to be in $\mathrm{C}$. For $\mathrm{m} 4$ we use again (2.43), this time for $g_{1}=g$ arbitrary and $g_{2}=g^{-1}$. The outcome can be written in the tangent groupoid as:

$$
1_{v}=\operatorname{hor}_{g}^{\mathrm{e}}(v) \circ \operatorname{hor}_{g^{-1}}^{\mathrm{e}}\left(g^{*} v\right)
$$

from which we deduce that hor $g^{-1}\left(g^{*} v\right)$ is the inverse of $\operatorname{hor}_{g}(v)$ - i.e. precisely formula (2.45). 
Remark 2.3.9. As one sees from the discussions above, a multiplicative Ehresmann connection $\mathcal{C}$ on $t: \Sigma \rightarrow \mathbf{X}$ is also an Ehresmann connection on $s: \Sigma \rightarrow \mathbf{X}$. Consequently, an analogue of the lifting operation 2.39 is defined with respect to $s$. In the rest of the chapter, we will use the same notation for these two lifting operations; which one is being considered will always be clear from the context.

The final outcome of our discussion is that, for the (analogue of the) "Haefliger's bicomplex" (2.33) to exist on $\Sigma \rightrightarrows \mathbf{X}$, one needs a multiplicative flat connecton $\mathcal{C}$ on $\Sigma$. In the rest of this chapter, starting from the next section, we study pairs $(\Sigma, \mathcal{C})$ of this form. In particular, we will gain a more conceptual understanding of Haefliger's work and a generalization of it.

Before going on with this program, in the next subsection we describe the flat multiplicative connection on $J^{\infty} \Gamma$ underlying bicomplex (2.33); then, we conclude this section spending some more time with the Bott-Shulman complex.

\subsubsection{The multiplicative flat connection on $J^{\infty} \Gamma$}

To describe the flat multiplicative connection on $J^{\infty} \Gamma$ that underlies Haefliger's differentiable cohomology of a Lie pseudogroup, it is useful to go back to the tower (2.14)

$$
J^{\infty} \Gamma \rightarrow \cdots \rightarrow J^{l} \Gamma \rightarrow J^{l-1} \Gamma \rightarrow \cdots \rightarrow J^{1} \Gamma \rightarrow J^{0} \Gamma \rightrightarrows \mathbf{X}
$$

This is a tower of groupoids over $\mathbf{X}$ and actually, since we work with Lie pseudogroups $\Gamma$, a tower of Lie groupoids: the $J^{l} \Gamma^{\prime}$ s are Lie groupoids and each map is a surjective submersion and a morphism of groupoids. The definition of Lie pseudogroup can be summed up and is naturally expressed by saying that this tower is a normal pf-atlas for $J^{\infty} \Gamma$; the reader is referred to the Appendix. The flat multiplicative connection on $J^{\infty} \Gamma$, that we will denote by $\mathcal{C}^{\infty}$, is a smooth distribution in the profinite dimensional sense, i.e. a "limit" of smooth distributions on the groupoids $J^{l} \Gamma$ that are compatible with the tower projections.

To begin with, it is useful to observe that $J^{l} \Gamma$ is a subspace of the jet space $J^{l}(\mathbf{X}, \mathbf{X})$. A section $\sigma: \mathbf{X} \rightarrow J^{l}(\mathbf{X}, \mathbf{X})$ of the projection

$$
s: J^{l}(\mathbf{X}, \mathbf{X}) \rightarrow \mathbf{X}, \quad j_{x}^{l} f \rightarrow x
$$

is called holonomic when $\sigma=j^{l} f: x \rightarrow j_{x}^{l} f$ for some $f: \mathbf{X} \rightarrow \mathbf{X}$. It is possible to define a distribution $\mathrm{C}^{l}$ on $J^{l}(\mathbf{X}, \mathbf{X})$ via the request that holonomic sections are precisely its integral sections.

Definition 2.3.10. The Cartan distribution $\varrho^{l}$ on $J^{l}(\mathbf{X}, \mathbf{X})$ is the distribution on $J^{l}(\mathbf{X}, \mathbf{X})$ such that a section $\sigma: \mathbf{X} \rightarrow J^{l}(\mathbf{X}, \mathbf{X})$ is holonomic if and only if its image is an integral manifold of $\mathrm{C}^{l}$.

The Cartan distribution on jet spaces is a very well known object. In particular, it plays a central role in the geometric theory of PDEs; among the extensive literature, see for example [32, 41]. A more explicit definition can be given by adopting the dual point of view. One defines a one form $\omega^{l}$ on $J^{l}(\mathbf{X}, \mathbf{X})$ valued in the vertical bundle $\operatorname{Ker}(d p r)$ of the projection $p r: J^{l}(\mathbf{X}, \mathbf{X}) \rightarrow J^{l-1}(\mathbf{X}, \mathbf{X})$ by

$$
\omega_{j_{x}^{l} f}^{l}=d_{j_{x}^{l} f} p r-d_{j_{x}^{l} f}\left(j^{l-1} f \circ s\right)
$$


and then $\mathcal{C}^{l}=\operatorname{Ker}\left(\omega^{l}\right)$ can either be taken as an alternative definition (see $[15,55$, 69]) or proven as a lemma. What matters for us is the following

Proposition 2.3.11 ([55,69]). For any Lie pseudogroup $\Gamma$ and for any $l \geq 1$, the Cartan distribution $\mathrm{e}^{l}$ restricts to $J^{l} \Gamma$ as a regular multiplicative distribution (still denoted by $\left.\mathrm{e}^{l}\right)$.

The multiplicativity of the Cartan distribution on the jet groupoids $J^{l} \Gamma$ of a Lie pseudogroup is a key property when studying the geometry of PDEs. See [55] for a systematic study of "Cartan-like" multiplicative forms on groupoids and [69] for a modern take on Cartan's seminal work on pseudogroups, where the role of multiplicativity is made explicit.

The above discussion stays true when the positive natural number $l$ is replaced by $\infty$. That is, $J^{\infty} \Gamma$ is equipped with a multiplicative distribution $\mathrm{C}^{\infty}$ that comes as a restriction of an analogous distribution on $J^{\infty}(\mathbf{X}, \mathbf{X})$ detecting holonomic sections (albeit in a peculiar way, see remark 2.3 .1 below). $\mathrm{C}^{\infty}$ is a smooth object in the sense of pf-manifolds, i.e. the "limit" of the distributions $\mathrm{C}^{l}$. In fact, to make the above claims, as well as the following one, completely rigorous, one needs to work with profinite dimensional manifolds; see the Appendix and in particular Examples 2.A.13, 2.A.18, 2.A.26, 2.A.37 and 2.A.38. What one is able to see is that:

Theorem 2.3.12. The distribution $\mathrm{e}^{\infty}$ on $J^{\infty} \Gamma$ is

- multiplicative;

- a connection with respect to $s: J^{\infty} \Gamma \rightarrow \mathbf{X}$;

- involutive.

Proof. The proof is well known; we sketch it for the sake of completeness.

First of all, as explained in the Appendix, the pf-tangent space $T J^{\infty} \Gamma$ can be identified with the limit $\lim _{\longleftarrow} T J^{l} \Gamma$; as a consequence, the Cartan distribution can be identified with the sequence $\left\{\mathrm{C}^{l}\right\}$ of the finite dimensional Cartan distributions, which is compatible with (2.14) in the sense that $d p r\left(\mathrm{C}^{l}\right) \subset \mathrm{e}^{l-1}$. With this in mind, $\mathrm{C}^{\infty}$ straight away inherits its multiplicativity from the multiplicativity of the $e^{l}$ 's, which can be checked directly (for example using the forms $\omega^{l}$ 's introduced above [69]).

The fact that $\mathrm{C}^{\infty}$ is an involutive distribution complementary to $s$, i.e. an involutive connection, is hidden in the interplay between the projections $p r$ : $J^{l} \Gamma \rightarrow J^{l-1} \Gamma$ and the Cartan distributions. Using the terminology of Definition 6.2.4 in [55], the projections $p r: J^{l} \Gamma \rightarrow J^{l-1} \Gamma$ are Lie prolongations. In particular,

- $\operatorname{dpr}\left(\mathrm{e}^{l} \cap \operatorname{Ker}(d s)\right)=0$ for all $l \geq 1$;

- $\left[\operatorname{dpr}\left(\mathrm{C}^{l+1}\right), \operatorname{dpr}\left(\mathrm{e}^{l+1}\right)\right] \subset \mathfrak{C}^{l}$, for all $l \geq 1$.

The first property implies that $\mathrm{e}^{\infty}$ is a connection; the second property implies that $\mathrm{C}^{\infty}$ is involutive.

Definition 2.3.13. $\mathrm{C}^{\infty}$ is called the Cartan connection on $J^{\infty} \Gamma$. 
Remark 2.3.1. It is worth mentioning that the restriction of the Cartan distribution to jet spaces of Lie pseudogroups works in a peculiar way when considering infinite jets. In fact, if $\mathcal{C}^{\infty}$ is the Cartan distribution on $J^{\infty}(\mathbf{X}, \mathbf{X})$ and $\Gamma$ is a Lie pseudogroup over $\mathbf{X}$, then $\mathrm{C}_{g}^{\infty} \subset T_{g} J^{\infty} \Gamma$ for all $g \in J^{\infty} \Gamma$. On the other hand, if $l \in \mathbb{N}, l \geq 1$, the Cartan distribution on $J^{\mathfrak{l}}(\mathbf{X}, \mathbf{X})$ is, in general, not contained into $T J^{l} \Gamma$.

Surprisingly enough, the multiplicativity of $\mathrm{e}^{\infty}$ was the last property to appear explicitly in the literature; in fact, the multiplicativity of the finite dimensional Cartan distributions $\mathrm{C}^{l}$ is explicitely addressed for the first time in [55]. On the other hand, the transversality and involutivity properties of $\mathcal{C}^{\infty}$ have been known for a long time.

\subsubsection{A look back at the structure on the Bott-Shulman and re- lated complexes}

It is interesting to spell out the various structures that are floating around on the various complexes. We concentrate first on the Bott-Shulman complex; so, let us fix an arbitrary Lie groupoid $\Sigma \rightrightarrows \mathbf{X}$ and consider

$$
\Omega^{p, q}=\Omega^{q}\left(\Sigma^{(p)}\right) .
$$

First of all together with the wedge product of forms, the de Rham differential and the pull-backs along the face and degeneracy maps, $\left(\Omega^{*, *}, \wedge, d, d_{i}^{*}, s_{i}^{*}\right)$ is a cosimplicial DGA, i.e.

- for each $p,\left(\Omega^{p, *}, \wedge, d\right)$ is a DGA; hence

$$
-\wedge-: \Omega^{p, q} \times \Omega^{p, q^{\prime}} \rightarrow \Omega^{p, q+q^{\prime}}
$$

and $d: \Omega^{p, q} \rightarrow \Omega^{p, q+1}$ satisfies the Leibniz identity:

$$
d\left(\omega \wedge \omega^{\prime}\right)=d(\omega) \wedge \omega^{\prime}+(-1)^{q} \omega \wedge d\left(\omega^{\prime}\right)
$$

whenever $\omega$ and $\omega$ are of bidegrees $(p, q)$, and $\left(p, q^{\prime}\right)$, respectively.

- these DGAs are related by morphisms (of DGAs)

$$
d_{i}^{*}: \Omega^{p-1, *} \rightarrow \Omega^{p, *}, \quad s_{i}^{*}: \Omega^{p+1, *} \rightarrow \Omega^{p, *} \quad(\text { for } 0 \leq i \leq p)
$$

satisfying the cosimplicial identities (i.e. the dual of the simplicial identities (2.18)).

In turn, for any such cosimplicial DGA, one can construct the second differential (along $p$ ), $\delta=\sum(-1)^{i} d_{i}^{*}$ and, furthermore, the product structure (2.16); indeed, note that the maps first ${ }_{p}^{*}$ and last ${ }_{p^{\prime}}^{*}$ can be expressed in terms of the simplicial structure as

$$
\text { first }_{p}^{*}=\underbrace{d_{0}^{*} \ldots d_{0}^{*}}_{p^{\prime} \text { times }}, \quad \text { last }_{p^{\prime}}^{*}=d_{p^{\prime}+p}^{*} d_{p^{\prime}+p-1}^{*} \ldots d_{p^{\prime}+1}^{*},
$$

hence (2.16) and (2.25) make sense for any cosimplicial DGA. The outcome is a double-DGA $\left(\Omega^{p, q}, \cup, \delta, d\right)$ in the sense that it comes with: 
- a bigraded algebra operation

$$
-\cup-: \Omega^{p, q} \times \Omega^{p^{\prime}, q^{\prime}} \rightarrow \Omega^{p+p^{\prime}, q+q^{\prime}}
$$

- a differential $\delta: \Omega^{p, q} \rightarrow \Omega^{p+1, q}$ satisfying the horizontal Leibniz identity:

$$
\delta\left(\omega \cup \omega^{\prime}\right)=\delta(\omega) \cup \omega^{\prime}+(-1)^{p} \omega \cup \delta\left(\omega^{\prime}\right)
$$

whenever $\omega$ and $\omega$ are of bidegrees $(p, q)$, and $\left(p^{\prime}, q^{\prime}\right)$, respectively;

- a differential $d: \Omega^{p, q} \rightarrow \Omega^{p, q+1}$ satisfying the vertical Leibniz identity:

$$
d\left(\omega \wedge \omega^{\prime}\right)=d(\omega) \wedge \omega^{\prime}+(-1)^{q} \omega \wedge d\left(\omega^{\prime}\right)
$$

whenever $\omega$ and $\omega$ are of bidegrees $(p, q)$, and $\left(p^{\prime}, q^{\prime}\right)$, respectively.

Finally, for any double DGA $\left(\Omega^{p, q}, \cup, \delta, d\right)$ one can also pass to the total complex as in (2.15):

$$
\operatorname{Tot}^{k} \Omega^{*, *}:=\bigoplus_{p+q=k} \Omega^{p, q}, \quad D_{\text {tot }}=\delta+(-1)^{p} d \text { on } \Omega^{p, q}
$$

endowed with the signed product

$$
\omega \cup_{\text {tot }} \omega^{\prime}:=(-1)^{q p^{\prime}} \omega \cup \omega^{\prime}, \quad \text { for } \omega \in \Omega^{p, q}, \omega^{\prime} \in \Omega^{p^{\prime}, q^{\prime}} .
$$

Of course, the signs are chosen so that $D_{\text {tot }}$ satisfies the Leibniz identity w.r.t. the total degree:

$$
D_{\text {tot }}\left(\omega \cup_{\text {tot }} \omega^{\prime}\right)=D_{\text {tot }}(\omega) \cup_{\text {tot }} \omega^{\prime}+(-1)^{k} \omega \cup_{\text {tot }} D_{\text {tot }}\left(\omega^{\prime}\right)
$$

where $k=p+q$ is the total degree of $\omega \in \Omega^{q}\left(\mathcal{G}^{(p)}\right)$. Therefore one obtains a DGA:

$$
\left(\operatorname{Tot} \Omega^{*, *}, D_{\text {tot }}, \cup_{\text {tot }}\right) \text {. }
$$

Of course, with the appropriate (and rather obvious) notion of morphisms, the previous constructions can be seen as functors

$$
\text { cosimplicial DGAs } \rightarrow \text { double DGAs } \rightarrow \text { DGAs. }
$$

The conclusion is that the Bott-Shulman complex is a cosimplicial DGA and, therefore, can also be turned into a double DGA or, by passing to the total complex, into an ordinary DGA. The fact that the differentiable complex of a pseudogroup $\Gamma$ is a sub-complex of the Bott-Shulman complex of $\operatorname{Germ}(\Gamma)$ can be refined into the following result - which is actually less messy to prove:

Proposition 2.3.14. For any Lie pseudogroup $\Gamma$ on $\mathbf{X}$, the differentiable complex of $\Gamma$ is a sub-cosimplicial DGA of the Bott-Shulman complex of the corresponding groupoid $\mathcal{G}=\operatorname{Germ}(\Gamma) \rightrightarrows \mathbf{X}$ (via the inclusion $j^{*}: C_{\text {diff }}^{p, q}(\Gamma) \hookrightarrow \Omega^{q}\left(\mathcal{G}^{(p)}\right)$ ). In particular, the differentiable complex of $\Gamma$ carries an induced cosimplicial DGA structure. 


\subsection{Generalization: Haefliger cohomology}

To sum up the discussion from the previous section, we found out that to define the analogue of the "Haefliger's bicomplex" (2.33) for a (profinite dimensional) Lie groupoid $\Sigma \rightrightarrows \mathbf{X}$ we need a multiplicative flat connection $\mathcal{C}$ on $\Sigma$. The aim of the rest of this chapter is to recast Haefliger's construction in this generality, while providing a conceptual understanding of the various steps involved. Indeed, in Section 2.4 we will define the Haefliger cohomology of a pair $(\Sigma, \mathcal{C})$ given by a Lie groupoid together with a flat multiplicative connection; in our terminology, a flat (Cartan) groupoid. In Section 2.5 we will discuss the corresponding infinitesimal picture. In Section 2.6 we will construct a "van Est map" generalizing the isomorphism (2.32). There, we will also discuss a generalization of the characteristic map (2.34) that, remarkably, is not defined as the composition with an analogue of $j^{*}: H_{\text {diff }}^{*}(\Gamma) \rightarrow H_{d R}^{*}(\mathcal{G})$, but coincides with it whenever such an analogue is available.

A terminology remark: from now on, we will simply write "Lie groupoids" or "Lie algebroids" (the latter starting from the next section) meaning either the usual, finite dimensional, objects or the (strict) profinite dimensional ones, discussed in the Appendix (see Definition 2.A.34). We stress that our profinite dimensional groupoids (algebroids) will always be defined over a finite dimensional base $\mathbf{X}$, except when discussing the general setting for our van Est map, in Section 2.6 and, in particular, in Subsection 2.6.1. ${ }^{2}$

\subsubsection{Definitions and first examples}

The problem that we posed and the discussion around it slowly revealed the structure that is needed to define Haefliger cohomology - summarized in the definition below.

Definition 2.4.1. A Cartan groupoid is a Lie groupoid $\Sigma \rightrightarrows \mathbf{X}$ together with a subbundle $\mathcal{C} \subset T \Sigma$ satisfying the following:

- it is an Ehresman connection on $t: \Sigma \rightarrow \mathbf{X}$ (Definition 2.3.3),

- it is multiplicative (Definition 2.3.7).

We say that $(\Sigma, \mathcal{C})$ is a flat Cartan groupoid if $\mathcal{C} \subset T \Sigma$ is involutive.

Remark 2.4.2. Typically, distributions $\mathcal{C}$ as in the definition above appear as kernels of 1 -forms

$$
\omega \in \Omega^{1}\left(\Sigma, t^{*} E\right)
$$

with values in representations $E$ of $\Sigma$, with the property that $\omega$ is multiplicative in the following sense: for each composable pair $(g, h) \in \Sigma^{(2)}$,

$$
\left(m^{*} \omega\right)_{(g, h)}=\left(p r_{1}^{*} \omega\right)_{(g, h)}+g \cdot\left(p r_{2}^{*} \omega\right)_{(g, h)}
$$

\footnotetext{
${ }^{2}$ More precisely: in Subsection 2.6.1 we need to consider the action groupoid $\Sigma \ltimes P=\Sigma_{s} \times{ }_{\mu} P$ over $P$ associated to an action of a (pf) Lie groupoid $\Sigma \rightrightarrows \mathbf{X}$ on a (pf) manifold $P$ along a map $\mu: P \rightarrow \mathbf{X}$.
} 
where $m: \Sigma^{(2)} \rightarrow \Sigma$ is the multiplication map and $g \cdot: E_{s(g)} \rightarrow E_{t(g)}$ is the action of $g \in \Sigma^{(2)}$. Indeed, requiring also that $\omega$ is pointwise surjective and that

$$
\mathcal{C}_{\omega}:=\operatorname{Ker}(\omega) \subset T \Sigma
$$

is complementary to the $t$-fibers, it follows that $\left(\Sigma, \mathcal{C}_{\omega}\right)$ is a Cartan groupoid.

Given a Cartan groupoid $(\Sigma, \mathcal{C})$, a multiplicative 1 -form $\omega$ as above such that $\mathcal{C}=\operatorname{Ker}(\omega)$ will be called a Cartan form for $(\Sigma, \mathcal{C})$. It is not difficult to see (and details can be found, within a more general framework, in [55]) that such a Cartan form always exists, and is even unique up to the obvious notion of isomorphism.

Given a flat Cartan groupoid $(\Sigma, \mathcal{C})$ we consider the action (2.40) of $\Sigma$ on $T \mathbf{X}$, the induced actions on $\Lambda^{q} T^{*} \mathbf{X}$ and the corresponding operators

$$
\delta: C^{p}\left(\Sigma, \Lambda^{q} T^{*} \mathbf{X}\right) \rightarrow C^{p+1}\left(\Sigma, \Lambda^{q} T^{*} \mathbf{X}\right),
$$

and then the Ehresmann connections $\mathrm{C}^{(p)}$ on $t: \Sigma^{(p)} \rightarrow \mathbf{X}$ given by (2.41) and the induced operators

$$
d: \Omega_{\mathrm{hor}}^{q}\left(\Sigma^{(p)}\right) \rightarrow \Omega_{\mathrm{hor}}^{q+1}\left(\Sigma^{(p)}\right) .
$$

Definition 2.4.3. The Haefliger complex of a flat Cartan groupoid $(\Sigma, \mathcal{C}) \rightrightarrows \mathbf{X}$ is the bicomplex

$$
C_{\text {Haef }}^{p, q}(\Sigma, \mathcal{C}):=C^{p}\left(\Sigma, \Lambda^{q} T^{*} \mathbf{X}\right)=\Omega_{\text {hor }}^{q}\left(\Sigma^{(p)}\right)
$$

endowed with the differentials $\delta$ and $d$ described above. Its cohomology is denoted

$$
H_{\text {Haef }}^{*}(\Sigma, \mathcal{C})
$$

and is called the Haefliger cohomology of $(\Sigma, \mathcal{C})$.

Remark 2.4.4. Occasionally, when $\omega$ is a Cartan form for $(\Sigma, \mathcal{C})$, we write $H_{\text {Haef }}^{*}(\Sigma, \omega)$ in place of $H_{\text {Haef }}^{*}(\Sigma, \mathcal{C})$.

The fact that we deal, indeed, with a double complex, and also that the double complex carries the same structures as the Bott-Shulman one, follows from the proof of the Proposition below, which was essentially carried out in the previous section:

Proposition 2.4.5. For any flat Cartan groupoid $(\Sigma, \mathrm{C}) \rightrightarrows \mathbf{X}$, the Haefliger complex $C_{\text {Haef }}^{*, *}(\Sigma, \mathcal{C})$ becomes a cosimplicial DGA.

Example 2.4.6. Of course, the motivating example for us is the flat Cartan groupoid $\Sigma=J^{\infty} \Gamma$ associated to any Lie pseudogroup $\Gamma$; the distribution on it is the Cartan connection $\mathcal{C}^{\infty}$ described in Subsection 2.3.6. In this example, the Haefliger cohomology $H_{\text {Haef }}^{*}\left(J^{\infty} \Gamma, \mathrm{C}^{\infty}\right)$ from Definition 2.4.3 is realized as Haefliger's differentiable cohomology $H_{\text {diff }}^{*}(\Gamma)$ of $\Gamma$ from Definition 2.3.1.

Example 2.4.7. Lie groups $G$, interpreted as Lie groupoids over a point $G \rightrightarrows \star$, are automatically flat Cartan groupoids- the only possibility for $\mathcal{C}$ being, of course, $\mathrm{C}=0$. Note however that even this very simple example possesses an interesting Cartan 1-form (Remark 2.4.2): the Maurer-Cartan form $\omega_{M C} \in \Omega^{1}(G, \mathfrak{g})$. The resulting Haefliger cohomology is precisely the differentiable cohomology of $G$ :

$$
H_{\text {Haef }}^{*}(G, \mathcal{C} \equiv 0)=H_{\text {Haef }}^{*}\left(G, \omega_{M C}\right)=H_{\text {diff }}^{*}(G) .
$$


In this way the differentiable cohomology of Lie groups and the differentiable cohomology of Lie pseudogroups are placed in a common framework.

Example 2.4.8. Another interesting example is that of action groupoids $G \ltimes M \rightrightarrows$ $M$ associated to actions of Lie groups $G$ on manifolds $M$. Recall that $G \ltimes M=$ $G \times M$; a pair $(g, x)$ is viewed as an arrow from $x$ to $g x$ and the composition is given by

$$
(h, g x) \cdot(g, x)=(h g, x) .
$$

In this case the source map $s=\mathrm{pr}_{2}: G \times M \rightarrow M$ has an obvious flat Ehresman connection: $\mathrm{C}=\mathcal{C}_{\text {can }}$ consisting of the vectors tangent to the slices $G \times x$. It is not difficult to see that

$$
\left(G \ltimes M, \mathcal{C}_{\text {can }}\right)
$$

is, indeed, a flat Cartan groupoid. Note that the associated Haefliger complex is

$$
C^{p, q}=C^{p}\left(G, \Omega^{q}(M)\right),
$$

i.e. the bicomplex of differentiable cochains on $G$ with values in forms on $M$, where the two differentials are the Lie group differential (with coefficients) and the de Rham differential on $M$. Hence, in some sense, the cohomology is a combination of the de Rham cohomology of $M$ with the differentiable cohomology of $G$. In general, all these are related by a spectral sequence, and there are two extreme situations that can be made more explicit:

- when $M$ is contractible, one obtains the differentiable cohomology of $G$;

- when $G$ is compact (the typical case when differentiable cohomology vanishes in positive degrees) and connected, one obtains the de Rham cohomology of $M$.

Remark 2.4.9. Actually, in the finite dimensional case, under simple topological assumptions, a flat Cartan groupoid $\Sigma$ must be isomorphic to an action groupoid. This is the case e.g. if the base $\mathbf{X}$ is compact and 1-connected, and the s-fibers of $\Sigma$ are 1-connected. See e.g. [55], Section 2.6. This discussion will become more transparent in the next section, when moving to the infinitesimal picture.

\subsubsection{Almost geometric structures}

The notion of flat Cartan groupoid (and related notions) also shows up when dealing with the "almost" version of geometric structures. Here are two illustrations of "almost" structures that may be useful to have in mind:

- while symplectic structures on a manifold $M$ are non-degenerate 2-forms that are closed, almost symplectic structures are obtained when giving up the closedness condition;

- while complex structures on $M$ may be interpreted as morphisms $J: T M \rightarrow T M$ with $J^{2}=-$ Id and for which the Nijenhuis tensor $\mathcal{N}_{J}$ vanishes, almost complex structures give up on the last condition. 
While the notion of $\Gamma$-structure encompasses many of the standard geometric structures, the notion of "almost $\Gamma$-structure" appears as a very unifying general concept. When $\Gamma$ is transitive, one can express everything in terms of principal group bundles; under some assumptions on $\Gamma$, one ends up with the classical notions of $G$ structure (see, for example, [64]) and (almost) integrable $G$-structure. In general one needs to appeal to groupoids and one discovers the notion of flat Cartan groupoid $(\Sigma, \mathcal{C})$ from Definition 2.4.1 and $(\Sigma, \mathcal{C})$-structure that we recall below. Indeed, the notion of "flat Cartan" is implicit in [15], to which we refer for more details on "almost structures".

Remark 2.4.10. Following the terminology of [15], an "almost $\Gamma$-structure" can described as a flat $\left(J^{\infty} \Gamma, \mathrm{C}^{\infty}\right)$-structure (see Definition 2.4.11 below) if and only if it is formally integrable. Since the formally integrable case is the one we need to focus on, we require formal integrability in our Definition of almost $\Gamma$-structure; in fact, we define an almost $\Gamma$-structure as a flat $\left(J^{\infty} \Gamma, \mathrm{e}^{\infty}\right)$-structure, see Definition 2.4.16. See also Example 2.4.18.

In some sense, the next definition is a generalization of Definition 2.4.1 in which we replace Lie groupoids $\Sigma \rightrightarrows \mathbf{X}$ by principal $\Sigma$-bundles $\pi: P \rightarrow M$ (over some manifold $M$ ):

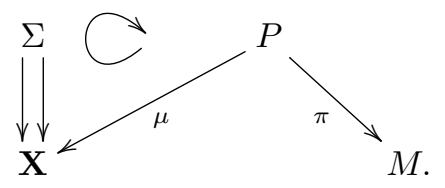

While before, in order to make sense of multiplicativity of $\mathcal{C} \subset T \Sigma$, we made use of the differential of the multiplication and other structure maps of $\Sigma$, now we use the differential of the action map

$$
a: \Sigma \ltimes P \rightarrow P, \quad(g, p) \mapsto g p \quad \text { (defined on }\{(g, p): s(g)=\mu(p)\})
$$

to obtain an action of $T \Sigma$ on $T P$. In particular, for $\mathcal{C} \subset T \Sigma, \mathcal{C}_{P} \subset T P$ we can consider

$$
\mathcal{C} \cdot \mathcal{C}_{P}:=\left\{(d a)(v, w): v \in \mathcal{C}, w \in \mathcal{C}_{P} \text { with } d s(v)=d \mu(w)\right\}
$$

Definition 2.4.11. Given a Cartan groupoid $(\Sigma, \mathcal{C}) \rightrightarrows \mathbf{X}$, a principal $(\Sigma, \mathcal{C})$ bundle on a manifold $M$ is any principal $\Sigma$-bundle $\pi: P \rightarrow M$ endowed with an Ehresmann connection $\mathcal{C}_{P} \subset T P$ with the property that $\mathcal{C} \cdot \mathcal{C}_{P} \subset \mathcal{C}_{P}$. If additionally $\operatorname{Ker}(d \mu) \cap \mathcal{C}_{P}$ is an involutive distribution, the principal $(\Sigma, \mathcal{C})$-bundle is called a $(\Sigma, \mathcal{C})$-structure. A principal $(\Sigma, \mathcal{C})$-bundle $/(\Sigma, \mathcal{C})$-structure is called flat if $\mathcal{C}_{P}$ is involutive.

Remark 2.4.12. As one sees from Example 2.4.14, principal $(\Sigma, \mathcal{C})$-bundles are more general objects than $(\Sigma, \mathcal{C})$-structures. That said, under flatness assumption, the two notions coincide - and the flat case is the one we focus on for the rest of this chapter, since it encompasses all the geometric examples that we have in mind (Example 2.4.18). 
Remark 2.4.13. Similar to Remark 2.4.2, while $\mathcal{C}$ arises as the kernel of a multiplicative 1-form $\omega \in \Omega^{1}\left(\Sigma, t^{*} E\right)$ with coefficients in a representation $E$ of $\mathcal{G}, \mathcal{C}_{P}$ arises as the kernel of a 1 -form

$$
\theta \in \Omega^{1}\left(P, \mu^{*} E\right)
$$

such that the action $a: \Sigma \ltimes P \rightarrow P$ satisfies the multiplicativity property:

$$
\left(a^{*} \theta\right)_{(g, p)}=\left(p r_{1}^{*} \omega\right)_{(g, p)}+\left(g \cdot p r_{2}^{*} \theta\right)_{(g, p)}
$$

for all $(g, p) \in \Sigma \ltimes_{\mu} P$.

For more details about multiplicative actions we refer to [15].

Example 2.4.14. When $\Sigma=G$ is a Lie group as in Example 2.4.7, then principal $(G, 0)$-bundles are the same thing as principal $G$-bundles $\pi: P \rightarrow M$ endowed with a principal connection (in the standard sense). For instance, using the point of view of 1 -forms as in the previous remark, since the 1-form corresponding to $G$ is the Maurer-Cartan form, for $P$ one is looking at $\omega_{P} \in \Omega^{1}(P, \mathfrak{g})$ satisfying

$$
a^{*}(\theta)-g \cdot p r_{2}^{*}(\theta)=p r_{1}^{*}\left(\omega_{M C}\right)
$$

Evaluating on pairs $(0, v)$, where $v$ is any vector tangent to $P$, one obtains

$$
L_{g}^{*}(\theta)=g \cdot(\theta)
$$

i.e. $G$-equivariance of $\theta$. Similarly, evaluating on pairs $(v, 0)$, with $v \in \mathfrak{g} \cong T_{e} G$, one finds

$$
\theta\left(\hat{v}_{p}\right)=v
$$

where $\hat{v}$ is the vertical vector at $p$ induced by $v$. As for $(G, 0)$-structures, they correspond precisely to flat connections; see Remark 2.4.12.

Example 2.4.15. Any Cartan groupoid $(\Sigma, \mathcal{C})$ acting on itself by left multiplication along the target map is a principal $(\Sigma, \mathcal{C})$-bundle and a $(\Sigma, \mathcal{C})$-structure, since $\operatorname{Ker}(d t) \cap \mathcal{C}=\operatorname{Ker}(d s) \cap \mathcal{C}$ by multiplicativity and $\operatorname{Ker}(d t) \cap \mathcal{C}=0$ because $\mathcal{C}$ is an Ehresmann connection. Moreover, $(\Sigma, \mathcal{C})$ is flat as a $(\Sigma, \mathcal{C})$-structure if and only if it is a flat Cartan groupoid. In fact, the multiplicativity of $\mathcal{C}$ is exactly the multiplicativity of the action with respect to $\mathcal{C}$ itself, and the action is clearly principal.

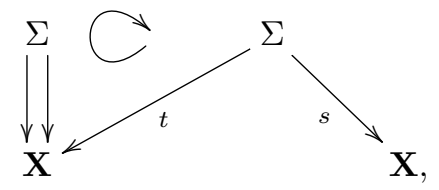

Definition 2.4.16. Given a pseudogroup $\Gamma$ on $\mathrm{X}$, a almost $\Gamma$-structure on a manifold $M$ is a flat $\left(J^{\infty} \Gamma, \mathrm{C}^{\infty}\right)$-structure $\left(P, \mathcal{C}_{P}\right) \rightarrow M$.

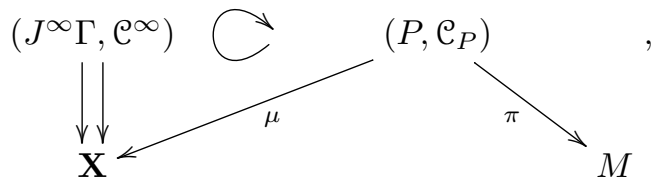


Remark 2.4.17. The flatness request in the definition above is motivated by the fact that the definiton of almost $\Gamma$-structure is meant to capture the idea of "infinite jet of a $\Gamma$-structure". In all the examples with geometric meaning, $P$ is a (a subset of) an infinite jet space and the corresponding $\mathcal{C}_{P}$ is obtained as a limit of distributions and automatically flat, as in the proof Proposition 2.3.12. See the example below.

Example 2.4.18. As indicated by the terminology and stressed in the previous remark, the motivating examples of almost $\Gamma$-structure arise when looking at the " $\infty$-order data" induced by $\Gamma$-structures. To explain this, let us first look at the case when $\operatorname{dim}(M)=\operatorname{dim}(\mathbf{X})$, when $\Gamma$-structures are encoded in $\Gamma$-atlases $\mathcal{A}$. If $\mathcal{A}$ is a maximal $\Gamma$-atlas then the infinite jets of elements of $\mathcal{A}$,

$$
J^{\infty} \mathcal{A}:=\left\{j_{x}^{\infty} f: f \in \mathcal{A}, x \in M\right\} \subset J^{\infty}(M, \mathbf{X}),
$$

carries precisely the structure from the previous definition:

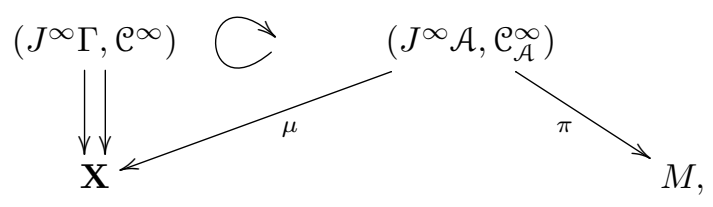

Here, $\mathcal{C}_{\mathcal{A}}^{\infty}$ is the canonical Cartan distribution on the infinite jet space $J^{\infty} \mathcal{A}$, see Example 2.A.18. $J^{\infty} \mathcal{A}$ fibers over $M$ via the map $\pi: j_{x}^{\infty} f \mapsto x$, over $\mathbf{X}$ via the map $\mu: j_{x}^{\infty} f \mapsto f(x)$, and it carries a left-action of $J^{\infty} \Gamma$ :

$$
j_{f(x)}^{\infty} \phi \cdot j_{x}^{\infty} f=j_{x}^{\infty}(\phi \circ f) \quad(\text { for } \phi \in \Gamma, f \in \mathcal{A}) .
$$

The smooth structure on $J^{\infty} \mathcal{A}$ is profinite dimensional, i.e. obtained as the "limit" of the smooth structures on the finite jet spaces $J^{k} \mathcal{A}, k \in \mathbb{N}$; see the appendix, and in particular Example 2.A.13). The Cartan distribution or form on $J^{\infty}(M, \mathbf{X})$ (see Examples 2.A.18 and 2.A.26 for the general constructions) induce the desired distribution (or form) on $J^{\infty} \mathcal{A}$, making it into a flat $\left(J^{\infty} \Gamma, \mathrm{C}^{\infty}\right)$-structure. Actually, note that the entire diagram and all the operations involved sit inside the principal bundle given by the action of the (larger) groupoid $\Pi^{\infty} \rightrightarrows \mathbf{X}$ of infinite jets of diffeomorphisms (see the appendix) on the space $\Pi^{\infty}(M, \mathbf{X})$ of jets of (local) diffeomorphisms between $M$ and $\mathbf{X}$ :

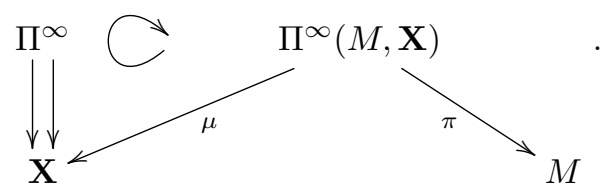

Let us now look at the general case (i.e. when $M$ and $\mathbf{X}$ may have different dimensions). For an intrinsic approach, we represent $\Gamma$-structures by principal $\mathcal{G}$-bundles $\mathcal{P}$ over $M(\mathcal{G}=\operatorname{Germ}(\Gamma))$. Then we define

$$
\mathcal{P}^{\infty} \subset J^{\infty}(M, \mathbf{X})
$$


consisting of jets of maps of type $\mu \circ \sigma$ where $\sigma$ is a section of $\pi: P \rightarrow M$ and one proceeds as above, obtaining all the desired structure from that of $J^{\infty}(M, \mathbf{X})$. One should be aware however that, if one embeds everything into a larger diagram (like the last one), one replaces $\Pi^{\infty}(M, \mathbf{X})$ by the similar space $\operatorname{Subm}^{\infty}(M, \mathbf{X})$ defined using submersions (to ensure that the resulting action is free) but, even so, the action is not transitive. Hence, in general, it is only after we restrict to the $\Gamma$-version of the diagram,

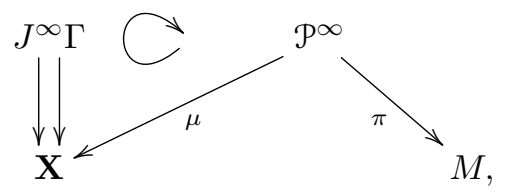

that we obtain a principal bundle (and a $\left(J^{\infty} \Gamma, \mathrm{C}^{\infty}\right)$-structure).

Note also that one could also proceed less intrinsically, using $\Gamma$-cocycles. The actual construction is, in principle, rather obvious: representing a $\Gamma$ structure on $M$ by a $\Gamma$-cocycle $c: M_{u} \rightarrow \operatorname{Germ}(\Gamma)$, just compose it with the obvious morphism $\operatorname{Germ}(\Gamma) \rightarrow J^{\infty} \Gamma$ to get a $J^{\infty} \Gamma$-cocycle, hence a principal $J^{\infty} \Gamma$-bundle. Proceeding this way however, one still has to exhibit the Cartan distribution and prove the independence of the choice of the cocycle.

Definition 2.4.19. An almost $\Gamma$-structure is said to be integrable if it is induced by a $\Gamma$-structure.

Remark 2.4.20. As we will discuss in Subsection 2.6.2 and prove in Proposition 2.6.3, the differentiable characteristic maps

$$
\kappa^{\mathcal{P}}: H_{\text {diff }}^{*}(\Gamma) \rightarrow H^{*}(M)
$$

associated to $\Gamma$-structures $P$ on a manifold $M$ (where $\Gamma$ is a Lie pseudogroup) only depend on the underlying almost $\Gamma$-structures. In other words, one has similar characteristic maps

$$
\kappa^{P}: H_{\text {diff }}^{*}(\Gamma) \rightarrow H^{*}(M)
$$

associated to any almost $\Gamma$-structure $P$ on $M$. And the construction actually makes sense more generally, for any flat Cartan groupoid $(\Sigma, \mathcal{C}) \rightrightarrows \mathbf{X}$ and flat $(\Sigma, \mathcal{C})$-structure $\left(P, \mathcal{C}_{P}\right) \rightarrow M$, with associated characteristic maps defined on the Haefliger cohomology:

$$
\kappa^{P}: H_{\text {Haef }}^{*}(\Sigma, \mathcal{C}) \rightarrow H^{*}(M)
$$

\subsection{The infinitesimal version of Haefliger cohomol- ogy}

\subsubsection{Lie algebroids}

In this section, we investigate the infinitesimal data associated with (flat) Cartan groupoids. This can be seen as part of the "Lie philosophy" of linearizing the global group-like structures. Of course, the starting point is the infinitesimal 
counterpart of Lie groupoids: Lie algebroids, i.e. vector bundles $A \rightarrow \mathbf{X}$ endowed with a Lie bracket $[\cdot, \cdot]$ on the space $\Gamma(A)$ of section of $A$ satisfying the Leibiniz type identity

$$
[\alpha, f \cdot \beta]=f \cdot[\alpha, \beta]+L_{\rho(\alpha)}(f) \beta \quad \text { for all } \alpha, \beta \in \Gamma(A), f \in C^{\infty}(\mathbf{X})
$$

for some vector bundle morphism $\rho: A \rightarrow T \mathbf{X}$ (necessarily unique, and called the anchor of $A$ ). The Lie algebroid $A=\operatorname{Lie}(\Sigma)$ of a Lie groupoid $\Sigma \rightrightarrows \mathbf{X}$ is defined as

$$
A:=\operatorname{Ker}(d s)_{\mathbf{X}}
$$

with the anchor

$$
\rho:=\left.d t\right|_{A}: A \rightarrow T \mathbf{X},
$$

and with Lie bracket obtained by identifying the sections of $A$ with right invariant vector fields on $\Sigma$. For the last notion we consider arrows $g: x \rightarrow y$, the corresponding right multiplication $R_{g}: s^{-1}(x) \rightarrow s^{-1}(y)$ and the induced tangent map

$$
d R_{g}: s^{-1}(x) \rightarrow s^{-1}(y) .
$$

With this, a vector field $X$ on $\Sigma$ is called right invariant if

- it is $s$-vertical;

- one has $X_{h g}=d R_{g}\left(X_{h}\right)$ for all the composable pairs $(h, g)$.

The key remark is that any section $\alpha$ of $A$ gives rise to the right invariant vector field $\alpha^{R}$ given by:

$$
\alpha_{g}^{R}=d R_{g}\left(\alpha_{t(g)}\right)
$$

and then $[\alpha, \beta]$ is defined by the condition that

$$
[\alpha, \beta]^{R}=\left[\alpha^{R}, \beta^{R}\right]
$$

Next, various notions and constructions valid for Lie groupoids $\Sigma \rightrightarrows \mathbf{X}$ have an infinitesimal counterpart, defined for Lie algebroids $A \rightarrow \mathbf{X}$. A basic example is the notion of representation which, on the infinitesimal side, takes us to representations of $A$, i.e. vector bundles $E \rightarrow \mathbf{X}$ endowed with a bilinear operator

$$
\nabla: \Gamma(A) \times \Gamma(E) \rightarrow \Gamma(E), \quad(\alpha, \sigma) \mapsto \nabla_{\alpha}(\sigma)
$$

which is $C^{\infty}(\mathbf{X})$-linear in the first argument, satisfies the Leibniz rule

$$
\nabla_{\alpha}(f \sigma)=f \nabla_{\alpha}(\sigma)+L_{\rho(\alpha)}(f) \sigma \quad \text { for all } \alpha \in \Gamma(A), \sigma \in \Gamma(E) \text { and } f \in C^{\infty}(\mathbf{X}),
$$

and is flat in the sense that

$$
\nabla_{[\alpha, \beta]}=\left[\nabla_{\alpha}, \nabla_{\beta}\right]
$$

Giving up the last condition one talks about $A$-connections on $E$ and then the difference of the two terms from the flatness condition defines a tensor

$$
k_{\nabla} \in \operatorname{Hom}\left(\Lambda^{2} A^{*}, \operatorname{End}(E)\right),
$$


called the curvature of $\nabla$.

If $A=\operatorname{Lie}(\Sigma)$ and $E$ is a representation of $\Sigma$, then $E$ can be turned into a representation of $A$ by defining

$$
\nabla_{\alpha}(\sigma)(x)=\left.\frac{d}{d \epsilon}\left(\phi_{\alpha}^{\epsilon}(x)^{-1} \cdot \sigma\left(\phi_{\rho(\alpha)}^{\epsilon}(x)\right)\right)\right|_{\epsilon=0}
$$

for all $\alpha \in \Gamma(A), \sigma \in \Gamma(E)$ and $x \in \mathbf{X}$; here, $\phi_{\alpha}^{\epsilon}$, called the flow of $\alpha$, is a one parameter family of bisections such that

$$
\left.\frac{d}{d \epsilon} \phi_{\alpha}^{\epsilon}(x)\right|_{\epsilon=0}=\alpha(x)
$$

for all $x \in \mathbf{X}$, while $\phi_{\rho(\alpha)}^{\epsilon}$ is the flow of the vector field $\rho(\alpha)$. A basic well-known result says that this construction $\operatorname{Rep}(\Sigma) \rightarrow \operatorname{Rep}(A)$ is injective if $\Sigma$ is $s$-connected (i.e. the $s$-fibers are connected) and 1-1 if the $s$-fibers are 1-connected (connected and simply connected).

Example 2.5.1. In Subsection 2.3.2, we have seen that, for any Cartan groupoid $(\Sigma, \mathcal{C}) \rightrightarrows \mathbf{X}, T \mathbf{X}$ is a representation of $\Sigma$. Next to this, there is another natural representation of $\Sigma$ : $A$ itself! Indeed,

$$
\Sigma_{s} \times_{\pi} A \rightarrow A, \quad\left(g, \alpha_{s(g)}\right) \rightarrow d R_{g^{-1}}\left(d m\left(h o r_{g}^{s}\left(\rho\left(\alpha_{s(g)}\right)\right), \alpha_{s(g)}\right)\right.
$$

defines a representation of $\Sigma$ on $A$.

This also allows us to expand Remark 2.4.2, where we pointed out that $\mathcal{C} \subset T \Sigma$ can be described as the kernel of a multiplicative 1-form $\omega \in \Omega^{1}\left(\Sigma, t^{*} E\right)$ with coefficients in some representation $E$ of $\Sigma$. Since $\omega$ is pointwise surjective and its kernel is complementary to $\operatorname{Ker}(d t)$, it follows that $E$ is actually isomorphic to $\operatorname{Ker}(d t) \mid \mathbf{x}$ hence, using the inversion, also to $\operatorname{Ker}(d s) \mid \mathbf{x}=A$. Using the multiplicativity condition, one can check that this is actually an isomorphism of representations, so that one may say (as in Corollary 2.5.10) that

$$
\omega \in \Omega^{1}\left(\Sigma, t^{*} A\right)
$$

Another illustration of the Lie philosophy is the infinitesimal counterpart of differentiable cohomology- the cohomology of Lie algebroid $A \rightarrow \mathbf{X}$ with coefficients in representations $E=(E, \nabla)$ : in degree $p$ one defines $C^{p}(A, E)=$ $\Gamma\left(\Lambda^{p} A^{*} \otimes E\right)$, and then the Koszul differential

$$
d: C^{p}(A, E) \rightarrow C^{p+1}(A, E)
$$

is defined by

$$
\begin{array}{r}
d \omega\left(\alpha_{1}, \ldots \alpha_{p+1}\right)=\sum_{i<j}(-1)^{i+j} \omega \\
\left(\left[\alpha_{i}, \alpha_{j}\right], \alpha_{1}, \ldots \hat{\alpha}_{i}, \ldots, \hat{\alpha}_{j}, \ldots, \alpha_{q+1}\right) \\
+\sum_{i}(-1)^{i} \nabla_{\alpha_{i}}\left(\alpha_{1}, \ldots, \hat{\alpha}_{i}, \ldots \alpha_{q+1}\right)
\end{array}
$$


The cohomology of the cochain complex $\left(C^{p}(A, E), d\right)$ denoted by $H^{*}(A, E)$ is the algebroid cohomology with coefficents in $E$. Lie algebra cohomology and de Rham cohomology are two examples, obtained respectively when $A=\mathfrak{g}$ (the Lie algebroid over a point) and $A=T \mathbf{X}$ (where the anchor is the identity and the bracket is the Lie bracket of vector fields). Finally, if $A=\operatorname{Lie}(\Sigma)$ and $E$ comes from a representation of $\Sigma$, there is a natural cohomology map (known as the van Est map)

$$
V E: H_{d}^{*}(\Sigma, E) \rightarrow H^{*}(A, E)
$$

Here, the left hand side is the differentiable cohomology of $\Sigma \rightrightarrows \mathbf{X}$ with coefficents in $E \rightarrow \mathbf{X}$, defined as for Lie groups: the cohomology of the subcomplex of groupoid cochains with coefficents in $E$ (that is, sections of $t^{*} E$, where $t: \Sigma^{(p)} \rightarrow \mathbf{X}$ takes the target of the first element) that are smooth. If $\Sigma$ has cohomologically $n$-connected $s$ fibers, the map is an isomorphism in degree lower than $n$ and injective in degree $n+1$. This was proven in [66] for Lie groups and generalized in [21] to Lie groupoids.

\subsubsection{Cartan algebroids and (infinitesimal) Haefliger cohomol- ogy}

We now proceed with the discussion of the infinitesimal counterpart of Cartan groupoids and their Haefliger cohomology. First of all, it may not come as a surprise that, instead of Ehresman connection on groupoids, one now considers linear (vector-bundle) connections on algebroids:

$$
\nabla: \mathfrak{X}(\mathbf{X}) \times \Gamma(A) \rightarrow \Gamma(A), \quad(X, \alpha) \mapsto \nabla_{X}(\alpha) .
$$

While an Ehresman connection gave rise to the "quasi-action" (2.40) of $\Sigma$ on $T M$, $\nabla$ will give rise to a "quasi-action" of $A$ on $T \mathbf{X}$, i.e. an $A$-connection. Namely:

$$
\begin{aligned}
\nabla^{T \mathbf{X}}: \Gamma(A) \times \Gamma(T \mathbf{X}) & \rightarrow \Gamma(T \mathbf{X}) \\
(\alpha, X) & \rightarrow[\rho(\alpha), X]+\rho\left(D_{X}(\alpha)\right) .
\end{aligned}
$$

Incidentally, let us point out that also the action of $\Sigma$ on $A$ from Example 2.5.1 has an infinitesimal counterpart, namely the $A$-connection on $A$

$$
\begin{aligned}
\nabla^{A}: \Gamma(A) \times \Gamma(A) & \rightarrow \Gamma(A) \\
\left(\alpha, \alpha^{\prime}\right) & \rightarrow D_{\rho\left(\alpha^{\prime}\right)} \alpha+\left[\alpha, \alpha^{\prime}\right] .
\end{aligned}
$$

What is a bit more subtle is to find what the infinitesimal analogue of the multiplicativity condition is. For the connection $\mathcal{C}$ on $\Sigma$, being closed under multiplication was encoded in the equation (2.43), which can be thought as the vanishing of some kind of 2-cochain on $\Sigma$ :

$$
\Sigma^{(2)} \ni\left(g_{1}, g_{2}\right) \mapsto d m\left(\operatorname{hor}_{g_{1}}(-), \operatorname{hor}_{g_{2}}\left(g_{1^{-}}^{*}\right)\right)-\operatorname{hor}_{g_{1} g_{2}}(-) \in \operatorname{Hom}\left(T_{t\left(g_{1}\right)} \mathbf{X}, A_{s\left(g_{2}\right)}\right) .
$$

One may think of this 2-cochain as some kind of "multiplicativity curvature" of $\mathcal{C}$. For linear connection the situation is a bit similar but we end up with 2-cochains on $A$. 
Definition 2.5.2. Given a Lie algebroid $A \rightarrow \mathbf{X}$ and a connection $\nabla$ on $A$, the basic curvature of $\nabla$ is defined as the tensor

$$
\begin{gathered}
k_{\nabla}^{\mathrm{bas}} \in C^{2}(A, \operatorname{Hom}(T \mathbf{X}, A)), \\
k_{\nabla}^{\mathrm{bas}}\left(\alpha, \alpha^{\prime}\right) X:=\nabla_{X}\left[\alpha, \alpha^{\prime}\right]-\left[\nabla_{X}(\alpha), \alpha^{\prime}\right]-\left[\alpha, \nabla_{X} \alpha^{\prime}\right]-\nabla_{\nabla_{\alpha^{\prime}}^{T M}(X)} \alpha+\nabla_{\nabla_{\alpha}^{T M}(X)} \alpha^{\prime},
\end{gathered}
$$

where $\nabla^{T \mathbf{X}}$ is given by (2.55). We say that $\nabla$ is infinitesimally multiplicative if $k_{\nabla}^{\text {bas }}=0$.

Note that the basic curvature is related to the $A$-curvature of $\nabla^{T \mathbf{X}}$ by:

$$
k_{\nabla^{T} \mathbf{x}}=\rho \circ k_{\nabla}^{\text {bas }}
$$

and, similarly, to the $A$-curvature of $\nabla^{A}$ by

$$
k_{\nabla^{A}}=k_{\nabla}^{\text {bas }} \circ \rho .
$$

Definition 2.5.3. A Cartan algebroid is an algebroid $A$ together with a connection $\nabla$ on it which is infinitesimally multiplicative (cf. the previous definition). It is called flat if $\nabla$ is a flat connection.

Remark 2.5.4. The name "Cartan algebroid" comes from [8], where the author considers infinitesimally multiplicative connections on Lie algebroids as a generalization of Cartan geometries [58].

We deduce the following.

Corollary 2.5.5. For any Cartan algebroid $(A, \nabla),(2.55)$ and (2.56) make $T \mathbf{X}$ and $A$ into representations of $A$. Moreover, $\rho: A \rightarrow T \mathbf{X}$ is A-equivariant.

Example 2.5.6. The infinitesimal analogue of Example 2.4.6 is the (pf) Lie algebroid $A^{\infty}$ of the (pf) Lie groupoid $J^{\infty} \Gamma$; we refer to our appendix, and in particular to Examples 2.A.37 and 2.A.38, for more details and terminology. A (strict) atlas for $A^{\infty}$ is given by the Lie algebroids $A^{k} \rightarrow \mathbf{X}$ associated to $J^{k} \Gamma$, $k \in \mathbb{N}$, together with the maps $A^{k} \rightarrow A^{k-1}$ obtained deriving the projections $J^{k} \Gamma \rightarrow J^{k-1} \Gamma$. Since we assume normality, $A^{\infty} \cong \lim _{\longleftarrow} A^{k}$. The flat infinitesimally multiplicative connection $\nabla$ on $A^{\infty}$ is the restriction of the Spencer operator (2.73) (see also [63] for a discussion of Spencer operators). We notice here that $A^{\infty}$ can be defined as the space of infinite jets of (local) $\Gamma$-vector fields (see Definition 2.2.8), that is

$$
A^{\infty}=\left\{j_{x}^{\infty} X: x \in \operatorname{Dom}(X), X \text { is a } \Gamma \text {-vector field }\right\} .
$$

One can give an explicit description of the action of $J^{\infty} \Gamma$ on $A^{\infty}$ from example 2.5.1 (which can be derived to provide a formula for the representation of $A^{\infty}$ on itself). Let $\alpha_{x} \in A_{x}^{\infty}$; we can write

$$
\alpha_{x}=j_{x}^{\infty}\left(\left.\frac{d}{d t}\right|_{t=0} \phi^{t}\right)
$$


with $\phi^{t} \in \Gamma$ and $\phi^{0}=i d$. If $j_{x}^{\infty} f \in J^{\infty} \Gamma$ then one has

$$
j_{x}^{\infty} f \cdot \alpha_{x}=j_{f(x)}^{\infty}\left(\left.\frac{d}{d t}\right|_{t=0} f \circ \phi^{t} \circ f^{-1}\right)
$$

Finally, let us notice that $A^{\infty}$ is also a bundle of Lie algebras; in fact, the fiber $A_{x}^{\infty}$ is precisely the space $\mathfrak{a}_{x}(\Gamma)$ of almost $\Gamma$-vector fields from Definition 2.2.8 whose bracket is given by the formula

$$
\left[j_{x}^{\infty} X, j_{x}^{\infty} Y\right]=j_{x}^{\infty}[X, Y]
$$

The existence of this fiberwise Lie bracket is not a coincidence; an analogous structure exists on any Cartan algebroid, as we explain in the next example.

Example 2.5.7. The infinitesimal analogue of Example 2.4.8 is that of action algebroids $\mathfrak{g} \ltimes \mathbf{X}$ associated to infinitesimal actions $\rho: \mathfrak{g} \rightarrow \mathfrak{X}(\mathbf{X})$ of Lie algebras. Recall that, as a vector bundle, $\mathfrak{g} \ltimes \mathbf{X}$ is the product bundle $\mathfrak{g} \times \mathbf{X}$, the anchor is given by the infinitesimal action and the bracket is the unique Lie bracket satisfying the Leibniz identity and the identity $\left[c_{v}, c_{w}\right]=c_{[v, w]}$, for all constant sections $c_{v}, c_{w}$ with $v, w \in \mathfrak{g}$. Endowed with the canonical flat connection, it becomes a flat Cartan algebroid.

As in Remark 2.4.9, these action algebroids exhaust most of the examples in the finite dimensional case. The key remark here is that, given a flat Cartan algebroid $(A, \nabla)$, each fiber of $A$ has a natural structure of Lie algebra. In fact, the bracket

$$
\{,\}_{\mathrm{pt}}: \begin{array}{cl}
\Gamma(A) \times \Gamma(A) & \rightarrow \Gamma(A) \\
(\alpha, \beta) & \rightarrow[\alpha, \beta]-\nabla_{\rho(\alpha)}(\beta)+\nabla_{\rho(\beta)}(\alpha)
\end{array}
$$

is $C^{\infty}(\mathbf{X})$-linear in its entries and satisfies Jacobi. Therefore, it makes $\left(A,\{,\}_{\mathrm{pt}}\right)$ into a bundle of Lie algebras (explaining also the notation "pt" to indicate "pointwise bracket").

Definition 2.5.8. For $x \in \mathbf{X}$, the extended isotropy Lie algebra of $(A, \nabla)$ at $x \in \mathbf{X}$, denoted $\mathfrak{a}_{x}(A)$, is $A_{x}$ endowed with the pointwise bracket (2.58) evaluated at $x$.

The terminology indicates the fact that $\mathfrak{a}_{x}(A)$ contains, as a Lie subalgebra the isotropy Lie algebra of $A$ at $x, \mathfrak{g}_{x}(A)$ (defined for any Lie algebroid as the kernel of the anchor equipped with the restriction of the bracket on $\Gamma(A))$.

Note also that $\nabla$ acts by derivations w.r.t. $\{,\}_{\mathrm{pt}}$. In particular, the parallel transport w.r.t. $\nabla$ induces Lie algebra isomorphisms between the extended isotropy Lie algebras at different points. Hence, when the base is simply connected, fixing a point $x_{0}$ one obtains an isomorphism of $A$ with $\mathfrak{a}_{x_{0}} \times \mathbf{X}$ and it is straightforward to check that, actually, this is an isomorphism of algebroids.

Finally, the precise relationship with Cartan groupoids:

Theorem 2.5.9. For any Cartan groupoid $(\Sigma, \mathcal{C}) \rightrightarrows \mathbf{X}$ its Lie algebroid $A$ becomes a Cartan algebroid with $\nabla^{\mathfrak{e}}: \mathfrak{X}(\mathbf{X}) \times \Gamma(A) \rightarrow \Gamma(A)$ given by:

$$
\nabla_{X}^{\mathfrak{e}}(\alpha)=\left.\left[\operatorname{hor}^{\mathfrak{e}}(X), \alpha^{R}\right]\right|_{\mathbf{x}} .
$$


Moreover, $\nabla^{\mathfrak{C}}$ is flat if $\mathcal{C}$ is so.

When $\Sigma$ has 1-connected s-fibers, the construction

$$
\mathcal{Q} \mapsto \nabla^{\mathcal{e}}
$$

is a 1-1 correspondence between Cartan connections on $\Sigma$ and infinitesimally multiplicative connections on $A$.

The theorem above is a particular case of Theorem 1 in [23] (see also [55]). Using Example 2.5.1 and the explicit formula for computing infinitesimal actions induced from groupoid actions (see above), one deduces the first two parts of the following. The last part will be discussed in greater generality in Proposition 2.5.18 below.

Corollary 2.5.10. If $\left(A, \nabla=\nabla^{\mathcal{C}}\right)$ is the Cartan algebroid of a Cartan groupoid $(\Sigma, \mathcal{C})$,

(1) $\nabla^{T \mathbf{X}}$ and $\nabla^{A}$ (i.e. (2.55) and (2.56)) are induced by corresponding representations of $\Sigma$ (cf. (2.40) and Example 2.5.1).

(2) The Cartan form of $(\Sigma, \mathrm{C})$ can be realized as a multiplicative form

$$
\omega \in \Omega^{1}\left(\mathcal{G}, t^{*} A\right) .
$$

(3) If $(\Sigma, \mathrm{C})$ is flat, using the de Rham differential on $\Sigma$ with coefficients in the (flat) pull-back bundle $t^{*} A$ (with connection $t^{*} \nabla$ ), and the pointwise bracket (2.58), $\omega$ satisfies the Maurer-Cartan equation

$$
d_{t^{*} A}(\omega)+\frac{1}{2}\{\omega, \omega\}_{p t}=0
$$

With the previous discussions in mind, the infinitesimal version of the Haefliger cohomology from Definition 2.4.3 is pretty clear. Given a flat Cartan algebroid $(A, \nabla)$ we first consider the infinitesimal action of $A$ on $T \mathbf{X}$ given by (2.55), the induced actions on $\Lambda^{q} T^{*} \mathbf{X}$ and the corresponding Koszul operators

$$
d_{A}: C^{p}\left(A, \Lambda^{q} T^{*} \mathbf{X}\right) \rightarrow C^{p+1}\left(A, \Lambda^{q} T^{*} \mathbf{X}\right) .
$$

Similarly, we consider the de Rham differential on $\mathbf{X}$ with coefficients in the flat bundles $\Lambda^{p} A^{*}$ (endowed with the flat connections induced by $\nabla$ )

$$
d: \Omega^{q}\left(\mathbf{X}, \Lambda^{p} A^{*}\right) \rightarrow \Omega^{q+1}\left(\mathbf{X}, \Lambda^{p} A^{*}\right) .
$$

Definition 2.5.11. The Haefliger complex of a flat Cartan algebroid $(A, \nabla) \rightarrow \mathbf{X}$ is the bicomplex

$$
C_{\text {Haef }}^{p, q}(A, \nabla):=C^{p}\left(A, \Lambda^{q} T^{*} \mathbf{X}\right)=\Omega^{q}\left(\mathbf{X}, \Lambda^{p} A^{*}\right)
$$

endowed with the differentials $d_{A}$ and $d$ described above. Its cohomology is denoted

$$
H_{\text {Haef }}^{*}(A, \nabla)
$$

and is called the (infinitesimal) Haefliger cohomology of $(A, \nabla)$.

We leave it to the reader to check that, indeed, this is a double complex (an argument arises as a "bonus" of the discussion from the next subsection). As we shall see (and as expected), this cohomology will be related to $H_{\text {Haef }}^{*}(\Sigma, \mathcal{C})$ via a van Est map, which is an isomorphism under the usual topological conditions on the $s$-fibers of $\Sigma$. 


\subsubsection{Matched pairs and the double}

Here we point out that flat Cartan algebroids and their Haefliger cohomology fits perfectly in the framework of matched pairs. Very briefly, a matched pair of Lie algebroids is a pair $\left(A_{1}, A_{2}\right)$ of Lie algebroids (over the same manifold $\mathbf{X}$ ) together with a Lie algebroid structure on their direct sum, $\left(D:=A_{1} \oplus A_{2},[-,-]_{D}, \rho_{D}\right)$, such that $A_{1}$ and $A_{2}$ are sub-algebroids. This condition forces the anchor to be

$$
\rho_{D}\left(\alpha_{1}, \alpha_{2}\right)=\rho_{A_{1}}\left(\alpha_{1}\right)+\rho_{A_{2}}\left(\alpha_{2}\right) .
$$

However, while the bracket $[-,-]_{D}$ is determined by the brackets of the two algebroids when applied on elements of type $\left(\alpha_{1}, 0\right)$ or of type $\left(0, \alpha_{2}\right)$, one still have some freedom when combining the two types of elements. Actually, writing the components of $\left[\left(\alpha_{1}, 0\right),\left(0, \alpha_{2}\right)\right]_{D}$ as

$$
\left[\left(\alpha_{1}, 0\right),\left(0, \alpha_{2}\right)\right]_{D}=\left(-\nabla_{\alpha_{2}}^{2} \alpha_{1}, \nabla_{\alpha_{1}}^{1} \alpha_{2}\right)
$$

one finds the explicit description of matched pairs:

Definition 2.5.12. A matched pair of Lie algebroids consists of two Lie algebroids $A_{1}$ and $A_{2}$, a flat $A_{1}$-connection $\nabla^{1}$ on $A_{2}$ and a flat $A_{2}$-connection $\nabla^{2}$ on $A_{1}$ such that the identities

i)

$$
\left[\rho_{1}(\alpha), \rho_{2}(\beta)\right]=-\rho_{1}\left(\nabla_{\beta}^{2} \alpha\right)+\rho_{2}\left(\nabla_{\alpha}^{1} \beta\right)
$$

ii)

$$
\nabla_{\alpha}^{1}\left[\beta^{1}, \beta^{2}\right]=\left[\nabla_{\alpha}^{1} \beta^{1}, \beta^{2}\right]+\left[\beta^{1}, \nabla_{\alpha}^{1} \beta^{2}\right]+\nabla_{\nabla_{\beta^{2}}^{2} \alpha}^{1} \beta^{1}-\nabla_{\nabla_{\beta^{1}}^{2} \alpha}^{1} \beta^{2}
$$

iii)

$$
\nabla_{\beta}^{2}\left[\alpha^{1}, \alpha^{2}\right]=\left[\nabla_{\beta}^{2} \alpha^{1}, \alpha^{2}\right]+\left[\alpha^{1}, \nabla_{\beta}^{2} \alpha^{2}\right]+\nabla_{\nabla_{\alpha^{2}} \beta^{2}}^{2} \alpha^{1}-\nabla_{\nabla_{\alpha^{1}} \beta^{2}}^{2} \alpha^{2}
$$

hold.

It is well-known $[46,50]$, and straightforward to check that, indeed, these conditions are equivalent to the fact that the resulting bracket and anchor on $A_{1} \oplus$ $A_{2}$ make it into a Lie algebroid. The key remark for us is:

Theorem 2.5.13. There is a one to one correspondence between flat Cartan algebroids $(A, \nabla)$ and matched pairs where one of the two algebroids is $T \mathbf{X}$.

Proof. First, let us start with a matched pair formed by $\left(A, \nabla^{T \mathbf{X}}\right)$ and $(T \mathbf{X}, \nabla)$. We consider the three conditions from Definition 2.5.12.

- The first condition is

$$
[\rho(\alpha), X]=\nabla_{\alpha}^{T \mathbf{X}} X-\rho\left(\nabla_{X} \alpha\right)
$$

for $\alpha \in \Gamma(A), X \in \mathfrak{X}(M)$. 
- The second condition is

$$
\nabla_{\alpha}^{T \mathbf{X}}[X, Y]=\left[\nabla_{\alpha}^{T \mathbf{X}} X, Y\right]+\left[X, \nabla_{\alpha}^{T \mathbf{X}} Y\right]+\nabla_{\nabla_{Y} \alpha}^{T \mathbf{X}} X-\nabla_{\nabla_{X} \alpha}^{T \mathbf{X}} Y
$$

for $\alpha \in \Gamma(A), X, Y \in \mathfrak{X}(\mathbf{X})$.

- Finally, the third condition is

$$
\nabla_{X}[\alpha, \beta]=\left[\nabla_{X} \alpha, \beta\right]+\left[\alpha, \nabla_{X} \beta\right]+\nabla_{\nabla_{\beta}^{T} \mathbf{x}_{X}} \alpha-\nabla_{\nabla_{\alpha}^{T} \mathbf{x}_{X}} \beta
$$

for $\alpha, \beta \in \Gamma(A), X \in \mathfrak{X}(\mathbf{X})$.

The fact that $\nabla$ is a representation of $T \mathbf{X}$ on $A$ tells us precisely that it is a flat connection. The third condition above is the compatibility condition 2.57 between $\nabla$ and $[$,$] . Hence, (A, \nabla)$ is a flat Cartan algebroid. As for the second condition, one sees that it follows automatically from the first one, the fact that $\nabla$ is a representation and the Jacobi identity. The first condition is simply the formula of the representation on $T \mathbf{X}$ associated with the flat Cartan algebroid $(A, \nabla)$.

If viceversa we start with a flat Cartan algebroid $(A, \nabla)$, the first and third conditions above are satisfied chosing $\nabla^{T \mathbf{X}}$ to be the representation of $A$ on $T \mathbf{X}$. As for the second one, it is identically satisfied by the same reasons as before ( $\nabla$ is a flat connection, the definition of $\nabla^{T \mathbf{X}}$ and the Jacobi identity).

This discussion reveals the presence of a larger algebroid associated to flat Cartan algebroids:

Definition 2.5.14. The double of a flat Cartan algebroid $(A, \nabla)$, denoted $\mathcal{D}(A, \nabla)$, is the Lie algebroid corresponding to the matched pair $(A, T \mathbf{X})$. Explicitly,

$$
\mathcal{D}(A, \nabla)=A \oplus T \mathbf{X},
$$

with anchor

$$
\rho_{\mathcal{D}}(\alpha, X)=\rho_{A}(\alpha)+X
$$

and with bracket

$$
[(\alpha, X),(\beta, Y)]_{\mathcal{D}}=\left([\alpha, \beta]+\nabla_{X} \beta-\nabla_{Y} \alpha,[X, Y]+\nabla_{\alpha}^{T \mathbf{X}_{Y}}-\nabla_{\beta}^{T \mathbf{X}} X\right)
$$

With this, one obtains the following re-interpretation of the Haefliger cohomology:

Proposition 2.5.15. For any flat Cartan algebroid $(A, \nabla)$, its Haefliger cohomology is equal to the usual cohomology of the double algebroid $\mathcal{D}(A, \nabla)$ :

$$
H_{\text {Haef }}^{*}(A, \nabla)=H^{*}(\mathcal{D}(A, \nabla)) .
$$

This holds true in full generality for matched pairs $\left(A_{1}, A_{2}\right)$; it is obtained by decomposing the algebroid complex of $A=A_{1} \oplus A_{2}$

$$
\Omega^{k}(A)=\bigoplus \Gamma\left(\Lambda^{p} A_{1}^{*} \otimes \Lambda^{q} A_{2}^{*}\right)
$$

Actually, one has the following, which also shows that flat Cartan algebroids are precisely what is needed in order to define the Haefliger double complex (compare with the similar discussion for groupoids in Section 2.3!). 
Theorem 2.5.16. Consider the quadruple $\left(A_{1}, \nabla^{1}, A_{2}, \nabla^{2}\right)$, where $\nabla^{1}$ is a representation of $A_{1}$ on $A_{2}$ and similarly for $\nabla^{2}$. Consider also the diagram

$$
\begin{array}{cc}
\Gamma\left(\Lambda^{k}\left(A_{1}\right)^{*} \otimes \Lambda^{q+1}\left(A_{2}\right)^{*}\right) \stackrel{d_{A_{1}}}{\longrightarrow} \Gamma\left(\Lambda^{k+1}\left(A_{1}\right)^{*} \otimes \Lambda^{q+1}\left(A_{2}\right)^{*}\right) \\
d_{A_{2}} \uparrow \\
\Gamma\left(\Lambda^{k}\left(A_{1}\right)^{*} \otimes \Lambda^{q}\left(A_{2}\right)^{*}\right) \stackrel{d_{A_{1}}}{\longrightarrow} \Gamma\left(\Lambda^{k+1}\left(A_{1}\right)^{*} \otimes \Lambda^{q}\left(A_{2}\right)^{*}\right)
\end{array}
$$

where $d_{A_{1}}$ and $d_{A_{2}}$ are the algebroid differentials associated to the induced representations on the exterior bundles.

The quadruple $\left(A_{1}, \nabla^{1}, A_{2}, \nabla^{2}\right)$ is a matched pair if and only if the diagram above is commutative for all $k, q \in \mathbb{N}$. Moreover, the resulting double complex is isomoprhic to the complex of algebroid cochains of $A_{1} \oplus A_{2}$.

For a proof, see [43].

Remark 2.5.17. The double algebroid $\mathcal{D}(A, \nabla)$ associated to a flat Cartan algebroid clarifies also the extended isotropy Lie algebras from Definition 2.5.8: it is isomorphic to the isotropy Lie algebra (i.e. the kernel of the anchor) of $\mathcal{D}(A, \nabla)$; the isomorphism is simply $\alpha_{x} \rightarrow\left(\alpha_{x},-\rho\left(\alpha_{x}\right)\right)$. The remark from Example 2.5.7 that all the fiberwise Lie algebras are isomorphic can also be seen as a particular case of the fact that the isotropy Lie algebras of any transitive Lie algebroid over a connected base are isomorphic to each other.

\subsubsection{Flat $(\Sigma, \mathcal{C})$-structures}

In Subsection 2.4.2 we discussed the notions of principal $(\Sigma, \mathcal{C})$-bundle and $(\Sigma, \mathcal{C})$ structure, where $(\Sigma, \mathcal{C}) \rightarrow \mathbf{X}$ is a flat Cartan groupoid, as a general framework for "almost geometric structures". We also pointed out that flat principal $(\Sigma, \mathcal{C})$ bundles are actually the same as flat $(\Sigma, \mathcal{C})$-structures, and that examples coming from geometry are always flat. As we have seen, $(\Sigma, \mathcal{C})$ comes with its associated Cartan algebroid $(A, \nabla)$. Here we reformulate the definition of flat principal $(\Sigma, \mathcal{C})$-bundle $/(\Sigma, \mathcal{C})$-structure using only the infinitesimal data $(A, \nabla)$.

Proposition 2.5.18. Let $(\Sigma, \mathrm{C}) \rightarrow \mathbf{X}$ be a Cartan groupoid, with associated Cartan algebroid $(A, \nabla)$. Assume also that $\pi: P \rightarrow M$ is a principal $\Sigma$-bundle,

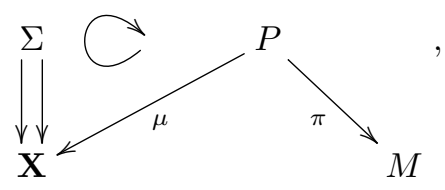

and denote by $a: \mu^{*} A \rightarrow T P$ the induced infinitesimal action. Then any sub-bundle $\mathcal{C}_{P} \subset T P$ making $P$ into a flat $(\Sigma, \mathcal{C})$-structure arises as the kernel of a 1-form

$$
\theta \in \Omega^{1}\left(P, \mu^{*} A\right)
$$

with the following two properties:

$$
\theta(a(\alpha))=\alpha \quad \text { for all } \alpha \in \Gamma(A),
$$




$$
d_{\mu^{*} A}(\theta)+\frac{1}{2}\{\theta, \theta\}_{p t}=0
$$

where, as in Corollary 2.5.10, $\{\cdot, \cdot\}_{p t}$ is the pointwise bracket (2.58) and $d_{\mu^{*} A}$ is de Rham differential on $P$ with coefficients in the flat pull-back bundle $\mu^{*} A$.

Furthermore, if the s-fibers of $\Sigma$ are connected, this provides a 1-1 correspondence between such sub-bundles and 1-forms with these two properties.

Proof. We denote $\widetilde{A}:=\mu^{*} A$, by $\widetilde{\rho}$ its anchor, by $\widetilde{\nabla}$ the pull-back of the connection $\nabla$ (making $\mu^{*} A$ itself a flat Cartan algebroid).

First of all we will need an infinitesimal characterization of multiplicativity - characterization that was worked out in greater generality in Proposition 5.3.4 from [15]. Here is the part that is of interest for us. The idea is that the multiplicativity property (2.51) of $\theta$, rewritten as

$$
\left(a^{*} \theta\right)_{(g, p)}-\left(g \cdot p r_{2}^{*} \theta\right)_{(g, p)}=\left(p r_{1}^{*} \theta\right)_{(g, p)},
$$

is an equality of multiplicative forms on the action groupoid $\Sigma \ltimes P$. Hence one can just look at the equality of the corresponding infinitesimal counterparts,

$$
\nabla^{\text {left }}, \nabla^{\text {right }}: \mathfrak{X}(P) \times \Gamma(\tilde{A}) \rightarrow \Gamma(\tilde{A}),
$$

for the left and the right hand side of (2.60), respectively. Computing the two operators we find

$$
\begin{gathered}
\nabla_{X}^{\text {left }}(\beta)=\widetilde{\nabla}_{\widetilde{\rho}(\theta(X))}(\beta)+[\beta, \theta(X)]_{\widetilde{A}}-\theta([\widetilde{\rho}(\beta), X]), \\
\nabla_{X}^{\text {right }}(\beta)=\widetilde{\nabla}_{X}(\beta)
\end{gathered}
$$

for all $X \in \mathfrak{X}(P), \beta \in \Gamma(\widetilde{A})$ (see [15] for more details). While the second satisfies Leibniz, the first one does if and only if (2.59) is satisfied.

Next, we concentrate on the Maurer-Cartan expression

$$
M C:=d_{\mu^{*} A}(\theta)+\frac{1}{2}\{\theta, \theta\}_{\mathrm{pt}} \in \Omega^{2}(P, \widetilde{A}),
$$

and we compute it on several types of tangent vectors:

- for $X, Y$ in the image of $\widetilde{\rho}$, we find immediately that $M C(X, Y)=0$;

- if only $Y$ is in the image of $\widetilde{\rho}$ and $X \in \operatorname{Ker}(\theta)$, writing $Y=\widetilde{\beta}$, a careful but simple computation gives

$$
M C(X, \widetilde{\beta})=\nabla_{X}^{\text {left }}(\beta)-\nabla_{X}^{\text {right }}(\beta)
$$

- $X, Y \in \operatorname{Ker}(\theta)$ we find right away $M C(X, Y)=-\theta([X, Y])$.

Hence we see that $M C=0$ encodes both infinitesimal multiplicativity as well as the involutivity of $\mathcal{C}_{P}=\operatorname{Ker}(\theta)$. 
Remark 2.5.19. As we see in the proof, $M C=0$ encodes more than just the infinitesimal characterization of multiplicativity of $\theta$. From that point of view, the condition $\nabla^{\text {left }}=\nabla^{\text {right }}$ is a better characterization. However, the minimal way to encode multiplicativity infinitesimally is to remove all the conditions that hold anyway. We see that what is left is $M C(X, \widetilde{\beta})=0$ whenever $X$ is killed by $\theta$, i.e.:

$$
\widetilde{\nabla}_{X}(\beta)=\theta([X, \widetilde{\rho}(\beta)]) \quad \text { for all } X \in \Gamma\left(\mathcal{C}_{P}\right), \beta \in \Gamma(\widetilde{A}) .
$$

Also this condition alone implies (2.59) and then the decomposition

$$
T P=\operatorname{Im}(a) \oplus \mathcal{C}_{P}
$$

(where $a$ is the infinitesimal action). On the other hand one can further rewrite the last equation on $\theta$ without any reference to $\theta$, by applying it to horizontal lifts and to $\beta=\mu^{*} \alpha$. We find that the infinitesimal counterpart of the multiplicativity of $\mathcal{C}_{P}$ is

$$
[\operatorname{hor}(V), a(\alpha)]=a\left(\nabla_{V}(\alpha)\right) \oplus \operatorname{hor}([V, \rho(\alpha)]) \quad \text { for all } V \in \mathfrak{X}(\mathbf{X}), \alpha \in \Gamma(A) .
$$

Remark 2.5.20. The form $\theta$ and its properties can be packed together sightly differently, using the corresponding double $\mathcal{D}=\mathcal{D}(A, \nabla)$. First of all, we re-interpret $\theta$ as a $\mathcal{D}$-valued form, where, in principle, we use $d \mu$ for the $T \mathbf{X}$-component. However, to obtain compatibility with anchors, we arrange the terms to be:

$$
\tilde{\theta}:=(d \mu-\rho \circ \theta, \theta): T P \rightarrow \mathcal{D} .
$$

Then the Maurer-Cartan equation for $\theta$ translates into a similar Maurer-Cartan equation for $\tilde{\theta}$ which, in turn, just encodes the fact that $\tilde{\theta}$ is a morphism of Lie algebroids. See also the proof of Proposition 2.6.5.

Remark 2.5.21. The previous remark is undoubtedly related to the discussion in [69] concerning Cartan's seminal work on Lie pseudogroups and more precisely his "three fundamental theorems". A precise exposition of the material is out of the scope of this chapter, but the point that we want to stress is that the Maurer-Cartan equation for $(\Sigma, \mathcal{C})$-structures mentioned in the remark above proves, together with the flat Cartan algebroid structure on $A$, that a flat Cartan groupoid $(\Sigma, \mathcal{C})$ is a very special case of Cartan's realization in the sense of [69], Definition 5.2.11. One considers the automorphism of vector bundles

$$
\Phi_{\mathcal{D}}: \mathcal{D} \rightarrow \mathcal{D}, \quad(X, \alpha) \rightarrow(X+\rho(\alpha), \alpha)
$$

There is a unique bracket $[[]$,$] such that the above map is an isomorphism of Lie$ algebroids

$$
\Phi_{\mathcal{D}}:(D,[,]) \rightarrow(\mathcal{D},[[,]]) .
$$

We can look at the flat $(\Sigma, \mathcal{C})$-structure given by the action of $(\Sigma, \mathcal{C})$ on itself and consider the form $\tilde{\omega}=(d t-\rho \circ \omega, \omega)$ as in the previous remark, where now $\omega$ is the form dual to $\mathcal{C}$. It follows from [69], Proposition 5.3.7, that the form $\Omega=\Phi_{\mathcal{D}} \circ \tilde{\omega}=(d t, \omega)$ satisfies Cartan's structure equation

$$
d \Omega+\frac{1}{2}[[\Omega, \Omega]]=0
$$


which is manifestly of Maurer-Cartan type. Getting rid of $\Phi_{\mathcal{D}}$, one finds the Maurer-Cartan equation for $\tilde{\omega}$ mentioned in the previous remark. On the other hand, when $\left(P, \mathcal{C}_{P}\right)$ is a flat $(\Sigma, \mathcal{C})$-structure and $\theta$ is the form dual to $\mathcal{C}_{P}$, the Maurer-Cartan equation for $\tilde{\theta}=(d t-\rho \circ \theta, \theta)$ can be read, composing with $\Phi_{\mathcal{D}}$, as a Cartan's structure equation in the sense of [69], Definition 5.2.11.

\subsection{Van Est maps}

\subsubsection{A very general setting}

In this subsection, given a proper action (not necessarily principal) of a flat Cartan groupoid $(\Sigma, \mathcal{C}) \rightrightarrows B$ on a space $P$ (not necessarily carrying extra-structure)

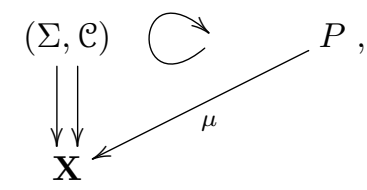

we will construct a natural map

$$
V E_{P}: H_{\text {Haef }}^{*}(\Sigma, \mathcal{C}) \rightarrow H_{\Sigma}^{*}(P)
$$

from the Haefliger cohomology of the Cartan groupoid to the cohomology corresponding to the subcomplex

$$
\Omega^{*}(P)^{\Sigma} \subset \Omega^{*}(P)
$$

of de Rham complex of $P$ consisting of $\Sigma$-invariant differential forms on $P$.

Here we are using that $\Sigma$ acts not only on $T \mathbf{X}$ but also on the tangent space $T P$ of any $\Sigma$-space $P$. Indeed, the action groupoid is itself a flat Cartan groupoid with the Cartan distribution pulled back from $\Sigma$ :

$$
\Sigma_{P}:=\Sigma \ltimes P \rightrightarrows P, \quad \mathcal{C}_{P}:=\mathrm{pr}_{1}^{-1} \mathcal{C}
$$

and we are just considering the induced action on the tangent space of the base. Working out the action one finds the explicit description: the action of $g: x \rightarrow y$ on $v_{p} \in T_{p} P\left(p \in \mu^{-1}(x)\right)$ is

$$
g \cdot v_{p}:=(d a)_{g, p}\left(\operatorname{hor}_{g}\left(d \mu\left(v_{p}\right)\right), v_{p}\right) \in T_{g p} P .
$$

The first projection from the action groupoid gives rise to a pull-back map between the corresponding Haefliger cohomologies (defined already at the level of complexes)

$$
\operatorname{pr}_{1}^{*}: H_{\text {Haef }}^{*}(\Sigma, \mathcal{C}) \rightarrow H_{\text {Haef }}^{*}\left(\Sigma_{P}, \mathcal{C}_{P}\right)
$$

Lemma 2.6.1. If the action of $\Sigma$ on $P$ is proper then the inclusion (2.62) of $\Omega^{*}(P)^{\Sigma}$ in the $p=0$ row of the Haefliger complex of $\left(\Sigma_{P}, \mathcal{C}_{P}\right)$ induces an isomorphism:

$$
H_{\Sigma}^{*}(P) \cong H_{\text {Haef }}^{*}\left(\Sigma_{P}, \mathcal{C}_{P}\right)
$$


Proof. Replacing $\Sigma_{P}$ by $\Sigma$, we may assume that $\Sigma \rightrightarrows \mathbf{X}$ is a proper groupoid and we are comparing $C_{\text {Haef }}^{*}(\Sigma)$ with $\Omega^{*}(X)^{\Sigma}$. The main point is that each row $C_{\text {Haef }}^{*, q}(\Sigma)$ computes the differentiable cohomology of the proper groupoid $\Sigma$ with various coefficients. But that is know to vanish in all positive degree, and give the $\Sigma$-invariant part in degree zero, if $\Sigma$ is proper (see Lemma 2.A.35 in the Appendix). Hence, by a standard double complex argument, the conclusion follows.

Combining the two maps above, i.e. $\mathrm{pr}_{1}^{*}$ and the isomorphism from the Lemma, we obtain the desired map (2.61).

Proposition 2.6.2. If the action of $\Sigma$ on $P$ is proper, $\mu$ is a submersion and the $\mu$ fibers of $P$ are cohomologically l-connected then $V E_{P}: H_{\text {Haef }}^{*}(\Sigma, \mathcal{C}) \rightarrow H_{\Sigma}^{*}(P)$ is an isomorphism up to degree $l$ and injective in degree $l+1$.

Proof. Let us call the property from the statement (iso $l$ ). We have to check (iso $l$ ) for the map induced in cohomology by map of double complexes

$$
\operatorname{pr}_{1}^{*}: C_{\text {Haef }}^{*, *}(\Sigma, \mathcal{C}) \rightarrow C_{\text {Haef }}^{*, *}\left(\Sigma_{P}, \mathcal{C}_{P}\right)
$$

If $A \hookrightarrow B$ is any inclusion of double complexes and $C$ denotes the quotient, from the long exact sequence in cohomology we see that $\left(\mathrm{iso}_{l}\right)$ for the map in cohomology is equivalent to $C$ having trivial cohomology up to degree $k$. Furthermore if such a property holds for all columns, then it also holds for the total complex.

By this double complex argument, it suffices to show that, in each column $p$, our map

$$
\operatorname{pr}_{1}^{*}: \Gamma\left(\Sigma^{(p)}, \Lambda^{*} \mathcal{C}_{p}^{*}\right) \rightarrow \Gamma\left(\Sigma_{P}^{(p)}, \Lambda^{*} \widetilde{\mathfrak{C}}_{P}^{*}\right)
$$

satisfies (iso $)_{l}$ ) in cohomology. Fixing $p$ and denoting

$$
\mathbb{P}:=\Sigma_{P}^{(p)}, \quad \mathbb{M}=\Sigma^{(p)}, \quad \pi=\operatorname{pr}_{1}
$$

we find ourselves precisely in the setting of Proposition 2.A.36 from the Appendix.

\subsubsection{The case of flat $(\Sigma, \mathcal{C})$-structures (and almost structures)}

The differentiable cohomology of pseudogroups serves as the domain of differentiable characteristic maps (2.34)

$$
\kappa_{\text {diff }}^{\mathcal{P}}: H_{\text {diff }}^{*}(\Gamma) \rightarrow H^{*}(M)
$$

associated to $\Gamma$-structures $\mathcal{P}$ - see Definition 2.3.1. Here we point out that these maps do not depend on $\mathcal{P}$ but just on the induced almost $\Gamma$-structure. Actually, these maps are defined in the general setting of flat Cartan groupoids $(\Sigma, \mathcal{C}) \rightrightarrows \mathbf{X}$ and flat $(\Sigma, \mathcal{C})$-structures $\left(P, \mathcal{C}_{P}\right) \rightarrow M$. This exploits the general van Est map (2.61) in the more special case when $P$ is actually a flat $(\Sigma, \mathcal{C})$-structure. In this case we have available the horizontal lift hor with respect to the Ehresmann connection $\mathcal{C}_{P}$, which induces a map

$$
\text { hor* }: \Omega^{*}(P)^{\Sigma} \rightarrow \Omega^{*}(M),
$$




$$
\operatorname{hor}^{*}(\alpha)\left(v_{x}^{1}, \ldots, v_{x}^{q}\right):=\alpha\left(\operatorname{hor}_{p}\left(v_{x}^{1}\right), \ldots, \operatorname{hor}_{p}\left(v_{x}^{q}\right)\right),
$$

where $p \in P$ is a /any element in the fiber above $x$; thanks to the invariance of $\alpha$, the definition is independent of the choice of $p$. Composing the induced map in cohomology with the general van Est map (2.61) gives rise to a map

$$
\kappa_{\text {Haef }}^{P}: H_{\text {Haef }}^{*}(\Sigma, \mathcal{C}) \rightarrow H^{*}(M)
$$

called the Haefliger characteristic map associated to the $(\Sigma, \mathcal{C})$-structure $\left(P, \mathcal{C}_{P}\right)$. As promised, we have:

Proposition 2.6.3. For Cartan groupoids $(\Sigma, \mathcal{C})$ and $(\Sigma, \mathcal{C})$-structures induced by pseudogroups $\Gamma$ and $\Gamma$-structures $\mathcal{P}$

$$
\Sigma=J^{\infty} \Gamma, \quad P=J^{\infty} \mathcal{P},
$$

the Haefliger characteristic map (2.63) becomes the differentiable characteristic map (2.34) of the $\Gamma$-structure.

Proof. We start by recalling that, by definition, $\kappa_{\text {diff }}^{\mathcal{P}}$ is constructed by composing the map

$$
j^{*}: H_{\mathrm{diff}}^{*}(\Gamma) \rightarrow H_{\mathrm{dR}}^{*}(\mathcal{G}) \quad(\mathcal{G}=\operatorname{Germ}(\Gamma))
$$

with the map (2.24)

$$
\gamma^{*}: H_{\mathrm{dR}}^{*}(\mathcal{G}) \rightarrow H^{*}(M) .
$$

We have the map of double complexes (we stick to the notation $\mathcal{G}=\operatorname{Germ}(\Gamma)$ )

$$
\operatorname{pr}_{1}^{*}: C^{p}\left(\mathcal{G}, \Omega_{\mathbf{X}}^{q}\right) \rightarrow C^{p}\left(\mathcal{G} \ltimes \mathcal{P}, \Omega_{\mathcal{P}}^{q}\right)
$$

Using properness of the principal action, the cohomology of the right hand side is seen to be isomorphic to $H_{\mathcal{G}}^{*}(\mathcal{P})$ (the cohomology of $\mathcal{G}$-invariant forms on $\mathcal{P}$; this goes as in Lemma 2.6.1). The projection $\pi: \mathcal{P} \rightarrow M$ is étale, because so is $\mathcal{G}$ and $\mathcal{P}$ is a principal $\mathcal{G}$-bundle, hence we see that $H_{\mathcal{G}}^{*}(\mathcal{P}) \cong H^{*}(\mathbf{X})$. We get a map

$$
\hat{\gamma}^{*}: H_{\mathrm{dR}}^{*}(\mathcal{G}) \rightarrow H^{*}(\mathbf{X})
$$

fitting in the diagram

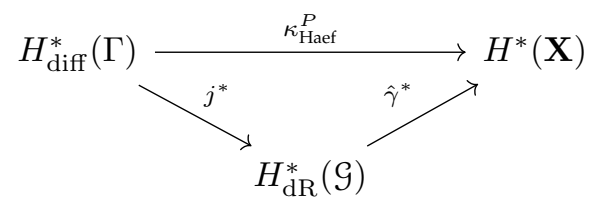

which is commutative by construction. Of course, $\hat{\gamma}^{*}$ has to be compared with $\gamma^{*}$ and (2.65) has to be compared with

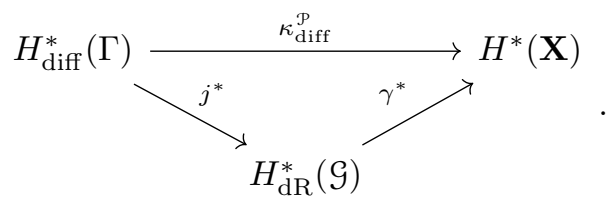


Hence, it is clear that the equality $\gamma^{*}=\hat{\gamma}^{*}$ implies our claim. To prove it, let us fix a cover $\mathcal{U}$ of $M$. We consider the map

$$
\bar{\gamma}^{*}: C^{p}\left(\mathcal{G} \ltimes \mathcal{P}, \Omega_{\mathcal{P}}^{q}\right) \rightarrow C^{p}\left(M_{\mathcal{U}}, \Omega_{M}^{q}\right), \quad c \rightarrow \bar{\gamma}^{*}(c)
$$

where

$$
\bar{\gamma}^{*}(c)_{i_{0}, \ldots, i_{p}}(x)=\sigma_{i_{p}}^{*} c\left(\left(\gamma_{i_{p-1} i_{p}}(x),\left[\sigma_{i_{p-1}}\right]_{x}\right), \ldots,\left(\gamma_{i_{0} i_{1}}(x),\left[\sigma_{i_{0}}\right]_{x}\right)\right) .
$$

Here the $\sigma_{i}$ 's are the sections of the principal $\mathcal{G}$-bundle $\mathcal{P}$ which correspond to the chosen $\mathcal{G}$-cocycle on $M$, and the $\left[\sigma_{i}\right]_{x}$ 's are the corresponding germs at $x$ (which can be identified with the points $\sigma_{i}(x) \in \mathcal{P}$ since $\pi$ is étale). More explicitely, $\sigma_{i}: U_{i} \rightarrow \mathcal{P}$ is the section of $\mathcal{P}$ which is the inverse of $\pi$ around $\left[\gamma_{i i}\right]_{x} \in \mathcal{P}$, the germ of $\gamma_{i i}$ at $x$ (in particular, $\left[\gamma_{i i}\right]_{x}=\sigma_{i}(x)$ ). This is a map of double complexes. The claim follows thanks to the commutative diagram below (we leave to the reader to check that $\bar{\gamma}$ induces in cohomology the isomorphism $H_{\mathcal{G}}^{*}(\mathcal{P}) \cong H^{*}(\mathbf{X})$ used to define $\hat{\gamma}^{*}$ ):

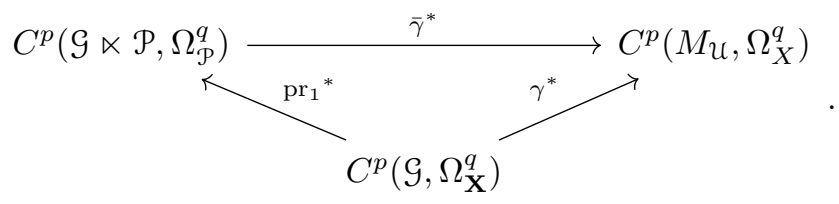

Remark 2.6.4. This new insight may make the characteristic maps useful also in detecting almost structures that are not integrable. The plan would be to use the commutative diagram

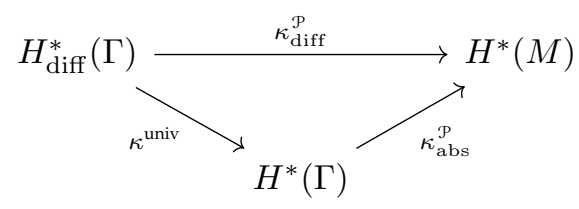

which is simply (2.31) for a general pseudogroup $\Gamma$ (notice that we see $\kappa_{\text {abs }}^{\mathcal{P}}$ as defined on $H^{*}(\Gamma)$ using Theorem 2.2.15). Step 1 is to detect classes

$$
u \in H_{\text {diff }}^{*}(\Gamma)
$$

that are killed when viewed as cocycles in $H^{*}(B \Gamma)$, i.e. are in the kernel of the universal map

$$
\kappa^{\text {univ }}: H_{\text {diff }}^{*}(\Gamma) \rightarrow H^{*}(\Gamma) \cong H^{*}(B \Gamma)
$$

Then step 2 is to exhibit almost $\Gamma$-structures $P$ whose corresponding $u$ characteristic class

$$
u(P)=\kappa_{\text {diff }}^{\mathcal{P}}(u) \in H^{*}(M)
$$

does not vanish. From the previous commutative diagram one concludes immediately that $P$ cannot arise from an actual $\Gamma$-structure $\mathcal{P}$. 
We conjecture that this plan can be implemented for certain pseudogroups $\Gamma$. Note however that already step 1 is a delicate matter. For instance, even for $\Gamma=\Gamma^{q}$ (when the previous plan cannot work because of the Frobenius theorem) one may expect that the universal characteristic map is injective but, despite some early announcement [30], that is still an open problem (this was pointed out in [20] - see e.g. the comments following Theorem 5, and the further references therein). On the other hand, it may be easier to find elements in the kernel of $\kappa^{\text {univ }}$ than proving that the map is injective; of course, one has to look at pseudogroups for which formal integrability does not imply integrability.

\subsubsection{The van Est map into infinitesimal Haefliger cohomology}

Next, we exploit the general van Est map (2.61) in another special case: when $P$ is $\Sigma$ itself.

Proposition 2.6.5. For a Cartan groupoid $(\Sigma, \mathcal{C}) \rightrightarrows \mathbf{X}$, taking $P=\Sigma$ endowed with the left action of $\Sigma$, the resulting complex $\Omega^{*}(\Sigma)^{\Sigma}$ is isomorphic to the infinitesimal Haefliger complex $C_{\text {Haef }}^{*}(A, \nabla)$ of the flat Cartan algebroid $(A, \nabla) \rightarrow \mathbf{X}$ of $(\Sigma, \mathcal{C})$.

Therefore, one obtains a canonical map

$$
V E: H_{\text {Haef }}^{*}(\Sigma, \mathcal{C}) \rightarrow H_{\text {Haef }}^{*}(A, \nabla)
$$

and this map is an isomorphism up to degree $l$ if the s-fibers of $\Sigma$ are cohomologically l-connected.

Proof. We will be using the description of the infinitesimal Haefliger complex as the algebroid complex of the double $\mathcal{D}(A, \nabla)$ (see Definition 2.5.14), appealing to Proposition 2.5.15. Recall that the total space of $\mathcal{D}(A, \nabla)$ is $A \oplus T \mathbf{X}$. We have (identifying $A$ with $\left.\operatorname{Ker}(d s)\right|_{M}$ via $\omega$ )

$$
A_{t(g)} \oplus T_{t(g)} \mathbf{X}=T_{g} \Sigma
$$

and we notice that a vector field left invariant for the action of $\Sigma \ltimes \Sigma$ on $T \Sigma$ is completely determined by its value over $u(\mathbf{X}) \subset \Sigma$. Moreover, as a representation of $\Sigma \ltimes \Sigma, T \Sigma$ is the direct sum representation of $t^{*} A$ and $t^{*} T \mathbf{X}$; if $(\alpha, X) \in \Gamma(A \oplus$ $T \mathbf{X})$ then

$$
V_{g}=\left(d m\left(\operatorname{hor}_{g}^{\mathfrak{e}}(d t(\alpha)), \alpha\right), d m\left(\operatorname{hor}_{g}^{\mathfrak{e}}(d t(X)), X\right)\right)
$$

defines a left invariant vector field $V \in \mathfrak{X}(\Sigma)$. This gives a bijection between sections of $A \oplus T \mathbf{X}$ and such invariant vector fields. Furthermore

$$
V_{g}=\left(\hat{\alpha}_{g}, \operatorname{hor}_{g}^{\mathrm{e}}(X)\right)
$$

where $\hat{\alpha}$ and $\operatorname{hor}_{g}^{\mathrm{e}}(X)$ are the left invariant sections of $t^{*} A$ and $t^{*} T \mathbf{X}$ associated to $\alpha \in \Gamma(A)$ and $X \in \Gamma(T \mathbf{X})$. In particular, $\hat{\alpha}$ is $s$-vertical and $\operatorname{hor}^{\mathcal{e}}(X)$ is the unique $s$-projectable vector field tangent to $\mathcal{C}$ and extending $X$ at $u(\mathbf{X})$. Notice that the map

$$
\begin{aligned}
\Phi: A \oplus T \mathbf{X} & \rightarrow A \oplus T \mathbf{X} \\
\left(\alpha_{x}, v_{x}\right) & \rightarrow\left(-\alpha_{x}, v_{x}+\rho\left(\alpha_{x}\right)\right)
\end{aligned}
$$


is an isomorphism of vector bundles. We will show that the composition of $\Phi$ with the map $(\alpha, X) \rightarrow\left(\hat{\alpha}\right.$, hor $\left.^{\mathbb{C}}(X)\right)$ gives an isomorphism of Lie algebras.

First of all, for all $\alpha, \beta \in \Gamma(A)$ and $X, Y \in \mathfrak{X}(\mathbf{X})$ (notice that $\rho(\hat{\alpha})=\operatorname{hor}^{\mathrm{e}}(\rho(\alpha))$, and the same holds for $\beta$ ),

$$
\begin{aligned}
& {\left[\left(-\hat{\alpha}, \operatorname{hor}^{\mathrm{e}}(X)+\rho(\hat{\alpha})\right),\left(-\hat{\beta}, \operatorname{hor}^{\mathrm{e}}(Y)+\rho(\hat{\beta})\right)\right]=[-\hat{\alpha},-\hat{\beta}]+\left[-\hat{\alpha}, \operatorname{hor}^{\mathrm{e}}(Y)\right]} \\
& +[-\hat{\alpha}, \rho(\hat{\beta})]+\left[\operatorname{hor}^{\mathcal{C}}(X),-\hat{\beta}\right]+\left[\operatorname{hor}^{\mathfrak{e}}(X), \operatorname{hor}^{\mathfrak{C}}(Y)\right] \\
& +\left[\operatorname{hor}^{\mathcal{C}}(X), \rho(\hat{\beta})\right]+[\rho(\hat{\alpha}),-\hat{\beta}]+\left[\rho(\hat{\alpha}), \operatorname{hor}^{\mathbb{C}}(Y)\right]+[\rho(\hat{\alpha}), \rho(\hat{\beta})] .
\end{aligned}
$$

Since the right hand side of the equality above is left invariant, we just have to show that it coincide with $\Phi([(\alpha, X),(\beta, Y)])$ when restricted to $\mathbf{X}$. Following our argument for Lemma 2.6.6 below, one sees that

$$
\left.[\hat{\alpha}, \hat{\beta}]\right|_{\mathbf{X}}=-[\alpha, \beta]+\nabla_{\rho(\alpha)}(\beta)-\nabla_{\rho(\beta)}(\alpha)
$$

Notice also that, for any $Z \in \mathfrak{X}(\mathbf{X})$, hor $^{\mathrm{e}}(Z)$ is tangent to $\mathbf{X}$. This implies that

$$
\left.\left[-\hat{\alpha}, \operatorname{hor}^{\mathfrak{e}}(Y)\right]\right|_{\mathbf{x}}=\nabla_{Y}(\alpha), \quad\left[\operatorname{hor}^{\mathfrak{e}}(X),-\hat{\beta}\right]=-\nabla_{X}(\beta)
$$

and that

$$
\left.[-\hat{\alpha}, \rho(\hat{\beta})]\right|_{\mathbf{x}}=\nabla_{\rho(\beta)}(\alpha),\left.\quad[\rho(\hat{\alpha}),-\hat{\beta}]\right|_{\mathbf{x}}=-\nabla_{\rho(\alpha)}(\beta)
$$

In conclusion,

$$
\begin{array}{r}
{\left.\left[\left(-\hat{\alpha}, \operatorname{hor}^{\mathcal{E}}(X)+\rho(\hat{\alpha})\right),\left(-\hat{\beta}, \operatorname{hor}^{\mathcal{E}}(Y)+\rho(\hat{\beta})\right)\right]\right|_{\mathbf{X}}=} \\
\left(-[\alpha, \beta]+\nabla_{Y}(\alpha)-\nabla_{X}(\beta),[X, Y]+[\rho(\alpha), Y]+[X, \rho(\beta)]+[\rho(\alpha), \rho(\beta)]\right)
\end{array}
$$

that coincides with $\Phi([(\alpha, X),(\beta, Y)])$.

\subsubsection{Application to the transitive case}

When $(\Sigma, \omega)$ is transitive, there is yet another natural choice of a $\Sigma$-space $P$ to which one can apply Proposition 2.6.2: the $s$-fiber $s^{-1}(x)$, for $x \in \mathbf{X}$.

Lemma 2.6.6. There is an isomorphism

$$
\Omega^{*}\left(s^{-1}(x)\right)^{\Sigma} \simeq C^{*}\left(\mathfrak{a}_{x}(A)\right)
$$

where $\mathfrak{a}_{x}(A)$ is the extended isotropy Lie algebra from Definition 2.5.8. Therefore, there is a canonical map in cohomology

$$
V E_{x}: H_{\text {Haef }}^{*}(\Sigma, \omega) \rightarrow H^{*}\left(\mathfrak{a}_{x}(A)\right)
$$

and this map is an isomorphism up to degree lif the isotropy group $\Sigma_{x}$ is cohomologically l-connected.

Proof. This proof is best approached working with a multiplicative pointwise surjective form $\omega \in \Omega^{1}\left(\Sigma, t^{*} A\right)$ whose kernel is $\mathcal{C}$, see Remark 2.4.2 and Corollary 2.5.10. In particular, we will need the Maurer-Cartan equation from Corollary 2.5.10 or, more precisely, the one for the restriction of $\omega$ to $s^{-1}(x)$; see Proposition 2.5.18 (of course, $\left(s^{-1}(x),\left.\omega\right|_{s^{-1}(x)}\right)$ is a flat $(\Sigma, \omega)$-structure, compare with 
Example 2.4.15). We will keep the notation $t$ for the restriction of the target map $t: \Sigma \rightarrow \mathbf{X}$ to $s^{-1}(x)$, which is surjective because $\Sigma$ is transitive.

Notice that we have a map

$$
r_{1_{x}}: \mathfrak{X}\left(s^{-1}(x)\right)^{\Sigma} \rightarrow A_{x},\left.\quad X \rightarrow\left(\left.\omega\right|_{s^{-1}(x)}(X)\right)\right|_{1_{x}}
$$

One sees immediately that this is a linear isomorphism. Observe that the inverse of $r_{1_{x}}$ is given by

$$
i_{x}: \alpha_{x} \in A_{x} \rightarrow i_{x}\left(\alpha_{x}\right) \in \Gamma\left(T s^{-1}(x)\right)
$$

where, for all $g \in s^{-1}(x)$,

$$
\left(i_{x}\left(\alpha_{x}\right)\right)_{g}=d m\left(\operatorname{hor}_{g}^{\mathcal{e}}\left(d t\left(\omega_{1_{x}}^{-1}\left(\alpha_{x}\right)\right)\right), \omega_{1_{x}}^{-1}\left(\alpha_{x}\right)\right) \in T_{g} s^{-1}(x) .
$$

We can choose $\omega$ so that $\omega_{1_{x}}$ is the identity map on $s$-vertical vectors and the formula for $i_{x}$ simplifies to

$$
\left(i_{x}\left(\alpha_{x}\right)\right)_{g}=d m\left(\operatorname{hor}_{g}^{\mathrm{e}}\left(\rho\left(\alpha_{x}\right)\right), \alpha_{x}\right) \in T_{g} s^{-1}(x) .
$$

We claim that $r_{1_{x}}$ is an anti-Lie algebra map, so that composing with the map

$$
A_{x} \rightarrow A_{x}, \quad \alpha_{x} \rightarrow-\alpha_{x}
$$

we get the desired isomorphism.

As anticipated above, this follows because $\left.\omega\right|_{s^{-1}(x)}$ satisfies the Maurer-Cartan equation

$$
d_{t^{*} A}\left(\left.\omega\right|_{s^{-1}(x)}\right)+\frac{1}{2}\left\{\left.\omega\right|_{s^{-1}(x)},\left.\omega\right|_{s^{-1}(x)}\right\}_{p t}=0 .
$$

To keep the notation simple, we will use the same symbols as in Proposition 2.5.18: $\widetilde{\nabla}$ will denote the infinitesimally multiplicative flat connection on $t^{*} A$ obtained pulling back $\nabla$ via $t, \widetilde{A}$ will denote $t^{*} A$ and we rebaptise $\left.\omega\right|_{s^{-1}(x)}$ as $\theta$, so that the Maurer-Cartan equation becomes

$$
d_{\widetilde{A}}(\theta)+\frac{1}{2}\{\theta, \theta\}_{p t}=0
$$

Let us recall that, for any $X_{1}, X_{2} \in \Gamma\left(T s^{-1}(x)\right)$,

$$
d_{\widetilde{A}}(\theta)\left(X_{1}, X_{2}\right)=\widetilde{\nabla}_{X_{1}}\left(\theta\left(X_{2}\right)\right)-\widetilde{\nabla}_{X_{2}}\left(\theta\left(X_{1}\right)\right)-\theta\left(\left[X_{1}, X_{2}\right]\right) .
$$

Consequently, from the Maurer-Cartan equation we see that for each pair of invariant vector fields $X_{1}, X_{2}$, it holds

$$
\left\{\theta\left(X_{1}\right), \theta\left(X_{2}\right)\right\}_{p t}=-\widetilde{\nabla}_{X_{1}}\left(\theta\left(X_{2}\right)\right)+\widetilde{\nabla}_{X_{2}}\left(\theta\left(X_{1}\right)\right)+\theta\left(\left[X_{1}, X_{2}\right]\right)
$$

As it is shown in Proposition 5.3.4 from [15], the following identity (already recalled while proving Proposition 2.5.18) holds for all $X_{1}, X_{2} \in \Gamma\left(T s^{-1}(x)\right)$ :

$$
\widetilde{\nabla}_{X_{1}}\left(\theta\left(X_{2}\right)\right)=\widetilde{\nabla}_{\widetilde{\rho}\left(\theta\left(X_{1}\right)\right)}\left(\theta\left(X_{2}\right)\right)+\left[\theta\left(X_{2}\right), \theta\left(X_{1}\right)\right]_{\widetilde{A}}-\theta\left(\left[\widetilde{\rho}\left(\theta\left(X_{2}\right)\right), X_{1}\right]\right)
$$

where we observe that the first two terms of the right hand side correspond to the action of $\theta\left(X_{2}\right)$ on $\theta\left(X_{1}\right)$ using the representation of $\widetilde{A}$ on itself (see formula (2.56), 
taking into account that $(\widetilde{A}, \widetilde{\nabla})$ is a flat Cartan algebroid). We claim that, if $X_{1}$ and $X_{2}$ are left invariant, then $\theta\left(X_{1}\right)$ and $\theta\left(X_{2}\right)$ are left invariant for the representation of $\Sigma \ltimes s^{-1}(x)$ on its algebroid $\widetilde{A}$ (see (2.54)). Assuming for a moment that this claim is true, using the formula recalled above we see that

$$
\widetilde{\nabla}_{X_{1}}\left(\theta\left(X_{2}\right)\right)=-\theta\left(\left[\widetilde{\rho}\left(\theta\left(X_{2}\right)\right), X_{1}\right]\right), \quad \widetilde{\nabla}_{X_{2}}\left(\theta\left(X_{1}\right)\right)=-\theta\left(\left[\widetilde{\rho}\left(\theta\left(X_{1}\right)\right), X_{2}\right]\right)
$$

for any pair of left invariant vector fields $X_{1}, X_{2}$ over $s^{-1}(x)$. Still using Proposition 5.3 .4 of [15], one has that $\theta$ is the inverse of $\widetilde{\rho}$, hence $-\widetilde{\nabla}_{X_{1}}\left(\theta\left(X_{2}\right)\right)+$ $\widetilde{\nabla}_{X_{2}}\left(\theta\left(X_{1}\right)\right)=-2 \theta\left(\left[X_{1}, X_{2}\right]\right)$ holds and we have

$$
\left\{\theta\left(X_{1}\right), \theta\left(X_{2}\right)\right\}=-\theta\left[X_{1}, X_{2}\right]
$$

i.e. the map

$$
\mathfrak{X}\left(s^{-1}(x)\right)^{\Sigma} \rightarrow A_{x}, \quad X \rightarrow-\theta(X)_{1_{x}}
$$

is an isomorphism of Lie algebras, which was our original claim.

We are left to show that if $X$ is left invariant then $\theta(X)$ is left invariant, which follows by proving that $\theta$ is a map of $\Sigma \ltimes s^{-1}(x)$-representations. Of course, this uses multiplicativity. In the following computation, we see $\widetilde{A}=t^{*} A$ as the fibered product $A \times \mathbf{X} s^{-1}(x)$ and $m$ denotes both the multiplication on $\Sigma$ and the one on the action groupoid:

$$
\begin{aligned}
\left(g, 1_{x}\right) \cdot\left(\theta\left(X_{x}\right), 1_{x}\right)= & d R_{\left(g^{-1}, g\right)} d m\left(\operatorname{hor}_{\left(g, 1_{x}\right)}^{p r_{1}^{-1} \mathcal{e}}\left(\widetilde{\rho}\left(\theta\left(X_{x}\right)\right)\right),\left(\theta\left(X_{x}\right), 1_{x}\right)\right)= \\
& d R_{\left(g^{-1}, g\right)} d m\left(\left(\operatorname{hor}_{g}^{\mathcal{e}}\left(\rho\left(X_{x}\right)\right), \widetilde{\rho}\left(\theta\left(X_{x}\right)\right),\left(X_{x}, 1_{x}\right)\right)=\right. \\
& d R_{\left(g^{-1}, g\right)}\left(d m\left(\operatorname{hor}_{g}^{\mathcal{e}}\left(\rho\left(X_{x}\right)\right), X_{x}\right), 1_{x}\right)=\left(\theta\left(X_{g}\right), g\right) .
\end{aligned}
$$

The last step follows from multiplicativity, which implies

$$
\theta\left(X_{g}\right)=g \cdot \theta\left(X_{x}\right)=d R_{g^{-1}} d m\left(\operatorname{hor}_{g}^{\mathrm{e}}\left(\rho\left(X_{x}\right)\right), X_{x}\right)
$$

We can then state the following theorem.

Theorem 2.6.7. Let $(\Sigma, \mathcal{C})$ be a transitive flat Cartan groupoid. If $K \subset \Sigma_{x}$ is a compact Lie subgroup with the property that $\Sigma_{x} / K$ is contractible, then the map from the previous Lemma factors though an isomorphism

$$
H_{\text {Haef }}^{*}(\Sigma, \mathcal{C}) \stackrel{\sim}{\rightarrow} H^{*}\left(\mathfrak{a}_{x}(A), K\right) .
$$

Remark 2.6.8. In examples coming from geometric structures, there is always a natural choice of $K$, even in the profinite dimensional case (see Example 2.6.9).

Proof. The triple $\left(s^{-1}(x), t, \Sigma_{x}\right)$ is a principal bundle and we know that $s^{-1}(x) / K$ has contractible fibers. Then, we can apply Proposition 2.6.2 to the $\Sigma$-space $s^{-1}(x) / K$. We have to prove that $\Sigma$-invariant forms on $s^{-1}(x) / K$ are isomorphic to the Lie algebra cochains in $A_{x}$ relative to $K$. For this, one just uses the fact that the isomorphism constructed in Lemma 2.6.6 descends to the basic subcomplexes; that is, it respects the contraction and the Lie derivative with respect to elements of $\mathfrak{k}=\operatorname{Lie}(K)$. 
Composing the isomorphism from the above Theorem with map (2.63), one sees that, if $(\Sigma, \mathcal{C})$ is a transitive flat Cartan groupoid, a $(\Sigma, \mathcal{C})$-structure comes with a natural map

$$
\kappa^{P}: H^{*}\left(\mathfrak{a}_{x}(A), K\right) \rightarrow H^{*}(M) .
$$

Example 2.6.9. When $\Gamma$ is a transitive Lie pseudogroup and $\Sigma=J^{\infty} \Gamma$, the resulting isomorphism becomes

$$
H_{\text {diff }}^{*}(\Gamma) \cong H^{*}\left(\mathfrak{a}_{x}(\Gamma), K\right),
$$

see Definition 2.2.8 and Example 2.5.6. For $k \geq 2$, the projections

$$
p r^{k, k-1}: J^{k} \Gamma \rightarrow J^{k-1} \Gamma
$$

are affine bundles projections; consequently, one can always choose a subgroup $K$ as in Theorem 2.6.7.

In particular, for $\Gamma=\operatorname{Diff}_{\text {loc }}\left(\mathbb{R}^{q}\right)$ and $\Sigma=J^{\infty} \operatorname{Diff}_{\text {loc }}\left(\mathbb{R}^{q}\right)$ the group $K$ can be identified with the orthogonal group $O(q)$. This is a phenomenon slightly more general than Diff loc $\left(\mathbb{R}^{q}\right)$ that we explain in Example 2.6.10. As a consequence, the isomorphism from Theorem 2.6.7 reduces to (2.32), with codomain given by the relative Gel'fand-Fuks cohomology of formal vector fields.

Going back to a transitive $\Gamma$, the characteristic map (2.67) corresponding to an almost $\Gamma$-structure $\mathcal{P} \infty$

$$
\kappa^{\mathcal{P} \infty}: H^{*}\left(a_{x}(\Gamma), K\right) \rightarrow H^{*}(M)
$$

is seen to be defined on the relative Gel'fand-Fuks cohomology of $\Gamma$-vector fields.

The characteristic map (2.68) for transitive Lie pseudogroups had been addressed in [11] and in [6,7]. We adapt the construction of [6, 7] to our setting; the outcome is a description of map (2.67) which is in the same spirit as the geometric characteristic map of flat principal bundles (see Subsection 2.2.2), i.e. of Chern-Weil nature. We use the description from Proposition 2.5.18 of $\mathcal{C}_{P}$ in terms of $\theta \in \Omega^{1}\left(P, \mu^{*} A\right)$. Restricting to

$$
Q:=\mu^{-1}(x),
$$

we obtain a principal $\Sigma_{x}$-bundle $Q \rightarrow M$ and

$$
\theta_{Q}:=\left.\theta\right|_{Q} \in \Omega^{1}\left(P, \mathfrak{a}_{x}(A)\right)
$$

will satisfy the Maurer-Cartan equation (cf. Proposition 2.5.18). The form $\theta_{Q}$ induces a map

$$
\theta_{Q}^{*}: C^{*}\left(\mathfrak{a}_{x}(A)\right) \rightarrow \Omega^{*}(Q) .
$$

This works as in the standard situation of flat principal bundles. Passing to $K$ basic elements and using that the fibers of $Q / K \rightarrow M$ are contractible, one obtains an induced map (compare with (2.5)):

$$
\kappa^{\theta_{Q}}: H^{*}\left(\mathfrak{a}_{x}(A), K\right) \rightarrow H^{*}(M) .
$$


Example 2.6.10. To further elaborate on Example 2.6.9, let us consider the transitive pseudogroup $\Gamma_{G}$ on $\mathbb{R}^{q}$ induced by some Lie subgroup $G \subset G L_{q}(\mathbb{R})$ as follows:

$$
\Gamma_{G}=\left\{\varphi \in \operatorname{Diff}_{\mathrm{loc}}\left(\mathbb{R}^{q}\right): d_{x} \varphi \in G, x \in \operatorname{dom}(\varphi)\right\}
$$

Notice that this pseudogroup is defined by a first order condition; more compactly it is of first order, which means that $\varphi \in \Gamma_{G}$ if and only if $j_{x}^{1} \varphi \in J^{1} \Gamma_{G}$ for all $x \in \operatorname{dom}(\varphi)$. As a consequence, a group $K$ as in Theorem 2.6.7 is induced by a maximal compact subgroup $\bar{K}$ of the isotropy group $\left(J^{1} \Gamma_{G}\right)_{x} \cong G$. In fact, one defines $K$ to be the group of infinite jets at $x$ of linear diffeomorphisms $\varphi$ fixing $x$ and such that $d_{x} \varphi \in \bar{K}$.

When $\operatorname{dim}(M)=q$, a $\Gamma_{G}$-structure on $M$ is the same thing as a flat (or integrable) $G$-structure; a $\left(J^{\infty} \Gamma_{G}, \mathrm{e}^{\infty}\right)$-structure on $M$, as in example 2.4 .18 , is a formally integrable $G$-structure (see, for example, [64] for $G$-structures and [15] for a discussion about formal integrability that is close to the formalism of this chapter). When $\operatorname{dim}(M)=n>q$, a $\Gamma_{G}$-structure is a codimension $q$ foliation equipped with a transverse flat $G$-structure.

\section{Appendix}

\section{A Pro-finite manifolds}

\section{A.1 Pf-manifolds $(\mathrm{pf}=$ pro-finite $)$}

Pro-finite manifolds are, in principle, infinite dimensional manifolds together with a "tail" of finite dimensional ones, a tail that allows one to export the usual (finite dimensional) concepts to the infinite dimensional setting. The basic example is that of infinite jets, where the "tail" is the sequence of jets spaces of finite orders.

Definition 2.A.1. A tower of manifolds $\mathbb{M}_{\bullet}$ is a sequence

$$
\mathbb{M}_{\bullet}: \quad \ldots \longrightarrow \mathbb{M}_{k} \stackrel{\pi_{k}}{\longrightarrow} \mathbb{M}_{k-1} \longrightarrow \ldots \longrightarrow \mathbb{M}_{2} \stackrel{\pi_{2}}{\longrightarrow} \mathbb{M}_{1} \stackrel{\pi_{1}}{\longrightarrow} \mathbb{M}_{0}
$$

consisting of smooth, finite dimensional manifolds $\mathbb{M}_{k}$ and surjective submersions between them.

A pf-atlas on a set $\mathbb{M}$, denoted

$$
a: \mathbb{M} \rightarrow \mathbb{M}_{\bullet}
$$

is a tower of manifolds $\mathbb{M}$. together with a collection $a=\left\{a_{k}: \mathbb{M} \rightarrow \mathbb{M}_{k}\right\}$ of surjections compatible with the tower projections $\left(\pi_{k} \circ a_{k}=a_{k-1}\right)$.

Remark 2.A.2. Given such a pf-atlas $a: \mathbb{M} \rightarrow \mathbb{M}_{\bullet}$, any $x \in \mathbb{M}$ has associated components w.r.t. $a$, namely:

$$
x_{k}=a_{k}(x) \in \mathbb{M}_{k} .
$$

But note that, in principle, two distinct points may have the same components. 
Perhaps a better name for the components $x_{k}=a_{k}(x)$ would be that of "truncations of $x^{\prime \prime}$. They are not arbitrary - actually each one of them determines the previous ones by:

$$
x_{k-1}=\pi_{k}\left(x_{k}\right) \text { for all } k .
$$

However, there may be sequences $\left(y_{k}\right)$ satisfying this compatibility but which do not arise from an element of $x \in \mathbb{M}$.

All these remarks can be packed together by considering the inverse limit of the tower

$$
\lim _{\longleftarrow} \mathbb{M}_{k}:=\left\{\left(x_{k}\right)_{k \geq 0}: x_{k} \in \mathbb{M}_{k}, \pi_{k}\left(x_{k}\right)=x_{k-1}\right\}
$$

and interpreting $a$ as a map

$$
a: \mathbb{M} \rightarrow \underset{k}{\lim } \mathbb{M}_{k}
$$

And we were just saying that this map may fail to be injective (different elements may have same components) or surjective (compatible sequences may not have a limit in $\mathbb{M})$.

Definition 2.A.3. A pf-atlas $a: \mathbb{M} \rightarrow \mathbb{M}_{\bullet}$ is said to be normal if the induced map $\mathbb{M} \rightarrow \underset{\longleftarrow}{\lim } \mathbb{M}_{k}$ is a bijection.

Example 2.A.4. One can always achieve normality by replacing $\mathbb{M}$ by $\lim _{k} \mathbb{M}_{k}$. However, there are natural examples which are not necessarily normal. For instance, $J^{\infty} \Gamma$, for certain choices of $\Gamma$ (see Example 2.A.38). A standard example is given by the pseudogroup of analytic diffeomorphisms of $\mathbb{R}^{q}$; see Example 2.A.38 and Remark 2.A.40.

Definition 2.A.5. Consider $a: \mathbb{M} \rightarrow \mathbb{M}_{\bullet}$ and $b: \mathbb{N} \rightarrow \mathbb{N}_{\bullet}$ be two pf-atlases. A set theoretical function $f: \mathbb{M} \rightarrow \mathbb{N}$ is said to be a smooth map w.r.t. $a$ and $b$, or we say that $f:(\mathbb{M}, a) \rightarrow(\mathbb{N}, b)$ is a smooth map of pf-atlases if, for each $k$, the $k$-th component

$$
b_{k} \circ f: \mathbb{M} \rightarrow \mathbb{N}_{k}
$$

comes from a smooth map defined on some $\mathbb{M}_{m_{k}}$ for some $m_{k}$ large enough:

$$
b_{k} \circ f=f_{k}^{m_{k}} \circ a_{m_{k}}, \quad \text { with } f_{k}^{m_{k}} \in C^{\infty}\left(\mathbb{M}_{m_{k}}, \mathbb{N}_{k}\right) .
$$

Remark 2.A.6. The outcome is very much related to the notion of morphism between towers of manifolds. Seeing towers as particular cases of projective systems of (finite dimensional) manifolds, one obtains an abstract but very standard description of the resulting hom-spaces:

$$
\operatorname{Hom}\left(\mathbb{M}_{\bullet}, \mathbb{N}_{\bullet}\right):={\underset{n}{n}}_{\lim } \underset{m}{\lim } C^{\infty}\left(\mathbb{M}_{m}, \mathbb{N}_{n}\right) \text {. }
$$

Working this out and using that the $\pi_{k}$ 's are surjective submersions, one obtains the following description which is related to the previous definition.

First of all, let us call a concrete morphism between two towers $\mathbb{M}$. and $\mathbb{N}$. any collection of smooth maps

$$
f_{\bullet}=\left\{f_{k}: \mathbb{M}_{m_{k}} \rightarrow \mathbb{N}_{k}\right\}_{k \geq 1} \quad \text { with } m_{1} \leq m_{2} \leq \ldots
$$


which is compatible in the sense that that it makes the resulting squares commutative:

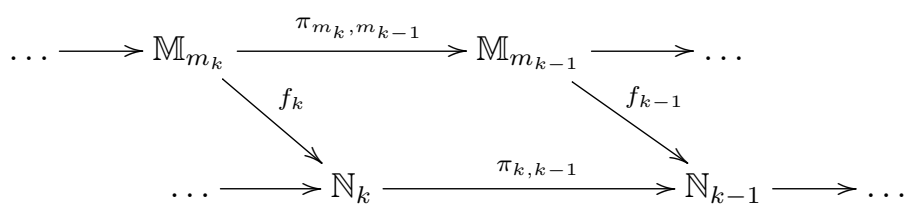

Note that, for the maps $f_{k}^{m_{k}}$ arising in the previous definition, this compatibility is automatically satisfied. Therefore, the condition from the previous definition can be reformulated by requiring that $f: \mathbb{M} \rightarrow \mathbb{N}$ is compatible with a concrete morphism of towers $f_{\bullet}$ from $\mathbb{M}_{\bullet}$ to $\mathbb{N}_{\bullet}$.

Next, given a second concrete morphism $g_{\bullet}=\left\{g_{k}: \mathbb{M}_{l_{k}} \rightarrow \mathbb{N}_{k}\right\}$ as above, we say that $f_{\bullet}$ and $g_{\bullet}$ are equivalent concrete morphisms if for each $k, f_{k}$ and $g_{k}$ become equal after composing them with the projections $\mathbb{M}_{K} \rightarrow \mathbb{M}_{m_{k}}$ and $\mathbb{M}_{K} \rightarrow$ $\mathbb{M}_{l_{k}}$, respectively, for some $K$-large enough (which actually may be chosen to be $\left.\max \left\{m_{k}, l_{k}\right\}\right)$. With this, a morphism of towers is an equivalence class of concrete morphisms.

And, again, it is useful to look back at the previous definition: while there the actual $f_{k}=f_{k}^{m_{k}}$ are far from unique (they are just required to exist), we see that any two choices are equivalent; hence one has a unique, well-defined induced morphism of towers

$$
f_{\bullet} \in \operatorname{Hom}\left(\mathbb{M}_{\bullet}, \mathbb{N}_{\bullet}\right) .
$$

Similar to the previous discussion about coordinates, two different $f$ 's may still induce the same morphism of towers, or there are morphism of towers that are not induced by an $f$. However, neither of these can happen when the two atlases are normal.

Example 2.A.7. It is straightforward now to define composition of morphisms of towers obtaining, therefore, the category of towers (of finite dimensional smooth manifolds). With the resulting notion of isomorphism we see that, given $\mathbb{M}_{\bullet}$, if we sample the tower via an increasing sequence of indices $m_{0}<m_{1}<m_{2}<\ldots$, then

$$
\ldots \longrightarrow \mathbb{M}_{m_{k}} \stackrel{\pi_{k}}{\longrightarrow} \mathbb{M}_{m_{k-1}} \longrightarrow \ldots \longrightarrow \mathbb{M}_{m_{2}} \stackrel{\pi_{2}}{\longrightarrow} \mathbb{M}_{m_{1}} \stackrel{\pi_{1}}{\longrightarrow} \mathbb{M}_{m_{0}}
$$

is isomorphic to $\mathbb{M}_{\bullet}$ itself.

Given Example 2.A.4, it is interesting to consider atlases that are not normal. However, to make our life easier (and be able to just quote existing results), from now on we will start imposing the normality condition.

Definition 2.A.8. A pf-manifold is a set $\mathbb{M}$ together with an equivalence class of normal pf-atlases, where two pf-atlases

$$
a: \mathbb{M} \rightarrow \mathbb{M}_{\bullet}, \quad a^{\prime}: \mathbb{M} \rightarrow \mathbb{M}_{\bullet}^{\prime}
$$

are said to be equivalent if the identity map $\mathrm{id}_{\mathbb{M}}$ is a smooth map of pf-atlases (in the sense of Definition 2.A.5) both as a map from (M,a) to $\left(\mathbb{M}, a^{\prime}\right)$ as well as the 
other way around. Equivalently, we require the existence of an isomorphism of towers

$$
c_{a, a^{\prime}} \in \operatorname{Hom}\left(\mathbb{M}_{\bullet}, \mathbb{M}_{\bullet}^{\prime}\right) \quad \text { ("change of coordinates") }
$$

that is compatible with $a$ and $a^{\prime}$.

Remark 2.A.9 (the underlying topological space). Note that any pf-manifold carries a natural topology: the smallest one which makes all the projection $a_{k}$ continuous (using a/any atlas $a$ ). As terminology:

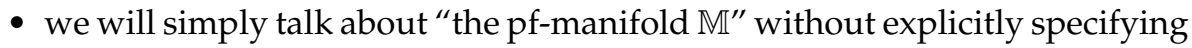
the (equivalence class) of the atlas in the notation.

- when thinking about $\mathbb{M}$ just as a topological space we will use the notation $|\mathbb{M}|$.

Definition 2.A.10. A smooth pf-map $f: \mathbb{M} \rightarrow \mathbb{N}$ between two pf-manifolds is any set theoretical map with the property that, for a/any atlas $a$ of $\mathbb{M}$ and $b$ of $\mathbb{N}$, $f$ is a smooth map w.r.t. $a$ and $b$ (in the sense of Definition 2.A.5). We will denote by $C^{\infty}(\mathbb{M}, \mathbb{N})$ the space of such smooth maps.

Example 2.A.11. When $\mathbb{N}=\mathbb{R}$ we obtain the algebra $C^{\infty}(\mathbb{M})$ of smooth functions on the pf-manifold $\mathbb{M}$; working out the previous definition we see that they are continuous functions

$$
f: \mathbb{M} \rightarrow \mathbb{R}
$$

with the property that there exists $m_{0}$ such that

$$
f=g \circ a_{m_{0}}, \quad \text { with } g: \mathbb{M}_{m_{0}} \rightarrow \mathbb{R} \text { smooth in the usual sense. }
$$

Abstractly, we deal with

$$
\underset{m}{\lim _{m}} C^{\infty}\left(\mathbb{M}_{m}\right)=\cup_{m} C^{\infty}\left(\mathbb{M}_{m}\right),
$$

viewed as a subspace of $C(\mathbb{M})$ via the pull-back by $a$. Concretely, we think of functions $f=f(x)$ on $\mathbb{M}$ as functions that may depend on infinitely many variables

$$
x_{0}=a_{0}(x), \quad x_{1}=a_{1}(x), \quad \ldots, \quad x_{k}=a_{k}(x), \quad \ldots
$$

while smooth functions depend only on a finite number of variables $m_{0}$ in a smooth way.

One can also require this condition only locally- and that give rise to another notion that we would call "smooth function on the space $|\mathbb{M}|$ " (for the notation see Remark 2.A.9); this gives rise to a sequence of (strict) inclusions

$$
C^{\infty}(\mathbb{M}) \subset C^{\infty}(|\mathbb{M}|) \subset \operatorname{Cont}(|\mathbb{M}|)
$$

Example 2.A.12. Of course, any finite dimensional manifold $M$ is a pf-manifold: just use the constant tower $\mathbb{M}_{\bullet}=M$. 
Example 2.A.13. The basic examples of towers and pf-manifolds are provided by jets and PDEs. Let us choose a fibration (meaning a surjective submersion)

$$
\pi: R \rightarrow M
$$

between finite dimensional manifolds. Associated to such a fibration there is an entire tower of jet spaces

$$
J^{\infty} R: \quad \ldots \rightarrow J^{2} R \rightarrow J^{1} R \rightarrow J^{0} R=R
$$

where $J^{k} R$ denotes the space of $k$-jets of sections of $\pi: R \rightarrow M$. Each such space with $k$ finite has a canonical smooth structure, while

$$
J^{\infty} R=\lim _{\longleftarrow} J^{k} R
$$

becomes a pf-manifold. A particular case of this is when $R=M \times N$ is a product and $\pi$ is the projection on the first factor. Then sections of $R$ are identified with smooth functions from $M$ to $N$ and one obtains the jet-spaces

$$
J^{k}(M, N):=J^{k}\left(\mathrm{pr}_{1}: M \times N \rightarrow M\right) .
$$

On the other hand one can also proceed more generally, for PDEs on (sections of) fibrations $\pi: R \rightarrow M$; an order $k$-PDE is then encoded into a subspace $P \subset$ $J^{k} R$ and the standard regularity assumption is that $P$ is a submanifold and $P \rightarrow$ $M$ is still a fibration. Then one can consider the jet spaces of $P$ itself, $J^{l} P \subset$ $J^{l} J^{k} R$; the intersection with $J^{k+l} R \subset J^{l} J^{k} R$ are called the l-prolongations of $P$ :

$$
P^{(l)} \subset J^{k+l} R
$$

All together these give a tower of spaces

$$
P^{(\infty)}: \quad \ldots \rightarrow P^{(2)} \rightarrow P^{(1)} \rightarrow P^{(0)}=P
$$

but the maps may fail to be surjective. The space $P^{(\infty)}$ plays the role of the space of "formal solutions" of the PDE $P$. One says that $P$ is formally integrable if all these maps are surjective submersions. In that case $P^{(\infty)}$ becomes a pf-manifold and the inclusions

$$
P^{(l)} \subset J^{(k+l)} R
$$

provide a natural example of concrete morphism of towers that does not preserve the degree. Of course, they represent the inclusion

$$
P^{(\infty)} \hookrightarrow J^{\infty} R
$$

as a morphism of pf-manifolds. Note also that, interpreting $P^{(l)}$ itself as a PDE of order $k+l$, one obtains

$$
\left(P^{(l)}\right)^{(\infty)} \cong P^{(\infty)}
$$

as pf-manifolds. 


\section{A.2 Direct pf-vector bundles (and sheaves)}

We now move to vector bundles and sheaves. As we shall explain, there are two interesting but different types of vector bundles to consider. In this section we look at the first type, called "direct". Because of the examples we have in mind, we concentrate on vector bundles; for sheaves the discussion is entirely similar.

Definition 2.A.14. A (direct) pf-vector bundle over a pf-manifold $\mathbb{M}$ consists of a pf-manifold $\mathbb{E}$ and a smooth pf-map $p: \mathbb{E} \rightarrow \mathbb{M}$, together with a vector bundle equivalence classes of $\mathrm{pf}$-vector bundle atlases where:

- a pf-vector bundle atlas for $p: \mathbb{E} \rightarrow \mathbb{M}$ is an atlas $\tilde{a}: \mathbb{E} \rightarrow \mathbb{E}$. for $\mathbb{E}$, an atlas $a: \mathbb{M} \rightarrow \mathbb{M}$. for $\mathbb{M}$ together with the structure of tower of vector bundles

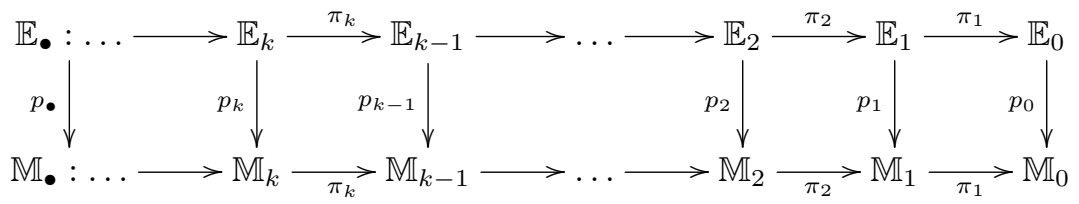

(hence each $\mathbb{E}_{k} \rightarrow \mathbb{M}_{k}$ is a smooth, finite dimensional vector bundle and each tower map $\mathbb{E}_{k} \rightarrow \mathbb{E}_{k-1}$ is a vector bundle morphisms covering the tower map $\mathbb{M}_{k} \rightarrow \mathbb{M}_{k-1}$ ) that represents $p$

- two pf-vector bundle atlases, $\tilde{a}$ and $\tilde{a}^{\prime}$, are said to be equivalent (as pf-vector bundle atlases) if they are equivalent as atlases of $\mathbb{E}$, with the corresponding isomorphism of towers $c_{\tilde{a}, \tilde{a}^{\prime}}: \mathbb{E}_{\bullet} \rightarrow \mathbb{E}_{\bullet}^{\prime}$ being represented by morphisms of vector bundles.

A (pf-)section of $\mathbb{E}$ is any smooth pf-map $\sigma: \mathbb{M} \rightarrow \mathbb{E}$ satisfying $p \circ \sigma=\mathrm{id}$. We denote by $\Gamma(\mathbb{E})$ the space of such sections.

Remark 2.A.15 (representing sections). Given a vector bundle atlas $a: \mathbb{E} \rightarrow \mathbb{E}_{\bullet}$, the best case scenario is when we deal with sections $\sigma$ that are represented by sections $\sigma_{k}$ of $\mathbb{E}_{k}$. It is not difficult to see that, given any section $\sigma$, one can find an atlas $a$ which realises the best case scenario for the given $\sigma$. However, this is of very little use since $a$ usually changes with $\sigma$.

In practice, we usually have a fixed (preferred) vector bundle atlas $a: \mathbb{E} \rightarrow$ $\mathbb{E}_{\bullet}$; given that, any section of $\mathbb{E}$ can be represented by a concrete morphism (cf. Remark 2.A.6)

$$
\sigma_{\bullet}=\left\{\sigma_{k}: \mathbb{M}_{m_{k}} \rightarrow \mathbb{E}_{k}\right\}_{k \geq 1} \text { with } m_{1}<m_{2}<\ldots
$$

and the section condition is that $p_{k} \circ \sigma_{k}$ is the projection $\mathbb{M}_{m_{k}} \rightarrow \mathbb{M}_{k}$; i.e. $\sigma_{k}$ comes from a section of the pull-back of $\mathbb{E}_{k}$ via the tower projection $\pi: \mathbb{M}_{m_{k}} \rightarrow \mathbb{M}_{k}$

$$
s_{k} \in \Gamma\left(\mathbb{M}_{m_{k}}, \pi^{*} \mathbb{E}_{k}\right) \text {. }
$$

On the other hand, the underlying topological space $|\mathbb{E}|$ (cf. Remark 2.A.9) is itself a topological vector bundle over $|\mathbb{M}|$ : the fiber $\mathbb{E}_{x}$ above any $x \in|\mathbb{M}|$ inherits 
a structure of vector space (actually a pf-vector space) and the corresponding maps (addition and multiplication by scalars) are continuous. And, of course, sections of $\mathbb{E} \rightarrow \mathbb{M}$ are, in particular, continuous sections of $|\mathbb{E}| \rightarrow|\mathbb{M}|$ :

$$
\Gamma(\mathbb{E}) \subset \Gamma_{\text {cont }}(|\mathbb{E}|)
$$

To recognize when a section

$$
\sigma:|\mathbb{M}| \rightarrow|\mathbb{E}|
$$

is a section of the pf-vector bundle $\mathbb{E}$, let us fix a vector bundle atlas $\tilde{a}: \mathbb{E} \rightarrow \mathbb{E}$. We look at the $\tilde{a}$-components $\left(\sigma^{0}, \sigma^{1}, \ldots\right)$ of $\sigma$ and we view them as functions depending on the $a$-components $\left(x_{0}, x_{1}, \ldots,\right)$ of $x$,

$$
\sigma_{k}=\sigma_{k}\left(x_{0}, x_{1}, \ldots\right) \in \mathbb{E}_{k} \quad\left(\text { a section over }|\mathbb{M}| \text { of the pull-back of } \mathbb{E}_{k}\right)
$$

Then $\sigma$ is a section of the pf-vector bundle $\mathbb{E}$ if and only if $\sigma_{k}$ depends only on a finite number of entries: there exists $m_{k} \geq k$ large enough and $s_{k}$ as in (2.70) such that

$$
\sigma_{k}(x)=s_{k}\left(x_{n_{k}}\right) \quad \forall x \in|\mathbb{M}| .
$$

Note that the last condition (existence of $s_{k}$ ) can also be required only locally and that gives rise to what we would call "smooth sections of $|\mathbb{E}|$ ", and resulting (strict) inclusions

$$
\Gamma(\mathbb{E}) \subset \Gamma_{\text {smooth }}(|\mathbb{E}|) \subset \Gamma_{\text {cont }}(|\mathbb{E}|) .
$$

Example 2.A.16. In Example 2.A.13, if $\pi: E \rightarrow M$ is a vector bundle (everything is finite dimensional), then each $J^{k} E$ is a vector bundle over $M$ and then

$$
\pi_{\infty}: J^{\infty} E \rightarrow M
$$

becomes a pf-vector bundle (for $M$ see Example 2.A.12).

Remark 2.A.17 (the structure of $\Gamma(\mathbb{E})$ ). The immediate structure on $\Gamma(\mathbb{E})$, the space of sections of a vector bundle $\mathbb{E} \rightarrow \mathbb{M}$ over a pf-finite manifold, is the algebraic one: it is a vector space, and a module over the ring $C^{\infty}(\mathbb{M})$ of smooth functions.

On the other hand, each vector bundle atlas $a: \mathbb{E} \rightarrow \mathbb{E} \bullet$ gives rise to a decreasing filtration of $\Gamma(\mathbb{E})$ :

$$
\cdots \subset \mathfrak{f}_{2} \subset \mathfrak{f}_{1} \subset \mathfrak{f}_{0} \subset \mathfrak{f}_{-1}=\Gamma(\mathbb{E}),
$$

where $\mathfrak{f}_{k}$ consists of those sections whose $k$-th component vanishes. To obtain a structure that is independent of the atlas, one considers the induced, $\mathfrak{f}_{\bullet}$-adic topology on $\Gamma(\mathbb{E})$, that is: the linear topology for which $\mathfrak{f}_{k} s$ form a basis of neighborhoods of the origin. It is a simple exercise to check that this topology does not depend on the choice of the atlas.

In conclusion, $\Gamma(\mathbb{E})$ is a topological vector space and $C^{\infty}(\mathbb{M})$-module. Of course, one could also say that $\Gamma(\mathbb{E})$ is a topological $C^{\infty}(\mathbb{M})$-module, where $C^{\infty}(\mathbb{M})$ is endowed with the discrete topology. 


\section{A.3 The tangent bundle and vector fields}

There are two basic examples of vector bundles to have in mind. First of all the trivial line bundle which, where we recover the notion of smoothness and the sequence of inclusions (2.72) becomes (2.69).

The other basic example is the tangent bundle of a pf-manifold M. For an intrinsic approach one defines $T_{x} \mathbb{M}$ as the space of derivations at $x, \delta_{x}: C^{\infty}(\mathbb{M}) \rightarrow$ $\mathbb{R}$, and then one sets $T \mathbb{M}=\cup_{x} T_{x} \mathbb{M}$. It follows that, for any pf-atlas $a: \mathbb{M} \rightarrow \mathbb{M}_{\bullet}$, one obtains a pf-atlas $T \mathbb{M} \rightarrow T \mathbb{M}_{\bullet}$, where the tangent tower of $\mathbb{M}_{\bullet}$ is, of course,

$$
T \mathbb{M}_{\bullet}: \quad \ldots \longrightarrow T \mathbb{M}_{k} \stackrel{d \pi_{k}}{\longrightarrow} T \mathbb{M}_{k-1} \longrightarrow \ldots \longrightarrow T \mathbb{M}_{2} \stackrel{d \pi_{2}}{\longrightarrow} T \mathbb{M}_{1} \stackrel{d \pi_{1}}{\longrightarrow} T \mathbb{M}_{0} .
$$

The fact that $T \mathbb{M}=\lim _{\longleftarrow} T \mathbb{M}_{k}$ is shown in [33] and can also serve as definition of $T \mathrm{M}$ (a less intrinsic but perhaps more practical one). $|\mathbb{M}|$ :

The tangent bundle gives rise to the notion of smooth vector fields of $\mathbb{M}$ and

$$
\mathfrak{X}(\mathbb{M}) \subset \mathfrak{X}(|\mathbb{M}|) .
$$

As a particular case of sections of vector bundles, $\mathfrak{X}(\mathbb{M})$ is a topological module over $C^{\infty}(\mathbb{M})$. As in the standard situation, vector fields act on smooth functions; and, using [33], Thm 3.26, one finds

$$
\mathfrak{X}(\mathbb{M}) \cong \operatorname{Der}\left(C^{\infty}(\mathbb{M})\right), \quad \mathfrak{X}(|\mathbb{M}|) \cong \operatorname{Der}\left(C^{\infty}(|\mathbb{M}|)\right) .
$$

In particular, vector fields still come endowed with a Lie bracket satisfying the standard Leibniz identity. With the notion of tangent bundle and the Lie bracket of vector fields at hand, one can talk about distributions and involutivity in pfmanifolds $\mathbb{M}$, Ehresmann connections, etc.

Example 2.A.18 (the Cartan distribution). We continue with the notations from Example 2.A.13, for a fibration $\pi: R \rightarrow M$. Any section $\sigma \in \Gamma(R)$ induces a section $j^{k} \sigma$ of $J^{k} R \rightarrow M$, and sections of $J^{k} R$ of this type are called holonomic. The Cartan distribution

$$
\mathcal{C}_{k} \subset T J^{k} R
$$

is defined so that it detects which sections of $J^{k} R$ are holonomic: they must be tangent to $\mathcal{C}_{k}$. Actually one can force this property into the definition and define $\mathcal{C}_{k}$ as the space of vectors that are tangent to such holonomic sections (another description using 1-forms will be recalled below). All together define

$$
\mathcal{C}_{\infty} \subset T J^{\infty} R
$$

as a distribution on the pf manifold; unlike its finite versions $\mathcal{C}_{k}, \mathcal{C}_{\infty}$ is involutive.

Example 2.A.19. And here is an interesting example of pf-connection. Consider the pf-vector bundle $J^{\infty} E \rightarrow M$ associated to a vector bundle $E \rightarrow M$ as in Example 2.A.16. It is well-known that $J^{\infty} E$ carries a canonical connection

$$
\nabla: \mathfrak{X}(M) \times \Gamma\left(J^{\infty} E\right) \rightarrow \Gamma\left(J^{\infty} E\right)
$$

which can be used, again, to detect the holonomic sections as the ones that are flat w.r.t. $\nabla$. Actually, as with the Cartan distribution, this property can be used 
to force the definition of $\nabla$. It is interesting to remark that $\nabla$ is of a true pf-nature: at each level $k$ it does not descend to a connection on $J^{k} E$ but to an operator

$$
\nabla^{k}: \mathfrak{X}(M) \times \Gamma\left(J^{k} E\right) \rightarrow \Gamma\left(J^{k-1} E\right)
$$

which is a connection "relative to $l: J^{k} E \rightarrow J^{k-1} E^{\text {" }}$ in the sense that the Leibniz identity takes the form

$$
\nabla_{X}^{k}(f \sigma)=f \nabla_{X}^{k}(\sigma)+L_{X}(f) l(\sigma) .
$$

Similar to the involutivity of the Cartan distribution, $\nabla$ is a flat connection. In the literature, it is known as the Spencer operator on $J^{\infty} E$. The list of literature concerning Spencer operators is extensive; see, for example, [63].

\section{A.4 Differential forms}

We now move to differential forms. We are interested in the general case with coefficients.

Definition 2.A.20. Let $\mathbb{M}$ be a pf-manifold and let $\mathbb{E}$ be a pf-vector bundle over $\mathbb{M}$. A $q$-differential form on $\mathbb{M}$ with coefficients in $\mathbb{E}$ is any $q$-alternating, $C^{\infty}(\mathbb{M})$ multilinear map

$$
\omega: \underbrace{\mathfrak{X}(\mathbb{M}) \times \ldots \mathfrak{X}(\mathbb{M})}_{q \text { times }} \rightarrow \Gamma(\mathbb{E})
$$

which is continuous w.r.t. the natural ( $\mathfrak{f}$-adic) topology on $\mathfrak{X}(\mathbb{M})$ and $\Gamma(\mathbb{E})$. We denote by $\Omega^{q}(\mathbb{M}, \mathbb{E})$ the space of such. When $\mathbb{E}$ is the trivial line bundle, we talk about $q$-forms on $\mathbb{M}$ and we use the notation $\Omega^{q}(\mathbb{M})$.

Remark 2.A.21. Note that, according to this definition, the continuity of $q$-forms

$$
\omega: \underbrace{\mathfrak{X}(\mathbb{M}) \times \ldots \mathfrak{X}(\mathbb{M})}_{q \text { times }} \rightarrow C^{\infty}(\mathbb{M})
$$

appeal to the discrete topology on $C^{\infty}(\mathbb{M})$. Without thinking at general coefficients, that may seem odd, hence it may be interesting to point out that the actual topology on smooth functions does not have such a big influence: any other topology that is linear (i.e. makes the vector space operations continuous) and for which the origin has a neighborhood that does not contain any line, would produce the same result.

Let us concentrate on forms with trivial coefficients $\mathbb{R}$. For a pointwise description, we will be looking at topological vector spaces $V$ and at $q$-alternating, $\mathbb{R}$-multilinear, continuous functionals on $V$

$$
\mathfrak{a l t}^{q}(V):=\{\omega: \underbrace{V \times \ldots \times V}_{q \text { times }} \rightarrow \mathbb{R}: \text { alternating, } \mathbb{R} \text {-multilinear, continuous }\}
$$

(where, again, $\mathbb{R}$ will be endowed with the discrete topology). Of course, we will be applying this to the tangent spaces $T_{x} \mathbb{M}$ of a pf-manifold $\mathbb{M}$ with their natural f-topology. 
Remark 2.A.22. When $V$ is finite dimensional endowed with the discrete topology, $\mathfrak{a l t}^{q}(V)$ is simply $\Lambda^{q} V^{*}$ - but this is a notation that may be confusing if used in the infinite dimensional case (note that we are not even interested in making sense of $\Lambda^{q} W$ for a general pf-space $W$, and then apply it to some topological dual $V^{*}$ of $V$ ).

Remark 2.A.23. When looking at topological vector spaces $V$ whose topology can be described as the $\mathfrak{f}$-adic topology induced by a filtration

$$
\ldots \subset \mathfrak{f}_{2} \subset \mathfrak{f}_{1} \subset \mathfrak{f}_{0} \subset \mathfrak{f}_{-1}=V
$$

consisting of vector subspaces of finite codimension, then the continuity of a form $\omega$ on $V$ is equivalent to the fact that $\omega$ factors through one of the (finite dimensional) quotients:

$$
V_{k}:=V / \mathfrak{f}_{k},
$$

In other words, considering the sequence of inclusions induced by the canonical projections, one has:

$$
\mathfrak{a l t}^{q}\left(V_{0}\right) \hookrightarrow \mathfrak{a l t}^{q}\left(V_{1}\right) \hookrightarrow \ldots \hookrightarrow \cup_{k} \mathfrak{a} \mathfrak{l t}^{q}\left(V_{k}\right)=\mathfrak{a l t}^{q}(V)
$$

where each $V_{k}$ is finite dimensional hence $\mathfrak{a l t}^{q}\left(V_{k}\right)$ is the standard $\Lambda^{q} V_{k}^{*}$.

Proposition 2.A.24. Let $\mathbb{M}$ be a pf-manifold. Then:

(a) any $\omega \in \Omega^{q}(\mathbb{M})$ is pointwise, i.e. there exists a family

$$
\left\{\omega_{x} \in \mathfrak{a l t}^{q}\left(T_{x} \mathbb{M}\right): x \in \mathbb{M}\right\},
$$

inducing it in the sense that

$$
\omega\left(X^{1}, \ldots, X^{q}\right)(x)=\omega_{x}\left(X_{x}^{1}, \ldots, X_{x}^{q}\right)
$$

for all $X^{1}, \ldots, X^{q} \in \mathfrak{X}(\mathbb{M}), x \in \mathbb{M}$.

(b) if a $: \mathbb{M} \rightarrow \mathbb{M}_{\bullet}$ is a pf-atlas for $\mathbb{M}$ then any usual differential form at some level $k$, $\eta \in \Omega^{q}\left(\mathbb{M}_{k}\right)$, can be promoted to a form on $\mathbb{M}, \tilde{\eta} \in \Omega^{q}(\mathbb{M})$, uniquely characterised by:

$$
\tilde{\eta}_{x}=\eta_{a_{k}(x)} \circ\left(\left(d a_{k}\right)_{x}, \ldots,\left(d a_{k}\right)_{x}\right)
$$

Moreover, the resulting forms exhaust all the differential forms on $\mathbb{M}$ :

$$
\Omega^{q}\left(\mathbb{M}_{0}\right) \hookrightarrow \Omega^{q}\left(\mathbb{M}_{1}\right) \hookrightarrow \ldots \hookrightarrow \bigcup_{k} \Omega^{q}\left(\mathbb{M}_{k}\right)=\Omega^{q}(\mathbb{M})
$$

In this statement, the "inclusions" $\Omega^{q}\left(\mathbb{M}_{k-1}\right) \hookrightarrow \Omega^{q}\left(\mathbb{M}_{k}\right)$ are, of course, induced by the projection maps $\pi: \mathbb{M}_{k} \rightarrow \mathbb{M}_{k-1}$, but we will omit $\pi^{*}$ from the notation. Also, in the previous construction, $\tilde{\eta}$ deserves the name $a_{k}^{*}(\eta)$ but, again, in what follows we will be omitting $a_{k}^{*}$ (as well as the tilde) from the notation. 
Proof. First note that any $v \in T_{x} \mathbb{M}$ can be written as $v=X_{x}$ for some $X \in \mathfrak{X}(\mathbb{M})$. To see this, at each level $k$ we look at $v_{k}=\left(d a_{k}\right)_{x}(v) \in T_{x_{k}} \mathbb{M}_{k}$ and we consider $X_{k} \in \mathfrak{X}\left(\mathbb{M}_{k}\right)$ extending $v_{k}$. However, one can proceed inductively and, at each stage, arrange that $X_{k}$ is projectable to $X_{k-1}$. Then the $X_{k}$ 's together define a vector field $X$ with the desired properties (see also [33], Lemma 3.15).

This implies the uniqueness of each $\omega_{x}$. For the existence we are left with checking that $\omega(X,-, \ldots,-)(x)=0$ whenever $X$ vanishes at $x$. Using an atlas $a: \mathbb{M} \rightarrow \mathbb{M}_{\bullet}$, since $\omega$ is continuous, it follows that it vanishes on some $\mathfrak{f}_{k}$, for some $k$. Using $C^{\infty}(\mathbb{M})$-linearity it suffices to show that

$$
X \equiv f \cdot Y \bmod \mathfrak{f}_{k}, \text { for some } f \in C^{\infty}(\mathbb{M}) \text { vanishing at } x, Y \in \mathfrak{X}(\mathbb{M}) \text {. }
$$

For that: look at the $k$-th component $X_{k} \in \Gamma\left(\mathbb{M}_{m_{k}}, \pi^{*} T \mathbb{M}_{k}\right)$ and write it as $f \cdot Y_{k}$ for some section $Y_{k}$ of the same bundle and $f \in C^{\infty}\left(\mathbb{M}_{k}\right)$ vanishing at $a_{k}(x)$; then extend $Y_{k}$ to a $Y \in \mathfrak{X}(\mathbb{M})$ and also interpret $f$ as a smooth function on $\mathbb{M}$. For (b) we have to check that the resulting $\tilde{\eta}$ is continuous, i.e. that it vanishes on some of the members of the filtration; but it clearly vanishes on $\mathfrak{f}_{k}$.

Finally, to see that any $\omega \in \Omega^{q}(\mathbb{M})$ arises as some $\tilde{\eta}$ (living at some level $k$ ) choose, of course, the $k$ so that $\omega$ vanishes on $\mathfrak{f}_{k}$. And, by the same arguments as above, note that any $X_{k} \in \mathfrak{X}\left(\mathbb{M}_{k}\right)$ can be realised as the $k$-th component of some $X \in \mathfrak{X}(\mathbb{M})$ (of course unique modulo $\mathfrak{f}_{k}$ ).

Note that all the usual operations and formulas valid for differential forms now make sense in the pf-setting. The ones that are relevant for us in the main body are: wedge products, interior products, Cartan magic formula, de Rham differential and the Koszul formula.

Returning now to forms with general coefficients $\mathbb{E}$, it is pretty straightforward to extend the previous discussion. The bottom line will be that the continuity condition for a form (2.75) translates into the fact that, for any pf-vector bundle atlas $\tilde{a}: \mathbb{E} \rightarrow \mathbb{E}_{\bullet}$, and for any $k$, the $k$-component $\omega_{k}$ (taking values in $\left.\Gamma\left(\mathbb{E}_{k}\right)\right)$, comes from a form at a finite step $m_{k}$

$$
\omega_{k} \in \Omega^{q}\left(\mathbb{M}_{m_{k}}, \mathbb{E}_{k}\right) .
$$

More formally,

$$
\Omega^{q}\left(\mathbb{M}_{\bullet}, \mathbb{E}_{\bullet}\right)=\varliminf_{k} \underset{m \geq k}{\lim } \Omega^{q}\left(\mathbb{M}_{m}, \pi^{*} \mathbb{E}_{k}\right)
$$

where, of course, $\pi=\pi_{m, k}: \mathbb{M}_{m} \rightarrow \mathbb{M}_{k}$ is the tower projection.

Example 2.A.25. As in the finite dimensional setting, connections $\nabla$ on $\mathbb{E}$ can be interpreted as operators

$$
d_{\nabla}: \Gamma(\mathbb{E}) \rightarrow \Omega^{1}(\mathbb{M}, \mathbb{E}),
$$

and then they are in 1-1 correspondence with operators on $\Omega^{*}(\mathbb{M}, \mathbb{E})$ satisfying the Leibniz identity. Here all the objects are in the pf-sense hence, in principle, also the connections do not have to come from connections on vector bundles $\mathbb{E}_{k}$. That is a phenomenon that we have seen already in the case of the standard connection (2.73) on the infinite jet space $J^{\infty} E$. Note that, in that example, $d_{\nabla}$ is reflected at level $k$ as

$$
d_{\nabla^{k}}: \Gamma\left(J^{k} E\right) \rightarrow \Omega^{1}\left(M, J^{k-1} E\right)
$$


Example 2.A.26 (Cartan forms). Let us return to a fibration $\pi: R \rightarrow M$ as in Example 2.A.13 and we recall the description of the Cartan distribution (see Example 2.A.18) as the kernel of a 1-form, the so-called Cartan form

$$
\omega \in \Omega^{1}\left(J^{\infty} R ; \mathcal{V}\right)
$$

First of all, $\mathcal{V}$ is the vector bundle over $J^{\infty} R$ which, at level $k$, is the vertical bundle of $J^{k} R \rightarrow M$ :

$$
V^{k}:=T^{\mathrm{vert}} J^{k} R \text {. }
$$

With this, the components of $\omega$ will be of type

$$
\omega^{k} \in \Omega^{1}\left(J^{k} R ; \pi^{*} v^{k-1}\right)
$$

(hence again of pf-nature!), where $\pi$ is the projection $\pi_{k, k-1}$ from $J^{k} R$ to $J^{k-1} R$. In particular, for the jet spaces of type $J^{k}(M, N)$ (see Example 2.A.13) $\omega^{k}$ is given by

$$
\omega_{j_{x}^{k} f}^{k}=d_{j_{x}^{k} f}\left(p^{k, k-1}\right)-d_{x}\left(j^{k-1} f\right) \circ d_{j_{x}^{k} f}(s)
$$

Finally, note that the differential forms in the previous sense are not part of the sheaf on $\mathbb{M}$ viewed as a topological space (which we denote by $|\mathbb{M}|$ ) but rather of a pf-sheaf on $\mathbb{M}$ viewed as a pf-manifold. Of course,

$$
\mathcal{O} p(|\mathbb{M}|) \ni U \mapsto \Omega^{q}(U)
$$

is a preseheaf.

Definition 2.A.27. The sheaf of $q$-forms on $|\mathbb{M}|$, denoted $\Omega_{|\mathbb{M}|}^{q}$, is the sheaf on $|\mathbb{M}|$ associated to the sheaf (2.78). The space of its global sections is denoted $\Omega^{q}(|\mathbb{M}|)$. Similarly for forms with coefficients.

While the elements of $\Omega^{q}(\mathbb{M})$ are $q$-alternating, $C^{\infty}(\mathbb{M})$-multilinear maps (2.76) which are required to come from a $q$-form on some $\mathbb{M}_{k}$, the elements of $\Omega^{q}(|\mathbb{M}|)$ can be described similarly, but imposing the same condition only locally.

\section{A.5 Inverse pf-sheaves (and vector bundles)}

We now move on and discuss the other type of vector bundles and sheaves on pf-manifolds: the inverse ones. Here, differently from the case of direct vector bundles/sheaves, the discussion for sheaves is simpler. Because of this and given the examples that we have in mind, we concentrate on sheaves first. In Example 2.A.33 we will clarify the relation between direct and inverse vector bundles/sheaves.

Definition 2.A.28. A sheaf $\mathcal{S}_{\bullet}$ on a tower $\mathbb{M}_{\bullet}$ is a sequence of sheaves $\mathcal{S}_{k}$ on $\mathbb{M}_{k}$ together with sheaf morphisms $\phi_{k}: \pi_{k}^{*} \mathcal{S}_{k-1} \rightarrow \mathcal{S}_{k}$ for each $k$.

Given a second sheaf $\mathcal{S}_{\bullet}^{\prime}$ on another tower $\mathbb{N}_{\bullet}$, a concrete morphism of sheaves $F_{\bullet}: \mathcal{S}_{\bullet} \rightarrow \mathcal{S}_{\bullet}^{\prime}$ consists of a strict morphism of towers

$$
f_{\bullet}=\left\{f_{k}: \mathbb{M}_{m_{k}} \rightarrow \mathbb{N}_{k}\right\}_{k \geq 1} \quad \text { with } m_{1}<m_{2}<\ldots
$$


together with morphisms of sheaves over $\mathbb{M}_{m_{k}}$

$$
F_{k}: f_{k}^{*} \mathcal{S}_{k}^{\prime} \rightarrow \mathcal{S}_{m_{k}}
$$

satisfying the coherence condition (for the notations, see the explanation below).

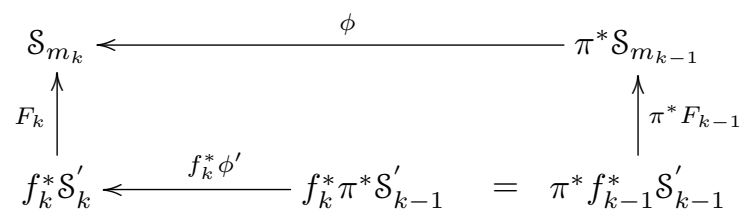

Given another concrete morphism of sheaves $G_{\bullet}: \mathcal{S}_{\bullet} \rightarrow \mathcal{S}_{\bullet}^{\prime}$, with concrete morphism of towers

$$
g_{\bullet}=\left\{g_{k}: \mathbb{M}_{l_{k}} \rightarrow \mathbb{N}_{k}\right\}_{k \geq 1} \quad \text { with } l_{1}<l_{2}<\ldots,
$$

we say that $F_{\bullet}$ and $G_{\bullet}$ are equivalent if for each $k$ there exists $K$ larger than $n_{k}$ and $l_{k}$ such that, as in the definition of equivalence of strict morphisms of towers, one has a commutative diagram

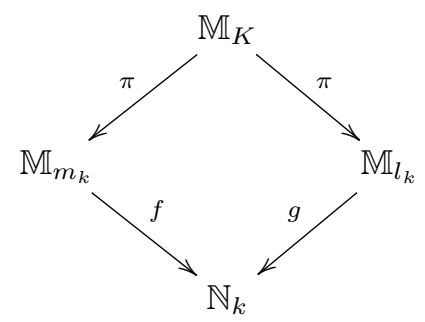

as well as a commutative diagram of sheaves over $\mathbb{M}_{K}$ :

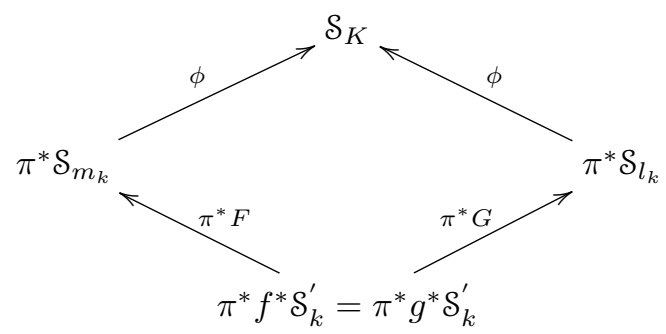

Finally, a morphism between two sheaves (over towers) is an equivalence class of concrete morphisms.

As a convention for our diagrams:

- for notational simplicity we omit the missing indices for $\phi, \phi^{\prime}$ and the tower projections $\pi$; on the diagram they should be clear by looking at the domains of the arrows.

- we also denote by $\pi$ and $\phi$ the composition of consecutive $\pi_{k}{ }^{\prime}$ s and $\phi_{k}$, respectively. 
And now we move to pf-manifolds.

Definition 2.A.29. Let $\mathbb{M}$ be a pf-manifold and let $\mathcal{S}_{|\mathbb{M}|}$ be a sheaf on the topological space $|\mathbb{M}|$ (underlying $\mathbb{M}$ ). A pf-atlas for $\mathcal{S}_{|\mathbb{M}|}$ is a pf-atlas $a: \mathbb{M} \rightarrow \mathbb{M} \bullet$ of $\mathbb{M}$, together with a sheaf $\left(\mathcal{S}_{\bullet}, \phi_{\bullet}\right)$ on the tower $\mathbb{M}_{\bullet}$ and an isomorphism of sheaves over $|\mathbb{M}|$ :

$$
\tilde{a}: \lim _{\longrightarrow} a_{k}^{*} \mathcal{S}_{k} \cong \mathcal{S}_{|\mathbb{M}|} \cdot
$$

Two such pf-atlases $\tilde{a}$ and $\tilde{a}^{\prime}$ are said to be equivalent (as pf-atlases for $\mathcal{S}_{|\mathbb{M}|}$ ) if they are equivalent as sheaves of towers, by isomorphisms that are compatible with $\tilde{a}$ and $\tilde{a}^{\prime}$.

Finally, a pf-sheaf $\mathcal{S}$ on $\mathbb{M}$, is a sheaf $\mathcal{S}_{|\mathbb{M}|}$ over $|\mathbb{M}|$ together with an equivalence class of pf-atlases.

Note that the isomorphism (2.79), hence also the inductive limit there, is within the category of sheaves; and the connecting maps are induced by the $\phi_{k}{ }^{\prime}$ s. This means that we have morphisms of sheaves

$$
\tilde{a}_{k}: a_{k}^{*} \mathcal{S}_{k} \rightarrow \mathcal{S}_{|\mathbb{M}|}
$$

which are compatible with the $\phi_{k} \mathrm{~s}$ :

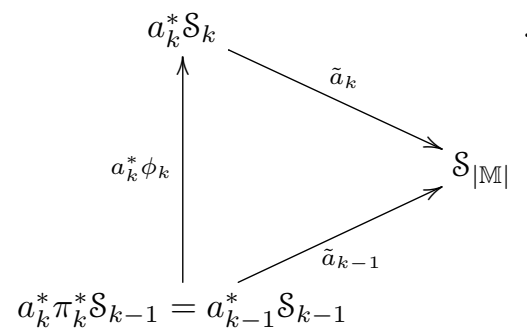

Furthermore, $\mathcal{S}_{|\mathbb{M}|}$ is universal among sheaves equipped with such diagrams. The fact that $\tilde{a}=\lim \tilde{a}_{k}$ is an isomorphism of sheaves boils down to a similar stalkwise statement. However, such isomorphisms do not hold at the level of sections; what happens there is the following:

- the spaces $\Gamma\left(\mathbb{M}_{k}, \mathcal{S}_{k}\right)$ form an inductive system

$$
\phi: \Gamma\left(\mathbb{M}_{k}, \mathcal{S}_{k}\right) \rightarrow \Gamma\left(\mathbb{M}_{k+1}, \mathcal{S}_{k+1}\right),
$$

- each $\Gamma\left(\mathbb{M}_{k}, \mathcal{S}_{k}\right)$ injects into $\Gamma\left(|\mathbb{M}|, \mathcal{S}_{|\mathbb{M}|}\right)$ via the map $\tilde{a}_{k}$ (and the base map $\left.a_{k}\right)$

$$
\tilde{a}_{k}: \Gamma\left(\mathbb{M}_{k}, \mathcal{S}_{k}\right) \hookrightarrow \Gamma\left(|\mathbb{M}|, \mathcal{S}_{|\mathbb{M}|}\right)
$$

and, together, one obtains

$$
\underset{k}{\lim } \Gamma\left(\mathbb{M}_{k}, \mathcal{S}_{k}\right) \cong \bigcup_{k} \tilde{a}_{k}\left(\Gamma\left(\mathbb{M}_{k}, \mathcal{S}_{k}\right)\right) \subset \Gamma\left(|\mathbb{M}|, \mathcal{S}_{|\mathbb{M}|}\right) .
$$

It is not difficult that the resulting subspace of $\Gamma(\mathbb{M}, \mathcal{S})$ does not depend on the atlas $\tilde{a}$ of $\mathcal{S}$. 
Definition 2.A.30. For any pf-sheaf $\mathcal{S}$ on a pf-manifold $\mathbb{M}$, the resulting subspace of $\Gamma\left(|\mathbb{M}|, \mathcal{S}_{|\mathbb{M}|}\right)$ is called the space of (pf-sections) of $\mathcal{S}$, denoted

$$
\Gamma(\mathbb{M}, \mathcal{S}) \subset \Gamma\left(|\mathbb{M}|, \mathcal{S}_{|\mathbb{M}|}\right)
$$

Hence it consists of sections $s$ of $\mathcal{S}_{|\mathbb{M}|}$ with the property that for a/any pf-atlas of $\mathcal{S}$, there exists a $k$ and a section $s_{k} \in \Gamma\left(\mathbb{M}_{k}, \mathcal{S}_{k}\right)$ such that

$$
s(x)=\tilde{a}_{k}\left(s_{k}\left(a_{k}(x)\right)\right) .
$$

Note that the entire discussion about sheaves, starting with Definition 2.A.28 and 2.A.29, makes sense if we replace the word "sheaf" by "vector bundles". The resulting notions will be coined as inverse vector bundles on towers and on pfmanifolds.

Example 2.A.31. The basic example is, of course, the pf-sheaf of $q$-forms, denoted $\Omega_{\mathbb{M}^{1}}^{q}$. The underlying sheaf on $|\mathbb{M}|$ is $\Omega_{|\mathbb{M}|}^{q}$ from Definition 2.A.27 and pf-atlases are obtained from atlases $a: \mathbb{M} \rightarrow \mathbb{M}$ • of $\mathbb{M}$, using $\Omega_{\mathbb{M}_{k}}^{q}$ over each $\mathbb{M}_{k}$ (and the required sheaf morphisms are given by pull-back of forms).

Example 2.A.32. Any (inverse) pf-vector bundle $\mathbb{E}$ on $\mathbb{M}$ gives rise to a (inverse) pf-sheaf $\Gamma_{\mathbb{E}}$ of sections.

Example 2.A.33. Any of the usual operations with vector bundles which are covariant in all arguments (e.g. direct sums, tensor products, tensor powers, etc) can be lifted right away to both (direct/inverse) types of pf-vector bundles. The situation is different for constructions that are contravariant in one of the termsthe basic example being that of taking duals. That is precisely the operation that changes direct vector bundles into inverse ones, and the other way around.

In particular, we see that for any pf-manifold $\mathbb{M}$, we can talk about $T^{*} \mathbb{M}$, as well as $\Lambda^{q} T^{*} \mathbb{M}$, as inverse vector bundles over $\mathbb{M}$. Passing to sections (cf. the previous example), one recovers the sheaf of $q$-forms.

\section{A.6 Oids in the pro-finite context}

Most of the basic concepts on Lie groupoids and Lie algebroids can be adapted to the pro-finite setting in a manner that is rather straightforward, though occasionally tedious. Furthermore, given the examples we have in mind, and to avoid further complications, in the main body of the chapter we will not even use some of the most general concepts. For instance, the most general concept of pf-groupoid $\Sigma \rightrightarrows \mathbb{M}$ comes with $\Sigma_{k} \mathrm{~s}, \mathbb{M}_{k} \mathrm{~s}$ and the structure maps (source/target, multiplication) are just pf-maps (hence may lower the index $k$ ). However, the examples that we encounter in the main body are most of the time over a finite dimensional base $\mathbb{M}$ and they are always "strict" in the following sense.

Definition 2.A.34. A strict Lie pf-groupoid is a topological groupoid $\Sigma \rightrightarrows \mathbb{M}$, where $\Sigma$ and $\mathbb{M}$ are pf-manifolds which admit strict pf-atlases, i.e. atlases $\tilde{a}: \Sigma \rightarrow$ $\Sigma_{\bullet}, a: \mathbb{M} \rightarrow \mathbb{M}_{\bullet}$, where each $\Sigma_{k}$ is a Lie groupoid over $\mathbb{M}_{k}$ with structure maps compatible with those of $\Sigma$ (towers of Lie groupoids). We say that $\Sigma$

- is proper if all the $\Sigma_{k} \rightrightarrows \mathbb{M}_{k}$ may be chosen to be proper. 
- has (cohomologically) l-connected fibers, if all the $\Sigma_{k} \rightrightarrows \mathbb{M}_{k}$ may be chosen to have this property.

A Lie pf-subgroupoid of $\Sigma$ is any subgroupoid $\Upsilon \subset \Sigma$ which is itself a (strict) Lie pf-groupoid, such that $\Sigma$ admits a strict pf-atlas $\tilde{a}: \Sigma \rightarrow \Sigma_{\bullet}$ with the property that $\left.\tilde{a}\right|_{\Upsilon}: \Upsilon \rightarrow \Upsilon$ • is a strict pf-atlas of $\Upsilon$, where each $\Upsilon_{k}:=\tilde{a}_{k}(\Upsilon)$ is a Lie subgroupoid of $\Sigma_{k}$.

Similarly, a strict action of such a groupoid on a pf-manifold $P$ is a topological action of $\Sigma$ on $P$ along some pf-map $\mu: P \rightarrow \mathbb{M}$, such that there exists atlases for $\Sigma$ and $\mathbb{M}$ as above, and for $P$, such that the action factors through actions of $\Sigma_{k}$ on $P_{k}$. We define strict principal $\Sigma$-bundles and strict (direct/inverse) representations of $\Sigma$ on pf-vector bundles in an analogous way.

Cochains on $\Sigma$ with values in a representation $\mathbb{E}$ are defined as in the finite dimensional case, see (2.19), except that we use sections in the pf-setting; hence $C^{*}(\Sigma, \mathbb{E})$ is a subcomplex of $C^{*}(|\Sigma|,|\mathbb{E}|)$. The same is true when defining multiplicative forms.

Concerning pf-algebroids $A \rightarrow \mathbb{M}$, the discussion is again quite similar, although here we will not restrict entirely to strict ones. Hence the Lie bracket on $\Gamma(A)$ may come from "brackets" $\Gamma\left(A_{k}\right) \times \Gamma\left(A_{k}\right) \rightarrow \Gamma\left(A_{k-1}\right)$ (hence the Jacobi identity makes sense only on the entire $\Gamma(A)$ ). Nevertheless, if $\Sigma \rightrightarrows \mathbb{M}$ is a strict pf-groupoid, one defined its Lie algebroid $A=\operatorname{Lie}(\Sigma)$ precisely as in the finite dimensional case, and the outcome will be a strict pf-agebroid. Actually, it is not difficult to see that, using strict pf-atlases as in Definition 2.A.34, the Lie algebroids $A_{k}=\operatorname{Lie}\left(\Sigma_{k}\right) \rightarrow \mathbb{M}_{k}$ can be used as strict atlases for $A$ (in particular $A=\lim _{\leftarrow} A_{k}$ ).

On the other hand one has to be a bit careful with the Lie functor at the level of representations, $\operatorname{Rep}(\Sigma) \rightarrow \operatorname{Rep}(A)$, since the defining formula (2.53) uses flows. For that one just remarks that that formula defining $\nabla_{\alpha}(\sigma)(x)$ can be described as the differential at $1_{x}$ of the map

$$
s^{-1}(x) \rightarrow \mathbb{E}_{x}, \quad g \mapsto g \cdot \sigma(t(g)) \in \mathbb{E}_{x},
$$

applied to $\alpha(x)$.

Here are two results that we use in the main body of the chapter.

Lemma 2.A.35. If $\Sigma \rightrightarrows \mathbb{M}$ is a proper strict pf-groupoid and $\mathbb{E}$ is any pf-representation, then $H^{p}(\Sigma, \mathbb{E})=0$ when $p \neq 0$, while when $p=0$ it is the space of invariant sections $\Gamma(\mathbb{E})^{\Sigma}$.

Proof. When the coefficients are trivial (or constant) we can just appeal to the similar finite dimensional result from [21] since, in that case, the cochains are the union of cochains defined at each step of the tower. This is also enough if the coefficent space is an inverse sheaf. For coefficients given by a direct vector bundle $E$ we have to be slightly more careful. We fix an atlas $\Sigma \rightarrow \Sigma_{\text {e consisting }}$ of proper groupoid, with $E_{k} \rightarrow \mathbb{M}_{k}$ representation of $\Sigma_{k} \rightrightarrows \mathbb{M}_{k}$. Let $c$ be a $p$ cochain with $p \neq 0$ such that $\delta(c)=0$ and let $n_{0}<n_{1}<\ldots$ so that the $k$-th component of $c$ comes from $\Sigma_{n_{k}}$ :

$$
c_{k} \in C^{p}\left(\Sigma_{n_{k}}, \mathbb{E}_{k}\right) .
$$


Using the properness of $\Sigma_{n_{k}}$ we can write

$$
c_{k}=\delta\left(u_{k}\right) \quad \text { with } u_{k} \in C^{p-1}\left(\Sigma_{n_{k}}, \mathbb{E}_{k}\right)
$$

We just have to make sure that one can arrange that the two types of resulting $E_{k}$-valued cochains over $\Sigma_{n_{k+1}}$,

$$
\pi^{*}\left(u_{k}\right)=u_{k} \circ(\pi, \ldots, \pi), \quad \pi \circ u_{k+1} \in C^{p-1}\left(\Sigma_{n_{k+1}}, \mathbb{E}_{k}\right)
$$

coincide (where the first $\pi$ is the one of the tower $\Sigma_{\bullet}$, while the second is of the tower $\left.\mathbb{E}_{\bullet}\right)$. We proceed inductively. Once $u_{k}$ was chosen, the previous condition determines part of $u_{k+1}$. Actually, let us decompose $\mathbb{E}_{k+1}=\tilde{E}_{k} \oplus V_{k}$, where $V_{k}$ is the kernel of the map $\mathbb{E}_{k+1} \rightarrow \mathbb{E}_{k}$, and $\tilde{E}_{k}$ is a complement which is $\Sigma_{n_{k+1}}$-invariant (the existence of such a complement is another consequence of properness- see [25]). The elements $v \in \mathbb{E}_{k+1}$ will be decomposed as $v=v^{\prime}+v^{\prime \prime}$ according to this direct sum. Then, for the components of the element $u_{k+1}$ that we are looking for: $u_{k+1}^{\prime}$ is uniquely determined by the condition $\pi\left(u_{k+1}^{\prime}\right)=$ $\pi^{*}\left(u_{k}\right)$, while when choosing $u_{k+1}^{\prime \prime}$ the only condition comes from the requirement $\delta\left(u_{k+1}\right)=c_{k+1}$, i.e.

$$
\delta\left(u_{k+1}^{\prime \prime}\right)=c_{k+1}-\delta\left(u_{k+1}^{\prime}\right)
$$

Note that the right hand side is a cocycle with values in $V_{k}$ (since $\delta\left(u_{k}\right)=c_{k}$ ). Hence using again the result from the finite dimensional case (for the representation $V_{k}$ of $\left.\Sigma_{n_{k+1}}\right)$, one does find the desired $V_{k}$-valued cochain $u_{k+1}^{\prime \prime}$.

The following is a particular case of Theorem 2 from [21] (applied to foliations) extended to the pf-setting.

Proposition 2.A.36. Let $\pi: \mathbb{P} \rightarrow \mathbb{M}$ be a submersion between $p f$-manifolds. Let $\mathcal{C} \subset$ $T \mathrm{M}$ be an involutive distribution, consider its inverse

$$
\widetilde{\mathcal{C}}:=\pi^{!}(\mathcal{C}):=(d \pi)^{-1}(\mathcal{C}) \subset T \mathbb{P} .
$$

Assume that the fibers of $\pi$ are cohomologically l-connected in the pf-sense i.e. after passing to pf-atlases, $\pi$ can be represented by maps whose fibers are cohomologically $l$ connected in the usual sense. Then the map induced by pull-back in the $\mathrm{C}$-cohomologies (the Lie algebroid cohomologies of the subalgebroids $\mathrm{C} \hookrightarrow T \mathrm{M}$ and $\widetilde{\mathrm{C}} \hookrightarrow T \mathbb{P}$ )

$$
\pi^{*}: H^{*}(\mathcal{C}) \rightarrow H^{*}(\widetilde{\mathcal{C}}),
$$

is an isomorphism up to degree $l$ and is injective in degree $l+1$.

Proof. In principle, we just follow the proof of Theorem 2 from [21] in the particular case of foliations (second part of the proof there), taking care of the pf-version. As there, using the same construction of the spectral sequence, one reduces everything to proving that

$$
H^{i}\left(\mathcal{F}_{\pi}, \mathbb{E}\right)=0 \quad \text { for all } i \in\{1, \ldots, l\},
$$

where

$$
\mathcal{F}_{\pi}:=\operatorname{Ker}(d \pi)
$$


(another involutive distribution) and $\mathbb{E}$ is any pf-vector bundle over $\mathbb{M}$. Here the coefficients $\pi^{*} \mathbb{E}$ are endowed with the canonical $\mathcal{F}_{\pi}$-connection, uniquely determined by the condition that pull-back sections are flat (and of relevance for us are the cases $\mathbb{E}=\Lambda^{q} \mathcal{C}$ ).

As in the proof of the previous result, this follows in principle from its finite dimensional version. To see this, start with any cocycle $\omega \in C^{i}\left(\mathcal{F}_{\pi}, \pi^{*} E\right)$, look at its $k$-components $\omega^{k}$, each one of them comes from the complex over some $\mathbb{P}_{n_{k}}$, and apply the finite dimensional result there to write $\omega_{k}=d\left(\eta_{k}\right)$ (for that we need the fibers of $\mathbb{P}_{n_{k}} \rightarrow \mathbb{M}_{n_{k}}$ to be cohomologically l-connected). Again, one has to make sure that the consecutive $\eta_{k} \mathrm{~s}$ are chosen compatible, but that is done precisely as in the previous proof, just that the situation is a bit simpler now, because we just need vector bundle complements inside each $E_{k+1}$.

Example 2.A.37. The main examples of groupoids that we encounter in this chapter there are groupoids of germs (finite dimensional but possibly huge and nonHausdorff) and groupoids of jets. The second type sit inside the tower of full jet groupoids associated to (finite dimensional) manifolds $\mathbf{X}$

$$
\Pi^{k}=\Pi^{k}(\mathbf{X}):=\left\{j_{x}^{k} f: f \in \operatorname{Diff}_{\mathrm{loc}}(\mathbf{X}), x \in \operatorname{domain}(f)\right\},
$$

which are groupoids over $\mathbf{X}$ with source $s\left(j_{x}^{k} f\right)=x$, target $t\left(j_{x}^{k} f\right)=f(x)$ and multiplication

$$
j_{f(x)}^{k} g \cdot j_{x}^{k} f=j_{x}^{k}(f \circ g) .
$$

For $k$ finite $\Pi^{k}$ inherits a smooth structure from the fact that it sits as an open in the $k$-jet space $J^{k}(\mathbf{X}, \mathbf{X})$. All-together, one has a tower of Lie groupoids

$$
\Pi^{\infty}: \ldots \rightarrow \Pi^{2} \rightarrow \Pi^{1} \rightarrow \Pi^{0}=\mathbf{X} \times \mathbf{X}
$$

and $\Pi^{\infty} \rightrightarrows B$ becomes a strict pf-groupoid.

Corresponding to this there is a pf-vector bundle over $\mathbf{X}$,

$$
\mathfrak{T}^{\infty}: \ldots \rightarrow \mathfrak{T}^{2} \rightarrow \mathfrak{T}^{1} \rightarrow \mathfrak{T}^{0}=T \mathbf{X},
$$

where

$$
\mathfrak{T}^{k}:=J^{k} T \mathbf{X} \rightarrow \mathbf{X}
$$

consists of $k$-jets of vector fields. Each $\mathfrak{T}^{k}$ with $k$ finite carries a canonical structure of Lie algebroid over $\mathbf{X}$ - the unique one with anchor the projection $\pi=\pi_{k, 0}$ : $\mathfrak{T}^{k} \rightarrow \mathfrak{T}^{0}=T \mathbf{X}$ and with bracket satisfying

$$
\left[j^{k} X, j^{k} Y\right]=j^{k}[X, Y] \text {. }
$$

Of course, when $k=\infty$, the previous discussion makes $\mathfrak{T}^{\infty}$ into a strict pfalgebroid over $\mathbf{X}$. Actually, this is precisely the Lie algebroid corresponding to the jet groupoids: the identification of $j_{x}^{k} X$ with a tangent vector to the $s$-fiber of $\Pi^{k}$, at the unit, is

$$
\left.j_{x}^{k} X \mapsto \frac{d}{d t}\right|_{t=0} j_{x}^{k}\left(\phi_{t}^{X}\right) .
$$

The general discussion of Cartan distributions and forms (see Example 2.A.18 and 2.A.26), applied to the first projection $R=\mathbf{X} \times \mathbf{X} \rightarrow \mathbf{X}$ gives rise to similar objects on $\Pi^{\infty}$. Note that the vertical bundle from Example 2.A.18 becomes 
the pull-back via $t$ of $\mathfrak{T}^{\infty}$; hence using our convention for denoting forms with coefficients in representations, the Cartan form becomes

$$
\omega_{\infty} \in \Omega^{1}\left(\Pi^{\infty}, \mathfrak{T}^{\infty}\right)
$$

(and, over $\Pi^{k}$, it becomes a form with values in $\mathfrak{T}^{k-1}$ ).

The structure that emerges is related to the general discussion of flat Cartan groupoids from the main body of the chapter, as $\Pi^{\infty} \rightrightarrows \mathbf{X}$ becomes a flat Cartan groupoid. The corresponding flat Cartan algebroid is $\left(\mathfrak{T}^{\infty}, \nabla\right)$ where $\nabla$ is the Spencer operator (2.73) in the case $E=T \mathbf{X}$.

Example 2.A.38 (Lie pseudogroups). A similar discussion applies to general (Lie) pseudogroups on $\mathbf{X}$ (the previous example corresponds to the full pseudogroup $\operatorname{Diff}_{\text {loc }}(\mathbf{X})$ ). First of all, for any pseudogroup $\Gamma$ on $\mathbf{X}$, restricting our attention at jets of diffeomorphisms from $\Gamma$, one obtains the tower of groupoids

$$
J^{\infty} \Gamma: \ldots \rightarrow J^{2} \Gamma \rightarrow J^{1} \Gamma \rightarrow J^{0} \Gamma,
$$

where each $J^{k} \Gamma$ sits inside $\Pi^{k}$. The only "problem" is that the groupoids $J^{k} \Gamma$ may fail to be smooth manifolds and the maps in the tower may fail to be submersions.

Definition 2.A.39. A Lie pseudogroup on $\mathbf{X}$ is any pseudogroup with the property that $J^{\infty} \Gamma$ is a smooth pf-subgroupoid of $\Pi^{\infty}$.

It follows that the corresponding algebroids

$$
A^{k}:=\operatorname{Lie}\left(J^{k} \Gamma\right)
$$

are Lie sub-algebroids of $\mathfrak{T}^{k}$, that $A^{\infty}$ becomes a pf-sub-algebroid of $\mathfrak{T}^{\infty}$ and that the action of $\Pi^{k}$ on $\mathfrak{T}^{k-1}$ restricts to an action of $J^{k} \Gamma$ on $A^{k-1}$. Furthermore, the Cartan forms restrict to similar forms

$$
\omega_{k} \in \Omega^{1}\left(J^{k} \Gamma, t^{*} A^{k-1}\right), \quad \omega_{\infty} \in \Omega^{1}\left(J^{\infty} \Gamma, t^{*} A^{\infty}\right),
$$

and the same happens for the Spencer operator on $\mathfrak{T}^{\infty}$.

Remark 2.A.40. Given our convention that al the pf-manifolds are normal, the condition that $\Gamma$ is a Lie subgroupoid includes the condition $J^{\infty} \Gamma=\lim J^{k} \Gamma$. For this reason, it would be desirable to remove normality: this condition is often satisfied, but there are interesting examples when it is not - e.g. for the pseudogroup $\Gamma_{\text {an }}^{n}$ of analytic diffeomorphisms on $\mathbb{R}^{n}$. In fact, $J^{k} \Gamma_{\text {an }}^{n}=J^{k} \Gamma^{n}$, but $J^{\infty} \Gamma_{\text {an }}^{n}$ is strictly contained into $J^{\infty} \Gamma^{n}$ : there are formal power series that are not convergent.

On the other hand, for Lie pseudogroups, $J^{\infty} \Gamma$ is not necessarily infinite dimensional; pseudogroups for which it has finite dimension are called of finite type. For instance, consider the pseudogroup in $\mathbb{R}$ consisting of (locally defined) diffeomorphisms of type

$$
\phi: x \mapsto \frac{a x+b}{c x+d}, \quad \text { with } a, b, c, d \in \mathbb{R} \text { with } a d-b c \neq 0 .
$$

One has

$$
J^{1} \Gamma=\Pi^{1}, \quad J^{2} \Gamma=\Pi^{2}
$$


and, for all $k \geq 2$, the projection $J^{k+1} \Gamma \rightarrow J^{k} \Gamma$ is an isomorphism. This follows from the fact that any diffeomorphism $\phi$ of the previous type satisfies

$$
\phi^{\prime \prime \prime}=\frac{3\left(\phi^{\prime \prime}\right)^{2}}{2 \phi^{\prime}},
$$

hence all the higher derivatives can be expressed in terms of the first two. Notice that from this it also follows that the Cartan distribution on $J^{3} \Gamma$ is involutive. Moreover, the isomorphisms $J^{k+1} \Gamma \rightarrow J^{k} \Gamma$, for $k \geq 3$, induce isomorphisms of the Cartan distributions. This means that the projection

$$
\left(J^{\infty} \Gamma, \omega^{\infty}\right) \rightarrow\left(J^{3} \Gamma, \omega^{3}\right)
$$

is an isomorphism of flat Cartan groupoids. 


\title{
3 On regular actions of pseu- dogroups
}

\author{
II-Eh mi, credevo che galleggiava... \\ - Ma se solo il cerchione pesa trenta chili!
}

- Aldo Baglio e Giovanni Storti, Tre uomini e

una gamba

In this chapter, we review the classical finite order problem for natural bundles $[28,54]$ as a problem concerning actions of étale groupoids that satisfy suitable continuity and smoothness conditions. We recall the correspondence between étale groupoids $\mathcal{G} \rightrightarrows \mathbf{X}$ and pseudogroups $\Gamma$ on $\mathbf{X}$ [36] and use Morita equivalences to describe natural $\Gamma$-bundles [5] in terms of actions of groupoids. The finite order problem is formulated in terms of factorizing the action through Cartan towers $[55,69]$ possessing the same abstract properties of the jet tower of $\Gamma$. A regularity condition on natural $\Gamma$-bundles is encoded by means of a diffeology and a suitable notion of convergence on the étale groupoid corresponding to $\Gamma$. Finally, the classical approach to a proof of the finite order theorem for regular natural $\Gamma$-bundles $[5,28,40,54,61,70]$ is reviewed and some relevant topological insights are discussed.

\subsection{Introduction}

The notion of "natural bundle" in differential geometry can be traced back to Nijenhuis [53]. In simple terms, a natural bundle is a functor $E$ from the category of $n$-manifolds and open embeddings to the category of fiber bundles that is also a section of the base functor; i.e., it sends a manifold $\mathbf{X}$ to a fiber bundle $E \mathbf{X} \rightarrow \mathbf{X}$. The most immediate example of such a functor is the tangent functor, sending $\mathbf{X}$ to the tangent bundle $T \mathbf{X} \rightarrow \mathbf{X}$ and an open embedding $f: \mathbf{X}_{1} \rightarrow \mathbf{X}_{2}$ to its tangent map $T f: T \mathbf{X}_{1} \rightarrow T \mathbf{X}_{2}$. A second example is $F r$, associating to a manifold $\mathbf{X}$ the frame bundle

$$
\operatorname{Fr}(\mathbf{X})=\left\{\left(x, T_{0} \phi\right): x \in \mathbf{X}, \phi:\left(\mathbb{R}^{n}, 0\right) \rightarrow(\mathbf{X}, x) \text { is an open embedding }\right\}
$$

and to an open embedding $f: \mathbf{X}_{1} \rightarrow \mathbf{X}_{2}$ the map $\operatorname{Fr}(f)$ induced by the differential. In a similar way $F r^{k}$, which takes the $k$-th order frame bundle

$$
F r^{k}(\mathbf{X})=\left\{j_{0}^{k} \phi: x \in \mathbf{X}, \phi:\left(\mathbb{R}^{n}, 0\right) \rightarrow(\mathbf{X}, x) \text { is an open embedding }\right\},
$$


is a natural bundle.

These examples are archetypical also in the sense that the total space of the tangent/frame bundle encodes properties of its base manifold; this is essentially true for any natural bundle. In fact, the trivializations and transition functions of the total space of $E \mathbf{X}$ can be canonically constructed out of an atlas of $\mathbf{X}$ : if $\left\{\left(U_{i}, f_{i}\right)\right\}_{i \in I}$, is such an atlas, with transition functions $\left\{\phi_{i j}\right\}_{i, j \in I}$, then $\left.E \mathbf{X}\right|_{U_{i}}$ is diffeomorphic to $E U_{i}$ via $E f_{i}$ and the $E \phi_{i j}$ 's provide a way to glue the $E U_{i}$ 's back and reconstruct $E \mathbf{X}$. In simpler words, the theory of natural bundles is local.

Natural bundles provide a suitable framework for a functorial, geometric approach to PDEs and variational problems with symmetries [29, 40], which explains the attention they received in the past.

The first actual theorem concerning natural bundles that appeared in the literature was the so called finite order theorem. This is the statement we focus on. The starting observation is that, when $f: \mathbf{X}_{1} \rightarrow \mathbf{X}_{2}$ is an open embedding, the map $(E f)_{x}:\left(E \mathbf{X}_{1}\right)_{x} \rightarrow\left(E \mathbf{X}_{2}\right)_{f(x)}$ induced on the fibers by the lift depends only on the germ of $f$ at $x$; this is simply another manifestation of locality. The finite order theorem is a strengthening of locality:

Theorem. Let $E$ be a natural bundle. There exists $k \in \mathbb{N}$ such that, for any open embedding $f: \mathbf{X}_{1} \rightarrow \mathbf{X}_{2}$ between $n$-dimensional manifolds and $x \in \mathbf{X}_{1}$, the map

$$
(E f)_{x}:\left(E \mathbf{X}_{1}\right)_{x} \rightarrow\left(E \mathbf{X}_{2}\right)_{f(x)}
$$

depends only on the $k$-jet of $f$ at $x$.

The natural number $k$ is called the order of $E$. In the spirit of locality of natural bundles, the finite order theorem implies that the transition functions of $\mu_{\mathbf{X}}$ : $E \mathbf{X} \rightarrow \mathbf{X}, E$ being a natural bundle, depend only on the transition functions of $\mathbf{X}$ and their derivatives up to order $k$.

One sees, for example, that $T$ and $F r$ have order 1 , while $F r^{k}$ has order $k$. Any action of the Lie group

$$
G L_{n}^{k}(\mathbb{R})=\left\{j_{0}^{k} \phi: \phi:\left(\mathbb{R}^{n}, 0\right) \rightarrow\left(\mathbb{R}^{n}, 0\right) \text { is a diffeomorphism }\right\}
$$

on some manifold $F$ determines a natural bundle of order $k$. In fact, for any $n$-manifold $\mathbf{X}, F r^{k}(\mathbf{X})$ is a principal $G L_{n}^{k}(\mathbb{R})$-bundle and one can set

$$
E \mathbf{X}:=F r^{k}(\mathbf{X}) \times_{G L_{n}^{k}(\mathbb{R})} F,
$$

the bundle with fiber $F$ associated to $F r^{k}(\mathbf{X})$ through the action of $G L_{n}^{k}(\mathbb{R})$. The finite order theorem can be rephrased by saying that these kind of examples are the only ones possible: a natural bundle comes with an integer $k \in \mathbb{N}$ and necessarily takes values in bundles associated to $k$-th order frame bundles.

The finite order theorem was discussed for the first time in [65] in the particular case of natural vector bundles. In [54] the general case was addressed, additionally assuming $E$ to send smooth families of open embeddings to smooth families of isomorphisms of bundles. Finally, Epstein and Thurston [28] proved the finite order theorem in full generality ${ }^{1}$, getting the smoothness assumption

\footnotetext{
${ }^{1}$ Actually, in far greater generality: they depart from the category of infinitely differentiable manifolds working with functors $E$ sending a $C^{r}$ manifold $\mathbf{X}$ to a $C^{s}$ map $\mu_{\mathbf{X}}: E \mathbf{X} \rightarrow \mathbf{X}, s \leq r$, which are local in the above sense. Furthermore, they prove that $E \mathbf{X}$ is a $C^{s}$-manifold and $\mu_{X}$ is a $C^{s}$-fiber bundle, instead of assuming it.
} 
of [54] as a consequence of the other axioms. A lot of very interesting literature, e.g. $[5,27,40,61,62,70]$, is available concerning generalizations of the statements and arguments of [54] and [28].

Our interest for the finite order theorem comes from our work with Lie pseudogroups and étale groupoids.

The key remark for us is that any natural bundle $E$ associates manifolds to bundles $\mu_{\mathbf{X}}: E \mathbf{X} \rightarrow \mathbf{X}$ that are $\Gamma^{\mathbf{X}}$-spaces, where $\Gamma^{\mathbf{X}}=\operatorname{Diff}_{\text {loc }}(\mathbf{X})$ is the set of germs of embeddings between opens of $\mathbf{X}$. That is, there is an action of the groupoid $\operatorname{Germ}\left(\Gamma^{\mathbf{X}}\right) \rightrightarrows \mathbf{X}$ of germs of elements of $\Gamma^{\mathbf{X}}$ along the map $\mu_{\mathbf{X}}$. Furthermore, the functor $E$ can be reconstructed from the datum of a manifold $\mathbf{X}$ and the $\Gamma^{\mathbf{X}}$-space $\mu_{\mathbf{X}}: E \mathbf{X} \rightarrow \mathbf{X}$. The groupoid $\operatorname{Germ}\left(\Gamma^{\mathbf{X}}\right) \rightrightarrows \mathbf{X}$ comes with an associated tower of groupoids

$$
\operatorname{Germ}\left(\Gamma^{\mathbf{X}}\right) \rightarrow J^{\infty} \Gamma^{\mathbf{X}} \rightarrow \ldots \rightarrow J^{k} \Gamma^{\mathbf{X}} \rightarrow J^{k-1} \Gamma^{\mathbf{X}} \rightarrow \ldots \rightarrow J^{1} \Gamma^{\mathbf{X}} \rightarrow J^{0} \Gamma^{\mathbf{X}} \rightrightarrows \mathbf{X}
$$

where $J^{k} \Gamma^{\mathbf{X}} \rightrightarrows \mathbf{X}$ is the groupoid of $k$-jets of elements of $\Gamma^{\mathbf{X}}$. The finite order theorem can be restated by saying that any $\Gamma^{\mathbf{X}}$-space is actually a $J^{k} \Gamma^{\mathbf{X}}$-space for some $k$.

The tower above is a tower of Lie groupoids where each arrow except the first one is a surjective submersive Lie groupoid map. It is called the canonical Cartan tower of $\Gamma^{\mathbf{X}}$ and plays a fundamental role in the geometric study of PDEs. Such an object makes sense for any pseudogroup $\Gamma$, i.e. any subset of the set $\Gamma^{\mathbf{X}}$ which satisfies group-like and sheaf-like properties, ensuring that $\operatorname{Germ}(\Gamma) \rightrightarrows$ $\mathbf{X}$ can be given the structure of étale groupoid. In old-fashioned terms, $\Gamma$ is a transformation group in the sense of Lie and Engel [44]. We call it Lie pseudogroup (or continuous transformation group), when its canonical Cartan tower

$$
J^{\infty} \Gamma \rightarrow \ldots \rightarrow J^{k} \Gamma \rightarrow J^{k-1} \Gamma \rightarrow \ldots \rightarrow J^{1} \Gamma \rightarrow J^{0} \Gamma \rightrightarrows \mathbf{X}
$$

is a tower of Lie groupoids where each map is a surjective submersive groupoid map. One then sees that the finite order problem makes perfect sense for any Lie pseudogroup: actions of $\operatorname{Germ}(\Gamma)$ can be recasted as natural bundle-like functors, see the $\Gamma$-natural bundles of [5], and one has the

Question. Which conditions on $\Gamma$ ensure that, given a $\operatorname{Germ}(\Gamma)$ action, there is some $k \in \mathbb{N}$ such that the action factors through an action of $J^{k} \Gamma$ ?

This is the problem that we address. We aim at understanding what is needed for a generalization of the arguments of [54] and how everything can be formulated in groupoid-theoretical terms. We do not claim any originality concerning results and/or techniques ${ }^{2}$, but we think that our approach can shed some light on the nature of the problem.

A first advantage of our approach is that it works also for generalized pseudogroups, i.e. subsets of the set of local bisections of Lie groupoids with properties analogous to the pseudogroup ones. This has some unifying power. For instance we treat classical pseudogroups whose orbit spaces exhibit singularity without

\footnotetext{
${ }^{2}$ In particular, the generalization of the finite order problem to categories of manifolds and $a$ subset of the set of open embeddings is not new $[5,40]$.
} 
changing framework. Moreover, generalized pseudogroups include, for example, "pseudogroups" of automorphisms of principal bundles; consequently, a formalism for the finite order problem in the context of generalized pseudogroups includes as a particular case the finite order theorem for gauge natural bundles [27, $29,40]$.

Furthermore, some topological aspects of the finite order problem, implicit in the arguments of [54] and [28], are clarified and made explicit in our discussion. In particular, there are different topologies on a germ groupoid $\operatorname{Germ}(\Gamma)$, and understanding their hierarchy plays a key role for understanding the problem.

In Section 3.2, we recall the basic definitions of pseudogroup and groupoid and the relation between the two; in particular, we recall that a pseudogroup is essentially the same as an étale groupoid and viceversa. We define groupoid actions and Morita equivalences and discuss their relationship with natural bundles and the finite order problem.

In Section 3.3 we define the support of an étale groupoid $\mathcal{G} \rightrightarrows \mathbf{X}$. A support is a finite dimensional Lie groupoid $\Sigma \rightrightarrows \mathbf{X}$ that realizes $\operatorname{Bis}_{\text {loc }}(\mathcal{G})$ as a subset of its set of bisections; when $\mathcal{G}=\operatorname{Germ}(\Gamma)$ and $\Gamma$ is a classical pseudogroup, the pair groupoid $\mathbf{X} \times \mathbf{X} \rightrightarrows \mathbf{X}$ is the most natural choice. A support allows one to introduce some sort of smooth structure on $\mathcal{G}$ and $\mathrm{Bis}_{\text {loc }}(\mathcal{G})$. This is made precise through diffeologies. The elementary theory of diffeologies that we need is recalled in Appendix 3.C.

In Section 3.4 we introduce Cartan resolutions, of which the canonical Car$\tan$ tower is the main example, in full generality. To handle them rigorously we need the theory of profinite dimensional manifolds, discussed briefly in Appendix 3.A. Furthermore, everything is made much simpler by working with Pfaffian groupoids, a concept conceived in [55] as an abstract framework for the study of the geometry of PDEs; the theory relevant to our purposes is recalled in Appendix 3.B. In Section 4 we also discuss some topology induced by a Cartan resolution on $\operatorname{Germ}(\Gamma)$, which will allow to treat the finite order problem more conceptually. We then formulate one of the key Axioms that $\Gamma$ needs to satisfy in order for $\Gamma$-spaces to be of finite order.

Finally, in Section 3.5 we define regular $\Gamma$-spaces as those $\Gamma$-spaces which satisfies a smoothness condition (in the diffeological sense of Section 3.3) and a continuity assumption (with respect to a convergence notion introduced in Section 3.4). Then, we run through the arguments of [54] explaining how they fit into our theoretical framework. The outcome is a proof of Theorem 3.5.2 - which represents an answer to the finite order problem formulated in terms of étale groupoids.

\subsection{Pseudogroups, actions and natural bundles}

Definition 3.2.1. A pseudogroup on a manifold $X$ is a subset $\Gamma$ of the set $\operatorname{Diffl}_{\text {loc }}(\mathbf{X})$ of locally defined diffeomorphisms of $\mathbf{X}$ satisfying:

- a group-like property: $\Gamma$ is closed under composition, inversion and it contains the identity $i d_{\mathbf{X}}$;

- sheaf-like properties: 
- $\Gamma$ is closed with respect to restrictions

- if $\left\{U_{i}\right\}_{i \in I}$ covers an open $U, f_{i}$ are elements of $\Gamma$ such that $\operatorname{Dom}\left(f_{i}\right)=$ $U_{i}$ and there exists $f \in \operatorname{Diff}_{\text {loc }}(\mathbf{X})$ such that $\left.f\right|_{U_{i}}=f_{i}$, then $f \in \Gamma$.

The foundations of pseudogroups can be attributed to Lie and Engel [44] and their theory of "transformation groups" on open subsets of Euclidean spaces.

Example 3.2.2. The simplest example of a pseudogroup is $\Gamma^{\mathbf{X}}:=\operatorname{Diff}_{\text {loc }}(\mathbf{X})$ itself. When $\mathbf{X}=\mathbb{R}^{n}$, we will use the notation $\Gamma^{n}$.

Example 3.2.3. If $\mathbf{X}$ has an analytic structure, the pseudogroup $\Gamma_{\mathrm{an}}^{\mathbf{X}}$ of analytic diffeomorphisms is defined. When $\mathbf{X}=\mathbb{R}^{n}$, we will use the notation $\Gamma_{\text {an }}^{n}$.

Example 3.2.4. When $(\mathbf{X}, \omega)$ is a symplectic manifold, local symplectomorphisms form a pseudogroup on $\mathbf{X}$. Similiarly, if $(\mathbf{X}, g)$ is a Riemannian manifold, local isometries form a pseudogroup. More generally, pseudogroups on $\mathbf{X}$ should be thought of as sets of symmetries of some geometric structure on $\mathbf{X}$.

A large class of examples on $\mathbb{R}^{n}$ is associated to Lie subgroups $G \hookrightarrow G L_{n}(\mathbb{R})$; the set

$$
\Gamma_{G}=\left\{\varphi \in \operatorname{Diff}_{\mathrm{loc}}\left(\mathbb{R}^{n}\right): d_{x} \varphi \in G, x \in \operatorname{dom}(\phi)\right\}
$$

is a pseudogroup. Notice that this example encodes the pseudogroup of local symplectomorphisms of the standard symplectic structure ( $n=2 k$ is even and $G=S p(k)$ ), the pseudogroup of local isometries of the standard metric $(G=O(n))$ and, more generally, all the pseudogroups of symmetries of geometric structures on $\mathbb{R}^{n}$ which can be encoded into a group of linear transformations.

The pseudogroup $\Gamma_{\text {cont }}$ of local contactomorphisms of the standard contact structure on $\mathbb{R}^{2 n+1}$ is an example that cannot be described by such a linear picture.

Example 3.2.5. If $p r: \mathbb{R}^{n+k} \rightarrow \mathbb{R}^{n}$ is the projection on the first $n$-coordinates, the set of locally defined diffeomorphisms of $\mathbb{R}^{n+k}$ projectable over $\mathbb{R}^{n}$ is a pseudogroup on $\mathbb{R}^{n+k}$, denoted by $\Gamma^{n, k}$. $\Gamma^{n, k}$ can actually be described via a Lie subgroup of $G L_{n+k}(\mathbb{R})$.

Example 3.2.6. One has also the pseudogroup of diffeomorphisms of $\mathbb{R}^{n+k}$ vertical over $\mathbb{R}^{n}$. This is a particular case of the pseudogroup $\Gamma^{\mathcal{F}}$ of leafwise diffeomorphisms of a foliated manifold $(\mathbf{X}, \mathcal{F})$.

\subsubsection{Pseudogroups and étale groupoids}

The group-like and sheaf-like properties of pseudogroups can be encoded by means of groupoids and in particular, effective étale groupoids. The idea is quite simple: one looks at the space of germs of elements of $\Gamma$, equipped with the étale topology. Set theoretically, this is the set $\operatorname{Germ}(\Gamma)$ of germs of diffeomorphisms in $\Gamma$. The group-like properties of $\Gamma$ then imply that the set of germs of its elements is a groupoid $\operatorname{Germ}(\Gamma) \rightrightarrows \mathbf{X}$ where

- the source and target maps

$$
s, t: \operatorname{Germ}(\Gamma) \rightarrow \mathbf{X}
$$

send an element $\gamma:=\operatorname{germ}_{x}(\varphi)$ to $x$ and $\varphi(x)$ respectively; 
- compatible arrows $\gamma_{1}, \gamma_{2} \in \operatorname{Serm}(\Gamma)$, i.e. $s\left(\gamma_{1}\right)=t\left(\gamma_{2}\right)$, can be composed to give $\gamma_{1} \cdot \gamma_{2} \in \operatorname{Germ}(\Gamma)$ : one takes representatives $\varphi_{1}, \varphi_{2} \in \Gamma$ such that $\varphi_{2}\left(\operatorname{Dom}\left(\varphi_{2}\right)\right) \subset \operatorname{Dom}\left(\varphi_{1}\right)$ (which is always possible due to the sheaf-like properties of $\Gamma$ ) and defines $\gamma_{1} \cdot \gamma_{2}$ as the germ of the composition $\varphi_{1} \circ \varphi_{2}$ at $x$;

- the inverse of a germ is the germ of the inverse.

$\operatorname{Germ}(\Gamma)$ has two further properties:

- $\operatorname{Germ}(\Gamma)$ can be given the structure of étale groupoid, i.e. a topology structure such that $s, t$ are local homeomorphisms;

- as an étale groupoid, $\operatorname{Germ}(\Gamma)$ is effective, i.e. the functor $\operatorname{Germ}(\Gamma) \rightarrow$ $\operatorname{Germ}\left(\Gamma^{\mathbf{X}}\right)$ is faithful.

The functor $\operatorname{Germ}(\Gamma) \rightarrow \operatorname{Germ}\left(\Gamma^{\mathbf{X}}\right)$ is the obvious one, obtained by seeing elements of a pseudogroups as locally defined diffeomorphisms of $\mathbf{X}$, i.e. elements of $\Gamma^{\mathbf{X}}$. That said, this notion of effectiveness makes sense for any étale groupoid G; this is explained below.

Recall that a Lie groupoid $\Sigma \rightrightarrows \mathbf{X}$ is a groupoid such that $\Sigma$ and $\mathbf{X}$ are smooth manifolds, all the structure operations are smooth and the source and target maps are surjective submersions. $\Sigma$ is called the arrow space of $\Sigma \rightrightarrows \mathbf{X}$.

Convention 1. Throughout this chapter, unless otherwise specified (in particular, see Convention 2 on page 159 below)

- smooth manifolds are assumed to be second countable Hausdorff topological spaces;

- the arrow space $\Sigma$ of a Lie groupoid $\Sigma \rightrightarrows \mathbf{X}$ is allowed to be a non-Hausdorff and non second-countable manifold.

An étale groupoid over a smooth manifold $\mathbf{X}$ can always be given a Lie groupoid structure: the coordinates on the arrow space are constructed by pullback via the source and target maps.

Remark 3.2.7. Germ groupoids of pseudogroups might fail to have Hausdorff and/or second countable arrow spaces; for example $\operatorname{Germ}\left(\Gamma^{n}\right)$ is neither Hausdorff nor second countable. This explains the second point in our Convention 1 above.

Any, not necessarily étale, Lie groupoid $\Sigma \rightrightarrows \mathbf{X}$ has an associated set of smooth local bisections $\operatorname{Bisloc}_{\text {loc }}(\Sigma)$, i.e. the set of local smooth sections $\sigma$ of $s$ such that $t \circ \sigma$ is a diffeomorphism. By definition, the target map induces a map $\operatorname{Bis}_{\text {loc }}(\Sigma) \rightarrow \Gamma^{\mathbf{X}}$ respecting restrictions, hence a map

$$
\operatorname{Germ}\left(\operatorname{Bis}_{\text {loc }}(\Sigma)\right) \rightarrow \operatorname{Germ}\left(\Gamma^{\mathbf{X}}\right) \text {. }
$$

When $\Sigma=\mathcal{G}$ is étale, then $\mathcal{G}$ is tautologically homeomorphic to the étale space $\operatorname{Germ}\left(\operatorname{Bis}_{\text {loc }}(\Sigma)\right)$; effectiveness for $\mathcal{G}$ means that the induced map $\mathcal{G} \rightarrow \operatorname{Germ}\left(\Gamma^{\mathbf{X}}\right)$ is a monomorphism.

This also reveals that the image by $t$ of the set of local bisections of any Lie groupoid $\Sigma \rightrightarrows \mathbf{X}$ defines a pseudogroup on $\mathbf{X}$; our discussion shows that for effective étale Lie groupoids one can "invert" this and recover the groupoid. More precisely, we state the following proposition, usually attributed to Haefliger [36]. 
Proposition 3.2.8. One has the correspondence (or, more precisely, the equivalence of categories):

$$
\left\{\begin{array}{c}
\text { pseudogroups } \\
\Gamma \text { on } \mathbf{X}
\end{array}\right\} \stackrel{1-1}{\longleftrightarrow}\left\{\begin{array}{c}
\text { effective étale groupoids } \\
\mathcal{G} \rightrightarrows \mathbf{X}
\end{array}\right\}
$$

Many notions involving pseudogroups become simpler by passing to groupoids, and this is the approach we take throughout the chapter. One example is the notion of action, which is at the core of the origin of pseudogroups but it is more elegantly expressed using groupoids. Recall that a left action of a Lie groupoid $\Sigma \rightrightarrows \mathbf{X}$ on a manifold $P$ along a map $\mu: P \rightarrow \mathbf{X}$ is a smooth map

$$
a: \Sigma_{s} \times{ }_{\mu} P \rightarrow P
$$

satisfying the usual action axioms, that is

- $a\left(g_{2}, a\left(g_{1}, p\right)\right)=a\left(g_{2} g_{1}, p\right), g_{i} \in \Sigma, p \in P$ such that $s\left(g_{2}\right)=t\left(g_{1}\right)$ and $\mu(p)=$ $s\left(g_{1}\right)$

- $a\left(1_{x}, p\right)=p, x \in \mathbf{X}, p \in P$ such that $\mu(p)=x$.

We will write $g \cdot p$ in place of $a(g, p)$.

Convention 2. Throughout this chapter, when $P$ is a $\Sigma$-space along $\mu: P \rightarrow \mathbf{X}$, the manifold $P$ is allowed to be non-Hausdorff and non-second countable (recall Convention 1 on page 158).

Convention 3. Throughout this chapter, when $P$ is a $\Sigma$-space along $\mu: P \rightarrow \mathbf{X}, \mu$ is assumed to be a surjective submersion.

Definition 3.2.9. Let $\Gamma$ be a pseudogroup on $\mathbf{X}$. A $\Gamma$-space $E$ is a surjective submersion $\mu: E \rightarrow \mathbf{X}$ along which $\operatorname{Germ}(\Gamma)$ acts smoothly, i.e. a $\operatorname{Germ}(\Gamma)$-space.

Remark 3.2.10. This is a first instance where working with groupoids proves to be useful. A definition of $\Gamma$-space in terms of the set $\Gamma$ itself would look more involved.

Example 3.2.11. For any $\Gamma, t: \operatorname{Germ}(\Gamma) \rightarrow \mathbf{X}$ itself is a $\Gamma$-space by left multiplication.

Remark 3.2.12. Because of Remark 3.2.7, the example above needs Convention 2.

Example 3.2.13. $T \mathbf{X}$ is always a $\Gamma^{\mathbf{X}}$-space, and actually a $\Gamma$-space for each $\Gamma \subset$ $\Gamma^{\mathbf{X}}$. The same happens for each fiber bundle associated to the frame bundle $\operatorname{Fr}(\mathbf{X})$, i.e. of the form $\operatorname{Fr}(\mathbf{X}) \times F / \sim$ where $F$ is a left $G L_{n}(\mathbb{R})$-space and the quotient is by the diagonal action.

Remark 3.2.14. The equivalence of categories between pseudogroups and effective étale groupoids encodes nicely the sheaf-like and group-like structure of pseudogroups. However, as subsets of $\operatorname{Diff}_{\text {loc }}(\mathbf{X})$, pseudogroups on $\mathbf{X}$ carry some additional structure; namely, the sets of elements of a pseudogroup over some open have a topology (let's say the $C^{\infty}$-topology). Even more, there is a clear notion of smooth families. A smooth family of elements of $\Gamma$ defined over 
an open subset $U \subset \mathbf{X}$ and parametrized by an open subset $W \in \mathbb{R}^{d}, d \in \mathbb{N}$, is a smooth map

$$
F: W \times U \rightarrow \mathbf{X}
$$

such that $F\left(w,{ }_{-}\right) \in \Gamma$ for each $w \in W$. When $W$ is some open interval in $\mathbb{R}$, one recovers isotopies between elements of $\Gamma$ through elements of $\Gamma$.

The correspondence from Proposition 3.2.8 is somehow unsatisfactory since these aspects are lost when passing to étale groupoids. To encode them, one has to look at diffeomorphisms as bisections of the non-étale Lie groupoid $\mathbf{X} \times \mathbf{X}$. We work this out in the next section.

\subsubsection{Lie pseudogroups and (finite) order}

One of Lie's important insights concerning pseudogroups of local symmetries (Example 3.2.4) was that they are characterized as the set of solutions of some (system of) PDE; these pseudogroups were called "continuous transformation groups" [44] and correspond, in modern terms, to Lie pseudogroups.

We need now to recall the basics concerning jet spaces.

- Recall that, when $\pi: Y \rightarrow \mathbf{X}$ is a surjective submersion, one has the tower of $k$-jets of $\pi$ :

$$
\ldots \rightarrow J^{k} Y \rightarrow J^{k-1} Y \rightarrow \ldots \rightarrow Y \rightarrow \mathbf{X}
$$

where an element $j_{x}^{k} \sigma$ of $J^{k} Y$ is an equivalence class of local sections $\sigma$ of $\pi$ defined around the point $x \in \mathbf{X}$ modulo the relation identifying two local sections around $x$ if their Taylor expansions of order $k$ at $x$ agree. Notice that all the maps of the tower are surjective submersions.

- The surjective submersions $J^{k} Y \rightarrow \mathbf{X}$ possess a distinguished class of local sections, called holonomic: $\theta: \mathbf{X} \rightarrow J^{k} Y$ is holonomic if and only if $\theta(x)=$ $j_{x}^{k} \sigma$ for any $x \in \operatorname{dom}(\theta)$ and some local section $\sigma$ of $\pi$. Compactly: $\theta=j^{k} \sigma$.

Moreover, $J^{k} Y$ is equipped with the canonical Cartan distribution $\mathrm{e}^{k}$ (see Appendix 3.A, Example 3.A.18). The literature concerning the Cartan distribution is extensive; see [69] for a modern discussion.

Definition 3.2.15. Let $Y \rightarrow \mathbf{X}$ be a surjective submersion. The Cartan distribution $\mathrm{C}^{k}$ on $J^{k} Y$ is the regular distribution arising as the kernel of the one form

$$
\omega_{j_{x}^{k} \sigma}^{k}:=d_{j_{x}^{k} \sigma} p r-d_{x} j^{k-1} \sigma \cdot d_{j_{x}^{k} \sigma} \pi
$$

where

$$
p r: J^{k} Y \rightarrow J^{k-1} Y, \quad j_{x}^{k} \sigma \rightarrow j_{x}^{k-1} \sigma
$$

and

$$
\pi: J^{k} Y \rightarrow \mathbf{X}, \quad j_{x}^{k} \sigma \rightarrow x
$$

are the canonical projections.

The following Lemma is well known to those working in geometry of PDEs.

Lemma 3.2.16. A section $\theta: U \subset \mathbf{X} \rightarrow J^{k} Y$ is holonomic if and only if the image of $\theta$ is tangent to $\mathrm{C}^{k}$. 
Applying the jet construction to $p r_{1}: \mathbf{X} \times \mathbf{X} \rightarrow \mathbf{X}$ one gets the tower of jets of locally defined smooth maps from $\mathbf{X}$ to $\mathbf{X}$. This can be restricted to locally defined diffeomorphisms, i.e. elements of $\Gamma^{\mathbf{X}}=\operatorname{Diff}_{\text {loc }}(\mathbf{X})$. The outcome is the tower

$$
\ldots \rightarrow J^{k} \Gamma^{\mathbf{X}} \rightarrow J^{k-1} \Gamma^{\mathbf{X}} \rightarrow \ldots \rightarrow J^{0} \Gamma^{\mathbf{X}} \cong \mathbf{X} \times \mathbf{X} \rightrightarrows \mathbf{X}
$$

which is now a tower of Lie groupoids, i.e. all the maps are surjective submersive Lie groupoid maps. Furthermore, the Cartan distributions restrict nicely i.e. as regular distributions. At this point the reader may have a look at Appendix 3.B, and in particular at the definition of Pfaffian groupoid, Definition 3.B.6 and of Lie prolongation of a Pfaffian groupoid 3.B.12. In fact, the tower $\left(J^{*} \Gamma^{\mathbf{X}}, \mathrm{C}^{*}\right)$ is a tower of prolongation of Pfaffian groupoids. Let us summarize here what we mean.

- The groupoid structure on $J^{k} \Gamma^{\mathbf{X}}$ interacts with $\mathrm{C}^{k}$, making the pair $\left(J^{k} \Gamma^{\mathbf{X}}, \mathrm{e}^{k}\right)$ into a Pfaffian groupoid. By this we mean that

i) $\mathcal{C}^{k}$ is transverse to the source projection onto $\mathbf{X}$, i.e. $\mathcal{C}^{k}+\operatorname{Ker}(d s)=$ $T J^{k} \Gamma^{\mathbf{X}}$;

ii) the intersection $\mathrm{C}^{k} \cap \operatorname{Ker}(d s)$ is involutive;

iii) $\mathrm{e}^{k} \cap \operatorname{Ker}(d s)=\mathfrak{e}^{k} \cap \operatorname{Ker}(d t)$;

iv) $\mathcal{C}^{k}$ is multiplicative, i.e. $\mathcal{C}^{k} \subset T J^{k} \Gamma^{\mathbf{X}}$ defines a subgroupoid of the tangent groupoid $T J \Gamma^{\mathbf{X}} \rightrightarrows \mathbf{X}$.

- The projections $\mathrm{pr}^{k+1, k}: J^{k+1} \Gamma^{\mathbf{X}} \rightarrow J^{k} \Gamma^{\mathbf{X}}, k \geq 1$, are morphisms of Pfaffian groupoids, i.e.:

i) their tangent maps send $\mathrm{C}^{k+1}$ to $\mathrm{C}^{k}$;

ii) the diagrams

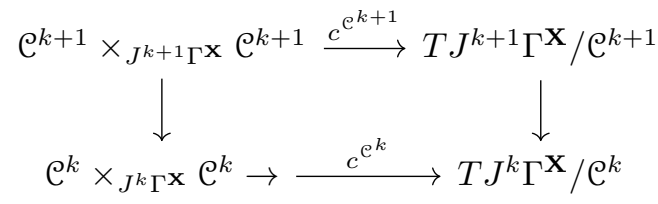

commute, where

$$
\begin{gathered}
c^{\mathfrak{e}^{k+1}}: \mathfrak{C}^{k+1} \times_{J^{k+1} \Gamma^{\mathbf{x}}} \mathcal{C}^{k+1} \rightarrow T J^{k+1} \Gamma^{\mathbf{x}} / \mathcal{C}^{k+1}, \\
c^{\mathfrak{e}^{k}}: \mathfrak{C}^{k} \times_{J^{k} \Gamma^{\mathbf{x}}} \mathfrak{C}^{k} \rightarrow T J^{k} \Gamma^{\mathbf{x}} / \mathfrak{C}^{k}
\end{gathered}
$$

are the curvatures of the distributions $\mathrm{e}^{k+1}, \mathrm{e}^{k}$ and the vertical arrows are induced by $d p r^{k+1, k}$;

- The morphisms of Pfaffian groupoids $p r^{k+1, k}: J^{k+1} \Gamma^{\mathbf{X}} \rightarrow J^{k} \Gamma^{\mathbf{X}}, k \geq 1$ are Lie prolongations i.e.:

i) they are surjective submersions;

ii) $d p r^{k+1, k}\left(\mathfrak{C}^{k+1} \cap \operatorname{Ker}(d s)\right)=0$; 
iii) for all $v, w \in \mathrm{e}^{k+1}, c^{\mathfrak{e}^{k}}\left(d p r^{k+1, k}(v), d p r^{k+1, k}(w)\right)=0$, where $c^{\mathrm{e}^{k}}$ is the curvature of $\mathrm{e}^{k}$;

iv) the map

$$
\left.\operatorname{Ker}(d s)\left|\mathbf{x} /\left(\operatorname{Ker}(d s) \cap \mathrm{e}^{k+1}\right)\right|_{\mathbf{x}} \rightarrow \operatorname{Ker}(d s)\right|_{\mathbf{x}}
$$

induced by $d p r^{k+1, k}$ is an isomorphism (on the right hand side, $s$ : $J^{k} \Gamma^{\mathbf{X}} \rightarrow \mathbf{X}$ is the source map of $\left.J^{k} \Gamma^{\mathbf{X}}\right)$.

According to Proposition 3.B.21, the set theoretical $\operatorname{limit} \underset{\leftarrow}{\lim } J^{k} \Gamma^{\mathbf{X}}$ is a pf-Lie groupoid (Appendix 3.A) equipped with a flat multiplicative connection $\mathrm{C}^{\infty}=$ $\lim _{\leftarrow} \mathcal{C}^{k}$ (see also Example 3.A.18). Moreover, $\lim _{\leftarrow} J^{k} \Gamma^{\mathbf{X}} \cong J^{\infty} \Gamma^{\mathbf{X}}$, the space of formal power series of elements in $\Gamma^{\mathbf{X}}$.

Any pseudogroup $\Gamma$ on $\mathbf{X}$ defines an analogous tower

$$
\ldots \rightarrow J^{k} \Gamma \rightarrow J^{k-1} \Gamma \rightarrow \ldots J^{0} \Gamma \rightrightarrows \mathbf{X}
$$

which is called the canonical Cartan tower of $\Gamma$. However, there is no reason for the $J^{k} \Gamma^{\prime}$ s to be smooth manifolds or for the maps $J^{k} \Gamma \rightarrow J^{k-1} \Gamma$ to be submersive. Furthermore $J^{\infty} \Gamma$ is not necessarily isomorphic to the limit of the tower.

Definition 3.2.17. A Lie pseudogroup $\Gamma$ over $\mathbf{X}$ is a pseudogroup $\Gamma$ such that

- $J^{k} \Gamma$ is a Lie subgroupoid of $J^{k} \Gamma^{\mathbf{X}}, k \in \mathbb{N}$;

- the projections $J^{k} \Gamma \rightarrow J^{k-1} \Gamma$ are submersive.

It is called strongly Lie if the canonical map $J^{\infty} \Gamma \rightarrow \lim J^{k} \Gamma$ is a bijection. A Lie pseudogroup is called of order $k$ when a locally defined diffeomorphism $\varphi$ on $\mathbf{X}$ is in $\Gamma$ if and only if $j^{k} \phi \in J^{k} \Gamma$, and $k$ is minimal with this property.

In the terminology of Appendix 3.A, $\Gamma$ is a Lie pseudogroup if and only if $J^{\infty} \Gamma$ is a pf subgroupoid of $J^{\infty} \Gamma^{\mathbf{X}} ; \Gamma$ is strongly Lie when it is also normal.

Remark 3.2.18. Intuitively, submanifolds of jet spaces are a geometrization of PDEs. This interpretation is quite standard; see [69] for a clear, modern discussion. Having this in mind, Lie pseudogroups are indeed defined by systems of PDEs, namely those encoding the inclusions $J^{k} \Gamma \subset J^{k} \Gamma^{\mathbf{X}}$. If $\Gamma$ is a Lie pseudogroup of order $k$, the PDE(s) of order $k$ encoded by $J^{k} \Gamma \subset J^{k} \Gamma^{\mathbf{X}}$ defines $\Gamma$; intuitively, the higher order inclusions are just differential consequences.

Remark 3.2.19. The distinction between strongly Lie pseudogroup from Lie pseudogroup in Definition 3.2.17 plays a role in the finite order problem that we will address later on, see Question 1 below. Equivalently, a Lie pseudogroup is strongly Lie if the map $\operatorname{Germ}(\Gamma) \rightarrow \lim J^{k} \Gamma$ is surjective. This can be in turn interpreted as a request of topological closure for $J^{\infty} \Gamma$ in $\lim _{\leftarrow} J^{k} \Gamma$, with respect to a topology different from the limit one, Definition 3.4.20. This last interpretation, in the infinitesimal setting of Lie algebra sheaves (Definition 3.3.14), can be read also in [60]. We will go back to this in Remark 3.4.19 and Example 3.4.35. 
Example 3.2.20. The pseudogroup $\Gamma^{\mathbf{X}}$, as well as all the pseudogroups from Example 3.2.4, are Lie. They are also strongly Lie. On the other hand, $\Gamma_{\mathrm{an}}^{\mathbf{X}}$ is Lie but not strongly Lie: $J^{k} \Gamma_{\text {an }}^{\mathbf{X}}=J^{k} \Gamma^{\mathbf{X}}$ for each $k$, but $J^{\infty} \Gamma_{\text {an }}^{\mathbf{X}}$ is strictly contained in $\lim _{\leftarrow} J^{k} \Gamma^{\mathbf{X}}$, since formal power series may fail to converge.

Example 3.2.21. To find examples of pseudogroups which are not Lie, one can start by looking at $J^{0} \Gamma$. For any $\Gamma, J^{0} \Gamma$ is a subgroupoid of the pair groupoid $\mathbf{X} \times \mathbf{X}$ that we call the orbit groupoid of $\Gamma$. The reason for the name lies in the fact that $(x, y) \in J^{0} \Gamma$ if and only if there exists $\varphi \in \Gamma$ with $\varphi(x)=y$. The groupoid $J^{0} \Gamma$ is a Lie subgroupoid of $\mathbf{X} \times \mathbf{X}$ if and only if the quotient of $\mathbf{X}$ by the orbit relation $\left(x \sim y\right.$ if and only if $\left.(x, y) \in J^{0} \Gamma\right)$ is a smooth manifold onto which $\mathbf{X}$ projects submersively; see Godement's theorem in [57, p. 92]. This implies, for example, that the pseudogroup of leafwise diffeomorphisms of the singular foliation on $\mathbf{X}=\mathbb{R}^{n}$ by concentric spheres is not Lie.

Convention 4. Throughout this chapter, unless otherwise specified, all our pseudogroups are Lie.

Remark 3.2.22. This rules out pseudogroups whose orbit spaces exhibit singularities. However, a large class of examples with such a behaviour can be treated with the same formalism as Lie generalized pseudogroups, see Subsection 3.2.5.

Notice that if $\Gamma$ is a Lie pseudogroup, then the Cartan distribution, Definition 3.2.15, on $J^{k}(\mathbf{X} \times \mathbf{X} \rightarrow \mathbf{X})$ restricts to $J^{k} \Gamma$ as a regular distribution. We will keep calling it the Cartan distribution. As for $\Gamma^{\mathbf{X}}=\operatorname{Diff}_{\text {loc }}(\mathbf{X})$, the tower $\left(J^{*} \Gamma, \mathcal{C}^{*}\right)$ is a tower of prolongations of Pfaffian groupoids, see Appendix 3.B, Definition 3.B.20 and the discussion above.

To sum up: the groupoid $\operatorname{Germ}(\Gamma)$ of a Lie pseudogroup comes with a "resolution" by Lie groupoids

$$
j: \operatorname{Germ}(\Gamma) \rightarrow J^{\infty} \Gamma \rightarrow \ldots \rightarrow J^{k} \Gamma \rightarrow \ldots
$$

where $j$ is the map sending a germ at $x$ to the corresponding infinite jet. This "resolution" possesses additional structure: it is a tower of Lie prolongations, Definitions 3.B.12 and 3.B.20 in Appendix 3.B. All of these will be explored later on; for now, we look at the interaction of this "resolution" with $\Gamma$-spaces.

Definition 3.2.23. Let $\Gamma$ be a Lie pseudogroup over $\mathbf{X}$. A $\Gamma$-space $\mu: E \rightarrow \mathbf{X}$ (cf. Definition 3.2.9) is called formally determined if the action of $\operatorname{Germ}(\Gamma)$ factors through $J^{\infty} \Gamma$. It is called of finite order $k$ if the action of $\operatorname{Germ}(\Gamma)$ factors through $J^{k} \Gamma$ and $k$ is minimal with this property.

Our aim is to discuss the finite order problem i.e. the following

Question 1. Which conditions on $\Gamma$ ensures that (a large subcategory of) $\Gamma$-spaces are of finite order?

Example 3.2.24. All the $\Gamma$-spaces described in Example 3.2.13 are of finite order 1. 
Example 3.2.25. More generally, one can consider the higher order frame bundles

$$
\operatorname{Fr}^{k}(\mathbf{X})=\left\{j_{0}^{k} \phi: \phi: \mathbb{R}^{n} \rightarrow \mathbf{X} \text { is a diffeomorphism on its image }\right\}
$$

where $k \in \mathbb{N}$. $F r^{k}(\mathbf{X}) \rightarrow \mathbf{X}$ is a (right, by convention) principal bundle with structure group

$$
G L_{n}^{k}(\mathbb{R})=\left\{j_{0}^{k} f: f: \mathbb{R}^{n} \rightarrow \mathbb{R}^{n} \text { is a diffeomorphism, } f(0)=0\right\}
$$

Notice that $F r^{1}(\mathbf{X})=F r(\mathbf{X})$, the usual bundle of frames of $\mathbf{X}$ with its principal $G L_{n}(\mathbb{R})$-bundle structure. Notice also that $F r^{k}(\mathbf{X})$ is a $\Gamma^{\mathbf{X}}$-space of order $k$. Indeed, the action of $[\varphi]_{x} \in \Gamma^{\mathbf{X}}$ is given by

$$
j_{\phi^{-1}(x)}^{k} \phi^{-1} \rightarrow j_{\phi^{-1}(x)}^{k}\left(\varphi \circ \phi^{-1}\right) .
$$

Notice that $\left(F r^{k}(\mathbf{X})\right)_{x}$ is sent to $\left(F r^{k}(\mathbf{X})\right)_{\varphi(x)}$ by the action of $\varphi$, and that such an action defines (the germ of) a local diffeomorphism of $F r^{k}(\mathbf{X})$; we denote such a local diffeomorphism by $F r^{k}(\varphi)$. For all $k \in \mathbb{N}, F r^{k}(\mathbf{X})$ is a $\Gamma^{\mathbf{X}}$-space, and in fact a $\Gamma$-space for any Lie pseudogroup $\Gamma$, of order $k$.

If a manifold $F$ carries a (left) action of $G L_{n}^{k}(\mathbb{R})$, we can form the associated bundle

$$
E:=F r^{k}(\mathbf{X}) \times F / \sim
$$

where the quotient is by the diagonal left action

$$
j_{0}^{k} f:\left(j_{0}^{k} \phi, e\right) \rightarrow\left(j_{0}^{k}\left(\phi \circ f^{-1}\right), j_{0}^{k} f \cdot e\right), \quad j_{0}^{k} f \in G L_{n}^{k}(\mathbb{R}) .
$$

The structure of $J^{k} \Gamma^{\mathbf{X}}$-space descends to $E$. Therefore, to any $G L_{n}^{k}(\mathbb{R})$-space one can associate a $\Gamma^{\mathbf{X}}$-space of order $k$.

In fact, any $\Gamma^{\mathbf{X}}=\operatorname{Diff}_{\text {loc }}(\mathbf{X})$-space of order $k$ arises as a bundle associated to $F r^{k}(\mathbf{X})$ [54]. Let $\mu: E \rightarrow \mathbf{X}$ be a $\Gamma^{\mathbf{X}}$-space of order $k$-i.e. a $J^{k} \Gamma^{\mathbf{X}}$-space. Let us fix $x \in \mathbf{X}$ and consider the manifold $\mu^{-1}(x)$. The action of $J^{k} \Gamma^{\mathbf{X}}$ on $E$ induces a left action of the isotropy group $\left(J^{k} \Gamma^{\mathbf{X}}\right)_{x}$ on $\mu^{-1}(x)$; but $\left(J^{k} \Gamma^{\mathbf{X}}\right)_{x} \cong G L_{n}^{k}(\mathbb{R})$, so we are in the situation of the exampe above and we can form the $\Gamma^{\mathbf{X}}$-space of order $k$

$$
E^{\prime}:=\operatorname{Fr}^{k}(\mathbf{X}) \times \mu^{-1}(x) / \sim
$$

We see that $E$ and $E^{\prime}$ are naturally isomorphic as $J^{k} \Gamma^{\mathbf{X}}$-spaces. The isomorphism is given by

$$
E \rightarrow E^{\prime}, \quad e_{x} \rightarrow\left[\left(j_{0}^{k} i d, e_{x}\right)\right]
$$

Furthermore, any $\Gamma^{\mathbf{X}}$-space is a bundle associated to $F r^{k}(\mathbf{X})$ for some $k \in \mathbb{N}$, because any $\Gamma^{\mathbf{X}}$-space has order $k$ for some $k \in \mathbb{N}$. This is the main result of [28, 54] (see also the discussion after Question 2 closing Subsection 3.2.4).

\subsubsection{Geometric structures: natural bundles via Morita equiva- lences}

Inspired by the group-like properties of pseudogroups and proceeding by analogy with groups, one can give the following definition. 
Definition 3.2.26. Let $\Gamma$ be a pseudogroup on $\mathbf{X}$ and let $M$ be another manifold. A $\Gamma$-cocycle on $M$ consists of a cover $\mathcal{U}=\left\{U_{i}\right\}_{i \in I}$ of $M$ and smooth maps

$$
\gamma_{i j}: U_{i} \cap U_{j} \rightarrow \operatorname{Germ}(\Gamma)
$$

such that:

- for $i=j$, each $\gamma_{i i}(x)$ is a unit (hence can be seen as a point in $\mathbf{X}$ ),

- for arbitrary $i$ and $j$ and $x \in U_{i} \cap U_{j}, \gamma_{i j}(x)$ is a germ from $\gamma_{i i}(x)$ to $\gamma_{j j}(x)$,

and such that the cocycle condition

$$
\gamma_{j k}(x) \circ \gamma_{i j}(x)=\gamma_{i k}(x) .
$$

is satisfied for all $x \in U_{i} \cap U_{j} \cap U_{k}$.

The notion of $\mathcal{G}$-cocycles, where $\mathcal{G} \rightrightarrows \mathbf{X}$ is any étale groupoid over $\mathbf{X}$, is defined similarly, by replacing $\operatorname{Germ}(\Gamma)$ with $\mathcal{G}$.

The first condition in Definition 3.2.26 can be rewritten as the existence of maps

$$
f_{i}:=\gamma_{i i}: U_{i} \rightarrow \mathbf{X}
$$

the second condition tells that, for each $x \in U_{i} \cap U_{j}$, one can find $\varphi_{i j}^{x} \in \Gamma$ representing $\gamma_{i j}(x)$ and such that, in a neighbourhood of $x$,

$$
f_{j}=\varphi_{i j}^{x} \circ f_{i} .
$$

Remark 3.2.27. If the $f_{i}$ 's are submersions, the cocycle condition from Definition 3.2.26 follows automatically.

One can introduce an equivalence relation on $\Gamma$-cocycles on $M$, in a way analogous to the one used for group cocycles: two $\Gamma$-cocycles indexed by $I$ and $J$ are equivalent if they are part of a larger $\Gamma$-cocycle indexed by $I \sqcup J$.

When $\operatorname{dim}(M)=\operatorname{dim}(\mathbf{X})$ and all the $f_{i}{ }^{\prime}$ s are diffeomorphisms onto their image, $\Gamma$-cocycles are actually $\Gamma$-atlases; in this case equivalence classes - i.e. maximal atlases - give rise to $\Gamma$-structures.

Definition 3.2.28. A $\Gamma$-atlas on $M$ is a smooth atlas modelled on opens in $\mathbf{X}$ whose transition functions are elements of $\Gamma$. An equivalence class of $\Gamma$-atlases on $M$ is called a $\Gamma$-structure on $M$.

Convention 5. From now on, unless otherwise specified, all $\Gamma$-cocycles are understood to be $\Gamma$-atlases and $\Gamma$-structures are represented by $\Gamma$-atlases.

Example 3.2.29. The simplest example arises taking $\mathbf{X}=\mathbb{R}^{n}$ and $\Gamma=\Gamma^{n}$; see Example 3.2.2 for the notation. In that case, a $\Gamma$-cocycle on $M$ is just an ordinary atlas. The associated $\Gamma$-structure is the smooth structure of $M$. When $\Gamma=\Gamma_{\mathrm{an}}^{n}$, a cocycle is an analytic atlas and the induced structure is an analytic structure on $M$ compatible with the smooth one. 
Example 3.2.30. In some sense, a $\Gamma$-structure on $M$ is a geometric structure modelled on some other structure on $\mathbf{X}$, meaning that the structure on $M$ is locally pulled back from that on $\mathbf{X}$ and the resulting local structures are "glued" correctly by means of the cocycle conditions. This is particularly clear looking at pseudogroups from Example 3.2.4. For instance, when $\mathbf{X}=\mathbb{R}^{2 n}$ and $\Gamma=\Gamma_{S p(n)}$ is the pseudogroup of symplectomorphisms of the standard symplectic structure, a $\Gamma$-structure on an $2 n$-dimensional manifold $M$ is the same thing as a symplectic structure on $M$. Similarly for $\Gamma=\Gamma_{O(n)}$; in this case $\Gamma$ structures on $M$ are flat Riemannian structures on $M . \Gamma_{G}$-structures on $M$ are flat $G$-structures on $M, \Gamma_{\text {cont }}{ }^{-}$ structures are contact structures, $\Gamma^{n, k}$-structures are codimension $k$-foliations, etc.

The clear analogy with group valued cocycles suggests to globalize the discussion by looking at principal bundles. An advantage of this approach is that it is more geometric; furthermore, it allows one not to worry anymore about the equivalence relation between cocycles. Let $\Sigma \rightrightarrows \mathbf{X}$ be a Lie groupoid. If $P$ is a $\Sigma$-space, we denote by $P / \Sigma$ the quotient of $P$ by the equivalence relation identifying $p_{1}$ and $p_{2}$ whenever there is $g \in \Sigma$ such that $g \cdot p_{1}=p_{2}$. We endow it with the quotient topology.

Definition 3.2.31. Let $\Sigma \rightrightarrows \mathbf{X}$ be a Lie groupoid and $M$ be a manifold. A principal $\Sigma$-bundle over $M$ is a $\Sigma$-space $\mu: P \rightarrow \mathbf{X}$ together with a surjective submersion $\pi: P \rightarrow M$ such that

- $\pi$ is $\Sigma$-invariant, i.e. $\pi(g \cdot p)=\pi(p)$ for all $g \in \Sigma, p \in P$ such that $s(g)=\mu(p)$;

- the action is free and proper, i.e.:

- the action is free: for all $p \in P, g \in \Sigma$ such that $s(g)=\mu(p)$ if $g \cdot p=p$ then $g=1_{\mu(p)}$;

- the action is proper: the map

$$
\Sigma_{s} \times{ }_{\mu} P \rightarrow P \times P, \quad(g, p) \rightarrow(g \cdot p, p)
$$

is a proper map.

When $P$ is a principal $\Sigma$-bundle over $M$, then $P / \Sigma$ possesses a canonical smooth structure such that quotient projection is a surjective submersion. Furthermore, the principal bundle projection $\pi: P \rightarrow M$ induces a map $P / \Sigma \rightarrow M$ which is a diffeomorphism when $P / \Sigma$ is given such a smooth structure.

Definition 3.2.32. A morphism of principal $\Sigma$-bundles $\mu_{1}: P_{1} \rightarrow \mathbf{X}, \mu_{2}: P_{2} \rightarrow \mathbf{X}$ over $M_{1}$ and $M_{2}$ respectively, is a smooth map $F: P_{1} \rightarrow P_{2}$ lifting a smooth map $f: M_{1} \rightarrow M_{2}$ and commuting with the action of $\Sigma$, i.e.

- $\pi_{2} \circ F=f \circ \pi_{1}$, where $\pi_{i}: P_{i} \rightarrow M_{i}$ is the principal bundle projection of $P_{i}$ to $M_{i}$;

- $\mu_{2} \circ F=\mu_{1}$;

- $F(g \cdot p)=g \cdot F(p)$ for all $g \in \Sigma, p \in P_{1}, \mu_{1}(p)=s(g)$. 
Let $\mathcal{G} \rightrightarrows \mathbf{X}$ be an étale groupoid over $\mathbf{X}$ and $\mathcal{P} \rightarrow M$ be a principal $\mathcal{G}$-bundle over $M$. One chooses a cover $\left\{U_{i}\right\}_{i \in I}$ of $M$ by opens $U_{i}$ on which $\mathcal{P}$ admits sections $\sigma_{i}: U_{i} \rightarrow \mathcal{P}$ and then, on overlapping points $x \in U_{i} \cap U_{j}$, one has

$$
\sigma_{i}(x)=\gamma_{i j}(x) \cdot \sigma_{j}(x)
$$

for some $\gamma_{i j}(x) \in \mathcal{G}$. One also gets the maps

$$
f_{i}=\mu \circ \sigma_{i}: U_{i} \rightarrow \mathbf{X}
$$

and, putting all together, a $\mathcal{G}$-cocycle. Proceeding as for groups (see [52]), one obtains the 1-1 correspondence

$$
\left\{\begin{array}{c}
\mathcal{G} \text {-cocycles on } M \\
\text { (up to equivalence) }
\end{array}\right\} \stackrel{1-1}{\longleftrightarrow}\left\{\begin{array}{c}
\text { principal } \mathcal{G} \text {-bundles on } M \\
\text { (up to isomorphism) }
\end{array}\right\}
$$

Corollary 3.2.33. Given the pseudogroup $\Gamma$ over $\mathbf{X}$, there is a 1-1 correspondence between:

- $\Gamma$-structures on $M$;

- isomorphism classes of principal $\operatorname{Germ}(\Gamma)$-bundles $\pi: \mathcal{P} \rightarrow M$ for which $\mu: \mathcal{P} \rightarrow$ $\mathbf{X}$ (along which $\operatorname{Germ}(\Gamma)$ acts) is an étale map.

Example 3.2.34. When $\Gamma=\Gamma^{\mathrm{X}}$, the pseudogroup of all locally defined diffeomorphisms Diff ${ }_{\text {loc }}(\mathbf{X})$, and we consider a $\Gamma^{\mathbf{X}}$-structure on $M$, the associated principal bundle is (isomorphic to)

$$
\mathcal{P}:=\Pi(M, \mathbf{X}),
$$

where $\Pi(M, \mathbf{X})$ denotes the space of germs of diffeomorphisms from opens in $M$ to opens in $\mathbf{X}$. More generally, for a pseudogroup $\Gamma$ on $\mathbf{X}$ and a $\Gamma$-structure on $M, \mathcal{P}$ is obtained by choosing a $\Gamma$-atlas and taking the saturation in $\Pi(M, \mathbf{X})$ of the set of germs of the maps $f_{i}: U_{i} \rightarrow \mathbf{X}$ (see Definition 3.2.28) with respect to the action of $\operatorname{Germ}(\Gamma)$.

$\Gamma$-structures, Definition 3.2.28, form a category with morphisms as in the following definition, where $\mathcal{P}_{1}$ and $\mathcal{P}_{2}$ are the principal bundles that arise by applying Corollary 3.2.33.

Definition 3.2.35. A morphism of $\Gamma$-structures $\mathcal{P}_{1}$ and $\mathcal{P}_{2}$ over $M_{1}$ and $M_{2}$, respectively, is a morphism of principal bundles $F: \mathcal{P}_{1} \rightarrow \mathcal{P}_{2}$.

Recall that $F: \mathcal{P}_{1} \rightarrow \mathcal{P}_{2}$ lifts a smooth map $f: M_{1} \rightarrow M_{2}$, see Definition 3.2.32.

Remark 3.2.36. A morphism of $\Gamma^{n}$-structures is simply a local diffeomorphism from $M_{1}$ to $M_{2}$. In fact, any local diffeomorphism $f: M_{1} \rightarrow M_{2}$ induces a lift $F: \Pi\left(M_{1}, \mathbf{X}\right) \rightarrow \Pi\left(M_{2}, \mathbf{X}\right)$ (recall that we use the notation $\Pi(M, \mathbf{X})$ for the space of germs of diffeomorphisms from opens of $M$ to opens of $\mathbf{X})$. For more general $\Gamma^{\prime}$ s, one can encode a morphism in a map $f: M_{1} \rightarrow M_{2}$ preserving the geometric structure induced by $\mathcal{P}_{1}, \mathcal{P}_{2}$. This can be made precise using atlases, in the same sense in which smoothness of a map is defined using atlases. If $\left\{\left(U_{i}, f_{i}\right)\right\}_{i \in I}$ is an atlas for $\mathcal{P}_{1}$ with changes of coordinates given by $\left\{\varphi_{i j}\right\}_{i, j \in I}$ and $\left\{\left(V_{k}, g_{k}\right)\right\}_{k \in K}$ is an atlas for $\mathcal{P}_{2}$ with changes of coordinates $\left\{\psi_{k h}\right\}_{k, h \in K}$, then one requires $g_{k} \circ f \circ$ $f_{i}^{-1}$ to be an element of $\Gamma$ for any pair $(i, k) \in I \times K$. A map with this property induces a map $\mathcal{P}_{1} \rightarrow \mathcal{P}_{2}$ (see Example 3.2.34 and the example below). 
Example 3.2.37. A morphism of $\Gamma_{S p(n)}$-structures between two $2 n$-dimensional symplectic manifolds $M_{1}, M_{2}$ is a symplectic map from $M_{1}$ to $M_{2}$. In the spirit of the previous remark, notice that choosing atlases of $M_{1}, M_{2}$ by symplectic charts, a map $f: M_{1} \rightarrow M_{2}$ is symplectic if and only if its coordinate representations are symplectomorphisms of $\mathbb{R}^{2 n}$ with the standard symplectic structure, i.e. elements of $\Gamma_{S p(n)}$.

Closely related to the notion of principal bundle is the notion of Morita equivalence (see, for instance, [49]).

Definition 3.2.38. Two (not necessarily étale) Lie groupoids $\Sigma_{1} \rightrightarrows \mathbf{X}, \Sigma_{2} \rightrightarrows \mathbf{X}_{2}$ are called Morita equivalent if there exists a principal $\left(\Sigma_{1}, \Sigma_{2}\right)$-bibundle $\mathbf{X}_{1} \leftarrow$ $P \rightarrow \mathbf{X}_{2}$, i.e. a principal left action of $\Sigma_{1}$ along $P \rightarrow \mathbf{X}_{1}$ with projection $P \rightarrow$ $\mathbf{X}_{2}$ and a principal right action of $\Sigma_{2}$ along $P \rightarrow \mathbf{X}_{2}$ with projection $P \rightarrow \mathbf{X}_{1}$ commuting with each other. Diagrammatically ${ }^{3}$ :

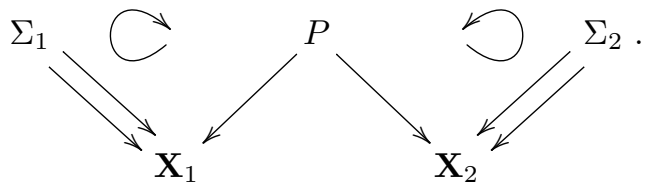

Morita equivalence is an equivalence relation. The notion of Morita equivalence captures the transverse geometry of a Lie groupoid; if two Lie groupoids are Morita equivalent, then they the induce the same geometry "transversally to their orbits". This is at the core of the relation between Lie groupoids and differentiable stacks, see for instance [24].

Given a principal groupoid bundle

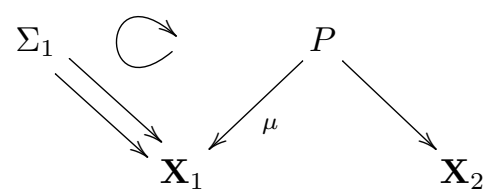

there is always a Morita complement for $\Sigma_{1}$, i.e. a Lie groupoid $\Sigma_{2} \rightrightarrows \mathbf{X}_{2}$ turning $P$ into a principal $\left(\Sigma_{1}, \Sigma_{2}\right)$-bibundle (recall Convention 3: we assume $\mu$ to be a surjective submersion). Explicitly, one takes $\Sigma_{2}$ to be the gauge groupoid $P \otimes_{\Sigma_{1}}$ $P \rightrightarrows \mathbf{X}_{1}$ of $P$, i.e. the quotient of the fibered product groupoid $P \times_{\mu} P \rightrightarrows P$ with respect to the diagonal action of $\Sigma_{1}$.

Since a $\Gamma$-structure can be encoded in a principal $\operatorname{Germ}(\Gamma)$-bundle $\mathcal{P} \rightarrow M$, it makes sense to look at the gauge groupoid $\mathcal{P} \otimes_{\operatorname{Germ}(\Gamma)} \mathcal{P} \rightrightarrows M$. In the proposition below we set $\mathcal{G}:=\operatorname{Germ}(\Gamma)$ to simplify the notation. We stress that our Conventions 3 and 5, see page 159 and page 165 respectively, play a role.

Lemma 3.2.39. $\mathcal{P} \otimes g \mathcal{P} \rightrightarrows M$ is an effective étale Lie groupoid.

Proof. Étaleness of the gauge groupoid follows from a general property of Morita equivalences: given two Morita equivalent groupoids $\Sigma_{1}, \Sigma_{2}$ and $\mathbf{X}_{1} \leftarrow \mathcal{P} \rightarrow \mathbf{X}_{2}$, $\Sigma_{2}$ is étale if and only if the quotient map $\mathcal{P} \rightarrow \mathbf{X}_{1}$ is an étale map. From this

\footnotetext{
${ }^{3}$ We are pleased to stress the resemblance of the diagram with a butterfly.
} 
one sees that étaleness of $\mathcal{P} \otimes_{\mathcal{G}} \mathcal{P}$ is equivalent to étaleness of $\mu$ (notice here the importance of Convention 5).

We claim that effectiveness of $\mathcal{P} \otimes_{\mathcal{G}} \mathcal{P}$ is then equivalent to effectiveness $\mathcal{G}$. First, observe that any (germ of) local bisection $\sigma$ of $\mathcal{G}$ induces a (germ of) local diffeomorphism of $\mathcal{P}$, denoted by $\varphi_{\sigma}$ and sending $p \in \mu^{-1}(\operatorname{Dom}(\sigma)$ to $\sigma(\mu(p)) \cdot p$. Let us consider a pair $\left(V, V^{\prime}\right)$ of opens in $\mathcal{P}$ projecting to the same open $W$ in $\mathbf{X}$ via $\mu$ and over opens $U, U^{\prime}$ in $M$ via $\pi$. It is clear that any (germ of) local bisection $\mathcal{P} \otimes \mathfrak{g} \mathcal{P}$ induces such a pair. Conversely, a (germ of) local bisection of $\mathcal{P} \otimes_{\mathcal{g}} \mathcal{P}$ is uniquely determined by the class of such a pair under the equivalence relation identifying $\left(V_{1}, V_{1}^{\prime}\right)$ with $\left(V_{2}, V_{2}^{\prime}\right)$ if there is a bisection $\sigma$ of $\mathcal{G}$ defined over $W$ and sending $\left(V_{1}, V_{1}^{\prime}\right)$ to $\left(V_{2}, V_{2}^{\prime}\right)$ via the diagonal action of $\varphi_{\sigma}$. Observe that $\left(V, V^{\prime}\right)$ represents the same class as $\left(V, \varphi_{\sigma}\left(V^{\prime}\right)\right)$ if and only if $\varphi_{\sigma}$ is the identity. This makes clear that $\mathcal{P} \otimes \mathcal{G} \mathcal{P}$ fails to be effective if and only if there are non-trivial bisections of $\mathcal{G}$ lifting the identity diffeomorphism of $W$, i.e. if and only if $\mathcal{G}$ is not effective.

Remark 3.2.40. The proof above shows that the gauge groupoid is étale even if the effectiveness assumption is removed.

Notice also that

Lemma 3.2.41. If $\mathcal{G} \rightrightarrows \mathbf{X}$ is an étale groupoid and $\mu_{i}: \mathcal{P}_{i} \rightrightarrows \mathbf{X}$ are principal bundles over $M_{i}$ and $F: \mathcal{P}_{1} \rightarrow \mathcal{P}_{2}$ is a morphism of principal $\mathcal{G}$-bundles, Definition 3.2.32, lifting $f: M_{1} \rightarrow M_{2}$, then the map

$$
\Phi_{F}: \mathcal{P}_{1} \otimes_{\mathcal{G}} \mathcal{P}_{1} \rightarrow \mathcal{P}_{2} \otimes_{\mathcal{G}} \mathcal{P}_{2}, \quad\left[\left(p_{1}, p_{1}^{\prime}\right)\right] \rightarrow\left[\left(F\left(p_{1}\right), F\left(p_{1}^{\prime}\right)\right)\right]
$$

induced by $F$, is a morphism of groupoids.

Via the construction of Proposition 3.2.8, $\mathcal{P} \otimes g_{\mathcal{G}} \mathcal{P}$ corresponds to a pseudogroup on $M$, which we denote by $\Gamma_{\mathcal{P}}$. Explicitely, if we pass to cocycles and consider the maps $f_{i}=\mu \circ \sigma_{i}: U_{i} \rightarrow \mathbf{X}$, we have the set

$$
\left\{\varphi \in \operatorname{Diff}_{\mathrm{loc}}(M): f_{j} \circ \varphi \circ f_{i}^{-1} \in \Gamma, i, j \in I\right\} ;
$$

here, when writing the composition $f_{j} \circ \varphi \circ f_{i}^{-1}$, the restriction of $\varphi \circ f_{i}^{-1}$ to $\operatorname{Dom}\left(f_{j}\right) \cap \operatorname{Im}\left(\varphi \circ f_{i}^{-1}\right)$ is understood. The set above generates the pseudogroup $\Gamma_{\mathcal{P}}$ by taking the closure with respect to sheaf-like structure on $\Gamma^{M}$, i.e. by forcibly adding all possible restrictions and gluings of elements of the set.

Example 3.2.42. When $\Gamma=\Gamma^{n}=\operatorname{Diff}_{\text {loc }}\left(\mathbb{R}^{n}\right), \mathcal{P}=\Pi(M, \mathbf{X})$ then $\Gamma_{\mathcal{P}}=\Gamma^{M}=$ $\operatorname{Diff}_{\text {loc }}(M)$.

Example 3.2.43. More generally, the pseudogroup $\Gamma_{\mathcal{P}}$ is essentially the pseudogroup of symmetries of the geometric structure on $M$ induced by the existence of $\mathcal{P}$ (see Examples 3.2.29 and 3.2.34). For instance, when the $\Gamma$-structure $\mathcal{P}$ that we consider is a symplectic structure on the $2 n$-dimensional manifold $M$, working out the definition one sees that $\Gamma_{\mathcal{P}}$ is the pseudogroup of local symplectomorphisms of $M$.

To sum up: 
Proposition 3.2.44. Let $\Gamma$ be some pseudogroup on $\mathbf{X}$. A $\Gamma$-structure $\mathcal{P}$ over a manifold $M$ induces a pseudogroup $\Gamma_{\mathcal{P}}$ on $M$ whose germ groupoid $\operatorname{Germ}\left(\Gamma_{\mathcal{P}}\right)$ is Morita equivalent to $\operatorname{Germ}(\Gamma)$ via $\mathcal{P}$.

A consequence of what we discussed so far is the Proposition 3.2.45 below. To make sense of the statement, given two étale groupoids $\mathcal{G}_{1} \rightrightarrows \mathbf{X}_{1}$ and $\mathcal{G}_{2} \rightrightarrows \mathbf{X}_{2}$, let us explain what do we mean by map from a $\mathcal{G}_{1}$-space $\mu_{1}: E_{1} \rightarrow \mathbf{X}_{1}$ to a $\mathcal{G}_{2}$-space $\mu_{2}: E_{2} \rightarrow \mathbf{X}_{2}$ respecting the actions. It is a pair consisting of

- a morphism of groupoids $\Phi: \mathcal{G}_{1} \rightarrow \mathcal{G}_{2}$ (that, in particular, lifts a smooth $\operatorname{map} f: \mathbf{X}_{1} \rightarrow \mathbf{X}_{2}$ );

- a map $F: E_{1} \rightarrow E_{2}$ commuting with the actions, i.e.

$$
\begin{aligned}
& \text { - } \mu_{2} \circ F=f \circ \mu_{1} ; \\
& \text { - } F\left(g_{1} \cdot e_{1}\right)=\Phi\left(g_{1}\right) \cdot F\left(e_{1}\right), g_{1} \in \mathcal{G}_{1}, e_{1} \in E_{1}, \mu\left(e_{1}\right)=s\left(g_{1}\right) .
\end{aligned}
$$

In particular, if $\mathcal{G}:=\mathcal{G}_{1}=\mathcal{G}_{2}, \Phi$ is the indentity and $E_{i}=\mathcal{P}_{i}$ are principal $\mathcal{G}$-bundles, one recovers the notion of morphism of principal $\mathcal{G}$-bundles, Definition 3.2.32. In the following proposition, we denote by $\Gamma$-sp the category of $\Gamma$-spaces and by $\Gamma$-st the category of $\Gamma$-structures.

Proposition 3.2.45. Let $\Gamma$ be a pseudogroup on $\mathbf{X}$. There is a bifunctor

$$
\Gamma \text {-sp } \times \Gamma \text {-st } \rightarrow \mathrm{Fib}^{\mathrm{n}}
$$

where $\mathrm{Fib}^{\mathrm{n}}$ is the category of surjective submersions over $n$-manifolds.

Proposition 3.2.45 holds true since Morita equivalent Lie groupoids have equivalent action categories (this is well known; see, for example, the proof of Theorem 4.2 in [68], or Theorem 6.6 in [42] for the particular case of groupoid representations). The bifunctor from Proposition 3.2.45 associates to a pair $(E \rightarrow \mathbf{X}, \mathcal{P})$ consisting of a $\Gamma$-space $E \rightarrow \mathbf{X}$ and a $\Gamma$-structure $\mathcal{P}$ over $M$ the $\mathcal{P} \otimes_{\operatorname{germ}(\Gamma)} \mathcal{P}$-space $E M \rightarrow M$ obtained from the Morita equivalence between $\operatorname{Germ}(\Gamma)$ and $\mathcal{P} \otimes \operatorname{Germ}(\Gamma) \mathcal{P}$. To see that one really gets a bifunctor - which, in particular, is natural with respect to arrows in $\Gamma$-st - one makes use of Lemma 3.2.41.

In particular, Proposition 3.2.45 tells us that there is a 1-1 correspondence between $\Gamma$-spaces and natural $\Gamma$-bundles. First, let us explain the terminology. We start by assuming $\Gamma=\Gamma^{n}=\operatorname{Diff}_{\text {loc }}\left(\mathbb{R}^{n}\right)$; we denote by $\operatorname{Man}^{\mathrm{n}}$ the category of $n$ dimensional manifolds with morphisms given by open embeddings (i.e. diffeomorphisms onto their images) and by $\mathrm{Fib}^{\mathrm{n}}$ the category of surjective submersions over $n$-manifolds, with morphisms given by smooth fibered maps that lifts open embeddings. The proposition above can be restated by saying that any $\Gamma$-space $E \mathbb{R}^{n} \rightarrow \mathbb{R}^{n}$ induces a functor $E: \mathrm{Man}^{n} \rightarrow \mathrm{Fib}^{n}$ which has the following two properties:

- $E M$ fibers over $M$ via a submersion $\mu_{M}$;

- if $\phi: M_{1} \rightarrow M_{2}$ is an open embedding (that in particular is a map of $\Gamma^{n_{-}}$ structures) $E(\phi)$ lifts $\phi$. 
In other words, $E$ is a section of the base functor Fib ${ }^{n} \rightarrow$ Man $^{n}$. Vice versa, any such functor determines a $\Gamma^{n}$-space, as one can readily see (the only check to be made is that, for all $x \in \mathbb{R}^{n}$ and all $\varphi \in \Gamma^{n}$ the map $\left.E(\varphi)\right|_{\mu_{M}^{-1}(x)}$ depends only on the germ of $\varphi$ at $x$ ).

These kind of functors were introduced by Nijenhuis (see the survey [53]). They are called natural bundles and have been studied by several authors. We see that natural bundles correspond precisely to $\Gamma^{n}$-spaces. Analogously, $\Gamma$ spaces are a generalization of natural bundles in the obvious sense. Let us denote by $\operatorname{Man}^{\Gamma}$ the category whose objects are manifolds equipped with a $\Gamma$-structure and whose arrows are morphisms of $\Gamma$-structures that lift open embeddings (see Definition 3.2.35). Let also $\mathrm{Fib}^{\top}$ be the category whose objects are surjective submersions over $n$-manifolds with a $\Gamma$-structure and whose arrows are fibered maps over arrows of $\operatorname{Man}^{\Gamma}$ (see Remark 3.2.36).

Definition 3.2.46. A natural $\Gamma$-bundle is a functor $E$ from the category $\operatorname{Man}^{\Gamma}$ to the category $\mathrm{Fib}^{\Gamma}$ which is a section of the base functor. We will write $\mu_{M}: E M \rightarrow$ $M$ to denote the object $E(M)$.

See also [5]. One also observes that a map of $\Gamma$-spaces induces a natural transformation of the corresponding natural $\Gamma$-bundles (this is still due to the fact that Morita equivalent Lie groupoids have equivalent action categories). Thanks to Proposition 3.2.45 we have:

Proposition 3.2.47. There is an equivalence of categories between $\Gamma$-spaces and natural $\Gamma$-bundles.

\subsubsection{Finite order problem for natural $\Gamma$-bundles}

Let now $\Gamma$ be a Lie pseudogroup. We are going to phrase explicitely the finite order problem for $\Gamma$-spaces in terms of natural $\Gamma$-bundles. This is the way in which the problem appeared originally. The key point is the following remark.

Remark 3.2.48. As explained in Example 3.2.34, when $\mathcal{P}$ is a $\Gamma$-structure over $M$, one can assume that $\mathcal{P}$ is embedded in $\Pi(M, \mathbf{X})$. One can consider the spaces $\Pi^{\infty}(M, \mathbf{X})$ and $\Pi^{k}(M, \mathbf{X}), k \in \mathbb{N}$, of jets of embeddings from opens in $M$ to opens in $\mathbf{X}$, and the subspaces obtained projecting $\mathcal{P}$. If the pseudogroup $\Gamma$ is a Lie pseudogroup, these are all smooth manifolds (profinite dimensional in the case of $\left.\Pi^{\infty}(M, \mathbf{X})\right)$ and carry an action of $J^{\infty} \Gamma$ and $J^{k} \Gamma$ respectively. These actions are principal over $M$.

With the above remark at hand, one can proceed as in the previous subsection and use Morita complements to show that $\Gamma$-spaces of order $k$ (resp. formally determined) are the same as natural $\Gamma$-bundles of order $k$ (resp. formally determined).

Definition 3.2.49. A natural $\Gamma$-bundle is of finite order $k$ (resp. formally determined) if for any arrow $\phi: M_{1} \rightarrow M_{2}$ of $\operatorname{Man}^{\Gamma}$ the map

$$
\left.E(\phi)\right|_{x}:\left(E M_{1}\right)_{x} \rightarrow\left(E M_{2}\right)_{\phi(x)}
$$

depends only on $j_{x}^{k} \phi\left(\right.$ resp. $\left.j_{x}^{\infty} \phi\right)$. 
We then have, again from a Morita equivalence argument,

Proposition 3.2.50. Under the equivalence from Proposition 3.2.47, finite order $\Gamma$ spaces correspond to finite order natural $\Gamma$-bundles.

The original version $[5,28,40,54,65]$ of the finite order problem is then

Question 2. Which condition on $\Gamma$ ensure that (a large subcategory of) natural $\Gamma$ bundles are of finite order?

The first case studied in the literature was that of natural bundles [28, 54, 65], i.e. $\Gamma=\Gamma^{n}:=\operatorname{Diff}_{\text {loc }}\left(\mathbb{R}^{n}\right)$. In [65] it is shown that continuous natural vector bundles have finite order; there, $E$ is called continuous when it sends sequences converging in the $C^{\infty}$-topology on $\mathbb{R}^{n}$ to sequences converging in the compact open topology on (local) maps on $E \mathbb{R}^{n}$. In [54] the vector bundle assumption was removed, while the continuity one was replaced by a smoothness request (still called continuity): $E$ was assumed to send smooth $k$-parameter families of open embeddings to smooth $k$-parameter families of isomorphisms (onto their images). Both in [65] and [54] the continuity and smoothness assumptions were conjectured to be redundant. This was proven in [28], where the authors finally showed that all natural bundles have finite order. As for generalizations to natural $\Gamma$-bundles, there are various attempts and results in the literature; see for example [5] or [40, 62]. In general, assumptions have to be made on $\Gamma$ and $E$ to ensure that the arguments from $[28,54]$ can be generalized. In [5], the author assumes $E$ to be smooth in the sense of [54]; furthermore, they restrict to the case where $\Gamma$ is transitive (i.e., given $x \in \mathbf{X}$ for each $y \in \mathbf{X}$ there is $\varphi \in \Gamma$ such that $\varphi(x)=y$ ) and admissible. This last assumption is of more technical nature and we refer the reader to [5] for more details. On the other hand, in [62] the author deals with a generalization of the arguments of [28] needed to remove the smoothness assumption (see also [40]).

Understanding Question 2 was our original motivation. In the rest of this chapter, we explain our findings in detail.

\subsubsection{A more general setting: generalized pseudogroups}

We will discuss the finite order problem in a slightly greater generality. Namely, we will work with a generalization of pseudogroups that, via ideas similar to those from Proposition 3.2.8, can be studied by means of étale groupoids that are not necessarily effective.

We begin by observing that the set $\operatorname{Bis}(\Sigma)$ of bisections of a Lie groupoid $\Sigma \rightrightarrows$ $\mathbf{X}$ has a group structure. In fact, if $\sigma_{1}, \sigma_{2} \in \operatorname{Bis}(\Sigma)$ one sets

$$
\sigma_{1} \cdot \sigma_{2}: x \rightarrow \sigma_{1}\left(t\left(\sigma_{2}(x)\right)\right) \cdot \sigma_{2}(x)
$$

Notice that this is, as claimed, a group multiplication. The identity is the unit section $\mathbf{X} \rightarrow \Sigma$ sending $x$ to $1_{x}$. Notice also that the diffeomorphism of $\mathbf{X}$ induced by $\sigma_{1} \cdot \sigma_{2}$ is the composition of the one induced by $\sigma_{1}$ with the one induced by $\sigma_{2}$. The set of locally defined bisections $\operatorname{Bis}_{\text {loc }}(\Sigma)$ can be equipped with a grouplike structure starting from the group structure of $\operatorname{Bis}(\Sigma)$, similarly to what is done on $\operatorname{Diff}_{\text {loc }}(\mathbf{X})$ starting from the composition of diffeomorphisms in Diff $(\mathbf{X})$. Moreover, $\operatorname{Bis}_{\text {loc }}(\Sigma)$ is clearly closed under restrictions.

These two facts together make $\operatorname{Bis}_{\text {loc }}(\Sigma)$ look quite similar to $\operatorname{Diff}_{\text {loc }}(\mathbf{X})$. 
Definition 3.2.51. Let $\Sigma \rightarrow \mathrm{X}$ be a Lie groupoid. A generalized pseudogroup on X supported by $\Sigma$ is a subset $\Gamma \subset \operatorname{Bisloc}_{\text {loc }}(\Sigma)$ of the set of local bisections of $\Sigma$ which

- is closed under multiplication and inversion of bisections and contains the unit bisection $1_{\mathbf{X}}$;

- is closed under restrictions;

- is such that, if $\left\{U_{i}\right\}_{i \in I}$ covers $U, \sigma_{i}: U_{i} \rightarrow \Sigma$ belongs to $\Gamma$ for all $i^{\prime}$ s and there exists $\sigma \in \operatorname{Bis}_{\text {loc }}(\Sigma)$ restricting to $\sigma_{i}$ over $U_{i}$, then $\sigma \in \Gamma$.

From now on, if we need to stress that $\Gamma$ is a pseudogroup of locally defined diffeomorphisms, we will use the terminology classical pseudogroups.

Generalized pseudogroups show up naturally when associating a classical pseudogroup to an étale groupoid: the pseudogroup is simply the image by $t$ of the generalized pseudogroup of bisections of $\mathcal{G}$. As for classical pseudogroups, a generalized pseudogroup $\Gamma$ over $\mathbf{X}$ has an associated germ groupoid $\operatorname{Germ}(\Gamma) \rightrightarrows$ $\mathbf{X}$, where the target is now defined by $t\left(\operatorname{germ}_{x}(b)\right)=t(b(x))$. When equipped with the étale topology, $\operatorname{Germ}(\Gamma) \rightrightarrows \mathbf{X}$ becomes an étale groupoid. Furthermore, if $\mathcal{G} \rightrightarrows \mathbf{X}$ is an étale groupoid,

$$
\Gamma_{\mathcal{G}}:=\operatorname{Bis}_{\text {loc }}(\mathcal{G})
$$

is a generalized pseudogroup over $\mathbf{X}$ supported by $\mathcal{G}$ itself.

Proposition 3.2.52. Let $\Gamma$ be a generalized pseudogroup supported by a Lie groupoid $\Sigma \rightrightarrows \mathbf{X}$. There is a natural bijection

$$
\Psi: \Gamma \rightarrow \Gamma_{\operatorname{germ}(\Gamma)}
$$

such that

- $\operatorname{Dom}(\Psi(\sigma))=\operatorname{Dom}(\sigma), \sigma \in \Gamma$;

- $\Psi$ commutes with restrictions;

- $(t \circ \Psi)(\sigma)=t \circ \sigma, \sigma \in \Gamma$;

- $\Psi\left(\sigma_{1} \cdot \sigma_{2}\right)=\Psi\left(\sigma_{1}\right) \cdot \Psi\left(\sigma_{2}\right), \sigma_{i} \in \Gamma, t\left(\sigma_{2}\right)(x) \subset \operatorname{Dom}\left(\sigma_{1}\right)$ for all $x \in \operatorname{Dom}\left(\sigma_{2}\right)$.

Proof. Consider the sheaf $\mathcal{S}=\Gamma\left(\Sigma,{ }_{-}\right)$of local sections of the source map of $\Sigma$. If $\sigma \in \Gamma(\Sigma, \operatorname{Dom}(\sigma))$, then the map

$$
x \in \operatorname{Dom}(\sigma) \rightarrow \operatorname{germ}_{x}(\sigma) \in \operatorname{Germ}(\mathcal{S})
$$

defines a section of the étale space $\operatorname{Germ}(\mathcal{S})$. We declare it to be $\Psi(\sigma)$ and obtain the map of sheaves - preserving domains and commuting with restrictions -

$$
\Psi: \mathcal{S} \rightarrow \Gamma\left(\mathcal{G e r m}(\mathcal{S}),_{-}\right) .
$$

Observe that $(t \circ \Psi)(\sigma)=t \circ \sigma$ follows from the definition. As a consequence, $\Psi$ restricts to a map, denoted by the same symbol,

$$
\Psi: \operatorname{Bis}_{\text {loc }}(\Sigma) \rightarrow \operatorname{Bis}_{\text {loc }}\left(\operatorname{Germ}\left(\operatorname{Bis}_{\text {loc }}(\Sigma)\right)\right),
$$


and, in fact, to a map

$$
\Psi: \Gamma \rightarrow \Gamma_{\mathcal{G e r m}(\Gamma)},
$$

which preserves domains, commutes with restrictions and is such that $(t \circ \Psi)(\sigma)=$ $t \circ \sigma$, for all $\sigma \in \Gamma$. $\Psi$ preserves the product of bisections: if $\sigma_{1}, \sigma_{2}$ are bisections of $\Gamma$ such that $t \circ \sigma_{2}\left(\operatorname{Dom}\left(\sigma_{2}\right)\right) \subset \operatorname{Dom}\left(\sigma_{1}\right)$ then $\Psi\left(\sigma_{2} \cdot \sigma_{1}\right)$ is the map

$$
x \in \operatorname{Dom}\left(\sigma_{2}\right) \rightarrow \operatorname{germ}_{x}\left(\sigma_{1} \cdot \sigma_{2}\right)=\operatorname{germ}_{t\left(\sigma_{2}(x)\right)}\left(\sigma_{1}\right) \cdot \operatorname{germ}_{x}\left(\sigma_{2}\right),
$$

which shows that $\Psi\left(\sigma_{2} \cdot \sigma_{1}\right)=\Psi\left(\sigma_{2}\right) \cdot \Psi\left(\sigma_{1}\right)$. It remains to show that $\Psi$ is bijective. We prove it directly; notice that the argument below is equivalent to showing that $\Psi$ is bijective at the level of sheaf of sections of the source maps and then arguing that the map induced on $\Gamma$ takes values in $\Gamma_{\mathcal{G e r m}(\Gamma)}$.

Injectivity of $\Psi: \Gamma \rightarrow \Gamma_{\operatorname{germ}(\Gamma)}$ follows since, given $\sigma_{1} \neq \sigma_{2} \in \Gamma$, either $\operatorname{Dom}\left(\sigma_{1}\right) \neq \operatorname{Dom}\left(\sigma_{2}\right)$ or there is $x \in \operatorname{Dom}\left(\sigma_{1}\right)=\operatorname{Dom}\left(\sigma_{2}\right)$ such that $\sigma_{1}(x) \neq$ $\sigma_{2}(x)$, which implies $\operatorname{germ}_{x}\left(\sigma_{1}\right) \neq \operatorname{germ}_{x}\left(\sigma_{2}\right)$, hence $\Psi\left(\sigma_{1}\right) \neq \Psi\left(\sigma_{2}\right)$. Of course, this is argument independent from the fact that the $\sigma_{i}{ }^{\prime}$ 's are bisections.

$\Psi: \Gamma \rightarrow \Gamma_{\operatorname{Germ}(\Gamma)}$ is also surjective. Let

$$
b: \operatorname{Dom}(b) \rightarrow \operatorname{Germ}(\Gamma)
$$

be a bisection; then, for each $x \in \operatorname{Dom}(b), b(x)=\operatorname{germ}_{x}\left(\sigma_{x}\right)$, where $\sigma_{x}$ is an element of $\Gamma$ defined on $\operatorname{Dom}\left(\sigma_{x}\right) \subset \operatorname{Dom}(b)$ such that $x \in \operatorname{Dom}\left(\sigma_{x}\right)$. We obtain a family $\left\{\sigma_{x}\right\}_{x \in \operatorname{Dom}(b)}$. Since $\operatorname{Germ}(\Gamma)$ has the étale topology and $b$ is continuous, we have

$$
\sigma_{x}=\sigma_{y}, \quad \text { over } \operatorname{Dom}\left(\sigma_{x}\right) \cap \operatorname{Dom}\left(\sigma_{y}\right) .
$$

It follows that the $\sigma_{x}$ 's glue to a section $\sigma$ of $\Sigma$ defined over $\operatorname{Dom}(b)$, and $\Psi(\sigma)=b$. However, $b$ is a bisection; since $(t \circ \Psi)(\sigma)=t \circ \sigma$, it follows that $\sigma$ is a bisection as well. Furthermore, since $\Gamma$ is a pseudogroup, $\sigma \in \Gamma$.

In a sense, the proposition above seems to tell us that generalized pseudogroups correspond to not necessarily étale Lie groupoids by the same construction used to prove Haefliger's correspondence from Proposition 3.2.8. This is not correct though, since the support $\Sigma \rightrightarrows \mathbf{X}$ is part of the definition of generalized pseudogroup and the above construction changes it from $\Sigma$ to $\operatorname{Germ}(\Gamma)$.

Even in the case of classical pseudogroups, when one has the correspondence with effective étale Lie groupoids, passing to groupoids some information is lost, as we already pointed out (Remark 3.2.14).

Remark 3.2.53. Let $\Gamma$ be a pseudogroup supported by $\Sigma$. Part of the structure that $\Sigma$ adds to $\Gamma$ (the part that is relevant for our purposes) is essentially a(n interesting) topology - even better, a diffeology - on the space of elements of $\Gamma$ defined over some open. Roughly speaking, $\Sigma$ allows to talk about smooth families in $\Gamma$. By smooth family over an open $U \subset \mathbf{X}$ parametrized by some open $W \subset \mathbb{R}^{d}$ we mean a smooth map

$$
F: W \times U \rightarrow \Sigma
$$

such that, for all $t \in W, F\left(t,{ }_{-}\right): U \rightarrow \Sigma$ is a bisection of $\Sigma$ defined over $U$ and lying in $\Gamma$. The smooth structure of $\Sigma$ influence the existence of such families. 
For example, if the generalized pseudogroup $\Gamma$ is supported by its corresponding étale groupoid $\operatorname{Germ}(\Gamma)$, there are no non trivial smooth families. On the other hand, if the support is a non étale Lie groupoid $\Sigma \rightarrow \mathbf{X}$, such non trivial families usually exist; for example, in the case of $\Gamma^{\mathbf{X}}$, which is naturally supported by $\mathbf{X} \times \mathbf{X}$.

Smooth families play a role in the finite order problem. See the discussion about continuity and smothness of natural bundles after Question 2 in Subsection 3.2.4. This means that we cannot simply replace generalized pseudogroups with étale groupoids $\mathcal{G} \rightrightarrows \mathbf{X}$, because in this way some relevant information is lost. This is the same phenomenon as the one described in Remark 3.2.14. See also the next remark.

Remark 3.2.54. A characterization of classical pseudogroups among generalized ones arises by observing that they are (generalized) pseudogroups of bisection of the pair groupoid. The key remark is that local bisections of the pair groupoid $\mathbf{X} \times \mathbf{X} \rightrightarrows \mathbf{X}$ corresponds precisely to local diffeomorphisms of $\mathbf{X}$. Hence, if the support $\Sigma \rightrightarrows \mathbf{X}$ of a generalized pseudogroup $\Gamma$ can be chosen to be the pair groupoid $\mathbf{X} \times \mathbf{X} \rightrightarrows \mathbf{X}$, the germ groupoid $\operatorname{Germ}(\Gamma)$ is necessarily effective. Viceversa, if an étale groupoid $\mathcal{G}$ is effective, then its generalized pseudogroup of bisections is isomorphic to a pseudogroup of local diffeomorphisms of $\mathbf{X}$, i.e. a generalized pseudogroup supported by $\mathbf{X} \times \mathbf{X} \rightrightarrows \mathbf{X}$.

Example 3.2.55. A generalized pseudogroup over a point correspond to a (discrete) subgroup $\Gamma$ of some Lie group $G, G$ playing the role of support. In fact, a bisection whose domain is a point corresponds to a point and the group structure on the set of bisections is the group structure of $\Gamma$. In this case, the topology on $\Gamma$ induced by the support is the topology induced by $G$.

Example 3.2.56. We will use the notation $\Gamma^{\Sigma}$ to denote the generalized pseudogroup of all local bisections of some Lie groupoid $\Sigma$. Of course, $\Gamma^{\mathbf{X} \times \mathbf{X}} \cong \Gamma^{\mathbf{X}}=$ $\operatorname{Diff}_{\text {loc }}(\mathbf{X})$.

Example 3.2.57 (Automorphisms of principal bundles). An example which is already quite interesting is the one where $\Sigma$ is a transitive Lie groupoid. It leads to sets of automorphisms of principal bundles satisfying multiplicativity and gluing conditions analogous to those of classical pseudogroups. Recall here that transitivity means that the map

$$
\Sigma \rightarrow \mathbf{X} \times \mathbf{X}, \quad g \rightarrow(s(g), t(g))
$$

is a surjective submersion. The surjectivity condition can be rephrased by saying that for each $x \in \mathbf{X}$ and $y \in \mathbf{X}$ there is $g \in \Sigma$ with $s(g)=t(y)$.

Let us assume $\Sigma \rightrightarrows \mathbf{X}$ to be transitive. A consequence of transitivity is that $\Sigma \rightrightarrows \mathbf{X}$ is Morita equivalent via the $s$-fiber $s^{-1}(x)$ to its isotropy group $G_{x}:=$ $s^{-1}(x) \cap t^{-1}(x)$ (notice that the choice of $x$ is irrelevant - i.e. changing point in $\mathbf{X}$ leads to isomorphic principal bundles).

It is well known that working with principal $G$-bundles is equivalent to working with transitive Lie groupoids. The key point is the gauge construction. Given a transitive Lie groupoid $\Sigma \rightrightarrows \mathbf{X}$ we have the principal $G_{x}$-bundle $s^{-1}(x)$, and, 
given a principal $G$-bundle $\pi: P \rightarrow \mathbf{X}$, we have its gauge groupoid $P \otimes_{G} P \rightrightarrows \mathbf{X}$ - the quotient of the pair groupoid $P \times P \rightrightarrows P$ with respect to the diagonal action.

This construction sends a (local) bisection of a transitive Lie groupoid $\Sigma$ to a (local) automorphism of the principal $G$-bundle $\pi: P \rightarrow \mathbf{X}$ (we set $G:=G_{x}$, $x \in \mathbf{X}$ ). If $\sigma: \mathbf{X} \rightarrow P \otimes_{G} P$ is a bisection of the gauge groupoid, then the corresponding automorphism of $P$ is defined by

$$
\phi_{\sigma}: P \rightarrow P, \quad p \rightarrow q:[(q, p)]=\sigma(\pi(p))
$$

and a local automorphism $\phi \in \operatorname{Aut}_{\text {loc }}(P)$ induces the bisection

$$
\sigma_{\phi}: \mathbf{X} \rightarrow P \otimes_{G} P, \quad x \rightarrow[(\phi(p), p)] .
$$

Notice also that, under this correspondence, the product of bisections corresponds to the composition of automorphisms. Consequently, generalized pseudogroups supported by transitive Lie groupoids can be described as sets $\Gamma$ of locally defined (on $\mathbf{X}$ ) automorphisms of $P$. The conditions ensuring that $\Gamma$ is a generalized pseudogroup are equivalent to asking that $\Gamma$ contains $i d_{P}$, is closed with respect to composition, inversion and restriction of locally defined automorphisms of $P$ and is such that a locally defined automorphism $\phi$ of $P$ belongs to $\Gamma$ if and only if $\left.\phi\right|_{U_{i}} \in \Gamma$ for all $i \in I$, where $\left\{U_{i}\right\}_{i \in I}$ is a cover of $\operatorname{Dom}(\phi)$.

As for classical pseudogroups, $\Gamma$-spaces are defined as $\operatorname{Germ}(\Gamma)$-spaces. The definition of Lie pseudogroups and the discussion about order is repeated verbatim. Let $\Gamma$ be supported by $\Sigma \rightrightarrows \mathbf{X}$.

One looks at the tower of jets of the submersion $s: \Sigma \rightarrow \mathbf{X}$. Restricting to jets of bisections, we get the tower

$$
J^{\infty} \Sigma \rightarrow \ldots \rightarrow J^{l} \Sigma \rightarrow J^{l-1} \Sigma \rightarrow \ldots \rightarrow J^{1} \Sigma \rightarrow J^{0} \Sigma \cong \Sigma
$$

where

$$
J^{k} \Sigma=\left\{j_{x}^{k} \sigma: x \in \mathbf{X}, \sigma \in \operatorname{Bis}_{\mathrm{loc}}(\Sigma), x \in \operatorname{Dom}(\sigma)\right\} .
$$

The above tower is a tower of Lie groupoids and all the maps are surjective submersive Lie groupoid maps; notice also that the natural map $J^{\infty} \Sigma \rightarrow \lim _{\leftarrow} J^{l} \Sigma$ is a bijection. Restricting once more to bisections in $\Gamma$ we get the tower

$$
J^{\infty} \Gamma \rightarrow \ldots \rightarrow J^{l} \Gamma \rightarrow J^{l-1} \Gamma \rightarrow \ldots \rightarrow J^{1} \Gamma \rightarrow J^{0} \Gamma .
$$

Definition 3.2.58. A generalized pseudogroup $\Gamma$ supported by $\Sigma$ is called Lie pseudogroup when

- $J^{k} \Gamma$ is a Lie subgroupoid of $J^{k} \Sigma$ for all $k \in \mathbb{N}$;

- the projections $J^{k} \Gamma \rightarrow J^{k-1} \Gamma$ are submerisve.

It is called strongly Lie if the natural map $J^{\infty} \Gamma \rightarrow \lim _{\leftarrow} J^{l} \Gamma$ is a bijection. A generalized Lie pseudogroup $\Gamma$ supported by $\Sigma$ is of order $k$ if $\sigma \in \Gamma$ if and only if $j_{x}^{k} \sigma \in \Gamma$, for all $x \in \operatorname{Dom}(\sigma)$, and $k$ is minimal with this property. 
Definition 3.2.59. Let $\Gamma$ be a Lie (generalized) pseudogroup supported by $\Sigma$. The tower

$$
\ldots \rightarrow J^{k} \Gamma \rightarrow J^{k-1} \Gamma \rightarrow \ldots \rightarrow J^{1} \Gamma \rightarrow J^{0} \Gamma \rightrightarrows \mathbf{X}
$$

is called canonical Cartan tower of $\Gamma$.

On the $k$-th jet space of $s: \Sigma \rightarrow \mathbf{X}$ we have the Cartan distribution $\mathcal{C}^{k}$, Definition 3.2.15. $\complement^{k}$ can be restricted to $J^{k} \Gamma$; when $\Gamma$ is a Lie pseudogroup, we get a regular distribution $[55,69]$, still denoted by $\complement^{k}$, on $J^{k} \Gamma$. As in the classical case, the canonical Cartan tower is a tower of Lie prolongations of Pfaffian groupoids, see Definitions 3.B.6, 3.B.12 and 3.B.20, and the discussion in Subsection 3.2.2. We will write

$$
\ldots \rightarrow\left(J^{k} \Gamma, \mathrm{e}^{k}\right) \rightarrow\left(J^{k-1} \Gamma, \mathrm{e}^{k-1}\right) \rightarrow \ldots \rightarrow\left(J^{1} \Gamma, \mathrm{e}^{1}\right) \rightarrow J^{0} \Gamma \rightrightarrows \mathbf{X} .
$$

Example 3.2.60. Generalized pseudogroup can be interesting not only as a generalization of classical pseudogroups but also as a mean to "lift" non-Lie pseudogroups to Lie ones. For instance, consider the pseudogroup $\Gamma_{\text {rot }}$ of rotations in $\mathbb{R}^{2}$. Its germ groupoid injects into the germ groupoid of the pseudogroup of leafwise diffeomorphisms of the singular foliation of $\mathbb{R}^{2}$ by concentric circles, see Example 3.2.21. The two pseudogroups have the same orbits, which implies that $\Gamma_{\text {rot }}$ is not Lie, because $J^{0} \Gamma_{\text {rot }}$ is not a Lie subgroupoid of the pair groupoid. However, one can consider the generalized pseudogroup $\hat{\Gamma}_{\text {rot }}$ of constant bisection of the action groupoid $\mathbb{S}^{1} \ltimes \mathbb{R}^{2}$, where the action is by rotations (hence the orbits of the groupoid are the orbits of $\left.\Gamma_{\text {rot }}\right)$. It turns out that $\hat{\Gamma}_{\text {rot }}$ is a Lie pseudogroup; notice that $J^{0} \hat{\Gamma}_{\text {rot }} \cong \mathbb{S}^{1} \ltimes \mathbb{R}^{2}$.

Then, one also generalizes the following concepts.

i) $\Gamma$-spaces, formally determined $\Gamma$-spaces and $\Gamma$-spaces of order $k$. They are, respectively: $\operatorname{Germ}(\Gamma)$-spaces, $\operatorname{Germ}(\Gamma)$-spaces such that the action factors through $J^{\infty} \Gamma$ and $\operatorname{Germ}(\Gamma)$-spaces such that the action factors through $J^{k} \Gamma$. The finite order problem is formulated as before: which conditions on $\Gamma$ ensures that (a large subcategory of) $\Gamma$-spaces are of finite order?

ii) $\Gamma$-atlases and $\Gamma$-structures. We define the notion of generalized $\Gamma$-structure on a manifold $M$ as (an isomorphism class of) principal Germ $(\Gamma)$-bundles $\mathcal{P} \rightarrow M$ where the map $\mu: \mathcal{P} \rightarrow \mathbf{X}$ along which $\operatorname{Germ}(\Gamma)$ acts is étale (which implies $\operatorname{dim}(\mathbf{X})=\operatorname{dim}(M)$ ). As before, such principal bundles can be encoded into equivalence classes of $\Gamma$-atlases. In this setting, $\Gamma$-atlases are defined as the datum of a cover $\mathcal{U}=\left\{U_{i}\right\}_{i \in I}$ of $M$ together with smooth maps

$$
\gamma_{i j}: U_{i} \cap U_{j} \rightarrow \operatorname{Germ}(\Gamma), \quad i, j \in I
$$

such that the $\gamma_{i i}$ 's are unit valued and diffeomorphisms onto their images

$$
\gamma_{i i}: U_{i} \rightarrow \mathbf{X}, \quad i \in I
$$

and the cocycle conditions

$$
\gamma_{j k}(x) \cdot \gamma_{i j}(x)=\gamma_{i k}(x)
$$

holds for all $x \in U_{i} \cap U_{j} \cap U_{k}$ and $i, j, k \in I$, where $\cdot$ is the product of bisections. 
Example 3.2.61. Looking back at Example 3.2.57, let us consider the generalized pseudogroup of locally defined automorphisms of the trivial principal $G$-bundle $\mathbb{R}^{n} \times G \rightarrow \mathbb{R}^{n}$ (here, $G$ acts on $\mathbb{R}^{n} \times G$ from the right). We denote this pseudogroup by $\Gamma^{n, G}$. An automorphism of $\mathbb{R}^{n} \times G \rightarrow \mathbb{R}^{n}$ defined over some open subset $U \subset \mathbb{R}^{n}$ is the same as a pair $(f, \rho)$ given by a diffeomorphism $f: U \rightarrow V \subset \mathbb{R}^{n}$ and a map $\rho: U \rightarrow G$. On the one hand, given such a pair, the corresponding automorphism of $\mathbb{R}^{n} \times G \rightarrow \mathbb{R}^{n}$ defined over $U$ is given by $(x, g) \rightarrow(f(x), \rho(x) \cdot g)$. On the other hand, a local automorphism $\varphi$ of $\mathbb{R}^{n} \times G \rightarrow \mathbb{R}^{n}$ defined over $U$ induces the pair $\left(f_{\varphi}, \rho_{\varphi}\right)$ where, for $x \in U, f_{\varphi}(x)=\left(p r_{1} \circ \varphi\right)(x, e)$ and $\rho_{\varphi}(x)=$ $\left(p r_{2} \circ \varphi\right)(x, e)$. Notice also that the composition $\varphi \circ \psi$ of two local automorphisms $\varphi, \psi$ corresponds to the pair $\left(f_{\varphi} \circ f_{\psi}, \rho_{\varphi} \cdot \rho_{\psi}\right)$.

Let now $\left\{U_{i}\right\}_{i \in I}$ be an open cover of a manifold $M$ and $\left\{\gamma_{i j}\right\}_{i, j \in I}$ be a collection of maps

$$
\gamma_{i j}: U_{i} \cap U_{j} \rightarrow \operatorname{Germ}\left(\Gamma^{n, G}\right)
$$

defining a $\Gamma^{n, G}$-atlas on $M$. In particular, we have the charts of $M$

$$
\gamma_{i i}: U_{i} \rightarrow \mathbb{R}^{n}, \quad i \in I
$$

For all $x \in U_{i} \cap U_{j}, \gamma_{i j}(x)=\operatorname{germ}_{\gamma_{i i}(x)}\left(\sigma_{i j}\right)$, where $\sigma_{i j}$ is a bisection of $\operatorname{Germ}\left(\Gamma^{n, G}\right)$ defined on $\gamma_{i i}\left(U_{i} \cap U_{j}\right)$; by the discussion in Example 3.2.57 and by Proposition 3.2.52, we have that $\sigma_{i j}$ is equivalently described as an automorphism $\varphi_{i j}$ of $\mathbb{R}^{n} \times G \rightarrow \mathbb{R}^{n}$ defined over $\gamma_{i i}\left(U_{i} \cap U_{j}\right)$. Moreover, $\varphi_{i j}$ is equivalently described as a pair $\left(f_{\varphi_{i j}}, \rho_{\varphi_{i j}}\right)$ as in the discussion above. We observe what follows.

- The family $\left\{\left(U_{i}, \phi_{i}\right)\right\}_{i \in I}$, where $\phi_{i}:=\gamma_{i i}, i \in I$, defines an atlas of $M$ with transition functions

$$
f_{\varphi_{i j}}: \gamma_{i i}\left(U_{i} \cap U_{j}\right) \rightarrow \gamma_{j j}\left(U_{i} \cap U_{j}\right), \quad i, j \in I .
$$

- The maps

$$
g_{i j}:=\left.\rho_{\varphi_{i j}} \circ \gamma_{i i}\right|_{U_{i} \cap U_{j}}: U_{i} \cap U_{j} \rightarrow G, \quad i, j \in I,
$$

define a $G$-cocycle on $M$; the cocycle conditions for the $g_{i j}$ 's hold thanks to the cocycle conditions for the $\gamma_{i j}$ 's.

Consequently, we see that a $\Gamma^{n, G}$-atlas on $M$ is equivalent to assigning:

- an atlas $\left\{U_{i}, \phi_{i}\right\}_{i \in I}$ on $M$;

- a $G$-cocycle on $M$ over the open cover $\left\{U_{i}\right\}_{i \in I}$.

It follows that a $\Gamma^{n, G}$-structure on $M$ consists of a smooth structure on $M$ together with the datum of a principal $G$-bundle (up to isomorphisms) $P \rightarrow M$.

iii) Natural $\Gamma$-bundles. Here we use the fact, observed after discussing Lemma 3.2.39, that, when $\mathcal{G}$ is an étale groupoid, the gauge groupoid $\mathcal{P} \otimes_{\mathcal{G}} \mathcal{P}$ is also étale. Consequently, it induces a generalized pseudogroup $\Gamma_{\mathcal{P}}$ on $M$; to be precise,

$$
\Gamma_{\mathcal{P}}=\operatorname{Bis}_{\text {loc }}\left(\mathcal{P} \otimes_{\mathcal{G}} \mathcal{P}\right)
$$


This leads us to generalizing Subsection 3.2.3 and Subsection 3.2.4. In fact, Propositions 3.2.44 and 3.2.45 still hold true; hence, $\Gamma$-spaces can be re-encoded as functors, that we keep calling natural $\Gamma$-bundles. The only difference is that, when $\Gamma$ is not classical, arrows in $\operatorname{Man}^{\Gamma}$ cannot be encoded purely in terms of maps between manifolds satisfying some additional properties. See the example below.

Example 3.2.62. When $\Gamma=\Gamma^{n, G}, G$ being a Lie group (see Example 3.2.61 above), the functors corresponding to $\Gamma$-spaces are known in the literature $[27,40]$ as gauge-natural bundles. They are functors $E$

- from the category $P(n, G)$ of principal $G$-bundles over $n$-dimensional manifolds, with arrows given by principal bundle morphisms lifting open embeddings;

- to the category $\mathrm{Fib}^{\mathrm{n}}$ of fibrations over $n$-dimensional manifolds, with arrows given by fibered maps lifting open embeddings;

- such that $E(P \rightarrow M)$ fibers over $M$;

- if $\psi: P_{1} \rightarrow P_{2}$ is a principal bundle morphism lifting an embedding $\phi$ : $M_{1} \rightarrow M_{2}$, then $E(\phi)$ lifts $\phi$.

These functors have been extensively studied in the literature, due to their relevance for the mathematical formulation of classical gauge field theories; they were introduced in [27] and a finite order result holds for them. This was proven in [27] assuming the functor to send smooth families to smooth families (see the discussion at the end of Subsection 3.2.4); this smoothness assumption was later removed, see [40].

\subsection{Smooth supports for étale groupoids. Lie algebra sheaves.}

We introduced in Subsection 3.2.5 the idea of support for a generalized pseudogroup $\Gamma$ : a Lie groupoid $\Sigma \rightrightarrows \mathbf{X}$ such that $\Gamma$ is a subset of $\Gamma^{\Sigma}=\operatorname{Bis}_{\text {loc }}(\Sigma)$ in a way respecting the group-like and sheaf-like structures (Definition 3.2.51) Rewriting this in terms of groupoids,

Definition 3.3.1. A support for an étale groupoid $\mathcal{G} \rightrightarrows \mathbf{X}$ is a Lie groupoid $\Sigma \rightrightarrows \mathbf{X}$ together with a Lie groupoid morphism

$$
i: \mathcal{G} \rightarrow \Sigma
$$

such that the induced map

$$
\operatorname{Bis}_{\mathrm{loc}}(\mathcal{G}) \rightarrow \operatorname{Bis}_{\mathrm{loc}}(\Sigma), \quad \sigma \rightarrow[x \rightarrow i(\sigma(x))]
$$

is injective. 
With supports at hand, one can generalize rigorously Haefliger's correspondence from Proposition 3.2.8:

$$
\left\{\begin{array}{c}
\text { generalized pseudogroups } \Gamma \text { on } \mathbf{X} \\
\text { with support }
\end{array}\right\} \stackrel{1-1}{\longleftrightarrow}\left\{\begin{array}{c}
\text { étale groupoids } \mathcal{G} \rightrightarrows \mathbf{X} \\
\text { with support }
\end{array}\right\}
$$

Remark 3.3.2. Let $\Gamma$ be a generalized pseudogroup on $\mathbf{X}$ supported by $\Sigma \rightrightarrows \mathbf{X}$. Consider the germ groupoid $\operatorname{Germ}(\Gamma) \rightrightarrows \mathbf{X}$. Then $\Sigma$ is a support for $\operatorname{Germ}(\Gamma)$. The Lie groupoid morphism $\operatorname{Germ}(\Gamma) \rightarrow \Sigma$ is given by

$$
i: \operatorname{Germ}(\Gamma) \rightarrow \Sigma, \quad \operatorname{germ}_{x}(\sigma) \rightarrow \sigma(x) .
$$

On the other hand, a support $\Sigma^{\prime}$ for $\operatorname{Germ}(\Gamma)$ can be thought of as an alternative support for $\Gamma$, thanks to Proposition 3.2.52.

Remark 3.3.3. It is clear that an étale groupoid admits in general many supports. In Remark 3.2.54 we pointed out that effective étale Lie groupoids are precisely those supported by the pair groupoid $\mathbf{X} \times \mathbf{X} \rightrightarrows \mathbf{X}$, but already here one sees that the étale groupoid $\operatorname{Germ}\left(\Gamma^{\mathcal{F}}\right)$, where $\mathcal{F}$ is a foliation of positive codimension of $\mathbf{X}$ (see Example 3.2.4), is also supported by a strict subgroupoid of $\mathbf{X} \times \mathbf{X}$. This is also evident in the case of discrete groups $\Gamma$ (Example 3.2.55); a, possibly positive dimensional, Lie group structure on $\Gamma$ defines a support (the identity being the group morphism from Definition 3.3.1), but so is any group injection $\Gamma \hookrightarrow G$, where $G$ is a possibly positive dimensional Lie group.

Inspired by the remark above, one could try to redefine a support for $\mathcal{G}$ as any Lie groupoid $\Sigma$ such that $\operatorname{Bis}_{\text {loc }}(\mathcal{G}) \rightarrow \operatorname{Bis}_{\text {loc }}(\Sigma)$ is a bijection, but there are subtleties involved. Consider for example the pseudogroups $\Gamma_{G}$ from Example 3.2.4, where $G \hookrightarrow G L_{n}(\mathbb{R})$. As for any classical pseudogroup, a natural choice for a support is the pair groupoid $\mathbb{R}^{n} \times \mathbb{R}^{n} \rightrightarrows \mathbb{R}^{n}$. It is not clear how to produce a support $\Sigma \rightrightarrows \mathbb{R}^{n}$ inducing the same notion of smooth families (Remark 3.2.14) but whose pseudogroup of bisection is (in bijection with) $\Gamma_{G}$.

However, as we anticipated in Remark 3.2.14 and Remark 3.2.53, for us the role of supports on pseudogroups $\Gamma$ is

- to make precise the notion of generalized pseudogroup;

- to provide a way to talk about smooth families on $\Gamma$.

Similarly, we are still interested in talking about smooth families of locally defined bisections of étale groupoids; our Definition 3.3.1 allows to do so in a satisfactory way. Details are provided in this section, by means of diffeologies. We also introduce a notion of convergence which is needed for discussing the finite order problem. Finally, we introduce Lie algebra sheaves supported by Lie algebroids as the infinitesimal counterpart of generalized pseudogroups (supported by Lie groupoids). To make sense of "infinitesimal", a support is needed. This infinitesimal approach will be useful to handle the finite order problem.

\subsubsection{Diffeology induced by a support, $D_{\Sigma}$-topology and $C^{\infty}$ - convergence.}

We introduce here the following notation: when $\Sigma \rightrightarrows \mathbf{X}$ is a groupoid, we write $g_{x} \in \Sigma$ to indicate that $x=s(g)$. 
Let $\mathcal{G} \rightrightarrows \mathbf{X}$ be an étale groupoid. There is a homeomorphism

$$
\mathcal{G} \rightarrow \operatorname{Germ}\left(\operatorname{Bis}_{\mathrm{loc}}(\mathcal{G})\right)
$$

where the right hand side is equipped with the sheaf (étale) topology. From now on, we will occasionally make use of this and write $g_{x}=[\gamma]_{x}$, where $g_{x} \in \mathcal{G}$ and $\gamma$ is some local bisection of $\mathcal{G}$ defined around $x$ whose germ at $x$ is the image of $g_{x}$ under the homeomorphism above.

Definition 3.3.4. The étale topology on an étale groupoid $\mathcal{G} \rightrightarrows \mathrm{X}$ is the given topology on the space of arrows $\mathcal{G} \rightrightarrows \mathbf{X}$.

Let $\left\{g_{x^{n}}^{n}\right\}_{n \in \mathbb{N}}$ be a sequence in an étale groupoid $\mathcal{G}$. Such a sequence converges to $g_{x}=[\gamma]_{x}$ in the étale topology if and only if $x^{n} \longrightarrow x$ and $g_{x^{n}}^{n}=[\gamma]_{x_{n}}$ (for $n$ sufficently big). We will write

$$
g_{x^{n}}^{n} \underset{\text { germ }}{\rightarrow} g_{x}
$$

When $\mathcal{G} \rightrightarrows \mathbf{X}$ is equipped with a support $\Sigma \rightrightarrows \mathbf{X}$, for each $g_{x} \in \mathcal{G}$ we can write $g_{x}=[\gamma]_{x}$, with $\gamma \in \Gamma^{\Sigma}=\operatorname{Bis}_{\mathrm{loc}}(\Sigma)$. We will construct an alternative topology and a diffeology on $\mathcal{G}$ that keeps track of the fact that its elements are germs of sections of a Lie groupoid $\Sigma \rightrightarrows \mathbf{X}$. We need just the basic understanding of diffeologies for our purposes. We provided the key notions in Appendix 3.C. We suggest to the reader not familiar with them to have a look there and at references quoted therein. The following construction is the one from Example 3.C.16.

The pseudogroup $\Gamma^{\Sigma}=\operatorname{Bis}_{l o c}(\Sigma)$ has the subset diffeology, see Example 3.C.6, coming from the standard functional diffeology, see Definition 3.C.14, on the set of locally defined maps $C_{\mathrm{loc}}^{\infty}(\mathbf{X}, \Sigma)$. Let us summarize briefly what we mean - see the Appendix for details.

- A diffeology on a set $S$, Definition 3.C.1 for details, is a set $D$ of parametrizations, called plots, $\varphi: U \rightarrow S$, where $U \subset \mathbb{R}^{n}$ for some $n \in \mathbb{N}$ is open, such that $D$ :

- contains constant parametrisations;

- is closed under gluing;

- is closed under pullback by smooth functions.

- Whenever $\iota: S \hookrightarrow S^{\prime}$ is an inclusion and $S^{\prime}$ possesses a diffeology, $S$ inherits the subset diffeology (Example 3.C.6), whose plots $\phi$ are parametrizations such that $\iota \circ \phi$ is a plot of $S$.

- Any space of locally defined smooth functions possesses a standard diffeology (Definition 3.C.14), which is obtained, roughly speaking, by declaring as plots those parametrisations for which the evaluation map is smooth. More precisely, if $\mathbf{X}_{1}, \mathbf{X}_{2}$ are smooth manifolds and $U \subset \mathbb{R}^{n}$ open, $f: U \rightarrow$ $C_{\mathrm{loc}}^{\infty}\left(\mathbf{X}_{1}, \mathbf{X}_{2}\right)$ is a plot in the standard functional diffeology when, for each $u \in U$, for each $x \in \operatorname{Dom}(f(u))$ there is an open neighbourhood $W \subset U$ of $u$ of $\mathrm{u}$ and an open neighbourhood $V$ of $x$ such that

$$
\text { - } V \subset \operatorname{Dom}(f(v)) \text {, for all } v \in W \text {; }
$$


- the evaluation map $e v(f): W \times V \rightarrow \mathbf{X}_{2}$ is smooth.

Recall that we have an inclusion

$$
\operatorname{Bis}_{\mathrm{loc}}(\mathcal{G}) \hookrightarrow \operatorname{Bis}_{\mathrm{loc}}(\Sigma) .
$$

We will equip $\operatorname{Bis}_{\text {loc }}(\mathcal{G})$ with the subset diffeology.

The groupoid $\mathcal{G}$ can be understood as the quotient of a subspace of $\mathbf{X} \times$ $\operatorname{Bis}_{\text {loc }}(\mathcal{G})$. More precisely, one sets

$$
S(\mathcal{G})=\left\{(x, \sigma) \in \mathbf{X} \times \operatorname{Bis}_{\text {loc }}(\mathcal{G}): x \in \operatorname{Dom}(\sigma)\right\}
$$

and consider the equivalence relation identifying sections with the same germ at a point. The quotient by such a relation can be identified with $\mathcal{G}$. Consequently, $\mathcal{G}$ inherits the quotient diffeology, Example 3.C.7. Briefly

- if $S^{\prime}$ is a diffeological space and is an equivalence relation on it, the quotient diffeology on $S:=S^{\prime} /$ is the one where plots are parametrisations that can locally be lifted to plots of $S^{\prime}$.

See also Example 3.C.16. To sum up, the intuition should be that a $k$-parameter family of local bisections of $\mathcal{G}$ is smooth if it is smooth as a family of local bisections of $\Sigma$.

Definition 3.3.5. The diffeologies on $\operatorname{Bis}_{\text {loc }}(\mathcal{G})$ and $\mathcal{G} \rightrightarrows \mathbf{X}$ constructed above are called the diffeologies induced by the support $\Sigma \rightrightarrows \mathbf{X}$.

Associated to any diffeology $D$ on a set $S$ one has the so called $D$-topology. It is the final topology on $S$ with respect to the plots; that is, the finest topology such that all plots are continuous. Recall that this is equivalent to say that $A \subset S$ is open if and only if all the preimages of $A$ via plots are open. See Definition 3.C.18 in Appendix 3.C.

If $C_{\mathrm{loc}}^{\infty}(X, \Sigma)$ is equipped with the $D$-topology of the standard functional diffeology, then $\operatorname{Bis}_{\text {loc }}(\mathcal{G})$ inherits the subspace topology. Furthermore $\mathbf{X} \times \operatorname{Bis}_{\text {loc }}(\mathcal{G})$ has the product topology and the subset $S(\mathcal{G}) \subset \mathbf{X} \times \operatorname{Bisloc}_{\text {loc }}(\mathcal{G})$ above inherits the subspace topology. Finally, the étale groupoid $\mathcal{G} \rightrightarrows \mathbf{X}$ can be equipped with the quotient topology.

Definition 3.3.6. The $D_{\Sigma}$-topology on $\mathcal{G}$ (respectively on $\operatorname{Bis}_{\operatorname{loc}}(\mathcal{G})$ ) is the topology from the paragraph above. write

If a sequence $\left\{g_{x^{n}}^{n}\right\}_{n \in \mathbb{N}}$ in $\mathcal{G}$ converges to $g_{x} \in \mathcal{G}$ in the $D_{\Sigma^{-}}$-topology, we will

$$
g_{x^{n}}^{n} \underset{D_{\Sigma}}{\rightarrow} g_{x}
$$

and similarly for elements of the pseudogroup $\operatorname{Bis}_{\text {loc }}(\mathcal{G})$.

Remark 3.3.7. We could also equip $\mathcal{G} \rightrightarrows \mathbf{X}$ (respectively $\operatorname{Bis}_{\text {loc }}(\mathcal{G})$ ) with the $D$ topology of the diffeology induced by a support $\Sigma \rightrightarrows \mathbf{X}$, cf. Definition 3.3.5.

The topology obtained in this way is not equivalent to the $D_{\Sigma}$-topology, because of the phenomenon illustrated in Example 3.C.27 in Appendix 3.C the $D$-topology of a subset diffeology is in general finer than the subspace topology. See also the example below. 
Example 3.3.8. If $\Gamma$ is an abstract group admitting a Lie group structure of positive dimension, denoted by $G$, we can take $G$ itself as a support. Notice that $C^{\infty}(p t, G) \cong G$; the standard functional diffeology on $C^{\infty}(p t, G)$ is simply the diffeology induced by the smooth structure on $G$, i.e. plots are precisely smooth maps from opens of Euclidean spaces to $G$ (Example 3.C.4). The induced diffeology on $\Gamma$ is precisely the smooth structure $G$ on $\Gamma$, and the $D$-topology of the induced diffeology coincide with the $D_{G}$-topology.

However, if $\Gamma$ is a subgroup of a Lie group $G$ of positive dimension, the $D$ topology of the induced diffeology and the $D_{G}$-topology may be different. This happens, for example, in the case of $\mathbb{Q} \subset \mathbb{R}$, where $\mathbb{R}$ has the usual smooth structure (Example 3.C.27). The diffeology induced by $\mathbb{R}$ on $\mathbb{Q}$ is the discrete one, i.e. the only plots are the constant functions (Example 3.C.3). The $D$-topology of such a diffeology is of course the discrete topology; on the other hand, the $D_{\mathbb{R}}$-topology on $\mathbb{Q}$ is the subspace topology, which makes $\mathbb{Q}$ dense in $\mathbb{R}$.

A key remark for our purposes is the following.

Proposition 3.3.9. Let $\mathcal{G} \rightrightarrows \mathbf{X}$ be an étale groupoid supported by $\Sigma \rightrightarrows \mathbf{X}$. The étale topology on $\mathcal{G}$ is sequentially finer than the $D_{\Sigma}$-topology.

Proof. In fact, if $\left\{g^{n}\right\}_{x^{n}}$ is a sequence in $\mathcal{G}, g_{x^{n}}^{n} \underset{\text { germ }}{\rightarrow} g_{x} \in G$ and $g_{x}=[\gamma]_{x}$, then $[\gamma]_{x^{n}}=g_{x^{n}}^{n}$ for $n$ big enough. This means that our sequence of points in $\mathcal{G}$ can be represented, for $n$ big enough, by a constant sequence of bisections in $\Sigma$ defined over some open $U$. Such a constant sequence converge in the $D$-topology on local maps $\mathbf{X} \rightarrow \Sigma$ (it defines a plot, which is smooth hence continuous in the $D$-topology); this implies $g_{x^{n}}^{n} \underset{D_{\Sigma}}{\rightarrow} g_{x}$.

The $D_{\Sigma}$-topologies, and the corresponding converging sequences, can look a bit mysterious. However, they turn out to be related to well known topologies on function spaces.

Let us recall that the weak Whitney $C^{\infty}$-topology on the space of sections of a surjective submersion $p: Y \rightarrow \mathbf{X}$ is the union of the weak Whitney $C^{k}$ topologies [37]. The weak Whitney $C^{k}$-topology is the subspace topology induced by the compact-open topology on the space of sections of $J^{k} Y \rightarrow \mathbf{X}$. Observe that when writing "subspace" we are using the jet maps

$$
j^{k}: \Gamma(Y, \mathbf{X}) \rightarrow \Gamma\left(J^{k} Y, \mathbf{X}\right), \quad k \in \mathbb{N}
$$

whose images are holonomic sections; here, $\Gamma(Y, \mathbf{X})$ is the space of sections of $p$ : $Y \rightarrow \mathbf{X}$, and similarly for $\Gamma\left(J^{k} Y, \mathbf{X}\right)$. A sequence of sections $\left\{\sigma^{n}\right\}_{n \in \mathbb{N}}$ converges to $\sigma \in \Gamma(Y, \mathbf{X})$ in the weak Whitney topology if and only if it converges uniformly on compact sets with all its derivatives.

The strong Whitney $C^{\infty}$-topology on the space of sections of $p: Y \rightarrow \mathbf{X}$ is the union of the strong Whitney $C^{k}$-topologies. The strong Whiney $C^{k}$-topology (see [37], where it is simply called the Whitney topology) is the topology induced by the graph topology on the space of sections of $J^{k} Y \rightarrow \mathbf{X}$, i.e. the topology where a sub-basis of neighbourhoods of $\sigma: \mathbf{X} \rightarrow J^{k} Y$ is generated (in the obvious sense, see Appendix 3.C, Subsection 3.C.5) by open neighbourhoods of the graph of $\sigma$. A sequence of sections $\{\sigma\}_{n \in \mathbb{N}}$ converges to $\sigma$ in the strong Whitney topology if and 
only if there exists a compact set $K$ such that $\left.\sigma^{n}\right|_{K}$ converges to $\left.\sigma\right|_{K}$ uniformly with all its derivatives and $\left.\sigma^{n}\right|_{\mathbf{X}-K}=\sigma_{\mathbf{X}-K}$ for all $n \in \mathbb{N}$. When a sequence of sections $\sigma^{n}$ of $p$ converges to $\sigma$ in the strong Whitney topology, we will write

$$
\sigma^{n} \underset{C^{\infty}}{\rightarrow} \sigma
$$

Definition 3.3.10. Let $\mathcal{G} \rightrightarrows \mathbf{X}$ be an étale group supported by $\Sigma \rightrightarrows \mathbf{X}$. We say that a sequence $\left\{g_{x^{n}}^{n}\right\}_{n \in \mathbb{N}}$ in $\mathcal{G} C^{\infty}$-converges to $g_{x} \in \mathcal{G}$ if and only if $x^{n} \rightarrow x$ and there are

- an open set $U \subset \mathbf{X}$ containing $x$ and $x^{n}$ for all $n$ sufficently big;

- bisections of $\mathcal{G}, \gamma^{n}, n \in \mathbb{N}$ and $\gamma$, representing the $g_{x^{n}}^{n}$ 's and $g_{x}$ respectively and defined on $U$ for $n$ big enough;

such that $\gamma^{n}$ converges to $\gamma$ in the strong Whitney $C^{\infty}$-topology on the space of sections of $\Sigma$ defined on $U$. We will write

$$
g_{x^{n}} \underset{C^{\infty}}{\rightarrow} g_{x}
$$

Reasoning as in Proposition 3.3.9, one has

Proposition 3.3.11. If $g_{x^{n}}^{n} \underset{\text { germ }}{\rightarrow} g_{x}$, then $g_{x^{n}}^{n} \underset{C^{\infty}}{\rightarrow} g_{x}$.

Furthermore, the following fact holds true.

Proposition 3.3.12. Let $\left\{g_{x^{n}}^{n}\right\}_{n \in \mathbb{N}}$ be a sequence in $\mathcal{G}$ and $g_{x} \in \mathcal{G}$. If $g_{x^{n}}^{n} \underset{C_{\infty}^{\infty}}{\rightarrow} g_{x}$ then $g_{x^{n}}^{n} \underset{D_{\Sigma}}{\rightarrow} g_{x}$

Proof. The proof of the statement is based on the results of [19], which we recalled also in Appendix 3.C, Subsection 3.C.5. To be precise, in [19] the authors show that the diffeological topology on the space of functions $C^{\infty}(M, N)$, where $M, N$ are manifolds and the diffeology is the standard one, is in general finer than the weak Whitney $C^{\infty}$-topology but coarser than the strong Whitney $C^{\infty}$-topology [37]. When $M$ is compact, the weak Whitney topology is known to be equivalent to the strong Whitney topology, which implies that the $D$-topology is equivalent to both.

Let's assume $\left\{g_{x^{n}}^{n}\right\}_{n \in \mathbb{N}}$ to be $C^{\infty}$-convergent to $g_{x}$. We choose representatives $\gamma^{n}, n \in \mathbb{N}$ and $\gamma$; by definition of $C^{\infty}$-convergence we can assume that they are all defined on a neighbourhood $U$ of $x$ and $U$ can be taken arbitrarily small. Still by definition of $C^{\infty}$-convergence, we have $\gamma^{n} \underset{C^{\infty}}{\rightarrow} \gamma$. By the aforementioned results of [19], $\gamma^{n} \rightarrow \gamma$ in the $D$-topology of the standard functional diffeology on $C^{\infty}(U, \Sigma)$, which is the subspace diffeology inherited from the standard functional diffeology on $C_{l o c}^{\infty}(\mathbf{X}, \Sigma)$. Since the $D$-topology of a subspace is finer than the subspace topology, $\gamma^{n} \underset{D_{\Sigma}}{\rightarrow} \gamma$. Since there is a sequence of representatives converging in the $D_{\Sigma}$-topology, we can conclude that $g_{x^{n}}^{n} \underset{D_{\Sigma}}{\rightarrow} g_{x}$.

A second point which will be relevant in our later discussions concerns separability. First of all, two points $x, y$ of a topological space $X$ are non separable if 
there is a finite sequence $x_{0}, \ldots, x_{k}$ satisfying $x_{0}=x, x_{k}=y$ and such that there are sequences $\left\{x_{i}^{n}\right\}_{n \in \mathbb{N}}, i=0, \ldots, k-1$, with the property that $x_{i}^{n}$ converges to both $x_{i}$ and $x_{i+1}$. Of course, the existence of non separable points implies nonHausdorffness. When two elements $g_{x}, g_{y}^{\prime}$ of $\mathcal{G}$ cannot be separated in the étale (respectively $D_{\Sigma^{-}}$) topology, we will write $g_{x} \underset{\text { germ }}{\sim} g_{y}^{\prime}$ (respectively $g_{x} \underset{D_{\Sigma}}{\sim} g_{y}^{\prime}$ ). We can also consider the Hausdorff quotients of $\mathcal{G}$ with respect to the two topologies, i.e. the quotients with respect to the relations identifying non separable elements in the étale and $D_{\Sigma}$-topology respectively. We denote them by Haus (G, germ) and $\operatorname{Haus}\left(\mathcal{G}, D_{\Sigma}\right)$. An immediate consequence of the proposition above is

Corollary 3.3.13. Non separability in the étale topology implies non separability in the D-topology. In particular, there is a continuous surjection $\operatorname{Haus}(\mathcal{G}$, germ $) \rightarrow$ $\operatorname{Haus}\left(\mathcal{G}, D_{\Sigma}\right)$.

We also have a notion of $C^{\infty}$-non separability, defined analogously, and the corresponding quotient $\operatorname{Haus}\left(\mathcal{G}, C^{\infty}\right)$.

\subsubsection{The infinitesimal counterpart of pseudogroups: Lie alge- bra sheaves}

When $\Sigma \rightrightarrows \mathbf{X}$ is a Lie groupoid, it is possible to construct a linear object that captures it infinitesimally. This object is a Lie algebroid $(A, \rho,[]$,$) over \mathbf{X}$. Recall that this means that

- $A$ is a vector bundle over $\mathbf{X}$;

- one has a vector bundle map $\rho: A \rightarrow T \mathbf{X}$;

- one has a Lie bracket on the space of sections $[]:, \Gamma(A) \times \Gamma(A) \rightarrow \Gamma(A)$;

such that the Leibniz identity

$$
[\alpha, f \beta]=f[\alpha, \beta]+L_{\rho(\alpha)}(f) \beta
$$

is satisfied for all $f \in C^{\infty}(\mathbf{X}), \alpha, \beta \in \Gamma(A)$. Notice that the bracket [ , ] makes the sheaf $\Gamma_{\text {loc }}(A)$ of local sections of $A$ into a sheaf of Lie algebras. One explicit way to attach a Lie algebroid $A$ to $\Sigma$ is the following.

- Set $A:=\left.\operatorname{Ker}(d s)\right|_{\mathbf{x}}$.

- Set $\rho:=d t: A \rightarrow T \mathbf{X}$.

- For $\alpha \in \Gamma(A)$, consider the right invariant vector field $\alpha^{R} \in X(\Sigma)$ defined as

$$
\alpha_{g}^{R}=d R_{g} \cdot \alpha_{t(g)} .
$$

where $R_{g}: s^{-1}(t(g)) \rightarrow s^{-1} s(g)$ is the right multiplication by $g \in \Sigma$. Notice that $\alpha^{R}$ is $s$-vertical since the right multiplication $R_{g}$ sends $s$-fibers to $s$ fibers. Then, set

$$
[\alpha, \beta]:=\left.\left[\alpha^{R}, \beta^{R}\right]\right|_{\mathbf{X}} .
$$

Since $\left[\alpha^{R}, \beta^{R}\right]$ is $s$-vertical and right invariant on $\Sigma,[\alpha, \beta]$ is an element of $\Gamma(A)$. 
This construction is clearly reminiscent of the Lie algebra of a Lie group, and it is indeed a generalization. We write $A=\operatorname{Lie}(\Sigma)$. The classical Lie theory can be generalized to this setting, but, differently from the case of Lie groups and Lie algebras, not every Lie algebroid is integrable - i.e. arises as the Lie algebroid of some Lie groupoid. The obstructions to integrability are well understood; see [22] and references therein for more details.

We aim at defining an infinitesimal version of generalized pseudogroups and supports by looking at the Lie algebroid of the support itself. We start by the following remark.

Let $C_{\mathbf{X}}^{\infty}$ be the sheaf of smooth functions on $\mathbf{X}$. If $A \rightarrow \mathbf{X}$ is a Lie algebroid, then its sheaf of sections

$$
\Gamma\left(A, \_\right)
$$

is a sheaf of $C_{\mathbf{X}}^{\infty}$-modules and a sheaf of Lie algebras; here we use $\Gamma(A, U)$ to denote $\Gamma\left(\left.A\right|_{U}\right)$, the space of sections of $A$ defined over $U$. Furthermore, the anchor induces a map of sheaves $\Gamma\left(A,{ }_{-}\right) \rightarrow X_{\mathbf{X}}$, where $X_{\mathbf{X}}$ is the sheaf of vector fields of $\mathbf{X}$, and the two structures interact with each other via the (sheaf version of) the Leibniz rule ${ }^{4}$.

Definition 3.3.14. A generalized Lie algebra sheaf (LAS) supported by a Lie algebroid $(A, \rho,[]$,$) on \mathbf{X}$ is a $C_{\mathbf{X}}^{\infty}$-submodule sheaf $\mathcal{A}$ of the sheaf $\Gamma\left(A, \_\right)$which is closed with respect to the Lie algebroid bracket.

Remark 3.3.15. "Closed with respect to the Lie bracket" means, of course, that if $s_{1}, s_{2} \in \mathcal{A}(U)$ then $\left[s_{1}, s_{2}\right] \in \mathcal{A}(U), U \subset \mathbf{X}$ open.

Remark 3.3.16. Any LAS is a sheaf of $C_{\mathrm{X}}^{\infty}$-modules and comes with an "anchor map" of LAS $\rho: \mathcal{A} \rightarrow X_{\mathbf{X}}$. These two structures are compatible with the bracket in the sense that the Leibniz identity coming from the supporting Lie algebroid has to be satisfied on each open $U$ in $\mathbf{X}^{5}$. This remark suggests a direction to generalize Definition 3.3.14.

When $\mathcal{A}$ is a LAS, we use $\operatorname{Germ}(\mathcal{A})$ to denote its étale space of germs. The fiber of $\operatorname{Germ}(\mathcal{A}) \rightarrow \mathbf{X}$ over $x \in \mathbf{X}$, the stalks of $\mathcal{A}$ at $x \in \mathbf{X}$, is denoted by $\mathcal{A}_{x}$. Notice that $\mathcal{A}_{x}$ is a Lie algebra; the Lie bracket is defined as

$$
\left[\operatorname{germ}_{x}\left(\alpha_{1}\right), \operatorname{germ}_{x}\left(\alpha_{2}\right)\right]=\operatorname{germ}_{x}\left[\left.\alpha_{1}\right|_{V},\left.\alpha_{2}\right|_{V}\right], \quad \operatorname{germ}_{x}\left(\alpha_{i}\right) \in \mathcal{A}_{x},
$$

where $V=\operatorname{Dom}\left(\alpha_{1}\right) \cap \operatorname{Dom}\left(\alpha_{2}\right)$. Furthermore, if $\mathcal{F}_{x}:=\left(C_{\mathbf{X}}^{\infty}\right)_{x}$ is the stalk at $x$ of the sheaf $C_{\mathbf{X}}^{\infty}$, we have that $\mathcal{A}$ is an $\mathcal{F}_{x}$-module by setting

$$
\operatorname{germ}_{x}(f) \cdot \operatorname{germ}_{x}(\alpha)=\operatorname{germ}_{x}\left(\left.\left.f\right|_{W} \cdot \alpha\right|_{W}\right), \quad \operatorname{germ}_{x}(\alpha) \in \mathcal{A}, \operatorname{germ}_{x}(f) \in \mathcal{F}_{x},
$$

where $W=\operatorname{Dom}(f) \cap \operatorname{Dom}(\alpha)$. And finally, the following version of the Leibniz identity

$$
\begin{aligned}
{\left[\operatorname{germ}_{x}\left(\alpha_{1}\right), \operatorname{germ}_{x}(f) \cdot \operatorname{germ}_{x}\left(\alpha_{2}\right)\right]=} & \operatorname{germ}_{x}(f) \cdot\left[\operatorname{germ}_{x}\left(\alpha_{1}\right), \operatorname{germ}_{x}\left(\alpha_{2}\right)\right] \\
& +\operatorname{germ}_{x}\left(L_{\rho\left(\alpha_{1}\right)}(f) \alpha_{2}\right)
\end{aligned}
$$

\footnotetext{
${ }^{4}$ To be precise, $\Gamma\left(A,{ }_{-}\right)$is a sheaf of Lie-Rinehart algebras over $C_{\mathbf{X}}^{\infty}$.

${ }^{5}$ In other words, any LAS is a sheaf of Lie-Rinehart algebras over $C_{\mathbf{X}}^{\infty}$.
} 
holds, for all $\operatorname{germ}_{x}\left(\alpha_{i}\right) \in \mathcal{A}_{x}, \operatorname{germ}_{x}(f) \in \mathcal{F}_{x}$.

The word "generalized" in Definition 3.3.14 is chosen so that it matches the terminology used for pseudogroups. Notice also that, when $A$ is $T \mathbf{X}$ with the usual Lie bracket of vector fields, the notion of LAS from Definition 3.3.14 is closely related to the notion of weak Lie algebra sheaf from [60]. In what follows, when $A=T \mathbf{X}$ we will use the terminology classical LAS.

Definition 3.3.17. A map of LAS $\mathcal{A}_{1} \rightarrow \mathcal{A}_{2}$ over a manifold $\mathrm{X}$ is a map of sheaves of Lie algebras over $\mathbf{X}$ that respects the anchor maps and the action by $C_{\mathbf{X}}^{\infty}$.

Let $\Gamma$ be a generalized pseudogroup supported by $\Sigma \rightrightarrows \mathbf{X}$ and $\operatorname{Germ}(\Gamma) \rightrightarrows \mathbf{X}$ be the corresponding germ groupoid. The key and motivating example of LAS for us comes from the sheaf of $\Gamma$-sections (Definition 3.3.19) of the Lie algebroid $\operatorname{Lie}(\Sigma)$. First, we recall the definition of flow of bisections defined by a section of $A=\operatorname{Lie}(\Sigma)$. This generalizes the notion of flow of a vector field. Moreover, it allows one to introduce an exponential map for $A=\operatorname{Lie}(\Sigma)$. Recall that, for any Lie groupoid $\Sigma \rightrightarrows \mathbf{X}$, we have the unit bisection

$$
x \in \mathbf{X} \rightarrow 1_{x} \in \Sigma,
$$

where $1_{x}$ is the unit over $x$ for the multiplicaton in $\Sigma$. Since the unit bisection is an embedding, we can interpret $\mathbf{X}$ as an embedded submanifold of $\Sigma$.

Definition 3.3.18. Let be $A=\operatorname{Lie}(\Sigma)$ and $\alpha \in \Gamma(A, \mathbf{X})$ be a section with compact support. The flow of $\alpha$ is the restriction to $\mathbb{R} \times \mathbf{X} \subset \mathbb{R} \times \Sigma$ of the flow

$$
\Phi_{\alpha^{R}}: \mathbb{R} \times \Sigma \rightarrow \Sigma
$$

of the right invariant vector field $\alpha^{R}$ corresponding to $\alpha$. We will use the notation $\Phi_{\alpha}$.

For each $t \in \mathbb{R}$, one gets a smooth map

$$
\Phi_{\alpha}^{t}: \mathbf{X} \rightarrow \Sigma, \quad x \rightarrow \Phi_{\alpha^{R}}^{t}\left(1_{x}\right) .
$$

Observe that the flow of $\alpha^{R}$ can be recovered by that of $\alpha$, since $\Phi_{\alpha^{R}}^{t}(g)=$ $\Phi_{\alpha}^{t}(t(g)) \cdot g$, because $\alpha^{R}$ is right invariant.

Observe also that, for $t \in \mathbb{R}, \Phi_{\alpha R}^{t}$ is an $s$-vertical diffeomorphism of $\Sigma$. It follows that $\Phi_{\alpha}^{t}$ can be represented by the left multiplication $L_{\sigma_{t}}$ via a bisection $\sigma: \mathbf{X} \rightarrow \Sigma$. Since $\Phi_{\alpha R}^{t}$ is a flow, $\sigma_{t}$ is a smooth family of bisections, and a one parameter group, in the sense that $\sigma_{t} \cdot \sigma_{s}=\sigma_{t+s}$ and $\sigma_{0}=1_{\mathbf{X}}$. Setting $\bar{\phi}^{t}:=t \circ \sigma_{t}$, we get an induced one parameter group of diffeomorphisms of $\mathbf{X} ; \bar{\phi}^{t}$ is precisely the flow of $\rho(\alpha)$.

With this machinery, and writing $\Gamma_{\text {cpt }}(A, \mathbf{X})$ for the set of sections of $A$ with compact support, one can define the exponential map of $A$ as

$$
\exp : \Gamma_{\operatorname{cpt}}(A, \mathbf{X}) \rightarrow \operatorname{Bis}(\Sigma), \quad \alpha \rightarrow \Phi_{\alpha}^{1}
$$

i.e. $\exp (\alpha)=\Phi_{\alpha}^{1}$. Note that $\Phi_{\alpha}^{t}=\exp (t \alpha)$.

Finally, observe that this construction can be made local, meaning that if $\alpha$ is a local section defined on some open $U$ and with compact support, $\alpha^{R}$ is defined 
over $t^{-1}(U)$ and the flow of $\alpha$ is still defined as a flow over $U$; the corresponding exponential map takes values into local bisections of $\Sigma$ over $U$. If one removes the assumption that $\alpha$ has compact support, its flow is defined only locally in $U$.

We can now give the following definition.

Definition 3.3.19. Let $\Gamma$ be a pseudogroup over $\mathbf{X}$ supported by $\Sigma \rightrightarrows \mathbf{X}$ and let be $A=\operatorname{Lie}(\Sigma)$. A local $\Gamma$-section of $A$ over an open $U \subset \mathbf{X}$ is a local section $\alpha \in \Gamma(A, U)$ whose (local) flow of bisections is by elements of $\Gamma$.

We will use the notation $\operatorname{Sec}_{A}^{\Gamma}$ to denote the set of local $\Gamma$-sections of $A=$ $\operatorname{Lie}(\Sigma)$. Notice that such a set defines a presheaf of sets

$$
U \rightarrow \operatorname{Sec}_{A}^{\Gamma}(U)=\left\{\alpha \in \operatorname{Sec}_{A}^{\Gamma}: \operatorname{Dom}(\alpha)=U\right\} .
$$

We will keep the notation $\operatorname{Sec}_{A}^{\Gamma}$ for this presheaf.

Example 3.3.20. If $\Gamma$ is an abstract group whose support consists of $\Gamma$ itself equipped with a Lie group structure, denoted by $G, \Gamma$-sections are simply elements of the Lie algebra $\mathfrak{g}$ of $G$.

Example 3.3.21. When $\Gamma$ is a classical pseudogroup and $\Sigma=\mathbf{X} \times \mathbf{X}, A=\operatorname{Lie}(\Sigma)=$ $T \mathbf{X}$ and $\Gamma$-sections correspond to $\Gamma$-vector fields, i.e. local vector fields on $\mathbf{X}$ whose flow is by elements of $\Gamma$. We denote the set of $\Gamma$-vector fields by $X_{\mathbf{X}}^{\Gamma}$.

Let $\Gamma$ be a pseudogroup over $\mathbf{X}$ supported by $\Sigma \rightrightarrows \mathbf{X}$. We have the natural question: is the sheaf of $\Gamma$-sections a LAS supported by $\operatorname{Lie}(\Sigma)$ ? The answer is not obvious.

Example 3.3.22. Let us choose $\mathbf{X}=p t$. We have that $\Gamma$ is a group and $\Sigma$ is a Lie group $G$ containing $\Gamma$ as a subgroup. The Lie algebroid Lie $(\Sigma)$ is simply the Lie algebra $\mathfrak{g}$ of $G$. In this setting, a local $\Gamma$-section is a vector $v$ in $\mathfrak{g}$ such that, for $t$ small enough, the smooth curve $t \rightarrow \exp (t v) \in G$ takes values in $\Gamma$. Let us denote by $T$ the set of vectors with such a property. We can interpret $T$ as a candidate for the tangent space of $\Gamma$ at the identity (notice that, a priori, $\Gamma$ is just a subgroup of $G$ ). $\Gamma$-sections form a LAS supported by $\mathfrak{g}$ precisely when $T$ is a Lie subalgebra of $\mathfrak{g}$.

Example 3.3.23. When $\Gamma$ is a classical pseudogroup, some sufficient conditions for $\Gamma$-vector fields to form a classical LAS are discussed in [67]. A possible option is to require the pseudogroup $\Gamma$ to be closed with respect to $C^{\infty}$-convergence (with respect to the weak topology), see Theorem 4.1 in [67] for details, but also Remark $b$ ) after Definition 1.4 in [60].

Alternatively, one has to compare $X_{\mathbf{X}}^{\Gamma}$ with the presheaf of vector fields tangent to local $C^{1}$-isotopies in $\Gamma$, Remark 4.3 .3 in [67]. If they coincide, $X_{\mathbf{X}}^{\Gamma}$ is a LAS.

We formulate now the following

Axiom 1. Given a pseudogroup $\Gamma$ over $\mathbf{X}$ supported by $\Sigma \rightrightarrows \mathbf{X}$, the $\operatorname{set}^{\operatorname{Sec}_{A}^{\Gamma} \text { of }}$ local $\Gamma$-sections of $A=\operatorname{Lie}(\Sigma)$ is a LAS supported by $A$. 
Remark 3.3.24. The discussion in this subsection can be carried out for an étale groupoid $\mathcal{G} \rightrightarrows \mathbf{X}$ supported by $\Sigma \rightrightarrows \mathbf{X}$ in the sense of Definition 3.3.1. The role of $\Gamma$ is played by $\operatorname{Bis}_{\text {loc }}(\mathcal{G})$, which is naturally supported by $\Sigma \rightrightarrows \mathbf{X}$. In particular, one can define the presheaf $\operatorname{Lie}\left(\operatorname{Bis}_{\text {loc }}(\mathcal{G})\right)$, which is denoted simply by $\operatorname{Lie}(\mathcal{G})$, and formulate Axiom 1 for étale groupoids.

Finally, we point out that the discussion in Subsection 3.3.1 can be repeated verbatim for LAS supported by Lie algebroids. That is, $\mathcal{A}$ carries a natural diffeology coming from the one on local sections of $A$, a $D_{A}$-topology can be defined and analogous statements about hierarchy and separability can be formulated.

\subsection{Cartan resolutions of étale groupoids}

While introducing generalized Lie pseudogroups in Section 3.2, we mentioned that each such a generalized pseudogroup $\Gamma$ has an associated canonical Cartan tower

$$
\cdots \rightarrow J^{k} \Gamma \rightarrow J^{k-1} \Gamma \rightarrow \ldots \rightrightarrows \mathbf{X} .
$$

Each jet space is a subspace of the space $J^{k} \Sigma$ (assuming $\Gamma$ to be supported by $\Sigma$ ), and as such is equipped with the restriction $\mathcal{C}^{k}$ of the canonical Cartan distribution, Definition 3.2.15. Recall that the holonomic bisections of $J^{k} \Sigma \rightrightarrows \mathbf{X}$-i.e. those bisections whose image is tangent to $\mathcal{C}^{k}-$ are in one to one correspondence with bisections of $\Sigma$. We defined Lie pseudogroups, Definition 3.2.58, as those pseudogroups such that the Cartan tower is a smooth subtower of the tower of the support

$$
\cdots \rightarrow J^{k} \Sigma \rightarrow J^{k-1} \Sigma \rightarrow \ldots \rightrightarrows \mathbf{X}
$$

and strongly Lie pseudogroups as those with the additional property $\lim _{\leftarrow} J^{k} \Gamma \cong$ $J^{\infty} \Gamma$. We also mentioned that the tower actually becomes a tower of pairs $\left(J^{k} \Gamma, \mathrm{C}^{k}\right)$, meaning that each map is a Lie prolongation in the sense of Definition 3.B.12 from Appendix 3.B. As explained in Appendix 3.A and 3.B, Proposition 3.B.21 (and as mentioned in Subsection 3.2.2), $J^{\infty} \Gamma$ is a pf Lie groupoid equipped with a multiplicative flat connection $\mathrm{e}^{\infty}$. The distributions $\mathrm{e}^{k}$ allow one to define the subsets of holonomic bisections $\operatorname{Bis}_{\text {loc }}^{\mathrm{e}^{k}}\left(J^{k} \Gamma\right)$, for each $k=1, \ldots, \infty$.

The multiplicativity property of $\mathcal{C}^{k}$ implies that $\operatorname{Bis}_{\text {loc }}^{\mathcal{e}^{k}}\left(J^{k} \Gamma\right)$ is a generalized pseudogroup supported by $J^{k} \Gamma \rightrightarrows \mathbf{X}$. The corresponding germ groupoid inherit a diffeology as in Subsection 3.3.1. We denote the germ groupoid of $\operatorname{Bis}_{\text {loc }}^{\mathcal{e}^{k}}\left(J^{k} \Gamma\right)$ by $\operatorname{Germ}\left(J^{k} \Gamma, \mathrm{C}^{k}\right)$. We spell out the following property of the canonical Cartan tower since it plays a role in the finite order problem.

Proposition 3.4.1. Let $\Gamma$ be a generalized pseudogroup on $\mathbf{X}$ supported by $\Sigma \rightrightarrows \mathbf{X}$. The jet maps induce injective maps of étale groupoids

$$
j^{k}: \operatorname{Germ}(\Gamma) \rightarrow \operatorname{Germ}\left(J^{k} \Gamma, \mathrm{C}^{k}\right), \quad \operatorname{germ}_{x}(\sigma) \rightarrow \operatorname{germ}_{x}\left(j^{k} \sigma\right)
$$

for $k \geq 1$. The $j^{k}$ 's are compatible with the maps

$$
\operatorname{Germ}\left(J^{k+1} \Gamma, \mathcal{C}^{k+1}\right) \rightarrow \operatorname{Germ}\left(J^{k} \Gamma, \mathcal{C}^{k}\right), \quad \operatorname{germ}_{x}\left(j^{k+1} \sigma\right) \rightarrow \operatorname{germ}_{x}\left(j^{k} \sigma\right)
$$


induced by the projections and smooth with respect to the diffeologies induced by the supports $\Sigma, J^{k} \Sigma$.

The following is a property of the canonical Cartan tower of generalized strongly Lie pseudogroups (Definition 3.2.58).

Proposition 3.4.2. Let $\Gamma$ be a generalized pseudogroup on $\mathbf{X}$ supported by $\Sigma \rightrightarrows \mathbf{X}$. $\Gamma$ is strongly Lie if and only if the canonical jet map

$$
j: \operatorname{Germ}(\Gamma) \rightarrow \lim _{\leftarrow} J^{k} \Gamma, \quad \operatorname{germ}_{x}(\sigma) \rightarrow\left(\ldots, j_{x}^{k} \sigma, j_{x}^{k-1} \sigma, \ldots \sigma(x), x\right)
$$

is surjective.

A last relevant observation is

Proposition 3.4.3. Let $\Gamma$ be a generalized pseudogroup on $\mathbf{X}$ supported by $\Sigma \rightrightarrows \mathbf{X}$. A pseudogroup $\Gamma$ is of order $k \in \mathbb{N}$ if and only if $\operatorname{Germ}(\Gamma) \cong \operatorname{Germ}\left(J^{k} \Gamma, \mathrm{C}^{k}\right)$ for some finite $k$ which is minimal with this property. This implies $\operatorname{Germ}\left(J^{h} \Gamma, \mathrm{C}^{h}\right) \cong \operatorname{Germ}\left(J^{k} \Gamma, \mathrm{C}^{k}\right)$ for each $h \geq k$ (hence $\left.\operatorname{Germ}\left(J^{\infty} \Gamma, \mathrm{C}^{\infty}\right) \cong \operatorname{Germ}\left(J^{k} \Gamma, \mathrm{C}^{k}\right)\right)$.

Below we introduce some language to deal with towers of Lie groupoids attached to étale groupoids, that we call "resolutions". These satisfy the property from Propositions 3.4.1 above, and should be thought of as an abstract version of the canonical Cartan tower of a pseudogroup. The finite order problem can be formulated in terms of an étale Lie groupoid equipped with a support and with such a resolution. We think that this formulation helps shedding more light on the nature of the problem. The reader who prefers to keep thinking in terms of jets and pseudogroups can simply pick the notation from Subsection 3.4.1 and then proceed to the next ones. There, we introduce two more topologies on étale groupoids. These topologies, which are constructed out of a resolution, complete our set of tools to deal with the finite order problem. We will then proceed by quickly going through the infinitesimal counterparts of the objects introduced.

\subsubsection{Cartan resolutions}

Once more, we encourage the reader to have a look at Appendix 3.B where we discuss Pfaffain groupoids and Lie prolongation maps; see also Subsection 3.2.2.

From now on, following appendix 3.A if $\Sigma \rightarrow \mathbf{X}$ is a Lie groupoid, by tower of Lie groupoids $\Sigma^{*}$ over $\Sigma \rightrightarrows \mathbf{X}$ we mean a diagram of the form

$$
\cdots \rightarrow \Sigma^{k+1} \rightarrow \Sigma^{k} \rightarrow \cdots \rightarrow \Sigma \rightrightarrows \mathbf{X}, \quad k \geq 1
$$

where all the $\Sigma^{k \prime}$ s are Lie groupoids and all the maps are surjective submersions and groupoid maps. The projective limit $\lim _{\leftarrow} \Sigma^{k}$ of the tower is denoted by $\Sigma^{\infty}$ :

$$
\Sigma^{\infty}:=\lim _{\leftarrow} \Sigma^{k}
$$

We recall here the definition of Cartan tower $\left(\Sigma^{*}, \mathfrak{C}^{*}\right)$ over $\Sigma$, Definition 3.B.20 in appendix 3.C. 
Let $\left(\Sigma^{k}, \mathrm{e}^{k}\right), k \geq 1$ be Pfaffian groupoids (Definition 3.B.6), and $\Sigma \rightrightarrows \mathbf{X}$ be a Lie groupoid over $\mathbf{X}$. A Cartan tower is a tower of groupoids over $\Sigma \rightrightarrows \mathbf{X}$

$$
\cdots \rightarrow\left(\Sigma^{k+1}, \mathrm{e}^{k+1}\right) \rightarrow\left(\Sigma^{k}, \mathrm{e}^{k}\right) \rightarrow \cdots \rightarrow \Sigma \rightrightarrows \mathbf{X}, \quad k \in \mathbb{N}
$$

where all the maps

$$
p r^{k+1, k}:\left(\Sigma^{k+1}, \mathrm{e}^{k+1}\right) \rightarrow\left(\Sigma^{k}, \mathrm{e}^{k}\right)
$$

are Lie prolongations, Definition 3.B.12, and the symbol space $\mathcal{C}^{1} \cap \operatorname{Ker}(d s)$ of $\left(\Sigma^{1}, \mathcal{C}^{1}\right)$ (Definition 3.B.1) is vertical over $\Sigma$-i.e. it satisfies $d p r^{1,0}\left(\mathcal{C}^{1} \cap \operatorname{Ker}(d s)\right)=$ 0 . The multiplicative flat connection induced on the limit $\Sigma^{\infty}$ according to Proposition 3.B.21, is denoted by $\mathrm{C}^{\infty}$. The maps of a tower will be denoted simply by $p r$ if there is no danger of confusion.

Let now $(\Sigma, \mathcal{C})$ be a Pfaffian groupoid. Similarly to what we did for jets of pseudgroups, we can look at bisections of $\Sigma$ that are holonomic for $\mathcal{C}$, i.e. their image is tangent to $\mathcal{C}$. Since $\mathcal{C}$ is multiplicative - i.e. $\mathcal{C} \cdot \mathcal{C} \subset \mathcal{C}$, see also Definition 3.B.6 - holonomic bisections form a generalized pseudogroup, that we denote by $\operatorname{Bis}_{\text {loc }}^{\mathrm{e}}(\Sigma)$, supported by $\Sigma \rightrightarrows \mathbf{X}$. The corresponding germ groupoid is denoted by $\operatorname{Germ}(\Sigma, \mathcal{C})$. Since a Lie prolongation map of Pfaffian groupoids (see Definition 3.B.12) is in particular a groupoid morphism and preserves the Pfaffian distribution, the following fact holds true.

Lemma 3.4.4. A Lie prolongation map

$$
p:\left(\Sigma^{\prime}, \mathrm{C}^{\prime}\right) \rightarrow(\Sigma, \mathrm{C})
$$

of Pfaffian groupoids induces a map of groupoids

$$
p: \operatorname{Germ}\left(\Sigma^{\prime}, \mathrm{C}^{\prime}\right) \rightarrow \operatorname{Germ}(\Sigma, \mathcal{C}), \quad \operatorname{germ}_{x}(\sigma) \rightarrow \operatorname{germ}_{x}(p r \circ \sigma)
$$

still denoted by $p$.

Definition 3.4.5. A Cartan resolution of an étale Lie groupoid $\mathcal{G} \rightrightarrows \mathbf{X}$ supported by $\Sigma \rightrightarrows \mathbf{X}$ is a Cartan tower $\left(\Sigma^{*}, \mathrm{C}^{*}\right)$ over $\Sigma$ together with maps of étale groupoids

$$
j_{\Sigma^{*}}^{k}: \mathcal{G} \rightarrow \operatorname{Germ}\left(\Sigma^{k}, \mathfrak{C}^{k}\right), \quad k \in \mathbb{N}, k \geq 1
$$

which are diffeologically smooth (with respect to the diffeologies induced by the supports $\Sigma$ and $\Sigma^{k}$ respectively) and such that

- one has

$$
p r^{k, k-1} \circ j_{\Sigma^{*}}^{k}=j_{\Sigma^{*}}^{k-1},
$$

for all $k \in \mathbb{N}, k \geq 1$, where

$$
\begin{aligned}
p r^{k, k-1}: \operatorname{Germ}\left(\Sigma^{k}, \mathrm{C}^{k}\right) & \rightarrow \operatorname{Germ}\left(\Sigma^{k-1}, \mathrm{C}^{k-1}\right) \\
\operatorname{germ}_{x}(\sigma) & \rightarrow \operatorname{germ}_{x}\left(p r^{k, k-1} \circ(\sigma)\right)
\end{aligned}
$$

are the maps induced by the projections; 
- the smooth groupoid maps

$$
j_{\Sigma^{k}}: \mathcal{G} \rightarrow \Sigma^{k}, \quad k \in \mathbb{N}, k \geq 1,
$$

obtained by postcomposing the $j_{\Sigma^{*}}^{k}$ 's with the evaluation map

$$
\operatorname{germ}_{x}(\sigma) \in \operatorname{Germ}\left(\Sigma^{k}, \mathfrak{C}^{k}\right) \rightarrow \sigma(x) \in \Sigma^{k},
$$

are surjective.

The pair $(\mathcal{G}, \Sigma)$ is called of Lie type if $\mathcal{G}$ admits a Cartan resolution over $\Sigma$; we also say that $\mathcal{G}$ is of Lie type over $\Sigma$.

Of course, if $\mathcal{G}=\operatorname{Germ}(\Gamma)$ corresponds to a generalized Lie pseudogroup, then $\mathcal{G}$ is of Lie type.

By convention, we set $j_{\Sigma^{*}}^{0}$ in Definition 3.4.5 to be the map induced by the Lie groupoid map $\mathcal{G} \rightarrow \Sigma$ that is part of the definition of support, Definition 3.3.1.

Since the $j_{\Sigma^{*}}^{k}$ 's are compatible with the projections, we also get a groupoid map

$$
j_{\Sigma^{\infty}}: \mathcal{G} \rightarrow \Sigma^{\infty},
$$

where $\mathcal{G}$ has its original smooth structure and $\Sigma^{\infty}$ the profinite dimensional one. This is not to be confused with $j_{\Sigma^{*}}^{\infty}: \mathcal{G} \hookrightarrow \operatorname{Germ}\left(\Sigma^{\infty}, \mathrm{C}^{\infty}\right)$. By definition, $j_{\Sigma^{\infty}}$ is the composition of $j_{\Sigma^{*}}^{\infty}$ with the obvious map $\operatorname{Germ}\left(\Sigma^{\infty}, \mathrm{C}^{\infty}\right) \rightarrow \Sigma^{\infty}$. In general, $j_{\Sigma^{\infty}}$ may fail to be both injective (as it happens with the canonical Cartan tower of $\Gamma^{n}$ ) and surjective (as it happens with the canonical Cartan tower of $\Gamma_{\mathrm{an}}^{n}$ ). It is the behaviour of $j_{\Sigma \infty}$ that distinguish strongly Lie pseudogroups according to Definition 3.2.17 from Lie pseudogroups whose jet groupoid $J^{\infty} \Gamma$ is strictly contanined in $\lim _{\leftarrow} J^{k} \Gamma$.

Definition 3.4.6. A tight Cartan resolution of an étale groupoid $\mathcal{G}$ over a support $\Sigma$ is a Cartan resolution $\left(\Sigma^{*}, \mathrm{C}^{*}\right)$ such that

$$
j_{\Sigma \infty} \text { is surjective. }
$$

The groupoid $\mathcal{G}$ is called of strong Lie type (over $\Sigma$ ) if it admits a tight Cartan resolution (over $\Sigma$ ).

Remark 3.4.7. Observe that the map $j_{\Sigma^{*}}^{k}: \mathcal{G} \rightarrow \operatorname{Germ}\left(\Sigma^{k}, \mathrm{e}^{k}\right)$ from Definition 3.4.5 induces a map

$$
\operatorname{Bis}_{\text {loc }}(\mathcal{G}) \rightarrow \operatorname{Bis}_{\text {loc }}^{\mathrm{e}^{k}}\left(\Sigma^{k}\right)
$$

sending a bisection $\sigma \in \operatorname{Bis}_{\text {loc }}(\mathcal{G})$ to the bisection $x \rightarrow j_{\Sigma^{*}}^{k}(\sigma(x))$. We keep denoting this map by $j_{\Sigma^{*}}^{k}$. Notice that

$$
j_{\Sigma^{*}}^{k}: \operatorname{Bis}_{\text {loc }}(\mathcal{G}) \rightarrow \operatorname{Bis}_{\text {loc }} \mathrm{e}^{k}\left(\Sigma^{k}\right)
$$

is smooth with respect to the diffeologies induced by the supports $\Sigma$ and $\Sigma^{k}$, see Definition 3.3.5.

Furthermore, since $j_{\Sigma^{*}}^{0}: \operatorname{Bis}_{\mathrm{loc}}(\mathcal{G}) \rightarrow \operatorname{Bis}_{\mathrm{loc}}(\Sigma)$ is injective, all the maps

$$
j_{\Sigma^{*}}^{k}: \operatorname{Bis}_{\mathrm{loc}}(\mathcal{G}) \rightarrow \operatorname{Bis}_{\text {loc }}^{\mathcal{e}^{k}}\left(\Sigma^{k}\right), \quad k \in \mathbb{N}, k \geq 1
$$


are injective as well.

This implies that, given a Cartan resolution $\left(\Sigma^{*}, \mathcal{C}^{*}\right)$ of an étale groupoid $\mathcal{G} \rightrightarrows$ $\mathbf{X}$ over a support $\Sigma \rightrightarrows \mathbf{X}$, each groupoid $\Sigma^{k}$ in the underlying tower is a support for $\mathcal{G} \rightrightarrows \mathbf{X}$.

Remark 3.4.8. It is possible to equip $\operatorname{Bis}_{\text {loc }}^{e^{\infty}}\left(\Sigma^{\infty}\right)$ with the inverse limit diffeology (one should proceed analogously to what is done in Example 3.C.9) induced by the diffeologies on the $\operatorname{Bis}_{\text {loc }} e^{k}\left(\Sigma^{k}\right)^{\prime}$ s. Then the map

$$
j_{\Sigma^{*}}^{\infty}: \operatorname{Bis}_{\text {loc }}(\mathcal{G}) \hookrightarrow \operatorname{Bis}_{\text {loc }} \mathrm{e}^{\infty}\left(\Sigma^{\infty}\right)
$$

induced by the $j_{\Sigma^{*}}^{k}$ 's is diffeologically smooth.

Definition 3.4.9. The pair $(\mathcal{G}, \Sigma)$ is of finite order if there exists a tight Cartan resolution $\left(\Sigma^{*}, \mathrm{e}^{*}\right)$ and $k \in \mathbb{N}$ satisfying

$$
j_{\Sigma^{*}}^{k} \text { is surjective. }
$$

Remark 3.4.10. If property (3.4) holds for some $k, j_{\Sigma^{*}}^{h}$ is a groupoid isomorphism for all $h \geq k$. In particular

$$
\operatorname{Germ}\left(\Sigma^{h}, \mathrm{e}^{h}\right) \cong \operatorname{Germ}\left(\Sigma^{k}, \mathrm{e}^{k}\right), \quad h \geq k
$$

Example 3.4.11. If $\mathcal{G}=G^{\delta}$ is a discrete group admitting a Lie group structure denoted by $G$, the tower

$$
\cdots \rightarrow G \rightarrow \cdots \rightarrow G \rightarrow\{\star\}
$$

where all maps are the identity, is a tight Cartan resolution.

Example 3.4.12. If $\left(\Sigma^{*}, \mathrm{C}^{*}\right)$ is a Cartan resolution over $\Sigma$ of some étale groupoid $\mathcal{G}$, one can define a Cartan resolution $\left(\Sigma^{\prime *}, \mathrm{e}^{\prime *}\right)$ over $\Sigma^{k}, k \in \mathbb{N}$, by setting $\Sigma^{\prime h}=$ $\Sigma^{h+k}, h \in \mathbb{N}$. Applying this to the canonical Cartan tower of some Lie pseudogroup $\Gamma$ of order $k$, we get the "truncated" Cartan resolution

$$
\cdots \rightarrow J^{h} \Gamma \rightarrow \cdots \rightarrow J^{k} \Gamma \rightrightarrows \mathbf{X}
$$

over the support $J^{k} \Gamma$ of $\mathcal{G}$. This is not the canonical Cartan tower of $J^{k} \Gamma$. Notice that in order to detect sections of $\Gamma$ by looking at the tower $\left(J^{h} \Gamma, \mathrm{C}^{h}\right), h>k$, we only need to detect holonomic bisections of $\left(J^{k+1} \Gamma, \mathrm{e}^{k+1}\right)$. All the information about $\Gamma$ is already encoded into $J^{k} \Gamma$.

We are now going to show that the canonical Cartan tower is final among Cartan towers. For the sake of clarity, while dealing with the statement below we use the lower decoration "can" to refer to the canonical Cartan tower of a groupoid $\Sigma$. That is, we write $\mathcal{C}_{\text {can }}$ for the Cartan distribution, $p r_{\text {can }}$ for the projection maps, $j_{\text {can }}^{k}$ for the jet maps, and so on.

Proposition 3.4.13. Let $\left(\Sigma^{*}, \mathrm{C}^{*}\right)$ be a Cartan resolution of $\mathcal{G}$ supported by $\Sigma$. There is a unique map of Cartan towers $i^{*}:\left(\Sigma^{*}, \mathrm{C}^{*}\right) \rightarrow\left(J^{*} \Sigma, \mathcal{C}_{\text {can }}^{*}\right)$. 
By "map of Cartan towers" of Pfaffian groupoids over $\Sigma \rightrightarrows \mathbf{X},\left(\Sigma_{1}^{*}, \mathrm{C}_{1}^{*}\right) \rightarrow$ $\left(\Sigma_{2}^{*}, \mathcal{C}_{2}^{*}\right)$, we mean a collection of morphism of Pfaffian groupoids

$$
\left(\Sigma_{1}^{k}, \mathrm{e}_{1}^{k}\right) \rightarrow\left(\Sigma_{2}^{k}, \mathrm{e}_{2}^{k}\right), \quad k \geq 1
$$

over the identity $i d: \mathbf{X} \rightarrow \mathbf{X}$ that commute with the projections of the towers.

Proof. This is a quite classical statement about prolongation towers in PDE theory. We spell out a proof based on the work in [55], in particular Corollary 6.2.13 and Proposition 6.2.42. We proceed by induction over the length of the tower.

We start looking at the map $p r:\left(\Sigma^{1}, \mathrm{e}^{1}\right) \rightarrow \Sigma^{0}$. Define

$$
i^{1}: \Sigma^{1} \rightarrow J^{1} \Sigma, \quad g \rightarrow i^{1}(g)
$$

for $i^{1}(g): T_{s(g)} \mathbf{X} \rightarrow T_{p r(g)} \Sigma^{0}$ that sends $v_{s(g)}$ to $d p r\left(\hat{v}_{g}\right)$, where $\hat{v}_{g}$ is any $s$-lift of $v_{s(g)}$ to a vector lying in $\mathrm{C}^{1}$. Let us show that this map is well defined and takes values in $J^{1} \Sigma$.

- The symbol $\mathrm{e}^{1} \cap \operatorname{Ker}(d s)$ of $\left(\Sigma^{1}, \mathrm{e}^{1}\right)$ is vertical over $\Sigma^{0}$, which implies that the map is well defined.

- One has

$$
d t\left(i^{1}(g)\left(v_{s(g)}\right)\right)=d t\left(d p r\left(\hat{v}_{g}\right)\right)=d t\left(\hat{v}_{g}\right) .
$$

This implies that $d t \circ i^{1}(g)$ is a linear isomorphism for each $g \in \Sigma^{1}$, since $\mathrm{e}^{1} \cap \operatorname{Ker} d(s)=\mathcal{e}^{1} \cap \operatorname{Ker}(d t)$ (Definition 3.B.6). Consequently, $i^{1}$ takes values in $J^{1} \Sigma$.

Notice that one has $p r_{\text {can }} \circ i=p r$ by construction.

Furthermore, $i^{1}$ is a groupoid morphism. The fact that $i^{1}$ commutes with the source and target maps is clear. The multiplication is respected thanks to multiplicativity of $\mathrm{e}^{1}$. In fact, let $g, h \in \Sigma^{1}$ be compatible arrows, i.e. $s(h)=t(g)$ so that $h g$ is defined. One has $s(h g)=s(g)$. The image $i^{1}(h g)$ is given by the linear isomorphism

$$
i^{1}(h g): T_{s(g)} \mathbf{X} \rightarrow T_{p r(h g)} \Sigma^{0}, \quad v_{s(g)} \rightarrow \operatorname{dpr}\left(\hat{v}_{h g}\right)
$$

where $\hat{v}_{h g}$ is any $s$-lift of $v_{s(g)}$ tangent to $\mathrm{C}^{1}$ at $h g$. Let $v_{s(g)} \in T_{s(g)} \mathbf{X}$; if $\hat{v}_{g}$ is an $s$-lift at $g$ tangent to $\mathrm{C}^{1}$ and $\widehat{d t\left(\hat{v}_{g}\right)_{h}}$ is an $s$-lift of $d t\left(\hat{v}_{g}\right) \in T_{s(h)} \mathbf{X}$ tangent to $\mathcal{C}^{1}$, the product

$$
d m\left({\widehat{d t\left(\hat{v}_{g}\right)_{h}}}_{1} \hat{v}_{g}\right)
$$

is an $s$-lift of $v_{s(g)}$ tangent to $\mathcal{C}^{1}$ at $h g$, because $\mathrm{C}^{1}$ is closed with respect to multiplication. Since $p r$ is a groupoid morphism, we see that $i(h g)=i(h) \cdot i(g)$ as claimed.

Finally, $d i^{1}\left(\mathcal{C}^{1}\right) \subset \mathcal{C}_{\text {can }}^{1}$. We make use of the fact that $\mathcal{C}_{\text {can }}^{1}$ is the kernel of the one form (Definition 3.2.15)

$$
\omega_{j_{x}^{1} \sigma}^{1}:=d_{j_{x}^{1} \sigma} p r_{\mathrm{can}}-d_{x} \sigma \cdot d_{j_{x}^{1} \sigma} s .
$$

We show that $\omega^{1}\left(d i^{1}\left(v_{g}\right)\right)=0$ for any $v_{g} \in \mathcal{C}^{1}$ at $g \in \Sigma^{1}$.

$$
\omega^{1}\left(d i^{1}\left(v_{g}\right)\right)=d p r_{\mathrm{can}}\left(d i^{1}\left(v_{g}\right)\right)-i^{1}(g)\left(d s\left(d i^{1}\left(v_{g}\right)\right)\right)=d p r\left(v_{g}\right)-i^{1}(g)\left(d s\left(v_{g}\right)\right)=0
$$


since $v_{g} \in \mathcal{C}^{1}$ is an $s$-lift of $d s\left(v_{g}\right) \in T_{s(g)} \mathbf{X}$ at $g$ tangent to $\mathcal{C}^{1}$.

We will now make use of Proposition 6.2.42 in [55]: the category of Pfaffian groupoids is equipped with a classical prolongation functor that attaches to any Pfaffian groupoid $(\Sigma, \mathcal{C})$ a prolongation $p r_{c l}:\left(\mathcal{P}_{\mathcal{C}}(\Sigma), \mathcal{C}_{c l}\right) \rightarrow(\Sigma, \mathcal{C})$ (Definition 6.2 .25 in [55]) which is final among prolongations. We recalled the definition of the classical prolongation in the appendix, Definition 3.B.14, where we also give a precise formulation of the claim that $p r_{c l}:\left(\mathcal{P}_{\mathcal{C}}(\Sigma), \mathcal{C}_{c l}\right) \rightarrow(\Sigma, \mathcal{C})$ is final, Proposition 3.B.18.

There is a canonical embedding $\left(\mathcal{P}_{\mathcal{C}}(\Sigma), \mathcal{C}_{c l}\right) \hookrightarrow\left(J^{1} \Sigma, \mathcal{C}_{\text {can }}\right)$; in fact $\mathcal{P}_{\mathcal{C}}(\Sigma)$ is defined as a subspace of $J^{1} \Sigma$, Definition 3.B.14. If $(\hat{\Sigma}, \hat{\mathcal{C}})$ is any prolongation of $(\Sigma, \mathcal{C})$, the natural map from Proposition 3.B.18 $(\hat{\Sigma}, \hat{\mathcal{C}}) \rightarrow\left(\mathcal{P}_{\mathcal{C}}(\Sigma), \mathcal{C}_{c l}\right)$ is precisely $(\star)$.

With this statement at hand, let us assume that, for any $0 \leq h \leq k$, there are morphisms of Pfaffian groupoids

$$
i^{h}:\left(\Sigma^{h}, \mathrm{C}^{h}\right) \rightarrow\left(J^{h} \Sigma^{0}, \mathrm{C}_{\text {can }}^{h}\right)
$$

making the diagram

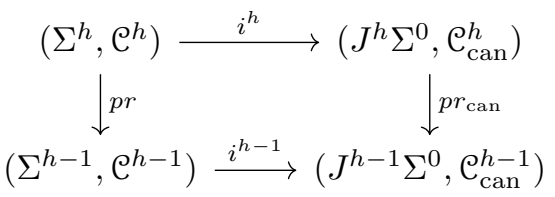

commutative. We aim at proving the existence of a morphism of Pfaffian groupoids

$$
i^{k+1}:\left(\Sigma^{k+1}, \mathrm{e}^{k+1}\right) \rightarrow\left(J^{k+1} \Sigma^{0}, \mathrm{C}_{\text {can }}^{k+1}\right)
$$

making the diagram

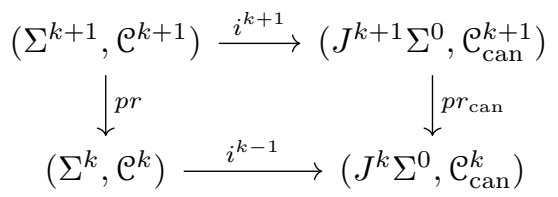

commutative.

We have a morphism of Pfaffian groupoids $i_{\mathrm{cl}}:\left(\Sigma^{k+1}, \mathrm{C}^{k+1}\right) \rightarrow\left(\mathcal{P}_{\mathrm{C}^{k}}\left(\Sigma^{k}\right), \mathcal{C}_{c l}\right)$ commuting with the projections to $\left(\Sigma^{k}, \mathrm{e}^{k}\right)$ (in fact, a map of prolongations, Proposition 3.B.18). Notice that $i^{k}:\left(\Sigma^{k}, \mathrm{C}^{k}\right) \rightarrow\left(J^{k} \Sigma, \mathrm{C}_{\mathrm{can}}^{k}\right)$ lifts to a map of Pfaffian groupoids

$$
j^{1}\left(j^{k}\right):\left(J^{1} \Sigma^{k}, \mathcal{C}_{\text {can }}\right) \rightarrow\left(J^{1} J^{k} \Sigma, \mathcal{C}_{\text {can }}\right)
$$

This map sends $\left(\mathcal{P}_{\mathcal{C}^{k}}\left(\Sigma^{k}\right), \mathcal{C}_{c l}\right)$ to $\left(J^{k+1} \Sigma, \mathcal{C}_{\text {can }}\right)$, as it follows from the definition of $\left(\mathcal{P}_{\mathcal{C}^{k}}\left(\Sigma^{k}\right), \mathcal{C}_{c l}\right)$, from the equality $\left(\mathcal{P}_{\mathcal{C}_{\text {can }}}\left(J^{k} \Sigma^{0}\right), \mathcal{C}_{c l}\right)=\left(J^{k+1} \Sigma, \mathcal{C}_{\text {can }}^{k+1}\right)$ and from the fact that $j^{1}\left(j^{k}\right)$ respects the Cartan distribution and its curvature.

Remark 3.4.14. Observe that in the proposition above $\mathcal{G}$ plays no role. It is a statement about Cartan towers. 


\subsection{2 $C_{\Sigma^{*}}^{\infty}$-convergence}

$C^{\infty}$-convergence on the space of diffeomorphisms of $\mathbb{R}^{n}$ has been a recurring ingredient in the finite order problem for natural bundles [28, 54, 65]. In [65], it was even part of the definition: natural bundles were asked to be continuous in the sense that the action functor $E$ was assumed to send $C^{\infty}$-converging sequences of diffeomorphisms to compact-open converging sequences. We already introduced a notion of $C^{\infty}$-convergence for étale groupoids with support, see definition 3.3.10. In this subsection, we are going to reformulate $C^{\infty}$-convergence in terms of general Cartan resolutions. Let $\left(\Sigma^{*}, \mathcal{C}^{*}\right)$ be a Cartan resolution of $\mathcal{G}$ over $\Sigma$. The idea is to define a topology on the spaces $\operatorname{Bis}(\mathcal{G}, U)$ of bisections of $\mathcal{G}$ over an open $U \subset \mathbf{X}$ by mimicking what is done with the strong Whitney topology. We will make use of the maps

$$
j_{\Sigma^{*}}^{k}: \operatorname{Bis}(U, \mathcal{G}) \rightarrow \operatorname{Bis}^{\mathrm{e}^{k}}\left(\Sigma^{k}, U\right) \subset C^{0}\left(U, \Sigma^{k}\right)
$$

induced by the Cartan resolution; here $\mathrm{Bis}^{\mathrm{e}^{k}}\left(\Sigma^{k}, U\right)$ is the set of holonomic bisection of $\Sigma^{k}$ defined over $U \subset \mathbf{X}$.

First, let us recall the strong topology on a space of continuous functions. Let $\mathbf{X}_{1}, \mathbf{X}_{2}$ be topological spaces. We can equip the space $C^{0}\left(\mathbf{X}_{1}, \mathbf{X}_{2}\right)$ of continuous functions with the strong topology. Recall that in such a topology a sub-basis of neighbourhoods of a function $f$ consists of sets of the form

$$
A(W)=\left\{g \in C^{0}\left(\mathbf{X}_{1}, \mathbf{X}_{2}\right): G_{g} \in W\right\}
$$

where $G_{g}$ is the graph of $g$ and $W \subset \mathbf{X}_{1} \times \mathbf{X}_{2}$ is an open neighbourhood of the graph $G_{f}$ of $f$ in $\mathbf{X}_{1} \times \mathbf{X}_{2}$. Convergence $f_{n} \rightarrow f$ of a sequence $\left\{f_{n}\right\}_{n \in \mathbb{N}}$ in such a topology means that there exists a compact set $K \subset \mathbf{X}_{1}$ such that $\left.\left.f_{n}\right|_{K} \rightarrow f\right|_{K}$ uniformly and $f_{n}\left|\mathbf{x}_{1-K}=f\right|_{\mathbf{x}_{1}-K}$ for $n$ big enough.

Now, we simply pull-back the strong topologies on the right hand sides of the maps

$$
j_{\Sigma^{*}}^{k}: \operatorname{Bis}(U, \mathcal{G}) \rightarrow \operatorname{Bis}^{\mathrm{e}^{k}}\left(\Sigma^{k}, U\right) \subset C^{0}\left(U, \Sigma^{k}\right) .
$$

We will call the topology obtained in this way the $C_{\Sigma^{*}}^{\infty}$-topology on $\operatorname{Bis}(\mathcal{G}, U)$; if a sequence $\left\{\gamma^{n}\right\}_{n \in \mathbb{N}}$ converges to $\gamma$ in such a topology we write

$$
\gamma^{n} \underset{C_{\Sigma^{*}}^{\infty}}{\rightarrow} \gamma
$$

Definition 3.4.15. We say that a sequence $\left\{g_{x^{n}}^{n}\right\}_{n \in \mathbb{N}}$ in $\mathcal{G} C_{\Sigma^{*}}^{\infty}$-converges to $g_{x}$ if and only $x^{n} \rightarrow x$ and there are

- an open set $U \subset \mathbf{X}$ with coordinates containing $x$ and $x^{n}$ for all $n$ sufficently big;

- bisections of $\mathcal{G}, \gamma^{n}, n \in \mathbb{N}$ and $\gamma$, representing the $g_{x^{n}}^{n}$ 's and $g_{x}$ respectively and defined on $U$ for $n$ big enough;

such that $\gamma^{n} \underset{C_{\Sigma^{*}}^{\infty}}{\rightarrow} \gamma$. We will write

$$
g_{x^{n}} \underset{C_{\Sigma^{*}}^{\infty}}{\rightarrow} g_{x} .
$$


Of course, Definition 3.4.15 becomes Definition 3.3.10 when we work with the canonical Cartan tower of $\mathcal{G}$.

A due lemma is the following one:

Lemma 3.4.16. If $g_{x^{n}}^{n} \underset{C_{\Sigma^{*}}^{\infty}}{\rightarrow} g_{x}$ then $g_{x^{n}}^{n} \underset{C^{\infty}}{\rightarrow} g_{x}$.

Proof. One uses the map $i$ from Proposition 3.4.13, and the fact that postcomposition with a continuous map preserve convergence in the strong topology on continuous maps.

\subsubsection{Topologies induced by a resolution}

When $\mathcal{G}$ is an étale groupoid supported by $\Sigma$ and $\mathcal{G}$ is of Lie type over $\Sigma$, we can choose a Cartan resolution $\left(\Sigma^{*}, \mathrm{C}^{*}\right)$ of $\mathcal{G}$ over $\Sigma$ (Definition 3.4.5). Such a resolution has a limit $\left(\Sigma^{\infty}, \mathrm{e}^{\infty}\right)$ and there is a map $j_{\Sigma^{\infty}}: \mathcal{G} \rightarrow \Sigma^{\infty}$. This map induces two further topologies on $\mathcal{G}$ that play a role in the finite order problem.

The first one is obtained by pulling back the limit topology on $\Sigma^{\infty}$ to $\mathcal{G}$.

Definition 3.4.17. The limit topology on $\mathcal{G}$ is the initial topology on $\mathcal{G}$ with respect to $j_{\Sigma^{\infty}}: \mathcal{G} \rightarrow \Sigma^{\infty}$.

Remark 3.4.18. Observe that a sequence $\left\{g_{x^{n}}^{n}\right\}_{n \in \mathbb{N}}$ converges to $g_{x}$ in the limit topology if and only if $j_{\Sigma^{\infty}}\left(g_{x^{n}}^{n}\right)$ converges to $j_{\Sigma^{\infty}}\left(g_{x}\right)$ in $\Sigma^{\infty}$, i.e. $p r^{\infty, i} \circ j_{\Sigma^{\infty}}\left(g_{x^{n}}^{n}\right)$ converges to $\mathrm{pr}^{\infty, i} \circ j_{\Sigma^{\infty}}\left(g_{x}\right)$ for all $i \in \mathbb{N}$. We will write

$$
g_{x^{n}}^{n} \underset{\lim }{\rightarrow} g_{x}
$$

The second topology we introduce looks quite similar to the previous one. We define a sub-basic system of neighbourhoods of $g_{x}^{\infty} \in \Sigma^{\infty}$ via

$$
U^{i}=g_{x}^{\infty} \cdot\left(p r^{\infty, i}\right)^{-1}(i d)=\left(p r^{\infty, i}\right)^{-1}\left(p r^{\infty, i}\left(g_{x}^{\infty}\right)\right)
$$

and consider the corresponding topology on $\Sigma^{\infty}$.

Remark 3.4.19. A strongly Lie pseudogroup, cf. Definition 3.2.17, can equivalently be defined as a pseudogroup such that $J^{\infty} \Gamma$ is closed in $\lim J^{k} \Gamma$ with respect to the topology constructed in this way. See also Example 3.4.33.

Definition 3.4.20. The vertical limit topology on $\mathcal{G}$ is the initial topology with respect to $j_{\Sigma^{\infty}}: \mathcal{G} \rightarrow \Sigma^{\infty}$, where $\Sigma^{\infty}$ is equipped with the topology introduced above.

Remark 3.4.21. A sequence $\left\{g_{x^{n}}^{n}\right\}_{n \in \mathbb{N}}$ in $\mathcal{G}$ converges to $g_{x}$ in the vertical limit topology if and only if $x^{n}=x$ for all $n \in \mathbb{N}$ and for all $i \in \mathbb{N}$ it holds $p r^{\infty, i}$ 。 $j_{\Sigma^{\infty}}\left(g_{x^{n}}^{n}\right)=p r^{\infty, i} \circ j_{\Sigma^{\infty}}\left(g_{x}\right)$ for all $n \geq n_{i}$ and some $n_{i} \in \mathbb{N}$. This justifies our choice of terminology. We will write

$$
g_{x^{n}}^{n} \underset{\operatorname{vlim}}{\rightarrow} g_{x}
$$


Remark 3.4.22. As will be clear from Subsection 3.4.4 and Subsection 3.4.5, in the infinitesimal setting there is a more transparent definition of the vertical limit topology as an adic topology on a module with respect to an ideal. In this global setting, we are morally considering the set of elements of $\Sigma^{\infty}$ over the isotropy group of $\Sigma=\Sigma^{0}$ as a ring and the $s$-fiber of $\Sigma^{\infty}$ over $x$ as a module over that ring. The set of elements of $\Sigma^{\infty}$ over the isotropy group of $\Sigma^{1}$ can then be thought of as an ideal and the vertical limit topology on the $s$-fiber of $\Sigma^{\infty}$ is the adic topology with respect to this ideal.

Remark 3.4.23. When the tower at hand is the canonical Cartan tower of $\Sigma$, we call the limit topology jet topology. In fact, $g_{x^{n}}^{n} \rightarrow g_{x}$ if and only if the infinite jets $j_{x^{n}}^{\infty} \gamma^{n}$ of a sequence of representatives converge to $j_{x}^{\infty} \gamma$, where $\gamma$ represents $g_{x}$. Analogously, we call the vertical limit topology vertical jet topology.

Proposition 3.4.24. The following facts hold true.

- $C_{\Sigma^{*}}^{\infty}$-convergence implies convergence in the limit topology.

- The vertical limit topology is sequentially finer than the limit topology.

Proof. If $g_{x^{n}}^{n} \underset{C_{\Sigma^{*}}^{\infty}}{\rightarrow} g_{x}$ then we have representatives $\gamma^{n}, \gamma$ for $g_{x^{n}}^{n}, g_{x}$, defined on some open $U$ (for $n$ big enough) and satisfying $\gamma^{n} \underset{C_{\Sigma^{*}}^{\infty}}{\rightarrow} \gamma$. Then either $\gamma^{n}\left(x^{n}\right)$ is constant for $n$ big enough or there exists a compact set $K$ containing $x$ and $x^{n}$ for $n$ big enough such that $\left(p r^{\infty, k} \circ j_{\Sigma^{\infty}}\right)\left(\left.\gamma^{n}\right|_{K}\right) \rightarrow\left(p r^{\infty, k} \circ j_{\Sigma^{\infty}}\right)\left(\left.\gamma\right|_{K}\right), k \in \mathbb{N}$, with respect to the compact open topology. This implies that $j_{\Sigma^{\infty}}\left(\gamma^{n}\right)(x) \rightarrow j_{\Sigma^{\infty}}(\gamma)(x)$, where $j_{\Sigma \infty}: \operatorname{Bis}_{\text {loc }}(\Sigma) \rightarrow \operatorname{Bis}_{\text {loc }}(\Sigma)$ and we are using the limit topology on $\Sigma^{\infty}$. Consequently, $g_{x^{n}}^{n} \underset{\lim }{\rightarrow} g_{x}$. The second statement is immediate.

Let us consider a sequence $\left\{g_{x^{n}}^{n}\right\}_{n \in \mathbb{N}}$ where $x^{n}=x$ for all $n \in \mathbb{N}$. With the following axiom we require that convergence in the vertical limit topology implies $C_{\Sigma^{*}}^{\infty}$-convergence.

Axiom 2. Consider a sequence $\left\{g_{x}^{n}\right\}_{n \in \mathbb{N}}$ such that for all $n \in \mathbb{N}$ it holds $p r^{\infty, n} \circ$ $j_{\Sigma^{\infty}}\left(g_{x}^{n}\right)=1_{x} \in \Sigma^{n}$; then the sequence $C_{\Sigma^{*}}^{\infty}$-converges to $1_{x} \in \mathcal{G}$.

Remark 3.4.25. Axiom 1, which was stated on page 188, is an axiom concerning the pair $(\mathcal{G}, \Sigma)$. Axiom 2 concerns the triple $\left(\mathcal{G}, \Sigma,\left(\Sigma^{*}, \mathfrak{e}^{*}\right)\right)$.

Example 3.4.26. In [54] it is shown that Axiom 2 is satisfied when $\mathcal{G}=\operatorname{Germ}\left(\Gamma^{n}\right)$ and the resolution $\left(\Sigma^{*}, \mathrm{e}^{*}\right)$ is the canonical Cartan tower.

We conclude this subsection by analizing separability in the limit and vertical limit topology. We will write $g_{x} \underset{\lim }{\sim} g_{y}^{\prime}, g_{x} \underset{\text { vlim }}{\sim} g_{y}^{\prime}$ when $g_{x}, g_{x}^{\prime}$ are non separable in the limit and vertical limit topology respectively. We will denote the correspondent Hausdorff quotients by $\operatorname{Haus}(\mathcal{G}, \lim ), \operatorname{Haus}(\mathcal{G}, \operatorname{vim})$.

Proposition 3.4.27. The following facts hold true.

- If $g_{x}$ and $g_{y}^{\prime}$ are non separable in the étale, limit or vertical limit topology, or if they are $C_{\Sigma^{*}}^{\infty}$-non-separable, then $j_{\Sigma^{\infty}}\left(g_{x}\right)=j_{\Sigma^{\infty}}\left(g_{y}^{\prime}\right)$. 
- If $j_{\Sigma \infty}\left(g_{x}\right)=j_{\Sigma \infty}\left(g_{y}^{\prime}\right)$ then $g_{x} \underset{\lim }{\sim} g_{y}^{\prime}$ and $g_{x} \underset{\operatorname{vlim}}{\sim} g_{y}^{\prime}$.

Consequently $\operatorname{Haus}(\mathcal{G}, \lim ) \cong \operatorname{Haus}(\mathcal{G}, \operatorname{vlim})$ and both are homeomorphic to a subspace of $\Sigma^{\infty}$. Moreover, there is a bijection $\operatorname{Haus}\left(\mathcal{G}, C_{\Sigma^{*}}^{\infty}\right) \rightarrow \operatorname{Haus}(\mathcal{G}, \lim )$.

Remark 3.4.28. The above statement needs Axiom 2 for the last point, while Axiom 1 plays no role.

Proof. First of all, by Proposition 3.4.24, the limit topology is sequentially coarser than the other topologies mentioned and $C_{\Sigma^{*}}^{\infty}$-convergence implies limit convergence; consequently, the first claim holds true. If $j_{\Sigma^{\infty}}\left(g_{x}\right)=j_{\Sigma^{\infty}}\left(g_{y}^{\prime}\right)$, the constant sequence $\left\{g_{x^{n}}^{n}=g_{x}\right\}_{x \in \mathbb{N}}$ in $\mathcal{G}$ converges to both $g_{x}$ and $g_{y}^{\prime}$ in the limit and vertical limit topology, proving the second point. The surjection $\operatorname{Haus}\left(\mathcal{G}, C_{\Sigma^{*}}^{\infty}\right) \rightarrow$ $\operatorname{Haus}(\mathcal{G}, \lim )$ exists because of Proposition 3.4.24. By the first two points, two elements $g_{x}, g_{y}^{\prime}$ are such that $g_{x} \underset{\lim }{\sim} g_{y}^{\prime}$ precisely when $g_{x} \underset{\text { vlim }}{\sim} g_{y}^{\prime}$; by Axiom 2 , if the latter happens then $g_{x} \underset{C_{\Sigma^{*}}^{\infty}}{\sim} g_{y}^{\prime}$, and this concludes the proof.

Remark 3.4.29. If Axiom 2 is dropped, one still has a surjective map $\operatorname{Haus}\left(\mathcal{G}, C_{\Sigma^{*}}^{\infty}\right) \rightarrow \operatorname{Haus}(\mathcal{G}, \lim )$.

Remark 3.4.30. The subspace of $\Sigma^{\infty}$ homeomorphic to $\operatorname{Haus}(\mathcal{G}, \lim )$ is $j_{\Sigma^{\infty}}(\mathcal{G})$. $\operatorname{Haus}(\mathcal{G}, \lim ) \cong \Sigma^{\infty}$ (meaning that they are homeomorphic) if and only if $j_{\Sigma^{\infty}}$ is surjective, i.e. the resolution is tight, Definition 3.4.6.

\subsubsection{The infinitesimal picture}

We now move to the infinitesimal picture. Recall that the infinitesimal counterpart of étale groupoids $\mathcal{G}$ supported by Lie groupoids $\Sigma$ are LAS $\mathcal{A}$ supported by Lie algebroids $A$ (Definition 3.3.14). In fact, we know that to any étale Lie groupoid with support $\Sigma$ we can associate the LAS $\operatorname{Sec}_{A}^{\Gamma}$ of $\Gamma$-sections of $A=$ $\operatorname{Lie}(\Sigma)$ (Definition 3.3.19 and Axiom 1). We aim at defining infinitesimal versions of Cartan resolutions; the reason is that in a linear setting it is simpler to deal with the finite order theorem - see the next section. Our starting point is the notion of Pfaffian algebroid $(A, D)$, Definition 3.B.22, and of Spencer tower, Definition 3.B.24 of Pfaffian algebroids over a Lie algebroid $A \rightarrow \mathbf{X}$ :

$$
\cdots \rightarrow\left(A^{k}, D^{k}\right) \rightarrow\left(A^{k-1}, D^{k-1}\right) \rightarrow \cdots \rightarrow A \rightarrow \mathbf{X},
$$

see appendix 3.B. As shown in [23], Theorem 1, and [55], Theorem 6.1.23 and Corollary 6.3.2, Pfaffian algebroids and Spencer towers are the infinitesimal counterparts of Pfaffian groupoids and Cartan towers; see also Theorem 3.B.29 in the appendix.

Recall here that, given two Lie algebroids $A \rightarrow \mathbf{X}$ and $E \rightarrow \mathbf{X}$, a structure of Pfaffian algebroid on $A \rightarrow \mathbf{X}$ relative to a Lie algebroid epimorphism $l: A \rightarrow E$ is given by an operator

$$
D: \Gamma(A) \rightarrow \Omega^{1}(\mathbf{X}, E)
$$

such that

- $D_{X}(f \alpha)=f D_{X}(\alpha)+L_{X}(f) l(\alpha)$ for $\alpha \in \Gamma(A), X \in X(\mathbf{X}), f \in C^{\infty}(\mathbf{X})$; 
- $D$ is infinitesimally multiplicative:

$$
\begin{gathered}
D_{X}[\alpha, \beta]-\left[D_{X}(\alpha), l(\beta)\right]_{E}-\left[l(\alpha), D_{X} \beta\right]_{E}= \\
D_{\rho\left(D_{X}(\beta)\right)+[\rho(\beta), X]}(\alpha)-D_{\rho\left(D_{X}(\alpha)\right)+[\rho(\alpha), X]}(\beta)
\end{gathered}
$$

for $\alpha, \beta \in \Gamma(A)$ and $X \in X(\mathbf{X})$, where $[,]_{E}$ denotes the bracket of $E$.

One has a notion of holonomic local sections over an open $U \subset \mathbf{X}$ of a Pfaffian algebroid $(A, D) \rightarrow \mathbf{X}$ relative to $l: A \rightarrow E$; namely, $\alpha: U \rightarrow A$ is holonomic if

$$
D(\alpha)=0
$$

as a map $T \mathbf{X} \rightarrow \Gamma(E)$. In view of infinitesimal multiplicativity, the sheaf of holonomic sections

$$
\Gamma^{D}\left(A,{ }_{-}\right)
$$

is a LAS supported by $A$.

Let $\left(A^{\prime}, D^{\prime}\right)$ be a Pfaffian algebroid relative to $l^{\prime}: A^{\prime} \rightarrow A$ and $(A, D)$ be a Pfaffian algebroid relative to $l: A \rightarrow E$. We say that $l^{\prime}:\left(A^{\prime}, D^{\prime}\right) \rightarrow(A, D)$ is a Lie prolongation map of Pfaffian algebroids if

- $D \circ l^{\prime}=l \circ D$;

- $D_{X} D_{Y}^{\prime}-D_{Y} D_{X}^{\prime}-l \circ D_{[X, Y]}^{\prime}=0$ for all $X, Y \in X(X)$;

- the map

$$
\operatorname{Ker}\left(l^{\prime}\right) \rightarrow \operatorname{Hom}(T \mathbf{X}, A), \quad w \rightarrow\left(v \rightarrow D_{v}^{\prime}(w)\right), w \in \operatorname{Ker}\left(l^{\prime}\right), v \in T \mathbf{X}
$$

is injective.

Prolongations induce maps of the LAS of holonomic sections.

Definition 3.4.31. A Spencer tower $\left(A^{*}, D^{*}\right)$ of Pfaffian algebroids over a Lie algebroid $A \rightarrow \mathbf{X}$ is a tower of prolongations of Pfaffian algebroids

$$
\ldots \stackrel{l^{k+2}}{\rightarrow}\left(A^{k+1}, D^{k+1}\right) \stackrel{l^{k+1}}{\rightarrow}\left(A^{k}, D^{k}\right) \rightarrow \ldots \stackrel{l^{1}}{\rightarrow} A \rightarrow \mathbf{X}
$$

over $A \rightarrow \mathbf{X}$ such that $\left(A^{1}, D^{1}\right)$ is relative to $l^{1}: A^{1} \rightarrow A$.

As in the global setting, a Spencer tower has a limit $\left(A^{\infty}, D^{\infty}\right)$ where $A^{\infty}:=$ $\lim _{\leftarrow} A^{k}$ is a pf-Lie algebroid and $D^{\infty}=\lim _{\leftarrow} D^{k}$ is a multiplicative flat (linear) connection on $A^{\infty}$, see Proposition 3.B.25.

The following definition is the infinitesimal counterpart of Definition 3.4.5.

Definition 3.4.32. A Spencer resolution of a $\operatorname{LAS} \mathcal{A}$ on $\mathbf{X} \operatorname{supported}$ by $(A, \rho,[]$, is a Spencer tower $\left(A^{*}, D^{*}\right)$ over $A$ together with diffeologically smooth morphisms of LAS

$$
j_{A^{*}}^{k}: \mathcal{A} \hookrightarrow \Gamma^{D^{k}}\left(A^{k},{ }_{-}\right)
$$

such that: 
- for all $k \geq 1$ the diagrams

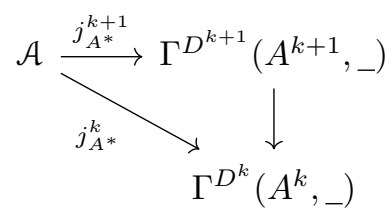

commute;

- the maps

$$
j_{A^{k}}: \operatorname{Germ}(\mathcal{A}) \rightarrow A^{k}
$$

obtained postcomposing (the map induced by) $j_{A^{*}}^{k}$ with the evaluation

$$
\operatorname{germ}_{x}(\alpha) \in \operatorname{Germ}\left(\Gamma^{D^{k}}\left(A^{k},{ }_{-}\right)\right) \rightarrow \alpha(x)
$$

are surjective.

A Spencer resolution is called tight if the induced map $j_{A^{\infty}}: \operatorname{Germ}(\mathcal{A}) \rightarrow A^{\infty}$ is surjective. The pair $(\mathcal{A}, A)$ is called of Lie type if $\mathcal{A}$ admits a Spencer resolution over $A$; we also say that $\mathcal{A}$ is of Lie type over $A$.

As in the groupoid setting, we set

$$
j_{A^{*}}^{0}: \mathcal{A} \hookrightarrow \Gamma\left(A,{ }_{-}\right)
$$

to be the map exhibiting $A \rightarrow \mathbf{X}$ as a support of $\mathcal{A}$. All the remarks made in Subsection 3.4.1 can be suitably adapted to this new setting. In particular a map

$$
j_{A^{\infty}}^{\infty}: \mathcal{A} \rightarrow A^{\infty}
$$

is induced by the $j_{A^{*}}^{k}$ 's.

Example 3.4.33. When $\Gamma$ is some classical Lie pseudogroup, we are in the setting of Axiom 1 on page 188, and we consider the sheaf of $\Gamma$-vector fields $X_{\mathbf{X}}^{\Gamma}$ (Example 3.3.23), the tower of jets (as sections of $T \mathbf{X} \rightarrow \mathbf{X}$ ) is a Spencer resolution of $x_{\mathbf{X}}^{\Gamma}$ over $T \mathbf{X}[55,63,69]$.

In general, if $\mathcal{A}$ is a LAS supported by $A$, then there is a clear candidate for a Spencer tower, namely the canonical Spencer tower

$$
\left(J^{\infty} \mathcal{A}, D^{\infty}\right) \rightarrow \cdots \rightarrow\left(J^{k} \mathcal{A}, D^{k}\right) \rightarrow\left(J^{k-1} \mathcal{A}, D^{k-1}\right) \rightarrow \cdots \rightarrow J^{0} \mathcal{A} \rightarrow \mathbf{X} .
$$

where

$$
J^{k} \mathcal{A}=\left\{j_{x}^{k} \alpha: x \in \mathbf{X}, \alpha \in \mathcal{A}(U), U \text { neighbourhood of } x\right\}
$$

and the $D^{k \prime}$ s are the restriction of the canonical Spencer operator on the jet tower of the vector bundle $A \rightarrow \mathbf{X}$, see $[55,63,69]$ for details. 
Definition 3.4.34. Let $\mathcal{A}$ be a LAS over $\mathbf{X}$ supported by $A \rightarrow \mathbf{X}$. We call $\mathcal{A}$ smooth LAS ${ }^{6}$ if $J^{\infty} \mathcal{A}$ is a smooth pf-subalgebroid of $J^{\infty} A$. Furthermore, if the canonical map

$$
\operatorname{Germ}(\mathcal{A}) \rightarrow J^{\infty} \mathcal{A}
$$

is surjective, $\mathcal{A}$ is called strongly smooth.

See appendix 3.A for details on the notion of pf-algebroid. Explicitly, a LAS $\mathcal{A}$ over $\mathbf{X}$ supported by $A \rightarrow \mathbf{X}$ is smooth when all the $J^{k} \mathcal{A}^{\prime}$ s are submanifolds of the $J^{k} A^{\prime}$ s and all the maps $j^{k} \mathcal{A} \rightarrow J^{k-1} \mathcal{A}$ are surjective submersions. Under these assumptions, the tower

$$
\cdots \rightarrow\left(J^{k} \mathcal{A}, D^{k}\right) \rightarrow\left(J^{k-1} \mathcal{A}, D^{k-1}\right) \rightarrow \cdots \rightarrow\left(J^{1} \mathcal{A}, D^{1}\right) \rightarrow J^{0} \mathcal{A} \rightarrow \mathbf{X} .
$$

above is automatically a Spencer resolution. If such a resolution is tight, we call $\mathcal{A}$ strongly smooth.

A version of Proposition 3.4.13 holds in the infinitesimal setting: the canonical Spencer tower is final among Spencer resolutions.

From now on, and as we did for pseudogroups, we assume all our LAS to be smooth unless otherwise specified. All the discussions in this section concerning $C^{\infty}$-convergence, limit and vertical limit topology can be adapted.

Remark 3.4.35. When $\mathcal{A}$ is classical and smooth, $J^{0} \mathcal{A}$ is a foliation on $\mathrm{X}$. This is the point of view adopted in [60]; there, strongly smooth classical Lie algebra sheaves are simply called Lie algebra sheaves. Moreover, in [60] the authors observe that the tightness request $J^{\infty} \mathcal{A} \cong \lim _{\leftarrow} J^{k} \mathcal{A}$ has to be understood as a condition of topological closure. See also Remark 3.2.19 and Remark 3.4.19.

Proposition 3.4.36. Let $\mathcal{G}$ be supported by $\Sigma$ and $\left(\Sigma^{*}, \mathrm{C}^{*}\right)$ be a Cartan resolution over $\Sigma \rightrightarrows \mathbf{X}$. The tower of Lie algebroids

$$
\cdots \rightarrow\left(\operatorname{Lie}\left(\Sigma^{k+1}\right), D^{k+1}\right) \rightarrow\left(\operatorname{Lie}\left(\Sigma^{k}\right), D^{k}\right) \rightarrow \cdots \rightarrow \operatorname{Lie}(\Sigma) \rightarrow \mathbf{X}
$$

where the maps are obtained by differentiation and $D^{*}$ is induced by $\mathcal{C}^{*}$ as explained in the Appendix 3.B, Theorem 3.B.29, is a Spencer resolution of $\mathcal{A}:=\operatorname{Lie}(\mathcal{G})$ over the support $A:=\operatorname{Lie}(\Sigma)$.

Proof. Theorem 3.B.29 from [55] tell us that the tower $\left(\operatorname{Lie}\left(\Sigma^{*}\right), D^{*}\right)$ is in fact a Spencer tower over $A \rightarrow \mathbf{X}$.

For the second point notice that holonomic sections of $A^{k}$ are obtained precisely from flows of holonomic bisections of $\Sigma^{k}$, as it follows from [55], Theorem 4.3.1. One then defines

$$
j_{A^{*}}^{k}: \Gamma\left(\mathcal{A},{ }_{-}\right) \rightarrow \Gamma^{D^{k}}\left(\operatorname{Lie}\left(\Sigma^{k}\right),{ }_{-}\right)
$$

by sending $\alpha \in \Gamma(\mathcal{A}, \underline{U})$ to the section of $\operatorname{Lie}\left(\Sigma^{k}\right)$ over $U$ whose flow is $j_{\Sigma^{*}}^{k}\left(\phi_{\alpha}^{t}\right)$, where $\phi_{\alpha}^{t}$ is the flow of $\alpha$ seen as a section of $\operatorname{Lie}(\Sigma)$.

\footnotetext{
${ }^{6}$ To keep the analogy with pseudogroups, one should use the terminology "Lie LAS", which doesn't sound right. A possible alternative could be "Lie type LAS", which not only doesn't sound right but is also already taken, see Definition 3.4.32.
} 
Later on, we will also make use of the following observation. The exponential map on compactly supported bisections of a Lie algebroid induces an exponential map at the level of towers. For each $k$ we have maps $\exp ^{\mathrm{k}}: \Gamma_{\mathrm{cpt}}\left(A^{k},{ }_{-}\right) \rightarrow$ $\operatorname{Bis}_{\text {loc }}\left(\Sigma^{k}\right)$ (see Subsection 3.3.2). These maps commute with the maps induced by the projections, because the projections of the Spencer tower are obtained from the projections of the Cartan tower by applying the Lie functor. That is, for each $k \in \mathbb{N}$

$$
p r^{k+1, k}: A^{k+1} \rightarrow A^{k},\left.\quad a \rightarrow \frac{d p r^{k+1, k}\left(\phi_{\alpha_{a}}^{t}\right)}{d t}\right|_{t=0}
$$

where $\alpha_{a}$ is a section of $A^{k+1}$ around $a$. This implies that there is an exponential map

$$
\exp ^{\infty}: \Gamma_{\text {cpt }}\left(A^{\infty},{ }_{-}\right) \rightarrow \operatorname{Bis}_{\text {loc }}\left(\Sigma^{\infty}\right)
$$

\subsubsection{Isotropies of a Cartan/Spencer resolution}

Let $\left(\Sigma^{*}, \mathrm{C}^{*}\right)$ be a Cartan resolution of an étale groupoid $\mathcal{G} \rightrightarrows \mathbf{X}$ over a support $\Sigma \rightrightarrows \mathbf{X}$. Recall that $\Sigma^{\infty}$ denotes the projective $\operatorname{limit} \lim \Sigma^{k}$ of the tower of groupoids

$$
\cdots \rightarrow \Sigma^{k+1} \rightarrow \Sigma^{k} \rightarrow \cdots \rightarrow \Sigma \rightrightarrows \mathbf{X}, \quad k \geq 1 .
$$

When writing $\operatorname{Ker}_{x}(f)$, for $f$ some map $\Sigma^{k} \rightarrow \Sigma^{i}$ where $i \in \mathbb{N}$ and $k$ can possibly be $\infty$, or $f: \mathcal{G} \rightarrow \Sigma^{i}$ where $i \in \mathbb{N}$, we mean $f^{-1}\left(1_{x}\right)$. We set:

- $G_{x}^{i}(\mathcal{G})=\operatorname{Ker}_{x}\left(p r^{\infty, i} \circ j_{\Sigma^{\infty}}: \mathcal{G} \rightarrow \Sigma^{\infty}\right), i \in \mathbb{N} \cup\{\infty\}$

- $G_{x}^{i}\left(\Sigma^{k}\right)=\operatorname{Ker}_{x}\left(p r^{k, i}: \Sigma^{k} \rightarrow \Sigma^{i}\right) \subset \Sigma^{k}, k \in \mathbb{N} \cup\{\infty\}, i \in \mathbb{N}$;

- $G_{x}^{i}=\operatorname{Ker}_{x}\left(p r^{\infty, i}: \Sigma^{\infty} \rightarrow \Sigma^{i}\right) \cap j_{\Sigma^{\infty}}(\mathcal{G}) \subset \Sigma^{\infty}, i \in \mathbb{N}$;

- $\mathcal{G}_{x}$ and $\Sigma_{x}^{k}, k \in \mathbb{N} \cup\{\infty\}$, for the isotropy groups at $x$.

Notice that:

- $G_{x}^{0}\left(\Sigma^{k}\right)$ is a subgroup of the isotropy group of $\Sigma^{k}$ at $x$ for all $k \in \mathbb{N} \cup\{\infty\}$;

- $G_{x}^{0}(\mathcal{G})$ is a subgroup of the isotropy group of $\mathcal{G}$ at $x$;

- $G_{x}^{0}$ is the isotropy group at $x$ of $j_{\Sigma^{\infty}}(\mathcal{G})$;

- the $G_{x}^{i}\left(\Sigma^{k}\right)^{\prime}$ s, for $i \in \mathbb{N}$ fixed and $k \in \mathbb{N}$ varying, form an inverse system of Lie groups;

- $G_{x}^{i}$ is a subgroup of the limit of the system above; if the chosen Cartan resolution is tight, i.e. $\Sigma^{\infty}=\lim _{\leftarrow} \Sigma^{i}, G_{x}^{i}$ is actually the limit;

- similarly, the isotropy Lie groups at $x \in \mathbf{X}$ also form an inverse system of Lie groups. 
A similar notation can be introduced in the infinitesimal setting, for a Spencer resolution $\left(A^{*}, D^{*}\right)$ of a LAS $\mathcal{A}$ over a support $A \rightarrow \mathbf{X}$; in this case, one has inverse systems of Lie algebras. Here, there is an additional interesting fact to point out: the fiber $A_{x}^{\infty}$ of the limit of the resolution is a Lie algebra for any $x \in \mathbf{X}$ (see also Appendix 3.B) and the map $j_{A^{\infty}}: \operatorname{Germ}(\mathcal{A})_{x} \rightarrow A_{x}^{\infty}$ is a Lie algebra map. We will be more explicit below. First, recall the notation from Subsection 3.3.2:

$$
\mathcal{A}_{x}:=\operatorname{Germ}(\mathcal{A})_{x}
$$

the stalk of $\mathcal{A}$ at $x \in \mathbf{X}$. We also set

$$
\mathfrak{a}_{x}:=A_{x}^{\infty}
$$

$\mathfrak{a}_{x}$ is called formal algebra of $\mathcal{A}$ at $x$. In fact, by Proposition 3.B.26 and the discussion preceding it in the Appendix,

Proposition 3.4.37. The limit $A^{\infty}$ of a Spencer tower is a bundle of Lie algebras when equipped with the bracket

$$
\begin{aligned}
\{,\}^{\infty}: \Gamma\left(A^{\infty}\right) \times \Gamma\left(A^{\infty}\right) & \rightarrow \Gamma\left(A^{\infty}\right) \\
(\alpha, \beta) & \rightarrow\{\alpha, \beta\}^{\infty}=[\alpha, \beta]^{\infty}-D_{\rho(\alpha)}^{\infty}(\beta)+D_{\rho(\beta)}^{\infty}(\alpha)
\end{aligned}
$$

Consequently, $\mathfrak{a}_{x}$ carries a Lie algebra structure.

$\mathcal{A}_{x}$ comes with a natural filtration. Recall that $\mathcal{F}_{x}:=\left(C_{\mathbf{X}}^{\infty}\right)_{x}$ is the stalk of the sheaf $C_{\mathbf{X}}^{\infty}$ of smooth functions on $\mathbf{X}$ and that, since $\mathcal{A}$ is a LAS, cf. Definition 3.3.14, $\mathcal{A}_{x}$ is a $\mathcal{F}_{x}$-module. $\mathcal{F}_{x}$ is naturally filtered by declaring $\mathcal{F}_{x}^{i}, i \in \mathbb{N}$, to be the stalk of functions vanishing with order $i$ at $x$ and $\mathcal{F}_{x}^{-1}=\mathscr{F}$. We declare $\mathcal{A}_{x, i}, i \in \mathbb{N}$, to be

$$
\mathcal{A}_{x, i}:=\mathcal{F}_{x}^{i} \cdot \mathcal{A}_{x}
$$

and

$$
\mathcal{A}_{x,-1}=\left\{\operatorname{germ}_{x}(\alpha) \in \mathcal{A}_{x}: \rho(\alpha)=0\right\}
$$

where $\rho$ is the anchor of the support $A \rightarrow \mathbf{X}$.

Notice that $\mathcal{A}_{x, i}$ is a Lie subalgebra of $\mathcal{A}_{x}$ and an $\mathcal{F}_{x}$-submodule; furthermore, the Leibniz identity that holds on $\mathcal{A}_{x}$, namely

$$
\begin{aligned}
{\left[\operatorname{germ}_{x}\left(\alpha_{1}\right), \operatorname{germ}_{x}(f) \cdot \operatorname{germ}_{x}\left(\alpha_{2}\right)\right]=} & \operatorname{germ}_{x}\left(L_{\rho\left(\alpha_{1}\right)}(f) \alpha_{2}\right) \\
& +\operatorname{germ}_{x}(f)\left[\operatorname{germ}_{x}\left(\alpha_{1}\right), \operatorname{germ}_{x}\left(\alpha_{2}\right)\right]
\end{aligned}
$$

for all $\operatorname{germ}_{x}\left(\alpha_{i}\right) \in \mathcal{A}_{x}$ and $\operatorname{germ}_{x}(f) \in \mathcal{F}_{x}$, still holds when restricting to $\operatorname{germ}_{x}\left(\alpha_{i}\right) \in \mathcal{A}_{x, i}$. As a consequence, one has:

Proposition 3.4.38. $\mathcal{A}_{x}$ admits the filtration

$$
\cdots \subset \mathcal{A}_{x, 1} \subset \mathcal{A}_{x, 0} \subset \mathcal{A}_{x,-1} \subset \mathcal{A}_{x},
$$

which induces a Lie algebra filtration, i.e.

$$
\left[\mathcal{A}_{x, i}, \mathcal{A}_{x, j}\right] \subset \mathcal{A}_{x, i+j}
$$

for any $i, j \in \mathbb{N}$. In particular, for any $i \in \mathbb{N}, \mathcal{A}_{x, i}$ is an ideal in $\mathcal{A}_{x, 0}$. 
We set $\operatorname{gr}\left(\mathcal{A}_{x,-1}\right)=\bigoplus \mathcal{A}_{x, i} / \mathcal{A}_{x, i+1}$, the associated graded Lie algebra. We also set the following notations.

- $\mathfrak{g}_{x}^{i}(\mathcal{A})=\operatorname{Ker}_{x}\left(p r \circ j_{A^{\infty}}: \mathcal{A} \rightarrow A^{i}\right), i \in \mathbb{N} \cup\{\infty\} ;$

- $\mathfrak{g}_{x}^{i}\left(A^{k}\right)=\operatorname{Ker}_{x}\left(p r: A^{k} \rightarrow A^{i}\right), k \in \mathbb{N} \cup \infty, i \in \mathbb{N}$;

- $\mathfrak{g}_{x}^{i}=\operatorname{Ker}_{x}\left(p r: A^{\infty} \rightarrow A^{i}\right) \cap j_{A^{\infty}}(\operatorname{Germ}(A)), i \in \mathbb{N}$;

- $\mathfrak{g}_{x}(\mathcal{A})$ and $\mathfrak{g}_{x}\left(A^{k}\right), k \in \mathbb{N}$, for the isotropy algebras at $x$.

Notice that all the spaces above are Lie algebras. In particular $\mathfrak{g}_{x}^{0}\left(A^{k}\right)$ is a subalgebra of the isotropy Lie algebra of $A^{k}$ over $x$. Notice also that $\mathfrak{g}_{x}^{i}\left(A^{k}\right)=$ $\operatorname{Ker}_{x}\left(\mathfrak{g}_{x}\left(A^{k}\right) \rightarrow \mathfrak{g}_{x}\left(A^{i}\right)\right)$.

Lemma 3.4.39. The map $j_{A^{\infty}}: \mathcal{A}_{x} \rightarrow \mathfrak{a}_{x}$ induced by

$$
j_{A^{\infty}}: \mathcal{A} \rightarrow A^{\infty}
$$

from Subsection 3.4.4, is a Lie algebra map.

Example 3.4.40. It is instructive to start looking at the case when $\mathcal{A}$ is a smooth LAS, cf. Definition 3.4.34, and the Spencer tower is the canonical one. In this case, the Lie algebra structure on the $\operatorname{stalk} \operatorname{Germ}(\mathcal{A})_{x}$ induces a Lie algebra structure on $J_{x}^{\infty} \mathcal{A}$ by setting

$$
\left[j_{x}^{\infty} \sigma_{1}, j_{x}^{\infty} \sigma_{2}\right]=j_{x}^{\infty}\left[\sigma_{1}, \sigma_{2}\right]
$$

and this is a subalgebra of the formal algebra $\mathfrak{a}_{x}=\left(\lim ^{k} \mathcal{A}\right)_{x}$ equipped with the "limit" bracket from Proposition 3.4.37. In fact, in the setting of this example, the "limit" bracket on $\left(\lim ^{k} J^{k} \mathcal{A}\right)_{x}$ is induced by the bilinear maps

$$
J_{x}^{k} \mathcal{A} \times J_{x}^{k} \mathcal{A} \rightarrow J_{x}^{k-1} \mathcal{A}, \quad\left[j_{x}^{k} \sigma_{1}, j_{x}^{k} \sigma_{2}\right]=j_{x}^{k-1}\left[\sigma_{1}, \sigma_{2}\right] .
$$

Of course, when $\mathcal{A}$ is "strongly" smooth, that is $J^{\infty} \mathcal{A}=\lim ^{k} J^{k}$, these two algebras coincide.

Let us prove Lemma 3.4.39.

Proof. One observes that, given a Spencer resolution $\left(A^{*}, D^{*}\right)$, the "limit" monomorphism of LAS

$$
j_{A^{*}}^{\infty}: \mathcal{A} \rightarrow \Gamma^{D^{\infty}}\left(A^{\infty},{ }_{-}\right)
$$

induces a fiberwise Lie algebra structure $\{,\}^{\prime}$ on $j_{A^{\infty}}\left(\operatorname{Serm}(\mathcal{A})_{x}\right)$ by setting

$$
\left\{j_{A^{\infty}}\left(\sigma_{1}\right)(x), j_{A^{\infty}}\left(\sigma_{2}\right)(x)\right\}^{\prime}=\left(j_{A^{\infty}}^{\infty}\left(\left[\sigma_{1}, \sigma_{2}\right]\right)\right)(x),
$$

where the $\sigma_{i}$ 's are sections of $\mathcal{A}$. This is a subalgebra of $A_{x}^{\infty}$ with the bracket $\{$, from Proposition 3.4.37. In fact, if $\alpha_{x}, \beta_{x} \in A_{x}^{\infty}$ lie in the image of $j_{A_{\infty}}$, there are horizontal sections $\alpha, \beta$ of $A^{\infty}$ such that $\alpha(x)=\alpha_{x}, \beta(x)=\beta_{x}$; they are the images via $j_{A^{*}}^{\infty}$ of the preimages of $\alpha_{x}, \beta_{x}$ in $\mathcal{A}$. Then,

$$
\left\{\alpha_{x}, \beta_{x}\right\}=[\alpha, \beta] .
$$


Moreover (by definition)

Lemma 3.4.41. The Lie algebra map $j_{A^{\infty}}: \mathcal{A}_{x} \rightarrow \mathfrak{a}_{x}$ sends $\mathfrak{g}^{i}(\mathcal{A})$ to $\mathfrak{g}^{i}$, for all $i \in$ $\mathbb{N} \cup\{\infty\}$.

Consequently, we have the following proposition.

Proposition 3.4.42. The formal algebra $\mathfrak{a}_{x}$ admits the filtration

$$
\cdots \subset \mathfrak{g}_{x}^{1} \subset \mathfrak{g}_{x}^{0} \subset \mathfrak{g}_{x}^{-1}=\mathfrak{g}_{x}\left(A^{\infty}\right) \subset \mathfrak{a}_{x}
$$

which is a Lie algebra filtration, i.e.

$$
\left[\mathfrak{g}_{x}^{i}, \mathfrak{g}_{x}^{j}\right] \subset \mathfrak{g}_{x}^{i+j}
$$

for any $i, j \in \mathbb{N}$. In particular, for each $i \in \mathbb{N}$, $\mathfrak{g}_{x}^{i}$ is an ideal of $A_{x}^{\infty}$.

Notice that, for $k>i$,

$$
\mathfrak{g}_{x}^{i}\left(A^{k}\right) \simeq \mathfrak{g}_{x}^{i} / \mathfrak{g}_{x}^{k} \simeq \mathfrak{g}_{x}^{i}(\mathcal{A}) / \mathfrak{g}_{x}^{k}(\mathcal{A})
$$

as Lie algebras. We set

$$
g r\left(\mathfrak{g}_{x}\left(A^{\infty}\right)\right)=\bigoplus \mathfrak{g}_{x}^{i} / \mathfrak{g}_{x}^{i+1}
$$

Corollary 3.4.43. For any $k \geq i, i \in \mathbb{N}, \mathfrak{g}^{i}\left(A^{k}\right)$ is nilpotent.

\subsection{Finite order problem à la Palais-Terng}

Let $E \mathbb{R}^{n} \rightarrow \mathbb{R}^{n}$ be a $\Gamma^{n}$-space, where we recall that $\Gamma^{n}=\operatorname{Diff}_{\text {loc }}\left(\mathbb{R}^{n}\right)$. In [54] the authors proved, under the assumptions of smothness (Definition 3.5.3 below), that $E \mathbb{R}^{n}$ is of finite order (Definition 3.2.23) The proof has essentially three steps.

- First, formal determinacy is proven, that is the action of $\operatorname{Germ}\left(\Gamma^{n}\right)$ descends to an action of $J^{\infty} \Gamma^{n}$. This makes use of an explicit argument that proves that the groupoid of flat diffeomorphisms, i.e. the kernel $\mathcal{F}$ in the short exact sequence of groupoids

$$
\mathbf{X} \rightarrow \mathcal{F} \rightarrow \operatorname{Germ}\left(\Gamma^{n}\right) \rightarrow J^{\infty} \Gamma^{n} \rightarrow \mathbf{X}
$$

consists of elements which are non-separable from the (germ of the) identity in the étale topology. In other words

$$
\operatorname{Haus}\left(\operatorname{Germ}\left(\Gamma^{n}\right), \operatorname{germ}\right) \cong J^{\infty} \Gamma^{n} .
$$

- Then, it is shown that for each $x \in \mathbf{X}$ and $e_{x} \in\left(E \mathbb{R}^{n}\right)_{x}$ there exists $k_{e_{x}} \in \mathbb{N}$ such that elements of $G^{k_{e_{x}}}$ (i.e., jets of elements coinciding with the identity up to order $k_{e_{x}}$ ) stabilize $e_{x}$. This property is called pointwise finite order [54]. It is proven by looking at the action of the isotropy group of $G_{x}^{0}$ at $x$ on the fiber $\left(E \mathbb{R}^{n}\right)_{x}$. The isotropy Lie algebra $\mathfrak{z}_{e_{x}}$ of such an action at some point $e_{x}$ in $\left(E \mathbb{R}^{n}\right)_{x}$ turns out to be 
- of finite codimension in the (isotropy) Lie algebra of $\Sigma_{x}^{\infty}$;

- closed in the vertical limit topology on $\operatorname{Lie}\left(\Sigma^{\infty}\right)$;

hence there exists $k_{e_{x}}$ such that $\mathfrak{z}_{e_{x}} \subset \mathfrak{g}_{x}^{k_{e_{x}}}$. Here one needs Axiom 2, but also the smoothness property of the action, i.e. the fact that the action sends smooth paths of (germs of) diffeomorphisms to smooth paths of (germs of) diffeomorphisms on $E \mathbb{R}^{n}$. This implies that the action sends $C^{\infty}$-converging sequences to sequences converging in the compact open topology, which is also used explicitely in the proof. Finally, in [54] the authors make use of the fact that $J^{\infty} \Gamma^{n}=\lim _{\leftarrow} J^{k} \Gamma^{n}$.

- To conclude, it is proven that there is an upper bound $k$ for $k_{e_{x}}$ 's which depends only on the dimension of the fiber of $E \mathbb{R}^{n} \rightarrow \mathbb{R}^{n}$.

Let now $E \rightarrow \mathbf{X}$ be a $\mathcal{G}$-space where $\mathcal{G} \rightrightarrows \mathbf{X}$ is some étale groupoid supported by $\Sigma \rightrightarrows \mathbf{X}$, in the sense of Definition 3.3.1. We assume here that $\mathcal{G}$ is of Lie type (Definition 3.4.5) and we fix a Cartan resolution $\left(\Sigma^{*}, \mathrm{C}^{*}\right)$ over $\Sigma$.

Definition 3.5.1. A $\mathcal{G}$-space $E \rightarrow \mathrm{X}$ is of finite order $k \in \mathbb{N}$ with respect to $\left(\Sigma^{*}, \mathrm{C}^{*}\right)$ if the action of $\mathcal{G}$ descends to an action of $\Sigma^{k}$ and is formally determined if the action descends to $j_{\Sigma^{\infty}}(\mathcal{G})$.

Then we can formulate the finite order problem as

Question 3. Under which conditions on $\mathcal{G}, \Sigma$ and $\left(\Sigma^{*}, \mathrm{e}^{*}\right)$ is it true that $\mathcal{G}$-spaces are of finite order with respect to $\left(\Sigma^{*}, \mathrm{C}^{*}\right)$ ?

Apart from the choice of a support $\Sigma \rightrightarrows \mathbf{X}$ for $\mathcal{G} \rightrightarrows \mathbf{X}$, the assumption that $\mathcal{G}$ is of Lie type over $\Sigma$, and the choice of a resolution $\left(\Sigma^{*}, \mathcal{C}^{*}\right)$ over $\Sigma \rightrightarrows \mathbf{X}$, we assume that

A1: $\mathcal{G}$ satisfies Axiom 1 (see Remark 3.3.24);

A2: $\mathcal{G}$ and the chosen tower satisfy Axiom 2;

A3: $E$ is a Hausdorff finite dimensional manifold that projects to $\mathbf{X}$ by a surjective submersion $\mu: E \rightarrow \mathbf{X}$ (i.e., Convention 3);

A4: the action on $E$ is regular; by this we mean that

- it is smooth in the diffeological sense (where the diffeology is induced by the support), Definition 3.5.3 below;

- it is continuous with respect to $C_{\Sigma^{*}}^{\infty}$-convergence on $\mathcal{G}$, that is $C_{\Sigma^{*}}^{\infty}$ converging sequences of germs in $\mathcal{G}$ induce sequences of maps on (a subset of) $E$ that converge in the compact open topology, Definition 3.5.6 below.

We elaborate on regularity of actions in the next subsections. Notice that assumption A3 means that for the rest of this section Convention 2 on page 159 is abandoned: $\Sigma$-spaces are Hausdorff manifolds.

In the end, following the approach to the case of $\Gamma^{n}$ from [54], we will show that 
Theorem 3.5.2. For $\mathcal{G}, \Sigma,\left(\Sigma^{*}, \mathrm{C}^{*}\right)$ satisfying $\mathrm{A} 1$ and $\mathrm{A} 2$, if

- the topological assumptions on the isotropy groups from Theorem 3.5.19 are satisfied;

- the algebraic condition (3.6) from Theorem 3.5.22 below is satisfied;

then regular $\mathcal{G}$-spaces satisfying A3 have finite order with respect to $\left(\Sigma^{*}, \mathrm{C}^{*}\right)$.

We will try to make clear the role of our assumptions (that in [54] were proven as propositions for $\Gamma^{n}$ ), and how the various topologies we introduced are involved.

\subsubsection{Regularity of $\mathcal{G}$-spaces}

Let $\mathcal{G} \rightrightarrows \mathbf{X}$ be an étale groupoid supported by $\Sigma \rightrightarrows \mathbf{X}$ and equipped with a Cartan resolution $\left(\Sigma^{*}, \mathfrak{C}^{*}\right)$ over $\Sigma \rightrightarrows \mathbf{X}$. Let $\mu: E \rightarrow \mathbf{X}$ be a $\mathcal{G}$-space. We assume to be in the setting of assumptions A1-A3 at the beginning of this section; we are going to give a precise definition of regularity, i.e. assumption $A 4$.

By definition, the action of $\mathcal{G}$ on $E$ is continuous with respect to the étale topology on $\mathcal{G}$. It makes sense to ask for the action to be smooth with respect to the diffeology induced by $\Sigma$, cf. Definition 3.3.5. Similar requests are known to be important for the finite order problem, see the conclusion of Subsection 3.2.4 and references therein.

Definition 3.5.3. A $\mathcal{G}$-space $\mu: E \rightarrow \mathbf{X}$ is smooth if the action map is smooth in the sense that it induces, for all $U \subset \mathbf{X}$ open, diffeologically smooth maps

$$
\operatorname{Bis}(\mathcal{G}, U) \rightarrow C^{\infty}\left(\left.E\right|_{U},\left.E\right|_{U}\right) .
$$

Example 3.5.4. If $\Gamma$ is a discrete group possessing a Lie group structure denoted by $G$, a $\Gamma$-space is smooth if and only if it is a smooth $G$-space.

The situation gets more involved when we assume $\Gamma$ to be a strict subgroup of some Lie group $G$. For instance, any action of $\mathbb{Q}$ supported by $\mathbb{R}$ with the usual smooth structure is smooth in the sense of Definition 3.5.3, because the induced diffeology is the discrete one, Examples 3.C.3 and 3.C.27.

Example 3.5.5. For a classical pseudogroup $\Gamma$, smoothness of a $\Gamma$ space means that a smooth family of elements of $\Gamma$ defined around $x \in \mathbf{X}$ defines a smooth family of locally defined diffeomorphisms of $E \mathbf{X}$ when composed with the action map. In terms of natural $\Gamma$-bundles, this was phrased as follows, see for example [40, 54, 62]. If $M, N \in \operatorname{Man}^{\Gamma}$ and $F: M \times U \rightarrow N$ is a smooth $k$ parameter family of arrows of $\operatorname{Man}^{\Gamma}$ (i.e. $U \subset \mathbb{R}^{k}$ is open, for all $t \in U$ the map $x \rightarrow f_{t}(x)=F(t, x)$ is a morphism of $\Gamma$-structures and $(x, t) \rightarrow F(x, t)$ is smooth), then the lift

$$
E F: E M \times U \rightarrow E N, \quad(e, t) \rightarrow E f_{t}
$$

is a smooth family (see Subsection 3.2.3 for the notation).

Definition 3.5.6. A $\mathcal{G}$-space $\mu: E \rightarrow \mathbf{X}$ is $C_{\Sigma^{*}}^{\infty}$-continuous if the action map respects $C_{\Sigma^{*}}^{\infty}$-convergence in the sense that $C_{\Sigma^{*}}^{\infty}$-converging sequences in $\mathcal{G}$ are sent to compact-open converging sequences in $C_{\mathrm{loc}}^{\infty}(E, E)$ by the action map. 
Observe that the above definition makes sense since the restriction map is continuous in the compact-open topology - i.e., for each pair of open sets $V, U$ in $\mathrm{X}$ such that $V \subset U$ the map

$$
C^{\infty}\left(\mu^{-1}(U), E\right) \rightarrow C^{\infty}\left(\mu^{-1}(V), E\right),\left.\quad f \rightarrow f\right|_{\mu^{-1}(V)}
$$

is continuous with respect to the compact open topology on both sides.

Remark 3.5.7. This continuity notion appears in [65] and [28] in the context of natural bundles. While in [65] it was part of the definition, in [28] it was proven as a consequence of the other axioms.

Definition 3.5.8. A $\mathcal{G}$-space $\mu: E \rightarrow \mathbf{X}$ is regular if it is smooth and $C_{\Sigma^{*}}^{\infty}$ continuous.

In [54], only smoothness was part of the assumptions. The point is that

Proposition 3.5.9. Let be $\mathcal{G}=\operatorname{Germ}\left(\Gamma^{\Sigma}\right), \Gamma^{\Sigma}=\operatorname{Bis}_{\mathrm{loc}}(\Sigma)$ and $\left(\Sigma^{*}, \mathcal{C}^{*}\right)=$ $\left(J^{*} \Sigma, \mathfrak{C}_{\text {can }}^{*}\right)$ be the canonical Cartan tower. Then smooth $\mathcal{G}$-spaces are $C^{\infty}$-continuous.

Proof. If $g_{x^{n}}^{n} \underset{C^{\infty}}{\rightarrow} g_{x}$, then there is a sequence of representatives $\left\{\gamma^{n}\right\}_{n \in \mathbb{N}}$ defined on some open $U \subset \mathbf{X}$ and such that $\gamma^{n} \rightarrow \gamma$, where $\gamma$ is defined over $U$ and represents $g_{x}$, in the strong Whitney topology. Consequently, $\gamma^{n} \rightarrow \gamma$ in the $D$ topology on the space $C^{\infty}(U, \Sigma)$ equipped with the standard functional diffeology. It is not restrictive to assume that $\gamma^{n}, \gamma$ can be extended, as bisections in $\Gamma^{\Sigma}$, to an open $V$ containing $U$ as a relatively compact set; both $U$ and $V$ can be taken as small as needed. We denote by $\operatorname{Bis}(\Sigma, U \subset V)$ the space of bisections defined over $U$ that can be extended to $V$.

We need to show that the topology induced on $\operatorname{Bis}(\Sigma, U \subset V)$ by the $D$ topology of $C^{\infty}(U, \Sigma)$ coincide with the $D$-topology of the subspace diffeology. We first do this for the larger space $\widehat{\operatorname{Bis}}(\Sigma, U \subset V)$ given by:

$\{\sigma: U \rightarrow \Sigma: s \circ \sigma, t \circ \sigma$ are diffeomorphisms, $\sigma$ can be extended to $V\}$.

Let $\sigma_{t}$ be a smooth path of maps $\sigma_{t}: U \rightarrow \Sigma$ such that $\sigma_{0} \in \widehat{\operatorname{Bis}}(\Sigma, U \subset V)$ and $\sigma_{0}$ can be extended to $V$. Then $\sigma_{t}$ can be extended to a smooth path of maps $V \rightarrow \Sigma$ for $t$ close to 0 . Since the path is smooth, this implies that for $t$ close to 0 , both $s \circ \sigma_{t}$ and $t \circ \sigma_{t}$ are a diffeomorphism onto their image (see [37], Lemma 1.3 in Chapter 2). Since the $D$-topology of a diffeology is characterized by curves in the sense of Proposition 3.C.19, this arguments shows that $\widehat{\operatorname{Bis}}(\Sigma, U \subset V)$ is open and that its $D$-topology coincide with the subspace topology as claimed before.

We now need to show that the topology induced by the inclusion of $\operatorname{Bis}(\Sigma, U \subset V)$ into $\widehat{\operatorname{Bis}}(\Sigma, U \subset V)$ is the same as the $D$-topology of the diffeology induced by the same inclusion. To show this, we observe that

$$
\widehat{\operatorname{Bis}}(\Sigma, U \subset V) \cong \operatorname{Emb}(U \subset V, \mathbf{X}) \times_{\mathbf{X}} \operatorname{Bis}(\Sigma, U \subset V),
$$

where $\operatorname{Emb}(U \subset V, \mathbf{X}) \times_{\mathbf{X}} \operatorname{Bis}(\Sigma, U \subset V)$ denotes

$$
\{(f, \gamma) \mid f \in \operatorname{Emb}(U \subset V, \mathbf{X}), \gamma \in \operatorname{Bis}(\Sigma, U \subset V), f(\operatorname{Dom}(f))=\operatorname{Dom}(\gamma)\},
$$


and $\operatorname{Emb}(U \subset V, \mathbf{X})$ is the space of diffeomorphisms onto their image defined on $U$ that can be extended to $V$. The bijection above sends $\sigma$ to $\left(s \circ \sigma, \sigma \circ(s \circ \sigma)^{-1}\right)$. The image of $\operatorname{Bis}(\Sigma, U \subset V)$ corresponds to the set of pairs where the first element is the restriction of the identity to $\operatorname{Dom}(\sigma)$. It is clear that a plot in $\widehat{\operatorname{Bis}}(\Sigma, U \subset V)$ with respect to the induced diffeology corresponds precisely to a plot in $\operatorname{Emb}(U \subset$ $V, \mathbf{X}) \times_{\mathbf{X}} \operatorname{Bis}(\Sigma, U \subset V)$ with respect to the diffeology induced by the product diffeology, i.e. the map above is smooth with smooth inverse. Consequently, it is a homeomorphism with respect to the $D$-topologies. If $O \subset \operatorname{Bis}(\Sigma, U)$ is open in the $D$-topology of the subspace diffeology, then $(\operatorname{Emb}(U \subset V, \mathbf{X}) \times O) \cap$ $\left(\operatorname{Emb}(U \subset V, \mathbf{X}) \times_{\mathbf{x}} \operatorname{Bis}(\Sigma, U \subset V)\right)$ is open in the induced topology, hence in the $D$-topology. It corresponds to an open $\hat{O}$ in $\widehat{\operatorname{Bis}}(\Sigma, U \subset V)$. By construction, the intersection of $\hat{O}$ with $\operatorname{Bis}(\Sigma, U \subset V)$ is precisely $O$.

Now, since the sequence $\left\{\gamma^{n}\right\}_{n \in \mathbb{N}}$ converges to $\gamma$ in the topology induced by the inclusion into $C^{\infty}(U, \Sigma)$, we now know it converges in the $D$-topology of the subspace diffeology. Since $\operatorname{Bis}(\Sigma, U \subset V)$ is a subspace of $\operatorname{Bis}(\Sigma, U)$, we know that $\gamma^{n} \rightarrow \gamma$ in the topology induced by the $D$-topology on $\operatorname{Bis}(\Sigma, U)$.

The claim is now proven by using that diffeologically smooth maps are continuous, Definition 3.5.3 and the fact that the D-topology on $C^{\infty}\left(\left.E\right|_{U},\left.E\right|_{U}\right)$ is finer than the compact open topology (again a consequence of Theorem 3.C.5 from [19]).

\subsubsection{Formal determinacy}

We can now address question 3 . Once more $\mathcal{G} \rightrightarrows \mathbf{X}$ is an étale groupoid acting on $\mu: E \mathbf{X} \rightarrow \mathbf{X}, \Sigma \rightrightarrows \mathbf{X}$ is a support for $\mathcal{G} \rightrightarrows \mathbf{X}$, cf. Definition 3.3.1, and $\left(\Sigma^{*}, \mathcal{C}^{*}\right)$ is a Cartan resolution of $\mathcal{G} \rightrightarrows \mathbf{X}$ over $\Sigma \rightrightarrows \mathbf{X}$, cf. Definition 3.4.5. We put ourselves in the setting of assumptions A1-A4, see the beginning of this section.

Since we assume $E$ to be Hausdorff (A3) and regular (A4), (in particular continuous, Definition 3.5.6), the following proposition is an immediate consequence of Proposition 3.4.27. We use the notion of formal determinacy from Definition 3.5.1.

Proposition 3.5.10. If $\operatorname{Haus}\left(\mathcal{G}, C_{\Sigma^{*}}^{\infty}\right) \cong \operatorname{Haus}(\mathcal{G}, \lim )$, then $\mathcal{G}$-spaces are formally determined (with respect to $\left(\Sigma^{*}, \mathrm{C}^{*}\right)$ ).

Consequently, Axiom 2 guarantees formal determinacy:

Theorem 3.5.11. Let $\mathcal{G} \rightrightarrows \mathbf{X}$ be an étale groupoid, $\Sigma \rightrightarrows \mathbf{X}$ be a support and $\left(\Sigma^{*}, \mathrm{C}^{*}\right)$ be a Cartan resolution over $\Sigma \rightrightarrows \mathbf{X}$. Let $E \rightarrow \mathbf{X}$ be a $\mathcal{G}$-space, satisfying assumptions A1-A4. Then $E$ is formally determined.

Remark 3.5.12. Let us reinterpret in our terms one of the main Lemmata from [54]:

Lemma 3.5.13. For any pair of disjoint closed subset $D^{0}$ and $D^{1}$ on $\mathbb{S}^{n-1}$, there exist a function $\varphi \in: \mathbb{R}^{n} \rightarrow \mathbb{R}$ such that

- $\varphi$ is everywhere smooth except at the origin; 
- for all $r^{\prime}$ s, for $x \rightarrow 0$ one has $D^{r} \varphi(x)=\mathcal{O}\left(|x|^{-r}\right)$ ( $D^{r}$ denotes the $r$-th order derivative of $\varphi$ );

- $\varphi$ restricted to $D_{i}$ is the constant $i(i \in\{0,1\})$;

- for $f \in C^{\infty}\left(\mathbb{R}^{n}\right)$ such that $[f]_{0}$ is flat, the function $\psi: x \rightarrow \varphi(x) f(x)$ is smooth and flat at 0 .

Via this lemma one constructs, for any diffeomorphism $\phi$ flat at $x$, a diffeomorphism $\psi$ defined around $x$ that coincides with $\phi$ on the radial saturation of some closed subset $D^{0} \subset \mathbb{S}^{n-1}$ and with the identity on the radial saturation of some closed subset $D^{1} \subset \mathbb{S}^{n-1}$. The existence of $\psi$ shows that the germ of $\phi$ is not separable from the germ of the identity in the étale topology. In other words, this Lemma shows that

$$
\operatorname{Haus}\left(\operatorname{Germ}\left(\Gamma^{n}\right), \text { germ }\right) \cong J^{\infty} \Gamma^{n} .
$$

In fact, proving $\operatorname{Haus}\left(\operatorname{Germ}\left(\Gamma^{n}\right)\right.$, germ $) \cong J^{\infty} \Gamma^{n}$ means precisely showing that flat elements (elements with the same infinite jet as the identity) are non separable from the identity. This immediately implies that the action on a $\Gamma^{n}$-space descends to $J^{\infty} \Gamma^{n}$ - the consequence of the Lemma that is used in [54].

\subsubsection{Pointwise finite order}

We move now to

Theorem 3.5.14. Let $E \rightarrow \mathbf{X}$ be a formally determined $\mathcal{G}$-space. Then, under certain connectedness assumptions on the resolution $\left(\Sigma^{*}, \mathrm{C}^{*}\right), E$ is pointwise of finite order, i.e.: for any $x \in \mathbf{X}$ and any $e_{x} \in E_{x}$ there exists $k_{e_{x}}$ such that $G^{k_{e_{x}}}$ lies in the stabilizer of $e_{x}$.

We will clarify later on the "connectedness assumptions" that we need (see the statement of Theorem 3.5.19). The proof that we start presenting follows conceptually [54], and makes explicit use of Axiom 2. Via this Axiom, one can prove an infinitesimal version of Theorem 3.5.14, and the additional connectedness assumptions are used to pass to the global result via the exponential map. Throughout the argument, we implement ideas from [70] which are fundamental for the discussion.

We begin by pointing out where Axiom 2 comes into play.

Lemma 3.5.15. Let $E \rightarrow \mathbf{X}$ be a formally determined $\mathcal{G}$-space such that the assumptions $\mathrm{A} 1-\mathrm{A} 4$ are satisfied. Then the action of $j_{\Sigma^{\infty}}(\mathcal{G})$ on $E$ is continuous in the vertical limit topology.

Remark 3.5.16. There is a slight abuse of notation in the statement above, since we defined the vertical limit topology as a topology on $\mathcal{G}$ pulled back from one on $\Sigma^{\infty}$, Definition 3.4.20. Here, we are using the same name for the topology on $\Sigma^{\infty}$ itself.

Proof. $\mathcal{G}$ acts continuously in the vertical limit topology thanks to Axiom 2. Consequently, a continuous action of the Hausdorff quotient $\operatorname{Haus}(\mathcal{G}, \operatorname{vlim})$ is induced. But $j_{\Sigma^{\infty}}(\mathcal{G})$ with the vertical limit topology is homeomorphic to $\operatorname{Haus}(\mathcal{G}, \operatorname{vlim})$ (Proposition 3.4.27) and the action of $j_{\Sigma^{\infty}}(\mathcal{G})$ corresponds, under this homeomorphism, to the continuous action of $\operatorname{Haus}(\mathcal{G}$, vlim). 
We denote by $Z_{e_{x}}$ the isotropy group of $\Sigma^{\infty} \ltimes E$ at $e_{x} \in E_{x}$ and by $\mathfrak{z}_{e_{x}}$ its Lie algebra. In what follows, the notation is from Subsection 3.4.5.

Lemma 3.5.17. The Lie algebra $\mathfrak{z} e_{x}$ has finite codimension in $\mathfrak{g}_{x}^{0}$.

Proof. Let $\alpha_{x} \in \mathfrak{g}_{x}^{0}(\mathcal{A})$, where $\mathcal{A}=\operatorname{Lie}(\mathcal{G})$. Any representative (denoted by $\alpha$ ) can be written as the time 0 derivative of a flow of bisections $\phi_{\alpha}^{t}$ of $\mathcal{G}$, seen as bisections of $\Sigma$. This induces (via the action map and using Definition 3.5.3) a smooth flow on $(E \mathbf{X})_{x}$ whose derivative at time 0 is a well defined tangent vector field at $e_{x}$, denoted by $a(\alpha)$. We end up having an infinitesimal action map defined from sections of $\mathcal{A}$ around $x$ that vanish at $x$ (i.e., such that their germ at $x$ belongs to $\mathfrak{g}_{x}^{0}(\mathcal{A})$ ) to local vector fields around $e_{x}$ in $(E \mathbf{X})_{x}$. This map respects the Lie brackets, because the action map sends products of bisections to compositions of diffeomorphisms of $E \mathbf{X}$. Now, by formal determinacy and passing to germs, we get a Lie algebra map defined from $\mathfrak{g}_{x}^{0}$ to germs of vector fields around $e_{x}$. The isotropy $\mathfrak{z} e_{x}$ is precisely the kernel of such a map. Since $(E \mathbf{X})_{x}$ is finite dimensional, this concludes the proof.

Since $\mathfrak{z}_{e_{x}}$ has finite codimension, there exists $k_{e_{x}} \in \mathbb{N}$ such that $\mathfrak{z}_{e_{x}}+\mathfrak{g}_{x}^{k_{e_{x}}}=$ $\mathfrak{z} e_{x}+\mathfrak{g}_{x}^{l}$ for all $l \geq k_{e_{x}}$.

Lemma 3.5.18. It holds $\mathfrak{g}_{x}^{k_{e x}} \subset \mathfrak{z} e_{x}$

Proof. The infinitesimal action map used in the proof of Lemma 3.5.17 descends to $j_{A^{\infty}}(\operatorname{Germ}(\mathcal{A}))$ by formal determinacy. Applying it to the equality

$$
\mathfrak{z}_{e_{x}}+\mathfrak{g}_{x}^{k_{e_{x}}}=\mathfrak{z} e_{x}+\mathfrak{g}_{x}^{l}, \quad l \geq k_{e_{x}},
$$

obtained above, one gets

$$
a\left(\mathfrak{g}_{x}^{k_{i}}\right)=a\left(\mathfrak{g}_{x}^{k_{i+1}}\right), \quad i \geq k_{e_{x}} .
$$

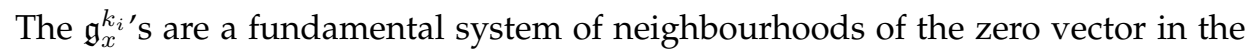
vertical limit topology; since the action on $E \mathbf{X}$ is continuous in the vertical limit topology, the claim follows.

The lemma above provides an infinitesimal version of Theorem 3.5.14. To get the global version, it is necessary to add some connectedness assumtpions to the groups $G^{i \prime}$ s (see the notation from Subsection 3.4.5). This issue has been studied in the literature in great generality, for inverse systems of Lie groups. In [28] the groups $G_{x}^{i}$ 's were assumed to be connected; this was mildly relaxed in [70], where the result is proven assuming the quotients $G_{x}^{i} / G_{x}^{i+1}$ to be connected. The complete statement of Theorem 3.5.14 is then

Theorem 3.5.19. If the Cartan tower $\left(\Sigma^{*}, \mathrm{C}^{*}\right)$ is such that, for each $i \in \mathbb{N}$ and $x \in \mathbf{X}$, $G_{x}^{i} / G_{x}^{i+1}$ is connected, then for any $e_{x} \in E \mathbf{X}$ there exists $k_{e_{x}}$ such that $G^{k_{e_{x}}}$ lies in the stabilizer of $e_{x}$.

Proof. We choose $k_{e_{x}}$ to be the integer obtained from the previous three lemmata (i.e. the finite order of the infinitesimal action at $e_{x}$ ). Recall that $Z_{e_{x}}$ denotes the stabilizer at $e_{x}$ for the action of $\Sigma^{\infty}$; we aim at showing $G^{k_{e_{x}}} \subset Z_{e_{x}}$. Let 
be $i \geq k_{e_{x}}$. Recall that we have an exponential map $\exp ^{\infty}: A^{\infty}=\operatorname{Lie}\left(\Sigma^{\infty}\right) \rightarrow$ $\Sigma^{\infty}$. We keep writing $\exp ^{\infty}$ to denote the restriction $\mathfrak{g}_{x}^{0} \rightarrow G_{x}^{0}$ which is the limit of the exponential maps for the finite dimensional Lie groups $G_{x}^{0}\left(\Sigma^{k}\right)$. One has $\exp \left(\mathfrak{g}_{x}^{i}\left(A^{i+1}\right)\right) \subset G_{x}^{i}\left(\Sigma^{i+1}\right)$ (since on $\mathfrak{g}_{x}^{i}\left(A^{i+1}\right)$ the map exp is by definition the usual exponential map). But we have $G_{x}^{i}\left(\Sigma^{i+1}\right) \cong G_{x}^{i} / G_{x}^{i+1}$, i.e. $G_{x}^{i}\left(\Sigma^{i+1}\right)$ is connected. Consequently, since its Lie algebra $\mathfrak{g}^{i}\left(A^{i+1}\right)$ is nilpotent, $G_{x}^{i}\left(\Sigma^{i+1}\right)$ is nilpotent as well and we have

$$
\exp \left(\mathfrak{g}_{x}^{i}\left(A^{i+1}\right)\right)=G_{x}^{i}\left(\Sigma^{i+1}\right)
$$

On the other hand, $\mathfrak{g}_{x}^{i}\left(A^{i+1}\right) \subset g_{x}^{i} \subset \mathfrak{z} e_{x}$. It is clear that $\exp \left(\mathfrak{z} e_{x}\right) \subset Z_{e_{x}}$; this concludes the proof because $G_{x}^{k_{e_{x}}}=\bigcup_{i \geq k_{e_{x}}} G_{x}^{i}\left(\Sigma^{i+1}\right)$.

Remark 3.5.20. In $[54,65]$, Axiom 2 for $\Gamma^{n}=\operatorname{Diff}_{\text {loc }}\left(\mathbb{R}^{n}\right)$ is stated as a lemma in the following terms:

Lemma 3.5.21. If $\phi: \mathbb{R}^{n} \rightarrow \mathbb{R}^{n}$ is a diffeomorphism such that $j_{0}^{k} \phi \simeq j_{0}^{k}$ id then, for any $\varepsilon>0$ there exists a diffeomorphism $\psi: \mathbb{R}^{n} \rightarrow \mathbb{R}^{n}$ such that $\operatorname{germ}_{0}(\phi) \simeq \operatorname{germ}_{0}(\psi)$ and $\left\|D^{\alpha}(\psi-i d)\right\| \leq \epsilon$ for all multi-indices $\alpha$ such that $|\alpha| \leq k$ (i.e., $\psi$ and $i d$ are close in the $C^{k}$-topology).

The connectedness assumptions in Theorem 3.5.19 are satisfied as well. Actually, in [54] the authors also prove that the isotropy Lie algebra is closed in the vertical limit topology, which, combined with Lemma 3.5.17, provides the infinitesimal version of Theorem 3.5.14. This makes use of the fact that the canonical Cartan tower of $\Gamma^{n}=\operatorname{Diff}_{\text {loc }}\left(\mathbb{R}^{n}\right)$ is a tight resolution.

\subsubsection{Bounding the order}

To prove that $\mathcal{G}$-spaces of pointwise finite order are actually of finite order, one needs to find a bound for the $k_{e_{x}}$ 's constructed above. Differently from what we did so far, finding such a bound seems to be a more example-specific task. In the case of $\Gamma^{n}$, the bound found in [54] is not sharp. A sharp bound for the order was provided in [28] (this was one of the main significant differences from [54] - the other one being the removal of the assumption of smoothness for natural bundles). In [70] the upper bound was achieved in greater generality, under certian assumptions on the tower of Lie algebras $\mathfrak{g}_{x}^{i}$ (the notation is from Subsection 3.4.5). There is quite some literature about generalizing the techniques from [70] and achieving sharp bounds; see, for example [40, 61, 71]. For the sake of completeness we give the result from [70] below (Theorem 3.5.22).

Again, $\mathcal{G} \rightrightarrows \mathbf{X}$ is an étale groupoid acting on $\mu: E \rightarrow \mathbf{X}, \Sigma \rightrightarrows \mathbf{X}$ is a support for $\mathcal{G} \rightrightarrows \mathbf{X}$, cf. Definition 3.3.1, and $\left(\Sigma^{*}, \mathrm{e}^{*}\right)$ is a Cartan resolution of $\mathcal{G} \rightrightarrows \mathbf{X}$ over $\Sigma \rightrightarrows \mathbf{X}$, cf. Definition 3.4.5. We put ourselves in the setting of

- assumptions A1-A4, see the beginning of this section;

- Theorem 3.5.19, i.e. for each $i \in \mathbb{N}$ and $x \in \mathbf{X}, G_{x}^{i} / G_{x}^{i+1}$ is connected.

Let $y \in \mu^{-1}(x)$. Let $\mathfrak{z}_{y} \subset \mathfrak{g}_{x}\left(A^{\infty}\right)$ be the isotropy algebra of the action (of $\Sigma^{\infty}$ ) at $y$. We can assume $E$ to be pointwise of finite order as in the previous subsection, so 
there exists a $k_{y}$ such that $\mathfrak{g}_{x}^{k_{y}} \subset \mathfrak{z}_{y}$. We can chose $k_{y}$ to be the minimum natural number with this property. Notice that $\mathfrak{z}_{y} / \mathfrak{g}_{x}^{k_{y}} \subset \mathfrak{g}_{x}\left(A^{k_{y}}\right)$; we denote by $\mathfrak{h}_{y}$ the quotient $\mathfrak{z}_{y} / \mathfrak{g}_{x}^{k_{y}}$. Notice that the (map induced by the) infinitesimal action map $a: \mathfrak{g}(\mathcal{A}) \rightarrow T_{y} E_{x}$ from Lemma 3.5.17 factors through a map

$$
\mathfrak{g}_{x}\left(A^{k_{y}}\right) \rightarrow T_{y} E_{x}
$$

whose kernel is $\mathfrak{h}_{y}$. Consequently, if we denote by $c_{y}$ the codimension of $\mathfrak{h}_{y}$ in $\mathfrak{g}_{x}\left(A^{k_{y}}\right)$, we have

$$
\operatorname{dim}\left(E_{x}\right) \geq c_{y}
$$

To obtain bounds on the order $k_{y}$, one needs to work with the grading from Proposition 3.4.42, e.g. as in the following theorem. The assumptions and the argument are those of $[61,70]$.

Theorem 3.5.22. Assume that, for all $x \in M$ and $i, j \in \mathbb{N}, i \neq j$, one has

$$
\left[\mathfrak{g}_{x}^{i}, \mathfrak{g}_{x}^{j}\right]=\mathfrak{g}_{x}^{i+j} .
$$

Then, for any $x \in M$ and $y \in E_{x}, k_{y} \leq 2 \operatorname{dim}\left(E_{x}\right)+1$.

Proof. First, we observe that the isotropy Lie algebra $\mathfrak{g}\left(A^{k_{y}}\right)$ is indeed filtered

$$
\mathfrak{g}\left(A^{k_{y}}\right) \supset \mathfrak{g}^{0}\left(A^{k_{y}}\right) \supset \mathfrak{g}^{1}\left(A^{k_{y}}\right) \cdots \supset \mathfrak{g}^{k_{y}}\left(A^{k_{y}}\right)=0
$$

and admits the grading

$$
\mathfrak{g}\left(A^{k_{y}}\right)=\bigoplus_{i=-1}^{k_{y}-1} \mathfrak{g}^{i}\left(A^{i+1}\right) ;
$$

where we set $\mathfrak{g}^{-1}\left(A^{0}\right)=\mathfrak{g}\left(A^{0}\right)$, the isotropy Lie algebra of $A^{0}$. This grading is precisely the grading associated to the filtration above since

$$
\mathfrak{g}^{i}\left(A^{i+1}\right) \cong \mathfrak{g}^{i}\left(A^{k_{y}}\right) / \mathfrak{g}^{i+1}\left(A^{k_{y}}\right) .
$$

By intersecting $\mathfrak{h}_{y}$ with the filtration of $\mathfrak{g}\left(A^{k_{y}}\right) \supset \mathfrak{h}_{y}$ we get a filtration

$$
\mathfrak{h}_{y} \supset \mathfrak{h}_{y}^{0} \supset \mathfrak{h}_{y}^{1} \cdots \supset \mathfrak{h}_{y}^{k_{y}-1} \supset 0
$$

where $\mathfrak{h}_{y}^{i}=\mathfrak{h}_{y} \cap \mathfrak{g}^{i}\left(A^{k_{y}}\right)$; notice that $\mathfrak{h}_{y}^{i} / \mathfrak{h}_{y}^{i+1} \subset \mathfrak{g}^{i}\left(A^{k_{y}}\right) / \mathfrak{g}^{i+1}\left(A^{k_{y}}\right)$. We have the corresponding graded algebra

$$
\operatorname{gr}\left(\mathfrak{h}_{y}\right)=\bigoplus_{i=-1}^{k_{y}-1} \mathfrak{h}_{y}^{i} / \mathfrak{h}_{y}^{i+1}
$$

Since $k_{y}$ is minimal such that $\mathfrak{g}_{x}^{k_{y}}\left(A^{\infty}\right) \subset \mathfrak{z}_{y}$, it follows that $\mathfrak{h}_{y}^{k_{y}-1} / \mathfrak{h}_{y}^{k_{y}}$ is strictly contained in $\mathfrak{g}^{k_{y}-1}\left(A^{k_{y}}\right)$. Consider the quotient projection

$$
\mathfrak{g}^{k_{y}-1}\left(A^{k_{y}}\right) \rightarrow\left(\mathfrak{g}^{k_{y}-1}\left(A^{k_{y}}\right)\right) /\left(\mathfrak{h}_{y}^{k_{y}-1} / \mathfrak{h}_{y}^{k_{y}}\right)
$$

and fix a basis $a$ belonging to the right hand side. 
For each pair $p, q$ such that $p+q=k_{y}$, we have that

$$
\left[\mathfrak{g}_{x}^{p}, \mathfrak{g}_{x}^{q}\right]=\mathfrak{g}_{x}^{k_{y}}
$$

because of condition (3.6). As a consequence, there are $a^{p} \in \mathfrak{g}_{x}^{p} / \mathfrak{g}_{x}^{p+1}, a^{q} \in \mathfrak{g}_{x}^{q} / \mathfrak{g}_{x}^{q+1}$ such that $\left[a^{p}, a^{q}\right]=a$. Since $a$ belongs to $\left(\mathfrak{g}^{k_{y}-1}\left(A^{k_{y}}\right)\right) /\left(\mathfrak{h}_{y}^{k_{y}-1} / \mathfrak{h}_{y}^{k}\right)$, then either $a^{p}$ does not belong to $\mathfrak{h}_{y}^{p} / \mathfrak{h}_{y}^{p+1}$ or $a^{q}$ does not belong to $\mathfrak{h}_{y}^{q} / \mathfrak{h}_{y}^{q+1}$.

However

$$
c_{y}=\sum_{i=-1}^{k_{y}-1} \operatorname{codim}\left(\mathfrak{h}_{y}^{i} / \mathfrak{h}_{y}^{i+1}\right) ;
$$

thanks to the argument above, by counting non ordered pairs $p, q$ such that $p \neq q$ and $p+q=k_{y}$ we have

$$
\sum_{i=-1}^{k_{y}-1} \operatorname{codim}\left(\mathfrak{h}_{y}^{i} / \mathfrak{h}_{y}^{i+1}\right) \geq \frac{1}{2}\left(k_{y}-1\right)
$$

and this concludes the proof.

Corollary 3.5.23. If the assumptions of the previous theorem are satisfied, then any pointwise finite order $\mathcal{G}$-space $E$ is of finite order lower or equal than $2 \operatorname{dim}\left(E_{x}\right)+1$.

Remark 3.5.24. The algebraic assumption (3.6) comes from the case $\mathcal{G}=$ $\operatorname{Germ}\left(\Gamma^{n}\right), \Gamma^{n}=\operatorname{Diff}_{\text {loc }}\left(\mathbb{R}^{n}\right)$, where it indeed holds, as we argue below. It seems clear to us that it is not the only possible algebraic assumption on $\mathfrak{a}_{x}$ ensuring the existence of a bound. However, as anticipated earlier, we are convinced that bounding the pointwise finite order is an example specific task.

The assumptions in Theorem 3.5.22 are satisfied by the canonical Cartan tower of the pseudogroup $\Gamma^{n}=\operatorname{Diff}_{\text {loc }}\left(\mathbb{R}^{n}\right)$. We spell out a proof below, see $[28,40,54$, 61].

To begin with, notice that $\operatorname{Lie}\left(\Gamma^{n}\right)=X_{\mathbb{R}^{n}}$, the sheaf of vector fields on $\mathbb{R}^{n}$, whose canonical Spencer tower is given by the jet tower

$$
\cdots \rightarrow J^{k+1} T \mathbb{R}^{n} \rightarrow J^{k} T \mathbb{R}^{n} \rightarrow \cdots \rightarrow T \mathbb{R}^{n} \rightarrow \mathbb{R}^{n} .
$$

In this setting the anchor $J^{k} T \mathbb{R}^{n} \rightarrow T \mathbb{R}^{n}$ is given by the map

$$
j_{x}^{k} X \in J^{k} T \mathbb{R}^{n} \in \rightarrow X_{x} \in T \mathbb{R}^{n} .
$$

The formal algebra at $x \in \mathbb{R}^{n}$

$$
\mathfrak{a}_{x}=J_{x}^{\infty} T \mathbb{R}^{n}
$$

is then isomorphic to the Lie algebra $\mathfrak{a}^{n}$ of formal vector fields on $\mathbb{R}^{n}$. Explicitly, a formal vector field is an expression

$$
\sum_{j=1}^{n} f^{j} \frac{\partial}{\partial x^{j}}
$$


where $x_{1}, \ldots x_{n}$ are coordinates on $\mathbb{R}^{n}$ and the $f^{j}$ 's are formal power series in the variables $x_{1}, \ldots, x_{n}$. The Lie bracket on $\mathfrak{a}_{x}$ can be identified with the Lie bracket on $\mathfrak{a}^{n}$

$$
\left[\sum_{j=1}^{n} f^{j} \frac{\partial}{\partial x^{j}}, \sum_{j=1}^{n} g^{j} \frac{\partial}{\partial x^{j}}\right]=\sum_{j=1}^{n} \sum_{l=1}^{n}\left(f^{l} \frac{\partial}{\partial x^{l}} g^{j}-g^{l} \frac{\partial}{\partial x^{l}} f^{j}\right)
$$

for each pair of formal vector fields $\sum_{j=1}^{n} f^{j} \frac{\partial}{\partial x^{j}}$ and $\sum_{l=1}^{n} g^{l} \frac{\partial}{\partial x^{l}}$. It follows that, for each $i \in \mathbb{N}$,

$$
\mathfrak{g}^{i}=\operatorname{Ker}\left(J^{\infty} T \mathbb{R}^{n} \rightarrow J^{i} T \mathbb{R}^{n}\right)
$$

can be identified with the subalgebra $\mathfrak{a}^{n, i}$ of formal vector fields whose coefficients have degree strictly higher than $i$, i.e. expressions of the form

$$
\sum_{j=1}^{n} h^{j} \frac{\partial}{\partial x^{j}}
$$

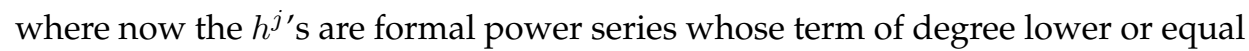
than $i$ have vanishing coefficients. Finally,

$$
\mathfrak{g}^{i}\left(J^{i+1} T \mathbb{R}^{n}\right)=\operatorname{Ker}\left(J^{i+1} T \mathbb{R}^{n} \rightarrow J^{i} T \mathbb{R}^{n}\right)
$$

is identified with the space $\mathfrak{p}^{i+1}$ of homogeneous polynomial vector fields of degree $i+1$, i.e. expressions of the form

$$
\sum_{j=1}^{n} p^{j} \frac{\partial}{\partial x^{j}}
$$

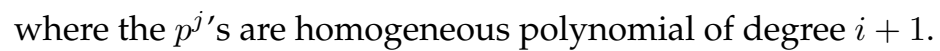

Lemma 3.5.25. It holds

$$
\left[\mathfrak{a}^{n, i}, \mathfrak{a}^{n, j}\right]=\mathfrak{a}^{n, i+j}
$$

for all $i \in \mathbb{N}, j \in \mathbb{N}, i \neq j$.

Proof. The argument that follows is the one from [61]. We make use of the grading

$$
\mathfrak{a}^{n, 0}=\bigoplus_{i=1}^{\infty} \mathfrak{p}^{i}
$$

We will prove

$$
\left[\mathfrak{p}^{i}, \mathfrak{p}^{j}\right]=\mathfrak{p}^{i+j-1}
$$

which implies the claim.

We use multi-indices denoted by capital latin letters and having the form

$$
A=\left(a_{1}, \ldots, a_{n}\right)
$$

where $a_{l} \in \mathbb{N}$; we use $|A|$ to denote $\sum_{l=1}^{n} a_{l}$. Sum of such multi-indices is defined term by term, i.e.

$$
A+B=\left(a_{1}+b_{1}, \ldots, a_{n}+b_{n}\right)
$$


moreover, when writing $1_{l}$ we mean the multi-index

$$
1_{i}=(0, \ldots, 0,1,0, \ldots 0)
$$

where the only non zero entry is the l-th one. The expression $x^{A}$ denotes $x_{1}^{a_{1}}$. $x_{2}^{a_{2}} \ldots x_{n}^{a_{n}}$, which has degree $|A|$.

Now let

$$
x^{C} \frac{\partial}{\partial x^{l}} \in \mathfrak{p}^{i+j-1}, \quad 1 \leq l \leq n,
$$

i.e. $|C|=i+j-1$. If we manage to show that $x^{C} \frac{\partial}{\partial x^{l}} \in\left[\mathfrak{p}^{i}, \mathfrak{p}^{j}\right]$, then we are done, because an element of this form are a basis of $\mathfrak{p}^{i+j-1}$. Observe that the formula

$$
\left[x^{A} \frac{\partial}{\partial x^{l}}, x^{B} \frac{\partial}{\partial x^{l}}\right]=\left(b_{i}-a_{i}\right) x^{A+B-1_{l}} \frac{\partial}{\partial x^{l}}
$$

holds. We are then lead to looking for multi-indices $A, B$ solving

$$
A+B-1_{l}=C
$$

under the constraints $|A|=i,|B|=j$, and $a_{l} \neq b_{l}$.

There are two cases:

- if $i=j$ then a solution might fail to exist;

- if $i \neq j$ a solution always exists.

The first point is seen easily already by taking $n=1$ and $i=j=1$. As for the second point, notice that

$$
a_{l}+b_{l}-1=c_{l}
$$

can be solved for each $c_{l} \in \mathbb{N}$ with $a_{l} \neq b_{l}$, provided $i \neq j$ (if $i=j$, then $c_{l}=$ $i+j-1$ forces $a_{l}=b_{l}$ ). Then, since

$$
\sum_{m=1}^{n} c_{m}=i+j-1
$$

the other entries of $A, B$ can be arranged in such a way that

$$
\sum_{m=1}^{n} a_{m}=i, \quad \sum_{m=1}^{n} b_{m}=j
$$

i.e. $|A|=i,|B|=j$.

Remark 3.5.26. In [54], the argument used to bound the "pointwise finite order" of $\Gamma^{n}=\operatorname{Diff}_{\text {loc }}\left(\mathbb{R}^{n}\right)$-spaces was phrased in a slightly different way, and relied on the observation that a diffeomorphism $\varphi$ of $\mathbb{R}$ induces the diffeomorphism

$$
\left(x_{1}, x_{2}, \ldots, x_{n}\right) \rightarrow\left(\varphi\left(x_{1}\right), x_{2}, \ldots, x_{n}\right)
$$

of $\mathbb{R}^{n}$. This induces a map

$$
\iota:\left(J^{*} \Gamma^{1}, \mathcal{C}^{*}\right) \rightarrow\left(J^{*} \Gamma^{n}, \mathcal{C}^{*}\right)
$$

The authors make use of this by proving the following 
Lemma 3.5.27. The normal subgroup of $G_{x}\left(J^{\infty} \Gamma^{n}\right)$ generated by $\iota\left(G_{x}^{k}\left(J^{\infty} \Gamma^{1}\right)\right)$ is $G_{x}^{k}\left(J^{\infty} \Gamma^{n}\right)$

This reduces the problem of bounding the order to base dimension 1 , where the authors argue by a direct computation. In fact, if $E \mathbb{R}^{n} \rightarrow \mathbb{R}^{n}$ is a $\Gamma^{n}$-space, the pullback via the inclusion

$$
x \rightarrow(x, 0, \ldots, 0)
$$

is a $\Gamma^{1}$-space. Consequently, if for some $k$ the image $\iota\left(G_{x}^{k}\left(J^{\infty} \Gamma^{1}\right)\right) \subset G_{x}^{k}\left(J^{\infty} \Gamma^{n}\right)$ stabilizes the fiber over $x \in \mathbf{X}$ of $E \mathbb{R}^{n}$ then, by the Lemma above, the same is true for $G_{x}^{k}\left(J^{\infty} \Gamma^{n}\right)$.

Example 3.5.28. Theorem 3.5.22 can be applied to the pseudogroup $\Gamma^{n, k}$ of locally defined diffeomorphisms of $\mathbb{R}^{n+k}$ projectable over $\mathbb{R}^{n}$. This was shown in [61].

\section{Appendix}

\section{A Some words on profinite dimensional differen- tial geometry}

This appendix is a subset of the appendix of [1], where pf-differential geometry is discussed more thoroughly. Here we present only the concepts that are relevant for the finite order problem. The material is well known in the community working with geometry of PDE, but there is not much background material written with a level of generality that suits us (at least not that we are aware of). A reference in this direction is [33]; however, our purposes need certain definitions to be different.

We point out that an important difference with the more complete appendix in [1] is that we do not restrict to normal pf-atlases, but only to atlases corresponding to an injection into the projective limit of a tower (Remark 3.A.2); this has some minor consequences when dealing with derivation functors, in particular when talking about tangent spaces and Lie algebroids associated to Lie groupoids.

\section{A.1 Pf manifolds}

A pro-finite manifold or profinite dimensional manifold is, roughly speaking, an infinite dimensional manifold equipped with a "tower" of finite dimensional manifolds. The tower makes the infinite dimensionality of a pf-manifold quite mild, since most of the classical differential geometry can be explicitely adapted. The basic example is given by the infinite jet space $J^{\infty}(M, N)$, where $M, N$ are finite dimensional manifolds. It is equipped with the tower

$$
\cdots \rightarrow J^{k}(M, N) \rightarrow J^{k-1}(M, N) \rightarrow \ldots J^{0}(M, N)
$$

Definition 3.A.1. A tower of manifolds $\mathbb{M}_{\bullet}$ is a sequence

$$
\mathbb{M}_{\bullet}: \quad \ldots \longrightarrow \mathbb{M}_{k} \stackrel{\pi_{k}}{\longrightarrow} \mathbb{M}_{k-1} \longrightarrow \ldots \longrightarrow \mathbb{M}_{2} \stackrel{\pi_{2}}{\longrightarrow} \mathbb{M}_{1} \stackrel{\pi_{1}}{\longrightarrow} \mathbb{M}_{0}
$$


consisting of smooth, finite dimensional manifolds $\mathbb{M}_{k}$ and surjective submersions between them.

A pf-atlas on a set $\mathbb{M}$, denoted by

$$
a: \mathbb{M} \rightarrow \mathbb{M}_{\bullet},
$$

is a tower of manifolds $\mathbb{M}$. together with a collection $a=\left\{a_{k}: \mathbb{M} \rightarrow \mathbb{M}_{k}\right\}$ of surjections compatible with the tower projections $\left(\pi_{k} \circ a_{k}=a_{k-1}\right)$.

Remark 3.A.2. A pf-atlas assigns to any $x \in \mathbb{M}$ "coordinates"

$$
x_{k}=a_{k}(x) \in \mathbb{M}_{k} .
$$

given by "truncating" $x$ at step $k$. They satisfy

$$
x_{k-1}=\pi_{k}\left(x_{k}\right) \text { for all } k \text {. }
$$

This means that if we consider the inverse limit

$$
\lim _{\longleftarrow} \mathbb{M}_{k}:=\left\{\left(x_{k}\right)_{k \geq 0}: x_{k} \in \mathbb{M}_{k}, \pi_{k}\left(x_{k}\right)=x_{k-1}\right\}
$$

a pf-atlas $a$ can be seen as a map

$$
a: \mathbb{M} \rightarrow \underset{k}{\lim } \mathbb{M}_{k}
$$

This map may fail to be surjective or injective, so the analogy with the usual notion of atlas should not be pushed too far.

There are natural examples where surjectivity does not hold. A standard one is given by the canonical Cartan tower of the pseudogroup $\Gamma_{\text {an }}^{n}$ of analytic diffeomorphisms of $\mathbb{R}^{n}$.

Definition 3.A.3. A pf-atlas $a: \mathbb{M} \rightarrow \mathbb{M} \bullet$ is said to be normal if the induced map $\mathbb{M} \rightarrow \underset{\leftarrow}{\lim } \mathbb{M}_{k}$ is a bijection.

Definition 3.A.4. Consider $a: \mathbb{M} \rightarrow \mathbb{M} \bullet$ and $b: \mathbb{N} \rightarrow \mathbb{N}$ • be two pf-atlases. We say that a set theoretical function $f: \mathbb{M} \rightarrow \mathbb{N}$ is a smooth map w.r.t. $a$ and $b$, or that $f:(\mathbb{M}, a) \rightarrow(\mathbb{N}, b)$ is a smooth map of pf-atlases if, for each $k$, the $k$-th component

$$
b_{k} \circ f: \mathbb{M} \rightarrow \mathbb{N}_{k}
$$

comes from a smooth map defined on $\mathbb{M}_{m_{k}}$ for $m_{k}$ large enough:

$$
b_{k} \circ f=f_{k}^{m_{k}} \circ a_{m_{k}}, \quad \text { with } f_{k}^{m_{k}} \in C^{\infty}\left(\mathbb{M}_{m_{k}}, \mathbb{N}_{k}\right) .
$$

Remark 3.A.5. Observe that if $f: \mathbb{M} \rightarrow \mathbb{N}$ is smooth w.r.t. atlases $a, b$ then the collection

$$
f_{\bullet}=\left\{f_{k}^{m_{k}}: \mathbb{M}_{m_{k}} \rightarrow \mathbb{N}_{k}\right\}_{k \geq 1} \quad \text { with } m_{1} \leq m_{2} \leq \ldots
$$

is compatible in the sense that

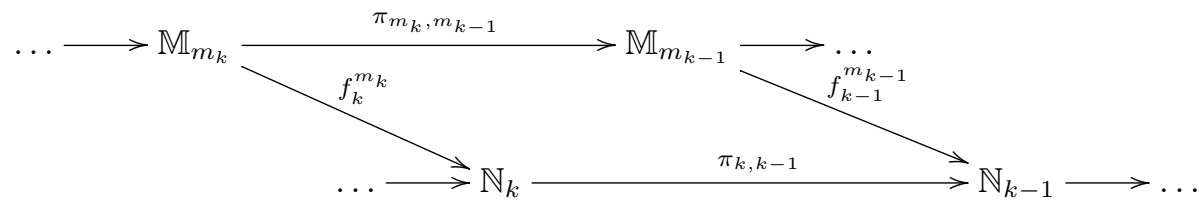


is commutative. In fact, the definition of smooth map $f: \mathbb{M} \rightarrow \mathbb{N}$ with respect to $a, b$ can be reformulated by requiring that there exists a compatible $f$ such that

$$
b_{k} \circ f=f_{k} \circ a_{m_{k}}, \quad \text { with } f_{k} \in C^{\infty}\left(\mathbb{M}_{m_{k}}, \mathbb{N}_{k}\right) .
$$

We declare two collections $f_{\bullet}, g_{\bullet}$ to be equivalent if, for any $k, f_{k}$ is equal to $g_{k}$ after composition with a projection $\mathbb{M}_{K} \rightarrow \mathbb{M}_{k}$, for $K$ big enough. It is not hard to see that if we have a map $f: \mathbb{M} \rightarrow \mathbb{N}$ and a compatible collection $f \bullet$ such that

$$
b_{k} \circ f=f_{k} \circ a_{m_{k}}, \quad \text { with } f_{k} \in C^{\infty}\left(\mathbb{M}_{m_{k}}, \mathbb{N}_{k}\right) .
$$

then the same happens (possibly with different $m_{k}$ 's) for any compatible collection $g_{\bullet}$ equivalent to $f_{\bullet}$. In conclusion $f: \mathbb{M} \rightarrow \mathbb{N}$ is smooth with respect to two atlases $a, b$ if and only if it induces an equivalence class of compatible collections

$$
f_{\bullet}=\left\{f_{k}: \mathbb{M}_{m_{k}} \rightarrow \mathbb{N}_{k}\right\}_{k \geq 1} \quad \text { with } m_{1} \leq m_{2} \leq \ldots
$$

or, in more compact terms, an element of

$$
\operatorname{Hom}\left(\mathbb{M}_{\bullet}, \mathbb{N}_{\bullet}\right):=\underbrace{\lim }_{m} \underset{n}{\lim } C^{\infty}\left(\mathbb{M}_{m}, \mathbb{N}_{n}\right) \text {. }
$$

Similar to the previous discussion about coordinates, two different $f^{\prime}$ 's may induce the same element of $\operatorname{Hom}\left(\mathbb{M}_{\bullet}, \mathbb{N}_{\bullet}\right)$, and there are elements of $\operatorname{Hom}\left(\mathbb{M}_{\bullet}, \mathbb{N}_{\bullet}\right)$ that are not induced by an $f$. Normality of the atlases rules out both these options.

Remark 3.A.6. The definition of $\operatorname{Hom}\left(\mathbb{M}_{\bullet}, \mathbb{N}_{\bullet}\right)$ above comes from seeing towers as particular instances of projective system of manifolds. An element of $\operatorname{Hom}\left(\mathbb{M}_{\bullet}, \mathbb{N}_{\bullet}\right)$ is a morphism of towers; it is straightforward to define composition of morphism of towers and to show that one gets a category of towers.

Because of the examples that we have in mind, we will restrict to the case where pf-manifolds admit atlases such that the map $a: \mathbb{M} \rightarrow \underset{k}{\lim } \mathbb{M}_{k}$ is injective; notice that this can always be achieved replacing $\mathbb{M}$ with $a(\mathbb{M}) \stackrel{\lim _{k}}{\subset} \mathbb{M}_{k}$.

Definition 3.A.7. A pf-manifold is a set $\mathbb{M}$ together with an equivalence class of pf-atlases, where two pf-atlases

$$
a: \mathbb{M} \rightarrow \mathbb{M}_{\bullet}, \quad a^{\prime}: \mathbb{M} \rightarrow \mathbb{M}_{\bullet}^{\prime}
$$

are said to be equivalent if the identity map id $\mathrm{d}_{\mathbb{M}}$ is a smooth map of pf-atlases (in the sense of Definition 3.A.4) both as a map from (M, $a)$ to $\left(\mathbb{M}^{\prime}, a^{\prime}\right)$ and as a map from $\left(\mathbb{M}^{\prime}, a^{\prime}\right)$ to $(\mathbb{M}, a)$. Equivalently, we require the existence of an isomorphism of towers

$$
c_{a, a^{\prime}} \in \operatorname{Hom}\left(\mathbb{M}_{\bullet}, \mathbb{M}_{\bullet}^{\prime}\right) \quad \text { ("change of coordinates") }
$$

that is compatible with $a$ and $a^{\prime}$. We call a pf-manifold normal if it admits a normal atlas (if it does, all atlases are normal).

As terminology, we will simply talk about "the pf-manifold $\mathbb{M}$ " without explicitly specifying the (equivalence class) of the atlas in the notation. 
Remark 3.A.8. Note that any pf-manifold carries a natural topology: the limit topology.

Example 3.A.9. Of course, any finite dimensional manifold $M$ is a pf-manifold: just use the constant tower $\mathbb{M}_{\bullet}=M$.

Example 3.A.10. The basic examples of towers and pf-manifolds are provided by jets and PDEs. Let us place ourselves in the setting of a fibration (in the sense of surjective submersion)

$$
\pi: R \rightarrow M
$$

between finite dimensional manifolds. Associated to such a fibration there is an entire tower of jet spaces

$$
J^{\infty} R: \quad \ldots \rightarrow J^{2} R \rightarrow J^{1} R \rightarrow J^{0} R=R
$$

where $J^{k} R$ denotes the space of $k$-jets of sections of $\pi: R \rightarrow M$. When $R=M \times N$ and $\pi=p r_{1}$ one recovers the spaces of jets of maps from $M \rightarrow N$.

Each $J^{k} R, k \in \mathbb{N}$, is canonically a smooth manifold; the infinite jet

$$
J^{\infty} R=\lim _{\longleftarrow} J^{k} R
$$

becomes a pf-manifold.

Definition 3.A.11. A smooth pf-map $f: \mathbb{M} \rightarrow \mathbb{N}$ between two pf-manifolds is any set theoretical map with the property that, for a/any atlas $a$ of $\mathbb{M}$ and $b$ of $\mathbb{N}$, $f$ is a smooth map w.r.t. $a$ and $b$ (in the sense of Definition 3.A.4). We will denote by $C^{\infty}(\mathbb{M}, \mathbb{N})$ the space of such smooth maps.

Example 3.A.12. When $\mathbb{N}=\mathbb{R}$ we obtain the algebra $C^{\infty}(\mathbb{M})$ of smooth functions on the pf-manifold $\mathbb{M}$. Working out the definition, we see that

$$
f: \mathbb{M} \rightarrow \mathbb{R}
$$

is smooth if it is continuous and there exists $m_{0}$ such that

$$
f=g \circ a_{m_{0}}, \quad \text { with } g: \mathbb{M}_{m_{0}} \rightarrow \mathbb{R} \text { smooth in the usual sense. }
$$

In other words, the algebra $C^{\infty}(\mathbb{M})$ is identified with the direct limit of the algebras $C^{\infty}\left(\mathbb{M}_{m}\right)$

$$
\underset{m}{\lim } C^{\infty}\left(\mathbb{M}_{m}\right)=\cup_{m} C^{\infty}\left(\mathbb{M}_{m}\right),
$$

via the pull-back by $a$. In concrete words, a function $f=f(x)$ on $\mathbb{M}$ is smooth if and only if it depends smoothly (in the usual sense) on a finite number variables.

\section{A.2 Pf-vector bundles}

Definition 3.A.13. A (direct) pf-vector bundle over a pf-manifold $\mathbb{M}$ consists of a pf-manifold $\mathbb{E}$ and a smooth pf-map $p: \mathbb{E} \rightarrow \mathbb{M}$, together with a vector bundle equivalence classes of $\mathrm{pf}$-vector bundle atlases where: 
- a pf-vector bundle atlas for $p: \mathbb{E} \rightarrow \mathbb{M}$ is an atlas $\tilde{a}: \mathbb{E} \rightarrow \mathbb{E}$. for $\mathbb{E}$, an atlas $a: \mathbb{M} \rightarrow \mathbb{M}$. for $\mathbb{M}$ together with the structure of tower of vector bundles

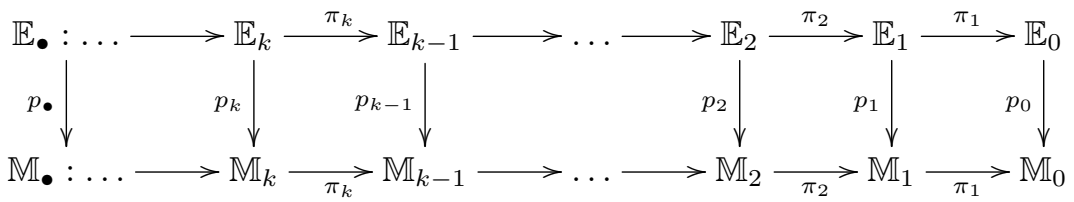

(hence each $\mathbb{E}_{k} \rightarrow \mathbb{M}_{k}$ is a smooth, finite dimensional vector bundle and each tower map $\mathbb{E}_{k} \rightarrow \mathbb{E}_{k-1}$ is a vector bundle morphisms covering the tower map $\mathbb{M}_{k} \rightarrow \mathbb{M}_{k-1}$ ) that represents $p$, i.e. $p_{\bullet} \circ \tilde{a}=a \circ p$;

- two such, $\tilde{a}$ and $\tilde{a}^{\prime}$, are said to be equivalent (as pf-vector bundle atlases) if they are equivalent as atlases of $\mathbb{E}$, with the corresponding isomorphism of towers $c_{\tilde{a}, \tilde{a}^{\prime}}: \mathbb{E}_{\bullet} \rightarrow \mathbb{E}_{\bullet}^{\prime}$ being a collection of morphisms of vector bundles.

A (pf-)section of $\mathbb{E}$ is any smooth pf-map $\sigma: \mathbb{M} \rightarrow \mathbb{E}$ satisfying $p \circ \sigma=\mathrm{id}$. We denote by $\Gamma(\mathbb{E})$ the space of such sections.

Remark 3.A.14. Given that, any section of $\mathbb{E}$ can be induces a morphism of tower, we can represent it via a collection (cf. Remark 3.A.5)

$$
\sigma_{\bullet}=\left\{\sigma_{k}: \mathbb{M}_{m_{k}} \rightarrow \mathbb{E}_{k}\right\}_{k \geq 1} \quad \text { with } m_{1}<m_{2}<\ldots
$$

and the section condition is that $p_{k} \circ \sigma_{k}$ is the projection $\mathbb{M}_{m_{k}} \rightarrow \mathbb{M}_{k}$; i.e. $\sigma_{k}$ comes from a section of the pull-back of $\mathbb{E}_{k}$ via the tower projection $\pi: \mathbb{M}_{m_{k}} \rightarrow \mathbb{M}_{k}$

$$
s_{k} \in \Gamma\left(\mathbb{M}_{m_{k}}, \pi^{*} \mathbb{E}_{k}\right) \text {. }
$$

Example 3.A.15. In Example 3.A.10, if $\pi: E \rightarrow M$ is a vector bundle (everything is finite dimensional), then each $J^{k} E$ is a vector bundle over $M$ and then

$$
\pi_{\infty}: J^{\infty} E \rightarrow M
$$

becomes a pf-vector bundle (for $M$ see Example 3.A.9).

Example 3.A.16. Of course, a basic example is the trivial line bundle. Smooth sections corresponds to smooth functions $\mathbb{M} \rightarrow \mathbb{R}$.

Example 3.A.17 (The tangent bundle). A fundamental example of pf-vector bundle is the tangent bundle of a pf-manifold $\mathbb{M}$. For an intrinsic approach one defines $T_{x} \mathbb{M}$ as the space of derivations at $x, \delta_{x}: C^{\infty}(\mathbb{M}) \rightarrow \mathbb{R}$, and then one sets $T \mathbb{M}=\cup_{x} T_{x} \mathbb{M}$. It follows that, for any pf-atlas $a: \mathbb{M} \rightarrow \mathbb{M}_{\bullet}$, one obtains a pf-atlas $T \mathbb{M} \rightarrow T \mathbb{M}_{\bullet}$, where the tangent tower of $\mathbb{M}_{\bullet}$ is, of course,

$$
T \mathbb{M}_{\bullet}: \quad \ldots \longrightarrow T \mathbb{M}_{k} \stackrel{d \pi_{k}}{\longrightarrow} T \mathbb{M}_{k-1} \longrightarrow \ldots \longrightarrow T \mathbb{M}_{2} \stackrel{d \pi_{2}}{\longrightarrow} T \mathbb{M}_{1} \stackrel{d \pi_{1}}{\longrightarrow} T \mathbb{M}_{0} .
$$

One has that $T \mathbb{M}=\lim T \mathbb{M}_{k}$. This is shown in [33] and provides a different definition of $T \mathbb{M}$ (less intrinsic but perhaps more practical). In particular, it shows that, according to our definition, the tangent bundle in the pf-setting depends only on 
the limit of a pf-atlas. A similar phenomenon happens with the Lie algebroid of a Lie pf-groupoid, see the next subsection.

The tangent bundle gives rise to the notion of smooth vector fields of $\mathbb{M}$. As in the standard situation, vector fields act on smooth functions; and, using [33], Thm 3.26, one finds

$$
\mathfrak{X}(\mathbb{M}) \cong \operatorname{Der}\left(C^{\infty}(\mathbb{M})\right)
$$

In particular, vector fields still come endowed with a Lie bracket satisfying the standard Leibniz identity. With this at hand, one can now define involutive distributions on a pf-manifold $\mathbb{M}$, Ehresmann connections, and so on.

Example 3.A.18 (The Cartan distribution). We continue with the notations from Example 3.A.10, for a fibration $\pi: R \rightarrow M$. Any section $\sigma \in \Gamma(R)$ induces a holonomic section $j^{k} \sigma$ of $J^{k} R \rightarrow M$. The Cartan distribution

$$
\mathrm{e}^{k} \subset T J^{k} R
$$

is defined so that a section of $J^{k} R$ is holonomic if and only if it is tangent to $\mathrm{C}^{k}$. More explicitly, one defines $\mathrm{C}^{k}$ as the space of vectors that are tangent to such holonomic sections. The distributions $\mathrm{e}^{k}$ are compatible with each other in the sense that

$$
d \pi_{k}\left(\mathrm{C}^{k}\right) \subset \mathrm{e}^{k-1} .
$$

In other words, they induce a distribution on the tower $J^{\bullet} R$, hence a distribution

$$
\mathrm{e}^{\infty} \subset T J^{\infty} R
$$

on the pf manifold $J^{\infty} R$. Unlike its finite versions $\mathrm{C}^{k}, \mathrm{e}^{\infty}$ is involutive.

Example 3.A.19 (The canonical Spencer operator). Let us consider here the pfvector bundle $J^{\infty} E \rightarrow M$ associated to a vector bundle $E \rightarrow M$ as in Example 3.A.15. Due to the vector bundle structure, one can detect holonomic sections using a pf-connection on $J^{\infty} E$

$$
D^{\infty}: \mathfrak{X}(M) \times \Gamma\left(J^{\infty} E\right) \rightarrow \Gamma\left(J^{\infty} E\right) .
$$

Holonomic sections are precisely those sections that are flat w.r.t. $D^{\infty}$; as with the Cartan distribution, this property can be used to force the definition of $D^{\infty}$. Notice that $D^{\infty}$, differently from the Cartan distribution $\mathcal{C}^{\infty}$, is not a limit of connections on the vector bundles $J^{k} E$. In fact, at each level $k D^{\infty}$ does not descend to a connection on $J^{k} E$ but to an operator

$$
D^{k}: \mathfrak{X}(M) \times \Gamma\left(J^{k} E\right) \rightarrow \Gamma\left(J^{k-1} E\right)
$$

which is a connection "relative to $l: J^{k} E \rightarrow J^{k-1} E^{\text {" }}$ in the sense that the Leibniz identity takes the form

$$
D_{X}^{k}(f \sigma)=f D_{X}^{k}(\sigma)+L_{X}(f) l(\sigma) .
$$

Similar to the involutivity of the Cartan distribution, $D^{\infty}$ is a flat connection. In the literature, it is known as the Spencer operator on $J^{\infty} E$. The list of references concerning Spencer operators is extensive; see, for example, [63]. 


\section{A.3 Lie pf-groupoids and algebroids}

Most of the basic concepts on groupoids and Lie algebroids can be adapted to the pro-finite setting in a manner that is rather straightforward, though occasionally tedious. For instance, the most general concept pf-groupoids $\Sigma \rightrightarrows \mathbb{M}$ comes with $\Sigma_{k} \mathrm{~s}, \mathbb{M}_{k} \mathrm{~s}$ and the structure maps (source/target, multiplication) are just pf-maps (hence may lower the index $k$ ). However, the examples that we encounter in the main body are over a finite dimensional base $\mathbf{X}$. Hence, we give the following

Definition 3.A.20. A Lie pf-groupoid is a topological groupoid $\Sigma \rightrightarrows \mathbf{X}$, where $\Sigma$ is a pf-manifolds and $\mathbf{X}$ is a finite dimensional manifold, which admit an atlas $a$ : $\Sigma \rightarrow \Sigma$. where each $\Sigma_{k}$ is a Lie groupoid over $\mathbf{X}$ with structure maps compatible with those of $\Sigma$ (i.e., the atlas is a tower of Lie groupoids).

A Lie pf-subgroupoid of $\Sigma$ is any subgroupoid $\Theta \subset \Sigma$ such that there is a pf-atlas $a: \Sigma \rightarrow \Sigma_{\bullet}$ with the property that $\left.a\right|_{\Theta}: \Theta \rightarrow \Theta_{\bullet}$ is a pf-atlas of $\Theta$, where each $\Theta_{k}:=a_{k}(\Theta)$ is a Lie subgroupoid of $\Sigma_{k}$.

Similarly, an action of a pf-Lie groupoid $\Sigma \rightrightarrows \mathbf{X}$ on a pf-manifold $P$ is a topological action of $\Sigma$ on $P$ along some pf-map $\mu: P \rightarrow \mathbf{X}$, such that there exists atlases for $\Sigma$ and $P$ such that the action projects to actions of the $\Sigma_{k}$ 's on the $P_{k}$ 's.

Principal $\Sigma$-bundles, where $\Sigma \rightrightarrows \mathbf{X}$ is a pf-Lie groupoid, are actions of $\Sigma \rightrightarrows$ $\mathrm{X}$ on a pf-manifold $P$ such that the quotient space with respect to the orbits of the action is a finite dimensional manifold $M$, the action of $\Sigma \rightrightarrows \mathbf{X}$ is free (and transitive) along the fibers of the quotient map $\pi: P \rightarrow M$, and there exist local sections of $\pi$ around any point of $M$.

Example 3.A.21. Pf-vector bundles over a finite dimensional base are an example of pf-Lie groupoid according to the definition above. The source and target maps coincide with the vector bundle projection.

Concerning Lie pf-algebroids $A \rightarrow \mathbf{X}$, the discussion is again quite similar, with one subtle difference. The Lie bracket on $\Gamma(A)$ is not necessarily a "limit" of actual Lie brackets on the spaces of sections of finite dimensional Lie algebroids $\Gamma\left(A^{k}\right)$, but may come from "brackets" $\Gamma\left(A_{k}\right) \times \Gamma\left(A_{k}\right) \rightarrow \Gamma\left(A_{k-1}\right)$ (in particular, the Jacobi identity makes sense only on $\Gamma(A))$. This is similar to the situation described in Example 3.A.19.

If $\Sigma \rightrightarrows \mathbf{X}$ is a Lie pf-groupoid, one defines its associated Lie algebroid as in the finite dimensional case, and the outcome is a Lie pf-algebroid. More explicitly, we consider as total space the source-vertical bundle of $\Sigma$ (defined straightforwardly), as anchor the differential of the target map, and so on. In this case, of course, the bracket on $\Gamma(A)$ is a "limit" of Lie brackets. In fact, it is not difficult to see that the Lie algebroids $A_{k}=\operatorname{Lie}\left(\Sigma_{k}\right) \rightarrow \mathbf{X}$ can be used as an atlas for $A$ (in particular $A=\lim A_{k}$ ). We write, as usual, $A=\operatorname{Lie}(\Sigma)$.

However, some words need to be said about dealing with groupoids $\Sigma \rightrightarrows \mathbf{X}$ that do not admit a normal atlas. Namely, we observe that the Lie algebroid of a Lie pf-groupoid depends, according to our definition, only on the limit of a pf-atlas. As a result, the Lie pf-algebroid of a non normal Lie pf-groupoid coincides with the Lie pf-algebroid of the limit of a pf-atlas. In the main body of the chapter, we deal with non normal pf-groupoids only when looking at non-tight Cartan resolutions of étale groupoids $\mathcal{G} \rightarrow \mathbf{X}$, see Definitions 3.4.5 and 3.4.6, and 
we never need the associated Lie pf-algebroids. In fact, in our setting we have a clearer infinitesimal counterpart for the possibly non normal Lie pf-groupoid $j_{\Sigma^{\infty}}^{\infty}(\mathcal{G})$ : the possibly non normal Lie pf-algebroid $j_{\operatorname{Lie}\left(\Sigma^{\infty}\right)}^{\infty}(\operatorname{Lie}(\mathcal{G}))$.

Example 3.A.22. The main groupoids that we encounter in this chapter are groupoids of germs (finite dimensional but possibly huge) and groupoids of jets. The second type sit inside the tower of full jet groupoids associated to (finite dimensional) manifolds $\mathbf{X}$

$$
J^{k} \Gamma^{\mathbf{X}}:=\left\{j_{x}^{k} f: f \in \Gamma^{\mathbf{X}}=\operatorname{Diff}_{\text {loc }}(\mathbf{X}), x \in \operatorname{dom}(f)\right\},
$$

which are groupoids over $\mathbf{X}$ with source $s\left(j_{x}^{k} f\right)=x$, target $t\left(j_{x}^{k} f\right)=f(x)$ and multiplication

$$
j_{y}^{k} g \cdot j_{x}^{k} f=j_{x}^{k}(f \circ g) .
$$

For $k$ finite, $J^{k} \Gamma^{\mathbf{X}}$ inherits a smooth structure from the fact that it sits as an open in the $k$-jet space $J^{k}(\mathbf{X}, \mathbf{X})$. All-together, one has a tower of Lie groupoids

$$
J^{\infty} \Gamma^{\mathbf{X}}: \ldots \rightarrow J^{2} \Gamma^{\mathbf{X}} \rightarrow J^{1} \Gamma^{\mathbf{X}} \rightarrow J^{0} \Gamma^{\mathbf{X}}=\mathbf{X} \times \mathbf{X}
$$

and $J^{\infty} \Gamma^{\mathbf{X}} \rightrightarrows \mathbf{X}$ becomes a pf-Lie groupoid.

Corresponding to this there is a pf-vector bundle over $\mathbf{X}$,

$$
A^{\infty}\left(\Gamma^{\mathbf{X}}\right): \ldots \rightarrow A^{2}\left(\Gamma^{\mathbf{X}}\right) \rightarrow A^{1}\left(\Gamma^{\mathbf{X}}\right) \rightarrow A^{0}\left(\Gamma^{\mathbf{X}}\right)=T \mathbf{X}
$$

where

$$
A^{k}\left(\Gamma^{\mathbf{X}}\right):=\operatorname{Lie}\left(J^{k} \Gamma^{\mathbf{X}}\right)=J^{k} T \mathbf{X} \rightarrow \mathbf{X}
$$

consists of $k$-jets of vector fields. Each $A^{k}\left(\Gamma^{\mathbf{X}}\right)$ with $k$ finite carries a canonical structure of Lie algebroid over $\mathbf{X}$ - the unique one with anchor the map $A^{k}\left(\Gamma^{\mathbf{X}}\right) \rightarrow$ $A^{0}\left(\Gamma^{\mathbf{X}}\right)=T \mathbf{X}$ given by the differential of the jet projection and with bracket satisfying

$$
\left[j^{k} X, j^{k} Y\right]=j^{k}[X, Y] .
$$

Of course, when $k=\infty$, the previous discussion makes $A^{\infty}\left(\Gamma^{\mathbf{X}}\right)$ into a pfalgebroid over $\mathbf{X}$. Actually, this is precisely the Lie algebroid corresponding to the jet groupoid: the identification of $j_{x}^{k} X$ with a tangent vector to the $s$-fiber of $J^{k} \Gamma^{\mathbf{X}}$, at the unit, is

$$
\left.j_{x}^{k} X \mapsto \frac{d}{d t}\right|_{t=0} j_{x}^{k}\left(\phi_{t}^{X}\right) .
$$

The general discussion of Cartan distributions (see Example 3.A.18), applied to the first projection $\mathbf{X} \times \mathbf{X} \rightarrow \mathbf{X}$ gives rise to a similar object on $J^{\infty} \Gamma^{\mathbf{X}}$. The corresponding algebroid $A^{\infty}\left(\Gamma^{\mathbf{X}}\right)$ carries the Spencer operator $D^{\infty}$ (3.8) in the case $E=T \mathbf{X}$.

However, a new property emerges here: multiplicativity. In simple words, the Cartan distribution on $J^{\infty} \Gamma^{\mathbf{X}}$ and the Spencer operator on $A^{\infty}\left(\Gamma^{\mathbf{X}}\right)$ are compatible with the composition of arrows and the bracket on sections respectively. The overall structure that one discovers is best described in terms of Pfaffian groupoids and towers of prolongations, which we discuss in the next appendix. 
Example 3.A.23 (Lie pseudogroups). A similar discussion applies to general (Lie) pseudogroups on $\mathbf{X}$ (the previous example corresponds to the full pseudogroup $\operatorname{Diff}_{\text {loc }}(\mathbf{X})$ ); see Subsection 3.2.2. For any pseudogroup $\Gamma$ on $\mathbf{X}$, restricting our attention at jets of diffeomorphisms from $\Gamma$, one obtains the tower of groupoids

$$
J^{\infty} \Gamma: \ldots \rightarrow J^{2} \Gamma \rightarrow J^{1} \Gamma \rightarrow J^{0} \Gamma
$$

where each $J^{k} \Gamma$ sits inside $\Pi^{k}$.. The only "problem" is that the groupoids $J^{k} \Gamma$ may fail to be smooth manifolds and the maps in the tower may fail to be submersions. One calls $\Gamma$ Lie pseudogroup when the above is a smooth tower of groupoids, i.e. $J^{\infty} \Gamma$ is a pf Lie subgroupoid of $J^{\infty} \Gamma^{\mathbf{X}}$.

Definition 3.A.24. A Lie pseudogroup on $\mathbf{X}$ is any pseudogroup with the property that $J^{\infty} \Gamma$ is a smooth pf-subgroupoid of $J^{\infty}\left(\Gamma^{\mathbf{X}}\right)$.

It follows that the corresponding algebroids

$$
A^{k}(\Gamma):=\operatorname{Lie}\left(J^{k} \Gamma\right)
$$

are Lie sub-algebroids of $A^{k}\left(\Gamma^{\mathbf{X}}\right)$ and that $A^{\infty}(\Gamma)$ becomes a pf subalgebroid of $A^{\infty}\left(\Gamma^{\mathbf{X}}\right)$. Furthermore, the Cartan distributions restrict to similar distributions on the $J^{k} \Gamma^{\prime}$ s the same happens for the Spencer operator on $A^{\infty}(\Gamma)$.

\section{B Pfaffian groupoids and their prolongations}

The theory of Pfaffian fibrations and Pfaffian groupoids was developed in [55]; see also [17]. Pfaffian fibrations encode the abstract properties of geometric PDEs, i.e. "nice" submanifolds of jet spaces, while Pfaffian groupoids encode the abstract properties of jet groupoids of Lie pseudogroups. The theory of Pfaffian groupoids is extensively used in the main body of this chapter. In particular, the notions of prolongation and of Cartan tower play a fundamental role in Section 3.4 and Section 3.5. Below, we briefly recall the main elements of the theory. We refer to $[15,17,55,69]$ for more details.

\section{B.1 Pfaffian groupoids}

Definition 3.B.1. A Pfaffian fibration $(P, \mathcal{C})$ is a surjective submersion $\pi: P \rightarrow \mathbf{X}$ together with a distribution $\mathcal{C}$ on $P$ which is $\pi$-transversal, i.e. $\mathcal{C}+\operatorname{Ker}(d \pi)=T P$, and $\pi$-involutive, i.e. the symbol space $\mathcal{C}^{s}:=\operatorname{Ker}(d \pi) \cap \mathcal{C}$ is an involutive subbundle of TP. The distribution $\mathcal{C}$ is called Pfaffian distribution.

Remark 3.B.2. In [55], Pfaffian fibrations were called Pfaffian bundles. The terminology "Pfaffian fibration" appeared first in the paper [17].

Definition 3.B.3. A section $\sigma: \mathbf{X} \rightarrow P$ of a Pfaffian fibration $(P, \mathcal{C})$ is called holonomic if it is tangent to $\mathrm{C}$.

Example 3.B.4. The Cartan distribution on a jet space from Example 3.A.18 is an example of Pfaffian distribution. A geometric PDE of order $k$ is an embedded submanifold $P \subset J^{k} R$ such that the restriction of the Cartan distribution of $J^{k} R$ 
to $P$ is a Pfaffian distribution on $P$. The name is justified by the fact that the local coordinate equations cutting out $P$ in $J^{k} R$ correspond formally to a PDE in the usual sense and holonomic sections of $P$ are in one to one correspondence with solution of the PDE.

Example 3.B.5. The $k$-th jet space $J^{k} \Gamma$ of a Lie pseudogroup $\Gamma$ is an example of geometric PDE. This example has two remarkable features. First, $J^{k} \Gamma$ possesses a multiplication and holonomic bisections are closed with respect to such a multiplication. Second, $J^{k} \Gamma$ projects on the PDE $J^{k-1} \Gamma$ in a way that interacts nicely with the distributions and their holonomic bisections.

It turns out that both the distinguished features of the above example can be encoded in properties of the distribution $\mathrm{C}$.

We start by looking at multiplicative structures. Recall that if $\Sigma \rightrightarrows T \mathbf{X}$ is a Lie groupoid, $T \Sigma \rightrightarrows T \mathbf{X}$ has a natural Lie groupoid structure where all the structure map are obtained by differentiation. We call it the tangent groupoid.

Definition 3.B.6. A Pfaffian groupoid $(\Sigma, \mathcal{C})$ is a Lie groupoid $\Sigma \rightrightarrows \mathbf{X}$ together with a distribution $\mathcal{C}$ which is Pfaffian with respect to the source projection, such that $\mathcal{C}^{s}=\mathcal{C}^{t}$ and multiplicative, i.e. $\mathcal{C} \subset T \Sigma$ is a subgroupoid of the tangent groupoid.

Remark 3.B.7. The multiplicativity of $\mathcal{C}$ equips the set of holonomic bisections of a Pfaffian groupoid with multiplicative structure that makes it into a generalized pseudogroup supported by $\Sigma$, cf. Definition 3.2.51.

Example 3.B.8. The Cartan distribution on the jet groupoid $J^{k} \Gamma$ of a Lie pseudogroup $\Gamma$ makes it into a Pfaffian groupoid (see $[55,69])$. Recall that the pseudogroup has order $k$ if $\varphi \in \Gamma(U)$ if and only if $j_{x}^{k} \varphi \in J^{k} \Gamma$ for each $x \in U$. In this case, the pseudogroup $\operatorname{Bis}_{\text {loc }}^{\text {hor }}\left(J^{k} \Gamma\right)$ of holonomic bisections of $J^{k} \Gamma$ is in bijection with $\Gamma$. In general, there is only an injective map from $\Gamma$ to $\operatorname{Bis}_{\text {loc }}^{\text {hor }}\left(J^{k} \Gamma\right)$.

For a Pfaffain bundle $(P, \mathcal{C})$ one has the projection $\pi^{\mathcal{C}}: T P \rightarrow T P / \mathcal{C}$ and the curvature map $c^{\mathcal{C}}: \mathcal{C} \times{ }_{P} \mathcal{C} \rightarrow T P / \mathcal{C}$, sending $(v, w)$ to $\pi^{\mathcal{C}}\left(\left[X_{x}, Y_{w}\right]_{p}\right)$; here $X_{v}$ and $Y_{w}$ are extensions of $v$ and $w$ as vector fields on $P$. When $(\Sigma, \mathcal{C})$ is a Pfaffian groupoid, then $T \Sigma / \mathcal{C} \cong t^{*}\left(\operatorname{Lie}(\Sigma) / \mathcal{C}^{s}\right)$ (where $\mathcal{C}^{s}$ is the symbol space, see Definition 3.B.1). The map $\pi^{\mathcal{E}}: T \Sigma \rightarrow t^{*}\left(\operatorname{Lie}(\Sigma) / \mathcal{C}^{s}\right)$ restricts to an epimorphism of vector bundles $l: \operatorname{Lie}(\Sigma) \rightarrow \operatorname{Lie}(\Sigma) / \mathcal{C}_{\mathbf{X}}^{s}$.

Proposition 3.B.9. There is a unique structure of Lie algebroid on $\operatorname{Lie}(\Sigma) / \mathcal{C}_{\mathbf{X}}^{s}$ such that $l$ is a Lie algebroid map.

See [55] for more details. In particular, when $\mathcal{C}^{s}=0, l$ is the identity. This is the case when $\mathcal{C}$ is a multiplicative connection on $\Sigma$. In this situation, the pair $(\Sigma, \mathcal{C})$ is also called Cartan groupoid.

Definition 3.B.10. A morphism of Pfaffian groupoids $p:\left(\Sigma_{1}, \mathcal{C}_{1}\right) \rightarrow\left(\Sigma_{2}, \mathrm{C}_{2}\right)$ over $\mathbf{X}$ is a map of Lie groupoids $p: \Sigma_{1} \rightarrow \Sigma_{2}$ such that

- $d p\left(\mathcal{C}_{1}\right) \subset \mathcal{C}_{2}$ (i.e., $p$ is a map of pairs $\left.\left(\Sigma_{1}, \mathcal{C}_{1}\right) \rightarrow\left(\Sigma_{2}, \mathcal{C}_{2}\right)\right)$;

- $p^{\mathcal{C}}\left(c^{\mathcal{C}_{1}}(v, w)\right)=c^{\mathcal{C}_{2}}(d p(v), d p(w))$ for all $v, w \in \mathcal{C}_{g}, g \in \Sigma_{1}$; 
where $p^{\mathcal{C}}: t^{*}\left(\operatorname{Lie}\left(\Sigma_{1}\right) / \mathcal{C}_{1}^{s} \rightarrow t^{*}\left(\operatorname{Lie}\left(\Sigma_{2}\right) / \mathcal{C}_{2}^{s}\right.\right.$ is the map induced by $\operatorname{Lie}(p)$ : $\operatorname{Lie}\left(\Sigma_{1}\right) \rightarrow \operatorname{Lie}\left(\Sigma_{2}\right)$ thanks to the fact that $p$ is a map of pairs.

More compactly, a morphism of Pfaffian groupoids is a map preserving the Pfaffian distribution and its curvature.

Example 3.B.11. The projections $p r^{k, k-1}:\left(J^{k} \Gamma, \mathrm{C}^{k}\right) \rightarrow\left(J^{k-1}, \mathrm{C}^{k-1}\right)$ between jet spaces of Lie pseudogroups are morphisms of Pfaffian groupoids.

The projections from the previous example satisfy some additional properties which are of great importance for the theory. We discuss them in the next subsections.

\section{B.2 Prolongations and Cartan towers}

The second distinguished feature of the jet groupoids of a Lie pseudogroup, a part for the multiplicativity of their Pfaffian form, is that the projection between them are prolongation maps.

Definition 3.B.12. A prolongation map, or Lie prolongation, $p r:\left(\Sigma^{\prime}, \mathrm{C}^{\prime}\right) \rightarrow$ $(\Sigma, \mathcal{C})$ between Pfaffian groupoids is a morphism of Pfaffian groupoids such that

- $p r$ is a surjective submersion;

- $d p\left(\left(\mathcal{C}^{\prime}\right)^{s}\right)=0$;

- for all $v, w \in \mathcal{C}_{g^{\prime}}^{\prime} g \in \Sigma^{\prime}$, it holds $c^{\mathcal{C}}(\operatorname{dpr}(v), \operatorname{dpr}(w))=0$;

- the map $\Phi: \operatorname{Lie}\left(\Sigma^{\prime}\right) /\left(\mathcal{C}^{\prime}\right)_{\mathbf{X}}^{S} \rightarrow \operatorname{Lie}(\Sigma)$ induced by the second point above is an isomorphism.

Example 3.B.13. When $\Gamma$ is a Lie pseudogroup, the canonical projection $p r^{k, k-1}$ : $\left(J^{k} \Gamma, \mathrm{C}^{k}\right) \rightarrow J^{k-1}\left(\Gamma, \mathrm{C}^{k-1}\right)$ is a prolongation map. It is a surjective submersion by definition of Lie pseudogroup. The second property follows because the symbol space of $\mathcal{C}$ is precisely $\operatorname{Ker}\left(d p r^{k, k-1}\right)$; this is a consequence of the fact that the same property is true for the Cartan distribution on $J^{k}(\mathbf{X}, \mathbf{X})$, where it can be checked in canonical coordinates. The same is true for the third property. The fourth property is then a consequence of $\operatorname{Ker}\left(d s: T J^{k} \Gamma \rightarrow T \mathbf{X}\right) / \operatorname{Ker}\left(d p r^{k, k-1}\right) \cong$ $\operatorname{Ker}\left(d s: T J^{k-1} \Gamma \rightarrow T \mathbf{X}\right)$. Observe that the first three properties hold for any projection $\mathrm{pr}^{h, k}: J^{h} \Gamma \rightarrow J^{k} \Gamma, h>k$, but the fourth one holds only when $h=$ $k+1$.

The category of Pfaffian groupoids is equipped with a classical prolongation functor that is final among prolongations. We make use of it in Proposition 3.4.13.

Definition 3.B.14. The classical prolongation of a Pfaffian groupoid $(\Sigma, \mathcal{C})$ is defined by

$\mathcal{P}_{\mathcal{C}}(\Sigma):=\left\{j_{x}^{1} \sigma \in J^{1} \Sigma \mid \sigma \in \operatorname{Bis}_{\mathrm{loc}}(\Sigma), d_{x} \sigma\left(T_{x} \mathbf{X}\right) \subset \mathcal{C}_{\sigma(x)}, c^{\mathcal{C}}\left(d_{x} \sigma\left({ }_{-}\right), d_{x} \sigma\left({ }_{-}\right)\right)=0\right\}$.

We say that it is smooth if $\mathcal{P}^{\mathcal{C}}(\Sigma)$ is a smooth submanifold of $J^{1} \Sigma$ and the induced projection $\mathcal{P}_{\mathfrak{C}}(\Sigma) \rightarrow \Sigma$ is a surjective submersion. We call $\mathcal{P}_{\mathfrak{C}}(\Sigma)$ the classical prolongation of $\Sigma$. 
The following facts hold true, and are proven in [55], Chapter 6.

Proposition 3.B.15. $\mathcal{P}_{\mathcal{C}}(\Sigma)$ is a subgroupoid of $J^{1} \Sigma$.

Proposition 3.B.16. Set $\mathcal{C}_{c l}:=\mathcal{C}^{1} \cap T \mathcal{P}_{\mathcal{C}}(\Sigma)$, where, as usual, $\mathrm{e}^{1}$ is the Cartan distribution on $J^{1} \Sigma$. If $\mathcal{P}_{\mathfrak{C}}(\Sigma)$ is smooth then $\left(\mathcal{P}_{\mathfrak{C}}(\Sigma), \mathcal{C}_{c l}\right)$ is a prolongation of $(\Sigma, \mathcal{C})$.

Proposition 3.B.17. $\left(\mathcal{P}_{\mathrm{C}^{k}}\left(J^{k} \Sigma\right), \mathcal{C}_{c l}\right)=\left(J^{k+1} \Sigma, \mathrm{e}^{k+1}\right)$ for any Lie groupoid $\Sigma \rightrightarrows \mathbf{X}$.

Proposition 3.B.18. The pair $\left(\mathcal{P}_{\mathfrak{e}}(\Sigma), \mathcal{C}_{c l}\right)$ is final among prolongation in the sense that if

$$
p r:\left(\Sigma^{\prime}, \mathfrak{C}^{\prime}\right) \rightarrow(\Sigma, \mathcal{C})
$$

is a prolongation map, then there exists a unique Lie groupoid map $j: \Sigma^{\prime} \rightarrow J^{1} \Sigma$ taking values in $\mathcal{P}_{\mathfrak{e}}(\Sigma)$, such that:

- $p r \circ j=p r_{\text {can, }}$ where we provisionally use $p r_{\text {can }}$ to denote the jet projection $J^{1} \Sigma \rightarrow$ $\Sigma$;

- for all $X, Y, Z \in X\left(\Sigma^{\prime}\right)$ such that $Y, Z$ are tangent to $\mathcal{C}^{\prime}$ it holds

$$
[X] \in T \Sigma^{\prime} / \mathfrak{C}^{\prime}=[\operatorname{dj}(X)] \in T P_{\mathcal{C}}(\Sigma) / \mathcal{C}_{c l}, \quad c^{\mathcal{C}^{\prime}}(Y, Z)=c^{\mathfrak{C}_{c l}}(\operatorname{dj}(Y), d j(Z))
$$

Remark 3.B.19. In the above proposition, smoothness of $P_{\mathfrak{C}}(\Sigma)$ is not an assumption.

We now define one of the key objects in the main body of the chapter.

Definition 3.B.20. A Cartan tower over a Lie groupoid $\Sigma \rightrightarrows \mathbf{X}$ is a tower of Pfaffian groupoids

$$
\cdots\left(\Sigma^{k}, \mathrm{C}^{k}\right) \stackrel{p r^{k, k-1}}{\rightarrow}\left(\Sigma^{k-1}, \mathrm{C}^{k-1}\right) \rightarrow \cdots \rightarrow \Sigma \rightrightarrows \mathbf{X}
$$

such that all the maps $p r^{k, k-1}$ are Lie prolongations and the symbol space $\left(\mathrm{C}^{1}\right)^{s}$ is vertical over $\Sigma$. We use the notation $\left(\Sigma^{*}, \mathrm{C}^{*}\right)$.

The fact that prolongations are Pfaffian morphisms impliy that the limit $\Sigma^{\infty}:=\lim \Sigma^{*}$ of a Cartan tower is equipped with a pf distribution $\mathrm{C}^{\infty}$; see also Example 3.A.18. $\mathrm{C}^{\infty}$ can be represented in the tower $\left(\Sigma^{*}, \mathrm{C}^{*}\right)$ by the sequence of distributions $\left\{\mathrm{C}^{k}\right\}_{k \in \mathbb{N}}$. The following fact is well known in the theory of geometry of PDEs and can be proven directly by using the prolongation conditions.

Proposition 3.B.21. $\mathrm{e}^{\infty}$ is an involutive Pfaffian pf distribution with vanishing symbol. In other words, it is a pf-flat multiplicative connection on $\Sigma^{\infty}$.

We conclude by pointing out that the universality of the classical prolongation can be used to get a universal prolongation tower over a Pfaffian groupoid, by following the lines of Proposition 3.4.13. 


\section{B.3 Spencer operators and Pfaffian algebroids}

The infinitesimal object associated to a Pfaffian distribution on a Lie groupoid is a Spencer operator on its Lie algberoid Lie $(\Sigma)$; see Theorem 3.B.29 at the end of the subsection.

Definition 3.B.22. Let $A \rightarrow \mathbf{X}, E \rightarrow \mathbf{X}$ be Lie algebroids and $l: A \rightarrow E$ be a Lie algebroid epimorphism. A Spencer operator on $A \rightarrow \mathbf{X}$ relative to $l: A \rightarrow E$ is an operator

$$
D: \Gamma(A) \rightarrow \Omega^{1}(\mathbf{X}, E)
$$

such that:

- $D$ is a connection relative to $l$, i.e. it satisfies the pseudo Leibniz rule

$$
D_{X}(f \alpha)=f D_{X}(\alpha)+L_{X}(f) l(\alpha)
$$

for $\alpha \in \Gamma(A), X \in X(\mathbf{X}), f \in C^{\infty}(\mathbf{X})$;

- $D$ is infinitesimally multiplicative, i.e. for all $\alpha, \beta \in \Gamma(A)$ and $X \in X(\mathbf{X})$ it holds

$$
\begin{aligned}
D_{X}[\alpha, \beta]-\left[D_{X}(\alpha), l(\beta)\right]_{E}-\left[l(\alpha), D_{X} \beta\right]_{E}= & D_{\rho\left(D_{X}(\beta)\right)+[\rho(\beta), X]}(\alpha) \\
& -D_{\rho\left(D_{X}(\alpha)\right)+[\rho(\alpha), X]}(\beta)
\end{aligned}
$$

where $[,]_{E}$ denotes the bracket of $E$.

The pair $(A, D)$ is called Pfaffian algebroid relative to $l$.

Observe that the space of sections $\Gamma(\operatorname{Ker}(l))$ is an ideal of $\Gamma(A)$ and $E \cong$ $A / \operatorname{Ker}(l)$. Morever, $\operatorname{Ker}(l)$ is also a bundle of Lie algebras, i.e. the restriction of the bracket of $A$ to $\operatorname{Ker}(l)$ is $C^{\infty}(M)$-linear. This follows from the fact that $\operatorname{Ker}(l) \subset$ $\operatorname{Ker}(\rho)$, because $l$ is a morphism of algebroids. For this same reason,

$$
D_{\rho(\beta)} \alpha=-l([\alpha, \beta]),
$$

holds for all $\alpha, \beta \in \Gamma(A)$. Compare with [55], where this last formula is part of the definition of Spencer operator since $E \rightarrow \mathbf{X}$ is allowed to be just a vector bundle, and $l: A \rightarrow E$ is a surjective vector bundle map. In [55], operators $D$ as in Definition 3.B.22 are called Lie-Spencer operators.

When $E=A$ and $l$ is the identity, $D$ is an infinitesimally multiplicative connection on $A$. The pair $(A, D)$ is also called Cartan algebroid and the operator $D$ is also called Cartan connection.

Of course, all the notions introduced above for Pfaffian groupoids have an infinitesimal counterpart. In particular, one can define holonomic sections as those sections $\alpha \in \Gamma_{\text {loc }}(A)$ such that $D_{X}(\alpha)=0$ for all $X \in X(\mathbf{X})$. Thanks to infinitesimal multiplicativity, holonomic sections of a Pfaffian algebroid define a LAS $\Gamma^{D}\left(A, \_\right)$(Definition 3.3.14).

We can also introduce prolongations of Pfaffian algebroids.

Definition 3.B.23. Let $\left(A^{\prime}, D^{\prime}\right),(A, D)$ be Pfaffian algebroids, relative to $l^{\prime}: A^{\prime} \rightarrow$ $A$ and $l: A \rightarrow E$ respectively. The morphism of Lie algebroids $l^{\prime}: A^{\prime} \rightarrow A$ is called prolongation of Pfaffian algebroids if 
- $D \circ l^{\prime}=l \circ D^{\prime}$;

- $D_{X} D_{Y}^{\prime}-D_{Y} D^{\prime} X-l \circ D_{[X, Y]}^{\prime}=0, X, Y \in X(\mathbf{X})$;

- the map $\operatorname{Ker}\left(l^{\prime}\right) \rightarrow \operatorname{Hom}(T \mathbf{X}, A)$ defined by

$$
v \rightarrow\left(X \rightarrow D_{X}(v)\right), \quad v \in \operatorname{Ker}\left(l^{\prime}\right), X \in T \mathbf{X}
$$

is injective.

One can check that this notion is indeed the infinitesimal counterpart of the notion of prolongation of Pfaffian groupoids by using the correspondence from Theorem 3.B.29.

One also has the notion of Spencer tower.

Definition 3.B.24. A Spencer tower over an algebroid $A \rightarrow \mathbf{X}$ is. a tower of prolongations of Pfaffian algebroids

$$
\ldots\left(A^{k}, D^{k}\right) \rightarrow\left(A^{k-1}, D^{k-1}\right) \rightarrow \cdots \rightarrow A \rightarrow \mathbf{X}
$$

over $A \rightarrow \mathbf{X}$ such that $\left(A^{1}, D^{1}\right)$ is relative to the epimorphism $A^{1} \rightarrow A$ of the tower.

Again, the Spencer operators $D^{k \prime}$ s induce some structure on the limit of the tower: a pf connection $D^{\infty}$.

Proposition 3.B.25. A spencer tower induces a Spencer operator $D^{\infty}$ on $A^{\infty}=\lim _{\leftarrow} A^{k}$ relative to the identity map and such that

$$
D_{[X, Y]}^{\infty}(\alpha)=\left[D_{X}^{\infty}, D_{Y}^{\infty}\right](\alpha), \quad X, Y \in X(\mathbf{X}), \alpha \in \Gamma\left(A^{\infty}\right) .
$$

In other words, $D^{\infty}$ is an infinitesimally multiplicative flat connection on $A^{\infty}$.

Observe that the Lie algebroid bracket on $A^{\infty}$ is the "limit" of the brackets on the $A^{k}$ 's. However, one also has $C^{\infty}(\mathbf{X})$-linear maps

$$
\begin{aligned}
\{,\}^{k}: \Gamma\left(A^{k}\right) \times \Gamma\left(A^{k}\right) & \rightarrow \Gamma\left(A^{k-1}\right) \\
(\alpha, \beta) & \rightarrow\{\alpha, \beta\}^{k}=l^{k}\left([\alpha, \beta]^{k}\right)-D_{\rho(\alpha)}(\beta)+D_{\rho(\beta)}(\alpha),
\end{aligned}
$$

where $[,]^{k}$ is the bracket of $A^{k}$ and $l^{k}: A^{k} \rightarrow A^{k-1}$ is the epimorphism of Lie algebroids to which $D^{k}$ is relative to. This bilinear maps induce a different pf Lie algebroid structure on $A^{\infty}$.

Proposition 3.B.26. $A^{\infty}$ is a bundle of Lie algebras with bracket

$$
\begin{aligned}
\{,\}^{\infty}: \Gamma\left(A^{\infty}\right) \times \Gamma\left(A^{\infty}\right) & \rightarrow \Gamma\left(A^{\infty}\right) \\
(\alpha, \beta) & \rightarrow\{\alpha, \beta\}^{\infty}=[\alpha, \beta]^{\infty}-D_{\rho(\alpha)}^{\infty}(\beta)+D_{\rho(\beta)}^{\infty}(\alpha) .
\end{aligned}
$$

Moreover, $D^{\infty}$ acts by derivations of $\{,\}^{\infty}$. 
Remark 3.B.27. By "bundle of Lie algebras" we mean that, for all $x \in \mathbf{X}$, the bracket

$$
\{,\}_{x}: A_{x}^{\infty} \times A_{x}^{\infty} \rightarrow A_{x}^{\infty}, \quad(\alpha, \beta) \rightarrow\left\{\alpha_{x}, \beta_{x}\right\}=\{\alpha, \beta\}^{\infty}(x),
$$

where $\alpha, \beta$ are any sections of $A^{\infty} \rightarrow \mathbf{X}$ extending $\alpha_{x}, \beta_{x}$, is well defined (and hence a Lie bracket). Of course, this is equivalent to saying that the Lie bracket $\{,\}^{\infty}$ is $C^{\infty}(\mathbf{X})$-linear. If $A^{\infty}$ is finite dimensional, then $D$ acting by derivations imply that $A^{\infty}$ is actually a Lie algebra bundle, i.e. locally trivial as a bundle of Lie algebras. In the pf setting local triviality is a trickier concept, but one can still see that all the algebras $A_{x}^{\infty}$ are isomorphic. See, for example, [1].

Example 3.B.28. The example we are mostly interested in is the Spencer tower of jets of $\Gamma$-sections, where $\Gamma$ is some classical Lie pseudogroup over $\mathbf{X}$. Recall that a Lie pseudogroup $\Gamma$ (satisfying Axiom 1) supported by $\Sigma$ carries the associated LAS $\mathcal{A}=\operatorname{Sec}_{A}^{\Gamma}$ (Definition 3.3.14); its sections are derivatives of flows of bisections of $\Sigma$ that lie in $\Gamma$. One can form the tower of jets

$$
\cdots \rightarrow J^{k} \mathcal{A} \rightarrow \cdots \rightarrow \mathbf{X}
$$

and it turns out that $J^{k} \mathcal{A}:=\operatorname{Lie}\left(J^{k} \Gamma\right)$. Via Theorem 3.B.29 the above tower becomes a Spencer tower.

As promised, we conclude the subsection with the following theorem, which shows that Pfaffian algebroids are the infinitesimal counterpart of Pfaffian groupoids.

Theorem 3.B.29. Let $(\Sigma, \mathrm{C})$ be a Pfaffian groupoid. Then the operator

$$
D: \Gamma(A) \times \mathcal{X}(\mathbf{X}) \rightarrow \Gamma\left(A / \mathcal{C}^{s} \mid \mathbf{x}\right), \quad D_{X}(\alpha)=\left[\operatorname{hor}^{s}(\mathcal{C}), \alpha^{R}\right]\left|\mathbf{x} \bmod \mathcal{e}^{\mathrm{s}}\right| \mathbf{x}
$$

is a Spencer operator on $\operatorname{Lie}(\Sigma)$ relative to the quotient projection $A \rightarrow A / \mathrm{e}^{s} \mid \mathbf{x}$, where $\operatorname{hor}^{s}(\mathcal{C})$ is any s-projectable vecor field over $X$ tangent to $\mathcal{C}$. If $\Sigma$ is s-simply connected, this defines a one to one correspondence between Pfaffian distributions on $\Sigma$ and Spencer operators on $\operatorname{Lie}(\Sigma)$.

Furthermore, the construction just described sends Cartan towers to Spencer towers.

Theorem 3.B.29 follows from Theorem 1 in [23] and is one of the main results of [55] (see Theorem 6.1.23 and Corollary 6.3.2 therein).

\section{C Basics on diffeologies}

Diffeologies provide a convenient way to talk about the differential structure on spaces of smooth functions and sections of fiber bundles. In particular, one can define smoothness of actions of pseudogroups (Definition 3.5.3) in terms of diffeologies. Below, we recall just the very basic elements of the theory, those that we use in the main body of the chapter. We follow [38] and refer to it for a complete discussion. We also follow [19] for what concern $D$-topologies. 


\section{C.1 Parametrisations, plots, diffeologies}

Definition 3.C.1. A parametrisation of a set $S$ is a function $f: U \rightarrow S$, where $U$ is an open of the Euclidean space $\mathbb{R}^{n}$ for some $n \in \mathbb{N}$. A diffeology on $S$ is a set of parametrisations $D$ on $S$ such that

- constant parametrisations are elements of $D$;

- if $f: U \rightarrow S$ is a parametrisation and for any $x \in U$ there is an open neighbourhood of $x, U_{x} \subset U$, such that $\left.f\right|_{U_{x}}$ belongs to $D$, then $f$ belongs to $D$;

- for any element $f: U \rightarrow S$ of $D$ and any smooth function $\phi: V \rightarrow U$ between open subset of Euclidean spaces (possibly of different dimension), one has $f \circ \phi \in D$.

More briefly: a diffeology is a subsheaf $D$ of the sheaf $U \rightarrow \operatorname{Hom}_{\mathrm{Set}}(U, S)$ on the category of open subsets of Euclidean spaces and smooth maps between them, such that $D\left(\mathbb{R}^{0}\right)=S$. The pair $(S, D)$ consisting of a set and a diffeology on it is called diffeological space. Parametrisations in $D$ are called plots.

Definition 3.C.2. A function $f: S_{1} \rightarrow S_{2}$ between diffeological spaces is smooth when it sends plots to plots. We denote the set of smooth maps from $S_{1}$ to $S_{2}$ by $C^{\infty}\left(S_{1}, S_{2}\right)$.

We usually omit $D$ when denoting a diffeological space $(S, D)$.

Example 3.C.3. Any set $S$ carries diffeology whose plots are precisely the constant maps from open of Euclidean spaces to $S$. It is called discrete diffeology.

Example 3.C.4. Any manifold $\mathbf{X}$ is naturally a diffeological space. The sheaf $D$ is simply the sheaf of smooth maps from open subsets of Euclidean spaces to $\mathbf{X}$. Smooth maps in the diffeological sense are just ordinary smooth maps. We call such a diffeology the diffeology induced by the smooth structure.

\section{C.2 Constructing diffeologies: pullback and pushforward}

The following proposition can be proven easily; see also 1.26 and 1.43 in [38]. In what follows we use the terms coarser, coarsest and finer, finest for diffeologies in the usual way; for example, a diffeology $D$ on $S$ is coarser than another diffeology $D^{\prime}$ on $S$ if $D \subset D^{\prime}$.

Proposition 3.C.5. Let $S$ be a diffeological space. For any set $S^{\prime}$ and map $g: S^{\prime} \rightarrow S$, the family of parametrisations $f: U \rightarrow S^{\prime}$ of $S^{\prime}$ such that $g \circ f$ is a plot forms a diffeology on $S^{\prime}$. It is the coarsest diffeology such that $g$ is smooth.

Let $S$ be a diffeological space. For any set $S^{\prime \prime}$ and map $h: S \rightarrow S^{\prime \prime}$, the family of parametrisations $f: U \rightarrow S^{\prime \prime}$ which are either constant or such that for any $x \in U$ there exists a neighbourhood $U_{x} \subset U$ of $x$ and a plot $f_{x}: U_{x} \rightarrow S$ of $S$ such that $\left.f\right|_{U_{x}}=h \circ f_{x}$ forms a diffeology of $S^{\prime \prime}$. It is the finest diffeology such that $h$ is smooth.

We call the diffeologies above pullback and pushforward diffeologies with respect to $g$ and $h$ respectively. 
Example 3.C.6. Subsets of diffeological spaces inherit a natural diffeology via pullback. Let $S$ be a diffeological space and $R \subset S$ be a subset; the pullback diffeology with respect to the inclusion is also called subset diffeology on $R$. In general, an injective map of diffeological spaces $i: R \rightarrow S$ is called induction when the diffeology on $R$ coincide with the pullback diffeology on $S$. The inclusion of a subset equipped with the subset diffeology is an induction.

Example 3.C.7. Similarly, quotients of diffeological spaces inherit a natural diffeology via pushforward. Let $S$ be a diffeological space, $\sim$ be an equivalence relation on $S$ and set $R:=S / \sim$; the pushforward diffeology with respect to the quotient projection is also called quotient diffeology on $R$. In general, a surjective map of diffeological spaces $\pi: S \rightarrow R$ is called subduction when the diffeology on $R$ coincides with the pushforward diffeology on $R$. The quotient projection equipped with the quotient diffeology is a subduction.

The following is discussed in 1.55-1.56 in [38].

Proposition 3.C.8. If $S_{1}, S_{2}$ are diffeological spaces, the family of parametrisations $f$ : $U \rightarrow S_{1} \times S_{2}$ such that $p r_{1} \circ f$ and $p r_{2} \circ f$ are plots is a diffeology on $S_{1} \times S_{2}$. It coincides with the intersection of the pullback diffeologies with respect to the projections $p r_{1}$ and $\mathrm{pr}_{2}$ and is the coarsest diffeology such that both the projections are smooth. Moreover, it makes both the projections into subductions.

An analogous statement holds for the product $\prod_{i \in I} S_{i}$ of a family of diffeological spaces $S_{i}$ indexed by a set of indices $I$.

We call the diffeology from the proposition above product diffeology on $S_{1} \times$ $S_{2}$ (or on $\prod_{i \in I} S_{i}$ ).

Example 3.C.9. Using products and pullbacks, one sees that profinite dimensional manifolds are diffeological spaces. We spell out the details below, but the idea is, as usual, quite simple: with respect to a pf-atlas on the manifold, plots are those parametrisations whose truncations are smooth maps (or, equivalently, plots with respect to the smooth structure on elements of the tower).

Let $\mathbb{M}$ be a pf-manifold and $a: \mathbb{M} \rightarrow \mathbb{M}$. be a pf-atlas. Recall that $a$ can be seen as a map

$$
a: \mathbb{M} \rightarrow \underset{k}{\lim } \mathbb{M}_{k}
$$

The projective limit $\lim \mathbb{M}_{k}$ can be equipped with the (projective) limit diffeology, which is simply the subspace diffeology on $\lim \mathbb{M}_{k}$ seen as a subspace of the product $\prod_{k \in \mathbb{N}} \mathbb{M}_{k}$ equipped with the product diffeology. The limit diffeology can also be characterized as the coarsest diffeology such that all the projections $p r_{\infty, i}: \lim _{k} \mathbb{M}_{k} \rightarrow \mathbb{M}_{i}$ are smooth. One can then equip the pf-manifold $\mathbb{M}$ with the pullback diffeology with respect to the map $a$. Alternatively, this is the coarsest diffeology on $\mathbb{M}$ such that all the truncations $a_{i}: \mathbb{M} \rightarrow \mathbb{M}_{i}$ are smooth (which is the explicit description mentioned above). By using the definition of equivalent pf atlases (see Definition 3.A.7), one can see that the induced diffeology on $\mathbb{M}$ does not change if we replace the pf-atlas $a$ with an equivalent one. Actually, 
this diffeology is "finer" than the pf-smooth structure, in the sense that $C^{\infty}(\mathbb{M})$ is contained in the algebra of diffeologically smooth maps $\mathbb{M} \rightarrow \mathbb{R}$ because all the truncations are smooth.

\section{C.3 Functional diffeologies}

Here we discuss the main example we are interested in: functional diffeologies. The following is discussed in 1.57 in [38].

Proposition 3.C.10. Let $S_{1}, S_{2}$ be diffeological spaces. Consider the set of parametrisations $f: U \rightarrow C^{\infty}\left(S_{1}, S_{2}\right)$ such that the map

$$
\hat{f}: U \times S_{1} \rightarrow S_{2}, \quad(x, s) \rightarrow f(x)(s)
$$

is smooth with respect to the product diffeology on $U \times S_{1}$. This set defines a diffeology on $C^{\infty}\left(S_{1}, S_{2}\right)$. Moreover, it can be characterized as the coarsest diffeology such that the evaluation map

$$
e v: C^{\infty}\left(S_{1}, S_{2}\right) \times S_{1} \rightarrow S_{2}, \quad(g, s) \rightarrow g(s)
$$

is smooth.

Definition 3.C.11. The diffeology from the proposition above is called standard functional diffeology on the space $C^{\infty}\left(S_{1}, S_{2}\right)$. More in general, a functional diffeology is any diffeology making the evaluation map ev smooth.

Example 3.C.12. If $\mathbf{X}_{1}, \mathbf{X}_{2}$ are manifolds, the standard functional diffeology $C^{\infty}\left(\mathbf{X}_{1}, \mathbf{X}_{2}\right)$ is the one such that plots are smooth $k$-parametric families of smooth functions in the usual sense. Notice that, if $\pi: E \rightarrow \mathbf{X}$ is a surjective submersion, the space of sections $\Gamma(E)$ of $\pi$ inherits a natural diffeology by taking the subspace diffeology of the standard functional diffeology on $C^{\infty}(\mathbf{X}, E)$.

In this paper we deal mainly with sets of locally defined functions. For this reason, we need to adapt a little bit the construction above. For simplicity we now restrict ourselves to the case when $S_{1}=\mathbf{X}_{1}$ and $S_{2}=\mathbf{X}_{2}$ are smooth finite dimensional manifolds. Let $C_{\mathrm{loc}}^{\infty}\left(\mathbf{X}_{1}, \mathbf{X}_{2}\right)$ be the set of locally defined smooth functions from $\mathbf{X}_{1}$ to $\mathbf{X}_{2}$. The following proposition follows from the arguments of 1.63 in [38].

Proposition 3.C.13. Consider the family of parametrisation $f: U \rightarrow C_{\mathrm{loc}}^{\infty}\left(\mathbf{X}_{1}, \mathbf{X}_{2}\right)$ such that, for each $u \in U$, for each $x \in \operatorname{dom}(f(u))$ there is an open neighbourhood $W \subset U$ of $u$ and an open neighbourhood $V$ of $x$ contained in $\operatorname{dom}(f(v))$ for all $v \in W$ such that

$$
W \times V \rightarrow \mathbf{X}_{2}, \quad(v, p) \rightarrow f(v)(p)
$$

is smooth. This family defines a diffeology on $C_{\mathrm{loc}}^{\infty}\left(\mathbf{X}_{1}, \mathbf{X}_{2}\right)$.

Definition 3.C.14. We call the diffeology from the proposition above standard functional diffeology on $C_{\mathrm{loc}}^{\infty}\left(\mathbf{X}_{1}, \mathbf{X}_{2}\right)$.

Example 3.C.15. As before, this construction allows one to define a natural diffeology on $\Gamma_{\text {loc }}(E)$, where $E \rightarrow \mathbf{X}$ is a surjective submersion, by taking the subspace diffeology with respect to the inclusion in $C_{\mathrm{loc}}^{\infty}(\mathbf{X}, E)$. 
Example 3.C.16. When $\Gamma$ is a generalized pseudogroup supported by a Lie groupoid $\Sigma \rightrightarrows \mathbf{X}$, we can equip both $\Gamma$ and the germ groupoid $\operatorname{Germ}(\Gamma) \rightrightarrows \mathbf{X}$ with a natural diffeology inherited from $\Sigma$. This construction plays an important role in the main body of the paper (Definition 3.3.5).

First of all, notice that the set of locally defined bisections $\operatorname{Bis}_{\mathrm{loc}}(\Sigma)$ has the subspace diffeology with respect to the inclusion in the set of all locally defined maps $\mathbf{X} \rightarrow \Sigma$. Since $\Gamma$ is a pseudogroup supported by $\Sigma$ (Definition 3.3.1) $\Gamma$ itself carries the subspace diffeology, that we call diffeology induced by the support $\Sigma$.

Then, the set $\mathbf{X} \times \Gamma$ can be equipped with the product diffeology and the set

$$
S(\Gamma)=\{(x, \sigma) \in \mathbf{X} \times \Gamma: x \in \operatorname{dom}(\sigma)\}
$$

inherits the subspace diffeology. The groupoid $\operatorname{Germ}(\Gamma)$ is a quotient of $S(\Gamma)$ with respect to the equivalence relation identifying $(x, \sigma)$ with $\left(y, \sigma^{\prime}\right)$ if and only if $x=y$ and $\sigma$ has the same germ at $x$ as $\sigma^{\prime}$. We equip $\operatorname{Germ}(\Gamma)$ with the quotient diffeology and call it the diffeology induced by the support $\Sigma$ as well.

Of course, the same can be done using the equivalent picture of étale groupoids where we consider an étale Lie groupoid $\mathcal{G} \rightrightarrows \mathbf{X}$ supported by the Lie groupoid $\Sigma \rightrightarrows \mathbf{X}$, and the generalized pseudogroup $\operatorname{Bis}_{\text {loc }}(\mathcal{G})$ supported by $\Sigma \rightrightarrows \mathbf{X}$. In this case, we use the notation $S(\mathcal{G})$ in place of $S(\Gamma)$.

Remark 3.C.17. We would like to point out that the relevance of the example above for our purposes was brought to our attention by [45], where the same idea was used to discuss holonomy groupoids of foliatied bundles.

\section{C.4 The $D$-topology of a diffeology}

By means of diffeologies, one introduces some sort of differentiable structure on a "naked" set $S$. It turns out that a diffeology on $S$ induces a topology; furthermore, if $S=\mathrm{X}$ is a manifold and the diffeology is the one induced by the smooth structure (cf. Example 3.C.4), the topology induced by the diffeology coincides with the topology supporting the smooth structure.

Definition 3.C.18. The $D$-topology or diffeological topology on a diffeological space $(S, D)$ is the final topology with respect to the plots, that is the finest topology on $S$ such that all the maps in $D$ are continuous (we assume their domains to be equipped with the standard topology).

Recall that the above definition means that a subset $A \subset S$ is declared open if and only if $f^{-1}(A)$ is open for any plot $f$ of $D$. In [19] it is proven what follows.

Proposition 3.C.19. The D-topology on $(S, D)$ is completely determined by curves, i.e. $A \subset S$ is open if and only if $c^{-1}(A)$ is open in $\mathbb{R}$ for any smooth curve $c: \mathbb{R} \rightarrow S$.

The following proposition is 2.9 in [38].

Proposition 3.C.20. Let $f: S_{1} \rightarrow S_{2}$ be a smooth map of diffeological spaces. Then $f$ is continuous with respect to the diffeological topologies. 
In other words there is a faithful functor from the category of diffeological spaces to the category of topological spaces. It turns out that this functor is a left adjoint.

Proposition 3.C.21. Let $T$ be a topological space. The set of continuous functions from opens of Euclidean spaces to $T$ is a diffeology on $T$.

Definition 3.C.22. The diffeology from the above proposition is called continuous diffeology on $T$.

Remark 3.C.23. Of course, given a diffeological space $S$, the continuous diffeology of the $D$-topology of $S$ is in general different from the diffeology of $S$. For example, if $S=\mathbf{X}$ is a smooth manifold, its continuous diffeology is much finer than its smooth structure. In fact, all continuous functions on $\mathbf{X}$ are smooth with respect to the continuous diffeology.

Since a continuous map of topological spaces $T_{1} \rightarrow T_{2}$ is smooth with respect to the continuous diffeologies, one gets a functor from the category of topological spaces to the category of diffeological spaces.

Let us denote by Diffeo the category of diffeological spaces and smooth maps and by Top the category of topological spaces and continuous functions.

Proposition 3.C.24. The functor D : Diffeo $\rightarrow$ Top induced by the D-topology construction is left adjoint to the functor $C:$ Top $\rightarrow$ Diffeo induced by the continuous diffeology construction.

See [59], Proposition 3.1. A consequence of this is the following

Corollary 3.C.25. Let $S$ be a diffeological space and $R=S / \sim$ the quotient set by an equivalence relation. The D-topology of the quotient diffeology on $R$ is the same as the quotient topology of the D-topology of $S$.

On the other hand, for our purposes it is important to stress that in general the D-topology of a subspace diffeology is not the subspace topology induced by the Dtopology. In general [19],

Lemma 3.C.26. The subspace topology induced by a D-topology is coarser than the D-topology of the subspace diffeology.

Example 3.C.27. Let us consider $\mathbb{R}$ with the diffeology associated to its smooth structure and the subset $\mathbb{Q} \subset \mathbb{R}$. The $D$-topology on $\mathbb{R}$ is the standard one, and the subspace topology on $\mathbb{Q}$ makes it dense. However, the subspace diffeology on $\mathbb{Q}$ has only constant plots and its $D$-topology is the discrete one.

\section{C.5 Functional diffeology and Whitney topologies}

We conclude by stating the results from [19] concerning the $D$-topology for functional diffeologies. We used these results in the main body of the paper, see Proposition 3.3.12.

Let $\mathbf{X}_{1}, \mathbf{X}_{2}$ be smooth manifolds. Recall that the compact-open topology on continuous maps $C^{0}\left(\mathbf{X}_{1}, \mathbf{X}_{2}\right)$ is the topology where a sub-basis of neighbourhoods consist of sets

$$
A(K, W)=\left\{f \in C^{0}\left(\mathbf{X}_{1}, \mathbf{X}_{2}\right): f(K) \subset W\right\}
$$


indexed by pairs $K, W$ where $K$ is non-empty and compact in $\mathbf{X}_{1}$ and $W$ is open in $\mathbf{X}_{2}$. The strong topology on continuous maps $C^{0}\left(\mathbf{X}_{1}, \mathbf{X}_{2}\right)$ is the topology where a sub-basis of neighbourhoods of a function $f$ consist of sets

$$
A(W)=\left\{g \in C^{0}\left(\mathbf{X}_{1}, \mathbf{X}_{2}\right): G_{g} \subset W\right\}
$$

where $G_{g}$ is the graph of $g$ and $W$ is an open neighbourhood of $G_{f}$ in $\mathbf{X}_{1} \times \mathbf{X}_{2}$. The above topology can be defined for spaces of continuous sections of surjective submersions as well, seeing the space of section of a surjective submersion as a subspace of the space of functions.

The weak Whitney $C^{k}$-topology on the space $C^{\infty}\left(\mathbf{X}_{1}, \mathbf{X}_{2}\right)$ is the subspace topology induced by the compact open topology on the space of continuous sections of $J^{k}\left(\mathbf{X}_{1}, \mathbf{X}_{2}\right)$. Analogously, the strong Whitney $C^{k}$-topology on $C^{\infty}\left(\mathbf{X}_{1}, \mathbf{X}_{2}\right)$ is the subspace topology induced by the strong topology on the space of continuous sections of $J^{k}\left(\mathbf{X}_{1}, \mathbf{X}_{2}\right)$. Here, $k$ can be taken to be $\infty$; again, one can use the subspace topology to deal with spaces of smooth sections.

It is possible to describe an explicit sub-basis for the weak and strong Whitney $C^{k}$-topologies. We are interested in the case when $k=\infty$. If $f \in C^{\infty}\left(\mathbf{X}_{1}, \mathbf{X}_{2}\right)$, for each chart $(\phi, U)$ of $\mathbf{X}_{1}$, compact set $K \subset U$, chart $(\psi, V)$ of $\mathbf{X}_{2}$ such that $f(K) \subset V$ and $\epsilon>0$, one has the set

$$
B^{r}(f ;(\phi, U), K,(\psi, V), \epsilon)
$$

defined as the set of functions $g \in C^{\infty}\left(\mathbf{X}_{1}, \mathbf{X}_{2}\right)$ such that

$g(K) \subset V,\left\|D^{k}\left(\psi \circ f \circ \phi^{-1}(x)\right)-D^{k}\left(\psi \circ g \circ \phi^{-1}(x)\right)\right\|<\epsilon$, for all $x \in \phi(K), k \in \mathbb{N}$.

The above sets, for any choice of $f,(\phi, U), K,(\psi, V)$, and $\epsilon$, define a sub-basis for the weak Whitney $C^{\infty}$-topology. Furthermore, for any choice of $f \in C^{\infty}\left(\mathbf{X}_{1}, \mathbf{X}_{2}\right)$, family of charts $\Phi=\left\{\phi_{i}, U_{i}\right\}_{i \in I}$ of $\mathbf{X}_{1}$ which is locally finite, family of compact sets $K=\left\{K_{i}\right\}_{i \in I}$, family of charts $\Psi=\left\{\psi_{i}, V_{i}\right\}_{i \in I}$ such that $f\left(K_{i}\right) \subset V_{i}$ and family of positive numbers $E=\left\{\epsilon_{i}\right\}_{i \in I}$, one has the set

$$
B^{r}(f ; \Phi, K, \Psi, E)
$$

defined as the set of functions $g \in C^{\infty}\left(\mathbf{X}_{1}, \mathbf{X}_{2}\right)$ such that, for all $i \in I$,

$g\left(K_{i}\right) \subset V_{i},\left\|D^{k}\left(\psi_{i} \circ f \circ \phi_{i}^{-1}(x)\right)-D^{k}\left(\psi_{i} \circ g \circ \phi_{i}^{-1}(x)\right)\right\|<\epsilon_{i}$, for all $x \in \phi_{i}(K), k \in \mathbb{N}$.

The family of sets as above is a sub-basis for the strong Whitney $C^{\infty}$-topology.

It is clear that the weak $C^{\infty}$-topology is coarser than or equal to the strong Whitney $C^{\infty}$-topology. If $\mathbf{X}_{1}$ is compact then these two topologies coincide. If $\mathbf{X}_{1}$ is non-compact and $\mathbf{X}_{2}$ has positive dimension, then the weak Whitney $C^{\infty}$ topology is strictly coarser.

In [19], it is proven that:

Theorem 3.C.28. The D-topology of the standard functional diffeology on $C^{\infty}\left(\mathbf{X}_{1}, \mathbf{X}_{2}\right)$ is finer than or equal to the weak Whitney $C^{\infty}$-topology and coarser than or equal to the strong Whitney $C^{\infty}$-topology. 


\section{Bibliography}

[1] L. Accornero and M. N. Crainic. Haefliger's differentiable cohomology. 2020. arXiv: 2012.07777 [math.DG] .

[2] C. Albert and P. Molino. Pseudogroupes de Lie transitifs. I. Structures principales. Travaux en Cours. Hermann, Paris, 1984, pp. iii+142. ISBN: 2-70565978-1.

[3] D. V. Alekseevsky and P. W. Michor. "Differential geometry of Cartan connections". In: Publ. Math. Debrecen 47.3-4 (1995), pp. 349-375. ISSN: 00333883.

[4] C. Arias Abad and M. N. Crainic. "Representations up to homotopy and Bott's spectral sequence for Lie groupoids". In: Adv. Math. 248 (2013), pp. 416-452. ISSN: 0001-8708. DOI: $10.1016 / \mathrm{j}$.aim.2012.12.022. URL: https://doi.org/10.1016/j.aim.2012.12.022.

[5] M. Benalili. "Fibrés naturels sur la catégorie des $\Gamma$-variétés". In: Rend. Circ. Mat. Palermo (2) 43.3 (1994), 309-328 (1995). ISSN: 0009-725X. DOI: 10 . 1007 /BF 02844245 . URL: https: / / doi .org/10.1007/BF02844245.

[6] I. N. Bernšteľn and B. I. Rosenfel'd. "Homogeneous spaces of infinitedimensional Lie algebras and the characteristic classes of foliations". In: Uspehi Mat. Nauk 28.4(172) (1973), pp. 103-138. ISSN: 0042-1316.

[7] I. N. Bernštel̆n and B. I. Rozenfel'd. "Characteristic classes of foliations". In: Funkcional. Anal. i Priložen. 6.1 (1972), pp. 68-69. ISSN: 0374-1990.

[8] A. D. Blaom. "Geometric structures as deformed infinitesimal symmetries". In: Trans. Amer. Math. Soc. 358.8 (2006), pp. 3651-3671. ISSN: 0002-9947. DOI: 10.1090/s0002-9947-06-04057-8. URL: https: / / doi.org/10 . 1090/S0002-9947-06-04057-8.

[9] R. Bott. "Lectures on characteristic classes and foliations". In: Lectures on algebraic and differential topology (Second Latin American School in Math., Mexico City, 1971). Notes by Lawrence Conlon, with two appendices by J. Stasheff. 1972, 1-94. Lecture Notes in Math., Vol. 279.

[10] R. Bott. "On characteristic classes in the framework of Gelfand-Fuks cohomology". In: "Analyse et Topologie" en l'Honneur de Henri Cartan (Orsay, 1974). 1976, 113-139. Astérisque, No. 32-33.

[11] R. Bott and A. Haefliger. "On characteristic classes of $\Gamma$-foliations". In: Bull. Amer. Math. Soc. 78.6 (Nov. 1972), pp. 1039-1044. URL: https : //projecteuclid.org:443/euclid.bams/1183534148. 
[12] A. Čap and J. Slovák. Parabolic geometries: background and general theory. AMS, 2009. ISBN: 978-0-8218-2681-2.

[13] É. Cartan. "Sur la structure des groupes infinis de transformation". fre. In: Annales scientifiques de l'École Normale Supérieure 21 (1904), pp. 153-206. URL: http://eudml.org/doc/81204.

[14] É. Cartan. "Sur la structure des groupes infinis de transformation (suite)". fre. In: Annales scientifiques de l'École Normale Supérieure 22 (1905), pp. 219308. URL: http: / / eudml .org/doc/81219.

[15] F. Cattafi. A general approach to almost structures in geometry. Ph.D. Thesis. 2020.

[16] F. Cattafi. "Cartan geometries and multiplicative forms". In: Differential Geom. Appl. 75 (2021), pp. 101722, 20. ISSN: 0926-2245. DOI: 10 . 1016 / j.difgeo.2021 . 101722. URL: https : / / doi .org / $10.1016 / j . d i f g e o .2021 .101722$.

[17] F. Cattafi, M. N. Crainic, and M. A. Salazar. "From PDEs to Pfaffian fibrations". In: L'Enseignement Mathématique 66.1 (2020), 187-250. ISSN: 00138584. DOI: $10.4171 / \mathrm{lem} / 66-1 / 2-10$. URL: http://dx.doi.org/10 . 4171/LEM/66-1/2-10.

[18] S. S. Chern. "The geometry of G-structures". In: Bull. Amer. Math. Soc. 72 (1966), pp. 167-219. ISSN: 0002-9904. DOI: 10.1090/S0002-9904-196611473-8. URL: https : / / doi .org/10 .1090/S0002-9904-1966$11473-8$.

[19] J. D. Christensen, G. Sinnamon, and E. Wu. “The D-topology for diffeological spaces". In: Pacific J. Math. 272.1 (2014), pp. 87-110. ISSN: 0030-8730. DOI: $10.2140 / \mathrm{pjm} .2014 .272$.87. URL: https://doi .org/10.2140/ pjm.2014.272.87.

[20] A. Connes and H. Moscovici. "Hopf algebras, cyclic cohomology and the transverse index theorem". In: Comm. Math. Phys. 198.1 (1998), pp. 199-246. ISSN: 0010-3616. DOI: 10.1007 / s002200050477. URL: https : / / doi . org/10.1007/s002200050477.

[21] M. N. Crainic. "Differentiable and algebroid cohomology, van Est isomorphisms, and characteristic classes". In: Comment. Math. Helv. 78.4 (2003), pp. 681-721. ISSN: 0010-2571. DOI: 10.1007 /s $00014-001-0766-9$. URL: https://doi.org/10.1007/s00014-001-0766-9.

[22] M. N. Crainic and R. L. Fernandes. "Integrability of Lie brackets". In: Ann. of Math. (2) 157.2 (2003), pp. 575-620. ISSN: 0003-486X. DOI: 10 . 4007 / annals.2003.157.575. URL: https://doi.org/10.4007/annals. 2003.157 .575$.

[23] M. N. Crainic, I. Struchiner, and M. A. Salazar. "Multiplicative forms and Spencer operators". In: Mathematische Zeitschrift 279 (Apr. 2015), pp. 939979. ISSN: 1432-1823. DOI: 10.1007 / s00209-014-1398-z. URL: https: //doi.org/10.1007/s00209-014-1398-z.

[24] M. L. del Hoyo. "Lie groupoids and their orbispaces". In: Portugaliae Mathematica 70.2 (2013), 161-209. ISSN: 0032-5155. DOI: 10 . $4171 / \mathrm{pm} / 1930$. URL: http://dx.doi.org/10.4171/PM/1930. 
[25] M. L. del Hoyo and R. L. Fernandes. "Riemannian metrics on Lie groupoids". In: J. Reine Angew. Math. 735 (2018), pp. 143-173. ISSN: 0075-4102. DOI: 10 . 1515 / crelle - 2015 - 0018. URL: https : //doi.org/10.1515/crelle-2015-0018.

[26] J. L. Dupont. Curvature and characteristic classes. Lecture Notes in Mathematics, Vol. 640. Springer-Verlag, Berlin-New York, 1978, pp. viii+175. ISBN: 3-540-08663-3.

[27] D. J. Eck. "Gauge-natural bundles and generalized gauge theories". In: Mem. Amer. Math. Soc. 33.247 (1981), pp. vi+48. ISSN: 0065-9266. DOI: 10 . $1090 /$ memo/0247. URL: https://doi.org/10.1090/memo/0247.

[28] D. B. A. Epstein and W. P. Thurston. "Transformation groups and natural bundles". In: Proc. London Math. Soc. (3) 38.2 (1979), pp. 219-236. ISSN: 00246115. DOI: 10.1112 /plms/s3-38 .2 .219. URL: https:// doi .org/ $10.1112 / \mathrm{plms} / \mathrm{s} 3-38.2 .219$.

[29] L. Fatibene and M. Francaviglia. Natural and Gauge Natural Formalism for Classical Field Theories. Springer Netherlands, 2003. ISBN: 978-94-017-23848.

[30] D. B. Fuks. "Non-trivialité des classes caractéristiques de g-structures. Applications aux variations des classes caractéristiques de feuilletages". In: $C$. R. Acad. Sci. Paris Sér. A-B 284.18 (1977), A1105-A1107. ISSN: 0151-0509.

[31] I. M. Gel'fand and D. B. Fuks. "Cohomology of the Lie algebra of formal vector fields". English. In: Math. USSR, Izv. 4 (1971), pp. 327-342. ISSN: 00255726. DOI: 10.1070 / IM1970v004n02ABEH000908.

[32] H. Goldschmidt. "Integrability criteria for systems of nonlinear partial differential equations". In: J. Differential Geometry 1 (1967), pp. 269-307. ISSN: 0022-040X. URL: http : / / projecteuclid . org / euclid.jdg / 1214428094.

[33] B. Güneysu and M. J. Pflaum. "The profinite dimensional manifold structure of formal solution spaces of formally integrable PDEs". In: SIGMA Symmetry Integrability Geom. Methods Appl. 13 (2017), Paper No. 003, 44. DOI: 10 . 3842 / SIGMA . 2017 .003. URL: https : / / doi .org/10 .3842 / SIGMA. 2017.003.

[34] A. Haefliger. Cohomology theory of étale topological groupoids. Unpublished.

[35] A. Haefliger. "Differential Cohomology". In: Differential Topology. Berlin, Heidelberg: Springer Berlin Heidelberg, 2011, pp. 19-70. ISBN: 978-3-64211102-0. DOI: 10 . 1007 /978-3-642-11102-0_3. URL: https: / / doi . org/10.1007/978-3-642-11102-0_3.

[36] A. Haefliger. "Structures feuilletées et cohomologie à valeur dans un faisceau de groupoïdes". In: Comment. Math. Helv. 32 (1958), pp. 248-329. ISSN: 0010-2571. DOI: 10 . 1007 / BF 02564582 . URL: https : / / doi . org/ 10 . $1007 / \mathrm{BF} 02564582$.

[37] M. W. Hirsch. Differential topology. Graduate Texts in Mathematics, No. 33. Springer-Verlag, New York-Heidelberg, 1976, pp. x+221. 
[38] P. Iglesias-Zemmour. Diffeology. Vol. 185. Mathematical Surveys and Monographs. American Mathematical Society, Providence, RI, 2013, pp. xxiv+439. ISBN: 978-0-8218-9131-5. DOI: 10 . 1090 / surv / 185. URL: https://doi.org/10.1090/surv/185.

[39] F. W. Kamber and P. Tondeur. Foliated bundles and characteristic classes. Lecture Notes in Mathematics, Vol. 493. Springer-Verlag, Berlin-New York, 1975, pp. xiv+208.

[40] I. Kolář, P. W. Michor, and J. Slovák. Natural operations in differential geometry. Springer-Verlag, Berlin, 1993, pp. vi+434. ISBN: 3-540-56235-4. DOI: 10 . 1007 / 978-3-662-02950-3. URL: https : / / doi .org/10 . 1007 / $978-3-662-02950-3$.

[41] J. Krasil'shchik and A. Verbovetsky. "Geometry of jet spaces and integrable systems". In: J. Geom. Phys. 61.9 (2011), pp. 1633-1674. ISSN: 0393-0440. DOI: $10.1016 / j$.geomphys.2010.10.012. URL: https: // doi.org/10 . $1016 / j$.geomphys.2010.10.012.

[42] N. P. Landsman. "Bicategories of operator algebras and Poisson manifolds". In: Mathematical physics in mathematics and physics (Siena, 2000). Vol. 30. Fields Inst. Commun. Amer. Math. Soc., Providence, RI, 2001, pp. 271-286.

[43] C. Laurent-Gengoux, M. Stiénon, and P. Xu. "Holomorphic Poisson manifolds and holomorphic Lie algebroids". In: Int. Math. Res. Not. IMRN (2008), Art. ID rnn 088, 46. ISSN: 1073-7928. DOI: 10.1093 / imrn/ rnn088. URL: https://doi.org/10.1093/imrn/rnn088.

[44] S. Lie. Theory of Transformation Groups I. Translation. Springer, 2015. ISBN: 978-3-662-46211-9.

[45] L. E. MacDonald. Hierarchies of holonomy groupoids for foliated bundles. 2021. arXiv: 2004.13929 [math.DG] .

[46] K. C. H. Mackenzie. "Ehresmann doubles and Drinfel'd doubles for Lie algebroids and Lie bialgebroids". In: J. Reine Angew. Math. 658 (2011), pp. 193245. ISSN: 0075-4102. DOI: 10 . 1515 / CRELLE . 2011 . 092. URL: https : //doi.org/10.1515/CRELLE.2011.092.

[47] J. N. Mestre. Differentiable stacks: stratifications, measures and deformations. Ph.D. Thesis. 2016.

[48] I. Moerdijk. "Proof of a conjecture of A. Haefliger". In: Topology 37.4 (1998), pp. 735-741. ISSN: 0040-9383. DOI: 10 .1016/s0040-9383 (97) $00053-0$. URL: https://doi.org/10.1016/S0040-9383(97)00053-0.

[49] I. Moerdijk and J. Mrčun. Introduction to foliations and Lie groupoids. Vol. 91. Cambridge Studies in Advanced Mathematics. Cambridge University Press, Cambridge, 2003, pp. x+173. ISBN: 0-521-83197-0. DOI: 10 . 1017 / CBO9780511615450. URL: https : / / doi .org/10 .1017/ CBO9780511615450.

[50] T. Mokri. “Matched pairs of Lie algebroids". In: Glasgow Math. J. 39.2 (1997), pp. 167-181. ISSN: 0017-0895. DOI: 10.1017 / S0 017089500032055. URL: https://doi.org/10.1017/s0017089500032055. 
[51] P. Molino. Riemannian foliations. Vol. 73. Progress in Mathematics. Translated from the French by Grant Cairns, With appendices by Cairns, Y. Carrière, É. Ghys, E. Salem and V. Sergiescu. Birkhäuser Boston Inc., Boston, MA, 1988, pp. xii+339. ISBN: 0-8176-3370-7. DOI: 10 . 1007 / $978-$ 1-4684-8670-4. URL: https: / / doi .org/10.1007/978-1-4684$8670-4$.

[52] J. Mrčun. Stability and invariants of Hilsum-Skandalis maps. Ph.D. Thesis. 1996. arXiv: 0506484 [math.DG].

[53] A. Nijenhuis. "Natural bundles and their general properties". In: Differential Geometry in Honor of K. Yano, Kinokuniya, Tokyo (1972), pp. 317-334.

[54] R. S. Palais and C. L. Terng. "Natural bundles have finite order". In: Topology 19.3 (1977), pp. 271-277. ISSN: 0040-9383. DOI: 10 . $1016 / 0040-$ 9383(77) 90008-8. URL: https: / / doi .org/10 .1016/0040$9383(77) 90008-8$.

[55] M. A. Salazar. Pfaffian groupoids. Ph.D. Thesis. 2013. arXiv: 1306.1164 [math.DG] .

[56] G. Segal. "Classifying spaces and spectral sequences". In: Inst. Hautes Études Sci. Publ. Math. 34 (1968), pp. 105-112. ISSN: 0073-8301. URL: http://www.numdam.org/item?id=PMIHES_1968_34_105_0.

[57] J.P. Serre and Harvard University. Lie Algebras and Lie Groups: 1964 Lectures Given at Harvard University. Lecture Notes in Mathematics No. 1500. Springer, 1992. ISBN: 9783540550082. URL: https: / / books . google.it/ books? id=3CQATGLDneUC.

[58] R.W. Sharpe. Differential geometry - Cartan's Generalization of Klein's Erlangen Program. Springer-Verlag New York, 1997. ISBN: 978-0-387-94732-7.

[59] K. Shimakawa, K. Yoshida, and T. Haraguchi. "Homology and cohomology via enriched bifunctors". In: Kyushu J. Math. 72.2 (2018), pp. 239-252. ISSN: 1340-6116. DOI: 10 . 2206 / kyushujm . 72 . 239. URL: https : / / doi . org/10.2206/kyushujm.72.239.

[60] I. M. Singer and S. Sternberg. "The infinite groups of Lie and Cartan. I. The transitive groups". In: J. Analyse Math. 15 (1965), pp. 1-114. ISSN: 0021-7670. DOI: 10 . 1007 / BF02787690. URL: https : / / doi . org / 10 . 1007 / $\mathrm{BF} 02787690$.

[61] J. Slovák. "Actions of jet groups on manifolds". In: Differential geometry and its applications (Brno, 1989). World Sci. Publ., Teaneck, NJ, 1990, pp. 178-186.

[62] J. Slovák. "Bundle functors on fibred manifolds". In: Ann. Global Anal. Geom. 9.2 (1991), pp. 129-143. ISSN: 0232-704X. DOI: 10 . 1007 / BF00776852. URL: https://doi.org/10.1007/BF00776852.

[63] D. C. Spencer. "Flat differential operators". In: Symposium on Several Complex Variables (Park City, Utah, 1970). 1971, 85-108. Lecture Notes in Math., Vol. 184.

[64] S. Sternberg. Lectures on differential geometry. Prentice-Hall Inc. Englewood Cliffs, N.J., 1964, pp. xv+390. 
[65] C. L. Terng. "Natural vector bundles and natural differential operators". In: Amer. J. Math. 100.4 (1978), pp. 775-828. ISSN: 0002-9327. DOI: 10 . 2307 / 2373910. URL: https://doi.org/10.2307/2373910.

[66] W. T. van Est. "Une application d'une méthode de Cartan-Leray". In: Nederl. Akad. Wetensch. Proc. Ser. A. 58 = Indag. Math. 17 (1955), pp. 542-544.

[67] R. Wang. On Integrable Systems \& Rigidity for PDEs with Symmetry. Ph.D. Thesis. 2017.

[68] P. Xu. "Morita equivalent symplectic groupoids". In: Symplectic geometry, groupoids, and integrable systems (Berkeley, CA, 1989). Vol. 20. Math. Sci. Res. Inst. Publ. Springer, New York, 1991, pp. 291-311. DOI: 10 . 1007 / $978-$ 1-4613-9719-9\_20. URL: https:// doi .org/10 .1007/978-14613-9719-9_20.

[69] O. Yudilevich. Lie pseudogroups à la Cartan from a modern perspective. Ph.D. Thesis. 2016.

[70] A. Zajtz. "Actions of some infinite Lie groups". In: Aequationes Math. 34.2-3 (1987), pp. 139-146. ISSN: 0001-9054. DOI: 10 . 1007 / BF 01830665. URL: https://doi.org/10.1007/BF01830665.

[71] A. Zajtz. "The sharp upper bound on the order of natural bundles of given dimensions". In: Bull. Soc. Math. Belg. Sér. B 39.3 (1987), pp. 347-357. ISSN: $0037-9476$. 


\section{Summary}

This thesis focuses on Lie pseudogroups. Lie pseudogroups evolved from the continuous transformation groups introduced by Sophus Lie in collaboration with Friedrich Engel [44]. They are, roughly speaking, sets of local transformations of manifolds that are solutions of some system of partial differential equations. They often arise as the sets of local symmetries of geometric structures. Here, a manifold is a "curved" geometric object, such as a sphere or a donut. A geometric structure on a manifold is some additional geometric data on such a curved object; for instance, a way to measure distances or a notion of area.

Let us try to be clearer.

- Manifolds are curved geometric objects that locally look like flat ones; that is, a manifold of dimension $n$ locally looks like the Euclidean space $\mathbb{R}^{n}$. A good way of visualizing this concept is to think of a sphere: around each point on the sphere we can cut out a small region and stretch it until it looks like a plane.

- Our geometric structures on manifolds always come with "flat versions" of themselves, living on the Euclidean space. For example, the flat version of a Riemannian metric - which one should think of as a notion of "length" - is simply the usual scalar product on the Euclidean space, corresponding to the standard/intuitive notion of "length". A geometric structure on a manifold is called integrable when it locally looks like its flat version. We can phrase this slightly differently: a geometric structure that is obtained by "patching" together some flat local models is called integrable.

- Our integrable geometric structures are, in a sense, solutions of some system of partial differential equations. For example, a notion of "length" on a curved space can be packed into an object called a tensor, which intuitively is a collection of functions locally defined on the space. In order to encode a "length", the tensor needs to be metric - i.e., the elements of the collection need to satisfy some algebraic relations. The integrable version of the notion of "length" on a manifold corresponds to a flat metric tensor; that is, a tensor satisfying some additional differential relations - i.e. relations involving the rates of change of the tensor itself along the manifold.

Given an integrable structure, the way in which the flat local models are glued together is controlled by a certain collection of locally defined transformations of the Euclidean space. Intuitively, each transformation encodes a rule to glue one 
local model to another. Such a collection of transformations has some additional properties involving composition, restriction and gluing of transformations. Furthermore, the transformations in the collection are solutions of some system of partial differential equations, determined by the geometric structure under consideration. A collection of transformations with such properties is called a Lie pseudogroup.

We use the notation $\Gamma$ to denote Lie pseudogroups. Integrable geometric structures whose "gluing rules" are elements of $\Gamma$ are called $\Gamma$-structures. Throughout this thesis, we often deal with the case when the geometric structure at hand is integrable only "up to a certain order". By this we mean, loosely speaking, that the elements of $\Gamma$ that form the "gluing rules" of the integrable version of the structure get replaced by transformations that are solutions of the differential equations underlying $\Gamma$ only up to order $k \in \mathbb{N}$. We call these almost T-structures.

In this thesis, we made use of some modern geometric tools to review, explore and generalize some classical results about pseudogroups, (almost) $\Gamma$-structures, and related matters. Below, we provide some more explanation.

\section{Chapter 1: transitivity of pseudogroups}

We call a pseudogroup $\Gamma$ transitive when, given two points in the Euclidean space, there is a transformation in the pseudogroup sending one point to the other. In the first chapter of this thesis, we investigate almost $\Gamma$-structures where $\Gamma$ is transitive.

We made use of tools such as Lie groupoids , Pfaffian forms, and the notion of (Pfaffian) Morita equivalence to show that almost $\Gamma$-structures can be re-encoded into simpler (and easier to handle) objects; in technical terms, principal bundles equipped with certain vector valued forms, which we call Cartan bundles.

Very loosely speaking, the reader may visualize a groupoid as a collection of arrows starting and ending at points of some fixed manifold. When a pseudogroup is given, one can construct several groupoids associated to it; the idea is that each transformation belonging to the pseudogroup and defined around a fixed point determines an arrow targeting the image of the point. This idea can be implemented in various ways. The main choice involves how much of the information carried by a transformation in the pseudogroup should be retained by the corresponding arrow in the groupoid (technically speaking, one takes either germ or a $k$-jet, for a chosen $k \in \mathbb{N}$, of the transformation around the chosen point). Since our pseudogroups are well behaved (they arise as sets of solutions of well behaved partial differential equations), the groupoids that one constructs out of them are also very well behaved; in particular, they are manifolds, and are called Lie groupoids.

The Lie groupoids constructed out of a pseudogroup are in general simpler objects than the pseudogroup itself, and carry less information. In a sense, (part of) the information needed to reconstruct the pseudogroup out of the groupoid is stored into a geometric object called a Pfaffian form. Studying Lie groupoids equipped with Pfaffian forms - which are called Pfaffian groupoids - is an efficient way of studying pseudogroups and geometric structures. An important part of the machinery is the notion of Pfaffian Morita equivalence, a relation between pairs 
of Pfaffian groupoids.

The key remark for us was that transitive Lie pseudogroups corresponds to Pfaffian groupoids which are Pfaffian Morita equivalent to very simple objects (technically, Lie groups equipped with two objects: a subalgebra of their Lie algebra, and a representation interacting nicely with the subalgebra). From this remark, we were able to discover Cartan bundles as a way to encode almost $\Gamma$-structures.

There are two very remarkable example of Cartan bundles: abstract $G$ structures and Cartan geometries. Both have been extensively studied in the literature, and can be traced back to pioneering work of Élie Cartan.

\section{Chapter 2: pseudogroups and foliations}

A codimension $q$ foliation, $q \in \mathbb{N}, 0 \leq q \leq n$, on a manifold $M$ of dimension $n$ is, loosely speaking, a partition of the manifold into submanifolds, called leaves, of dimension $n-q$, which locally looks like the partition of the Euclidean space $\mathbb{R}^{n}$ by "parallel" copies of $\mathbb{R}^{n-q}$.

An equivalent definition of foliation in terms of pseudogroups has been given by André Haefliger [36]. The intuition behind the construction is that a foliation always has some local symmetries, given by the fact that we can "reshuffle" the copies of $\mathbb{R}^{n-q}$ in $\mathbb{R}^{n}$ without changing the foliation itself. Intuitively, this corresponds to making a transformation of $\mathbb{R}^{n}$ that is induced by a transformation of the copy of $\mathbb{R}^{q}$ perpendicular to the leaves. Building on this, Haefliger has reencoded foliations in terms of an algebro-geometric object called $\Gamma^{q}$-cocycle; here, $\Gamma^{q}$ denotes the pseudogroup of all locally defined transformations of $\mathbb{R}^{q}$.

This reformulation of the notion of foliation turns out to be useful to discuss certain invariants of foliations (technically, certain cohomology classes). In particular, Haefliger has built an algebraic object (to be precise, the cohomology of a certain bicomplex) out of the pseudogroup $\Gamma^{q}$, and has proven that known invariants of foliations can be organized into a characteristic map defined on this object.

In the second chapter of this thesis we reviewed Haefliger's construction and extended it not only to arbitrary Lie pseudogroups but also to more general objects: Lie groupoids equipped with a flat multiplicative connection.

\section{Chapter 3: actions of pseudogroups}

When $G$ is a group, an action of $G$ on a manifold $E$ is a map associating to each element of $G$ a transformation of $M$ in a way compatible with the group structure of $G$. One can extend this notion to pseudogroups. Very loosely speaking, an action of $\Gamma$ on a manifold $E$ is a map associating to each element of $\Gamma$ a locally defined transformation of $E$; this map is compatible with the restriction and composition of transformations in $\Gamma$.

It turns out that this notion is better phrased by passing to the groupoid $\operatorname{Germ}(\Gamma)$ of germs of elements in $\Gamma$. Intuitively, given an element of $\Gamma$ - i.e. a locally defined transformation - its germ around a point packs the behaviour of the map in an arbitrarily small neighbourhood of the point. Among the various groupoids that one can attach to a pseudogroup, $\operatorname{Germ}(\Gamma)$ retains the most information; studying pseudogroups is the same as studying groupoids of germs. 
In the third chapter of this thesis we adopted this point of view. A first interesting observation one can make is that actions of a pseudogroup $\Gamma$ turn out to be equivalently described by $\Gamma$-natural bundles. When $\Gamma^{n}$ is the pseudogroup of local transformations of $\mathbb{R}^{n}$, we simply talk about natural bundles. Natural bundles can be traced back to Nijenhuis [53], and have been extensively studied.

A quite remarkable fact about natural bundles is the finite order property. Looking at natural bundles as actions of the pseudogroup of local transformations of $\mathbb{R}^{n}$, one sees, roughly speaking, that the action of a transformation $\phi$ depends only on the $k$-th order Taylor expansion of $\phi$. This is the core content of the finite order theorem. The first version of such a Theorem was proven by Palais and Terng [54]; a little time later, Epstein and Thurston proved a stronger statement [28].

We focused on the finite order theorem and its proof, reviewing them from the novel point of view of actions of groupoids. From such a point of view, the finite order theorem states that the action of the germ groupoid $\operatorname{Germ}\left(\Gamma^{n}\right)$ is completely determined by the action of the jet groupoid $J^{k} \Gamma^{n}$, for some $k \in \mathbb{N} . J^{k} \Gamma^{n}$ is the groupoid whose elements are $k$-jets of transformations at a point. Loosely speaking, arrows in $J^{k} \Gamma^{n}$ corresponds to the $k$-th order Taylor expansions of transformations in $\Gamma^{n}$; as a consequence, $J^{k} \Gamma^{n}$ is a simpler object than $\operatorname{Germ}\left(\Gamma^{n}\right)$, since arrows in $J^{k} \Gamma^{n}$ contain less information about elements of $\Gamma^{n}$ than arrows in $\operatorname{Germ}\left(\Gamma^{n}\right)$. For this reason, the finite order theorem is a remarkable result.

By adopting the point of view of groupoids, we were naturally lead to investigate for which pseudogroups the finite order theorem holds true. Furthermore, apart from shedding some new light on the nature of the problem, our approach allowed us to explicitly explore some interesting insights that are implicit in [54] and [28]. 


\section{Samenvatting}

Dit proefschrift richt zich op Lie pseudogroepen. Lie pseudogroepen zijn voortgekomen uit de continue transformatiegroepen geïntroduceerd door Sophus Lie in samenwerking met Friedrich Engel [44]. Het zijn, ruwweg, verzamelingen van lokale transformaties van variëteiten die oplossingen zijn van een stelsel van partiële differentiaalvergelijkingen. Ze ontstaan vaak als verzamelingen van lokale symmetrieën van geometrische structuren. Een variëteit is een "gekromd" geometrisch object, zoals een bol of een donut. Een geometrische structuur op een variëteit bestaat uit enkele aanvullende geometrische gegevens over zo'n gekromd object; bijvoorbeeld een manier om afstanden te meten of een notie van oppervlakte.

Laten we proberen duidelijker te zijn.

- Variëteiten zijn gekromd geometrische objecten die lokaal lijken op platte; dat wil zeggen, een variëteit van dimensie $n$ lokaal lijkt op de Euclidische ruimte $\mathbb{R}^{n}$. Een goede manier om dit concept te visualiseren, is door aan een bol te denken: ond elk punt op de bol kunnen we een klein gebied uitsnijden en uitrekken tot het op een vlak lijkt.

- Onze geometrische structuren op variëteiten komen altijd met "platte versies" van zichzelf, welke leven op de Euclidische ruimte. Bijvoorbeeld, de platte versie van een Riemanniaanse metriek - wat je kan zien als een begrip van "lengte" - is gewoon het gebruikelijke scalaire product op de Euclidische ruimte, corresponderend met de standaard/intuïtieve notie van "lengte". Een geometrische structuur op een variëteit wordt integreerbaar genoemd als hij er lokaal uitziet als zijn platte versie. We kunnen dit iets anders formuleren: een geometrische structuur die wordt verkregen door enkele vlakke lokale modellen samen te voegen, wordt integreerbaar genoemd.

- Onze integreerbare geometrische structuren zijn in zekere zin oplossingen van een stelsel van it partiële differentiaalvergelijkingen. Een notie van "lengte" op een gekromde ruimte kan bijvoorbeeld worden verpakt in een object met de naam tensor, dat intuitief een verzameling van functies is die lokaal in de ruimte zijn gedefinieerd. Om een "lengte" te coderen, moet de tensor metrisch zijn - d.w.z. de elementen van de verzameling moeten aan een aantal algebraïsche relaties voldoen. De integreerbare versie van het begrip "lengte" op een variëteit komt overeen met een platte metrische tensor; 
dat wil zeggen, een tensor die voldoet aan enkele aanvullende differentiaal relaties - d.w.z. relaties met betrekking tot de mate van verandering van de tensor zelf langs de variëteit.

Gegeven een integreerbare structuur, de manier waarop de platte lokale modellen aan elkaar worden gelijmd wordt bepaald door een bepaalde verzameling lokaal gedefinieerde transformaties van de Euclidische ruimte. Intuïtief codeert elke transformatie een instructie om het ene lokale model aan het andere te lijmen. Zo'n verzameling transformaties heeft enkele extra eigenschappen met betrekking tot samenstelling, restrictie en lijmen. Bovendien zijn de transformaties in de verzameling oplossingen van een stelsel van partiële differentiaalvergelijkingen bepaald door de geometrische structuur in kwestie. Een verzameling transformaties met dergelijke eigenschappen wordt een Lie pseudogroep genoemd.

We gebruiken de notatie $\Gamma$ om Lie pseudogroepen aan te duiden. Integreerbare geometrische structuren waarvan de "lijmregels" elementen zijn van $\Gamma$ worden $\Gamma$-structuren genoemd. In dit proefschrift behandelen we vaak het geval waarin de geometrische structuur in kwestie slechts "tot een bepaalde orde" kan worden geïntegreerd. Hiermee bedoelen we, ruwweg, dat de elementen van $\Gamma$ die de "lijmregels" van de integreerbare versie van de structuur vormen, worden vervangen door transformaties die oplossingen zijn van de differentiaalvergelijkingen die ten grondslag liggen aan $\Gamma$ alleen tot op volgorde $k \in \mathbb{N}$. We noemen deze bijna $\Gamma$-structuren.

In dit proefschrift hebben we gebruik gemaakt van enkele moderne meetkundige gereedschappen om enkele klassieke resultaten over pseudogroepen, (bijna) $\Gamma$-structuren en aanverwante zaken te herzien, te onderzoeken en te generaliseren. Hieronder geven we wat meer uitleg.

\section{Hoofdstuk 1: transitiviteit van pseudogroepen}

We noemen een pseudogroep $\Gamma$ transitief wanneer, gegeven twee punten in de Euclidische ruimte, er een transformatie is in de pseudogroep die hete ene punt naar het andere stuurt. In het eerste hoofdstuk van dit proefschrift onderzoeken we bijna $\Gamma$-structuren waarvoor $\Gamma$ transitief is.

We hebben gebruik gemaakt van hulpmiddelen zoals Lie groepoïden, Pfaffiaanse vormen en het begrip van (Pfaffiaanse) Morita-equivalentie om aan te tonen dat bijna $\Gamma$-structuren gehercodeerd kunnen worden in eenvoudigere (en gemakkelijker te hanteren) objecten; in technische termen hoofdbundels die zijn uitgerust met bepaalde vectorwaardige vormen, die we Cartan bundels noemen.

Ruwweg kan de lezer een groepoïde visualiseren als een verzameling pijlen die beginnen en eindigen op punten van een vaste variëteit. Gegeven een pseudogroep, kan men verschillende groepoïden construeren die eraan verbonden zijn; het idee is dat elke transformatie die tot de pseudogroep behoort en rond een vast punt is gedefinieerd, een pijl bepaalt die gericht is op het beeld van het punt. Dit idee kan op verschillende manieren worden geïmplementeerd. De belangrijkste keuze is hoeveel van de informatie die door een transformatie in de pseudogroep wordt gedragen, moet worden vastgehouden door de corresponderende pijl in de groepoïde (technisch gesproken neemt men ofwel kiem of een $k$-jet, voor een gekozen $k \in \mathbb{N}$, van de transformatie rond het gekozen punt). Om- 
dat onze pseudogroepen zich goed gedragen (ze ontstaan als reeksen oplossingen van goed gedragen partiële differentiaalvergelijkingen), gedragen de groepoïden die men eruit construeert zich ook zeer goed; in het bijzonder zijn het variëteiten, en worden Lie groepoïden genoemd.

De Lie groepoïden opgebouwd uit een pseudogroep zijn over het algemeen eenvoudiger objecten dan de pseudogroep zelf en bevatten minder informatie. In zekere zin wordt (een deel van) de informatie die nodig is om de pseudogroep uit de groepoïde te reconstrueren, opgeslagen in een geometrisch object genaamd een Pfaffiaanse vorm. Het bestuderen van Lie groepoïden uitgerust met Pfaffiaanse vormen - Pfaffiaanse groepoïden genoemd - is een efficiënte manier om pseudogroepen en geometrische structuren te bestuderen. Een belangrijk onderdeel van de machinerie is het begrip Pfaffiaanse Morita equivalentie, een relatie tussen paren van Pfaffiaanse groepoïden.

De belangrijkste opmerking voor ons was dat een transitieve Lie pseudogroepen overeenkomen met Pfaffiaanse groepoïden die Pfaffian Morita equivalent zijn aan zeer eenvoudige objecten (technisch gesproken, Lie groepen uitgerust met twee objecten: een subalgebra van hun Lie algebra, en een representatie die mooi samenwerkt met de subalgebra). Door deze opmerking konden we Cartan-bundels ontdekken als een manier om bijna $\Gamma$-structuren te coderen.

Er zijn twee zeer opmerkelijke voorbeelden van Cartan bundels: abstracte $G$ structuren en Cartan geometrieën. Beide zijn uitgebreid bestudeerd in de literatuur en zijn terug te voeren op het pionierswerk van Élie Cartan.

\section{Hoofdstuk 2: pseudogroepen en foliaties}

Een codimensie $q$ foliatie, $q \in \mathbb{N}, 0 \leq q \leq n$, op een variëteit $M$ van dimensie $n$ is, ruwweg, een partitie van het variëteit in subvariëteiten, genaamd bladeren, van dimensie $n-q$, die lokaal lijkt op de verdeling van de Euclidische ruimte $\mathbb{R}^{n}$ door "parallelle" kopieën van $\mathbb{R}^{n-q}$.

Een equivalente definitie van foliatie in termen van pseudogroepen is gegeven door André Haefliger [36]. De intuïtie achter deze constructie is dat een foliatie altijd enkele lokale symmetrieën heeft, gezien het feit dat we de kopieën van $\mathbb{R}^{n-q}$ in $\mathbb{R}^{n}$ kunnen "herschikken" zonder de foliatie zelf te wijzigen. Intuitief gezien zijn deze herschikkingen transformaties van $\mathbb{R}^{n}$ geïnduceerd door de kopie van $\mathbb{R}^{q}$ loodrecht ten opzichte van de bladeren te verschuiven. Op basis hiervan heeft Haefliger foliaties opnieuw gecodeerd in termen van een algebrogeometrisch object met de naam $\Gamma^{q}$-cocycle; $\Gamma^{q}$ is hier de pseudogroep van alle lokaal gedefinieerde transformaties van $\mathbb{R}^{q}$.

Deze herformulering van het begrip foliatie blijkt nuttig te zijn om bepaalde invarianten van foliatie te bespreken (technisch gezien bepaalde cohomologie classen). In het bijzonder heeft Haefliger een algebraïsch object gebouwd (om precies te zijn, de cohomologie van een bepaald bicomplex) uit de pseudogroep $\Gamma^{q}$, en heeft bewezen dat bekende invarianten van foliaties kunnen worden gegroepeerd in een karakteristieke afbeelding gedefinieerd op dit object.

In het tweede hoofdstuk van dit proefschrift hebben we de constructie van Haefliger besproken en deze niet alleen uitgebreid tot willekeurige Liepseudogroepen, maar ook tot meer algemene objecten: Lie-groepoïden uitgerust met een platte multiplicatieve verbinding. 


\section{Hoofdstuk 3: acties van pseudogroepen}

Als $G$ een groep is, bestaat een action van $G$ op een variëteit $E$ uit een afbeelding die aan elk element van $G$ een transformatie van $M$ associeert op een manier die compatibel is met de groepsstructuur van $G$. Men kan dit begrip uitbreiden tot pseudogroepen. Heel ruwweg is een actie van Gamma op een variëteit $E$ een afbeelding die aan elk element van Gamma een it lokaal gedefinieerde transformatie van $E$ associëren; deze afbeelding is compatibel met de beperking en samenstelling van transformaties in Gamma.

Het blijkt dat dit begrip beter geformuleerd kan worden door de groepoïde $\operatorname{Germ}(\Gamma)$ van kiemen van elementen in $\Gamma$ te beschouwen. Intuïtief, gegeven een element van $\Gamma$ - d.w.z. een lokaal gedefinieerde transformatie - beschrijft de kiem rond een punt het gedrag van de afbeelding in een willekeurig kleine omgeving van het punt. Van de verschillende groepoïden die men aan een pseudogroep kan koppelen, behoudt $\operatorname{Germ}(\Gamma)$ de meeste informatie; het bestuderen van pseudogroepen hetzelfde is als het bestuderen van groepoïden van kiemen.

In het derde hoofdstuk van dit proefschrift hebben we dit gezichtspunt aangenomen. Een eerste interessante observatie is dat acties van een pseudogroep $\Gamma$ equivalent kunnen worden beschreven door $\Gamma$-natuurlijke bundels. Als $\Gamma^{n}$ de pseudogroep is van lokale transformaties van $\mathbb{R}^{n}$, dan hebben we het gewoon over natuurlijke bundels. Natuurlijke bundels zijn terug te voeren op Nijenhuis [53] en zijn uitgebreid bestudeerd.

Een opmerkelijk feit over natuurlijke bundels is de eigenschap van eindige orde. Als we natuurlijke bundels beschouwen als acties van de pseudogroep van lokale transformaties van $\mathbb{R}^{n}$, dan zie je ruwweg dat de actie van een transformatie $\phi$ alleen afhangt van de $k$-de orde Taylor uitbreiding van $\phi$. Dit is de kern van de eindige orde stelling. De eerste versie van een dergelijke stelling werd bewezen door Palais en Terng [54]; iets later, bewezen Epstein en Thurston een sterkere stelling [28].

We hebben ons gericht op de stelling van de eindige orde en het bewijs daarvan, waarbij we ze hebben bekeken vanuit het nieuwe gezichtspunt van acties van groepoïden. Vanuit een dergelijk oogpunt stelt de eindige-ordestelling dat de actie van de kiemgroepoïde $\operatorname{Germ}\left(\Gamma^{n}\right)$ volledig bepaald blijkt te worden door de actie van de jet groepoïde $J^{k} \Gamma^{n}$, voor een $k \in \mathbb{N}$. $J^{k} \Gamma^{n}$ is de groepoïde waarvan de elementen $k$-jets van transformaties op een punt zijn. Ruwweg komen de pijlen in $J^{k} \Gamma^{n}$ overeen met de $k$-de orde Taylor-uitbreidingen van transformaties in $\Gamma^{n}$; als gevolg daarvan is $J^{k} \Gamma^{n}$ een eenvoudiger object dan $\operatorname{Germ}\left(\Gamma^{n}\right)$, aangezien pijlen in $J^{k} \Gamma^{n}$ minder informatie bevatten over elementen van $\Gamma^{n}$ dan pijlen in $\operatorname{Germ}\left(\Gamma^{n}\right)$. Om deze reden is de eindige-ordestelling een opmerkelijk resultaat.

Door het gezichtspunt van groepoïden in te nemen, werd het bestuderen van voor welke pseudogroepen de eindige orde stelling geldt een natuurlijke vervolg. Afgezien van het werpen van een nieuw licht op de aard van het probleem, stelde onze aanpak ons bovendien in staat expliciet enkele interessante inzichten te onderzoeken die impliciet aanwezig zijn in [54] en [28]. 


\title{
Acknowledgments
}

\author{
(I Il personaggio, Serpentieri, me lo molli un po'. \\ - René Ferretti, Boris, prima stagione
}

The reader familiar with Italian television won't fail to notice the enormous influence that René Ferretti and his artistic vision have had on this thesis. However, unlike most of Ferretti's work, this thesis has been greatly improved by many people who have sustained me before and during my Ph.D. in Utrecht. When writing "greatly" and "many", I really mean it; that's the reason why this acknowledgments section is so long.

First and foremost, my advisor, Marius. These four years under your supervision have been one hell of a ride. There have been ups and downs, and I would go through all of them again without thinking. I've greatly enjoyed doing my Ph.D. in Utrecht. I have a lot to thank you for: your guidance, countless discussions (only half of which were about science), teaching me how to do math, believing in me (it really helped), and, of course, allowing me to practice my espresso-making skills. Our communication has not always been perfect, but even the disagreements have taught me a lot, and I know that I am the one to blame for most of them. The last months have been very stressful, and yet you have supported and helped me without fail. And last but not least, thanks for creating such a nice and stimulating environment and for putting together such a wonderful group of people; many of them are mentioned below, and for good reason.

To the official and unofficial members of the reading committee, Christian Blohmann, Ieke Moerdijk, Janez Mrčun, María Amelia Salazar, Xiang Tang, Luca Vitagliano and Chenchang Zhu, thank you for the time and work you have invested in reviewing this thesis; I am aware that it is not an entertaining read.

Special thanks are due to Luca and María. I've appreciated enormously your detailed feedback, the interest that you've shown for my work, and your insightful suggestions. They have greatly improved the final outcome.

I am also very grateful to Ieke Moerdijk and Chengchang Zhu, who have stepped in with very short notice and have saved me from delaying the defense because of bureaucratic reasons.

In fact, dealing with the bureacracy to submit this thesis has felt, at times, like a Kafkaesque experience. I am glad that the committee has made an effort to show the flexibility that neither Utrecht University nor the administrative staff handling the process have shown. 
The nice part of being a younger student in a research group is that there are a lot of people to look up to; Álvaro, Francesco and Lauran, you've definitely been among those people.

Álvaro, you are an explosive combination of a remarkable mathematician and a genuinely joyful person. I've learned a lot from you, and have received great input from your turbolent flow of ideas. You've led me to explore a side of math that is way out of my comfort zone, and that I greatly enjoy. I've always found your style captivating and unique; thanks for all the suggestions you've given to me, I think they improved this thesis quite a bit. Your positive and supportive attitude has been of invaluable help during these four years.

Francesco, we met in Turin many years ago, when you were the teaching assistant of the multvariable calculus course; knowing you has led me to Utrecht, and to the past four years. Thanks for having been such an amazing officemate; it has been a pleasure to disturb the whole eight floor with our random conversations. You have taught me a good chunk of the math I now know; that has evolved into our current collaboration - and into the math that appears in the first chapter of this thesis. You've also given me a lot of precious feedback concerning this thesis, and I'm extremely grateful for that. And, of course, you've always been the person to go to when having doubts about bureaucratic procedures, or when needing advice concerning documents, travel, and all sort of practical things. You've always happily shared your great organizational skills, and I truly think that our group should build a statue in your honor for that.

Lauran, you are a kind and generous person, and a very smart guy. Your way of looking at math, and at everything else, has been of great inspiration. Thanks for taking the time to teach me a lot of cool math, many things about living in the Netherlands, and even how to properly ride a racing bike. You're the coolest, and I'm very happy that our paths have crossed. And thanks for listening to my endless - and often pointless - complaints about things, and for always being up for a chat.

While doing a Ph.D., being part of a research group with many fellow students is an unvaluable experience. Aldo, Maarten, Florian and Dušan, I'm really glad that we've shared this experience together.

Aldo, my Ph.D. started and finished in an office with you. We've made it together, despite all the attempts made by Utrecht University to prevent our graduation. Thanks for all the questions you have helped me to answer, but even more for all the questions that you have asked me. Even though we did not always agree, I have learned a lot from our discussions about politics and life, and they have been an engaging distraction when I needed a break from math. I greatly admire your "idealistic" attitude, and your will to fight and live according to your own ideals. And, of course, I'll be forever grateful to you for helping me with the dutch translation of the summary of this thesis.

Maarten, you started a little while after me as a Ph.D. student, but you have been part of Marius' group since before I arrived in Utrecht, and I've got to learn way more from you than you from me. Your precise and crystal clear way of doing math has always impressed me, and I have strived to put a little bit of that in what I have done in these four years. Thanks for getting me involved in the seminar on equivariant cohomology; I have greatly enjoyed preparing it, and it 
has taught me a lot of stuff, part of which appears here and there in this thesis. And thanks for all the discussions about academia and the future, which have become more and more frequent with the date of my defense approaching; they have been clarifying and comforting, albeit in their own uncomfortable way.

Dušan, even though you were not one of Marius' students, you've always been part of the eight floor group. We both are night owls, and it has been nice to have someone to talk to during my late night working on the eight floor. Thanks for all the mathematical discussions you've had with me; they've helped me greatly with the third chapter.

Florian, you were a student of Ionuţ in Nijmengen, but you have always been part of our group in Utrecht too. We have spent some good time together - at schools and conferences, but also during our free time - and I have greatly enjoyed your company in the last four years.

Chiara and Sergej: there are not enough words to express how happy I am that we have met each other, and how grateful I am that you have decided to "adopt" me. All those dinners together (for which I will forever be in debt with you!) have kept me sane through some difficult moments.

Chiara, you are one of a kind, and a true force of nature. Your explosive attitude never fails to make me smile, and your enthusiatic way of looking at things is truly contagious. I had great fun during our shopping trips to the Ikea and the Action, and I hope that there are many more to come!

Sergej, you're a clever mathematician and a good friend. I have enjoyed our seminar with Maarten and learned a lot from it. Thanks for all the videogames we've played together, and for that nice kayak trip! During the last period of my Ph.D., having lunch together always gave me the shot of energy that I needed to go through the day.

Davide, you graduated when I was still halfway through, yet we have spent quite some good time together, and I've always appreciated your easy going attitude and your guidance. You offered me a place to stay when I first came to Utrecht, explained to me what the van Est theorem is about, and gave me advice about all sort of things. Thanks for all of that, and for the decisive help that you've offered me during my job hunt.

Anto, you brought some amazing energy to the math department. Thanks for all the dinners we've had together right after the first lockdown due to the pandemic; they have been a much needed breath of fresh air.

There are some more experienced colleagues that have to be thanked for all the genuine interest that they have shown in my work; more than once, that has given me the boost of confidence that I was in need of.

Ivan, we met when my Ph.D. was barely started; I think I looked like a rather different person back then. I greatly enjoyed your Caipirinha (and I think you and Ori remember that quite well!), and all the chances we've had to interact during these four years. Thanks for the amazing project you have asked me and Mateus to be part of, it's cool math and I really like it.

María, even though I already thanked you as a member of the reading committee, I'd like to do it one more time. Thanks for all the discussions that we 
had while you were staying in Utrecht, and for listening to me when I was full of doubts.

Luca, I want to thank you twice as well. Thanks for all the feedback you've given me about the paper with Marius on Haefliger's cohomology, and for explaining to me many things about the way academia works.

There are some other members of Marius' extended group who have contributed to make my time in Utrecht a beautiful experience, with either a nice bike ride, help with some practical matter, an illuminating mathematical discussion, a beer together during a conference, a place to sleep or just a pleasant chat at the right moment. Thank you Arjen, Bas, Gil, Lukas, Ionuţ, João, Kirsten, Ori, Ralph and Roy.

All the Ph.D. students and postdocs at the math department deserve a huge "thank you" for making the environment so nice and welcoming. In particular, thank you Carlo, Giacomo, Harry, Jack, Jan-Willem, Jetze, Joost, Mar, Mireja, Shuntaro, Stefano and Yuqing for all the nice conversations we've had together.

To all the students of the 2019 Poisson geometry course and the 2020 Morse theory seminar: teaching to you has been a pleasure and an experience from which I have learned a whole lot myself. Your enthusiastic engagement has been a pleasure to see, and your deep questions have been a pleasure to receive, and very instructive to think about; you truly have taught me the material a second time.

Nina: thank you also for all those discussions we had - and the kind advice that you offered me - about some very delicate topics.

I want to thank the administrative staff of the math department too: you've always been ready to help, and with the friendliest of attitudes. I've always felt welcomed to drop by at your offices and ask for assistance, whatever the issue.

A special thank goes to all the contributors on math.stackexchange, mathoverflow and tex.stackexchange, who answered countless mathematical doubts and greatly improved the way this thesis looks like. In particular, the code that produces the "inspirational quotes" in this thesis has been posted by the user moewe on tex. stackexchange.

I had (had I?) a life before my Ph.D.; during these four years, the support I got from some of the people from back then has been amazing.

Marcella: you have believed in me like no one else ever since you were my Master's advisor, and that has boosted my confidence enough to get me through my Ph.D.. Thanks for all the work you've put into our papers together, and for all the interesting workshops you have organized and invited me to.

Daniele, Davide, Eric, Giulio, Luca and Simone: I'm very happy that our friendship did not end after our graduation in Turin. It's safe to say that this thesis would not be done, at least not in this form, without our chat group and our collective skype calls. They kept me sane-and able to do some work - during the first lockdown due to the pandemic.

Simone (the older one), it has been of great help to have a friend that could understand the experience of doing a Ph.D. in a foreign country, even though we feel somewhat different about our rather similar paths. Thanks for hosting me in 
Denmark after my thesis was done, I was really in need of decompressing a bit. I enjoyed my time there, and I hope the same is true for you and the time you spent at my place in The Netherlands.

Marco, there would have been no Ph.D. without a Bachelor's degree, and we both know that you're the guy to thank for my Bachelor's degree. Going back to those fun memories that we often recall together always puts a smile on my face.

Andrea, the story of our friendship has been full of twists; I'm happy that we have found our stable equilibrium. During my Ph.D., I have always been happy when receiving your (long!) vocal messages, which filled me with beautiful memories. Thanks for your friendship, and for all the support and advice you've given me during these four years.

Simone (the younger one): our Studio Ghibli movie marathon made it much easier to get through the lockdown in the spring 2020. During the last four years you've been a very good friend; thanks for listening to me whenever I needed someone to talk to.

Of course, and here I want to shift to Italian: grazie alla mia famiglia per il supporto infinito che mi ha dato.

Mamma e papà, grazie per avermi fatto fare quello che volevo, sempre sostenendomi al massimo, per essere stati sempre presenti per tutto e per avermi fatto crescere in un ambiente sereno, dove ho potuto capire quello che mi andava di fare e farlo senza pensieri. Mamma, grazie per confrontarti con me su tutte quelle questioni pratiche che sono essenziali per sopravvivere con un tetto sopra la testa. Papà, grazie per aver condiviso con me tante passioni che mi porto dietro tuttora, e che hanno reso più semplici questi quattro anni.

Michela, non c'è brutto pensiero di cui non mi dimentichi quando passo del tempo con te, e con Gian. Hai sempre avuto un modo unico di farmi sentire orgoglioso di me stesso, e questo è stato davvero prezioso durante il mio dottorato.

Un grazie enorme ai miei nonni, Franco e Maria, Alberto e Gianfranca; il vostro modo di sostenermi e credere in me mi ha spronato a dare il massimo.

Sara, confrontarmi con te, in merito al mio dottorato ed a molte altre cose, mi ha sempre dato grande serenità e uno sguardo più lucido sulle cose; spero lo stesso valga per te. Grazie per essere venuta a visitarmi ad Utrecht, mi ha reso molto felice.

Grazie ai miei zii - Mauro, Paolo, e Stefania - e ai miei altri tre cugini - Andrea, Elena, e Matteo - per avermi fatto sentire a casa durante le mie (sempre lunghe!) vacanze in Italia.

Un grazie speciale a mio zio Mauro: i nostri giri in bici sono stati una bella, e fondamentale, distrazione dal lavoro.

And, finally, Lucrezia. If I had to say all I want to say right now, this already huge section would get as big as Zeus the cat during winter. And, at the same time, I'm not sure I can find the necessary words. Despite me being me, we've been close since way before I started my Ph.D., and I owe to your friendship and support as much as I owe to myself. During these four years, I've often looked for your help, and you've never failed to comfort me and point me to the right direction. Thanks for all the precious time we've spent together, and all the beautiful memories; I'm looking forward to building new ones. 

O squillo acuto, o spirito possente,

che passi in alto e gridi, che ti segua!

Ma questo è il Fine, è l'Oceàno, il Niente...

e il canto passa ed oltre noi dilegua.

- Giovanni Pascoli, Alexandros 



\section{Curriculum Vitae}

Luca Accornero was born in Vercelli, Italy, on May 13, 1993, son to Gabriele and Simona. He has a little sister, Michela. During his childhood and adolescence, he lived in the italian village of Tronzano Vercellese. He completed high school in 2012 at Liceo Scientifico "Amedeo Avogadro" in Vercelli.

From 2012 to 2017 he attended the University of Torino, completing cum laude a Bachelor's track in Mathematics and a Master's track in Mathematics with focus on mathematical physics and differential geometry. In 2016 he lived at Collegio Universitario Renato Einaudi in Torino. In 2019 he was awarded a prize by the University of Torino for the best Master's thesis in Mathematics from the cohort 2016-2017.

In 2017 he moved to Utrecht, the Netherlands, and started a Ph.D. in differential geometry under the supervision of Prof. Marius Crainic. He defended his thesis, titled "Topics on Lie pseudogroups", on September 29, 2021. 